\title{
REDUCING BARRIERS TO THE USE OF HIGH-EFFICIENCY LIGHTING SYSTEMS
}

Final Report

January 22, 2001 through December 31, 2005

Prepared by

Peter Morante

July 2006

DE-FC26-01NT40986

Lighting Research Center

Rensselaer Polytechnic Institute

21 Union Street

Troy, NY 12180

518-687-7100

518-687-7120 (fax) 


\section{DISCLAIMER}

This report was prepared as an account of work sponsored by an agency of the United States Government. Neither the United States Government nor any agency thereof, nor any of their employees, makes any warranty, express or implied, or assumes any legal liability or responsibility for the accuracy, completeness, or usefulness of any information, apparatus, product, or process disclosed, or represents that its use would not infringe privately owned rights. Reference herein to any specific commercial product, process, or service by trade name, trademark, manufacturer or otherwise does not necessarily constitute or imply its endorsement, recommendation, or favoring by the United States Government or any agency thereof. The views and opinions of the authors expressed herein do not necessarily state or reflect those of the United States Government or any agency thereof. 


\section{ABSTRACT}

With funding from the U.S. Department of Energy (DOE), the Lighting Research Center (LRC) at Rensselaer Polytechnic Institute completed the four-year research project, Reducing Barriers to the Use of High-Efficiency Lighting Systems. The initial objectives were: (1) identifying barriers to widespread penetration of lighting controls in commercial/industrial (C/I) applications that employ fluorescent lamp technologies, and (2) making recommendations to overcome these barriers. The addition of a fourth year expanded the original project objectives to include an examination of the impact on fluorescent lamps from dimming utilizing different lamp electrode heating and dimming ratios. The scope of the project was narrowed to identify barriers to the penetration of lighting controls into commercial-industrial (C/I) applications that employ fluorescent lamp technologies, and to recommend means for overcoming these barriers.

Working with lighting manufacturers, specifiers, and installers, the project identified technological and marketing barriers to the widespread use of lighting controls, specifically automatic-off controls, occupancy sensors, photosensors, dimming systems, communication protocols and load-shedding ballasts. The primary barriers identified include cost effectiveness of lighting controls to the building owner, lack of standard communication protocols to allow different part of the control system to communicate effectively, and installation and commissioning issues.

Overcoming the identified barriers requires lighting control products on the market to achieve three main goals:

1. Achieve sufficient functionality to meet the key requirements of their main market.

2. Allow significant cost reduction compared to current market standard systems. Cost should consider: hardware capital cost including wiring, design time required by the specifier and the control system manufacturer, installation time required by the electrician, and commissioning time and remedial time required by the electrician and end user.

3. Minimize ongoing perceived overhead costs and inconvenience to the end user, or in other words, systems should be simple to understand and use.

In addition, we believe that no lighting controls solution is effective or acceptable unless it contributes to, or does not compromise, the following goals:

- Productivity - Planning, installation, commissioning, maintenance, and use of controls should not decrease business productivity;

- Energy savings - Lighting controls should save significant amounts of energy and money in relation to the expense involved in using them (acceptable payback period); and/or...

- $\quad$ Reduced power demand - Society as a whole should benefit from the lowered demand for expensive power and for more natural resources.

Discussions of technology barriers and developments are insufficient by themselves to achieve higher penetration of lighting controls in the market place. Technology transfer efforts must play a key role in gaining market acceptance. The LRC developed a technology transfer model to better understand what actions are required and by whom to move any technology toward full market acceptance. 


\section{TABLE OF CONTENTS}

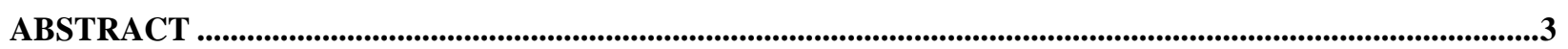

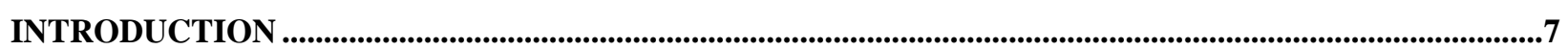

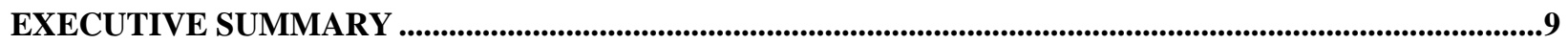

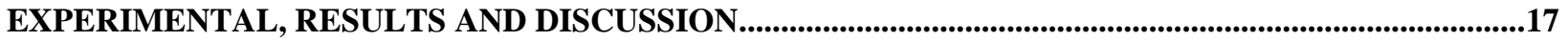

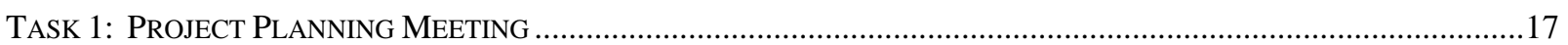

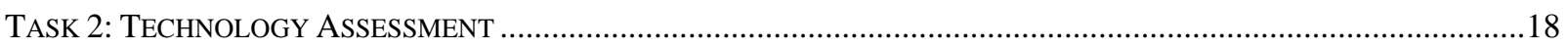

Task 2.1: Conduct searches and reviews of latest technical and patent literature related to photosensor, occupancy sensor, and dimming fluorescent lamp ballast technology and communications systems protocols..18

Task 2.2: Identify current controls manufacturers (occupancy sensors, photosensors, dimming electronic ballasts, and appropriate building control systems) and obtain up-to-date data on relevant new and existing technologies.

Task 2.3: Develop a list of these manufacturers and their control products (ballasts, photosensors, occupancy sensors, energy management systems) and which protocols they offer..............................................................21

Task 2.4: Obtain samples and data sheets for relevant products and technologies where necessary.................21

Task 2.5: Conduct exploratory investigations and analysis of operational parameters required for each of the control technologies (occupancy sensors, photosensors, and dimming electronic ballasts) in common commercial and industrial applications, such as private and open offices and warehouses. ............................21

Task 2.6: Protocol utilization share in commercial lighting applications ..........................................................24

Task 2.7: Review the types of whole building communication protocols used in the US including, but not limited

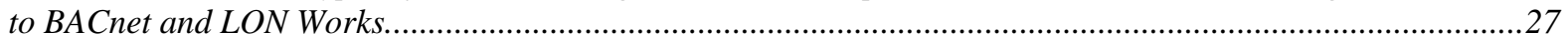

Task 2.8: Investigate to what extent the whole building communication protocols are currently integrated with

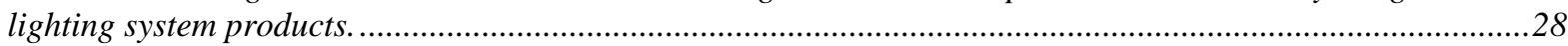

Task 2.9: Investigate strengths and limitations of each protocol as they apply to lighting system performance 30 Task 2.10: Interview contractors and installers to determine installation and commissioning issues, such as times and costs, and to identify other possible technology, market and application barriers to use of the control technologies under investigation (occupancy sensors, photosensors, dimming electronic ballasts) .....

Task 2.11: Interview key control manufacturers (occupancy sensors, photosensors, dimming electronic ballasts, and appropriate building control systems) to get their inputs regarding the information and analysis conducted.

Task 2.12: Meet with lamp manufacturers to get their inputs on lamp operating parameters that are key to maintaining performance when used with control technologies.

Task 2.13: Catalog any solutions that have been recommended for improving lighting and whole building control protocols.

Task 2.14: Develop draft performance criteria for the control technologies (occupancy sensor, photosensor, dimming ballasts) for selected commercial and industrial applications.....

Task 2.15: Summarize current state of the art for each component evaluated above; elucidate the technical and market barriers for each with an overall synopsis of potential opportunities; develop a peer review group consisting of industry leaders and authorities.

TASK 3: IDENTIFICATION OF MANUFACTURING PARTNERS ……................................................................5

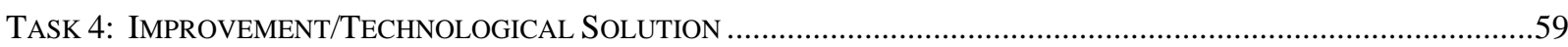

Task 4.1: Work with key manufacturers, industry associations, government agencies and National Laboratories to expand the best existing communications protocols, if necessary, to meet the needs of a fully integrated lighting and whole building control protocol.....

Task 4.2: Investigate ideal lamp light output dimming ratio required for different types of applications ..........62

Tasks 4.3, 4.4, and 4.5: Investigation of the effects of dimming on fluorescent lamp life ...... . .67 
Task 4.6: Develop recommendations for improved components and systems as evaluated in Task 2, based on defined performance characteristics, and defined acquisition, installation and commissioning costs .

Task 4.7: Provide technical foundation for market transformation groups to increase penetration of automatic shutoff controls.

Task 4.8: Provide technical foundation on behalf of manufacturers to overcome nagging installation barriers

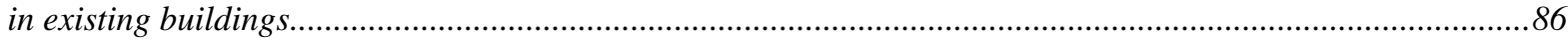

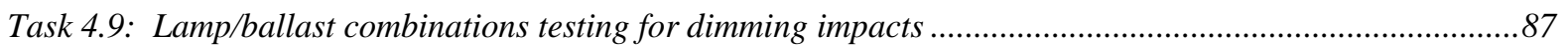

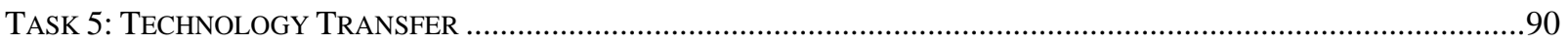

NATIONAL Lighting PRODUCT INFORMATION PROGRAM (NLPIP) ……...........................................................96

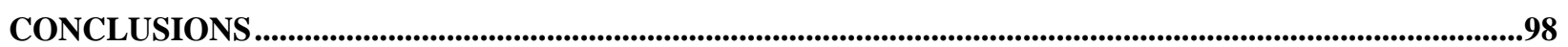

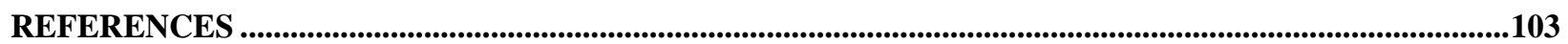

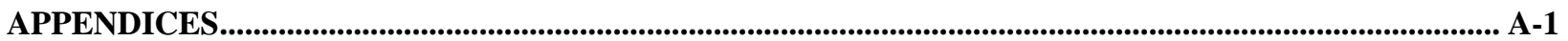

APPENDIX A: REPORT: CONDUCT SEARCHES AND REVIEWS OF LATEST TECHNICAL AND PATENT LITERATURE RELATED TO PHOTOSENSOR, OCCUPANCY SENSOR, AND DIMMING FLUORESCENT LAMP BALLAST TECHNOLOGY AND

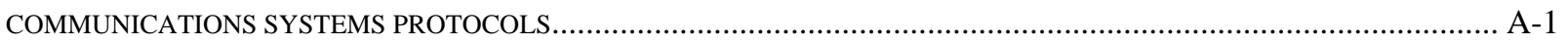

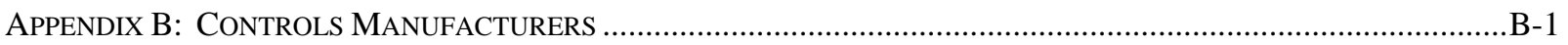

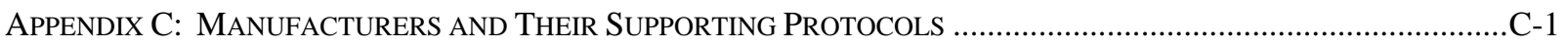

APPENDIX D: INVESTIGATIONS AND ANALYSIS OF OPERATIONAL PARAMETERS REQUIRED FOR EACH OF THE CONTROL TECHNOLOGIES (OCCUPANCY SENSORS, PHOTOSENSORS, DIMMING ELECTRONIC BALLASTS) IN COMMON

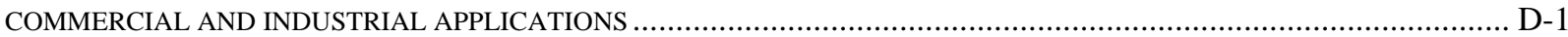

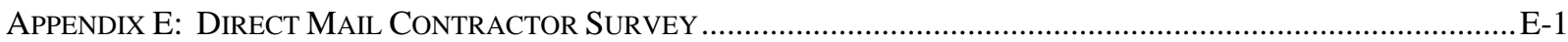

APPENDIX F: TECHNICAL AND MARKET BARRIERS TO FULL IMPLEMENTATION OF LOAD SHED BALLASTS,

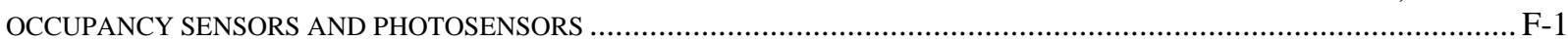

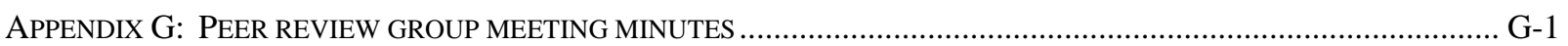

APPENDIX H: COMMUNICATION PROTOCOLS TO MEET THE NEEDS OF A FULLY INTEGRATED LIGHTING AND WHOLE BUILDING CONTROL PROTOCOL ..................................................................................................................

APPENDIX I: ENERGY SAVINGS FOR LOAD-SHEDDING BALLAST FOR FLUORESCENT LIGHTING SYSTEMS ................... I-1

APPENDIX J: THE EFFECT OF BIAS TOWARDS ENERGY SAVINGS ON OCCUPANTS' DIMMING REQUIREMENTS ..............J-1

APPENDIX K: INVESTIGATION OF THE EFFECTS OF DIMMING ON FLUORESCENT LAMP LIFE ................................ K-1

APPENDIX L: REDUCING BARRIERS TO THE USE OF LIGHTING CONTROLS; PROBLEMS ENCOUNTERED DURING

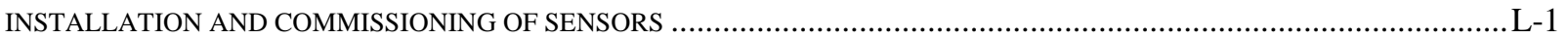

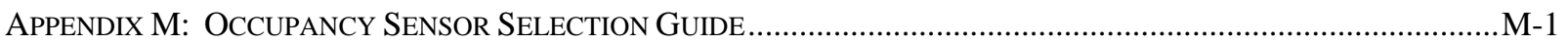

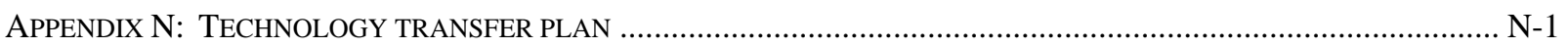

Appendix O: Association of Energy Service Professionals PreSENTATION ............................................. O-1

APPENDIX P: ASSOCIATION OF ENERGY SERVICE PROFESSIONALS, PAPER ....................................................... P-1

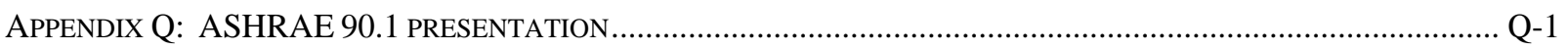

APPENDIX R: IESNA LIGHTING CONTROL PROTOCOL SUBCOMMITTEE PRESENTATION ….......................................R-1

APPENDIX S: PEAK LOAD MANAGEMENT ALLIANCE PRESENTATION ……...................................................... S-1

APPENDIX T: HUMAN FACTORS RESEARCH PRESENTATION, IESNA CONFERENCE .................................................T-1 


\section{FIGURES}

Figure 1. Potential versus distance for cold cathode and hot cathode discharges (60 Hz operation)..........................69

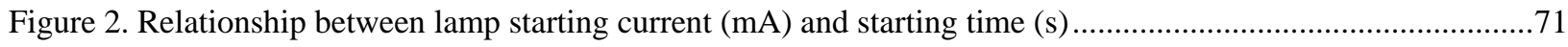

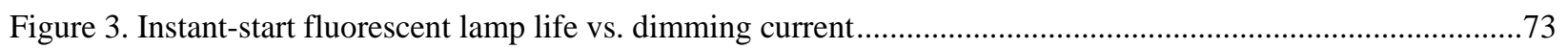

Figure 4. Dimming lamp/Ballast Compatibility Experimental Design.................................................................8

Figure 5. Lamp/dimming ballast compatibility life testing results (5,000 hours) ........................................................89

Figure 6. Technology transfer model: identification of key players and activities ..................................................90

Figure 7. Technology transfer model: transformation mechanisms...........................................................................91

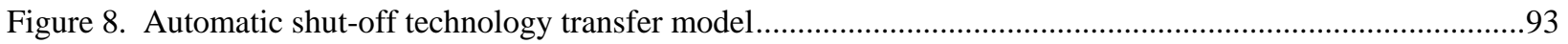

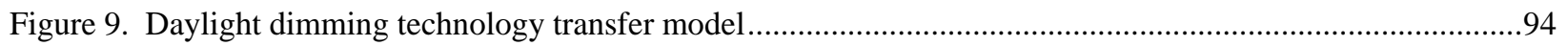

Figure 10. Load management technology transfer model........................................................................................95

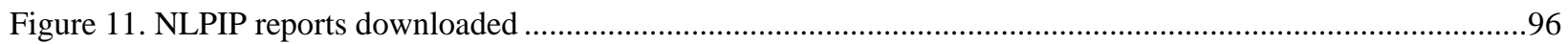

\section{TABLES}

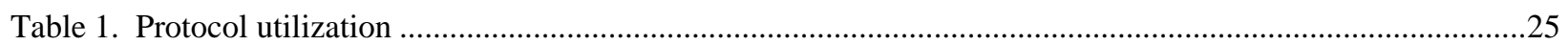

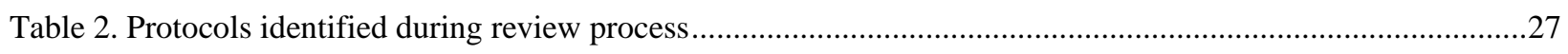

Table 3. Summary of lighting control manufacturers and the communication protocol supported............................29

Table 4. Building Control protocols: Lamp-ballast control techniques (protocols)......................................................32

Table 5. Key features and applications for individually addressable luminaires ${ }^{\mathrm{A}}$...................................................61

Table 6. Recommended energy saving values for occupancy sensors.....................................................................86 


\section{INTRODUCTION}

With funding from the U.S. Department of Energy (DOE), the Lighting Research Center (LRC) at Rensselaer Polytechnic Institute completed the four-year research project, Reducing Barriers to the Use of High-Efficiency Lighting Systems. The initial objectives were: (1) identifying barriers to widespread penetration of lighting controls in commercial/industrial (C/I) applications that employ fluorescent lamp technologies, and (2) making recommendations to overcome these barriers. The addition of a fourth year expanded the original project objectives to include an examination of the impact on fluorescent lamps from dimming utilizing different lamp electrode heating and dimming ratios.

Over the first year of the project (2001/2002), the LRC focused on identifying the barriers to the widespread use of lighting controls. These findings are presented as deliverables to Tasks 2.12.15. The LRC reviewed existing research, technologies, patents, and market data related to lighting controls, interviewed manufacturers, surveyed control installers, and conducted a peer group review to improve and validate a proposed set of recommendations. The LRC identified two distinct categories of effort, each with their own set of barriers: dimming controls (load-shed dimming and photosensor-activated daylight dimming) and automatic shut-off controls (occupancy sensors and time clocks). ${ }^{1}$ Participating experts in lighting agreed that the tasks associated with the latter category should be geared towards market transformation activities while the tasks to be performed under the first category should be focused on technological investigations and solutions.

The second-year scope of work focused on identifying and proposing solutions for each of the categories identified during the first year. ${ }^{1}$ In the category dealing with automatic shutoff controls, two tasks were undertaken. The first of these two tasks (Task 4.7) was directed toward developing fixed values for energy savings from occupant sensors so that state and regional agencies in the U.S. could use these values in their market transformation activities. Based upon an extensive review and analysis of the literature, the LRC developed fixed values for energy savings. The second of the two tasks (Task 4.8) was directed at developing a best-practice document that helps both manufacturers and installers improve the likelihood of installing and commissioning occupancy sensors. In the category dealing with dimming, which represented the majority of the effort for the second year, the focus was on investigating and proposing specific technological parameters for product improvement.

The LRC identified manufacturing partners to begin discussion about these technological solutions (e.g., development of load-shedding ballast) and conducted limited testing. The LRC focused on economic, perceptional (human), and technical issues associated with whole-building and local control strategies, and undertook five tasks. In addition to developing a review of the progress in whole-building control systems (Task 4.1), great progress continues to be made in developing communication systems for lighting control systems. Problems still exist, however, with standardization and cost. These systems have developed their own momentum and only time will tell when the U.S. market embraces whole-building lighting control systems.

\footnotetext{
${ }^{1}$ Architectural dimming is excluded from this project.
} 
Neglected in most discussions of lighting controls is the lamp-ballast system used. Task 4.1 through Task 4.5 concentrated on understanding lamp-ballast performance issues, which we believe must be at the core of developing any successful fluorescent lighting control system for $\mathrm{C} / \mathrm{I}$ applications. The major issues to consider are occupant response, initial equipment cost, and lamp life. Heating the electrodes prolongs lamp life by minimizing the damaging effects of sputtering from both switching and dimming. These systems are commonly three to five times more expensive than systems that do not provide heat to the electrode. Without question, these systems reduce lamp life due to sputtering from starting and dimming. Interestingly however, a non-linear relationship exists between operating current and life. The technical and economic issues associated with dimming instant start systems was a central focus for the latter part of this project.

Finally, the LRC reviewed the significance of dimming for occupants and has made recommendations for integrating them with lamp-ballast system performance. Task 4.6, develops recommendations for improved components and systems as evaluated in Task 2 (technology assessment) and integrates all of the information on Tasks 4.1 to 4.5.

The findings and conclusions from Years 1 and 2 became the basis for developing technology transfer plans during Year 3. A technology transfer model was developed to help the industry better understand where each technology currently rests, what is required to move market acceptance of the technology forward, and who the players are in these efforts (Task 5.1). Outreach efforts were successfully pursued with many papers, articles, presentations and seminars completed discussing the findings of this project (Tasks 5.2 and 5.3). Finally, a dedicated Web site was developed and launched. Currently this Web site resides on the LRC server. However, the LRC has the ability to transfer this Web site to the DOE upon request.

An additional sub-task (Task 4.9) was added during 2004, which extended the project until its end date of December 31, 2005. The purpose of this task was to determine the interaction and compatibility between fluorescent lamp electrode heating and differing lamp-operating currents (dimming) in order to provide the foundation for industry standards for lamps and dimming ballasts to ensure compatibility. The results of the dimming and lamp life studies are continuing outside the scope of this project with assistance from lamp and ballast manufacturers.

An additional inclusion of the Reducing Barriers to the Use of High-Efficiency Lighting Systems project was the sponsorship of the National Lighting Product Information Program (NLPIP) for one year. This program, with multiple sponsors, helps lighting professionals, contractors, designers, building managers, homeowners and other consumers find and effectively use efficient, quality products to meet their lighting needs. NLPIP disseminates objective, accurate, timely, manufacturer-specific information about energy-efficient lighting products.

A summary of the project is presented below with supporting materials included as appendices. Each task and sub-task is presented with findings, conclusions and, if appropriate, recommendations for overcoming barriers. 


\section{EXECUTIVE SUMMARY}

With funding from the U.S. Department of Energy (DOE), the Lighting Research Center (LRC) at Rensselaer Polytechnic Institute completed the four-year research project, Reducing Barriers to the Use of High-Efficiency Lighting Systems. The initial objectives were: (1) identifying barriers to widespread penetration of lighting controls in commercial/industrial (C/I) applications that employ fluorescent lamp technologies, and (2) making recommendations to overcome these barriers. The addition of a fourth year expanded the original project objectives to include an examination of the impact on fluorescent lamps from dimming utilizing different lamp electrode heating and dimming ratios. With the agreement of DOE, the focus of the project was narrowed to identify barriers to the penetration of lighting controls into commercial-industrial (C/I) applications that employ fluorescent lamp technologies, and to recommend means for overcoming these barriers. This agreement was based upon the fact that C/I applications are the largest market segment and are dominated by fluorescent technologies. Moreover, "static" fluorescent technologies, such as T8 electronic ballast fluorescent systems, were well established in $\mathrm{C} / \mathrm{I}$ installations and did not deserve special attention under this contract. Therefore, the meeting participants agreed to concentrate efforts on occupancy/motion sensors, photosensors used in conjunction with daylighting and dimming ballasts, and communication of control signals.

As readers examine this report, they must remember that many of these findings are now three and four years old. Much has occurred during this time within the lighting and lighting controls industry to overcome the barriers identified within this report. Occupancy sensors are now included in energy codes and standards, photosensor technology has moved to the digital age and a manufacturer has produced a load-shedding ballast for fluorescent lighting. However, the wide acceptance of lighting controls other than automatic shutoff remains at minimal levels

Marketing and technology efforts still have to be made for daylighting and its controls and the compatibility of fluorescent lamps and dimming ballasts.

The findings of this research were prepared with the assistance of and review by lighting manufacturers, specifiers, and installers. Their participation ensured the validity of this report. A review of the patents in a particular technology area is one method of assessing its status in terms of technological achievement. Counting and categorizing patents provides a good indication of the amount of economic investment and activity in a given technology area. Of the 272 patents found regarding lighting controls and ballast technologies, only 26 were for occupancy sensors and four were for photosensors. This indicates a low interest in the development of these technologies.

Lighting controls can be divided into local, central, and two-way controls. Local controls allow users to control light levels in their working area. Central controls turn the lights on or off in large areas, such as whole buildings, whole floors, or large sections of an open plan area. The status of the lighting is not known. For a two-way control system, the ballast sends and receives information to/from a centralized computer and information about the status of the lighting can be determined.

Communicating with lighting controls requires software protocols. There are many communication protocols in existence. All existing communication protocols require a separate set of communication wiring. This requirement adds cost to lighting control systems and is a 
to control, lighting requires communication with essentially each lighting fixture creating thousands of communication points within a building. Most of the communication protocols used by lighting equipment use systems developed for the HVAC industry like BACnet and LONWorks.

Lighting control systems require immediate response times, the ability to detect if occupants are still active in a space, and significantly more control points. Naturally, lighting control systems need to be sensitive to these differences.

\section{For a lighting control system to operate successfully the system must:}

- Minimize command strings aggregating control points

- Warn occupants of lighting system changes

- $\quad$ Sense delay override commands

- Interact with other sensors and controls within the office environment

These requirements often exclude existing building automation system protocols from being used to control the lighting because they have slow response speeds, cannot control many points, and if they did, they would be prohibitively expensive.

With the plethora of communication protocols in use and different manufacturers' equipment utilizing different protocols, it is difficult to develop a whole system approach to lighting controls where all components can talk to each other. The development of the digital addressable lighting interface (DALI) by the lighting industry should overcome this issue.

Through a direct-mail survey, lighting control installation contractors confirmed overall what the industry already knew regarding the barriers to the use of lighting controls. Two significant results were quite clear:

- The potential market for occupancy sensors, photosensors, and dimming electronic ballasts is much greater than the present market. Installers were willing, across the board, to say that they could install far more controls than they do if there were no barriers to market penetration.

- The universally cited barrier to market penetration was lack of customer demand, as expected. Although not directly asked, installers responded that they had difficulty proving that customers would see the payback from lighting controls.

The results of this survey seemed to show that customers must first be convinced that lighting controls will benefit their bottom line. If that happens, little else remains in the way of their widespread use.

Discussions with lighting manufacturers developed four categories of barriers that have been identified that include several barriers within each category: lamp-ballast compatibility, component compatibility, installation/commissioning assurance, and marketing. Penetration of lighting controls for fluorescent systems into C/I applications will not be simple or rapid. Indeed, to reach significant penetration into the market a cultural change will have to occur in the lighting industry. This cultural change can be characterized as developing effective communications among all stakeholders in electric energy efficiency and load management, 
particularly those charged with the responsibility of establishing rational social policies for power generation and transmission.

The minimum acceptable requirements for lighting controls are that they:

- Provide sufficient light for the users of a space-after all, we can achieve perfect control by simply turning out all the lights in a building, but that is not a productive solution.

- Avoid waste - that is, turn off lights when no one is there to benefit from them (except for security, display, etc.).

- Reduce lighting levels without compromising occupants' satisfaction and productivity.

In addition, no lighting controls solution is effective or acceptable unless it contributes to, or does not compromise, the following goals:

- Productivity - Planning, installation, commissioning, maintenance, and use of controls should not decrease business productivity;

- Energy savings - Lighting controls should save significant amounts of energy and money in relation to the expense involved in using them (acceptable payback period); and/or...

- Reduced power demand - Society as a whole should benefit from the lowered demand for expensive power and for more natural resources.

In general, technological problems pose relatively low market barriers for lighting controls. A failure to show reasonable payback periods and other economic benefits, however, limits the acceptance of controls in the marketplace. Without greater market demand for lighting controls, manufacturers have little incentive to eliminate the remaining, albeit small, technological problems.

Overcoming the identified barriers requires lighting control products on the market to achieve three main goals:

- Achieve sufficient functionality to meet the key requirements of their main market.

- Allow a significant cost reduction compared to current market standard systems. Cost should take into account: hardware capital cost including wiring, design time required by the specifier and the control system manufacturer, installation time required from the electrician, and commissioning time and remedial time required from the electrician and end user.

- Minimize ongoing perceived overhead costs and inconvenience to the end user, or in other words, systems should be simple to understand and use.

In the course of Task 2 of this project, three technologies were identified as having the potential to achieve greater market share if certain barriers could be overcome. These technologies were the prototyped load-shedding ballast, occupancy sensors, and an innovative, easily commissioned photosensor. To maximize their market potential, these technologies must all demonstrate their cost-effectiveness, their practicality in real installations, and their suitability to the market. Based on subsequent development of these technologies and analysis of the market, we recommend the following courses of action: 


\section{Load-shed ballasts:}

- Perform large-scale field trials of load-shedding ballasts. These trials should aim to verify the acceptability of illuminance reductions to occupants, both when the reasons for load shedding have been explained to occupants, and when they have not. The process of informing occupants of the environmental and cost benefits of load shedding is referred to as "biasing".

- Test the electromagnetic compatibility (EMC) of load-shed ballasts in their two normal operating states (i.e., full on and dimmed), and when switching between states.

- Work with one or more ballast manufacturers to produce a commercially viable load-shed ballast based on electronic instant-start technology. To be financially attractive to customers, the price of this ballast should be no more than $\$ 9$ more than a regular instantstart ballast.

- Test the prototype ballasts as part of the LRC's National Lighting Product Information Program (NLPIP), and make results of the testing available on the NLPIP website.

- Work with one or more ballast manufacturers to develop a suitable method to carry signals from a central controller within the building to the individual ballasts ("stage 2" signaling). This signal should not cause disruption (e.g., flicker) to the other lighting in the building.

- Ensure that the signals sent out by electricity suppliers are suitable to be received by lighting load-shed controllers ("stage 1" signaling). Both the format and the medium for these signals should be considered in detail.

- Develop a performance specification for load-shedding ballasts that can be easily followed by commercial ballast manufacturers, yet ensures minimum performance standards.

- Leverage current and future market forces to create demand for load-shedding ballasts. This can be achieved by promoting the electricity cost savings achieved using loadshedding ballasts, and by promoting load-shedding ballasts as an environmentally friendly and therefore desirable feature of a building, especially new builds. This may take place through published articles, seminars, demonstration projects and voluntary accreditation schemes.

\section{Occupancy sensors:}

- Publicize the aggregate figures for energy savings compiled as part of Task 4. Everyone in the building industry should be aware of typical figures for energy savings achievable by the use of occupancy sensors.

- Work with control system manufacturers to produce a commissioning standard for occupancy sensors. If all sensors can be commissioned using the same procedure, the likelihood of installers making mistakes on site will be reduced, and these mistakes account for many of the failings of systems in situ. 


\section{Easily commissioned photosensor:}

- Continue to use existing market channels, such as the LRC’s NLPIP program and outreach education, to support the use of photosensing in those applications for which it is commercially viable. This is usually only in owner-occupied buildings with large window area and high occupation density.

A major barrier to the use of dimming systems and the potential energy savings through the use of daylighting is the potential of reduced lamp life when fluorescent lamps are used in dimming systems. To better understand the technical issues associated with fluorescent lamps and dimming, the LRC along with the National Electrical Manufactures Association has undertaken a research effort to develop industry standards for lamps and dimming ballasts to ensure their compatibility.

Initial results of the life test at 5,000 hours of operation indicate early lamp failures with low lamp current and low cathode heating.

Discussions of technology barriers and developments are insufficient by themselves to achieve higher penetrations of lighting controls in the market place. Technology transfer efforts must play a key role in getting market acceptance. The LRC developed the following technology transfer model to better understand what actions are required and by whom to move any technology toward full market acceptance.

\section{Technology Transfer Model: Identification of Key Players and Activities}

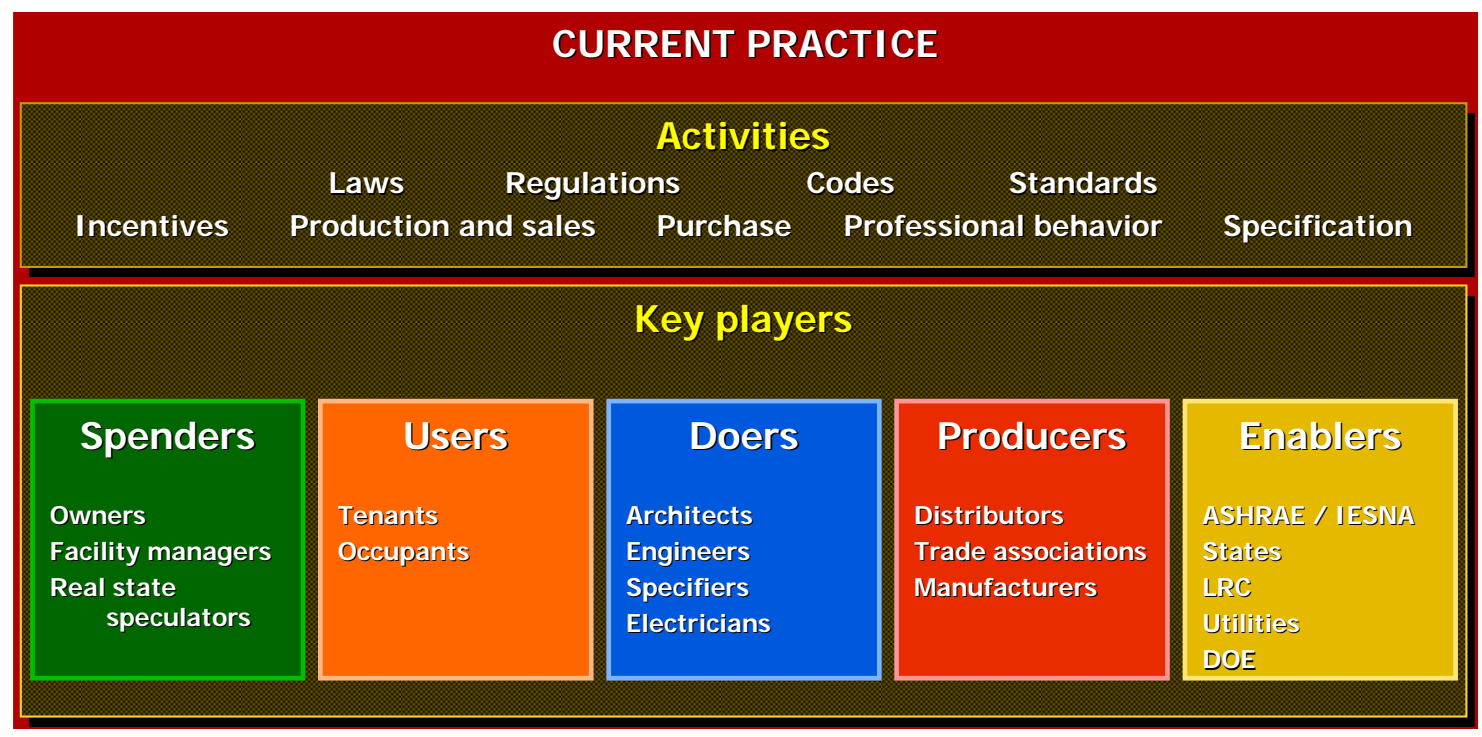

Key players include:

- $\quad$ Spenders - These are building owners, facility managers, real estate speculators and developers. They make the ultimate buying decision but are influenced by the other key players and what they read and see in different types of communication outlets.

- Users - These are the ultimate users of the technologies such as building tenants and occupants. They must be satisfied with the technology's performance and the ability to deliver its claims. 
- Doers - These are the architects, consulting engineers, specifiers and installers of the technology. They have great influence over the spenders. When doers recommend or install a technology, they risk their reputation on whether a product will perform as advertised.

- Producers - These people make and distribute the technology and their respective associations/organizations. They must have a reliable and marketable technology that will earn profits for the company. Besides the key role of manufacturing the technology, they act as marketer.

- Enablers - These are the organizations that write laws, regulations, codes and/or standards such as state and federal governments, i.e., American Society of Heating, Refrigeration and Air Conditioning Engineers (ASHRAE), National Electrical Manufacturers Association (NEMA) or Illuminating Engineering Society of North America (IESNA). Also included are organizations that influence law, regulation, and code and/or are standard writers such as Natural Resources Defense Council (NRDC) or American Council for an Energy Efficiency Environment (ACEEE). Enablers are also represented by organizations such as the Lighting Research Center who have relationships and partnerships with all of the key players within an industry and are able to bring these dissimilar types of players together in collaborative and mutually beneficial efforts to perform technology transfer successfully. Also included with this grouping are utilities and state/regional organizations such as Xcel Energy and the Northwest Energy Efficiency Alliance, which are charged with the responsibility for implementation of energy efficiency programs.

\section{Technology Transfer Model: Transformation Mechanisms}

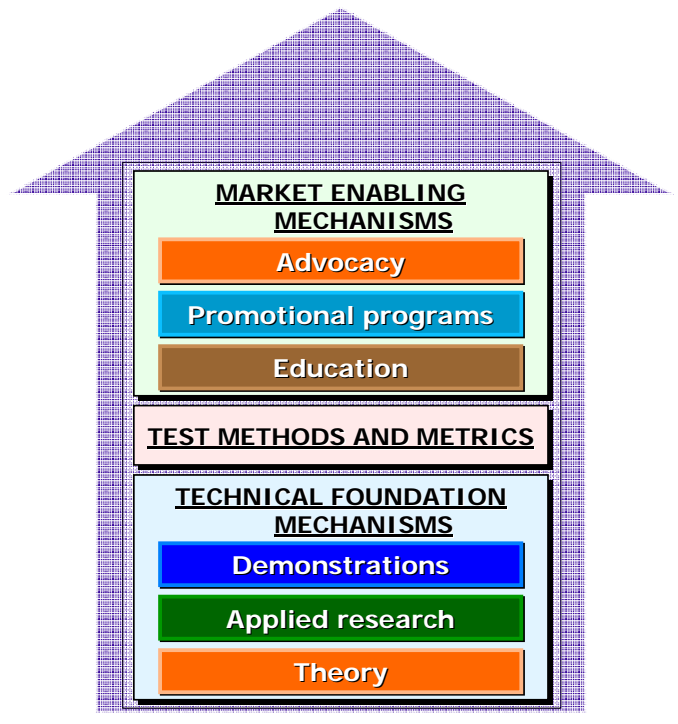

The second phase of the model outlines the transformation mechanisms in a methodical, step-bystep process. This process addresses the development, demonstration, evaluation, commercialization, and, finally, customer acceptance of a technology. The successful achievement of each step's goals is a prerequisite to moving onto the next step. Failure to meet 
these goals or an attempt to skip a step reduces the chances of the technology being accepted by the user. The mechanisms include:

- Theory - This mechanism identifies a possible technology to satisfy an unfulfilled customer or user need. The technology becomes defined and researchers determine if the technology is achievable at some reasonable cost to the user. While research organizations like the LRC and other producers are the primary players for this mechanism, input from and funding to the other key players is mandatory.

- Applied Research - This mechanism must prove the technical and economic concepts of the technology through technical and market research. Prototypes are developed as part of meeting this step's goals. Again, close collaboration with all key players is necessary as the research or manufacturing organizations endeavor to meet the goals of this mechanism. As one of its goals, the applied research mechanism identifies manufacturers willing to commercialize the technology and begin performing the necessary engineering for manufacturing.

- Demonstrations - Full-scale demonstrations are necessary to "show the world" the technology works and delivers the promised benefits. The demonstrations must answer any questions or concerns raised by users or spenders during the market research phase. The proof must be of the technology and its economics as applied to an actual, rather than laboratory, situation. Successful demonstrations with multiple locations or situations increase the acceptance of the technology. Funding and defining the measured metrics for this phase must come from other key players.

- Test Methods and Metrics - The method and metrics on which the evaluation is conducted of a technology, especially during the demonstration step, must be acceptable and agreed to by the research community as well as all the key players. Test results must withstand the rigors of review. While this mechanism may seem intuitive, it may be the most important step of all transformation mechanisms. It sets the stage as to how believable the claims made by the technology will be to the spenders, users, doers, producers, and enablers and, ultimately, how successful the technology will be in the marketplace. It also provides a uniform means to measure results utilizing the same technology in different labs and facilities.

- Education - The education mechanism is meant to be broad in scope to include the necessity to educate all the key players about the benefits, abilities, and limitations of the technology and why it should be adopted in the marketplace, i.e., what it will do to help society. This is the first step in bringing the technology to the market. Yet, many times organizations responsible for technology development do not view the educational function as part of their responsibilities. Funding for education should be included as part of any technology development or else the technology may never advance to the market.

- Promotional Programs - If the rigors of the test methods and metrics are met and if a successful education program is funded and executed, then, and only then, will the producers, enablers, utilities and state/regional agencies charged with the conduct of energy efficiency programs promote the use of the technology to the spenders, doers and other users. Promotional programs can take many forms such as utility incentive programs or endorsements. 
- Advocacy - This is the final step in the technology transfer model but does not occur until after the technology has reached some level of acceptance within the marketplace. It only occurs when there is public consensus of the use and benefits of the technology. Advocates then request that the benefits of the technology are included in regulations, codes and/or standards. 


\section{EXPERIMENTAL, RESULTS AND DISCUSSION}

\section{Task 1: Project Planning Meeting}

A kick off meting was held on December 13, 2000. The meeting was held at the Lighting Research Center in Troy, NY. Dr. Jim Brodrick and Mr. Ron Lewis from DOE, Mr. Ed Petrow a consultant to DOE and Dr. Mark Rea, Dr. Victor Roberts, Mr. Andrew Bierman, Mr. Russ Leslie, Mr. Dan Frering, Ms. Dorene Maniccia, and Ms. Mariana Figueiro of the LRC participated. This meeting set the stage for the project's objectives and deliverables.

The outcome of this meeting was agreement among all parties that the focus of this project would be to identify barriers to the penetration of lighting controls into commercial-industrial (C/I) applications that employ fluorescent lamp technologies, and to recommend means for overcoming these barriers. This agreement was based upon the fact that $\mathrm{C} / \mathrm{I}$ applications are the largest market segment and are dominated by fluorescent technologies. Moreover, "static" fluorescent technologies, such as T8 electronic ballast fluorescent systems, were well established in $\mathrm{C} / \mathrm{I}$ installations and did not deserve special attention under this contract. Therefore, the meeting participants agreed to concentrate efforts on occupancy/motion sensors, photosensors used in conjunction with daylighting and dimming ballasts and communication of control signals. As the project began, peak electric load reduction became an important topic for discussion because the real cost of electric power is highly dependent upon the time-of-day when the power is required. Lighting controls for fluorescent systems were quickly seen as potentially important technologies for load management, because electric load for lighting can be modulated more quickly and with less disruption to occupants than other electric technologies. Consequently, the advisory group agreed that efforts under this contract to establish load-shed strategies and technologies for fluorescent systems were appropriate.

Tasks 2 and 3 were discussed in detail, since these two tasks were assigned for the first year of the project. All participants agreed on the scope of work to be performed during the first year of the project as outlined below.

Task 2. Technology Assessment (photosensor, occupancy sensor, dimming ballast, control systems)

- Market assessment

- Technology assessment

- Identify barriers limiting use and application of components and systems

- Develop draft performance criteria

- Develop peer review group

Task 3. Identify Manufacturing Partners

- Obtain commitment for participation

- Conduct a technical and market assessment review of the information obtained in task 2

- Develop, plan and host a roundtable to review activities conducted during the first year of the project 


\section{TASK 2: Technology Assessment}

\section{Task 2.1: Conduct searches and reviews of latest technical and patent literature related to photosensor, occupancy sensor, and dimming fluorescent lamp ballast technology and communications systems protocols.}

A review of the patents in a particular technology area is one method of assessing its status in terms of technological achievement. Counting and categorizing patents provides a good indication of the amount of economic investment and activity in a given technology area. Patents cost money, including both application fees and the implied costs of defending one's patent rights when challenged by others. Therefore, we would expect to find that the areas of technology that are viewed in the business world as offering the greatest economic growth or activity would have the greatest amount of patent activity associated with them.

With this in mind, a U.S. patent search was conducted in the technology area of lighting controls and fluorescent lamp ballasts. The purpose of the search was two-fold: to seek out newly developed lighting technology that looks promising for increasing the efficiency of lighting systems, and to get a better understanding of technological state of the lighting controls and ballast industry.

\section{Search Results}

Two hundred seventy-one patents were found and deemed germane to the topic. By far, most of the patents found are concerned with ballast technology; 172 of the 271 patents and this despite limiting the search for ballast companies and ballast key words to only the past three years. These 172 patents were categorized into the following groups:

- 133 dealt with lamp operation, including, but not limited to efficiency, safety (with respect to the newer, small diameter T-5 fluorescent lamps), power quality, starting, lamp life, and novel circuits and materials.

- 35 dealt with ballast control, including, but not limited to dimming, addressing, and on/off power schemes involving control elements within the ballast.

- 4 dealt with mechanical fixtures for ballasts and electrical connectors. This category was not eliminated because of the possibility that better connectors and fastening systems could help reduce installation costs; a well know barrier to introducing new more efficient technology.

The remaining 111 patents were concerned with lighting controls. This group was categorized into the following groups and subgroups.

- 61 dealt with lighting control components

o 22 on dimming and/or switching including panel-level control among others

o 26 on occupancy sensors

o on photosensors 
o on combined sensor technology (e.g., combining a photosensor with an occupancy sensor)

o on window treatments (all having same assignee)

o 1 on time clocks

- 30 dealt with energy management systems including, but not limited to home automation products and schemes, control networks and methods of communication.

- 8 patents were categorized as design patents. These patents concerned the embodiment of a specific product. For example, some of these patents were on the physical shape of a switch or dimming knob used in a wall dimmer.

\section{Analysis}

The fact that most of the found patents concern ballast technology indicates that this technology area has received far more economic attention then other areas involving lighting controls. A relatively small number of the ballast patents, 35 , appeared from their abstracts to deal directly with control issues such as dimming, addressing, and the integration of control logic into the ballast. Most of the patents in this category deal with specific circuits for dimming fluorescent lamps. The few that are concerned with addressing and the integration of control logic into the ballast tend to be represented in these results by smaller companies such as Energy Savings, Inc. and JRS Technology, Inc. Examples of this technology include ballasts with microprocessors that receive control signals and perform control functions to switch-off or reduce lamp power, and a "modular" ballast design that allows it to receive many different types of control signals from sensors and controllers.

Looking at the 111 patents concerned directly with lighting controls, it is again obvious where most of the economic attention has been within this area; energy management systems and panellevel controls, followed closely by occupancy sensor components. Only a total of 13 patents were found on photosensors, combined sensor technology, window treatments and time clocks combined. The lack of patents in these lesser-used control areas signifies that there is not much investment in these areas, economic or research. Occupancy sensing, manual dimming, and panel-level switching seem to be the types of controls most actively pursued by the lighting industry.

The occupancy sensor patents are concerned with circuit and sensing improvements and added features such as lower power consumption, two-wire installation, and use as security system components. The photosensor patents include a scheme for semiautomatic calibration, a dualphotosensor feedback system, a skylight photosensor system and a circuit for using photoconductive cells as linear detectors. The patents that describe combining different sensors most often combine a photosensor with an occupancy sensor. One adds a dimmer and a thermostat to the combination. One patent describes a method to control lights from a remote desktop device.

The category of patents on dimming and switching can be further divided into three groups; one dealing with incandescent type dimming (e.g., phase chopping or triac dimming circuits), one concerning panel, or multi-zone controls and one concerning dimming of fluorescent lamps. Lutron Electronics Company has many patents in all these areas. Some of the panel-level and 
multi-zone control patents are closely related to the building energy management system patents and refer to their use in building automation systems.

Of the 30 patents dealing with energy management systems, eight have the word "home" in the titles and are not directly relevant to the commercial and industrial marketplace. Assignees of these patents include names such as Microsoft, IntelliNet, Inc., and Samsung Electronics Co., Ltd. Nevertheless, as far as the base technology is concerned, the technology and concepts used in home systems could likely be used in commercial and industrial settings as well. It is interesting that, because the home is a different market, it involves different companies than those traditionally associated with building control. The influx of new players in the building automation industry portends continued innovation in this area.

Of the eight patents concerning the physical communication layer, three employ power line carrier schemes and one employs a dedicated fiber optic carrier. A patent on "distributed intelligence,” assigned to Echelon Corporation, is also included in this group.

The appearance of only one patent dealing with communication protocols seems at first contrary to expectations. Patents that deal with communication methods and protocols in general are beyond the scope of this search, even though they might be applicable to building automation. On the other hand, the lack of patents found in this area having to do with lighting controls might be a reflection of the trend within the controls industry to gravitate towards "open systems" that use existing, open communication protocols.

The above patent search provides an indication that additional research and commercialization efforts are required for photosensor and communication protocol technologies associated with lighting controls. Both technologies offer opportunities to improve the efficiency of lighting systems.

The full report of the patent search findings, including a listing of all 271 germane patents can be found in Appendix A: Report; Conduct searches and reviews of latest technical and patent literature related to photosensor, occupancy sensor, and dimming fluorescent lamp ballast technology and communications systems protocols.

\section{Task 2.2: Identify current controls manufacturers (occupancy sensors, photosensors, dimming electronic ballasts, and appropriate building control systems) and obtain up-to-date data on relevant new and existing technologies.}

A search for current control manufacturers produced a list of 108 entities. These companies range in size from General Electric, OSRAM Sylvania to very small companies such as Blackbird. Many of the nations building systems control companies such as Honeywell and Johnson Controls are included on the list, as well as, companies that specialize in just lighting controls such as WattStopper and SensorSwitch. Some of the manufacturers produce multiple parts of the lighting control systems such as ballasts, photosensors and communication protocols such as Lutron. In fact, Lutron may be the only company to produce all necessary components to make a fully operational lighting control system. However, Lutron does not manufacture the fluorescent lamps that are utilized with their systems.

The decentralization of the lighting controls industry with different manufacturers producing different parts of the control system can be perceived as a barrier. With a dimming ballast from 
manufacturer A, a photosensor from manufacturer B, fluorescent lamps from manufacturer $\mathrm{C}$ and the building automation system from manufacturer $\mathrm{D}$, who does the buyer turn to if issues arise. In the eyes of the buyer, dealing with multiple manufacturers is a serious issue and can be considered a barrier to the use of lighting controls to improve the efficiency of lighting systems.

The full listing of controls manufacturers can be found in Appendix B: Controls Manufacturers.

\section{Task 2.3: Develop a list of these manufacturers and their control products (ballasts, photosensors, occupancy sensors, energy management systems) and which protocols they offer.}

Many software protocols are utilized by lighting control manufacturers. They can be subdivided into two categories: open protocol where any manufacturer can use the software at no cost to the manufacturer, and proprietary protocols which require a license to use. The most common open protocols used by the lighting industry are BACNet, DMX-512, LONWorks, X-10 and digital addressable lighting interface (DALI) — a system specifically created for lighting controls of different manufacturers to be able to talk with each other. The most common proprietary protocols for lighting controls are developed by control manufacturers such as Lutron, GE, and Leviton. A listing of lighting control manufacturers and which communication protocols they support are included in Appendix C: Manufacturers and Their Supporting Protocols.

All existing communication protocols require a separate set of communication wiring. This requirement adds cost to lighting control systems and is a barrier to their use. Unlike heating and air conditioning systems where there are only a few units to control, lighting requires communication with essentially each lighting fixture creating thousands of communication points within a building.

With the plethora of communication protocols in use and different manufacturers' equipment utilizing different protocols, it is difficult to develop a whole system approach to lighting controls where all components can talk to each other. The development of DALI by the lighting industry should overcome this issue.

\section{Task 2.4: Obtain samples and data sheets for relevant products and technologies where necessary.}

Two binders containing the data sheets for relevant products and technologies were submitted to Ron Lewis, Program Manager at USDOE, email: ronald.lewis@hq.doe.gov, on May 22, 2001.

Please refer to these binders for information.

Task 2.5: Conduct exploratory investigations and analysis of operational parameters required for each of the control technologies (occupancy sensors, photosensors, and dimming electronic ballasts) in common commercial and industrial applications, such as private and open offices and warehouses.

A convenient way of analyzing control devices is to consider a device as a system of inputs and outputs. Inputs to control devices are usually sensors and commissioning set points, and the 
outputs are control signals that govern the operation of the controlled equipment. Once the inputs and outputs are identified, then the operation of the control device is described by how the inputs affect the outputs. The input/output relationships can be either logical relationships, continuous functional relationships, or a combination of the two. This type of analysis is effective for both small, local control systems such as an occupancy sensor in a room, and for large, distributed control systems where the outputs of devices such as occupancy and photosensors are used as inputs to higher-level building automation control systems. In fact, communication protocols for building automation systems, such as BACnet and LonWorks, are specified in terms of inputs and outputs. This report focuses on occupancy sensors, photosensors, and dimming ballasts, describing them in terms of inputs and outputs.

\section{Occupancy sensors}

- Available sensor inputs:

o Passive infrared (PIR)

o Ultrasonic

o Acoustic

- Other inputs:

o Sensitivity adjustments (for PIR and ultrasonic)

o Time delay

- Outputs:

o On/off power relay

o Bi-level switching on/off power

o Low voltage logic signal (to be used a an input for another controller)

There are advantages and disadvantages to each input and output combinations. Some save additional energy while others are easier to install and calibrate. The user must decide which options are most important for their application.

\section{Photosensors}

- Available sensor inputs:

o Illuminance (wide field of view)

o Luminance (narrow field of view)

- Other inputs:

o Sensitivity (gain)

o Light level set point

o Hysteresis (dead band)

- Outputs:

o On/off power relay 


\section{o Bi-level switching on/off power \\ o Continuous dimming level (e.g. 0-10V)}

The input to a photosensor is optical radiation. Loosely speaking, the input is light, but because some photosensors respond to infrared (IR) and ultraviolet (UV) radiation as well, it is necessary to make a distinction between optical radiation that is visible light and other kinds of optical radiation. The response of a photosensor to optical radiation is fully described by the spatial response and the spectral response.

The spatial response describes the sensitivity of the photosensor to incident radiation from different directions - in other words, what the photosensor "sees" at different locations. Spatial response is analogous to a luminaire intensity distribution, but describes sensitivity instead of output.

The spectral response describes the sensitivity of the photosensor to optical radiation of different wavelengths. This is important because only a small part of the optical radiation spectrum is visible. Daylight and fluorescent lighting differ substantially in spectral composition. Daylight has a comparatively uniform distribution of energy over the near-UV, visible, and near-IR regions of the spectrum. Fluorescent lamps, on the other hand, have most of their output concentrated in the region of the spectrum where visual sensitivity is high. This is one reason fluorescent lighting is so efficient. Even though the exact spectrum of daylight changes depending on weather conditions, times of the day and season, as well as being affected by surrounding buildings and foliage, these differences are small compared to the relative differences in UV and IR content between daylight and fluorescent light sources. The greater UV and IR content of daylight, combined with the broader than ideal spectral response of most photosensors, makes most photosensors much more sensitive to daylight than to light from fluorescent lamps. A greater sensitivity means that a photosensor will respond as if more daylight were present than actually exists. This can lead to problems where precise switching or dimming levels need to be realized.

Illuminance sensors (wide field of view) - The signal produced from illuminance type sensors is useful for detecting ambient light levels. A wide spatial response corresponds closely to what an illuminance meter would measure.

Luminance sensors (narrow filed of view) - Not as common as illuminance type sensors, luminance sensors detect light from a particular direction and over a small field of view. They are used to detect brightness from a distant location; for example, to detect desktop luminance from a mounting position in the ceiling. 


\section{Dimming Ballasts}

- Available inputs:
o 1-10V analog signal
$0<0.5$ volt standby signal
o Phase chop angle power line signal
o Digital control interfaces (DALI and SuperDim)

- Outputs:
o Lamp power level
o Ballast status (e.g., lamp failure for DALI equipped ballasts)

Control inputs to ballasts are divided into analog and digital categories. Within each of these categories, different signaling protocols and/or conventions are used. Digital control inputs to ballasts have only recently been introduced on the market and currently comprise a very small market share. There is, however, considerable interest in and backing by different ballast manufacturers for the DALI communication protocol for ballasts. (For a detailed listing of the strengths and weaknesses of the different ballast control interfaces see Task 2.9 below)

Currently, analog control interfaces for ballasts are the most widely available with 0-10V control interfaces being most common. The $0-10 \mathrm{~V}$ interface was the first to be used when dimming electronic ballasts appeared on the market in the early 1990s. The control scheme itself dates back to the early 1970s where it originated in the theatrical lighting controls industry. In fact ANSI has recently approved a 0-10V standard for entertainment technology (Standard E1.32000). However, the implementation of $0-10 \mathrm{~V}$ control in ballasts is different from that used in theatrical controls. ANSI Standard E 1.3-2000 even specifically states that the standard does not apply to fluorescent dimming ballasts. Ballast manufacturers themselves have not adopted a standard for commercial use at this time and it seems likely at this time that none will ever come about. As a result, consistent behavior across different ballasts types or manufacturers is not assured.

A full report regarding the investigations and analysis of operational parameters of occupancy sensors, photosensors and dimming electronic ballasts can be found in Appendix D: Investigations and analysis of operational parameters required for each of the control technologies (occupancy sensors, photosensors, dimming electronic ballasts) in common commercial and industrial applications.

\section{Task 2.6: Protocol utilization share in commercial lighting applications}

Lighting controls can be divided into local, central and two-way controls. Local controls allow users to control light levels in their working area. Central controls turn the lights on or off in large areas, such as whole buildings, whole floors, or large sections of an open plan area. The status of the lighting is not known. For a two-way control system, the ballast sends and receives information to/from a centralized computer and information about the status of the lighting can be determined. 
There is no information available on the percentage of local, central, or two-way control installations in commercial/industrial buildings, but the last is certainly in the extreme minority. In addition, many buildings can have more than one type of control installation.

It is also very difficult to separate lighting control hardware and software when classifying lighting controls systems. A lighting control system protocol always implies a combination of hardware and software. Some types of hardware or software may be common to two different protocols, or the same hardware may be linked to two different types of software. Below is an estimation of the percentage of utilization of different protocols based on market observation and manufacturers' literature. The "trend" reflects the possible increase, decrease, or stability of the market penetration of a technology. These trends need to be put into perspective, however. According to lighting controls manufacturers, the market penetration of all lighting controls is approximately $2 \%$.

Table 1. Protocol utilization

\begin{tabular}{|c|c|c|c|}
\hline Protocol & \% Estimate & Trend & Comments \\
\hline Ethernet & $80 \%$ & Increase & $\begin{array}{l}\text { A majority of control companies offer } \\
\text { some means of interfacing with } \\
\text { computer LANs to share information } \\
\text { or allow for remote utilization of the } \\
\text { system. } \\
\text { As technology evolves, companies } \\
\text { will use the Ethernet protocol closer } \\
\text { to the device level replacing }\end{array}$ \\
\hline RS-232 & $90 \%$ & Flat & $\begin{array}{l}\text { Used by most companies as a } \\
\text { method to connect peripheral } \\
\text { devices, the majority of control } \\
\text { systems provide RS232 connection } \\
\text { at some point in their system. Due to } \\
\text { distance limitations, protocol is not } \\
\text { used for panel-to-panel connectivity. }\end{array}$ \\
\hline USB & $5 \%$ & increase & $\begin{array}{l}\text { Due to changes in the computer } \\
\text { industry, USB may replace RS-232 in } \\
\text { the future }\end{array}$ \\
\hline BACnet & $65 \%$ & Increase & $\begin{array}{l}\text { Appears to be gaining momentum as } \\
\text { the protocol of choice for } \\
\text { communication between } \\
\text { subsystems. }\end{array}$ \\
\hline LonMark -LonWork & $35 \%$ & $\begin{array}{l}\text { Flat to } \\
\text { increase }\end{array}$ & $\begin{array}{l}\text { Difficult to gauge trend. Although } \\
\text { most companies have invested in the } \\
\text { development of systems, few lighting } \\
\text { related companies actually } \\
\text { introduced products. Widespread } \\
\text { acceptance has been limited due to } \\
\text { silicon costs and commissioning } \\
\text { requirements. }\end{array}$ \\
\hline
\end{tabular}




\begin{tabular}{|c|c|c|c|}
\hline $\begin{array}{l}\text { Twisted Pair } \\
\text { RS-422 } \\
\text { RS-485 }\end{array}$ & $99 \%$ & $\begin{array}{l}\text { Flat to } \\
\text { decline }\end{array}$ & $\begin{array}{l}\text { These protocols are defined primarily by } \\
\text { their hardware characteristics. 2-wire } \\
\text { communication methods are the } \\
\text { dominant means to carry custom } \\
\text { protocols. Although marked as declining } \\
\text { it will be perhaps a few decades before } \\
\text { the market truly changes }\end{array}$ \\
\hline $0-10 \mathrm{~V}$ & $85 \%$ & $\begin{array}{l}\text { Flat to } \\
\text { increase }\end{array}$ & $\begin{array}{l}\text { The leading open protocol for } \\
\text { dimming fluorescent lamp ballast. } \\
\text { Most all architectural lighting control } \\
\text { providers offer a connection to } \\
\text { control 0-10Volt }\end{array}$ \\
\hline DMX-512 & $99 \%$ & Flat & $\begin{array}{l}\text { Control manufacturers participating } \\
\text { in the theatrical lighting control } \\
\text { segment implement DMX-512 }\end{array}$ \\
\hline Phase control & $30 \%$ & Increase & $\begin{array}{l}\text { Gaining momentum as the method of } \\
\text { choice for lighting control retrofit } \\
\text { applications and new installations } \\
\text { were simple fluorescent lamp } \\
\text { dimming control is required }\end{array}$ \\
\hline$X-10$ & $5 \%-$ & Flat & $\begin{array}{l}\text { Once seen as the technology with } \\
\text { the most potential (early } 80 \text { 's). The } \\
\text { X-10 powerline carrier technology } \\
\text { protocol had difficulty maintaining } \\
\text { momentum due to reliability issues } \\
\text { with noise interference. X-10 is still } \\
\text { used in residential and limited } \\
\text { commercial applications. }\end{array}$ \\
\hline ESI- SuperDIM & & & $\begin{array}{l}\text { Digital fluorescent lamp ballast } \\
\text { protocol recently introduced in the } \\
\text { market. To soon to predict the } \\
\text { acceptance level. }\end{array}$ \\
\hline DALI & & & $\begin{array}{l}\text { Digital fluorescent lamp ballast } \\
\text { protocol recently introduced in the } \\
\text { market. To soon to predict the } \\
\text { acceptance level in the US. Protocol } \\
\text { has been adopted as a IEC standard } \\
\text { for Europe and has the support of top } \\
\text { ballast manufacturers. }\end{array}$ \\
\hline
\end{tabular}




\begin{tabular}{|l|l|}
\hline Protocol & Comments \\
\hline ALC & \\
MODBUS & The large installed base of control equipment in both the HVAC and Lighting \\
Honeywell & segments with companies to continue offering devices or bridges to existing \\
Johnson Controls N2 & protocols. \\
ETC & \\
Leviton & \\
LUTRON & Of the custom protocols listed Johnson Controls-N2 and Seimens-Modbus \\
Lightolier Controls & have the largest market share within the HVAC Segment and Lutron has the \\
Watt Stopper / Horton & largest market share within the Lighting segment. \\
& \\
\end{tabular}

\section{Task 2.7: Review the types of whole building communication protocols used in the US including, but not limited to BACnet and LON Works.}

There is a plethora of communication protocols used within the building controls market. Every control system manufacturer had proprietary protocols prior to the late 1980's. As open protocols emerged, most companies started to support them.

It is important to note that a majority of the protocols identified here did not meet the project criteria [commercial/industrial (C/I), fluorescent lighting systems] and therefore were not included in the evaluation. A significant number of protocols are focused on the residential control and home electronics market. Only protocols specific to commercial lighting controls are discussed.

Proprietary protocol information was not available for review; however, due to the market share controlled by the many manufacturers we included them in the following list.

Table 2. Protocols identified during review process

Open Protocols: Residential and Commercial

\begin{tabular}{|l|l|l|l|}
\hline 1394ta & 5Ghz IAG & AHAM & BACnet \\
\hline Bluetooth & CableLabs & CEBus & DSL Forum \\
\hline DALI & EIB & ETI Alliance & Ethernet \\
\hline Konnex & HiperLAN2 & HAVi & HomeCNA \\
\hline HomePlug & Home Plug \& Play & HomePNA & HomeRF \\
\hline Jini & Jetsend & LonMark -LonWork & OSGi \\
\hline OFDM Forum & PLC & R7.1-R7.5 & RS-232 \\
\hline RS-485 & SC25/WG1 & Salutation & SCP \\
\hline SIA & SNMP & TCP/IP & Twisted Pair \\
\hline UPnP & WAP Forum & WECA & WLANA \\
\hline WGNA & & & \\
\hline
\end{tabular}


Lighting Related: Residential and Commercial

\begin{tabular}{|l|l|l|l|}
\hline $0-10 \mathrm{~V}$ & ACN & DMX-512 & ESI- SuperDIM \\
\hline DALI & $\mathrm{X}-10$ & & \\
\hline
\end{tabular}

Communication protocols meeting project requirements:

Building Control Communication Protocols (higher level):

OPEN

\begin{tabular}{|l|l|l|l|}
\hline BACnet & LonWorks & SNMP & Ethernet \\
\hline
\end{tabular}

PROPRIETARY

\begin{tabular}{|l|l|l|l|}
\hline ALC & Johnson Controls N2 & Honeywell & MODBUS \\
\hline
\end{tabular}

Lighting Communication Protocols (higher level):

OPEN

\begin{tabular}{|l|l|l|l|l|l|}
\hline $\begin{array}{l}\text { Advanced Control } \\
\text { Network }(\mathrm{ACN})\end{array}$ & DMX512 & 0-10V & X10 & DALI & ESI superDIM \\
\hline
\end{tabular}

PROPRIETARY

\begin{tabular}{|l|l|l|l|}
\hline GE & Douglas Controls & Horton/Watt Stopper & Ledalite \\
\hline Leviton & Lightolier Controls & LUTRON & $\mathrm{PCl}$ \\
\hline Sensorswitch & & & \\
\hline
\end{tabular}

It is important to point out that a majority of the C/I communication protocols used today for whole building communications evolved with a HVAC applications. Heating and cooling systems involve occupant control (feedback) combined with central building control. Lighting controls for the most part however, are usually only locally controlled loads using wall switches and control relays. Dimming control of fluorescent fixtures is usually confined to executive offices, conference rooms, and classrooms.

Task 2.8: Investigate to what extent the whole building communication protocols are currently integrated with lighting system products.

LON works and BACnet have the most support from the lighting control manufacturers. Devices range from protocol translators through control devices fitted to support the protocol directly. Proprietary systems from Johnson Controls, Honeywell and Modicon have the second largest following.

Ethernet should receive mention as the dominating computer network protocol. Several controls manufacturers have devices that connect directly to Ethernet. 
Table 3. Summary of lighting control manufacturers and the communication protocol supported

\begin{tabular}{|c|c|c|}
\hline Item & Company Name & Supporting Protocol \\
\hline 1 & ABB Control Inc. & Custom, BACnet, Modicon and LON \\
\hline 2 & Advance Transformer & 0-10VDC, 2-wire \\
\hline 3 & Advanced Control Technologies Inc & LonWorks Independent Developer \\
\hline 4 & Agilent Technologies & LAN/WAN Ethernet \\
\hline 5 & Alerton Technologies, Inc. & BACnet, Modbus, Ethernet \\
\hline 6 & Andover Controls Corporation & BACnet, Ethernet, LON \\
\hline 7 & Architectural Lighting Management & DMX-512 \\
\hline 8 & Automated Logic Corporation & $\begin{array}{l}\text { Supports all major communication protocols } \\
\text { including BACnet, LON works, MODbus and } \\
\text { SNMP. }\end{array}$ \\
\hline 9 & Cooper Lighting & ESI digital protocol \\
\hline 10 & Crestron & LAN/WAN/Internet connection \\
\hline 11 & Cutler Hammer (Eaton) & X-10, CEBus, RS232, INCOM \\
\hline 12 & Delta Controls Inc & BACnet and ORCAview \\
\hline 13 & Douglas Lighting Controls Inc & LonWorks \\
\hline 14 & Easylite & \\
\hline 15 & Echelon Corporation & LON works \\
\hline 16 & Electronic Theatre Controls Inc & $\begin{array}{l}\text { DMX 512, ETCnet, ETClink, MIDI, BACNet, } \\
\text { LON Works }\end{array}$ \\
\hline 17 & Elk Products Inc & RS232, RS485, SIMPLE \\
\hline 18 & Energy Savings Inc. & Digital - SuperDIM \\
\hline 19 & GE Industrial Systems & Ethernet, MODbus, RS485/232, LON works \\
\hline 20 & GE Lighting & See MagneTek \\
\hline 21 & Genlyte - Lightolier Controls & RS232, RS485, BACNet, DMX512 \\
\hline 22 & H I Solutions Inc & LAN Ethernet, RS485 \\
\hline 23 & Honeywell & $\begin{array}{l}\text { RS232, ARCnet, Ethernet, BACNet, Modbus, } \\
\text { Allen Bradley Data Highway, Modbus plus, } \\
\text { and a wide variety of other common protocols } \\
\text { are available. }\end{array}$ \\
\hline 24 & Horton Controls / Watt Stopper & LON works \\
\hline 25 & Hubbell and Hubbell Lighting & 0-10VDC, 2-wire \\
\hline 26 & Hunt Controls Systems Inc & 0-10VDC, 2-wire, ESU super dim \\
\hline 27 & Johnson Controls Inc & $\begin{array}{l}\text { BACnet, LON works, Ethernet and most other } \\
\text { major protocols. }\end{array}$ \\
\hline 28 & JRS Technology Inc & LON works, 0-10VDC \\
\hline 29 & LEAX Lighting controls & $\begin{array}{l}\text { LON works, DMX512, RS232, Analogue Input, } \\
\text { Volt free, 2wire, DALI, 0-10VDC, DSI }\end{array}$ \\
\hline 30 & Lehigh Electric Products & LON works, DALI, DSI, 1-10VDC \\
\hline 31 & Leviton (LON products group) & 0-10VDC, DMX512, LON works, BACnet \\
\hline 32 & Leviton Lighting Control Division & $\begin{array}{l}\text { LON works, BACnet, 0-10 VDC, LAN/WAN } \\
\text { Ethernet }\end{array}$ \\
\hline
\end{tabular}




\begin{tabular}{|l|l|l|}
33 & Lithonia Lighting (controls) & BACnet, DMX512, 0-10VDC, 10-30VDC \\
\hline 34 & Lutron Electronics Co Inc & $\begin{array}{l}\text { O-10VDC, 2-wire line voltage control, 3-wire } \\
\text { line voltage control, DMX 512 }\end{array}$ \\
\hline 35 & MagneTek Lighting Products Group & 0-10VDC, DMX 512, DALI \\
\hline 36 & MYTEC Corporation & 2-wire, 24VDC \\
\hline 37 & NexLight & 2-wire, BACnet \\
\hline 38 & Novitas Inc & 0-10VDC \\
\hline 39 & Osram Sylvania/ Ballast Division & 0-10VDC, DALI \\
\hline 40 & Panja Inc & DMX512, Ethernet, BACNet \\
\hline 41 & Pass \& Seymour/ Legrand & 2-wire \\
\hline 42 & PCI Lighting Control Systems Inc & RS232, RS 485, DMX 512 \\
\hline 43 & PLC Multipoint Inc & $\begin{array}{l}\text { Direct Serial RS232, FIBER OPTIC } \\
\text { COMMUNICATION, LOCAL MULTI-DROP } \\
\text { COMMUNICATION BUS }\end{array}$ \\
\hline 44 & SensorSwitch Inc & 1-10VDC \\
\hline 45 & Siemens Building Technologies Ltd & BACnet, MODbus, LON works, DMX 512 \\
\hline 46 & Smart America & Ethernet \\
\hline 47 & Square D Company & $\begin{array}{l}\text { RS232, RS 485, MODbus, Ethernet 10Base T } \\
\text { Connectivity with TCP/IP Protocols }\end{array}$ \\
\hline 48 & The Watt Stopper / Pass \& Seymour & 0-10VDC, LON works \\
\hline 49 & Tork & \\
\hline 50 & Touch-Plate Lighting Controls & BACnet \\
\hline 51 & Triatek Inc & $\begin{array}{l}\text { TRIATEK Link, BACnet, MODbus, Ethernet } \\
\text { TCP/IP, RS 485, DALI }\end{array}$ \\
\hline 52 & Tridonic Inc. (North America) & Digital - DSI, DALI \\
\hline 53 & Vantage & RS232 \\
\hline 54 & Xitron Technologies Inc & RS232 \\
\hline & &
\end{tabular}

\section{Task 2.9: Investigate strengths and limitations of each protocol as they apply to lighting system performance}

Protocol architecture for computer communication was developed in the early 70s and defined in the 7-layer Open Systems Interconnection (OSI) - Basic reference model (ISO 7498). OSI is an international standard that defines the structures for developers to achieve multi-vendor computer communications protocol standards. The benefit to following the layered structure approach is that minimal understanding of the other layers is required to develop a compatible/working interface.

One point to keep in mind is that there are substantial incremental costs associated with implementing all seven layers into products. Therefore, the complete OSI model is typically followed through interfaces plugging directly into computers.

Pertaining to the building and lighting control markets only the protocols categorized as backbone communications architecture contain every OSI. Ethernet is the most dominant 
protocol meeting these criteria. BACnet uses a 4-layer version of the OSI standard. LON Work's protocol also resembles the OSI standard.

Most control systems do not use the OSI standard because of the high cost to do so (hardware overhead and development time). Moreover, the speed, amount, type and portability of data communicated do not require the entire structure.

Because several communications methods are utilized within the building environment, compatibility between subsystems is an overriding issue. To overcome protocol in compatibility issues, routers, translators, bridges and other defined modules are usually required. There are obvious distinct differences between the protocols used for building controls and for lighting controls. This fact is independent of whether the protocols are open or proprietary. Building control systems typically control HVAC devices that do not require fast response times and do not require many control points. Lighting control systems require immediate response times, the ability to detect if occupants are still active in a space and significantly more control points. Naturally, lighting control systems need to be sensitive to these differences.

For a lighting control system to operate successfully the system must:

- Minimize command strings aggregating control points

- Warn occupants of lighting system changes

- Sense delay override commands

- Interact with other sensors and controls within the office environment

These requirements often exclude existing building automation system protocols from being used to control the lighting because they have slow response speeds, cannot control many points, and if they did, they would be prohibitively expensive.

Until recently, lighting control systems were predominately on/off control. Lighting systems that provided dimming control were limited to local room or area control, and not tied into a central lighting control system or building automation system. Recent introductions of digital fluorescent ballasts with communication protocols have created a new way for control manufacturers to approach the design of lighting control components.

Table 4 reviews the existing protocols and describes how well they are suited for use as lighting control. 
Table 4. Building Control protocols: Lamp-ballast control techniques (protocols)

\begin{tabular}{|c|c|}
\hline \multicolumn{2}{|c|}{$0-10$ volt } \\
\hline Strengths & Weaknesses \\
\hline $\begin{array}{l}\text { 1. Many suppliers already have products } \\
\text { that use it. It has been commercially } \\
\text { available since the early 1970's for } \\
\text { theatrical lighting controls ANSI has } \\
\text { recently approved 0-10V std. for } \\
\text { Entertainment Technology - published } \\
\text { standard E1.3-2001. } \\
\text { 2. Entered commercial electronic } \\
\text { fluorescent dimming ballast products in }\end{array}$ & $\begin{array}{l}\text { 1. Ballast manufacturers have not adopted } \\
\text { standard for commercial. As a result } \\
\text { consistent behavior across different ballast } \\
\text { types or manufacturers is not assured. For } \\
\text { example, } 5 \text { volts for one ballast } \\
\text { manufacturer might result in } 30 \% \text { dim level, } \\
\text { while the same 5-volt signal might result in } \\
\text { a 50\% dim level for another manufacturer's } \\
\text { ballast. }\end{array}$ \\
\hline
\end{tabular}
1993.

3. Conceptually simple - light output varies with control voltage. Three analog command regions $(-0.5 \mathrm{~V}$ to 0.3 volts $=$ "zero state: $0.3 \mathrm{~V}$ to $9.8 \mathrm{~V}=$ active range: $9.8 \mathrm{~V}$ to $30 \mathrm{~V}=$ full state)

4. Simple, inexpensive analog control devices for simple control functions. A variable resistor can provide dimming control, but for controlling multiple ballasts, a slightly more complicated circuit in the control device is needed.

5. Incremental cost to implement ballast input control circuit is low. (Not to be confused with cost associated with implementing overall dimming capabilities.)

6. Protocol defines control device function intended for controllers and dimmers

2. Small bandwidth (0-10Volt) creates difficulty obtaining enough space for control functions in addition to dimming resolution.

3. Control cable is polarity sensitive making it more difficult to install and troubleshoot.

4. Low dc voltage is susceptible to electrical interference from power lines and lamp leads. (e.g., running control lines in same conduit as ac power can cause lamps to flicker).

5. Total cable length becomes issues due to line loses.

6. Requires low voltage control wiring. This is more of a problem for retrofit installations.

7. The linking of a particular ballast (addressing), or group of ballasts, to a control function must be pre-planned and hardwired electrically. Ballast control is dependent on the control wire circuit.

8. One-way communication. Ballasts cannot communicate information back to the controller.

9. When multiple controls or sensors are used a master - satellite configuration is required to provide setting control hierarchy and avoid conflicts.

10. Does not define function of load device, so device could source or sink current. 


\begin{tabular}{|c|l|}
\hline \multicolumn{2}{|c|}{$\mathbf{X - 1 0}$ (power line communications) } \\
\hline Strengths & Weaknesses \\
\hline $\begin{array}{l}\text { 1. Uses existing power wiring as } \\
\text { control communication media. }\end{array}$ & $\begin{array}{l}\text { 1. } \begin{array}{l}\text { Due to inherent limitations and concern for } \\
\text { robustness ballast manufacturers have not } \\
\text { endorsed powerline communication } \\
\text { approach. }\end{array} \\
\text { 2. Very low cost communication } \\
\text { technique. }\end{array}$ \\
$\begin{array}{l}\text { 3. Good for residential and small } \\
\text { building applications (256 }\end{array}$ & 2. $\begin{array}{l}\text { Susceptible to power line noise } \\
\text { disturbances. }\end{array}$ \\
$\begin{array}{l}\text { addresses) } \\
\text { 4. Supported by several control device } \\
\text { manufacturers. }\end{array}$ & 3. Limited to 256 addresses \\
5. Proven technology & 5. Conflicts between other systems highly \\
\end{tabular}

\begin{tabular}{|c|c|}
\hline \multicolumn{2}{|c|}{ Two-wire (ac phase control dimming) } \\
\hline Strengths & Weaknesses \\
\hline $\begin{array}{l}\text { 1. No additional wiring required for } \\
\text { dimming control (reduces retrofit } \\
\text { costs). Uses existing line switch leg } \\
\text { and neutral wiring as control } \\
\text { communication. } \\
\text { 2. Uses conventional incandescent } \\
\text { type wall dimmers (although some } \\
\text { implementations require special } \\
\text { dimmer circuits). } \\
\text { 1. 3. High-level signals are less } \\
\text { sensitive to interference from } \\
\text { adjacent power wires and lamp } \\
\text { wiring in fixture, especially } \\
\text { compared to 0-10 volt systems at } \\
\text { low voltages. }\end{array}$ & $\begin{array}{l}\text { 1. No specification or standard to insure } \\
\text { consistent behavior across different } \\
\text { ballast types or manufacturers. } \\
\text { 2. Control/ballast manufacturers have not } \\
\text { developed standard for commercial use. } \\
\text { 3. Susceptible to power line power quality } \\
\text { disturbances. Phase-cut technology } \\
\text { creates power factor imbalance and } \\
\text { harmonic distortion on the neutral. } \\
\text { 4. Its use in large installations could } \\
\text { potentially cause power quality } \\
\text { problems. } \\
\text { 5. Interfacing to controllers requires ac line } \\
\text { voltage to low voltage signal interface } \\
\text { circuitry. } \\
\text { 6. The linking of a particular ballast, or } \\
\text { group of ballasts, to a control function } \\
\text { must be pre-planned and hardwired } \\
\text { from the controller. } \\
\text { 7. Ballast control is power circuit } \\
\text { dependent. } \\
\text { 8. One-way communication. Ballasts } \\
\text { cannot communicate back to controller. }\end{array}$ \\
\hline
\end{tabular}

\section{DMX-512 communications}




\begin{tabular}{|c|c|}
\hline Stre & Weaknesses \\
\hline $\begin{array}{l}\text { 1. Industry standard for theatrical lighting } \\
\text { control systems. Limited use in general } \\
\text { commercial lighting applications. } \\
\text { 2. Supported by all theatrical control } \\
\text { manufacturers } \\
\text { 3. High-speed wide bandwidth for control } \\
\text { of as many as } 512 \text { points per loop. } \\
\text { 4. Transmission speed 250k bites/sec. } \\
\text { 5. Simple command structure } 3 \text { data types } \\
\text { (Reset, Null Start code and valid } \\
\text { dimmer levels 0-255). Commands are } \\
\text { sent in an asynchronous continuous } \\
\text { serial format. }\end{array}$ & $\begin{array}{l}\text { 1. Not supported for fluorescent dimming } \\
\text { control by ballast manufacturers. } \\
\text { 2. Control circuit is expensive to incorporate } \\
\text { into ballast design. } \\
\text { 3. Continuous one-way transmission } \\
\text { 4. New standard is being reviewed to possibly } \\
\text { replace DMX512 called the Advanced } \\
\text { Control Network (ACN). } \\
\text { 5. Command structure limited and does not } \\
\text { support basic commercial lighting control } \\
\text { requirements. } \\
\text { 6. Standard does not define minimum } \\
\text { performance levels of connected equipment. }\end{array}$ \\
\hline
\end{tabular}

\begin{tabular}{|l|}
\hline \multicolumn{1}{|c|}{ Strengths } \\
\hline DALI Co \\
\hline 1. \\
Recognized standard in Europe IEC- \\
929 and supported by all major ballast \\
manufacturers. North American control \\
and ballast manufacturers are \\
supporting concept however have not \\
agreed to adopt as a standard.
\end{tabular}

2. Two-way communication - ballasts can report operational parameters and lamp status information back to the controller.

3. Individual ballast control through individual addressing, as well as the ability to configure groups for common operation.

4. Addresses are dynamically allocated no agreements between manufacturers are needed to ensure unique addresses, able to mix manufacturers products within one job.

5. Grouping ballasts with particular control functions and controls can be changed without any rewiring.

6. Uniform action in response to

\section{Weaknesses}

1. Commissioning of digital lighting systems is new to industry. Therefore a process is not in place to service and commission systems.

2. Potential for initial control cost to be more expensive for some applications until economies of scale are reached.

3. Commissioning burden shifted from designer/installer to facility manager/enduser. This could be considered a strength if new controls specialists are brought into the scene.

4. Setting up addresses requires a person to physically identify individual ballasts to the controller.

5. Newly introduced protocol in North America - lack of availability of products (both ballasts and controls) makes investment risky at first.

6. Lack of diverse control products that provide high level functions and preprogrammed control operations such as dimming for daylight or load shedding. This 
commands across different

manufacturers' products.

7. No analog interface circuitry when microprocessor controllers are used (reduces device cost and increases reliability of control systems).

8. Incremental cost to implement ballast input control circuit is low.

9. Cost effective way to provide individual luminaire control to office occupant. might be only a temporary, initial shortcoming.

7. Uses communication technique called 'Manchester code' making the use of standard UARTS difficult.

8. Protocol defines ballast definition but not control criteria. Opening the door for controls to be incompatible within a specific system.

9. Limitation of 64 addresses per loop increases requirement for additional hardware when implementing in larger spaces.

10. Control manufacturers are uncertain about performance of the product.

\section{Comments on DALI Functional Profiles -}

- Although 32 bit address has been developed, there is no way to control devices with this number, limiting total output control to 64 addresses per loop.

- No single command to reset load device to default values.

- No command priority structure leaving the scheme to the control input device.

- No single command available to clear a specific device address.

- No commands for passing variables between DALI loops.

- No commands to define function of the control devices.

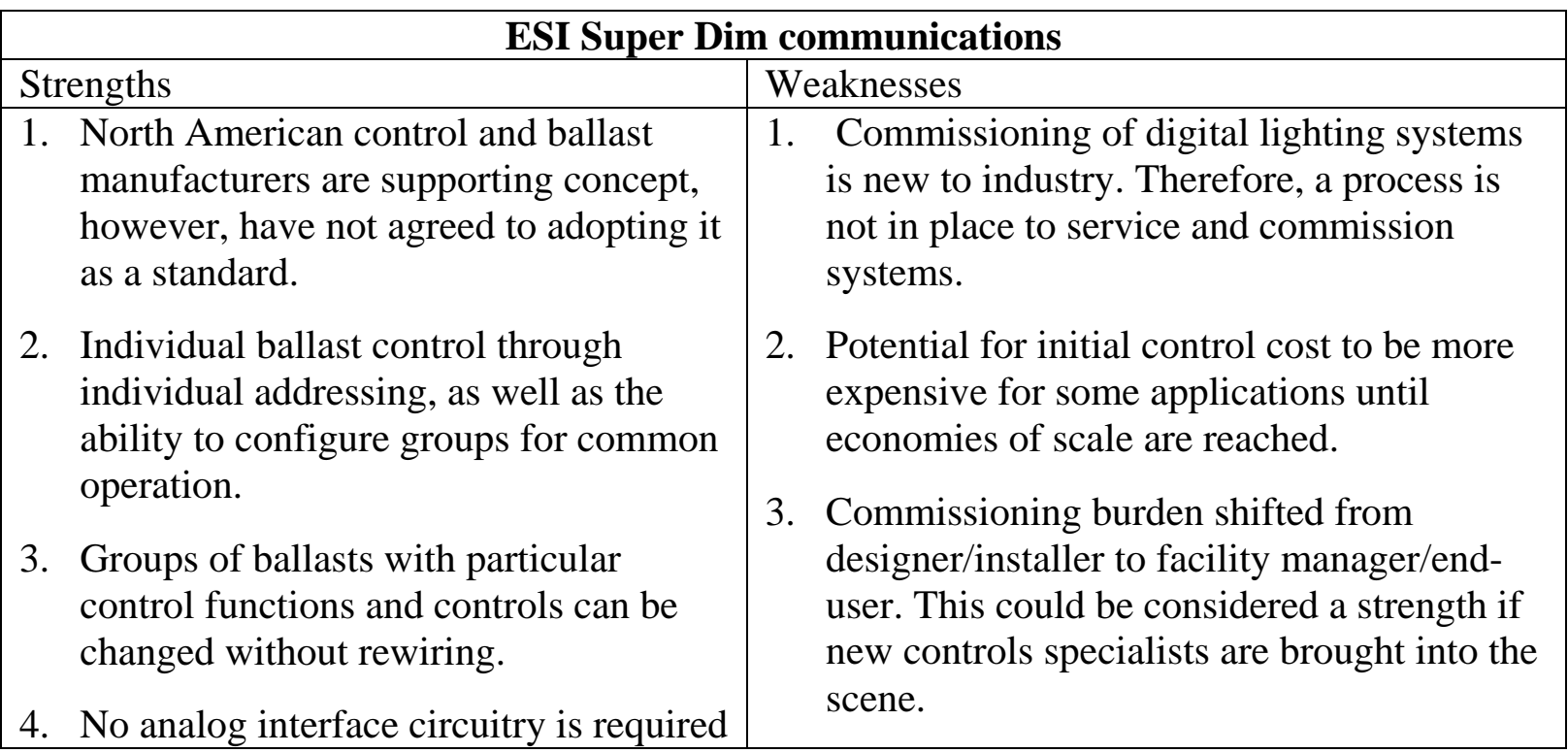




\begin{tabular}{|c|c|}
\hline $\begin{array}{l}\text { when microprocessor controllers are } \\
\text { used. This reduces device cost and } \\
\text { increases reliability of control systems. } \\
\text { 5. Incremental cost to implement ballast } \\
\text { input control circuit is low. } \\
\text { 6. Uniform action in response to } \\
\text { commands across different } \\
\text { manufacturers' products. } \\
\text { 7. Cost effective way to provide } \\
\text { individual luminaire control to office } \\
\text { occupants. }\end{array}$ & $\begin{array}{l}\text { 4. Setting up addresses requires a person to } \\
\text { physically identify individual ballasts to the } \\
\text { controller. } \\
\text { 5. Newly introduced protocol in North } \\
\text { America - lack of availability of products } \\
\text { (both ballasts and controls) makes } \\
\text { investment risky at first. } \\
\text { 6. Lack of diverse control products that } \\
\text { provide high level functions and pre- } \\
\text { programmed control operations such as } \\
\text { dimming for daylight or load shedding. This } \\
\text { might be only a temporary, initial } \\
\text { shortcoming. } \\
\text { 7. Presently, only one-way communication to } \\
\text { ballast. } \\
\text { 8. Addresses are factory assigned requiring } \\
\text { agreements between manufacturers to avoid } \\
\text { duplicate addresses. } \\
\text { 9. Protocol defines ballast definition but not } \\
\text { control criteria. Opening the door for } \\
\text { controls to be incompatible within a specific } \\
\text { system. }\end{array}$ \\
\hline \multicolumn{2}{|c|}{$\begin{array}{l}\text { Comments on ESI Functional Profiles - } \\
\text { - No single command to reset load device to default values. } \\
\text { - No command priority structure leaving the scheme to the control input device. } \\
\text { - No single command available to clear a specific device address. } \\
\text { - No commands to define function of the control devices. } \\
\text { - No commands for two way communications. } \\
\text { - No commands supporting device status. } \\
\text { - No device min or max commands. }\end{array}$} \\
\hline
\end{tabular}




\begin{tabular}{|c|c|}
\hline \multicolumn{2}{|c|}{ LON Work communications } \\
\hline Strengths & Weaknesses \\
\hline $\begin{array}{l}\text { 1. Recognized as a global standard for } \\
\text { building automation control devices. }\end{array}$ & $\begin{array}{l}\text { 1. Expensive for applications requiring large } \\
\text { number of points of control. }\end{array}$ \\
\hline $\begin{array}{l}\text { 2. Supported by many control device } \\
\text { manufacturers. }\end{array}$ & $\begin{array}{l}\text { 2. A fee is charged per address making the } \\
\text { technology cost prohibited for each ballast. }\end{array}$ \\
\hline 3. Introduced in the early 80's and is no & 3. As a result of the component costs, the \\
\hline 4. Supports lighting and HVAC control & suppliers. \\
\hline attributes. & 4. Increased installation complexity. \\
\hline $\begin{array}{l}\text { 5. Financially backed by large electronic } \\
\text { companies. }\end{array}$ & $\begin{array}{l}\text { 5. Setting up addresses requires a person to } \\
\text { physically identify individual ballasts to the }\end{array}$ \\
\hline $\begin{array}{l}\text { 6. High Speed communications (up to } 1.5 \\
\text { Mbits/sec.). }\end{array}$ & \\
\hline \multicolumn{2}{|c|}{ Comments on LonMark Functional Profiles - } \\
\hline \multicolumn{2}{|c|}{$\begin{array}{l}\text { Light Sensor: 1010, version } 1.1 \text { - Sensor output is defined in units of lux with valid ranges from } 1 \text { to } 65,535 \text { lux. } \\
\text { First, this is potentially confusing since the sensor will most likely be responding to ceiling illuminance rather than } \\
\text { work plane illuminance. Second, most ceiling light sensors for daylighting applications do not have cosine spatial } \\
\text { responses, so calling the variable lux is misleading. Third, ceiling illuminances typically are much lower than work } \\
\text { plane illuminances for common office lighting (such as ceiling troffers with parabolic reflectors), often by a factor } \\
\text { of five or more. Therefore, a resolution of one lux may not be sufficient for smooth dimming control. Since the } \\
\text { high end of the scale needs to include values as high as } 10,000 \text { lux, a data type with more dynamic range than an } \\
\text { unsigned, two-byte word is needed. Using the optional Reflection Factor and Field Calibration SCPTs could } \\
\text { overcome this limitation. The terminology is inconsistent with lighting terminology and potentially confusing since } \\
\text { more than just the reflectance of the of the surfaces is involved. A more suitable term for Reflection Factor is } \\
\text { ceiling/work plane illuminance ratio. }\end{array}$} \\
\hline \multicolumn{2}{|c|}{$\begin{array}{l}\text { Occupancy Sensor: 1060, version } 1.0 \text { - The Location Label SCPT is mandatory for the occupancy sensor and not } \\
\text { for the light sensor. The Debounce Time SCPT does not need a value longer than a few hundred milliseconds }\end{array}$} \\
\hline \multicolumn{2}{|c|}{$\begin{array}{l}\text { Lamp Actuator: 3040, version } 1.0 \text { - } 0 \text { to } 200 \text { correspond to } 0 \text { to } 100 \% \text { light output. } 255 \text { is undefined and rejected.; } \\
\text { values from } 201 \text { to } 254 \text { are undefined. The Energy Counter SNVT is in units of kWh but is not calibrated. It is } \\
\text { unclear whether the optional SCPT runHrAlarm is for re-lamping purposes. }\end{array}$} \\
\hline \multicolumn{2}{|c|}{$\begin{array}{l}\text { Constant Light Controller: } 3050-10 \text {, version } 1.0 \text { - The inputs for manual override and control seem well designed. } \\
\text { A constant set-point control algorithm (also known as reset control, or integral control) is all that this device is } \\
\text { capable of doing. This type of control algorithm has been shown to be inappropriate for daylighting control. } \\
\text { Control algorithms more appropriate for daylighting are open-loop proportional and closed-loop proportional (also } \\
\text { known as sliding set-point control). To be able to perform these other control algorithms additional SCPTs are } \\
\text { needed to allow proper commission. }\end{array}$} \\
\hline \multicolumn{2}{|c|}{$\begin{array}{l}\text { Occupancy Controller: } 3071 \text {, version } 1.0 \text { - Well designed inputs, especially having the capability to provide input } \\
\text { to a constant light controller, thereby combining manual switching, occupancy sensing and photocell control into } \\
\text { one control scheme. }\end{array}$} \\
\hline Partition Wall Controller: 3252, version 1.0 - Thi & $\begin{array}{l}\text { tion seems to have great potential for being able to redefine } \\
\text { controls. }\end{array}$ \\
\hline
\end{tabular}




\begin{tabular}{|c|c|}
\hline \multicolumn{2}{|c|}{ BACnet communications } \\
\hline Strengths & Weaknesses \\
\hline $\begin{array}{l}\text { 1. Recognized as a global standard for } \\
\text { building automation control devices. }\end{array}$ & $\begin{array}{l}\text { 1. Expensive for applications requiring large } \\
\text { number of points of control. }\end{array}$ \\
\hline 2. Supported by many control device & 2. Designed as a HVAC protocol. \\
\hline $\begin{array}{l}\text { manufacturers. } \\
\text { 3. Open Protocol. }\end{array}$ & $\begin{array}{l}\text { 3. Lighting protocol attributes are currently } \\
\text { under review for adoption into standard. }\end{array}$ \\
\hline 4. Preferred whole building (higher level) & 4. Installation complexity. \\
\hline $\begin{array}{l}\text { protocol. } \\
\text { 5. HVAC control attributes defined. } \\
\text { 6. High Speed communications (up to } 1.5 \\
\text { Mbits/sec.) }\end{array}$ & $\begin{array}{l}\text { 5. Expensive to implement into control } \\
\text { devices. Setting up addresses requires a } \\
\text { person to physically identify individual } \\
\text { ballasts to the controller. }\end{array}$ \\
\hline 7. 4 layer collapsed architecture. & \\
\hline $\begin{array}{l}\text { Comments on BACnet Functional Profile } \\
\text { - Priority assignments are supported in ar } \\
\text { - Trend logging and other monitoring fun }\end{array}$ & $\begin{array}{l}\text { - } \\
\text { iitecture. } \\
\text { ions supported. }\end{array}$ \\
\hline
\end{tabular}

\section{Proprietary/custom protocols}

Prior to the 1990's all lighting control systems and most building automation systems utilized custom or proprietary communication protocols.

Even today, there are several well-recognized manufacturers using custom communication protocols. Companies like JCI (N2-Metisys), Honeywell, Lutron, GE, PCI all use proprietary systems.

Johnson Controls provided a copy of their N2 protocol for our review. The N2 protocol is a simple protocol for exchanging information between devices on the N2 network. No specific commands are provided pertaining to lighting control or control strategies.

There are seven object types that make up the command record: 1) analog inputs, 2) binary inputs, 3) analog outputs, 4) binary outputs, 5) float internal values, 6) integer values, and 7) byte internal values.

In addition, JCI provided a copy of their METASYS system architecture. Their system overview is similar to other building automation system architecture reviewed.

As a result of not having access to other proprietary protocols, the LRC was not able to provide specific details regarding their performance. 


\section{Task 2.10: Interview contractors and installers to determine installation and commissioning issues, such as times and costs, and to identify other possible technology, market and application barriers to use of the control technologies under investigation (occupancy sensors, photosensors, dimming electronic ballasts)}

The LRC decided to conduct a direct-mail survey to contractors and control installers to try to identify market barriers affecting the acceptance of lighting controls. The Executive Summary of the survey is included below. The full survey report can be found in Appendix E: Direct Mail Contractor Survey.

\section{Executive Summary}

This report represents the results of a direct-mail survey conducted from May 15 to June 15, 2001, by the Lighting Research Center. The purpose of the survey was to determine contractors' and controls installers' attitudes about market barriers affecting the acceptance of lighting controls.

This survey confirmed overall what the industry already knows about lighting controls. Two significant results were quite clear:

- The potential market for occupancy sensors, photosensors, and dimming electronic ballasts is much greater than the present market. Installers were willing across the board to say that they could install far more controls than they do, if there were no barriers to market penetration.

- The universally cited barrier to market penetration was lack of customer demand, as expected. Although not directly asked, installers responded that they had difficulty proving that customers would see the payback from lighting controls.

Other market barriers seem relatively unimportant to this group:

- Lighting controls do not seem to cost installers much difficulty, either for time to install, or from returning to the job to adjust the controls. Everyone who completed the surveys commissions as well as installs the controls, so it can be assumed that all the installers are familiar with problems that arise in commissioning.

- Neither difficulty in finding skilled labor, nor a lack of compatible products seems to affect the number of controls this group installs. The majority of installers rate these barriers as "low" in importance across all types of controls, with only a few rating them "medium."

- Information about profitability was unclear; most installers have not broken out the cost of individual controls, as they use fixed contract pricing. However, for all four types of lighting controls, low profitability was cited as a major barrier no more than $20 \%$ of the time.

The results of this survey seem to show that customers must first be convinced that lighting controls will benefit their bottom line. If that happens, little else remains in the way of their widespread use. 


\section{Task 2.11: Interview key control manufacturers (occupancy sensors, photosensors, dimming electronic ballasts, and appropriate building control systems) to get their inputs regarding the information and analysis conducted.}

The LRC staff interviewed Steve Carson (Lightolier Controls), Pekka Hakarinen (Lutron), Brian Platner (Sensor Switch), Dave Peterson and Dorene Maniccia (Watt Stopper), Kevin Keefe (GE Industrial Systems), Troy Maeder (Automated Logic), and Brent Medearis (Siemans Lighting Control). Many of what was learned during the interviews was summarized in the white paper developed in task 2.15. Below is a summary of the notes taken during the interviews.

\section{Lightolier Controls. Key Interviewee: Steve Carson}

Carson noted that lighting is the most complex building system to control (compared to other building systems). Variable light levels are needed because “on” will not please everyone. Lighting controls involve many nodes, very subjective comfort levels, and often very different configurations, e.g., direct/indirect.

He is concerned about the lack of controls protocols and the lack of building automation systems that include lighting in the planning; these systems focus on HVAC; lighting is seen simply as a "load."

As for penetrating market barriers to lighting controls, he thinks energy savings companies (ESCOs) are the major influence for existing systems. They operate on guaranteed energy savings. Replacing existing lamps with T8s provides guaranteed savings, so ESCOs will always do this. But they find it difficult to sell unknown savings based on statistical usage.

Simple, standalone control devices need "perks" that must be tied into a centralized system, such as monitoring or load shed, but then the system cost is too great for ESCOs to sell easily.

For example, consider the installation of a \$10-15 individual addressable ballast; a gap exists between specifying it and making it work. No commissioning is included in the cost. These units need to be easily commissioned, and set up for "back end zoning."

The controls industry also needs a way to certify commissioning (not a problem for Lutron). Additionally, the commercial sector needs "systems integrators"-a new profession that does all the commissioning work. Such a professional could come from the security or audio-visual industry, as controls systems started with home theater. Carson believes that legislation should be undertaken to fill these needs.

\section{Lutron. Key Interviewee: Pekka Hakarinen}

Hakarinen says that lighting as an industry is not successful with architects (or building owners, or business operators); poor communication leads to poor penetration with these groups. She believes more education is needed. Communication about the value of lighting controls stops at the facility manager; because of this, the industry needs to work to get its messages to the right level.

The "first cost" mentality is a significant market barrier, in her view.

Technology is not an issue. Daylighting systems have been available since the 1980s. 
An additional market barrier is that certified electricians must be used for line voltage installations. Although Lutron has no problems in this area, having its own field service technicians for commissioning, other companies with different business models may have difficulty finding qualified people to commission their products.

Hakarinen believes that DALI is not the answer; how does one individually address and control each fixture? Also, DALI is incomplete because it doesn't address commissioning.

Commissioning is a difficulty that the industry has not yet overcome. The lighting industry needs to create the installer/commissioning professional and educate the owner that [lighting] is a good investment. This education is vital, because service charges for set-up and commissioning may be equal to or greater than the cost of the hardware.

Concerning building integration, she notes that technically, systems can meet the design intent, but they are not free.

Hakarinen says, "Specifiers understand the value of lighting, but we need to give them better tools to get the point across. If you see a value, then you won't mind paying for it.”

Payback is a problem (two years is too long). Lighting needs to have another value proposition. Opinion leaders play a big role in market development. For example, Governor Davis of California talking about lighting controls as a solution for the present energy crisis would be effective. This effort must be accompanied by financial incentives. She thinks the industry should stay away from products rebates. Products need to sell themselves. Product or technology rebates do not sustain a market. Some other incentive is needed.

\section{Sensor Switch. Key Interviewee: Brian Platner}

Platner points out that there is no lighting controls section or category in the AIA checklist for buildings. He believes that not enough attention is paid early on to lighting. Getting lighting into guidelines like the AIA will help it get a spot.

In penetrating markets, he says that up-front cost is critical; however, we need to get beyond first costs.

He does not agree that more legislation is the answer, because the existing laws are not enforced. People who are responsible for inspections do not know about lighting and lighting-related energy standards-they are only concerned with fire safety and related issues. Who is the policeman for energy code compliance? For example, a large engineering firm has no compliance form for energy codes for small jobs, but 30-35\% of its business is small jobs. A full $65-75 \%$ of the country's population already lives where energy codes exist. He thinks ASHRAE 90.1 is pretty good and explicit-it simply needs to be enforced. Building departments need, at a minimum, a form to be filled out by the builder that checks for lighting controls and energy compliance.

In Platner's opinion, lighting controls should be local controls. Central control for lighting is ludicrous. Building automation systems (BAS) need information for monitoring, but they don't need to control the lighting. However, centralized control for load shedding would be good.

He is unsure about the benefits of DALI. 
Platner would like to see hard data on actual case studies that show energy savings is needed, and believes that education is necessary to articulate the value of controls.

\section{Horton Controls (The WattStopper). Key Interviewees: Dave Peterson, Dorene Maniccia and Harold Jepsen}

Jepsen noted three barriers to the penetration of lighting controls:

1. Initial cost

2. Hassles involved in commissioning; in general, installers avoid hassles that are not required. In their world, lighting controls are a low priority compared to fire safety, HVAC security, etc.

3. Basic lack of knowledge: the unknown is risky, and people avoid risk.

Peterson commented that lighting controls are not mission-critical. They suffer from a lack of proof for payback, unlike T-8 replacement, which is guaranteed savings.

As for building automation systems and centralized control issues, Peterson says that

the best results for lighting controls come when control is done at the local level. Central monitoring offers perks, such as data for security, but this is not so meaningful for energy savings. Most energy savings come from stand-alone controls. Monitoring the whole building is important for spotting trouble and waste. All the members of this group said that the lighting controls should be local, with the data sent back to the central system.

Jepsen remarked that when direct digital controls (DDC) were introduced, a new profession came into being to commission the controls for HVAC-a "temperature control contractor," who works as a sub-contractor to the HVAC contractor. However, there is no analogous "lighting controls contractor." Warranty contracts are used in HVAC.

This group feels that DALI is a beneficial movement in controls protocols, since all manufacturers will build ballasts that work the same. Individual addressing is good, but major questions remain about commissioning-it is much more difficult than with non-addressable systems. However, nothing is being done beyond the ballast. The industry has no guidance on how to do system things. No one makes digital logic devices to use with DALI. DALI is focusing on "individual satisfaction" with the Ledalite Company and not energy savings.

The group also says that legislation is one way to get controls used--at least money is budgeted for controls. Rebates are good. They reduce payback. The industry should look at the past incentives for electronic ballasts, as these can work for speeding up penetration. However, manufacturers do not like on-again, off-again incentives.

On load shedding technology, they note that when lighting is already as low as $1 \mathrm{~W} / \mathrm{sq}$.ft., load shed offers little benefit. Their advice to the Department of Energy is to "get moving on ASHRAE 90.1.”

\section{GE Lighting Control Systems. Key Interviewee: Kevin Keefe}

Market:

What would you list as the top three primary market barriers restricting the increase demand and utilization of lighting control and fluorescent dimming systems? 
- Cost of retrofit

- Understanding of potential energy cost savings

- Lack of codes/code enforcement

If the LRC and or the DOE could assist in increasing demand for lighting control and fluorescent dimming systems what action would you recommend?

- $\mathrm{DOE}$ - financial incentives

From a cost perspective which part of the system is the largest contributor (initial design, product, installation, commissioning, warranty service)?

- In new construction, product \& commissioning

- In existing construction, installation

What is the number one issue the end user has for not purchasing lighting control and fluorescent dimming systems?

- In new construction, lack of lighting control strategy

- In existing construction, cost of installation

System:

What are the primary product barriers restricting the increase demand and utilization of lighting control and fluorescent dimming systems?

- Wireless systems to facilitate cost-effective system retrofits

How easily do systems support daylight and load shed control of lighting loads?

- This is a common application today (at least for GE)

Regarding your companies lighting control system what communication bus is supported? Does the protocol support on-off control and dimming?

- We currently have two protocols... One is GE proprietary, running on RS232 \& twisted pair. The other is LonWorks running on twisted pair. Both currently support ON/OFF only.

Installation and Service:

What percentage is retrofit vs. new construction?

- $85 \%$ new construction

What is the largest installation barrier?

- In a retrofit situation, it's pulling the wire.

Who performs the project commissioning?

- GE or GE Factory Authorized Service Teams

Who provides the service for your systems?

- Same as above. 


\section{Automated Logic. Key Interviewee: Troy Maeder}

Market:

What would you list as the top 3 primary market barriers restricting the increase demand and utilization of lighting control and fluorescent dimming systems?

- Automated Logic's dealers would be better able to answer this question. We manufacturer our BAS and our dealers sell, install, and service the systems.

If the LRC and or the DOE could assist in increasing demand for lighting control and fluorescent dimming systems what action would you recommend?

- Same answer as above.

From a cost perspective which part of the system is the largest contributor (initial design, product, installation, commissioning, warranty service)?

- $\quad$ Same answer as above.

What is the number one issue the end user has for not purchasing lighting control and fluorescent dimming systems?

- $\quad$ Same answer as above.

System:

What are the primary product barriers restricting the increase demand and utilization of lighting control and fluorescent dimming systems?

- Same answer as above.

How easily do systems support daylight and load shed control of lighting loads?

- It is not inherent in ALC's system but it is easy to do.

Regarding your companies lighting control system what communication bus is supported? Does the protocol support on-off control and dimming?

- BACnet is our native protocol. On-off control and dimming can be accomplished very easily using BACnet.

Installation and Service:

What percentage is retrofit vs. new construction?

- $\quad$ Same answer as \#1.

What is the largest installation barrier?

- $\quad$ Same answer as \#1.

Who performs the project commissioning?

- $\quad$ Same answer as \#1.

Who provides the service for your systems?

- $\quad$ Same answer as \#1. 


\section{Siemens Building Automation (Lighting Control), Key Interviewee: Brent Medearis}

Market:

What would you list as the top 3 primary market barriers restricting the increase demand and utilization of lighting control and fluorescent dimming systems?

- Cost

- Lack of nation-wide energy codes

- Lack of applications expertise by the consulting electrical engineer

If the LRC and or the DOE could assist in increasing demand for lighting control and fluorescent dimming systems what action would you recommend?

- Get ASHRAE 90.1-1999 incorporated into the UBC in all 50 states.

From a cost perspective, which part of the system is the largest contributor (initial design, product, installation, commissioning, warranty service)?

- Small jobs - commissioning

- Large jobs - product

System:

What are the primary product barriers restricting the increased demand and utilization of lighting control and fluorescent dimming systems?

- No common universal communication protocol (ModBus, BACnet, Lonworks, etc...) among manufacturers.

How easily do systems support daylight and load shed control of lighting loads?

- Load shedding is easily accomplished. Non-dimmed daylighting is easily accomplished

Regarding your companies lighting control system what communication bus is supported? Does the protocol support on-off control and dimming?

- Lighting Control - PCI, ModBus, SBT - Modbus, LonWorks, Johnson Controls N2, BACnet. Lon and BACnet do allow for dimming, but we do not implement it.

Installation and Service:

What percentage is retrofit vs. new construction?

- $\quad$ Lighting Control - 20/80

What is the largest installation barrier?

- Space in the electrical closet

Who performs the project commissioning?

- Our field service representatives

Who provides the service for your systems?

- Our field service representatives 


\section{Task 2.12: Meet with lamp manufacturers to get their inputs on lamp operating parameters that are key to maintaining performance when used with control technologies.}

LRC staff interviewed Ed Yandek (GE Lighting), John Wilson and Tom Leyh (Philips Lighting), and David Bay, Bob Horner, David Reid, and Walter Lapatovich (OSRAM SYLVANIA). The outcome of the interviews was incorporated in the white paper developed in task 2.15. Below is a brief summary of the interviews.

\section{GE Lighting, Key Interviewee: Ed Yandek}

Mr. Yandek stated that the technology of lighting controls is not a problem. "[We] can do anything technically," he remarked. However, problems arise with understanding the markets' requirements for these technologies. For example, there is a problem with dimming, in that there is no standardization or definition of basic requirements. "No one has defined what the system functionality has to be," said Yandek. GE can bring new products to market in less than five years, but "we need to know the requirements."

He stated that what manufacturers need, from his point of view, is a functional specification for a "box" that interfaces to lighting, and this needs to be standardized. How can this happen? In his opinion, some organization needs to take charge and hold meetings to drive this to closure. The first meetings should not include lighting designers or lighting scientists. He believes that the first attempt should be to figure out communications, then bring in lighting people. The "high level guys" for building automation want to grab onto lighting and use it without having to know all about it. They need this "box."

Current controls involve "front-end" design solutions that need extensive custom design work. The DALI protocol might be useful between ballast and ballast control modules (BCMs). BCMs have the intelligence to perform the control algorithms, and could potentially be the "box" Yandek referred to.

Yandek knows of no current work by ANSI on taking the lead for controls standardization. The acting panel of IEC that will look at standards needs industry agreement on minimum standards. The IEC panel is the only group doing work, but it suffers from a slow pace, and is not involving major building automation controls manufacturers.

Yandek also stated that lighting controls go way beyond daylighting.

GE works mainly with Universal (the old Magnetek).

\section{Philips Lighting. Key interviewees: John Wilson and Tom Leyh}

Major barriers for use of lighting controls:

- Lighting controls are not totally safe for all lamp/ballast systems; no known problems, but plenty of uncertainty

- Reliability of dimming systems are lower than reliability of on/off systems

- Lighting is too specific to applications, not everyone needs all types of controls 
- Lack of skilled labor force; lack of educated users

Ways to overcome these barriers

- Understanding in the marketplace the total cost of ownership; life of the system cost is not taken into consideration

- Increase interchangeability of lamps and ballasts

Opportunities for lighting controls

- Expect high growth in the next years

- More controls options will be available

- Reliability of lamp/ballast combinations will increase

They believe that central controls will be open protocol in the near future, but they are still unfamiliar about the future of DALI.

\section{OSRAM SYLVANIA. Key Interviewees: David Bay, Bob Horner, David Reid, Walter Lapatovich}

The group agreed that manufacturers perspective on lighting controls, and especially on dimming systems may be different than customers.

From their standpoint, the main barriers for widespread use of lighting controls are:

- Installation

- Perceived high cost ; full benefits are still not known

- Manufacturers do not fully understand the operating characteristics of lamp/ballast when operating in a dim condition

- Proprietary understanding of system performance; manufacturers are doing their internal research, but are not communicating their findings to the industry

In order to overcome some of the barriers they believe that industry should agree communicate a common message. With this in mind, they believe that DALI will be the "good housekeeping seal” that will assure good compatibility between different manufacturers.

They also believe that real time pricing will be a growing area, and that manufactures should work closely with utilities to develop load management strategies.

Overall, this group believed that lighting controls market would be growing in the next years, especially in the area of load management. They also believed that the technical barriers encountered today would be resolved in the near future. 


\section{Task 2.13: Catalog any solutions that have been recommended for improving lighting and whole building control protocols.}

\section{Practical Solutions for Improving the Penetration of Lighting Controls for Fluorescent Systems in Commercial/Industrial Applications}

The perceived difficulties and hidden economic barriers that may be hindering acceptance and demand for lighting controls are part of a broader picture. Any intervention to develop market demand should be considered in the widest social and economic context.

Naturally, the minimum acceptable requirements for lighting controls are that they:

- Provide sufficient light for the users of a space-after all, we can achieve perfect control by simply turning out all the lights in a building, but that is not a productive solution.

- Avoid waste - that is, turn off lights when no one is there to benefit from them (except for security, display, etc.).

- Reduce lighting levels, without compromising occupants' satisfaction and productivity.

In addition, we believe that no lighting controls solution is effective or acceptable unless it contributes to, or does not compromise, the following goals:

- Productivity - Planning, installation, commissioning, maintenance, and use of controls should not decrease business productivity.

- Energy savings - Lighting controls should save significant amounts of energy and money in relation to the expense involved in using them (acceptable payback period), and/or...

- Reduced power demand - Society as a whole should benefit from the lowered demand for expensive power and for more natural resources.

In general, technological problems pose relatively low market barriers for lighting controls. A failure to show reasonable payback periods and other economic benefits, however, limits the acceptance of controls in the marketplace. Without greater market demand for lighting controls, manufacturers have little incentive to eliminate the remaining, albeit small, technological problems.

Our recommendations for decreasing the barriers revolve around these economic arguments, laid out in detail in Section 2.15. The recommendations are also specifically centered on "on-off" technologies and load-shedding dimming as energy solutions, rather than on architectural dimming. These technologies are likely to provide the most economic benefit to society as a whole, not only by providing energy savings, but also by reducing peak demand and, concomitantly, the need to add more power-generating capacity (peak load reduction).

The technological barriers that do surround the lighting controls and applications discussed here are also those most likely to yield to attack under concerted market demand. For example, lamp/ballast compatibility is a problem that requires developing a full understanding of lamp/ballast system characteristics, but not one that is insuperable if sufficient market demand exists to make solving it worthwhile.

Within this context, we see opportunities for improving acceptance of each of the following lighting control solutions: 
Lamp/ballast compatibility - While not a control technology, compatibility is the sine qua non of dimming control solutions. Performance criteria for lamp/ballast systems would help ensure against premature failure of lamps when they are operated in the dimming mode.

- Benefits: Assured compatibility of lamps and ballasts would help improve acceptance of dimming control solutions. Facilities owners and managers often deem the control system itself unsuccessful when lamp life is shortened.

- Barriers: No criteria exist at present, and thus far, no organization has pressed for them. Additionally, compatibility criteria must examine performance within luminaires, not just still-air testing.

- Investments required: Technology research and development, campaign for the adoption of performance standards for lamps and ballasts.

Local control (manual controls for 'on' functions) - Nothing replaces local control, because no sensor or zoning plan can account for novel situations (a lone worker finishing a project late at night, unplanned meetings, etc.).

- Benefits: Provide the most occupant satisfaction, because once lights are turned on to occupants' desired level, no further action must be taken.

- Barriers: Provide the least guaranteed energy savings, as lights are more often on when they are not needed.

- Investments required: No additional investment, unless optional access improvements are provided to promote turning lights off.

Occupancy sensors - Occupancy sensor technology is robust, and works well for defined spaces. Obviously, it is not suitable for open-plan areas that require a level of continuity in the lighting, or where different categories of occupants come and go.

- Benefits: The major benefit of occupancy sensors is their ability to get some of the lights in a building completely off when people aren't in the space, without reducing occupant satisfaction. Because dimming is not involved, implementing occupancy sensor systems is simpler than implementing photoelectric dimming systems.

- Barriers: Hassles in commissioning remain; the technology must also overcome specifiers' inertia when considering controls solutions. Additionally, specifiers are loathe to accept claims of statistical rather than guaranteed savings.

- Investments required: Effective marketing campaigns.

Photoelectric dimming - New developments in simplified-commissioning photosensor technology (a new device developed and patented by the LRC) means that photoelectric dimming can be made to work cost effectively.

- Benefits: Photosensors are an obvious, though hard-to-implement solution, now rendered more or less hassle-free by the simplified-commissioning feature.

- Barriers: Simplified commissioning is not yet fully developed or commercially available. Without this feature, photosensors continue to involve commissioning and fine-tuning problems. 
- Investments required: Technology development and demonstrations, commercialization, and effective marketing campaigns

Time clocks/central control - Relay-panel products and the like are capable of reducing lighting loads, but only if the zoning is truly logical for the space. Such technology makes sense if a careful analysis of the zoning is carried out, yet the scope of the analysis required, and the need for exceptions to any zoning plan, militates against productivity.

- Benefits: The ability to get large groups of lights in a building guaranteed off, yet with fewer control nodes than occupancy sensor or photosensor systems.

- Barriers: Difficulties in zoning logically, hassles in planning and commissioning, lack of flexibility when space usage changes.

- Investments required: R\&D effort to improve ease of use, effective marketing campaigns.

Load-shedding ballasts - Electronic ballasts can be modified at minimal cost to reduce power by $20 \%$ or more to fluorescent lamps during peak demand. When developed, these load-shedding ballasts would allow a utility or building manager to lower the power to the lighting system "on demand" at a rate that minimally affects building occupants. Presently, it is envisioned that a non-dimming, programmed-start ballast can be redesigned to meet these objectives.

- Benefits: Load-shedding ballasts would not be as expensive as electronic dimming ballasts, and their installation would require minimum wiring. It is envisioned that utilities can control the load shed by signaling the customers from a remote location. Preliminary studies have shown that dimming up to $20 \%$ over a period of five seconds is acceptable to customers.

- Barriers: Load-shedding ballasts have not yet been developed and tested for commercial markets.

- Investments required: Technology research and development, demonstrations, commercialization, and effective marketing campaigns

Logical zoning and communications - Emerging controls protocols allow grouping lights together into a logical control scheme that minimizes the number of control nodes and sensors.

- Benefits: Logical zoning enables users to dim or shut large groups of lights off in specific locations, while being able to change zoning as their needs change. The ideal protocol would allow facilities managers to cope easily with high "churn rates," as a building's commercial tenants come and go.

- Barriers: The development of open, industry-accepted standards that allow lighting components to communicate with each other has been a lengthy process, but new standards such as DALI and BacNet might prove successful.

- Investments required: Technology development and commercial acceptance of standards, as well as effective marketing campaigns directed at producing societal change. 


\section{Task 2.14: Develop draft performance criteria for the control technologies (occupancy sensor, photosensor, dimming ballasts) for selected commercial and industrial applications}

Draft performance standards for a load shed ballast, occupancy sensor and photosensor are presented below.

\section{Draft Performance Specification - Load-shed Ballasts}

\section{Operational definition of load-shed}

What:

Why:

How:

When:

Where:

\section{Load-shed signal}

Source:

Information carried in signal:

Duration of signal:

Default state of ballast:

Time needed to receive signal from utility initiation:

Elapsed time with no signal to revert to full light output:

Staggered return to full power:
Reducing power demand

To avoid brownouts and blackouts, to avoid peak demand expenses (society or utility expenses)

Dimming fluorescent lighting or switching off lamps operated on multi-lamp ballasts

Only when required. Low frequency and duration of occurrence (less than 30 days per year, less than 6 hours duration)

All C/I applications except light-dependent critical tasks (e.g., hospital operating rooms)

Utility- or user-based in utility signal

Load shed on, or off

Continual (repeated) throughout load shedding condition

Full light output

5 minutes

15 minutes

(Some provision must be made so that all ballasts do not return to full power operation at the same time, causing a sudden demand condition. For example, a random time interval could be set before returning to full power.)

\section{Ballast performance non-load-shedding condition}

(The idea here is to make sure that a load-shed ballast is in every other way equivalent to the high quality ballasts now being specified and required in many areas. A load-shed ballast must meet or exceed most existing performance specifications so that it can be installed everywhere.)

Power factor: $\quad>0.9$

THD:

$<20 \%$ (ANSI definition) 
Ballast efficiency factor:

Power factor:

THD:
Multiple requirements dependent on lamp type and number of lamps operated by ballast (table needed)

$>0.8$ (to ensure that the demanded current is reduced along with the power in the event that load-shed is needed because of a transmission capacity situation. A little leeway is given so that current ballast designs can meet the spec even if, as expected, power factor changes slightly upon dimming or switching lamps.)

While load shedding, the root mean square (rms) of the current harmonics 2-33 shall not be greater than that during non-load-shedding operation. (THD as a percentage of fundamental current can increase as fundamental current drops without causing any more harm. What is important is that the absolute value of the distortion current not increase.)

How much power should be reduced during load-shed must be determined by consensus, and the following factors must be considered:

- How much demand reduction is needed to make load-shed worthwhile

- How much reduction in light output will building occupants tolerate, or if none is tolerated, how much can light output be reduced without awareness of dimming

Determining these factors will determine whether switching lamps of multiple lamp ballasts or dimming is the best strategy. The above specifications apply regardless of the actual power reductions agreed upon and regardless of the method used to obtain them. If occupant requirements prohibit the switching of lamps, then maximum dimming rates ( $<2 \%$ per second, say), and minimum light output dim levels (80\%, say) must be specified.

\section{Draft Performance Specification - Occupancy Sensors}

\section{Performance Requirements}

Coverage Area:

Field of view:

Extraneous Signal Sensitivity:
Minimum of $400 \mathrm{ft}^{2}$

Ceiling - Horizontal: 360; Vertical: up to 180

Wall - Horizontal: up to 180; Vertical: up to 90

Shall not be sensitive to:

Radio signals

Electromagnetic interference

Electrostatic discharge

Sudden changes in temperature and humanity

Noise or vibrations

Natural or forced air movement 
Failure Mode:

Maintained Detection:

Products Shall:

Operating Temperature Range:

Operating Humidity Range:

Time Delay:

Movement of inanimate objects

Occupancy or motion beyond the boundaries of the office

Manual on/off remains operable

Regardless of occupancy orientation to the sensor, location in the room, or speed of performing a motion, products shall detect: 1) small body motion (e.g., writing, typing, reading); 2) medium arm motion (e.g., reaching for telephone); 3) large body motion (e.g., walking)

Have silent operation

Unobtrusive status indicator

0 to $35 \mathrm{C}$

45 to $85 \%$

Adjustable

Demarcations clearly labeled

Calibrated and accurate within $\pm 10 \%$ of the set value

Minimum: 15 seconds

Maximum: 30 minutes

No user access to adjustment

Installation \& Commissioning: $\quad<30$ minutes

Power Requirements: > $\quad>1$ watt

\section{Draft Performance Specification - Photosensors}

\section{Performance Requirements}

Photosensor must:

- Independently adjust illuminance when dimming begins $\left(\mathrm{E}_{1}\right)$

o $1 \leq \mathrm{E}_{1} \leq 10,000$ lux

- Independently adjust illuminance when dimming stops $\left(\mathrm{E}_{2}\right)$

o $1 \leq \mathrm{E}_{2} \leq 10,000$ lux

- Any adjustment must not affect $\mathrm{E}_{1}$ or $\mathrm{E}_{2}$ by more than $5 \%$

$\mathrm{O} \quad \mathrm{E}_{2} \geq \mathrm{E}_{1}$

- $\quad$ Photosensor must be able to interface with a ballast such that $\phi_{2} \leq \phi_{1}$

- Gain must not deviate by more than $10 \%$ for $\left(E_{2}-E_{1}\right) / 0.8 \phi_{1}$ throughout the dimming range Provisions must be made during operation and commissioning for occlusion of the photosensor from direct illumination from natural and electrical light sources (shielding provisions is necessary). 
Subject to the previous provision, the light-sensitive element of the photosensor must have an unobstructed acceptance angle of at least $2 \Omega(\geq 50 \%$ maximum response).

Electric lighting levels must not dim at a rate faster than $1 \%$ per second so that the dimming rate is acceptable to occupants.

The device must have a spectral response that is photopic with a maximum $f_{1}$, error of $30 \%$. $\left(f_{1}\right.$, is defined in CIE Publication No. 69, "Method for characterizing illuminance and luminance meters," CIE, 1987).

Incremental adjustments of illuminance of the set points $E_{1}$ and $E_{2}<5 \%$ of $E_{1}$ and $E_{2}$ respectively.

Installation and Commissioning: > $\quad 25$ minutes

Failure Mode:

Lights on/full brightness

Products Shall:

Have silent operation

Operating Temperature Range: $\quad 0$ to $35^{\circ} \mathrm{C}$

Operating Humidity Range: $\quad 45$ to $85 \%$

Power Requirements: > > $\quad>$ watt

\section{Task 2.15: Summarize current state of the art for each component evaluated above; elucidate the technical and market barriers for each with an overall synopsis of potential opportunities; develop a peer review group consisting of industry leaders and authorities}

The goals of this project are to identify barriers to the penetration of lighting controls into commercial/industrial (C/I) applications that employ fluorescent lamp technologies, and to recommend means for overcoming those barriers. Preliminary recommendations have been developed after discussions with various stakeholders and examinations of published research and patents. Subsequent efforts over the next two years will refine, improve and validate the preliminary recommendations presented here.

There are four categories of barriers that have been identified that include several barriers within each category: lamp-ballast compatibility, component compatibility, installation/commissioning assurance, and marketing. Penetration of lighting controls for fluorescent systems into C/I applications will not be simple or rapid. Indeed, to reach significant penetration into the market a cultural change will have to occur in the lighting industry. This cultural change can be characterized as developing effective communications among all stakeholders in electric energy efficiency and load management, particularly those charged with the responsibility of establishing rational social policies for power generation and transmission.

The full report is found in Appendix F: Technical and Market Barriers to Full Implementation of Load Shed Ballasts, Occupancy Sensors and Photosensors. 


\section{Lamp - ballast compatibility}

The global lighting manufacturers are beginning to understand reliable operation of lamps on dimming ballasts, but progress is slow because of low market demand for dimming ballasts. Thus, there is a "Catch 22"; poor demand retards development and retarded development leads to poor demand. Nevertheless, there is some reason for optimism because all three global manufacturers are now taking responsibility for both the lamp and the ballast. And whereas there may not be a complete understanding of the underlying physics in operating lamps under dimmed conditions, it seems likely that the major manufacturers will eventually produce dimming ballasts that work well, if not perfectly, with any commodity fluorescent lamp. If market demand for dimming increased substantially, it seems very likely that reliable fluorescent dimming systems would be produced more quickly. But, again, without that demand, there is little reason to expect commodity fluorescent dimming systems to work reliably in the near future. However, three lamp and four ballast manufacturers have joined an LRC/DOE effort to understand the interaction of fluorescent lamps and dimming ballasts and to develop a series of metrics. This effort is reported in sub-task 4.9 below.

\section{Component compatibility}

One barrier that is being effectively addressed by the control manufacturers is the issue of interoperability of control components. On a local level, interoperability means attaining communication to control the ballast. More globally, interoperability concerns communication between individual control devices and, at a higher level, with whole building automation systems (BAS). Lighting controls manufacturers are working successfully to develop products that conform to open communication and command protocols (rather than proprietary systems) for all of the control technologies peripheral to the ballast. Several protocols are being discussed and it seems likely that standards will continue to be developed and refined in the next two years that will facilitate utilization of control components in larger systems.

\section{Installation/commissioning assurance}

The ability to install and commission controls ranges from very good to nonexistent. The reliable installation of on-off controls into C/I applications is simple and can be accomplished easily by trained technicians. Time controls, occupancy sensors, and manual switching are reliable and easy to install. There is not, however, significant market demand for on-off lighting controls beyond simple switches in $\mathrm{C} / \mathrm{I}$ applications.

Dimming systems, however, are both expensive to acquire and difficult to install. Commissioning photosensors is prohibitively time-consuming and, consequently, photosensors are almost never installed. Recent development by the Lighting Research Center of a simplifiedcommissioning photosensor to take advantage of daylight may, however, overcome this barrier.

Even if this photosensor technology becomes commercially available, there are still nagging small barriers to installing and commissioning lighting controls, particularly dimming systems. These barriers include wiring uncertainty, identification and zoning issues for groups of ballasts, the need for manual overrides, and the training of installation personnel.

\section{Marketing}

First cost remains a barrier for lighting controls. Often the person or department that pays for the installation of lighting equipment does not receive the benefit from the reduced electrical bills. Also, in existing buildings, managers do not want the "hassle factor" of implementing a change. 
First cost and hassle factor have been overcome for other energy-efficient lighting technologies with demand-side management (DSM) incentive programs, market transformation program procurement, shared-savings marketing by energy service companies (ESCOs), and new code requirements. Combinations of these approaches increase market demand for energy-efficient lighting products, leading to lower product cost and market transformation. Products such as T8 lamps, electronic ballasts, and LED exit signs are examples of successful lighting market transformations. Lighting controls, however, have yet to benefit substantially from these market forces because of the difficulty in predicting the energy savings resulting from control use.

DSM program developers and evaluators, code developers, and ESCOs do not have adequate information to predict expected savings by control measures. They need to know, for example, when and for how long the control switches off or dims the lights. There are case study examples, but robust, statistically significant control use factors need to be developed that will give the program developers confidence that a specific control installation will deliver a specific energy savings. With this confidence, controls will more likely find their way into lighting incentive programs, code provisions, and ESCO strategies.

The lighting industry is a fragmented collection of component manufacturers. No sector of the lighting community, private, public, or non-profit, is the recognized champion for lighting. Each agency works very hard to communicate the value of its own product or service. However, that communication is most often to other lighting component agents. Consequently, the lighting industry members do not and cannot communicate the value of lighting to specific markets such as auto manufacturers, health care professionals, sports and recreation, community development, etc. Remarkably, the industry remains largely uneducated about the perceived value of lighting to these sectors, focusing on market share in traditional markets rather than growing new markets. This is a significant cultural barrier to the penetration of fluorescent lighting controls into the $\mathrm{C} / \mathrm{I}$ market.

In particular, lighting controls manufacturers fail to communicate the value of lighting of their products. The lighting controls manufacturers are insulated not only from the public, but also from other sectors of the lighting industry (e.g., lamp and ballast manufacturers) and from specifiers. Lutron is one notable exception. They have established themselves not as a component or even system manufacturer, but, rather, they have successfully reached the specification community by providing assurances that their lighting controls will work and will deliver value to their customers. The majority of the lighting controls manufacturers remain focused on components, which drives down price. With little profit, there is little innovation or marketing. Consequently, there has been little penetration of lighting controls into the $\mathrm{C} / \mathrm{I}$ market.

\section{Preliminary Recommendations}

The following recommendations are ordered in terms of the relative effort needed to overcome identified barriers to the penetration of lighting controls into C/I markets. The first two recommendations are focused on existing, on-off lighting controls that have been demonstrated to be cost effective and reliable. These two recommendations can be implemented immediately. The remaining recommendations represent significant investment of resources to support widespread penetration of fluorescent dimming systems. As noted in this document, there are many significant barriers to the use of dimming fluorescent systems in $\mathrm{C} / \mathrm{I}$ applications. These barriers will not be overcome using traditional economic models, and should not be attempted 
without a much stronger financial incentive. A new economic model that incorporates the cost to society for building new power generation infrastructure does offer a realistic financial foundation for overcoming the barriers to fluorescent dimming systems.

1. Support data collection that provides fixed numbers for expected energy savings and load reductions for various static on-off lighting control technologies in various applications.

2. Support programs that overcome the inertia to first costs to static on-off lighting controls, working with building owners, professional societies, code developers, ESCOs, and conservation program developers.

3. Support functional and substantive means of communication among lamp-ballast manufacturers, controls manufacturers, controls installers, building automation system manufacturers, utilities, and government agencies charged with maintaining public benefit. The economics of load management needs to be widely understood by all stakeholders in lighting controls. The first task would be to identify key decision-makers in these different sectors, and then to provide incentives for those disparate groups to cooperatively work together. These incentives could be in the form of demonstration projects specifically designed to cooperatively link the key sectors. Incentives could also include support for cooperative technology development. In particular, two development projects are immediately envisioned:

3a. Develop an inexpensive, highly efficacious dimming ballast that responds to loadshed signals from the utility.

3b. Support development of open communications systems that link utility need for shedding load to building fluorescent dimming systems. This implies a standard ballast-utility interface for load shedding.

4. Support a human factors research program that establishes the principles for dimming levels and dimming rates in occupied spaces.

5. Develop a long-term labeling and testing program that provides assurances to all stakeholders that (a) the dimming ballast reliably operates the lamp under all conditions; (b) all control system components work reliably together; (c) installation and commissioning of dimming systems are reliable; and (d) articulates the value of lighting controls for energy and cost savings that reflect their social value by ensuring an inexpensive and reliable supply of electrical power. 


\section{TAsk 3: Identification Of MANufacturing Partners}

The LRC submitted the recommendations summarized in the white paper are presented in Tasks 2.14 and 2.15 to a peer review group. The members of the peer review group are: David Bay (OSRAM SYLVANIA), Jim Benya (Benya Lighting Design), Jim Gallagher (NYS Public Service Commission), Arthur Kressner (ConEdison), Ron Lewis (USDOE), Peter Morante (Northeast Utilities), Dave Peterson (The Wattstopper), Michael Stein (Consultant, Universal Ballast), Edward Yandek (GE Lighting).

A meeting with the peer review group was held on December $12^{\text {th }}$ 2001. Minutes of the meeting are included in Appendix G, Peer Review Group Meeting Minutes. In summary, the peer review group suggested that there are currently two distinct, viable categories of lighting controls: (1) automatic shut-off and (2) dimming controls. It was consensus that automatic shut-off controls are a robust technology ready for widespread installation, while dimming controls still have substantial technical barriers and are dependent upon changes in electricity pricing systems to be cost-effective in many applications. 


\section{TASK 4: IMPROVEMENT/TECHNOLOGICAL SOLUTION}

The Task 4 scope of work focused on identifying and proposing solutions for each of the categories identified during the first year. In the category dealing with automatic shutoff controls, two tasks were undertaken. The first of these two tasks (4.7) was directed toward developing fixed values for energy savings from occupant sensors so state and regional agencies in the U.S. could use these values in their market transformation activities. Based upon an extensive review and analysis of the literature, the LRC developed fixed values for energy savings. The second of the two tasks (4.8) was directed at developing a best practices document that helps both manufacturers and installers improve the likelihood of installing and commissioning occupancy sensors.

In the category dealing with dimming, which represented the majority of the effort for Task 4, the focus was on investigating and proposing specific technological parameters for product improvement. The LRC identified manufacturing partners to begin discussion about these technological solutions (e.g., development of load-shed ballast) and conducted limited testing. The LRC focused on economic, perceptional (human), and technical issues associated with whole-building and local control strategies, and undertook five tasks. In addition to developing a review of the progress in whole building control systems (4.1), great progress continues to be made in developing communication systems for lighting control systems. Problems still exist, however, with standardization and cost. These systems have developed their own momentum and only time will tell if and when the US market embraces whole-building lighting control systems. Neglected in most discussions of lighting controls is the lamp-ballast system used. Tasks 4.1, 4.2, 4.3, 4.4 and 4.5 concentrated on understanding lamp-ballast performance issues, which we believe must be at the core of developing any successful fluorescent lighting control system for $\mathrm{C} / \mathrm{I}$ applications. The major issues to consider are occupant response, initial equipment cost, and lamp life. Heating the electrodes prolongs lamp life by minimizing the damaging effects of sputtering from both switching and dimming. These systems are commonly three to five times more expensive than systems that do not provide heat to the electrode. Without question, these systems reduce lamp life due to sputtering from starting and dimming. Interestingly however, a non-linear relationship exists between operating current and life. A central focus of the effort this year was to explore the technical and economic issues associated with dimming instant start systems. Finally, the LRC reviewed the significance of dimming for occupants and made recommendations for integrating them with lamp-ballast system performance.

Task 4.6, develops recommendations for improved components and systems as evaluated in Task 2 (technology assessment) and integrates all of the information on tasks 4.1 to 4.5.

An additional task was added (4.9) to explore further the lamp-ballast compatibility issues associated with dimming systems. The goal of this task is to develop industry wide metrics to ensure lamps can reach reasonable life when used with full dimming ballasts. 


\section{Task 4.1: Work with key manufacturers, industry associations, government agencies and National Laboratories to expand the best existing communications protocols, if necessary, to meet the needs of a fully integrated lighting and whole building control protocol}

The success of lighting control products on the market must allow systems to achieve three main goals:

- Achieve sufficient functionality to meet the key requirements of their main market.

- Allow a significant cost reduction compared to current market standard systems. Cost should take into account: hardware capital cost including wiring, design time required by the specifier and the control system manufacturer, installation time required from the electrician, and commissioning time and remedial time required from the electrician and end user.

- Minimize ongoing perceived overhead costs and inconvenience to the end user, or in other words, systems should be simple to understand and use.

Local control functions do not need to be integrated into a whole building system in order to work effectively. For monitoring purposes, they might be connected, but their value for energy savings is not clear. Problems encountered when trying to integrate local control into large networks include:

- $\quad$ Too many control nodes: the large number of individual ballasts and lighting fixtures adds excessive cost and complexity to systems. Such individualism is usually not needed anyway because fixtures are mostly operated/controlled in groups as part of an overall lighting design.

- The need for immediate response/action places excessive demand on bandwidth and processing ability of a networked system. For example, a signal from an occupancy sensor must turn on a light within half a second or less. This is easily accomplished in a local system with a dedicated signal path, but in a networked system, a lot of processing and communication has to be done to determine which sensor is signaling, what action should be taken, addressing each component, receiving confirmations, carrying out network protocols, and gaining access to the communication medium. As more devices are added to the network, the reliability of getting a signal through within a given period of time diminishes.

- Attention to reliability is of much greater importance for an integrated system than for individual controls, which adds considerable cost. Problems with an integrated system threaten the whole operation of a building, while local control isolates problems to small areas.

Collective controls could benefit by being integrated with other building communication systems. With collective control schemes, the number of control nodes is greatly reduced by collecting individual lighting ballasts/fixtures into groups. This does not strain the limitations of existing networks. In addition, large groupings of fixtures usually do not require immediate action/response. The cost-benefit of integrating collective control is that existing hardware and network infrastructure can be shared across all building control systems. 
Trying to apply a single solution that is capable of handling all the requirements of the different control strategies is not cost effective. Such a strategy will result in an overbuilt system where most of the resources are hardly, if ever, used, and so consequently the system is too expensive and/or complicated to use. Therefore, it should be recognized that whole building lighting control systems might not be appropriate for all buildings now, until costs come down.

The ultimate level of control being pursued by the lighting industry is the individually addressable ballast, ideally with full-range dimming, as well as switching capabilities. Listed below (Table 5) are the key features and applications of a ballast/control system that meets this ideal. Meeting these features goes well beyond ballast design and hardware issues. They are also critically dependent upon the communication protocol used for implementing whole-building control, the commissioning of the system, and other building aspects such as daylight availability and auxiliary control devices.

It is not expected that all buildings need, or even desire this level of control, but the more features that can be packaged into a system presumably increases its market appeal, if other factors, namely cost and reliability, are not affected. The other advantage of an all-in-one approach is that makes life simpler for the specifier and improves interoperability because fewer product types need to be offered. Manufacturers also benefit from having to support fewer product lines. Most likely, though, cost and reliability are affected by making a system more universal. In this case, the market will eventually sort out what features are most beneficial and cost effective. Assuming that the trends for increasing space use intensification, energy costs, and state and federal building code legislation continue, many of the features that are now considered nonessential may become perceived as mandatory, as they already are to some extent in other countries for intensively used, urban office space.

Table 5. Key features and applications for individually addressable luminaires ${ }^{\mathrm{A}}$

\begin{tabular}{|l|l|}
\hline $\begin{array}{l}\text { Testing and monitoring of emergency } \\
\text { luminaires }\end{array}$ & $\begin{array}{l}\text { Photosensing } \\
\text { Control of window shading devices } \\
\text { Load shedding }\end{array}$ \\
$\begin{array}{l}\text { Lamp failure reporting } \\
\text { Interchangeability }\end{array}$ \\
$\begin{array}{l}\text { Reduced / zero wiring } \\
\text { * See notes for further explanation }\end{array}$ & \\
\hline
\end{tabular}

Notes:

A. Individually addressable luminaires allow end-users to organize workplace lighting into functional groups, so that switching / scene setting corresponds to the organization of the workplace. Intensively-used (usually urban) office space is frequently repartitioned or reorganized to adapt to the changing requirements of businesses and addressable systems allow the lighting to be reconfigured easily as this happens.

The end users should be able to either carry out simple lighting reconfiguration themselves (adding new luminaires, changing functional groups, changing scene settings), or have reconfiguration carried out by their regular qualified electrician, rather than having to contact the control system manufacturer. This is desirable both in order to reduce wasted time for the end-user, and to reduce the overhead costs of lighting controls manufacturers, which are recovered by adding (often very significantly) to the capital cost of lighting controls hardware, thus raising a barrier to more widespread use.

Because large buildings often house high salary workers at high occupancy densities, disruption is costly and, as far as possible, readdressing of luminaires should be carried out without access to the ceiling being required.

B. Lamp failure reporting facilitates quick replacement of failed lamps, which minimizes occupant dissatisfaction, maintains the architectural appearance of the building, and fulfils the end-user's health and safety obligations.

Assuming that he cost of electrical and electronic hardware will continue to fall in comparison to the cost of labor, the necessity of installing additional signal wiring will become an increasingly significant barrier to the use of control systems. This problem may soon be solved by wireless communications, but in the short term it is desirable for lighting control protocols either to allow the amount of wiring to be minimized, and/or for their signals to be sufficiently robust to allow signal wires to be run with lighting power in order to minimize the cost or cable or conduit. 
C. Under the National Electric Code (NEC) 2002, conductors of different circuits rated at 600V or less (both ac and dc) are permitted to run in the same cable (300.3(C1)), so - barring regional variations - wiring of this type, though not common, is technically feasible.

D. Interchangeable systems are those in which components from a variety of different suppliers can be used as part of an integrated system. Conversely, proprietary systems are those with which only a single supplier's components are compatible. Due to the technical difficulty of achieving interoperability, and the absence of a perceived need for interoperability among lighting specifiers, proprietary systems remain the worldwide norm for lighting (though not for HVAC systems, which don't require instant responses and usually require far fewer addresses). Nevertheless, interchangeability remains a desirable goal for clients nervous about having only a single source for future replacement or upgraded parts. However, it remains to be seen whether interchangeability will founder on the contractual rocks of having to allocate responsibility or blame when components turn out not to be interchangeable in real life. The related issues of "interoperable" systems are a partial solution not addressed in this report.

A full report regarding protocols and their value to lighting controls systems is included in Appendix H: Communication Protocols to Meet the Needs of a Fully Integrated Lighting and Whole Building Control Protocol.

\section{Task 4.2: Investigate ideal lamp light output dimming ratio required for different types of applications}

Task 4.2 is concerned with switch-dimming, step-dimming and continuous-dimming in spaces dominated by electric lighting. Switch-dimming is switching one or more lamps off within a luminaire; step-dimming is dropping power to all lamps within the luminaire. In every case, the discussion is limited to linear, T-8 fluorescent lighting systems operated with electronic ballasts. Although, consideration of daylight from windows and skylights is implicitly excluded from this task because, ideally, an occupant will not perceive reductions in light levels if a successful photoelectric dimming system is installed, the system performance discussion is applicable to photoelectric dimming as well.

\section{Lighting to meet human needs}

Prescribed illuminance levels are offered by sanctioning bodies such as the Illuminating Engineering Society of North America (IESNA) (Rea, 2000). It is formally acknowledged by the IESNA that a prescribed illuminance for nominally identical spaces will, in practice, vary. Naturally, photometric measurements show statistical variation and, moreover, humans are poor detectors of small changes in light levels, measured either in terms of subjective judgments of brightness or of objective measures of performance. Several recent studies by Kryszczuk (2001), Shikakura et al. (2001) and Akashi et al. (2002a, Appendix 2-A) found that people cannot reliably perceive reductions of up to $20 \%$ from the original illuminance level. A series of experiments by Rea and his colleagues $(1986,1987,1991)$ show visual performance, defined in terms of speed and accuracy of processing visual information, does not vary by more than a few percent for most reading materials found in commercial spaces (Dillon et al., 1987). Since variations in visual performance are small for the range of illuminances presently recommended (300 to $1000 \mathrm{~lx}$ ), task performance, which is dependent upon many non-visual factors, will vary even less (Boyce and Rea, 2001). Moreover, Rea et al. (1985) showed that people will perform compensatory behaviors (e.g., moving closer to the task) to ensure good performance while performing visual tasks. These studies indirectly support the IESNA position that variations of $+/-33 \%$ from the recommended illuminance can be considered as "the same" light level. Since these empirical data and the IESNA recommendations are consistent, it is reasonable to expect that occasional, modest dimming of the lights to lower levels to save energy or reduce electrical demand will be accepted by occupants and will not affect their performance. 
The IESNA also acknowledges variations in the difficulty of visual tasks performed in spaces as well as variations in the visual needs of different individuals. Further, there will always be some statistical uncertainty in any behavioral measurement. This provides still greater potential opportunity for dimming while continuing to meet occupant visual needs. Nevertheless, it must be acknowledged that for every opportunity to reduce recommended light levels, and thereby reduce energy or manage load, there is a risk that these reductions can penalize certain individuals performing certain visual tasks. Without question a dimming strategy can only be satisfactorily realized if those individuals and visual tasks are considered. This point is entirely consistent with the IESNA recommendations that lighting specifiers should not apply general recommendations without understanding the visual requirements in a specific application.

It is also important to realize that people are not rigid with regard to their preferences for changes in light level, even when the magnitude of those changes is reliably perceived. For example, most people find very dim lighting in high-end restaurants acceptable but, for nominally identical visual requirements, prefer much higher illuminance levels in cafeterias or lunchrooms. In a simulated commercial space, Akashi et al. (2002b, Appendix 2-B) showed that $80 \%$ of the people in his study would accept dimming to $62 \%$ of the original light level (a 38\% reduction, from $500 \mathrm{~lx}$ to $310 \mathrm{~lx}$ ). It is also clear that certain biases can be introduced into subjective judgments for one type of application, even in the same building space. For example, occupants will set illuminance levels in residential dining rooms differently depending upon the situation. Illuminance levels while feeding the family in the dining room will be typically higher than those while entertaining guests. In a simulated commercial space, Akashi et al. (2002b) showed that $80 \%$ of the subjects in his study were willing to accept dimming to $46 \%$ of the original light level (a 54\% reduction, from $500 \mathrm{~lx}$ to $230 \mathrm{~lx}$ ) under conditions where they felt they were helping their employer financially and helping the environment, both locally and globally.

As argued at the end of this task report, the evidence suggests that occasional dimming by 33\% will be readily acceptable to occupants and, if the purposes for dimming were explained well, occupants would also accept dimming by $50 \%$.

\section{Dimming Methods}

There are two approaches to dimming lamps within a building space, dimming all the lamps by a fixed amount or switching off some of the lamps. Although there is still much to learn about how lamp-ballast system combinations affect lamp life, much more is known about the impact of switching on lamp life than about the effects dimming on lamp life.

It is well established that on-off switching of fluorescent lamps will reduce lamp life (O'Rourke and Figueiro, 2000; Carriere and Rea, 1988). Sputtering is the primary failure mechanism associated with starting, and, except for very special cases, lamp life will be shorter the more frequently the system is switched on and off. Broadly, there are two ways to switch a lamp, with or without application of heat to the electrodes before starting. In general, heating the electrode before starting will reduce sputtering and thereby increase lamp life.

Instant start systems apply a high voltage across the lamp electrodes to start the lamp. These "cold starts" have been shown to increase sputtering of emissive coating on the electrodes which leads to shorten lamp life compared to continuous operation. Rapid start and program start systems apply heat to the electrodes prior to starting to minimize sputtering. Although the distinction between instant start and rapid start systems was useful for older T-12, magnetic ballast systems, this distinction is less clear today with T-8 electronic ballast systems. Empirical 
evidence has shown that lamp life is not significantly different for rapid-start and instant-start electronic ballast systems (Davis and Ji, 1998). The reason appears to be that although there is a distinction in how rapid-start and instant-start systems, as separate groups, actually start the lamps, there is little, if any, impact on lamp life. Although there is no formal definition for program start, commercially available systems called program start, in fact, control electrode heating prior to starting and significantly increase lamp life (Davis and Ji, 1998).

Switch-dimming can occur external to the ballast utilizing separate electrical circuits or with multiple-lamp ballast. Switch-dimming can be accomplished with tandem wiring ballasts of adjacent fixtures or with ballasts that control multiple lamps within a fixture. Separate electrical circuits, one of which could receive a signal to shed load, could supply these ballasts. This option is independent of the ballast, and any ballast type could be used, but this approach may be a better solution for new construction or a major renovation because rewiring an existing installation would not be cost effective. It is also possible to switch lamps independently of each other with multiple-lamp ballasts. Turning off one lamp in a two-lamp, three-lamp, or four-lamp ballast results in 50\%, 33\%, or 25\% dimming, respectively. Lamp life will, of course, depend on the frequency of switching, but it will also depend upon the particular lamp-ballast combination used, and although the relationship between switching and lamp life is better understood than the impact of step- or continuous-dimming on lamp life, there is still great uncertainty in predicting lamp life and, thus, life cycle costs.

The impact of continuous- and step-dimming on lamp life is less well known, as previously mentioned. However, the principles affecting lamp life remain the same. Namely, electrode heating is the key issue to consider during lamp operation in the dim mode. Filament temperatures below approximately $700^{\circ} \mathrm{C}$ will be associated with sputtering of the filament emissive coating, whereas temperatures above $1000^{\circ} \mathrm{C}$ will be associated with evaporation of the emissive coating. Between these two temperatures, the spatial-temporal effects of dimming are not well understood.

Rapid-start and program-start ballasts are currently the only ballasts used for dimming. These ballasts add heat to the electrodes while operating, as well as for starting, to minimize sputtering and extend lamp life. Dimming ballasts account for about 1\% of the fluorescent ballast market, presumably because of their relatively high cost. An instant-start ballast sells for between $\$ 8.00$ and $\$ 15.00$, while a dimming program start ballast, the most expensive type, may cost well over $\$ 50.00$. These ballasts also use more energy at full light output than an instant-start ballast because of electrode heating and the additional dimming interface circuitry, they may save energy relative to fixed-level systems if they are dimmed to at least $15 \%$ of full light output.

Instant-start ballasts make up $80 \%$ of the market of electronic ballasts sold today. This ballast design is the simplest and the cheapest, which is why it is also the most prevalent. Instant-start ballasts do not provide heat to the filaments while starting or operating the lamps when dimmed. The lower filament temperatures of instant-start systems are believed to cause greater sputtering of emissive coating from the lamp electrodes, which leads to shorter lamp life.

This logic would preclude the development of instant-start dimming ballasts and, indeed, an instant-start dimming ballast cannot be found in the specification market. Consider, however, that ballast factor simply describes the fixed level of dimming offered by the instant-start system. Since manufactured ballasts with different ballast factors have the same rated life, it should be 
possible to actively dim instant-start ballasts within the range of currently manufactured ballast factors.

Ballast factor (BF) is the ratio of the light output of a lamp or lamps operated by a specific ballast to the light output of the same lamp(s) operated by a reference ballast. BF can range from 0.73 to 1.50 (Lighting Research Center, 2000). Thus, the range of “fixed" dimming currently offered by instant-start systems is $73 \%(0.73 / 1.00)$ or $49 \%(0.73 / 1.50)$, depending upon the ballast used as the reference. Again, however, there appears to be a wide range of continuous- or step-dimming instant-start systems that are possible, but active dimming with these systems has not been explored. Naturally, the big advantage of instant-start dimming is the relatively low cost of the ballast. If it could be shown that lamp life was not significantly affected by continuous- or step-dimming of instant-start systems, then the life cycle cost of dimming would be significantly reduced, due to the low initial costs and high efficacy over the life of the system.

As mentioned several times, there remains uncertainty about the performance of switching modern electronic lamp-ballast systems. Dimming systems are even less well understood and there are no standards to guide the manufacturers or the specifiers to ensure satisfactory system performance. It is beyond the scope of this section to describe the physics of electrode heating, but it can be emphasized that reducing filament heat during dimming is a distinctly non-linear process, exacerbated by variations in manufacturing. So called “deep dimming” without heating the electrodes certainly adds complexity to the issue of dimming, but it appears that dimming by $33 \%$ or, perhaps, even 50\% may have no measurable impact on lamp life, for short periods of time (less than 100 hours/year).

\section{The optimum dimming ratio}

The optimum dimming ratio will be driven by maximizing lighting system performance while minimizing negative impacts on occupants within the building space. Human factors research suggests that occupants have difficulty in detecting dimming of $20 \%$ from recommended levels of illuminance, and that the majority (80\%) of people will readily accept dimming of 33\%.

Moreover, under some conditions the majority (80\%) will accept dimming of 50\% (Akashi et al., 2002a and 2002b). As noted several times, there are some uncertainties about lighting system performance with electronic ballast systems not found with the older, obsolete magnetic ballast systems. Without question, more research is needed to understand lamp-ballast interactions before a precise optimum dimming ratio can be deduced. Nevertheless, it is useful to develop some interim recommendations that could lead to more widespread use of technologies that should improve life cycle cost of fluorescent dimming systems through lighting controls.

Perhaps the most obvious approach is to utilize existing program start systems that maintain electrode heating during dimming. The major strength of this approach is that these systems ensure customers will achieve rated lamp life. They can also serve as a platform for "deep dimming" for maximizing photoelectric and user-controlled dimming, as well as any other control strategy. The largest barrier to this approach is that these systems are expensive to purchase, and they are slightly more expensive to operate because they have slightly higher energy expenditures due to lamp electrode heating. Since clear economic benefits of photoelectric dimming and of user-controlled dimming have yet to be established, this strategy will probably continue to capture only a few percent of the market.

A more cost effective strategy is switch-dimming, either with different circuits or with ballasts that switch out certain lamps. This approach reduces initial cost considerably but does not allow 
for added functionality achieved by the program start dimming system. If added functionality is not required, or expected, a switch-dimming approach appears to be a very logical line of development. It should be noted, again, that the effect of switching on lamp life is better understood, but there is still some uncertainty in the performance of electronic ballast and lamp systems and more research should be undertaken. There is also some uncertainty to this approach regarding user acceptance. One objection to switch-dimming and to step-dimming is that occupants have been shown to be dissatisfied with sudden transitions in light output (Boyce, 1984). Another objection might be that occupants do not like to see luminaires with deactivated lamps. Nevertheless, as shown by Akashi (2002b), it may be possible to adjust occupant expectations such that switch-dimming can be acceptable under some circumstances, but this has yet to be shown.

Another approach to dimming that has not been properly explored is continuous- or stepdimming of instant-start systems. The largest appeal to this approach is that these systems are the least expensive. Strategies for new construction and retrofit would seem to be cost effective if it was established that modest dimming to no more than $50 \%$ of maximum light output for less than 100 hours/year did not significantly affect lamp life. Preliminary evidence from our laboratory suggests that operating lamps in a dimmed mode ( $90 \mathrm{~mA}$, or a ballast factor of 0.44$)$ is in fact possible for periods longer than 100 hours/year. Certainly, commercially available ballasts with a ballast factor of 0.73 are warranted at rated lamp life, so operating them at $27 \%$ dimmed mode (BF of 1.00) appears to be readily achieved without significantly affecting lamp life.

Whatever approach is taken, it would be useful to standardize on dimming levels. We recommend that the industry standardize on $33 \%$ and $50 \%$ dimming. Both the lighting system performance data and the human factors data suggest that these values will have minimum negative impacts on lighting system performance and on occupant satisfaction and performance. The major advantage of this standard would be to accommodate switch-dimming of two, three and four lamp systems as well as both instant-start and program start dimming systems, utilizing either continuous- or step-dimming. ${ }^{2}$ Naturally, the cost of dimming will vary depending upon the lamp-ballast system, but every approach can be accommodated by this standard. The marketplace will determine which system will ultimately be more attractive.

To understand to potential for a load shedding ballast system used to reduce peak electrical demands, two studies were undertaken. These studies are presented in Appendix I: Energy Savings for Load-Shedding Ballast for Fluorescent Lighting Systems and Appendix J: The Effect of Bias Towards Energy Savings on Occupant's Dimming Requirements.

\footnotetext{
${ }^{2}$ It must be noted, however, that dimming light levels is only equal to dimming power for switch-dimming and for continuous- or step-dimming without electrode heating (i.e., instant start systems). For the same reduction in power using electrode heating (i.e., rapid start or program start), the light levels will always be relatively lower than they will be for instant start systems.
} 


\section{Tasks 4.3, 4.4, and 4.5: Investigation of the effects of dimming on fluorescent lamp life}

\section{Introduction}

The overall objective of these tasks are to identify and seek to reduce the barriers to wide acceptance and use of energy-saving daylighting, electric lighting, and control technologies, including occupancy sensors, photosensors, dimming electronic ballasts, and whole building integrated control systems. The following are detailed descriptions of the scope of work from tasks 4.3, 4.4, and 4.5:

- $\quad$ Task 4.3 - Investigate critical performance factors, such as optimal electrode voltage vs. percent rated current required maintaining electrode hot spot temperature. The focus will be on popular 32-watt, T8 lamps, and on the performance of rapid-start dimming ballasts.

- $\quad$ Task 4.4 - Investigate the impact on lamp life of starting lamps when dimmed, i.e., at lower than rated current. This is related to lamps starting under dimmed conditions.

- Task 4.5 - Investigate the allowable dimming range that can be achieved without additional electrode heating voltage. This will enable lower cost, instant-start dimming ballasts for load shedding, lumen maintenance, etc. This task requires investigation of instant-start dimming ballasts for load-shedding applications.

Overall, these three tasks required the LRC to investigate the interaction between dimming ballasts and fluorescent lamps, and to identify the impacts of dimming on fluorescent lamp life. This knowledge will be used to help reduce the barriers to widespread use of electronic dimming ballasts. A full report of the findings is included in Appendix K: Investigation of the Effects of Dimming on Fluorescent Lamp Life.

\section{Lamp failure mechanism}

The failure of fluorescent lamps is caused mainly by the loss of the electron emissive coating of the lamp electrodes (den Hoek, 2002, Verderber, 1985, Waymouth, 1971). Under certain circumstances, such as high frequency operation and frequent starting on instant-start ballasts ("cold ignition"), fracture of the tungsten coil is also observed, which causes the lamp to fail (Haverlag, 2002). Electrode temperature directly affects the evaporation and erosion of the emitting material and thus the lamp life. A very high electrode temperature (greater than $1000^{\circ} \mathrm{C}$ ) will reduce lamp life due to evaporation of the emitting material, and a low electrode temperature (less than $700^{\circ} \mathrm{C}$ ) will reduce lamp life due to erosion of the emitting material by sputtering ( $\mathrm{Ji}$, 1998).

With the introduction of low-mercury content lamps it is possible that lamp life, when over 20,000 hours, might also be influenced by a reduction of available mercury within the lamp. Since there is a strong movement in the industry to limit the mercury content of fluorescent lamps, some new lamp designs are manufactured with near the minimum amount of mercury needed for an efficient mercury discharge. Over time, free mercury in the lamp bonds to other materials within the lamp envelope, especially the sputtered and/or evaporated electrode material that gets deposited over the inside lamp walls (personal conversation with lamp manufacturer representative). For lamps with excess mercury added, mercury absorption is not an operational problem because the lamp electrodes limit life before the excess available mercury is removed. 
However, lamps with marginal amounts of mercury dosing, combined with severely sputtered and/or evaporated electrodes may show different end-of-life behavior.

\section{Optimum electrode heating as a function of the discharge current}

Fluorescent lamp electrodes are designed to operate at a temperature high enough to support thermionic emission of electrons when they are operated near the rated discharge current. Dimming a fluorescent lamp by reducing the lamp current will reduce the cathode hot spot temperature. The consequence will be an increase in cathode fall voltage that is sufficient to increase the electrode temperature again by increasing the energy of the ions reaching the electrode. This may result in a higher electrode sputtering rate and lead to an early lamp failure. At the same time, however, the reduced discharge current requires less electron emission from the cathode. This reduced requirement somewhat offsets the lack of cathode heating, but because cathode electron emission is highly non-linear with electrode temperature, cathode fall voltage still rises upon dimming. At lamp discharge currents in the range of a few percent of the rated current value, lamp life may drop down to values of only a few days.

In order to avoid these negative effects on lamp life when dimming, there is the possibility of applying supplemental electrode heating current, which goes through the electrode filament and heats it by resistance heating to the temperature that is necessary for thermionic emission. Obviously, the lower the dimming level at which the lamp is operated, the higher the supplemental electrode heating current required. However, the electrode heating current should not be too high. The guiding principles are:

- Too little heat reduces lamp life due to sputtering.

- Too much heat reduces lamp life due to a high rate of the emission-mix evaporation.

Following these arguments, an optimum supplemental electrode heating current (or electrode voltage) exists for lamp dimming as a function of the discharge current and must be maintained in order to keep lamp life within a reasonable range. Various approaches for the determination of this optimization are possible through the following measurements:

- Lamp life at various levels of the discharge current and different values for the electrode heating current

- $\quad$ Electrode temperature (overall hot spot location) at different discharge and supplemental heating currents

- Evaporation of barium from the electrode at different discharge and supplemental heating currents

- $\quad$ Cathode-fall voltage measurements at different settings of discharge and heating currents made by the following techniques:

o Langmuir probe

o Capacitive coupling

o Measuring the lamp operating voltage

The first method yields the most reliable results as it is a direct measure of life and can match conditions as closely as possible to the situation the lamp user would experience. Unfortunately, 
results from such measurements will only be available after two to three years of testing with much effort and at great expense.

\section{Impact on lamp life of starting lamps when dimmed}

During the instant-start and rapid-start starting processes, the lamp undergoes a transition from cold cathode glow discharge to hot cathode discharge. The cold cathode glow discharge is formed when the cathode is capable of emitting electrons only by ion-impact-induced secondary electron emission. Even though the discharge might be self-sustaining, the current near the cathode is carried mainly by positive ions. As a result, each electron that leaves the cathode must cause the production of many ions at a point near the cathode. For this to occur, a very high cathode-fall voltage must exist-often several hundred volts.

In the case of hot cathode discharge, the current at the cathode is carried mainly by thermionically-emitted electrons; positive ions are needed only to neutralize electron space charge and provide a modest electron-accelerating field at the cathode surface. The necessary ion production is much less than one ion per electron. The cathode fall voltage is therefore very small, typically on the order of the ionization potential of the atoms of the gas (5 to 10V). Figure 1 illustrates the potential versus distance for cold cathode and hot cathode discharges operated at the same current in similar tubes at the same gas pressure $(60 \mathrm{~Hz})$ (Waymouth, 1971). The positive column potential drop, $\mathbf{V}_{\mathbf{p}}$, and anode fall, $\mathbf{V}_{\mathbf{A}}$, are the same, but cathode fall voltage, and therefore total potential, are substantially greater for the cold cathode discharge.

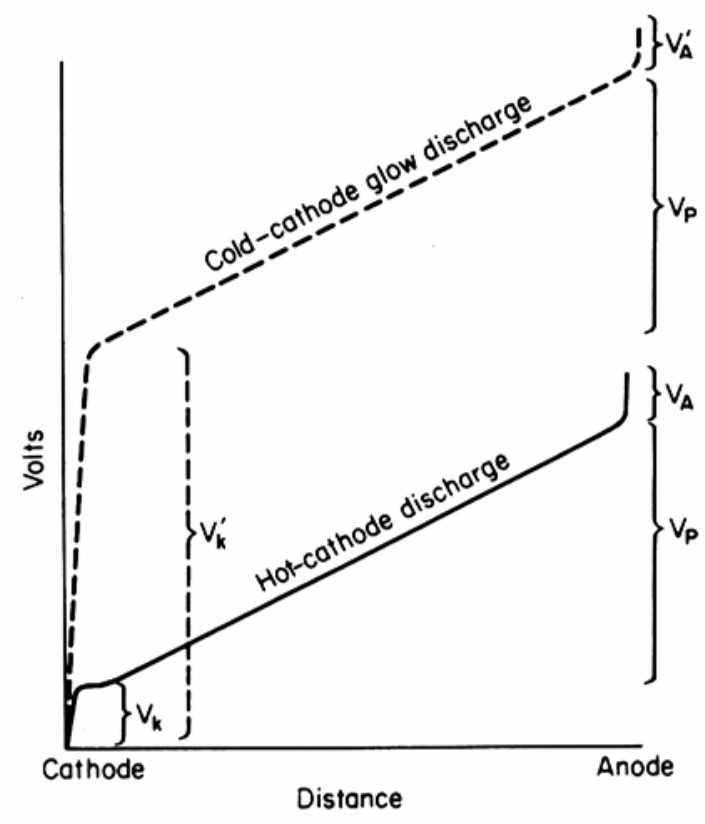

Figure 1. Potential versus distance for cold cathode and hot cathode discharges (60 Hz operation)

In the usual range of glow discharge conditions encountered in the process of lamp starting, the cathode fall voltage increases with rising current (Brown, 1959). This helps the transition from cold to hot cathode discharge by increasing the energy input to the cathode from the discharge. The positive ions reaching the cold cathode strike it with kinetic energies equal to the charge times the accelerating potential, which can be as high as the full cathode fall voltage. Most of this energy goes into heating the cathode, which increases the cathode temperature. The higher 
the cathode fall voltage and the ion current, the faster the cathode is heated to thermionic emitting temperature and the sooner the discharge is converted to a hot-cathode discharge.

When starting the lamp with an instant-start ballast at its rated lamp current, a sufficiently high potential is applied to the lamp to ionize the gas and to reach full operating current without a temporary pause in the glow state. The discharge current and lamp light output reach their normal operating values typically well before the $100 \mathrm{~ms}$ time limit measured after the application of the open-circuit voltage as defined by ANSI. The instantaneous energy input to the cathode during the transient glow-discharge-like condition that persists while current is increasing to normal operating current is very high. The rate of change of cathode surface temperature has been described as "almost of explosive violence" (Waymouth, 1971). As a result, during lamp starting, simple blasting off of chunks of cathode material by the drastic temperature rise will reduce the amount of coating remaining and would be expected to reduce lamp life. It is not known whether this potentially explosive heating reduces lamp life in practice, however. Most fluorescent lamp electrodes are designed with a coiled-coil or triple coil structure to help restrain the emission material so the lamp can last its rated life (typically 20,000 hours at 3 hours per start for T8 lamps) with no problem.

In the case of starting lamps when dimmed, i.e. at lower than rated current, the transition from cold-cathode glow discharge to hot-cathode discharge will most likely be extended due to the decrease of lamp current and the corresponding decrease in heating power available to the electrode. Although this may alleviate somewhat the violent cathode damage due to the explosive temperature rise, overall it is much more damaging on the cathodes because of the potential for a relatively extended period of glow discharge ion bombardment (sputtering). In the extreme case, when the lamp is dimmed to levels below about $20 \%$, the current in the steadystate glow discharge is too low to heat the cathode to a sufficient temperature to convert it to the hot cathode mode. The discharge will "hang up" in the glow state. The high-energy ion bombardment in the glow discharge knocks away surface atoms and erodes the cathode to the point of destruction in a short time. Waymouth (1971) explained this sputtering damage during glow discharge at starting as follows:

"Under glow-discharge conditions, with cathode fall voltages of $200 \mathrm{~V}$ or so, sputtering rates of 0.1 atom removed per incident ion would be quite typical. Besides, sputtering also damages the thermionic emission of the surface (even without significant material removal) in two ways. First, since the cathode is cold, atoms are sputtered away, bounced back on the cathode surface by the gas, and re-deposited helter-skelter instead of in an orderly way. Destruction of the ordered surface of the crystal must be expected to increase the work function and thus reduce thermionic emission. Second, tungsten atoms sputtered from the surface of exposed tungsten are deposited on the surface of the oxide almost as readily as on the tungsten, "plating” the oxide with a metallic covering and increasing its work function. The resulting reduction of accelerating field emission of the cathode means that the cathode fall voltage in the discharge must be higher to increase the ion current; the increased power input to the cathode increases its temperature to bring the electron emission up to the required level. The increased cathode fall voltage, ion current, and temperature persist for ten minutes to an hour after the damage, until the effects of the damage have been 'annealed out."' 
Figure 2 illustrates the relationship between lamp current and starting time for one Sylvania OCTRON FO32/735 T8 lamp operated by a high-frequency electronic ballast. It clearly indicates that start time increases exponentially with the reduction of lamp current.

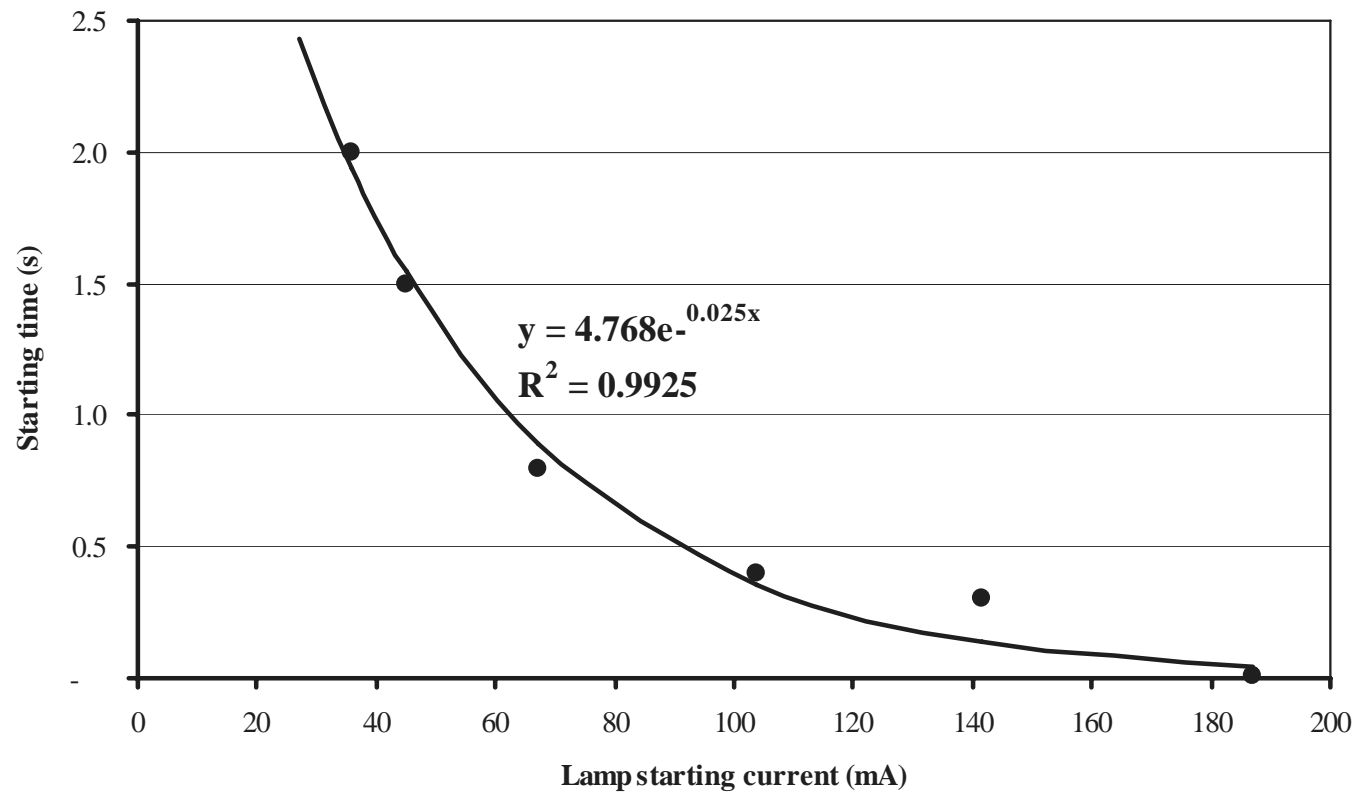

Figure 2. Relationship between lamp starting current (mA) and starting time (s)

Also, it is observed after each start that the cathode operates for a significant time at a higher temperature than it does under continuous-burning conditions. This period of increased temperature greatly increases the evaporation rate of cathode coating material, and consequently the overall loss rate of coating material, leading to shortened lamp life.

Technically, it is possible to apply supplemental electrode heating current to heat the electrode before starting lamps when dimmed. This is similar to the situation of rapid-start or programmed start fluorescent lamp. However, this is at the expense of efficiency, and/or initial cost. The possibility also exists that the intended supplemental electrode heating current is not effectively applied in all cases. LRC researchers found that in actual applications, connections and excessively long, tightly bundled wire leads reduce the amount of heating that actually takes place. On at least one LRC project this condition seriously reduced lamp life when traditional dimming ballasts that employ supplemental electrode heating were used (LRC, to be published).

Therefore, starting lamps when dimmed, i.e., at lower than rated current, is not recommended. To ensure long life, especially when supplemental electrode heating is not applied (e.g., instantstart), a procedure of starting the lamp at its nominal full output level and then dimming it down to the desired light levels is necessary. Starting at full power will minimize the damaging effects of starting. This is advisable whether supplemental electrode heating is applied as part of the starting sequence, but most critical without supplemental electrode heating.

\section{Allowable dimming range without additional electrode heating}

In ac operation, when no separate heating of the cathode is provided, most of the cathode heating arises from electron bombardment during the half-cycle when the electrode is acting as anode. 
Some additional heating results from ion bombardment and from the resistance losses (loss = $I^{2} R$ ) in the tungsten wire core caused by the discharge current flowing in through the leads and out through the cathode surface. In these cases, the cathode heat developed is positively related to the arc current being drawn. The main heat loss comes from the conduction to adjacent elements, the electron emission cooling, radiation cooling, and convection cooling of the electrode surface. Although dimming can reduce the electrode heat loss from the reduction of electron emission cooling, this reduction of heat loss does not compensate completely for the reduction of electrode heating.

Obviously, a certain amount of variation in lamp current is entirely permissible and results in no unfavorable performance. This is demonstrated by the fact that ballasts with ballast factors ranging from 1.3 to 0.71 are widely used and appear to maintain lamp life acceptably.

Nevertheless, any cathode operates best at some definite value of lamp current. A cathode that is properly designed for best operation at $225 \mathrm{~mA}$, for example, will run too hot at $400 \mathrm{~mA}$ and entirely too cold at $75 \mathrm{~mA}$. If such a cathode is operated at too high a current and temperature, results may include excessive vaporization of barium, discoloration in the form of anode spots, and reduced lamp life. If the cathode is operated at too low of a current, too high of a percentage of the total emission must be obtained by ion bombardment, field emission, or some mechanism other than thermionic emission. This method of operation is conducive to an excessive cathodefall voltage, greatly increased cathode sputtering by ion bombardment, and in extreme cases, very short life.

It has also been found that sputtering under continuous operating conditions is small under fairly wide variations of lamp current. Wehner (Stuart and Wehner, 1962) found that at a cathode fall voltage less than $20 \mathrm{~V}$, the electrode sputter field is quite small, about $10^{-6}$ atoms removed per incident ion. In addition, there is little cathode temperature dependence in the sputtering yield (Stuart and Wehner, 1962). A great deal of the electrode heating energy comes from the collection of electrons in the anode half-cycle, and due to thermal inertia, the electrode does not cool down between its half-cycle of cathode duty. During operation, evaporation normally occurs at about a factor of 20 times greater than the rate of removal by sputtering. Therefore, sputtering can be ignored entirely in comparison with evaporation as a cause of coating loss under continuous burning conditions at nominal lamp currents. A lamp should be able to preserve a reasonable life within fairly wide variations of lamp current if it is started at its full rated current, and then dimmed down to the desired light level after it has reached its full light output. Now the question is: how much dimming is allowable for such reasonable lamp life?

The LRC performed a series of experiments that continue even today to answer this question. As of November $6^{\text {th }}, 2002$, the lamps had accumulated 93 days of operation (2139 hours). By this date, all lamps operated at $49 \mathrm{~mA}$ and below had reached their median life (50\% had failed). At the other extreme, all lamps operated at or above $121 \mathrm{~mA}$ were still operating. For the group at $64 \mathrm{~mA}$ operating current, only one lamp had failed by this date. Figure 3 summarizes the results for the dimming testing of instant-start fluorescent systems. The results show that dimming to about one third of rated current (121 mA), a T8 fluorescent lamp operated with an instant-start circuit still keeps a reasonable long life (more than 2139 hours with no failures).

Using current electricity pricing practices and load shed programs, LRC researchers found that load shed-related dimming of 100 hours per year would capture most of the economic benefit. If the damage to the electrodes during dimmed operation is simply cumulative, meaning that no effects that accelerate the damage caused by dimming are occurring, then 2000 hours of dimmed 
operation is sufficient for load shedding applications. For example, a 20,000-hour lamp life corresponds to about 5.5 years of operation at 10 hours per day. If load-shed dimming amounts to 100 hours per year, then 550 hours of dimmed operation is required. This dimmed operation would be expected to reduce the life of the lamp by about $25 \%$ (550/2100), equivalent to a 15,000-hour life. A more exact calculation, taking into account the reduced dimmed operation time due to the reduced life yields a life reduction of only 19\%, corresponding to a life of 16,200 hours.

The $121 \mathrm{~mA}$ lamp operation has continued to remain in operation. All ten lamps that began the study continue to operate with no failures. They have accumulated over 30,000 hours of operation as of May 2006. This astonishing achievement leads the LRC to believe there will be no ill effects on lamp life from lamps operating on instant-start ballasts dimmed to $121 \mathrm{~mA}$.

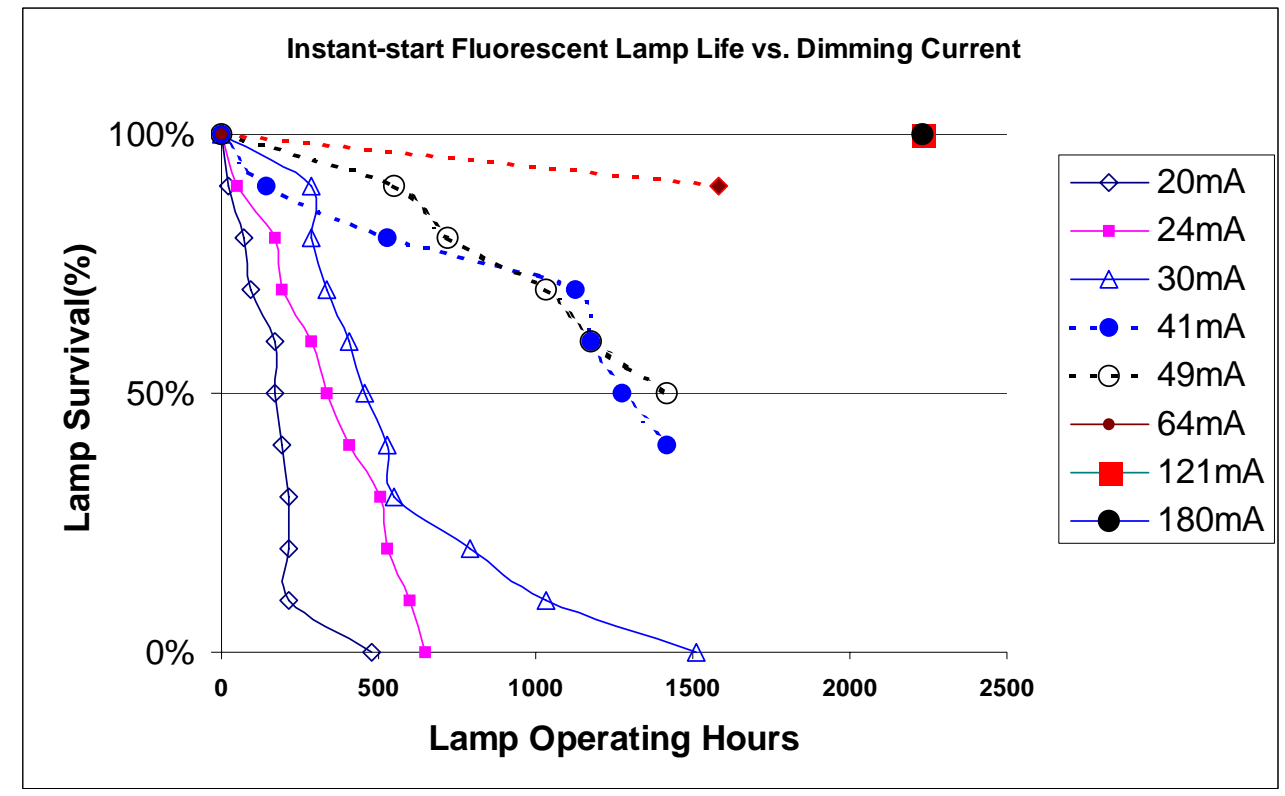

Figure 3. Instant-start fluorescent lamp life vs. dimming current

Task 4.6: Develop recommendations for improved components and systems as evaluated in Task 2, based on defined performance characteristics, and defined acquisition, installation and commissioning costs

\section{Summary of recommendations}

In the course of Task 2 of this project, three technologies were identified as having the potential to achieve greater market share, if certain barriers could be overcome. These technologies were the prototyped load-shed ballast, occupancy sensors, and an innovative easily-commissioned photosensor. To maximize their market potential, these technologies must all demonstrate their cost-effectiveness, their practicality in real installations, and their suitability to the market. Based on subsequent development of these technologies and analysis of the market, the following courses of action are recommended.

\section{Load-shed ballasts}


- $\quad$ Perform large-scale field trials of load-shed ballasts. These trials should aim to verify the acceptability of illuminance reductions to occupants, both when the reasons for load shedding have been explained to occupants, and when they have not. The process of informing occupants of the environmental and cost benefits of load shedding is referred to as "biasing”.

- Test the electromagnetic compatibility (EMC) of load-shed ballasts in their two normal operating states (i.e., full on and dimmed), and when switching between states.

- Work with one or more ballast manufacturers to produce a commercially viable load-shed ballast based on electronic instant-start technology. To be financially attractive to customers, the price of this ballast should be no more than $\$ 9$ more than a regular instantstart ballast.

- $\quad$ Test the prototype ballast as part of the LRC's National Lighting Product Information Program (NLPIP), and make the result of the testing available on the NLPIP website.

- Work with one or more ballast manufacturers to develop a suitable method to carry signals from a central controller within the building, to the individual ballasts. ("stage 2" signaling). This signal should not cause disruption (e.g., flicker) to the other lighting in the building.

- $\quad$ Ensure that the signals sent out by electricity suppliers are suitable to be received by lighting load-shed controllers ("stage 1" signaling). Both the format and the medium for these signals should be considered in detail.

- Develop a performance specification for load-shed ballasts that can be easily followed by commercial ballast manufacturers, yet ensures minimum performance standards.

- Leverage current and future market forces to create demand for load-shed ballasts. This can be achieved by promoting the electricity cost savings achieved using load-shed ballasts, and by promoting load-shed ballasts as an environmentally friendly and therefore desirable feature of a building, especially new-builds. This may take place through published articles, seminars, demonstration projects and voluntary accreditation schemes.

\section{Occupancy sensors}

- $\quad$ Publicize the aggregate figures for energy savings compiled as part of Task 4. Everyone in the building industry should be aware of typical figures for energy saving achievable by the use of occupancy sensors.

- Work with control system manufacturers to produce a commissioning standard for occupancy sensors. If all sensors can be commissioned using the same procedure, the likelihood of installers making mistakes on site will be much reduced, and these mistakes account for a lot of the failings of systems in situ.

\section{Easily-commissioned photosensor}

- Continue to use existing market channels, such as the LRC's NLPIP program and outreach education, to support the use of photosensing in those applications for which it is commercially viable. This is usually only in owner-occupied buildings with large window area and high occupation density. 


\section{Introduction}

Some technologies aimed at reducing lighting energy consumption and cost are already well accepted and reliably implemented by the building community, while others are constrained by real or perceived barriers to more widespread use, such as complexity, reliability or cost.

Examples of the former include "architectural” dimming, occupancy sensing in new-builds, programmed-start ballasts, time switching, and energy efficient lamps and luminaires. Examples of the latter include photosensing, occupancy sensing in retrofits, and load management systems. The LRC is primarily interested in determining which of these latter technologies may be able to graduate into the mainstream, with the help of technological improvements, human factors research, and market transformation efforts.

We are particularly interested in the comparatively neglected potential of innovative, low-cost, simple systems that may reach a wide market. Load-shed ballasts are a good example of a technology with low cost and potentially high benefit for consumers, electricity suppliers, and the country as a whole. The LRC's intention is to prepare the way for fast and sustainable market uptake of lighting load shedding systems, by ensuring that they are easy to specify and install, and that the cost-benefits calculations are highly persuasive - especially in an economic climate where companies have little spare cash for non-core investments.

This report deals mainly with load shedding systems. It is important to note that load-shed ballasts and other lighting control systems (such as occupancy sensing) are not mutually exclusive; either or both may be used in a space. Therefore, a concentration on load shedding is complementary to the LRC and DOE's existing commitments to promote other energy-saving strategies. Furthermore, most US office space is deep-plan therefore not amenable to daylight savings, and occupancy systems may not be appropriate in every space. Load shedding is therefore a complementary technology that may be added to other energy and cost-saving strategies.

This report goes on to deal with ways of expanding the market for occupancy sensors, and the market potential of a design for a new type of easily commissioned photosensor, which may be much easier to design, install and commission than existing types.

\section{Lighting opportunities in the deregulated power market}

At times of peak load, the cost of providing every additional kilowatt hour of power can be as much as \$1. This imposes a high cost on the nation, and should provide a high incentive for consumers to reduce load at peak times, but current electricity pricing structures prevent these costs being visible to consumers, so it is often not financially beneficial for building operators to install power-reducing systems. Forthcoming changes to the structure of electricity pricing, however, are likely to create a market for shedding load.

The Federal Energy Regulatory Commission (FERC) has proposed the following policy:

"Power markets should accommodate price-responsive load and demand resales, through market rules that give willing [wholesale] customers and their suppliers reasonable opportunity 
to adjust consumption in response to market conditions...FERC should promote transmission rate designs that reveal the cost of congestion across different times and locations"3

The cost of providing additional power to a consumer at a given location and time is known as the "locational marginal price" (LMP), and is usually measured in dollars per megawatt. LMP comprises the cost of generation, and the cost of transmission (including the fact that power may have to be transported to allow for regional disparities in capacity and consumption).

If consumers were charged the LMP for any extra capacity they required, many of them would ensure that their load was minimized at times of peak demand (and hence peak LMP). This would require an accurate and verifiable record to be kept of their electrical load hour-by-hour or minute-by-minute. Although verifiable timed metering is now comparatively cheap (typically less than \$200 to install), consumers have been reluctant to be charged the LMP for their additional power due to the possibility that it might increase their electricity bills and/or cause them to have to change their hours of work.

In the last two to three years, however, some regional transmission organizations (RTOs) have piloted "economic load response" schemes for large consumers (typically those whose peak demand exceeds $1 \mathrm{MW}$ ). Some of these schemes have gone on to allow consumers to bid in advance (typically one day in advance) for load reductions, thus creating a true market for power, with consumers able to engage in bilateral trading of available capacity. It is anticipated that participation in load response schemes will rapidly be extended to more and smaller consumers as hourly metering and data logging becomes less expensive.

In addition to these economic load response schemes, some RTOs have piloted emergency load shedding schemes ${ }^{4}$. These schemes are doubly useful in that they not only reduce generation costs, but also ensure adequate power quality and reliability, which are as important to RTOs as cost. The key difference is that, in the economic schemes, consumers have the prerogative to buy or sell capacity or capacity options at their own discretion, whereas in the emergency load shedding schemes, consumers must shed capacity when instructed to do so by the RTO.

Although a consumer may take part in both schemes, each separate load must be registered in either one scheme or the other - not both - because for the purposes of emergency load-shed, the RTO must be able to predict in advance the magnitude of the drop in demand.

Another key difference between economic and emergency load response schemes is that it may only be large consumers that find it worthwhile to participate in economic load response schemes, because of the overhead costs of personnel and of installing hourly metering in their premises. Emergency load shedding schemes, by contrast, because they are administered by the RTO instead of by the consumer, require little overhead cost and so comparatively small consumers may find it worthwhile to participate.

Small consumers are pivotal to the success of lighting load shedding because there are effectively no large lighting consumers; even a large office building with 1000 occupants will have the potential to shed no more than $50 \mathrm{~kW}$ - much smaller than the current minimum

\footnotetext{
${ }^{3}$ Federal Energy Regulatory Council (FERC). 4 January 2002. Demand-side resources and regional power markets: a roadmap for FERC (draft 2A). Accessed on 13 December 2002 at http://www.ferc.gov/electric/rto/mrkt-strct-comments/02-05-02/FERC-load-response-roadmap-02-05-02.pdf

${ }^{4}$ Such as PJM, a regional transmission operator (www.pjm.com)
} 
increment of $100 \mathrm{~kW}$ commonly used in current economic load response pilots. Therefore emergency load shedding schemes are more likely to be attractive to those seeking to save lighting electricity costs.

\section{Load-shed ballasts}

\section{Background: Who benefits from load shedding?}

Load shedding is not primarily a way of reducing energy consumption; rather it is a way of reducing the cost of providing power at times of peak electrical demand. Load shedding is only envisaged as occurring a few times per year, and only for brief periods ( $<100$ hours/year), so the number of kWh of electricity which can potentially be saved is small. Consequently, the environmental benefit is marginal, although it should be remembered that peak-demand electricity is particularly "dirty" to produce, because older, smaller, and therefore less efficient power stations are used to produce it, and that load shedding at peak times may obviate the need to build new power stations.

Load shedding also helps to relieve transmission congestion, and the need to transport electrical power from one region to another; hence, it reduces the inefficiencies associated with the transmission of power, and the need for extra capacity to cover contingencies such as the loss of transmission lines.

The direct beneficiaries of these cost savings would be electricity suppliers, although adjustments to the pricing structure of electricity should ensure that the cost savings are eventually passed on to consumers.

So load shedding would produce cost savings for the economy, and benefits for the environment. Lighting is an important part of any load-shedding strategy because it accounts for around $25 \%$ of national electricity consumption. To ensure that individual consumers find it profitable to include their lighting load in economic or emergency load response programs, the lighting industry (in the form of lamp and ballast manufacturers, and the LRC) should become involved in the discussions of pricing structure currently underway within the electricity industry.

\section{Human factors implications}

Load shedding using bi-level switching ballasts creates a comparatively fast reduction in workplace illuminance for occupants, taking place over only a few seconds, and lasting for around four hours. It is essential to determine whether these reductions are either noticeable or acceptable to occupants, before investing in the development of load shedding technology.

Experiments were conducted at the LRC to determine what magnitude of illuminance reduction is acceptable to office workers. The reductions took place over a period of around three seconds. The results showed that the degree to which subjects found illuminance reductions acceptable was affected by whether the reason for the reduction was explained beforehand. The reasons consisted of the potential for energy cost saving, and the environmental benefits. Subjects who had previously had the reasons explained to them were known as "biased" subjects. All of the "unbiased" subjects found a reduction in illuminance of $20 \%$ acceptable, and $80 \%$ of them found a reduction of $50 \%$ acceptable. All of the "biased" subjects found a reduction of 50\% acceptable, and $80 \%$ of them found a reduction of $75 \%$ acceptable. 
On the basis of this research, and previous research, the LRC has recommended ${ }^{5}$ that the development of load-shed ballasts should standardize on illuminance reductions of 33\% and/or $50 \%$, since these figures correspond to the switching off of one lamp in a three-lamp luminaire, or two lamps in a four-lamp (or one in a two-lamp) luminaire. These figures could be used as a basis for initial trials.

Field trials in real buildings would be required to verify the findings of the research already undertaken, and to ensure that "real world" factors such as the presence to daylight (perhaps as a brightness referent) do not result in lower acceptability ratings in practice.

\section{Power quality implications}

Sudden changes in electricity consumption, especially for inductive or capacitive loads, can cause harmonic distortions in the line voltage (electromagnetic interference), which can adversely affect other electronic equipment. The sudden reduction in electricity consumption caused by load shedding may produce effects of this kind. Further research should be carried out to ensure that harmonics do not adversely affect either the building's switchgear, or other connected loads.

\section{Route map for developing ballast technology}

The discussion above suggests that developing a load-shed ballast is both desirable and possible, though it must meet strict performance and cost targets.

The LRC's investigations into the effect of dimming on fluorescent lamp life ${ }^{6}$, and the constraints of cost (see below), suggest that electronic instant-start may be the preferred technology base for load-shed ballasts. Instant-start is substantially less expensive than other technologies (rapid-start, programmed-start), and does not appear to result in shorter lamp life under standard test conditions of 3 hours on, 20 minutes off.

Operating instant-start ballasts at a reduced ballast factor (i.e., dimmed), reduces lamp life marginally; in tests conducted at the LRC, dimming to $67 \%$ for 100 hours per year shortened life by less than $20 \%{ }^{7}$. These tests suggested that most fluorescent lamps can be operated without significantly reduced lamp life or efficiency, over a wide range of outputs, both below and above their rated output. The energy consumption of a ballast is described by its "ballast factor", where 1.0 means that the ballast is running at its rated output. Many lamps operate without detrimental effect on their life, at ballast factors between 0.7 to 1.3.

A further persuasive reason to build load-shed ballasts on an electronic instant-start platform is that instant-start currently constitutes the largest segment of the ballast market, and is therefore most likely to receive quick acceptance by consumers.

5 Lighting Research Center (LRC). November 2002. Reducing Barriers to Use of High Efficiency Lighting Systems, Task 4.2: Investigate ideal lamp light output dimming ratio required for different types of applications. Troy, NY. Report submitted to US Department of Energy.

6

6 Lighting Research Center (LRC). November 2002. Reducing Barriers to Use of High Efficiency Lighting Systems, Task 4.3-4.5: Investigation of the Effects of Dimming on Fluorescent Lamps. Troy, NY: Lighting Research Center. Report submitted to US Department of Energy.

${ }^{7}$ Ibid. 
Field trials in real buildings will require prototype ballasts to be developed, leading to a commercially viable mass-produced version, which should be tested under the National Lighting Product Information Program (NLPIP). The LRC will need to work closely with one or more ballast manufacturers in order to achieve this.

The report of Task 4.3 of this project projected that;

"If a load-shed ballast and its associated communication link can be sold...for an incremental cost of $\$ 9$ over an instant-start ballast...the market for this ballast would be approximately $10 \%$ of all ballasts sold in the new-construction / remodeling market." 8

\section{Route map for developing communication protocol(s)}

Experience from other lighting control technologies clearly shows that the communication protocol is a determining factor in the success or failure of a technology. A protocol must be immune to interference, must allow easy installation and reconfiguration, and compatible components must be widely available from a variety of competing suppliers. It must also be possible to add new components on to an existing system, so the protocol must be backwards compatible. This means that it is crucial to get the protocol right in the prototype stage, and avoid subsequent changes.

Two distinct communication signals are required within a load shedding system. Firstly the signal from the electricity supplier or RTO to the building's load-shed coupler (stage 1), and secondly the signal from the load-shed coupler to the individual ballasts (stage 2).

Some utility companies and RTOs have already developed stage 1 systems for sending out realtime price information to consumers via the utility's website, or via email ${ }^{9}$. Such a system could also send out instructions to shed load. For ease of commissioning, it may be preferable to use a standalone system such as cell phone short message service (SMS) to receive stage 1 signals, instead of attempting to link the load-shed coupler to the internet via a company's local area network. Stage 1 signaling should present no technical problems, and the nature of the signal will mainly be determined by the requirements of the electricity supplier or RTO - the LRC need not be overly concerned with stage 1 signals.

For maximum market penetration, stage 2 signals between the coupler and the load-shed ballasts should not require any additional wiring, and should require an absolute minimum of additional hardware, in order to minimize cost. The additional design effort required by electrical engineers and architects should also be minimal, in order not to create reluctance among specifiers toward the provision of load shedding ballasts. For these reasons, development will focus on the use of the building's electrical power network to carry signals to the load-shed ballasts; it would also be possible to implement the signal using wireless technology, but this remains expensive. The LRC recommends that stage 2 rather than stage 1 signaling is the focus of load-shed signaling development.

In a small number of buildings, there is already a whole-building lighting control system, connected to dimming ballasts via a protocol such as LONworks, BACnet or DALI. These

\footnotetext{
${ }^{8}$ Ibid, p.75

9 Independent System Operator New England (ISO-NE). 2002. ISO-NE Load Response Program Manual. This system sends information about forthcoming changes in the price of electricity, on an hour-by-hour basis. Load shedding would require minute-to-minute information.
} 
systems (especially DALI) may become more commonly used in the medium term, but it is worthwhile to check whether load shedding could be implemented in such a system using conventional dimming ballasts instead of instant-start load-shed ballasts. In this case, the lighting control system would simply instruct all ballasts to dim down so that their electricity consumption falls to $66 \%$ or $50 \%$ of maximum (their light output would be a slightly lower percentage of its maximum), so the result would be the same as in a building equipped with loadshed ballasts.

\section{Should specifications be developed for ballast and communication technology?}

Some kind of minimal specification for load-shed ballasts is required, for two reasons. First, if incentives or rebates are used to make load shedding attractive, then some minimum performance specification will be required in order to allow ballasts or luminaires to be classified as qualifying for the incentive. Secondly, if ballasts from different manufacturers are used in the same building, they must dim down to approximately the same level in order to preserve illuminance and luminance uniformity. The LRC currently recommends either $66 \%$ or $50 \%$ as the target light output level. However, a performance specification should be based around electricity consumption rather than light output, so that energy suppliers obtain a guaranteed reduction in load. The specification could simply require the ballast to reduce its electricity consumption to a certain target value within a narrow margin of error, while also achieving a target value for light output, with a slightly boarder margin of error.

Stage 1 signals are likely to be technologically easy to implement; to that extent, there is little need to worry about achieving a workable solution. Nevertheless, these signals warrant attention because it would be desirable for utility companies and RTOs to use the same medium and the same format for the load-shed instructions or real-time price information they send out to consumers. This would make it much cheaper and easier to implement lighting load shedding nationwide, rather than state-by-state or even provider-by-provider. This will require cooperation between a core of committed utility companies and RTOs at an early stage; the LRC and DOE are well placed to facilitate this process.

Stage 2 signals must be robust, should not cause lamps to flicker, and must not interfere with other building systems. During the early stages of development, it may be sufficient to engineer solutions on the fly using a loose specification, but at some stage, it will become necessary to develop a formal standard for the stage 2 protocol and signals, in order to ensure interchangeability between components from different suppliers. Developing a standard is a time-consuming process and all parties should be cognizant of the potential for this to delay the market penetration of load-shed ballasts.

\section{Field trials}

A prototype load-shed ballast has already been developed by the LRC and OSRAM SYLVANIA (OSI) for Connecticut Light and Power, based on a programmed-start ballast. Programmed-start ballasts maximize lamp life but may be too expensive to achieve the intended market share for load-shed. Development will therefore concentrate on cheaper technologies, but programmedstart remains an important complementary technology because it will allow load-shedding systems to be installed alongside other control systems, such as photosensing.

The power line signal used to control the existing prototype ballast is simple, requiring a significant change to the waveform for around two seconds; such a simple signal may however 
interfere with the operation of other connected loads, and might necessitate a completely separate circuit for load-shed ballasts - this would add highly undesirable costs.

For the forthcoming field trial, to be conducted in conjunction with the California Energy Commission, the envisioned prototype will be based on a cheaper instant-start ballast, and a more refined signal will be used. This prototype is likely to be trialed in the LRC's own offices.

One or more large-scale trials will be required, to ensure that the technology achieves the predicted cost savings, that it can be implemented on a large scale, and that the occupants of a large office behave in the way suggested by the human factors experiments already conducted, i.e., that they find the same magnitude of illuminance reduction acceptable. Such a field trial would have to be conducted with the active involvement of the building owners and occupants, to ensure that the occupants are informed of the reasons for the illuminance reductions.

It may be beneficial to work closely with a ballast manufacturer to find a suitable large office building in which to hold trials. The manufacturer could alert the LRC to forthcoming suitable sites on their order books.

In parallel, it may be desirable to trial a load shedding system implemented using a wholebuilding lighting control system. This also would require the LRC to liaise with lighting controls manufacturers.

\section{Market transformation}

Market forces (rather than legislation, tax breaks or other incentives) are the preferred vehicle for transforming markets. Government agencies repeatedly express their preference for existing or nascent market forces, as the means of bringing about market change ${ }^{10}$. The US in particular has a "successful policy of market reliance." 11 Energy efficiency standards or rebates should be considered only if there is no means by which the technology can be made commercially viable. In addition, technologies supported in significant part by legislation or government incentives remain commercially parlous because the structures that support them could be withdrawn or amended at any time.

One feature peculiar to the building industry is that the party buying or specifying the ballast is usually not the same party that will benefit from the electricity cost savings. This is especially true of buildings procured under design-and-build contracts, where no independent advocate for the client exists. This may present a hurdle to the use of market forces to expand demand for load-shed ballasts.

"Market forces" include:

- The electricity cost savings achieved by installing load-shed ballasts;

- The possibility of adding value to the building (in terms of resale or lease price or lettability) by installing a technology that is perceived as valuable either in its own right

\footnotetext{
${ }^{10}$ Simons, J. 1992. Energy efficiency standard in a market economy - regulation vs competition? In Proceedings of the International Energy Agency conference on Use of Efficiency Standards in Energy Policy 42-51. Paris 1992.

${ }^{11}$ Millhone, J. 1992. The Role of Efficiency Standards in the United States. In Proceedings of the International Energy Agency conference on Use of Efficiency Standards in Energy Policy 16-20. Paris 1992.
} 
(such as air conditioning) or as part of a voluntary building accreditation program (such as Leadership in Energy and Environmental Design (LEED)).

In the short term, before electricity price restructuring takes effect, it may be possible to begin to create demand for load-shed ballasts by including them into existing voluntary accreditation programs. These programs give each building a score based on an evaluation of its "green" credentials, and so allow building owners to more effectively market the added value of the energy-saving technology in which they have invested. So, tentatively, there are three stages in achieving widespread acceptance of load-shed ballasts by the building community:

1. Collect detailed data on the degree or type of price restructuring or incentive that utility companies, state energy agencies and RTOs offer consumers to install load-shed ballasts.

2. Communicate the financial and environmental benefits of load-shed ballasts to the building community; e.g., by reviewing successful projects in magazines and seminars.

3. Ensure that load-shed ballasts are mentioned in any future mandatory energy-saving legislation, best practice advice, and voluntary environmental accreditation programs.

The first of these stages is an ongoing activity of the $\mathrm{LRC}^{12}$. The second of these stages is referred to in the plan for the third year of the Reducing Barriers Project, although within that time frame there may not be any large completed projects. Continued funding will be required to ensure that successful installations are publicized.

The timescale for the third stage is somewhat determined by the slow natural frequency with which energy saving legislation and best practice advice are revised. Within this project, it may be possible to establish a precedent for incorporating ${ }^{13}$ load-shed ballasts into the LEED certification $^{14}$ for commercial interiors, a document which is still in draft form.

LEED is a voluntary, consensus-based national standard for developing high-performance, sustainable buildings. Acquiring LEED certification for a building demonstrates to potential buyers or tenants that the building has low energy consumption, a high degree or airtightness, and certain environment-oriented facilities of benefit to staff (such as bike racks, showers). The LEED certification is similar to the BREEAM energy rating method developed for commercial premises in the United Kingdom in the mid-1990s. Experience from the BREEAM project suggests that voluntary accreditation can work effectively if the certification is seen to benefit the occupier, and is sufficiently onerous to garner support from environmental pressure groups. Uptake of the BREEAM rating on new-build offices in the UK reached and maintained a level of $25 \%$ of floor space within three years of the scheme's inception, and it remains a widely known and respected indicator of environmental and build quality.

\footnotetext{
${ }^{12}$ Lighting Research Center (LRC). January 2002. Reducing Barriers to Use of High Efficiency Lighting Systems, Task 4.3 Appendix A: Market Analysis for Load Shedding Ballasts. Troy, NY: Lighting Research Center. Report submitted to US Department of Energy.

${ }^{13}$ The US Green Buildling Council has indicated that load-shed ballasts could initially be eligible for an "innovation credit” within the LEED scheme, and could then be further considered for formal inclusion.

${ }^{14}$ U.S. Green Buildings Council (USGBC). June 2002. Rating System for Commercial Interiors, Un-balloted Draft for Pilot Program. Accessed on 7 November 2002 at http://www.usgbc.org/docs/LEEDdocs/LEEDCIvPilot.pdf
} 
Voluntary accreditation schemes are complementary to price restructuring approach, since price restructuring would affect mainly owner-occupiers, while voluntary accreditation would affect mainly property developers and property owners.

\section{Development of the market for occupancy sensors}

The technology of occupancy sensors is mature enough to allow accurate data on its energysaving potential to be gathered. To expand the market, this dataset needs to be communicated to specifiers and end-users.

Figures compiled by the LRC from a wide variety of sources indicate that the potential energy savings achievable by occupancy sensing range from a mean of $25 \%$ for shared (e.g., open-plan) spaces, to $40 \%$ for privately "owned” spaces such as private or partitioned offices. These figures are for the energy saved in comparison with each area's lighting energy consumption prior to the installation of the occupancy sensing system, relative to an eight to ten-hour working day. In relation to these substantial savings, the occupancy sensor market remains notably underdeveloped.

Widespread publication of these figures in industry magazines and via other communication channels, will bolster market demand for occupancy sensing systems.

A previous investigation ${ }^{15}$ conducted by the LRC indicated that the difficulty of commissioning occupancy-sensing systems was a significant barrier to their more widespread use. To overcome this barrier, a commissioning standard should be developed in co-operation with key controls manufacturers (already identified ${ }^{16}$ ). This standard would include requirements for at least the following; the process by which sensors are commissioned; their functionality; and their labeling. The importance of generating support for this standard among lighting controls manufacturers cannot be overstated.

\section{Easily commissioned photosensor}

The LRC's design for a more easily commissioned photosensor, while innovative and low in cost, still depends on the use of expensive dimming ballasts to adjust the output of the luminaire in response to daylight.

Several reliability and efficiency issues hamper the widespread acceptance of full-range continuous dimming ballasts. First, many dimming ballasts have a low efficiency when operated at full load, due to the constant heating of the electrodes. Secondly, some ballasts have an adverse effect on lamp life, because they do not constantly heat the electrodes. Thirdly, dimming ballasts remain expensive in comparison to non-dimming ballasts. Until the price of dimming ballasts falls to a level where paybacks are achievable over a commercially-viable timeframe, the use of daylight-linked systems will remain limited to a few building owners; those willing to commit to a long return on investment, and those who require dimming ballasts anyway, for reasons other than energy saving. Codes and/or legislation requiring the use of daylight-linked

\footnotetext{
${ }^{15}$ Lighting Research Center (LRC). January 2002. Reducing Barriers to Use of High Efficiency Lighting Systems, Task 2.11: Interview key control manufacturers.... Troy, NY: Lighting Research Center. Report submitted to US Department of Energy.

16

6 Lighting Research Center (LRC). January 2002. Reducing Barriers to Use of High Efficiency Lighting Systems, Task 2.10: Interview contractors and installers to determine installation and commissioning issues.... Troy, NY: Lighting Research Center. Report submitted to US Department of Energy.
} 
systems could only be justified once the performance and price issues surrounding dimming ballasts have been resolved.

Meanwhile, it remains worthwhile to promote the value of daylighting (high internal daylight levels make photosensing more economic), and to ensure that photosensing is given appropriate credit in voluntary accreditation schemes such as LEED, and in lighting energy legislation. Moreover, photosensors should be considered as part of a daylight system of windows, light fixtures and controls. As such, the incremental cost of photosensors is small.

The LRC is inherently supportive of photosensing, because daylight-linked controls can contribute to integrated architectural lighting design. Daylight-linked controls give the designer the ability to control the balance between natural and electric light in a space, in order to maximize the visibility of daylight, or to minimize the contrast imbalances created by it.

Research suggests that the most effective way of reducing lighting energy use is to ensure that occupants do not turn the lights on in the first instance ${ }^{17}$, and these enhanced savings will only be consistently achieved with the advent of a much more subtle and research-based approach to lighting design and daylighting than commonly exists at present.

\section{Task 4.7: Provide technical foundation for market transformation groups to increase penetration of automatic shutoff controls}

\section{Background}

Automatic shutoff lighting controls consist of two main technologies: occupancy sensors and time clock devices. Occupancy sensors are designed to monitor small areas but can be linked together ${ }^{18}$ to control the lights in a large area. Due to their intrinsically small area of influence, occupancy sensors are valuable for controlling small spaces (private offices, restrooms) or for spaces that tend to be used periodically (storage closets, warehouse aisles). Manual controls or time clocks may be a more cost-effective automatic shutoff technology for large spaces (open plan offices) or spaces where the pattern of use is very regimented (classrooms, state offices).

In remodeling or retrofit applications, time clocks are usually impractical due to the need to provide extensive rewiring and/or local override switches. In these cases, occupancy sensors remain a viable option for reducing wasted lighting energy use.

Advice from Steering Committee members in December 2001 indicated that the market for occupancy sensors in new construction is successfully transformed in states with progressive energy codes such as California. However, feedback from the subsequent industry roundtable in February 2002 indicated that energy codes in much of the nation lag behind those of the western states, such that new construction is still an important area for consideration of automatic shutoff controls.

The roundtable group indicated that market transformation groups lack credible support to predict how much energy occupancy sensors will save. Market transformation experts suggested

\footnotetext{
${ }^{17}$ Lighting Research Center (LRC). 1998. A field study of lighting controls. Troy, NY: Lighting Research Center.

${ }^{18}$ One occupancy sensor controls a much smaller area than a standard electrical branch circuit. When multiple occupancy sensors must be connected to control a larger circuit, time clocks may be more cost-effective than tens of occupancy sensors.
} 
the need for a review of available literature about the effectiveness of occupancy sensors. They saw less need for a similar review of literature regarding time clock effectiveness. Based on this advice, the LRC focused on identifying expected savings percentages from occupancy sensors.

\section{Proposed Estimate of Energy Savings}

The LRC consulted its extensive resource collection to compile a group of 26 case studies and claims by manufacturers regarding the effectiveness of occupancy sensor technologies. We organized the data in different ways in the various studies, often by specific room type (restrooms, hallways, coffee break rooms, conference rooms). We reviewed the literature and organized studies into broader occupancy categories based upon private ("owned") vs. shared and scheduled vs. sporadic:

- Private (“Owned”) Spaces (such as single-person offices or spaces in which the user takes "ownership")

- Shared Spaces, Scheduled use (such as classrooms)

- Shared Spaces, Sporadically used (such as public spaces, open-plan offices, corridors, bathrooms, storage rooms)

Private (“Owned”), scheduled occupancy was not a legitimate category.

For private offices, all data were averaged leading to an average savings of $31.7 \%$. This average represents a wide range of hours of use, so we also examined the percentage of energy savings in those studies that were based upon documented "core hours" between 7.5 to 10 hours per day. This analysis yielded a lower average energy savings of $26 \%$, as expected because the former analysis was dominated by studies using longer hours of use as a base line. This latter percentage (26\%) is perhaps a better estimate of potential energy savings from occupancy sensors because, for buildings showing wasted energy use outside the core hours, other technologies such as time clocks are usually more cost effective than occupancy sensors. We recommend $25 \%$ as the best estimate of energy savings for private offices with sporadic use.

For shared spaces that are only used sporadically, the average savings was $40.8 \%$. Again, there was a wide range of hours of use, but it seems inappropriate to base energy savings on "core hours" ( 7.5 to 10 hours per day) because hallways, stairs, etc. can be used at any time day or night. Therefore, we recommend $40 \%$ energy savings as the best estimate for shared, sporadically occupied spaces. Note, however, that some spaces should be illuminated without occupancy if lighting is used to signal potential occupants that a business is open or if the lights being on provide occupants with a sense of security or safety.

For shared, scheduled spaces, in particular classrooms, it is often difficult to ascertain the hours of use. Classrooms are used not only for teaching during the day, but also for community activities during the night. Often more than one teacher uses the space but does not "own" the classroom. Since these spaces are sporadically used and do not have a clear "owner," occupancy sensors are a good choice for reducing wasted lighting energy. The average energy savings was $31.7 \%$. We recommend $30 \%$ energy savings as the best of estimate for shared, scheduled, spaces. Note, however, that one study of teacher "owned" classrooms reviewed for this task showed that more energy waste due to the time delays on the occupancy sensors. Consequently, the specifier must have a clear understanding of classroom use before applying occupancy sensors to reduce wasted energy. 
Table 6. Recommended energy saving values for occupancy sensors

Percent Savings from Occupancy Sensors (Mean Values)

\begin{tabular}{ll|c|} 
& \multicolumn{1}{c}{ Private ("Owned") } & Shared \\
\cline { 2 - 3 } Sporadically Use: & 25 & 40 \\
\cline { 2 - 3 } Scheduled Use: & & 30 \\
\cline { 2 - 3 } & &
\end{tabular}

\section{Task 4.8: Provide technical foundation on behalf of manufacturers to overcome nagging installation barriers in existing buildings}

\section{Practices for Installing Occupancy Sensors}

A roundtable conducted in February 2002 brought together manufacturers, market transformation groups, specifiers, government agencies, and utilities to discuss barriers to widespread penetration of automatic shut-off lighting controls. One of the recommendations from this group was to encourage, aggressively, the widespread deployment of automatic shutoff lighting controls in new and existing C/I buildings.

In addition to the roundtable, LRC staff participated in a field visit to witness occupancy sensor installations at a company in New Jersey. Professional installers conducted the installation and participating staff learned a number of lessons during the visit. A site visit report is attached in Appendix L: Reducing Barriers to the Use of Lighting Controls; Problems Encountered during Installation and Commissioning of Sensors.

Automatic shut-off controls turn off lamps when a signal is received from an occupancy sensor, a building automation system (BAS), or a timer located locally or remotely in a lighting circuit panel box. The main goal of automatic shut-off controls is to turn off the lights when no one is occupying the space. Based on recommendations from the roundtable, this task focused on best practices for installing occupancy sensors to help increase the penetration of these controls into C/I applications.

Automatic shut-off controls have a high penetration on new construction as a result of energycode provisions, ease of installation, and potential energy savings. Penetration of automatic shutoff controls in existing buildings is poor, however, with the "hassle factor," (aggravation) perceived or real, seen as the greatest barrier to acceptance. The hassle factor associated with automatic shut-off, particularly with occupancy sensors, may be a result of poor sensor positioning in the space, the wrong choice of sensor for the application, poor product labeling, lack of commissioning settings on sensors, or complexity and uniqueness of installation.

As an attempt to help overcome this specific barrier, the LRC, following the recommendations from the roundtable, developed the following best practice document for installing occupancy sensors. We performed this task as an attempt to fill gaps and/or simplify information currently available in the manufacturers' literature. Recommendations that will help address nagging installation barriers of occupancy sensors are given to manufacturers and installers. Although 
just a starting point, this can be a useful tool and can help reduce the real and perceived "hassle factor” associated with installing, commissioning, and using occupancy sensors.

\section{Best Practices for Manufacturers}

In order to minimize installation and commissioning time and hassle, manufacturers should:

- Supply circuit schematics or documentation explaining inputs and outputs to the sensors and power packs.

- Use the same wire color scheme across manufacturers. This will facilitate the installation of sensors and power packs or any other components that are not from the same manufacturer.

- Supply documentation on how to override the system after the power packs are installed. In general, power packs are installed before the sensor and installers do not have enough information about how to override the system after the sensor is installed.

- Develop a diagnostic interface that can be plugged in each power pack and sensor to speed up commissioning and diagnosing problems.

- Include zero-delay setting to the sensor to allow faster commissioning.

- Set up a 24-hour hotline customer service to allow nightshift installers to have customer support at night.

\section{Best Practices for Installers}

In order to properly install and commission occupancy sensors, the installers should:

- Never disable manual controls when installing occupancy sensors; existent light switch should be supplemented, not replaced, by the occupancy sensor.

- Provide a sufficient number of sensors, placed appropriately, in open plan offices. Saving money by reducing the number of occupancy sensors used in open plan area may result in poor performance and thus, dissatisfaction and rejection.

- Place occupancy sensors in areas where small movements are made (e.g., near desks, over bathroom stalls).

- Put two sensors in hallways, one at each end, pointing toward the center of the controlled area to provide good coverage and avoid false triggering.

- Use BX cable instead of conduits to ease the wiring process.

- While commissioning and re-commissioning, increase the time delay, if sensor sensitivity is turned down, to allow the lights to be on longer without movement.

In addition to the tips above, the LRC developed a prototype "laminated sheet" that can be used on site by installers. This prototype "laminated sheet" will help installers select the best occupancy sensor for the application and shows examples of installations in typical applications. Please refer to Appendix M: Occupancy Sensor Selection Guide to view an example of this reference.

\section{Task 4.9: Lamp/ballast combinations testing for dimming impacts}


The purpose of this task was to determine the interaction and compatibility between fluorescent lamp electrode heating and differing lamp operating currents (dimming) in order to provide the foundation for industry standards for lamps and dimming ballasts to ensure compatibility. The results of the dimming and lamp life studies are continuing outside the scope of this project with assistance from lamp and ballast manufacturers.

The LRC has been working with the lighting industry through the National Electrical Manufacturers Association (NEMA) and held a meeting at the LRC on June 4, 2004 to coordinate efforts. The lamp and ballast manufacturers agreed to provide in-kind services for the project including lamps, specialty ballasts and technical review. The value of these services is estimated to be $\$ 228,000$.

The technical approach was to develop an experimental design agreeable to all manufacturers (achieved June 2004); build testing apparatus and conduct pilot testing (achieved October 2004); construct testing apparatus based on pilot test results (achieved March 2005); characterize each dimming system (achieved May 2005); initiate dimming systems compatibility testing (started June 2005 and ongoing); and draft dimming standards (to be developed at end of testing). The experimental design sets a series of lamp currents $(20 \mathrm{~mA}, 50 \mathrm{~mA}, 80 \mathrm{~mA}, 110 \mathrm{~mA}$ and $180 \mathrm{~mA})$ and electrode heating ( 1.5 volts, 3.0 volts, 4.5 volts and 6.0 volts) points. Four samples of each lamp and ballast combination operate at each of these points for a total 864 test points. Ballast manufacturers built special ballasts that only operate at each of the test points. See Figure 4 for a graphic depiction of the experimental design.

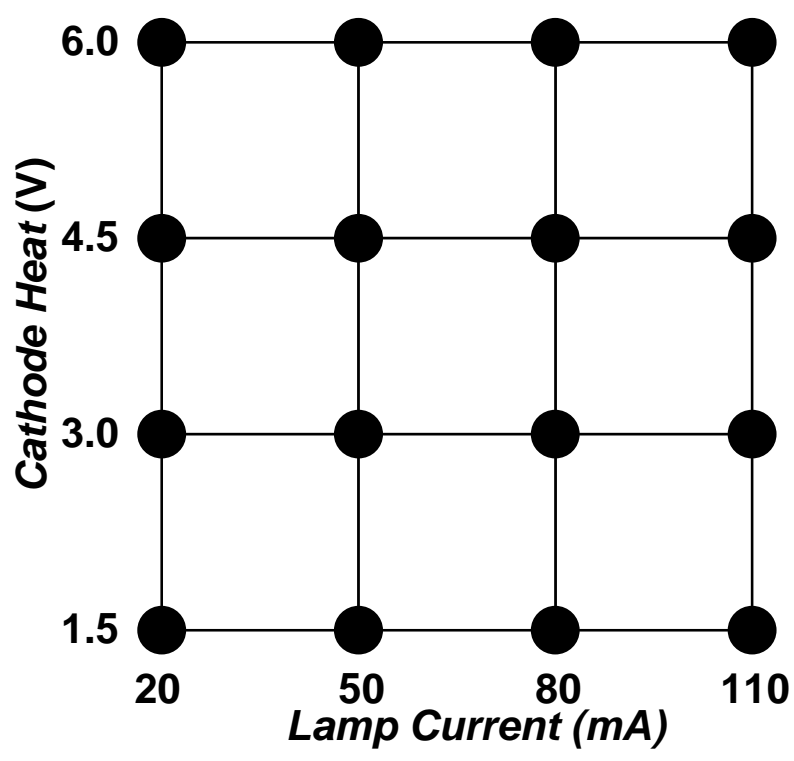

\author{
Each point \\ 4 ballast mfr. \\ 3 lamp mfr. \\ 4 samples each \\ Total 48 systems \\ Total points \\ 16 data points \\ 2 control points \\ $180 \mathrm{~mA} \& 3.0 \mathrm{~V}$ \\ $180 \mathrm{~mA} \& 0 \mathrm{~V}$ \\ Total 864 systems
}

Figure 4. Dimming lamp/Ballast Compatibility Experimental Design 
Initial system testing prior to beginning the life test was performed on each of the 864 lamp/ballast combinations. Data taken on each system included:

- electrode cold resistance

- electrode heating voltage

- lamp current

- lamp current crest factor

- lamp voltage

- lead currents (for sum-of-squares analysis)

- operating frequency

- optical emission at $811.5 \mathrm{~nm}$, phase resolved with lamp current

- wave form data

Initial results of the life tests at 5,000 hours of operation are shown on Figure 5. Early failures are where expected in the low lamp current/low cathode heating ranges. However, failures have also been detected in the low lamp current/high cathode heating range. This was not expected.

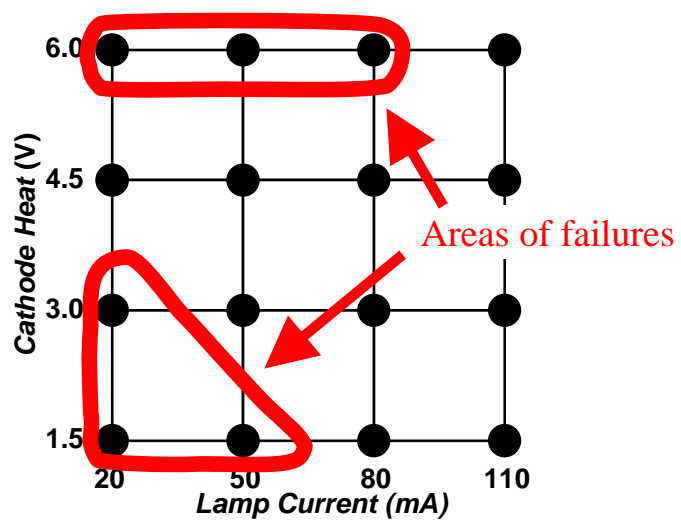

Figure 5. Lamp/dimming ballast compatibility life testing results (5,000 hours)

Life testing will continue through 2007. Once sufficient data has been collected regarding failures and causes, work will commence on developing metrics for lamps and ballasts used in dimming systems. This work will be conducted outside the scope of this project. 


\section{TASK 5: TechnOlOgy TRANSFER}

The LRC developed a technology transfer plan to bring to market improved, integrated lighting and control systems, which would significantly reduce energy used for lighting in commercial buildings. The plan, which was peer-reviewed, served as a roadmap for the actions taken during year 3 of the Reducing Barriers project. A detailed version of the technology transfer plan is included as Appendix N: Technology Transfer Plan.

The ultimate goal of technology transfer is to have the technology so widely used that the impact of the technology is written into laws, regulations, codes and/or standards. The LRC, after developing the technology transfer plan, utilized a technology transfer model to identify the technical foundation and marketing enabling mechanisms associated with each new technology, as well as the organizations, companies and representatives that interact with these mechanisms.

Figure 6, through identifying the key players, as well as the activities they pursue in today's current practices of technology transfer, represents the first phase of the technology transfer model.

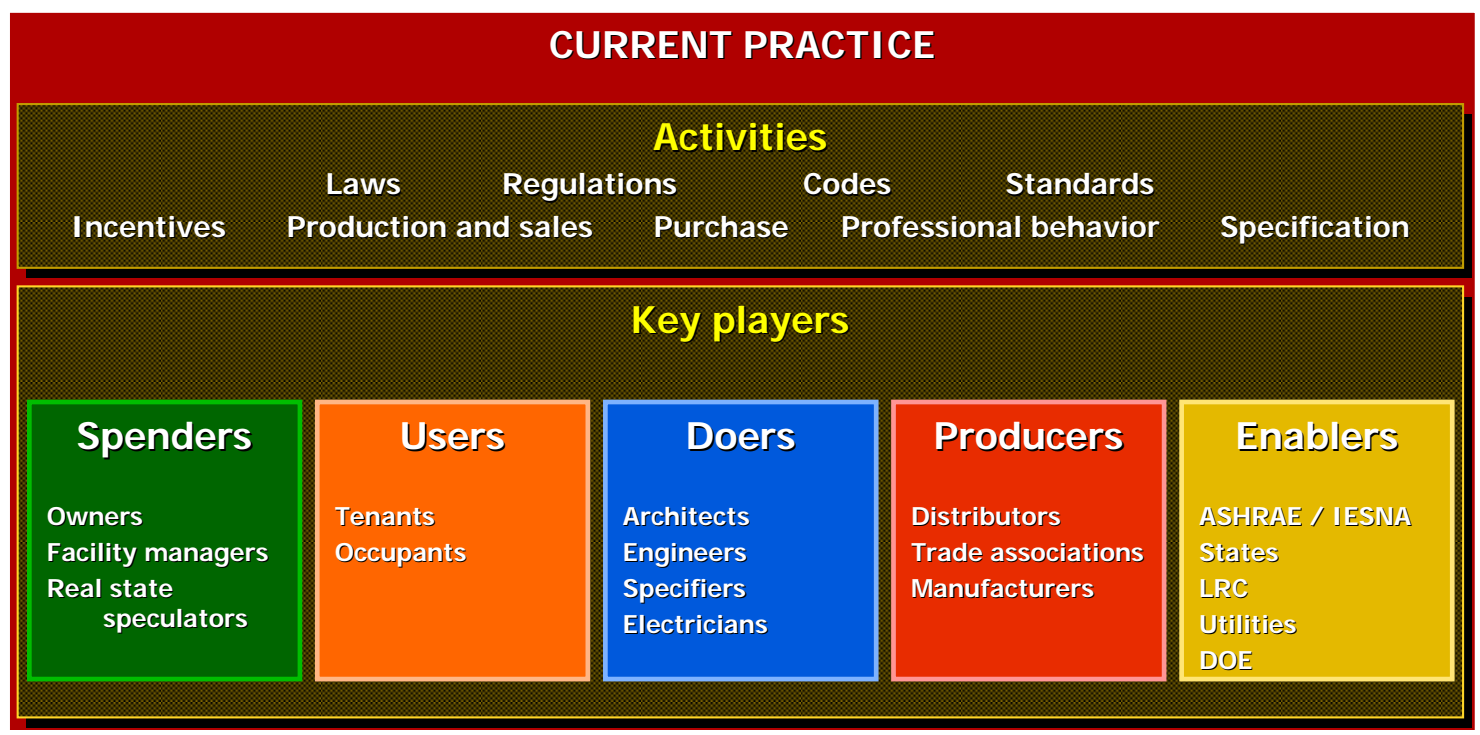

Figure 6. Technology transfer model: identification of key players and activities

\section{Key players include:}

- Spenders - These are building owners, facility managers, real estate speculators and developers. They make the ultimate buying decision but are influenced by the other types of key players and what they read and see in different types of communication outlets.

- Users - These are the ultimate users of the technologies such as building tenants and occupants. They must be satisfied with the technology's performance and the ability to deliver its claims.

- Doers - These are the architects, consulting engineers, specifiers and installers of the technology. They have great influence over the spenders. When doers recommend or 
install a technology, they risk their reputation on whether product will perform as advertised.

- Producers - These people make and distribute the technology and their respective associations/organizations. They must have a reliable and marketable technology that will earn profits for the company. Besides the key role of manufacturing the technology, they act as marketer.

- Enablers - These organizations write laws, regulations, codes and/or standards such as state and federal governments, American Society of Heating, Refrigeration and Air Conditioning Engineers (ASHRAE), National Electrical Manufacturers Association (NEMA) or Illuminating Engineering Society of North America (IESNA). Also included are organizations that influence law, regulation, and code and/or are standard writers such as Natural Resources Defense Council (NRDC) or American Council for an Energy Efficiency Environment (ACEEE). Enablers are also represented by organizations such as the Lighting Research Center who has relationships and partnerships with all of the key players within an industry and is able to bring these dissimilar types of players together in collaborative and mutually beneficial efforts to perform successful technology transfer. Also included with this grouping are utilities and state/regional organizations such as Xcel Energy and the Northwest Energy Efficiency Alliance, which are charged with the responsibility for implementation of energy efficiency programs.

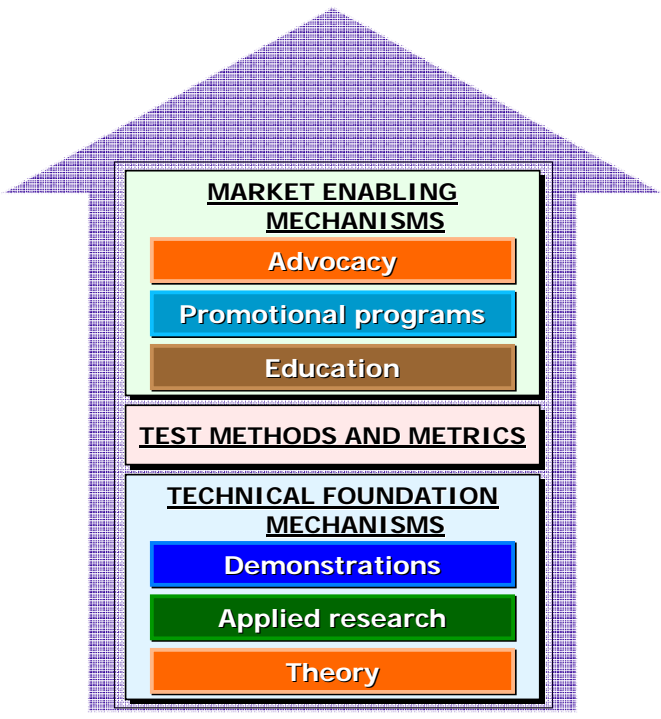

Figure 7. Technology transfer model: transformation mechanisms

Figure 7, which represents the second phase of the model, outlines the transformation mechanisms in a methodical, step-by-step process. This process addresses the development, demonstration, evaluation, commercialization and, finally, customer acceptance of a technology. The successful achievement of each step's goals is a prerequisite to moving onto the next step. Failure to meet these goals or an attempt to skip a step reduces the chances of the technology being accepted by the user. The mechanisms include: 
- Theory - This mechanism identifies a possible technology to satisfy an unfulfilled customer or user need. The technology gets defined and researchers determine if the technology is achievable at some reasonable cost to the user. While research organizations like the LRC and other producers are the primary players for this mechanism, input from and funding to the other key players is mandatory.

- Applied Research - This mechanism must prove the technical and economic concepts of the technology through technical and market research. Prototypes are developed as part of meeting this step's goals. Again, close collaboration with all key players is necessary as the research or manufacturing organizations endeavor to meet the goals of this mechanism. As one of its goals, the applied research mechanism identifies manufacturers willing to commercialize the technology and begin performing the necessary engineering for manufacturing.

- Demonstrations - Full-scale demonstrations are necessary to "show the world" the technology works and delivers the promised benefits. The demonstrations must answer any questions or concerns raised by users or spenders during the market research phase. The proof must be of the technology and its economics as applied to an actual, rather than laboratory, situation. Successful demonstrations with multiple locations or situations increase the acceptance of the technology. Funding and defining the measured metrics for this phase must come from other key players.

- Test Methods and Metrics - The method and metrics on which the evaluation is conducted of a technology, especially during the demonstration step, must be acceptable and agreed to by the research community as well as all the key players. Test results must withstand the rigors of review. While this mechanism may seem intuitive, it may be the most important step of all transformation mechanisms. It sets the stage as to how believable the claims made by the technology will be to the spenders, users, doers, producers and enablers and, ultimately, how successful the technology will be in the marketplace. Also, it provides a uniform means to measure results utilizing the same technology in different labs and facilities.

- Education - The education mechanism is meant to be broad in scope to include the necessity to educate all the key players about the benefits, abilities and limitations of the technology and why it should be adopted in the marketplace, i.e., what it will do to help society. This is the first step in bringing the technology to the market. Yet, many times organizations responsible for technology development do not view the educational function as part of their responsibilities. Funding for education should be included as part of any technology development or else the technology may never advance to the market.

- Promotional Programs - If the rigors of the test methods and metrics are met and if a successful education program is funded and executed, then, and only then, will the producers, enablers, utilities and state/regional agencies charged with the conduct of energy efficiency programs promote the use of the technology to the spenders, doers and other users. Promotional programs can take many forms such as utility incentive programs or endorsements.

- Advocacy - This is the final step in the technology transfer model but does not occur until after the technology has reached some level of acceptance within the marketplace. It 
only occurs when there is public consensus of the use and benefits of the technology. Advocates then request that the benefits of the technology are included in regulations, codes and/or standards.

\section{Deliverables}

Lighting controls included in this report (automatic shut-off, daylight dimming and load management) were analyzed using the technology transfer model. Figure 8 represents where each control system resided as of December 2004, the end of the original project. Actions taken to move technologies toward market acceptance are listed with each technology.

\section{Automatic Shut-off}

\begin{tabular}{|c|c|c|c|c|c|c|}
\hline & \multirow{2}{*}{ Status } & \multicolumn{5}{|c|}{ Key Players } \\
\hline & & Spenders & Users & Doers & Producers & Enablers \\
\hline Advocacy & Ongoing & & & AESP & & $\begin{array}{l}\text { ACEEE } \\
\text { CEE }\end{array}$ \\
\hline $\begin{array}{l}\text { Promotional } \\
\text { Programs }\end{array}$ & Ongoing & & & & & Utilities, \\
\hline Education & Ongoing & BOMA & BOMA & $\begin{array}{l}\text { ASHRAE, } \\
\text { IESNA }\end{array}$ & NEMA & $\begin{array}{c}\text { LRC } \\
\text { Utilities, } \\
\text { NYSERDA, }\end{array}$ \\
\hline $\begin{array}{l}\text { Test Methods \& } \\
\text { Metrics }\end{array}$ & Complete & & & & & \\
\hline Demonstrations & Complete & & & & & \\
\hline Applied Research & Complete & & & & & \\
\hline Theory & Complete & & & & & \\
\hline
\end{tabular}

Figure 8. Automatic shut-off technology transfer model

\section{Actions}

- Presentation regarding automatic shut-off conducted for the Association of Energy Service Professionals (AESP). See Appendix O: Association of Energy Service Professionals Presentation.

- Published a paper on findings about automatic shut-off in the conference proceedings of the AESP Meeting. See Appendix P: Association of Energy Service Professionals Paper.

- Presented information about automatic shut-off to the American Council for an Energyefficient Environment (ACEEE) and discussed the possibility of including materials in standards.

- Participated in ASHRAE 90.1 - Lighting Sub-committee meeting - advocated for inclusion of automatic shut-off in standards. See Appendix Q: ASHRAE 90.1 Presentation.

- Published article on occupancy sensors in Lighting Design and Application (LD\&A), a magazine published by the Illuminating Engineering Society of North America (IESNA).

- Participated and made a presentation on lighting controls at the Illuminating Engineering Society of North America (IESNA) Lighting Controls Protocol Sub-committee. See Appendix R: IESNA Lighting Control Protocol Subcommittee Presentation. 
- Presented “Best Practices” brochure to Utilities, System Benefit Charges organizations, and Consortium for Energy Efficiency (CEE) and advocated for them to place the document in their web site.

\section{Daylight Dimming}

\begin{tabular}{|c|c|c|c|c|c|c|}
\hline & \multirow{2}{*}{ Status } & \multicolumn{5}{|c|}{ Key Players } \\
\hline & & Spenders & Users & Doers & Producers & Enablers \\
\hline Advocacy & Not started & & & & & \\
\hline $\begin{array}{l}\text { Promotional } \\
\text { Programs }\end{array}$ & Not started & & & & & \\
\hline Education & Ongoing & & & AESP, & & CEE, NEEA \\
\hline $\begin{array}{l}\text { Test Methods \& } \\
\text { Metrics }\end{array}$ & Ongoing & & & & & \\
\hline Demonstrations & Ongoing & & & & & \\
\hline Applied Research & Complete & & & & & \\
\hline Theory & Complete & & & & & \\
\hline
\end{tabular}

Figure 9. Daylight dimming technology transfer model

\section{Actions:}

- Presentation regarding the self-commissioning photosensor and daylight dimming conducted at the Association of Energy Service Professionals (AESP) Meeting. See Appendix O, Association of Energy Service Professionals Presentation.

- Published a paper in the conference proceedings of the AESP Meeting. See Appendix P, Association of Energy Service Professionals Paper.

- Presented information on lighting controls at a Controls Summit organized by the Northwest Energy Efficiency Alliance (NEEA) and Seattle Design Lab.

- Presented information about the self-commissioning photosensor at the Consortium for Energy Efficiency (CEE) 2003 meeting.

\section{Load Management}




\begin{tabular}{|c|c|c|c|c|c|c|}
\hline & \multirow{2}{*}{ Status } & \multicolumn{5}{|c|}{ Key Players } \\
\hline & & Spenders & Users & Doers & Producers & Enablers \\
\hline Advocacy & Not started & & & & & \\
\hline $\begin{array}{l}\text { Promotional } \\
\text { Programs }\end{array}$ & Not started & & & & & \\
\hline Education & $\begin{array}{l}\text { Preliminary } \\
\text { activities }\end{array}$ & & & IESNA, AESP & & PLMA, IEA, \\
\hline $\begin{array}{l}\text { Test Methods \& } \\
\text { Metrics }\end{array}$ & Ongoing & & & & & \\
\hline Demonstrations & Proposed & & & & & LRC \\
\hline Applied Research & Ongoing & & & & & LRC \\
\hline Theory & Complete & & & & & \\
\hline
\end{tabular}

Figure 10. Load management technology transfer model

\section{Actions}

- $\quad$ Presented load management activities update at the Peak Load Management Alliance (PLMA), September 2003. See Appendix S: Peak Load Management Alliance Presentation.

- Presented findings on human factors research and dimming at the IESNA Annual Conference. See Appendix T, Human Factors Research Presentation; IESNA Conference.

- Published a paper on human factors research and dimming in the Journal of the Illuminating Society of North America (JIES).

- Published article on human factors research and dimming in Lighting Design and Application (LD\&A), a magazine published by the Illuminating Engineering Society of North America (IESNA).

- Presented load management activities update, and hosted dinner for the International Energy Agency - Demand Response subcommittee.

- Presented load management activities update at the National Energy Service Conference and Exposition.

- Published a paper on the proceedings of the National Energy Service Conference and Exposition.

The LRC developed a website currently linked to the LRC website. However, this site can be extracted in its entirety and transferred to whomever, DOE wishes to use to maintain the site. The home page of the site is shown below. It can be accessed at www.Irc.rpi.edu/researchtopics/ reducingbarriers/index.asp. 


\section{National Lighting Product Information Program (NLPIP)}

The National Lighting Product Information Program (NLPIP) was added to the technology transfer efforts for calendar year 2005. This program, with multiple sponsors, helps lighting professionals, contractors, designers, building managers, homeowners and other consumers find and effectively use efficient, quality products to meet their lighting needs. Three project reports were delivered during 2005.

NLPIP website was visited by 144,000 people during 2005 . The people visiting the site are further broken down by their occupation.

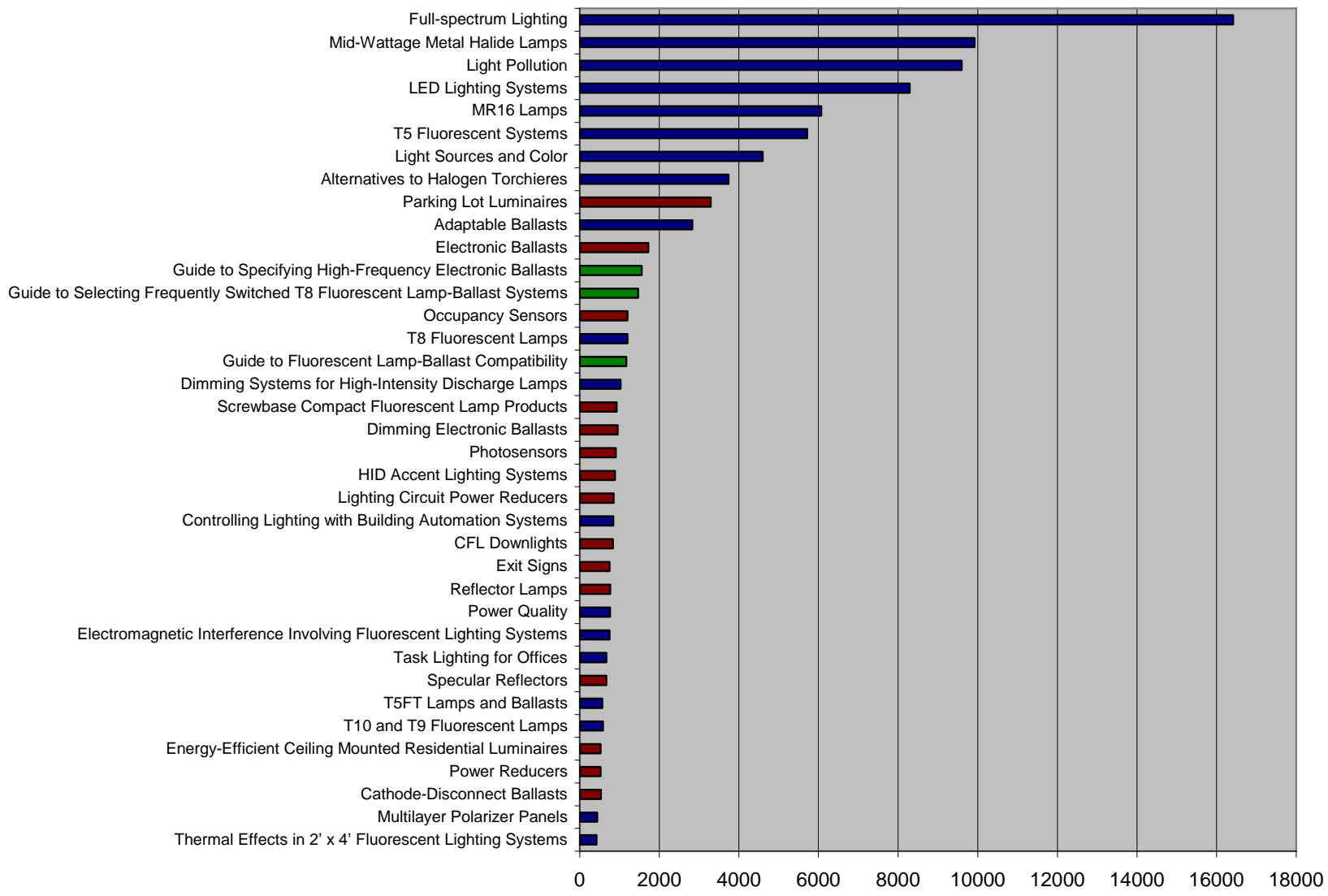

Figure 11. NLPIP reports downloaded

\section{Supplement to Specifier Report: Lighting Circuit Power Reducers}

Lighting circuit power reducers are retrofit devices designed to reduce energy use of a lighting circuit. The power reducers, also called lighting controllers, are installed at lighting panels between the circuit breaker and the lighting load to reduce the active power of the entire lighting circuit. Reducing the lighting load, however, reduces illuminance and may also reduce system efficacy.

The product evaluated for the 2005 supplement was an Electric City lighting power reducer. Power reductions were measured for four different lamp and ballast combinations using the suggested manufacturer setting of the controls. The magnetic ballast/T12 lamp showed a power reduction of $22.4 \%$ while the electronic ballast and T8 lamps had power reductions of $14.5 \%$ to 
19.2\%. Savings are dependant on whose lamp is used in the system. There are corresponding reductions in light output.

The full Specifier Report can be found on the NLPIP Web site: www.lrc.rpi.edu/programs/ NLPIP/publications.asp.

\section{Lighting Answers: Mid-wattage Metal Halide}

Lighting Answers: Mid-wattage Metal Halide helps lighting professionals to understand midwattage metal halide lamps and their most important performance characteristics. It explains how the lamps work, the difference between quartz and ceramic arc tubes, and the differences between probe start and pulse start technologies. It provides information about issues such as burning position, warm-up times, restrike, lumen maintenance, color rendering and color shift. Finally, this publication provides pointers to lighting professional on how to choose mid-wattage metal halide lamps.

The advantages of ceramic arc tubes include better efficacy, color rendering and color stability. Pulse start technologies increase lamp life, lumen maintenance and provide short warm-up times and faster restrike.

The full Specifier Report can be found on the NLPIP Web site: www.lrc.rpi.edu/programs/ NLPIP/publications.asp.

\section{Supplement to Specifier Report: Screwbase Compact Fluorescent Lamp Products}

This supplement is the eighth supplement to the Specifier Report: Screwbase Compact Fluorescent Lamp Products, 1999. This supplement differs from previous supplements in that it describes the results of both a long-term performance test and a life test of screwbase compact fluorescent lamp (CFL) products. This study was conducted to better understand how long-term CFL performance is affected by operating at different positions and by operating within a luminaire.

The full Specifier Report can be found on the NLPIP Web site: www.lrc.rpi.edu/programs/ NLPIP/publications.asp. 


\section{CONCLUSIONS}

- A search of patents regarding lighting controls and ballasts indicates minimal manufacturer interest in developing photosensors because of the low market penetration of dimming products.

- Most of the economic attention of manufacturers with regard to lighting controls has been directed toward the development of energy management systems and panel-level controls. This is followed by occupancy sensors.

- A review of existing patents provides an indication that additional research and commercialization efforts are required for photosensors and communication protocol technologies associated with lighting controls.

- The decentralization of the lighting controls industry with different manufacturers producing different parts of the control system can be perceived as a barrier. With a dimming ballast from manufacturer $\mathrm{A}$, a photosensor from manufacturer $\mathrm{B}$, fluorescent lamps from manufacturer $\mathrm{C}$ and the building automation system from manufacturer $\mathrm{D}$, who does the buyer turn to if issues arise.

- All existing communication protocols require a separate set of communication wiring. This requirement adds cost to lighting control systems and is a barrier to their use. Unlike heating and air conditioning systems where there are only a few units to control, lighting requires communication with essentially each lighting fixture creating thousands of communication points within a building.

- With the plethora of communication protocols in use and different manufacturers' equipment utilizing different protocols, it is difficult to develop a whole system approach to lighting controls where all components can talk to each other. The development of DALI by the lighting industry should overcome this issue.

- Currently, most communication protocols used for lighting control systems come from the heating and air conditioning control schemes such as BACnet and LONWorks.

- For a lighting control system to operate successfully the system must:

o Minimize command strings aggregating control points

o Warn occupants of lighting system changes

o Sense delay override commands

o Interact with other sensors and controls within the office environment

- Digital Addressable Lighting Interface (DALI) is a recognized standard that is beginning to be adopted within the United States as a lighting control protocol.

- Lighting control installing contractors believe the potential market for occupancy sensors, photosensors and dimming electronic ballasts is much larger than the present market.

- Customers must be convinced that lighting controls will benefit their bottom line before increased demand for lighting controls is achieved. 
- The minimum acceptable requirements for lighting controls are that they:

o Provide sufficient light for the users of a space—after all, we can achieve perfect control by simply turning out all the lights in a building, but that is not a productive solution.

o Avoid waste- that is, turn off lights when no one is there to benefit from them (except for security, display, etc.).

o Reduce lighting levels, without compromising occupants' satisfaction and productivity.

- No lighting controls solution is effective or acceptable unless it contributes to, or does not compromise, the following goals:

o Productivity - Planning, installation, commissioning, maintenance, and use of controls should not decrease business productivity.

o Energy savings - Lighting controls should save significant amounts of energy and money in relation to the expense involved in using them (acceptable payback period), and/or...

o Reduced power demand - Society as a whole should benefit from the lowered demand for expensive power and for more natural resources.

- In general, technological problems pose relatively low market barriers for lighting controls. A failure to show reasonable payback periods and other economic benefits, however, limits the acceptance of controls in the marketplace. Without greater market demand for lighting controls, manufacturers have little incentive to eliminate the remaining, albeit small, technological problems.

- One barrier that is being effectively addressed by the control manufacturers is the issue of interoperability of control components. On a local level, interoperability means attaining communication to control the ballast. More globally, interoperability concerns communication between individual control devices and, at a higher level, with whole building automation systems (BAS). Lighting controls manufacturers are working successfully to develop products that conform to open communication and command protocols (rather than proprietary systems) for all of the control technologies peripheral to the ballast. Several protocols are being discussed and it seems likely that standards will continue to be developed and refined in the next two years that will facilitate utilization of control components in larger systems.

- Dimming systems are both expensive to acquire and difficult to install. Commissioning photosensors is prohibitively time-consuming and, consequently, photosensors are almost never installed. Recent development by the Lighting Research Center of a simplifiedcommissioning photosensor to take advantage of daylight, however, may overcome this barrier.

- The success of lighting control products on the market must allow systems to achieve three main goals:

o Achieve sufficient functionality to meet the key requirements of their main market. 
o Allow a significant cost reduction compared to current market standard systems. Cost should take into account: hardware capital cost including wiring, design time required by the specifier and the control system manufacturer, installation time required from the electrician, and commissioning time and remedial time required from the electrician and end user.

o Minimize ongoing perceived overhead costs and inconvenience to the end user, or in other words, systems should be simple to understand and use.

- Local control functions do not need to be integrated into a whole building system in order to work effectively.

- Trying to apply a single solution that is capable of handling all the requirements of the different control strategies is not cost effective. Such a strategy will result in an overbuilt system where most of the resources are hardly, if ever, used, and so consequently the system is too expensive and/or complicated to use. Therefore, it should be recognized that whole building lighting control systems might not be appropriate for all buildings now, until costs come down.

- Starting lamps when dimmed, i.e., at lower than rated current, is not recommended. To ensure long life, especially when supplemental electrode heating is not applied (e.g., instant-start), a procedure of starting the lamp at its nominal full output level and then dimming it down to the desired light levels is necessary. Starting at full power will minimize the damaging effects of starting. This is advisable whether supplemental electrode heating is applied as part of the starting sequence, but most critical without supplemental electrode heating.

- It is possible to dim fluorescent lamps without supplemental heating of the electrode to at least $121 \mathrm{~mA}$ without adversely affecting lamp life.

- Some technologies aimed at reducing lighting energy consumption and cost are already well accepted and reliably implemented by the building community, while others are constrained by real or perceived barriers to more widespread use, such as complexity, reliability or cost. Examples of the former include "architectural" dimming, occupancy sensing in new-builds, programmed-start ballasts, time switching, and energy efficient lamps and luminaires. Examples of the latter include photosensing, occupancy sensing in retrofits, and load management systems.

- Some kind of minimal specification for load-shed ballasts is required for two reasons. First, if incentives or rebates are used to make load shedding attractive, then some minimum performance specification will be required in order to allow ballasts or luminaires to be classified as qualifying for the incentive. Second, if ballasts from different manufacturers are used in the same building, they must dim down to approximately the same level in order to preserve illuminance and luminance uniformity.

- Figures compiled by the LRC from a wide variety of sources indicate that the potential energy savings achievable by occupancy sensing range from a mean of $25 \%$ for shared (e.g., open-plan) spaces, to $40 \%$ for privately "owned" spaces such as private or partitioned offices. These figures are for the energy saved in comparison with each area's lighting energy consumption prior to the installation of the occupancy sensing system, 
relative to an eight to ten-hour working day. In relation to these substantial savings, the occupancy sensor market remains notably underdeveloped.

- It remains worthwhile to promote the value of daylighting (high internal daylight levels make photosensing more economic), and to ensure that photosensing is given appropriate credit in voluntary accreditation schemes such as LEED, and in lighting energy legislation. Moreover, photosensors should be considered as part of a daylight system of windows, light fixtures and controls. As such, the incremental cost of photosensors is small.

- The market for occupancy sensors in new construction is successfully transformed in states with progressive energy codes such as California. However, feedback from the subsequent industry roundtable in February 2002 indicated that energy codes in much of the nation lag behind those of the western states, such that new construction is still an important area for consideration of automatic shutoff controls.

- Market transformation groups lack credible support to predict how much energy occupancy sensors will save. Market transformation experts suggested the need for a review of available literature about the effectiveness of occupancy sensors.

- In order to minimize installation and commissioning time and hassle of occupancy sensors, manufacturers should:

o Supply circuit schematics or documentation explaining inputs and outputs to the sensors and power packs.

o Use the same wire color scheme across manufacturers. This will facilitate the installation of sensors and power packs or any other components that are not from the same manufacturer.

o Supply documentation on how to override the system after the power packs are installed. In general, power packs are installed before the sensor and installers do not have enough information about how to override the system after the sensor is installed.

o Develop a diagnostic interface that can be plugged in each power pack and sensor to speed up commissioning and diagnosing problems.

o Include zero-delay setting to the sensor to allow faster commissioning.

o Set up a 24-hour hotline customer service to allow nightshift installers to have customer support at night.

- In order to properly install and commission occupancy sensors, the installers should:

o Never disable manual controls when installing occupancy sensors; existent light switch should be supplemented, not replaced, by the occupancy sensor.

o Provide a sufficient number of sensors, placed appropriately, in open plan offices. Saving money by reducing the number of occupancy sensors used in open plan area may result in poor performance and thus, dissatisfaction and rejection.

o Place occupancy sensors in areas where small movements are made (e.g., near desks, over bathroom stalls). 
o Put two sensors in hallways, one at each end, pointing toward the center of the controlled area to provide good coverage and avoid false triggering.

o Use BX cable instead of conduits to ease the wiring process.

o While commissioning and re-commissioning, increase the time delay, if sensor sensitivity is turned down, to allow the lights to be on longer without movement.

- Initial lamp and dimming ballast compatibility testing indicates early failure of lamps with low lamp current and low electrode heating as well as low lamp current and high electrode heating.

- The ultimate goal of technology transfer is to have the technology so widely used that the impact of the technology is written into laws, regulations, codes and/or standards.

- The National Lighting Product Information Program (NLPIP) offers lighting professionals an unbiased resource for understanding lighting technologies and for their applications. 


\section{REFERENCES}

American National Standards Institute. C82.11 Consolidated-2002. ANSI standard for lamp ballast: High frequency fluorescent lamp ballasts, supplements. American National Standard Lighting Group, NEMA.

Bierman, A., and K. Conway. 1999. Characterizing daylight photosensor system performance to help overcome market barriers. Illuminating Engineering Society of North America 1999 Annual Conference: Proceedings. IESNA: New York, NY. 413-432.

Brown, S. C. 1959. Basic Data of Plasma Physics. Cambridge, MA: M.I.T. Press.

Buddenberg, A., and R. Wolsey. 1995. Compatibility test of dimming electronic ballasts used in daylighting and environment controls. Illuminating Engineering Society of North America 1995 Annual Conference: Proceedings. IESNA: New York, NY. 1-9.

Davis, R. G., and Y. Ji. 1998. Fluorescent lamp-ballast systems. ESEERCO Final Report 94-40, part 1. Troy, NY: Lighting Research Center.

Hammer, E. E. 1989. Comparative starting operating characteristics of typical F40 systems. Journal of the Illuminating Engineering Society 18, no. 1: 63-69.

- 1995. Cathode fall voltage relationship with fluorescent lamps. Journal of the Illuminating Engineering Society 24, no. 1: 116-122.

Hilscher, A. 2002. Determination of the cathode fall voltage in fluorescent lamps by measurement of the operating voltage. Journal of Physics D: Applied Physics 35: 17071715.

Kelly, D. H. 1961. Visual response to time-dependent stimuli. Journal of the Optical Society of America No. 51.

- 1971. Theory of flicker and transient responses, Journal of the Optical Society of America No. 61.

Kryszczuk, K. 2001. Detection of slow light level reduction. 2001 IESNA Conference Proceedings, 315-322.

Lighting Research Center. 1998. Report 10073: Photosensor Project Bibliography. Troy, NY: Lighting Research Center, Rensselaer Polytechnic Institute.

—. 2000. Photosensor Tutorial. Troy, NY: Lighting Research Center, Rensselaer Polytechnic Institute.

—. 2003. Integrated skylight luminaire. Field Test Delta 1, no. 1. Troy, NY: Lighting Research Center, Rensselaer Polytechnic Institute.

Lowry, E. F. 1951. Thermionic cathodes for fluorescent lamps and their behavior. Illuminating Engineering 46: 288-294.

Maniccia, D., A. Wadhwa, and R. Longtin. 1996. A new method for assessing occupancy sensor performance using robotics. Illuminating Engineering Society of North America 1996 Annual Conference: Proceedings. IESNA: New York, NY. 760-799.

Misono, K. 1991. Cathode fall voltage of low-current fluorescent lamps. Journal of the Illuminating Engineering Society 20, no. 2: 108-115. 
Mistrick, R. G., C. H. Chen, A. Bierman, and D. Felts. 1999. An analysis of photosensorcontrolled dimming systems in a small office. Illuminating Engineering Society of North America 1999 Annual Conference: Proceedings. IESNA: New York, NY. 433-448.

National Lighting Product Information Program. 1997. Specifier Reports: Occupancy Sensors 5, no.1. Troy, NY: Lighting Research Center, Rensselaer Polytechnic Institute.

—. 1998. Specifier Reports: Photosensors 6, no. 1. Troy, NY: Lighting Research Center, Rensselaer Polytechnic Institute. . 1999. Specifier Reports: Dimming Electronic Ballasts 7, no. 3. Troy, NY: Lighting Research Center, Rensselaer Polytechnic Institute.

—. 2000. Specifier Reports: Electronic Ballasts 8, no. 1. Troy, NY: Lighting Research Center, Rensselaer Polytechnic Institute.

Rea, M., and D. Maniccia. 1994. Lighting Controls: A Scoping Study, Reference Document. Troy, NY: Lighting Research Center, Rensselaer Polytechnic Institute.

Shikakura, T., H. Morikawa, and Y. Nakamura. 2001. Research on the perception of lighting fluctuation in a luminous offices environment. Journal of the Illuminating Engineering Institute of Japan, 85, 5: 346-351.

Soules, T. F., J. H. Ingold, A. K. Bhattacharya, and R. H. Springer. 1989. Thermal model of the fluorescent lamp electrode. Journal of the Illuminating Engineering Society 18, no. 2: 8192.

Stuart, R. V., and G. K. Wehner. 1962. Sputtering at very low bombarding energies. Journal of Applied Physics 33, no. 7: 2345-2352.

Tetri, E. 2000. Daylight linked dimming: effect on fluorescent lamp performance. Lighting Research and Technology 34, no.1: 3-10.

U. S. Census Bureau. 2001. Fluorescent lamp ballasts: 2001. Current Industrial Reports MQ335C (01)-5.

Watanabe, Y. 1995. Dependency of cathode temperature on operation frequencies in fluorescent lamps. Journal of the Illuminating Engineering Society 24, no. 1: 48-57.

Watanabe, Y., and S. Murayama. 1993. Cathode fall characteristics of fluorescent lamps under high-frequency operation. Japanese Journal of Applied Physics 32, Pt. 1, no. 8.

Waymouth, J. F. 1971. Electric Discharge Lamps. Cambridge, MA and London, England: M.I.T. Press. 


\title{
APPENDICES
}

\author{
APPENDIX A: REPORT: CONDUCT SEARCHES AND REVIEWS OF LATEST \\ TECHNICAL AND PATENT LITERATURE RELATED TO PHOTOSENSOR, OCCUPANCY \\ SENSOR, AND DIMMING FLUORESCENT LAMP BALLAST TECHNOLOGY AND \\ COMMUNICATIONS SYSTEMS PROTOCOLS
}

\section{Review of Recent U.S. Patents Related to Lighting Controls and Fluorescent Lamp Ballast Technology}

A review of the patents in a particular technology area is one method of assessing its status in terms of technological achievement. While patents may not provide answers on the significance of certain technological achievement, a counting and categorization of them provides a good indication of the amount of economic investment and activity in a given technology area. Patents cost money, including both application fees and the implied costs of defending one's patent rights when challenged by others. Therefore, we would expect to find that the areas of technology that are viewed in the business world as offering the greatest economic growth or activity would have the greatest amount of patent activity associated with them.

Of course, patents are certainly not the only measure of economic activity in a technology area. Many technology businesses survive on trade secrets, or just knowing a better way of doing business. Patent activity does not indicate how successful a business is, or will be either, but without any intellectual property in a technology area, it is doubtful that any economic growth will take place.

With this in mind, a U.S. patent search was conducted in the technology area of lighting controls and fluorescent lamp ballasts. The purpose of the search was to was two-fold: to seek out newly developed lighting technology that looks promising for increasing the efficiency of lighting systems, and to get a better understanding of technological state of the lighting controls and ballast industry.

\section{Method}

The Delphion.com website, operated by Delphion, Inc., was used to conduct the search. Delphion is a spin-off company from IBM making use of the extensive former IBM patent database, as well as providing other intellectual property management and services. At the time when this information was collected, the full Delphion database appeared to be freely available for searching. The LRC used these free services.

The search covered patents granted within the last five years (1996-2001) for lighting control technology, and the last three years (1998-2001) for fluorescent lamp ballast technology. The ballast technology search was limited to the past three years to reduce the relatively large volume of patents found in this area.

Searches were conducted by assignee, titles, and abstract fields using company names and/or a list of key control words. The list of company names and preliminary key words, organized alphabetically are given in Appendix 2.1 A. Additional product key words were generated as a result of a preliminary search of the technical literature using the IEEE Explore, Inspec, and Engineering Index Compendex databases. 


\section{Search Result Statistics}

About 350 patents were flagged as meeting the search criteria and appearing to be relevant to lighting control technology and/or fluorescent lamp ballast technology. Abstracts of these patents were printed and reviewed to make sure they still met the search criteria, and to categorize them into more specific technology areas. Of the 350 printed patent abstracts, 271 were deemed germane to the topic. Most of the 80 eliminated from the first pass filtering were concerned with HID ballasts, emergency lighting and similarly sounding technology such as lighted indicator switches.

By far, most of the patents found are concerned with ballast technology; 172 of the 271 patents and this despite limiting the search for ballast companies and ballast key words to only the past three years. These 172 patents were categorized into the following groups:

- 133 dealt with lamp operation, including, but not limited to efficiency, safety (with respect to the newer, small diameter T-5 fluorescent lamps), power quality, starting, lamp life, and novel circuits and materials.

- 35 dealt with ballast control, including, but not limited to dimming, addressing, and on/off power schemes involving control elements within the ballast.

- 4 dealt with mechanical fixtures for ballasts and electrical connectors. This category was not eliminated because of the possibility that better connectors and fastening systems could help reduce installation costs; a well know barrier to introducing new more efficient technology.

The remaining 111 patents were concerned with lighting controls. This group was categorized into the following groups and subgroups.

- 61 dealt with lighting control components

o 22 on dimming and/or switching including panel-level control among others

o 26 on occupancy sensors

o 4 on photosensors

o 5 on combined sensor technology (e.g., combining a photosensor with and occupancy sensor)

o 3 on window treatments (all having same assignee)

o 1 on time clocks

- 30 dealt with energy management systems including, but not limited to home automation products and schemes, control networks and methods of communication.

- 8 patents were categorized as design patents. These patents concerned the embodiment of a specific product. For example, some of these patents were on the physical shape of a switch or dimming knob used in a wall dimmer.

All of the 271 germane patents are listed in Appendix 2.1 B. They are cross referenced by U.S. patent number, assignee name, and the above technology categories. 


\section{Preliminary Analyses}

The fact that most of the found patents concern ballast technology indicates that this technology area has received far more economic attention then other areas involving lighting controls. The well-known ballast manufacturing companies (e.g., Motorola, Philips, GE, Magnetek and Howard) are all represented with patents, some more so than others. Interestingly, many patents in this area are also held by some very small companies, and even what appears to be private individuals. Most notably, Mr. Ole Nilssen of Barrington, IL, is represented with nine patents in the Ballast Operation category and one patent in the Ballast Control category. The question of whether such patents are important in any way is beyond the scope of this report, but it does signify that a lot research effort and/or legal claims are being pursued in the area of ballast technology, both by large companies and small.

A relatively small number of the ballast patents, 35, appeared from their abstracts to deal directly with control issues such as dimming, addressing and the integration of control logic into the ballast. Most of the patents in this category deal with specific circuits for dimming fluorescent lamps. The few that are concerned with addressing and the integration of control logic into the ballast tend to be represented in these results by smaller companies such as Energy Savings, Inc. and JRS Technology, Inc. Examples of this technology include ballasts with microprocessors that receive control signals and perform control functions to switch-off or reduce lamp power, and a "modular" ballast design that allows it to receive many different types of control signals from sensors and controllers.

Looking at the 111 patents concerned directly with lighting controls, it is again obvious where most of the economic attention has been within this area; energy management systems and panellevel controls, followed closely by occupancy sensor components. Only a total of 13 patents were found on photosensors, combined sensor technology, window treatments and time clocks combined. The lack of patents in these lesser-used control areas signifies that there is not much investment in these areas, economic or research. Occupancy sensing, manual dimming, and panel-level switching seem to be the types of controls most actively pursued by the lighting industry.

The occupancy sensor patents are concerned with circuit and sensing improvements and added features such as lower power consumption, two-wire installation, and use as security system component. The photosensor patents include a scheme for semiautomatic calibration, a dualphotosensor feedback system, a skylight photosensor system and a circuit for using photoconductive cells as linear detectors. The patents that describe combining different sensors most often combine a photosensor with an occupancy sensor. One adds a dimmer and a thermostat to the combination. One patent describes a method to control lights from a remote desktop device.

The category of patents on dimming and switching can be further divided into three groups; one dealing with incandescent type dimming (e.g., phase chopping or triac dimming circuits), one concerning panel, or multi-zone controls and one concerning dimming of fluorescent lamps. Lutron Electronics Company has many patents in all these areas. Some of the panel-level and multi-zone control patents are closely related to the building energy management system patents and make reference to their use in building automation systems.

Of the 30 patents dealing with energy management systems, eight have the word "home" in the titles and are not directly relevant to the commercial and industrial marketplace. Assignees of these patents include names such as Microsoft, IntelliNet, Inc., and Samsung Electronics Co., Ltd. Nevertheless, as far as the base technology is concerned, the technology and concepts used 
in home systems could likely be used in commercial and industrial settings as well. It is interesting that, because the home is a different market, it involves different companies than those traditionally associated with building control. The influx of new players in the building automation industry portends continued innovation in this area.

Breaking down the building automation patents into subcategories is difficult because there is not always a clear distinction between the different aspects of building automation. For example, software permeates all levels, but some patents deal more specifically with the software that others. Keeping this in mind, a somewhat subjective breakdown yields the following results:

- 10 appeared to deal with user interface and software issues

- 9 dealt with system-level designs

- 8 to concerned the physical communication layer

- 2 dealt with incorporating various sensors into the network

- 1 dealt with communication protocols

Johnson Controls Technology Company has a series of 6 patents all dealing with building automation control software. Terms such as "distributed-object" and "object oriented" and "standard-object" are used in the titles signifying that the recently developed, high-level software programming architectures are being incorporated into building automation systems.

Of the eight patents concerning the physical communication layer, three employ power line carrier schemes and one employs a dedicated fiber optic carrier. A patent on "distributed intelligence," assigned to Echelon Corporation, is also included in this group.

Hubble Corporation and Leviton Manufacturing Co., Inc., are the assignees for the two patents concerned with sensors. Both patents describe a multifunction sensor on a network having at least occupancy, ambient light level, and temperature sensing functions.

The appearance of only one patent dealing with communication protocols seems at first contrary to expectations. This result might reflect the inadequacy of this search, or the difficulty of placing these types of patents into the above categories. Patents that deal with communication methods and protocols in general are beyond the scope of this search, even though they might be applicable to building automation. On the other hand, the lack of patents found in this area having to do with lighting controls might be a reflection of the trend within the controls industry to gravitate towards “open systems” that use existing, open communication protocols.

\section{Appendix $2.1 \mathrm{~A}$}

List of Patent Assignee Names and Preliminary Key Words Used for Patent Search

\begin{tabular}{|l|}
\hline Company name \\
\hline ABB Control Inc. \\
\hline ACE Compact Electronics \\
\hline ADB Airfield Solutions \\
\hline Ademco Sensor Co (ASC) \\
\hline Advance Transformer \\
\hline Advanced Control Technologies Inc \\
\hline
\end{tabular}

\begin{tabular}{|l|}
\hline Agilent Technologies \\
\hline Alerton Technologies, Inc. \\
\hline American-De Rosa Lamparts Inc \\
\hline Amperlite Company Inc \\
\hline AMX (consolidated into PANJA Inc) \\
\hline Andover Controls Corporation \\
\hline Antron Compact Electronics Inc \\
\hline Architectural Lighting Management \\
\hline
\end{tabular}


Aromat (Panasonic)

Automated Logic Corporation

AVAB America Inc

B \& L Technologies Ltd

Blackbird

Bryant Electric (Hubbell)

Colortran (Leviton)

Cooper Lighting

Crestron

Cutler Hammer (Eaton)

Delta Controls Inc

Denki Corporation of North America

Douglas Lighting Controls Inc

EBW Electronics Inc

Echelon Corporation

Electronic Lighting Inc

Electronic Theatre Controls Inc

Elk Products Inc

Energy Savings Inc.

Fulham Company Inc

Future Wave Technologies

GE Industrial Systems

GE Lighting

Genlyte - Lightolier Controls

Genlyte / Thomas

GE-Smart

Gilbert Manufacturing Company Inc

H I Solutions Inc

Harrison Toshiba Lighting USA, Inc

High End Systems Inc

Honeywell

Horton Controls / Watt Stopper

Howard Industries Inc

HUB Electric/Vara-Light/Dimatronics

Hubbell and Hubbell Lighting

Hunt Controls Systems Inc

IBM Mylonas Lighting Company

Irideon Inc (purchased by ETC)

Johnson Controls Inc

JRS Technology Inc

Keegan Wireless

Kelar Controls Inc

Kingtec Inc
K-Tronic Ballasts

Lawrence Berkeley Labs

Company Name

LEAX Lighting controls

Lehigh Electric Products

Leviton (LON products group)

Leviton Lighting Control Division

Lighting \& Power Source, LP

Lithonia Lighting (controls)

Lithonia Lighting Canada

Lumion Corporation

Lutron Electronics Co Inc

MagneTek Lighting Products Group

Marco Electronics (now NSI Corp)

Marinco Industrial Group

Marlin Controls Inc

MaxLite

Microlites Scientific

Molex Incorporated

MYTEC Corporation

N.V. ADB-TV Tech S.A. (Siemens)

NexLight

North Point Engineering (now Nexlite)

Novar Controls Corporation

Novitas Inc

Osram Sylvania/ Ballast Division

Panja Inc

Paragon Electrical Products

Pass \& Seymour/ Legrand

PCI Lighting Control Systems Inc

PLC Multipoint Inc

RAB Electric Mfg Co Inc

Robertson Worldwide

Semper Fi Power Supply Inc

SensorSwitch Inc

Siebe Environmental Controls (Invensys)

Siemens Automation

Siemens Building Technologies Ltd

Sine Systems/Pyle Connector Corp

SLI Lighting Inc

SLS Industries/UNIDIM

Smart America

Square D Company 


\begin{tabular}{|l|}
\hline Sterner Controls \\
\hline Strand Lighting Canada Inc \\
\hline Strand Lighting Inc \\
\hline Sunpark Electronics Corporation \\
\hline The Watt Stopper / Pass \& Seymour \\
\hline Thomas Lighting \\
\hline Tork \\
\hline Touch-Plate Lighting Controls \\
\hline Trane \\
\hline Trend Group Inc \\
\hline Triatek Inc \\
\hline Tridonic Inc. (North America) \\
\hline Unenco (Hubbell) \\
\hline Vantage \\
\hline Vantage Lighting Inc \\
\hline Veam \\
\hline Visonic Inc \\
\hline X-10 USA Inc \\
\hline Xitron Technologies Inc \\
\hline \\
\hline Key Words \\
\hline Adaptation compensation \\
\hline Addressable ballasts \\
\hline BacNet \\
\hline Ballasts \\
\hline Building automation \\
\hline Commissioning \\
\hline Communications \\
\hline Control interoperability \\
\hline Control panels \\
\hline Controls \\
\hline Daylight \\
\hline Daylighting \\
\hline Dimming ballasts \\
\hline Dimming electronic ballasts \\
\hline Home Automation \\
\hline Intelligent controllable ballasts \\
\hline Lighting relay panels \\
\hline Load shedding \\
\hline LonWorks \\
\hline Lumen maintenance \\
\hline Manual dimming \\
\hline Occupancy \\
\hline
\end{tabular}

Peak demand limiting

Photosensors

Power line carriers

Programmable relay panels

Scheduling

Sensors

Smart building controls

Standard protocols

Task tuning

Theatrical controls

Time clocks

Timers

Tuning

Wall controllers

Whole building integrated control systems 


\section{Appendix 2.1 B}

\section{Patent Listing Sorted by Technology Categories}

\begin{tabular}{|c|c|c|c|c|}
\hline $\begin{array}{l}\text { U.S. Patent } \\
\text { Number. }\end{array}$ & Applicant/Company & Category & Subcategory & Patent Title \\
\hline 6121734 & Barna Szabados & Ballast & Ballast control & $\begin{array}{l}\text { Apparatus for dimming a fluorescent lamp } \\
\text { with a magnetic ballast }\end{array}$ \\
\hline 6072284 & Duro-Test Corporation & Ballast & Ballast control & $\begin{array}{l}\text { Three-way compact fluorescent lamp ballast } \\
\text { and lamp holder incorporating same }\end{array}$ \\
\hline 5828182 & Electronic Lighting Inc. & Ballast & Ballast control & $\begin{array}{l}\text { Apparatus for supplying power from a } \\
\text { ballast circuit to an auxiliary load }\end{array}$ \\
\hline 5936357 & Energy Savings, Inc. & Ballast & Ballast control & $\begin{array}{l}\text { Electronic ballast that manages switching } \\
\text { frequencies for extrinsic purposes }\end{array}$ \\
\hline 6137239 & Energy Savings, Inc. & Ballast & Ballast control & Electronic ballast with selective load control \\
\hline 6177769 & Energy Savings, Inc. & Ballast & Ballast control & $\begin{array}{l}\text { Electric Ballast with selective power } \\
\text { dissipation }\end{array}$ \\
\hline 6181072 & EZ Lighting, LLC & Ballast & Ballast control & $\begin{array}{l}\text { Apparatus and methods for dimming gas } \\
\text { discharge lamps using electronic ballast }\end{array}$ \\
\hline 5701059 & General Electric Company & Ballast & Ballast control & $\begin{array}{l}\text { Elimination of striations in fluorescent lamps } \\
\text { driven by high-frequency ballasts }\end{array}$ \\
\hline 5703441 & General Electric Company & Ballast & Ballast control & $\begin{array}{l}\text { Multi-function filament-heater power supply } \\
\text { for an electronic ballast for long-life } \\
\text { dimmable lamps }\end{array}$ \\
\hline 5965985 & General Electric Company & Ballast & Ballast control & $\begin{array}{l}\text { Dimmable ballast with complementary } \\
\text { converter switches }\end{array}$ \\
\hline 5994840 & General Electric Company & Ballast & Ballast control & $\begin{array}{l}\text { Controlling the transmission of light from } \\
\text { light sources }\end{array}$ \\
\hline 6008593 & International Rectifier Corp. & Ballast & Ballast control & $\begin{array}{l}\text { Closed-loop/dimming ballast controller } \\
\text { integrated circuits }\end{array}$ \\
\hline 5838116 & JRS Technology, Inc. & Ballast & Ballast control & $\begin{array}{l}\text { Fluorescent light ballast with information } \\
\text { transmission circuitry }\end{array}$ \\
\hline 6107755 & JRS Technology, Inc. & Ballast & Ballast control & $\begin{array}{l}\text { Modular, configurable dimming ballast for a } \\
\text { gas-discharge lamp }\end{array}$ \\
\hline 6181086 & JRS Technology, Inc. & Ballast & Ballast control & $\begin{array}{l}\text { Electronic ballast with embedded network } \\
\text { micro-controller }\end{array}$ \\
\hline 6218787 & JRS Technology, Inc. & Ballast & Ballast control & $\begin{array}{l}\text { Remote dimming control system for a } \\
\text { fluorescent ballast utilizing existing building } \\
\text { wiring }\end{array}$ \\
\hline 5949196 & Lumatech Corp. & Ballast & Ballast control & $\begin{array}{l}\text { Method and system for switchable light } \\
\text { levels in operating gas discharge lamps with } \\
\text { an inexpensive single ballast }\end{array}$ \\
\hline 6040661 & Lumion Corp. & Ballast & Ballast control & Programmable universal lighting system \\
\hline 5841239 & Lutron Electronics Co., Inc. & Ballast & Ballast control & $\begin{array}{l}\text { Circuit for dimming compact fluorescent } \\
\text { lamps }\end{array}$ \\
\hline 5864212 & Lutron Electronics Co., Inc. & Ballast & Ballast control & $\begin{array}{l}\text { Control system for providing power to a gas } \\
\text { discharge lamp }\end{array}$ \\
\hline 6060842 & $\begin{array}{l}\text { Ming-Chao Lin and Liu-Chih } \\
\text { Wang }\end{array}$ & Ballast & Ballast control & $\begin{array}{l}\text { Electronic ballast lighting power control } \\
\text { device }\end{array}$ \\
\hline
\end{tabular}




\begin{tabular}{|c|c|c|c|c|}
\hline $\begin{array}{l}\text { U.S. Patent } \\
\text { Number. }\end{array}$ & Applicant/Company & Category & Subcategory & Patent Title \\
\hline 6133697 & $\begin{array}{l}\text { Mitsubishi Denki Kabushiki } \\
\text { Kaisha }\end{array}$ & Ballast & Ballast control & Dimming apparatus for fluorescent lamps \\
\hline 6194841 & $\begin{array}{l}\text { Mitsubishi Denki Kabushiki } \\
\text { Kaisha }\end{array}$ & Ballast & Ballast control & Discharge lamp lighting device \\
\hline 6181085 & Ole K. Nilssen & Ballast & Ballast control & Electronic ballast with output control feature \\
\hline 6037722 & Pacific Scientific & Ballast & Ballast control & $\begin{array}{l}\text { Dimmable ballast apparatus and method for } \\
\text { controlling power delivered to a fluorescent } \\
\text { lamp }\end{array}$ \\
\hline 5866993 & Pacific Scientific Co. & Ballast & Ballast control & $\begin{array}{l}\text { Three-way dimming ballast circuit with } \\
\text { passive power factor correction }\end{array}$ \\
\hline 6011360 & $\begin{array}{l}\text { Philips Electronics North } \\
\text { America Corp. }\end{array}$ & Ballast & Ballast control & $\begin{array}{l}\text { High efficiency dimmable cold cathode } \\
\text { fluorescent lamp ballast }\end{array}$ \\
\hline 6118228 & Sandor Pal & Ballast & Ballast control & Electronic ballast for fluorescent lamps \\
\hline 6168289 & Suresh H. Shah & Ballast & Ballast control & $\begin{array}{l}\text { Straight fluorescent tube that has an } \\
\text { electronic ballast housed in the sockets on } \\
\text { both sides }\end{array}$ \\
\hline 6172466 & $\begin{array}{l}\text { The Hong Kong University of } \\
\text { Science and Technology }\end{array}$ & Ballast & Ballast control & Phase-controlled dimmable ballast \\
\hline 6208122 & Triatek, Inc. & Ballast & Ballast control & $\begin{array}{l}\text { High frequency pulse width modulation of } \\
\text { AC current for control of lighting load power }\end{array}$ \\
\hline 6094016 & Tridonic Bauelemente $\mathrm{GmbH}$ & Ballast & Ballast control & Electronic Ballast \\
\hline 6051938 & U. S. Philips Corp. & Ballast & Ballast control & $\begin{array}{l}\text { Dimmable ballast with active power } \\
\text { feedback control }\end{array}$ \\
\hline 6078147 & U. S. Philips Corp. & Ballast & Ballast control & $\begin{array}{l}\text { Discharge lamp ballast circuit with duty } \\
\text { cycle dimming control }\end{array}$ \\
\hline 6084362 & Wen-Shin Chao & Ballast & Ballast control & $\begin{array}{l}\text { Electronic ballast capable of linear and } \\
\text { stepless light regulation }\end{array}$ \\
\hline 6176594 & Herbert Lagin & Ballast & Ballast fixturing & $\begin{array}{l}\text { Streamlined fluorescent lamp ballast and } \\
\text { mounting assembly }\end{array}$ \\
\hline 5908235 & JRS Technology & Ballast & Ballast fixturing & Ballast fixture for fluorescent lighting \\
\hline 5788527 & MagneTek & Ballast & Ballast fixturing & $\begin{array}{l}\text { Electrical connector with improved safety } \\
\text { latching for a fluorescent-lighting ballast }\end{array}$ \\
\hline 5907218 & The Whitaker Corp. & Ballast & Ballast fixturing & $\begin{array}{l}\text { Fluorescent lighting assembly with integral } \\
\text { ballast }\end{array}$ \\
\hline 5872431 & Ahmed;Zahir M. & Ballast & Lamp operation & Efficient power transfer in electronic ballast \\
\hline 5900701 & $\begin{array}{l}\text { Allied Energy Services } \\
\text { International.Inc. }\end{array}$ & Ballast & Lamp operation & High frequency electronic ballast for lighting \\
\hline 6107751 & Billings;Keith & Ballast & Lamp operation & Current fed, parallel resonant ballast \\
\hline 5801492 & Bobel;Andrzej & Ballast & Lamp operation & $\begin{array}{l}\text { Electronic ballast for gas discharge lamp } \\
\text { having primary and auxiliary resonant } \\
\text { circuits }\end{array}$ \\
\hline 6175189 & Bruce Industries,Inc. & Ballast & Lamp operation & $\begin{array}{l}\text { Floating reference fault protection circuit for } \\
\text { arc discharge lamp ballast }\end{array}$ \\
\hline 6091210 & Cavolina: Alejandro & Ballast & Lamp operation & Electronic ballast with boost converter \\
\hline 6137233 & Electro-Mag International, Inc. & Ballast & Lamp operation & Ballast circuit with independent lamp control \\
\hline
\end{tabular}




\begin{tabular}{|c|c|c|c|c|}
\hline $\begin{array}{l}\text { U.S. Patent } \\
\text { Number. }\end{array}$ & Applicant/Company & Category & Subcategory & Patent Title \\
\hline 6028399 & Electro-Mag International,Inc. & Ballast & Lamp operation & \begin{tabular}{|l|} 
Ballast circuit with a capacitive and \\
inductive feedback path
\end{tabular} \\
\hline 6069455 & Electro-Mag International,Inc. & Ballast & Lamp operation & Ballast having a selectively resonant circuit \\
\hline 6100645 & Electro-Mag International,Inc. & Ballast & Lamp operation & Ballast having a reactive feedback circuit \\
\hline 6100648 & Electro-Mag International,Inc. & Ballast & Lamp operation & $\begin{array}{l}\text { Ballast having a resonant feedback circuit } \\
\text { for linear diode operation }\end{array}$ \\
\hline 6127786 & Electro-Mag International,Inc. & Ballast & Lamp operation & Ballast having a lamp end of life circuit \\
\hline 6160358 & Electro-Mag International,Inc. & Ballast & Lamp operation & $\begin{array}{l}\text { Ballast circuit with lamp current regulating } \\
\text { circuit }\end{array}$ \\
\hline 6181082 & Electro-Mag International,Inc. & Ballast & Lamp operation & Ballast power control circuit \\
\hline 6181083 & Electro-Mag International,Inc. & Ballast & Lamp operation & Ballast circuit with controlled strike/restart \\
\hline 6222326 & Electro-Mag International,Inc. & Ballast & Lamp operation & Ballast circuit with independent lamp control \\
\hline 5635825 & Electronic Lighting,Inc. & Ballast & Lamp operation & $\begin{array}{l}\text { Power factor corrected feed forward } \\
\text { coupled DC power supply }\end{array}$ \\
\hline 5789866 & Energy Savings, Inc. & Ballast & Lamp operation & $\begin{array}{l}\text { Electronic ballast with reversibly wound } \\
\text { filament winding }\end{array}$ \\
\hline 5493180 & Energy Savings,Inc. & Ballast & Lamp operation & Lamp protective, electronic ballast \\
\hline 5493181 & Energy Savings,Inc. & Ballast & Lamp operation & Capacitive lamp out detector \\
\hline 5500576 & Energy Savings,Inc. & Ballast & Lamp operation & Low height ballast for fluorescent lamps \\
\hline 5747941 & Energy Savings, Inc. & Ballast & Lamp operation & $\begin{array}{l}\text { Electronic ballast that monitors direct } \\
\text { current through lamp filaments }\end{array}$ \\
\hline 5925990 & Energy Savings,Inc. & Ballast & Lamp operation & Microprocessor controlled electronic ballast \\
\hline 5973455 & Energy Savings,Inc. & Ballast & Lamp operation & Electronic ballast with filament cut-out \\
\hline 5982113 & Energy Savings, Inc. & Ballast & Lamp operation & $\begin{array}{l}\text { Electronic ballast producing voltage having } \\
\text { trapezoidal envelope for instant-start lamps }\end{array}$ \\
\hline 6023132 & Energy Savings,Inc. & Ballast & Lamp operation & $\begin{array}{l}\text { Electronic ballast deriving auxiliary power } \\
\text { from lamp output }\end{array}$ \\
\hline 6049177 & Fulham Co. Inc. & Ballast & Lamp operation & $\begin{array}{l}\text { Single fluorescent lamp ballast for } \\
\text { simultaneous operation of different lamps in } \\
\text { series or parallel }\end{array}$ \\
\hline 5517086 & General Electric Company & Ballast & Lamp operation & $\begin{array}{l}\text { Modified Valley fill high power factor } \\
\text { correction ballast }\end{array}$ \\
\hline 5635800 & General Electric Company & Ballast & Lamp operation & $\begin{array}{l}\text { Ballast circuit with a japed transformer } \\
\text { flyback converter providing driving energy } \\
\text { for start, glow and run modes of a lamp }\end{array}$ \\
\hline 5703439 & General Electric Company & Ballast & Lamp operation & $\begin{array}{l}\text { Lamp power supply circuit with electronic } \\
\text { feedback circuit for switch control }\end{array}$ \\
\hline 5814976 & General Electric Company & Ballast & Lamp operation & High power factor electronic ballast \\
\hline 5838117 & General Electric Company & Ballast & Lamp operation & $\begin{array}{l}\text { Ballast circuit with synchronization and } \\
\text { preheat functions }\end{array}$ \\
\hline 5847508 & General Electric Company & Ballast & Lamp operation & $\begin{array}{l}\text { Integrated starting and running amalgam } \\
\text { assembly for an electrodeless fluorescent } \\
\text { lamp }\end{array}$ \\
\hline 5859504 & General Electric Company & Ballast & Lamp operation & $\begin{array}{l}\text { Lamp ballast circuit with cathode preheat } \\
\text { function }\end{array}$ \\
\hline
\end{tabular}




\begin{tabular}{|c|c|c|c|c|}
\hline $\begin{array}{l}\text { U.S. Patent } \\
\text { Number. }\end{array}$ & Applicant/Company & Category & Subcategory & Patent Title \\
\hline 5877595 & General Electric Company & Ballast & Lamp operation & $\begin{array}{l}\text { High power factor ballast circuit with } \\
\text { converter switches }\end{array}$ \\
\hline 5877596 & General Electric Company & Ballast & Lamp operation & $\begin{array}{l}\text { Universal electronic ballast for a family of } \\
\text { fluorescent lamps }\end{array}$ \\
\hline 5889373 & General Electric Company & Ballast & Lamp operation & $\begin{array}{l}\text { Fluorescent lamp ballast with current } \\
\text { feedback using a dual-function magnetic } \\
\text { device }\end{array}$ \\
\hline 5910708 & General Electric Company & Ballast & Lamp operation & $\begin{array}{l}\text { Gas discharge lamp ballast circuit with } \\
\text { complementary converter switches }\end{array}$ \\
\hline 5910709 & General Electric Company & Ballast & Lamp operation & \begin{tabular}{|l|} 
Fluorescent lamp ballast control for zero- \\
voltage switching operation over wide input \\
voltage range and over voltage protection
\end{tabular} \\
\hline 5917289 & General Electric Company & Ballast & Lamp operation & Lamp ballast with triggerless starting circuit \\
\hline 5952790 & General Electric Company & Ballast & Lamp operation & $\begin{array}{l}\text { Lamp ballast circuit with simplified starting } \\
\text { circuit }\end{array}$ \\
\hline 5986410 & General Electric Company & Ballast & Lamp operation & $\begin{array}{l}\text { Integrated circuit for use in a ballast circuit } \\
\text { for a gas discharge lamp }\end{array}$ \\
\hline 6011363 & General Electric Company & Ballast & Lamp operation & $\begin{array}{l}\text { Constant wattage electric ballast circuit for } \\
\text { lamp }\end{array}$ \\
\hline 6018220 & General Electric Company & Ballast & Lamp operation & $\begin{array}{l}\text { Gas discharge lamp ballast circuit with a } \\
\text { non-electrolytic smoothing capacitor for } \\
\text { rectified current }\end{array}$ \\
\hline 6051934 & General Electric Company & Ballast & Lamp operation & $\begin{array}{l}\text { Gas discharge lamp ballast circuit with high } \\
\text { speed gate drive circuitry }\end{array}$ \\
\hline 6111363 & General Electric Company & Ballast & Lamp operation & $\begin{array}{l}\text { Ballast shutdown circuit for a gas discharge } \\
\text { lamp }\end{array}$ \\
\hline 6144173 & General Electric Company & Ballast & Lamp operation & Single switch electronic ballast \\
\hline 6147455 & General Electric Company & Ballast & Lamp operation & $\begin{array}{l}\text { Gas discharge lamp ballast circuit with } \\
\text { electronic starter }\end{array}$ \\
\hline 6150769 & General Electric Company & Ballast & Lamp operation & $\begin{array}{l}\text { Gas discharge lamp ballast with tapeless } \\
\text { feedback circuit }\end{array}$ \\
\hline 5874809 & Hagen;Thomas & Ballast & Lamp operation & Constant light output ballast circuit \\
\hline 6031399 & Hewlett-Packard Company & Ballast & Lamp operation & $\begin{array}{l}\text { Efficient electronic ballast for fluorescent } \\
\text { tubes }\end{array}$ \\
\hline 5825139 & Hubbell Incorporated & Ballast & Lamp operation & $\begin{array}{l}\text { Lamp driven voltage transformation and } \\
\text { ballasting system }\end{array}$ \\
\hline 6166492 & Hubbell Incorporated & Ballast & Lamp operation & Lows loss, electronic ballast \\
\hline 35994 & IceCap,Inc. & Ballast & Lamp operation & Variable control, current sensing ballast \\
\hline 5828184 & $\begin{array}{l}\text { International Rectifier } \\
\text { Corporation }\end{array}$ & Ballast & Lamp operation & $\begin{array}{l}\text { Lamp ballast drive circuit having a resistor } \\
\text { in place of boot strap diode }\end{array}$ \\
\hline 5932974 & $\begin{array}{l}\text { International Rectifier } \\
\text { Corporation }\end{array}$ & Ballast & Lamp operation & $\begin{array}{l}\text { Ballast circuit with lamp removal protection } \\
\text { and soft starting }\end{array}$ \\
\hline 6002214 & $\begin{array}{l}\text { International Rectifier } \\
\text { Corporation }\end{array}$ & Ballast & Lamp operation & $\begin{array}{l}\text { Phase detection control circuit for an } \\
\text { electronic ballast }\end{array}$ \\
\hline 6005354 & $\begin{array}{l}\text { International Rectifier } \\
\text { Corporation }\end{array}$ & Ballast & Lamp operation & Ballast IC with shut-down function \\
\hline
\end{tabular}




\begin{tabular}{|c|c|c|c|c|}
\hline $\begin{array}{l}\text { U.S. Patent } \\
\text { Number. }\end{array}$ & Applicant/Company & Category & Subcategory & Patent Title \\
\hline 6008592 & $\begin{array}{l}\text { International Rectifier } \\
\text { Corporation }\end{array}$ & Ballast & Lamp operation & $\begin{array}{l}\text { End of lamp life or false lamp detection } \\
\text { circuit for an electronic ballast }\end{array}$ \\
\hline 6031342 & $\begin{array}{l}\text { International Rectifier } \\
\text { Corporation }\end{array}$ & Ballast & Lamp operation & Universal input warm-start linear ballast \\
\hline 6150773 & $\begin{array}{l}\text { International Rectifier } \\
\text { Corporation }\end{array}$ & Ballast & Lamp operation & $\begin{array}{l}\text { Module and method for high-frequency } \\
\text { electronic ballast design }\end{array}$ \\
\hline 6215253 & $\begin{array}{l}\text { International Rectifier } \\
\text { Corporation }\end{array}$ & Ballast & Lamp operation & Inductorless ballast \\
\hline 5519289 & $\begin{array}{l}\text { JRS Technology Associates, } \\
\text { Inc. }\end{array}$ & Ballast & Lamp operation & $\begin{array}{l}\text { Electronic ballast with lamp current } \\
\text { correction circuit }\end{array}$ \\
\hline 5930130 & JRS Technology, Inc. & Ballast & Lamp operation & Inrush protection circuit \\
\hline 6034484 & Korea Tronix Co, Ltd, & Ballast & Lamp operation & Piezoelectronic ballast for fluorescent lamp \\
\hline 6060844 & LapLaz Lighting Co, & Ballast & Lamp operation & $\begin{array}{l}\text { Method and apparatus of an improved } \\
\text { electronics ballast circuit }\end{array}$ \\
\hline 6083021 & Lau;Kenneth & Ballast & Lamp operation & $\begin{array}{l}\text { Fluorescent light ballast lamp mounting } \\
\text { socket construction }\end{array}$ \\
\hline 6094124 & Lee;Kyung-Soo & Ballast & Lamp operation & $\begin{array}{l}\text { Ballast for discharge lamp and method and } \\
\text { apparatus for manufacturing the same }\end{array}$ \\
\hline 6034488 & Lighting Control, Inc. & Ballast & Lamp operation & $\begin{array}{l}\text { Electronic ballast for fluorescent lighting } \\
\text { system including a voltage monitoring circui }\end{array}$ \\
\hline 551561 & Lumion Corporation & Ballast & Lamp operation & Power factor correction circuitry \\
\hline 6137240 & Lumion Corporation & Ballast & Lamp operation & Universal ballast control circuit \\
\hline 6111368 & Lutron Electronics Co, Inc. & Ballast & Lamp operation & $\begin{array}{l}\text { System for preventing oscillations in a } \\
\text { fluorescent lamp ballast }\end{array}$ \\
\hline 6114810 & Mass Technology(H.K) Ltd, & Ballast & Lamp operation & $\begin{array}{l}\text { Electronic ballast circuit for fluorescent } \\
\text { lamps which have a high Q factor and high } \\
\text { resonance voltage }\end{array}$ \\
\hline 5789871 & $\begin{array}{l}\text { Massachusetts Institute of } \\
\text { Technology }\end{array}$ & Ballast & Lamp operation & Series-capacitor electronic ballast \\
\hline 5920155 & Matsushita Electric Works, Ltd, & Ballast & Lamp operation & Electronic ballast for discharge lamps \\
\hline 6054815 & Matsusita Electric Industrial, Ltd, & Ballast & Lamp operation & Ballast for a discharge lamp \\
\hline 6008587 & Mills; Robert & Ballast & Lamp operation & $\begin{array}{l}\text { Fluorescent lamp electronic ballast control } \\
\text { circuit }\end{array}$ \\
\hline 6181087 & $\begin{array}{l}\text { Mitsubishi Denki Kabushiki } \\
\text { Kaisha; Mitsubishi Electric } \\
\text { Lighting Corporation }\end{array}$ & Ballast & Lamp operation & Discharge lamp operating device \\
\hline 5969483 & Motorola & Ballast & Lamp operation & $\begin{array}{l}\text { Inverter control method for electronic } \\
\text { ballasts }\end{array}$ \\
\hline 5770925 & Motorola Inc. & Ballast & Lamp operation & $\begin{array}{l}\text { Electronic ballast with inverter protection } \\
\text { and relamping circuits }\end{array}$ \\
\hline 5834924 & Motorola Inc. & Ballast & Lamp operation & $\begin{array}{l}\text { In-rush current reduction circuit for boost } \\
\text { converters and electronic ballast }\end{array}$ \\
\hline 5869935 & Motorola Inc. & Ballast & Lamp operation & $\begin{array}{l}\text { Electronic ballast with inverter protection } \\
\text { circuit }\end{array}$ \\
\hline 5869937 & Motorola Inc. & Ballast & Lamp operation & High efficiency electronic ballast \\
\hline
\end{tabular}




\begin{tabular}{|c|c|c|c|c|}
\hline $\begin{array}{l}\text { U.S. Patent } \\
\text { Number. }\end{array}$ & Applicant/Company & Category & Subcategory & Patent Title \\
\hline 5872430 & Motorola Inc. & Ballast & Lamp operation & $\begin{array}{l}\text { Single switch electronic ballast with low in- } \\
\text { rush current }\end{array}$ \\
\hline 5883473 & Motorola Inc. & Ballast & Lamp operation & $\begin{array}{l}\text { Electronic Ballast with inverter protection } \\
\text { circuit }\end{array}$ \\
\hline 5945788 & Motorola Inc. & Ballast & Lamp operation & Electronic ballast with inverter control circuit \\
\hline 5982109 & Motorola Inc. & Ballast & Lamp operation & $\begin{array}{l}\text { Electronic ballast with fault-protected series } \\
\text { resonant output circuit }\end{array}$ \\
\hline 5998930 & Motorola Inc. & Ballast & Lamp operation & $\begin{array}{l}\text { Electronic ballast with two-step boost } \\
\text { converter and method }\end{array}$ \\
\hline 5841241 & Nilssen; Ole K. & Ballast & Lamp operation & Electronic ballast for fluorescent lamps \\
\hline 5977721 & Nilssen; Ole K. & Ballast & Lamp operation & Controlled power-factor-corrected ballast \\
\hline 6002210 & Nilssen; Ole K. & Ballast & Lamp operation & $\begin{array}{l}\text { Electronic ballast with controlled-magnitude } \\
\text { output voltage }\end{array}$ \\
\hline 6107749 & Nilssen; Ole K. & Ballast & Lamp operation & FET-bipolar electronic ballast \\
\hline 6121733 & Nilssen; Ole K. & Ballast & Lamp operation & $\begin{array}{l}\text { Controlled inverter-type fluorescent lamp } \\
\text { ballast }\end{array}$ \\
\hline 61444445 & Nilssen; Ole K. & Ballast & Lamp operation & Electronic ballast products and systems \\
\hline 6150768 & Nilssen; Ole K. & Ballast & Lamp operation & Ballast with active power factor correction \\
\hline 6211619 & Nilssen; Ole K. & Ballast & Lamp operation & Electronic ballast cathode heating circuit \\
\hline 6211625 & Nilssen; Ole K. & Ballast & Lamp operation & $\begin{array}{l}\text { Electronic ballast with over-voltage } \\
\text { protection }\end{array}$ \\
\hline 5969484 & $\begin{array}{l}\text { Optimum Power Conversion, } \\
\text { Inc. }\end{array}$ & Ballast & Lamp operation & Electronic ballast \\
\hline 5821699 & Pacific Scientific & Ballast & Lamp operation & Ballast circuit for fluorescent lamps \\
\hline 5798617 & Pacific Scientific Company & Ballast & Lamp operation & $\begin{array}{l}\text { Magnetic feedback ballast circuit for } \\
\text { fluorescent lamp }\end{array}$ \\
\hline 5955841 & Pacific Scientific Company & Ballast & Lamp operation & Ballast circuit for fluorescent lamp \\
\hline 5982111 & Pacific Scientific Company & Ballast & Lamp operation & $\begin{array}{l}\text { Fluorescent lamp ballast having a resonant } \\
\text { output stage using a split resonating } \\
\text { inductor }\end{array}$ \\
\hline 6078144 & $\begin{array}{l}\text { Patent-Treuhand-Gesellschaft } \\
\text { fuer elektrische Gluehlampen } \\
\text { mbH }\end{array}$ & Ballast & Lamp operation & Electronic ballast with inrush current limiting \\
\hline 5834906 & $\begin{array}{l}\text { Philips Electronics North } \\
\text { America Corporation }\end{array}$ & Ballast & Lamp operation & $\begin{array}{l}\text { Instant start for an electronic ballast } \\
\text { preconditioned having an active power } \\
\text { factor controller }\end{array}$ \\
\hline 5907223 & $\begin{array}{l}\text { Philips Electronics North } \\
\text { America Corporation }\end{array}$ & Ballast & Lamp operation & $\begin{array}{l}\text { Two-frequency electronic ballast system } \\
\text { having an isolated PFC converter }\end{array}$ \\
\hline 5923126 & $\begin{array}{l}\text { Philips Electronics North } \\
\text { America Corporation }\end{array}$ & Ballast & Lamp operation & $\begin{array}{l}\text { Fluorescent lamp electronic ballast with } \\
\text { rapid voltage turn-on after preheating }\end{array}$ \\
\hline 5973437 & $\begin{array}{l}\text { Philips Electronics North } \\
\text { America Corporation }\end{array}$ & Ballast & Lamp operation & Scheme for sensing ballast lamp current \\
\hline 6020689 & $\begin{array}{l}\text { Philips Electronics North } \\
\text { America Corporation }\end{array}$ & Ballast & Lamp operation & $\begin{array}{l}\text { Anti-flicker scheme for a fluorescent lamp } \\
\text { ballast driver }\end{array}$ \\
\hline 6100646 & $\begin{array}{l}\text { Philips Electronics North } \\
\text { America Corporation }\end{array}$ & Ballast & Lamp operation & Ballast feedback scheme \\
\hline
\end{tabular}




\begin{tabular}{|c|c|c|c|c|}
\hline $\begin{array}{l}\text { U.S. Patent } \\
\text { Number. }\end{array}$ & Applicant/Company & Category & Subcategory & Patent Title \\
\hline 6100647 & \begin{tabular}{|l|} 
Philips Electronics North \\
America Corporation
\end{tabular} & Ballast & Lamp operation & $\begin{array}{l}\text { Lamp ballast for accurate control of lamp } \\
\text { intensity }\end{array}$ \\
\hline 6181079 & $\begin{array}{l}\text { Philips Electronics North } \\
\text { America Corporation }\end{array}$ & Ballast & Lamp operation & $\begin{array}{l}\text { High power electronic ballast wit an } \\
\text { integrated magnetic component }\end{array}$ \\
\hline 6184630 & $\begin{array}{l}\text { Philips Electronics North } \\
\text { America Corporation }\end{array}$ & Ballast & Lamp operation & $\begin{array}{l}\text { Electronic lamp ballast wit voltage source } \\
\text { power feedback to AC-side }\end{array}$ \\
\hline 6072282 & Power Circuit Innovations, Inc., & Ballast & Lamp operation & $\begin{array}{l}\text { Frequency controlled quick and soft start } \\
\text { gas discharges lamp ballast and method } \\
\text { therefore }\end{array}$ \\
\hline 6088249 & Power Circuit Innovations, Inc., & Ballast & Lamp operation & $\begin{array}{l}\text { Frequency modulated ballast with loosely } \\
\text { coupled transformer }\end{array}$ \\
\hline 6181066 & Power Circuit Innovations, Inc., & Ballast & Lamp operation & $\begin{array}{l}\text { Frequency modulated ballast with loosely } \\
\text { coupled transformer for parallel gas } \\
\text { discharge lamp control }\end{array}$ \\
\hline 6222322 & $\begin{array}{l}\text { Q Technology Incorporated; } \\
\text { New Athony, Inc., }\end{array}$ & Ballast & Lamp operation & $\begin{array}{l}\text { Ballast with lamp abnormal sensor and } \\
\text { method therefore }\end{array}$ \\
\hline 5977723 & $\begin{array}{l}\text { Samsung Display Devices Co., } \\
\text { Ltd., }\end{array}$ & Ballast & Lamp operation & Ballast circuit for fluorescent lamp \\
\hline 5770926 & Samsung Electronics Co., Ltd., & Ballast & Lamp operation & $\begin{array}{l}\text { Feedback control system of an electronic } \\
\text { ballast which detects arcing of a lamp }\end{array}$ \\
\hline 5786671 & Samsung Electronics Co., Ltd., & Ballast & Lamp operation & $\begin{array}{l}\text { Electronic ballast circuit having voltage } \\
\text { reducing transformer }\end{array}$ \\
\hline 6005355 & $\begin{array}{l}\text { Siao; Susan Yeung: Anne Chon } \\
\text { My }\end{array}$ & Ballast & Lamp operation & $\begin{array}{l}\text { Electronic ballast system for fluorescent } \\
\text { lamps }\end{array}$ \\
\hline 6091206 & $\begin{array}{l}\text { Siao;Susan Yeung:Anne Chon } \\
\text { My }\end{array}$ & Ballast & Lamp operation & $\begin{array}{l}\text { Electronic ballast system for fluorescent } \\
\text { lamps }\end{array}$ \\
\hline 5705894 & Siemens Aktiengesellschaft & Ballast & Lamp operation & $\begin{array}{l}\text { Method for operating at least one } \\
\text { fluorescent lamp with an electronic ballast, } \\
\text { as well as ballast therefore }\end{array}$ \\
\hline 5834899 & $\begin{array}{l}\text { Tapeswitch Corporation of } \\
\text { America }\end{array}$ & Ballast & Lamp operation & $\begin{array}{l}\text { Fluorescent apparatus and method } \\
\text { employing low-frequency excitation into a } \\
\text { conductive-resistive inductive medium }\end{array}$ \\
\hline 5930126 & The Genlyte Group Incorporated & Ballast & Lamp operation & $\begin{array}{l}\text { Ballast shut-down circuit responsive to an } \\
\text { unbalanced load condition in a single lamp } \\
\text { ballast or in either lamp of a two-lamp } \\
\text { ballast }\end{array}$ \\
\hline 5825137 & Titus;Charles H., & Ballast & Lamp operation & $\begin{array}{l}\text { Electronic ballasts for plural lamp } \\
\text { fluorescent lighting without feedback } \\
\text { circuitry }\end{array}$ \\
\hline 6107747 & $\begin{array}{l}\text { Toshiba Lighting \& Technology } \\
\text { Corporation }\end{array}$ & Ballast & Lamp operation & $\begin{array}{l}\text { Self ballasted fluorescent lamp and lighting } \\
\text { fixture }\end{array}$ \\
\hline 6166491 & $\begin{array}{l}\text { Toshiba Lighting \& Technology } \\
\text { Corporation }\end{array}$ & Ballast & Lamp operation & Lighting device and display equipment \\
\hline 5936359 & Trojan Technologies,Inc., & Ballast & Lamp operation & $\begin{array}{l}\text { Apparatus for efficient remote ballasting of } \\
\text { gaseous discharge lamps }\end{array}$ \\
\hline 6069453 & U.S. Philips Corporation & Ballast & Lamp operation & $\begin{array}{l}\text { Ballast circuit for reducing striations in a } \\
\text { discharge lamp }\end{array}$ \\
\hline
\end{tabular}




\begin{tabular}{|c|c|c|c|c|}
\hline $\begin{array}{l}\text { U.S. Patent } \\
\text { Number. }\end{array}$ & Applicant/Company & Category & Subcategory & Patent Title \\
\hline 6188184 & U.S. Philips Corporation & Ballast & Lamp operation & $\begin{array}{l}\text { Electronic ballast with reduced operating } \\
\text { frequency after lamp ignition }\end{array}$ \\
\hline 6147459 & $\begin{array}{l}\text { Vossloh-Schwabe Elektronik } \\
\text { GmbH, }\end{array}$ & Ballast & Lamp operation & $\begin{array}{l}\text { Voltage-controlled ballast for discharge } \\
\text { lamps }\end{array}$ \\
\hline 6011358 & Vossloh-Schwabe GmbH, & Ballast & Lamp operation & $\begin{array}{l}\text { Ballast for independent parallel operation of } \\
\text { low-pressure gas discharge lamps }\end{array}$ \\
\hline 6043612 & Vossloh-Schwabe GmbH, & Ballast & Lamp operation & Electronic ballast with automatic restarting \\
\hline 5834765 & $\begin{array}{l}\text { Ledalite Architectural Products, } \\
\text { Inc. }\end{array}$ & Controls & Combined sensors & $\begin{array}{l}\text { Integral ambient light and occupancy sensor } \\
\text { having a linear array of sensor element and } \\
\text { a segmented slit aperture device }\end{array}$ \\
\hline 435473 & Leviton Manufacturing Co., Inc. & Controls & Combined sensors & $\begin{array}{l}\text { Combined dimmer, switch, IR receiver, } \\
\text { thermostat, ambient light sensor and } \\
\text { passive infrared motion sensor }\end{array}$ \\
\hline 5406173 & The Watt Stopper & Controls & Combined sensors & $\begin{array}{l}\text { Apparatus and method for adjusting lights } \\
\text { according to the level of ambient light }\end{array}$ \\
\hline 5455487 & The Watt Stopper & Controls & Combined sensors & Movable desktop light controller \\
\hline 5598042 & The Watt Stopper & Controls & Combined sensors & Movable desktop load controller \\
\hline 6000810 & Eagle Energy Systems, Ltd. & Controls & Dim/Sw Systems & $\begin{array}{l}\text { Low voltage storage warehouse lighting } \\
\text { system }\end{array}$ \\
\hline 5530322 & Lutron Electronics Co., Inc. & Controls & Dim/Sw Systems & Multi-zone lighting control system \\
\hline 5637930 & Lutron Electronics Co., Inc. & Controls & Dim/Sw Systems & Wall-mountable switch and dimmer \\
\hline 5949200 & Lutron Electronics Co., Inc. & Controls & Dim/Sw Systems & $\begin{array}{l}\text { Wall mountable control system with virtually } \\
\text { unlimited zone capacity }\end{array}$ \\
\hline 5990635 & Lutron Electronics Co., Inc. & Controls & Dim/Sw Systems & Multi-zone lighting control system \\
\hline 6046550 & Lutron Electronics Co., Inc. & Controls & Dim/Sw Systems & Multi-zone lighting control system \\
\hline 6188181 & Lutron Electronics Co., Inc. & Controls & Dim/Sw Systems & $\begin{array}{l}\text { Lighting control system for different load } \\
\text { types }\end{array}$ \\
\hline 4972125 & Lee Colortran, Inc. & Controls & Dimming & $\begin{array}{l}\text { Plug-in dimmer module for lighting control } \\
\text { systems }\end{array}$ \\
\hline 5485058 & Leviton Manufacturing $\mathrm{C}_{0 .,}$ Inc. & Controls & Dimming & Touch dimmer system \\
\hline 5798581 & Lutron Electronics Co., Inc. & Controls & Dimming & $\begin{array}{l}\text { Location independent dimmer switch for use } \\
\text { in multiple location switch system and } \\
\text { switch system employing same }\end{array}$ \\
\hline 5808417 & Lutron Electronics Co., Inc. & Controls & Dimming & $\begin{array}{l}\text { Lighting control system with corrugated heat } \\
\text { sink }\end{array}$ \\
\hline 6005308 & Lutron Electronics Co., Inc. & Controls & Dimming & Electrical switch and dimmer control device \\
\hline 5585713 & Molex, Inc. & Controls & Dimming & $\begin{array}{l}\text { Light dimmer circuit with control pulse } \\
\text { stretching }\end{array}$ \\
\hline 5283516 & Pass and Seymore Legrand & Controls & Dimming & Low voltage dimmer with no-load protection \\
\hline 369348 & The Watt Stopper & Controls & Dimming & Light level controller \\
\hline 5402040 & The Watt Stopper & Controls & Dimming & Dimmable ballast control circuit \\
\hline 5742131 & The Watt Stopper & Controls & Dimming & Dimmable ballast control circuit \\
\hline 5699243 & Hubbell, Inc. & Controls & Occupancy sensor & $\begin{array}{l}\text { Motion sensing system with adaptive timing } \\
\text { for controlling lighting fixtures }\end{array}$ \\
\hline 5764146 & Hubbell, Inc. & Controls & Occupancy sensor & Multifunction occupancy \\
\hline
\end{tabular}




\begin{tabular}{|c|c|c|c|c|}
\hline $\begin{array}{l}\text { U.S. Patent } \\
\text { Number. }\end{array}$ & Applicant/Company & Category & Subcategory & Patent Title \\
\hline 5946209 & Hubbell, Inc. & Controls & Occupancy sensor & $\begin{array}{l}\text { Motion sensing system with adaptive timing } \\
\text { for controlling lighting fixtures }\end{array}$ \\
\hline 5984513 & Hubbell, Inc. & Controls & Occupancy sensor & $\begin{array}{l}\text { Very low current microcontroller operated } \\
\text { motion sensor }\end{array}$ \\
\hline 6151529 & Hubbell, Inc. & Controls & Occupancy sensor & $\begin{array}{l}\text { Motion sensing system with adaptive timing } \\
\text { for controlling lighting fixtures }\end{array}$ \\
\hline 5739753 & Leviton Manufacturing $\mathrm{C}_{0 .}$., Inc & Controls & Occupancy sensor & Detector system with adjustable field of view \\
\hline 5623172 & Leviton Manufacturing Co., Inc. & Controls & Occupancy sensor & $\begin{array}{l}\text { Two wire PIR occupancy sensor utilizing a } \\
\text { rechargeable energy storage device }\end{array}$ \\
\hline 5786644 & Leviton Manufacturing Co., Inc. & Controls & Occupancy sensor & $\begin{array}{l}\text { Two wire PIR occupancy sensor utilizing a } \\
\text { rechargeable energy storage device }\end{array}$ \\
\hline 5128654 & Lightolier, Inc. & Controls & Occupancy sensor & $\begin{array}{l}\text { Preset light controller including infrared } \\
\text { sensor operable in multiple modes }\end{array}$ \\
\hline 401175 & Mytech Corporation & Controls & Occupancy sensor & Occupancy sensor \\
\hline 404325 & Mytech Corporation & Controls & Occupancy sensor & Occupancy sensor \\
\hline 404326 & Mytech Corporation & Controls & Occupancy sensor & Occupancy sensor \\
\hline 430056 & Mytech Corporation & Controls & Occupancy sensor & Ultrasonic occupancy sensor \\
\hline 435798 & Mytech Corporation & Controls & Occupancy sensor & Ultrasonic occupancy sensor \\
\hline 5986357 & Mytech Corporation & Controls & Occupancy sensor & $\begin{array}{l}\text { Occupancy sensor and method of operating } \\
\text { same }\end{array}$ \\
\hline 6078253 & Mytech Corporation & Controls & Occupancy sensor & $\begin{array}{l}\text { Occupancy sensor and method of operating } \\
\text { same }\end{array}$ \\
\hline 6078253 & Mytech Corporation & Controls & Occupancy sensor & $\begin{array}{l}\text { Occupancy sensor and method of operating } \\
\text { same }\end{array}$ \\
\hline 6078253 & Mytech Corporation & Controls & Occupancy sensor & $\begin{array}{l}\text { Occupancy sensor and method of operating } \\
\text { same }\end{array}$ \\
\hline 6222191 & Mytech Corporation & Controls & Occupancy sensor & Occupancy sensor \\
\hline 5489827 & Philips Electronics N.A. Corp. & Controls & Occupancy sensor & Light controller with occupancy sensor \\
\hline 6215398 & Platner, Brian P. & Controls & Occupancy sensor & $\begin{array}{l}\text { Occupancy sensors for long range sensing } \\
\text { within a narrow field of view }\end{array}$ \\
\hline 360842 & The Watt Stopper & Controls & Occupancy sensor & Motion Sensor \\
\hline 5221919 & Unenco, Inc. & Controls & Occupancy sensor & Room \\
\hline 5973996 & Visonic Ltd. & Controls & Occupancy sensor & Ultrasound intrusion detector \\
\hline 6211522 & Visonic Ltd. & Controls & Occupancy sensor & Passive infra-red intrusion sensor \\
\hline 370863 & Visonic, Ltd. & Controls & Occupancy sensor & Passive infra-red sensor \\
\hline 6025679 & Harper; Raymond G. & Controls & Photosensor & Lighting space controller \\
\hline 4538218 & Honeywell Inc. & Controls & Photosensor & Skylight sensor and control system \\
\hline 5701058 & Honeywell Inc. & Controls & Photosensor & $\begin{array}{l}\text { Method of semiautomatic ambient light } \\
\text { sensor calibration in an automatic control } \\
\text { system }\end{array}$ \\
\hline 5039853 & Multipoint Control Systems, Inc. & Controls & Photosensor & $\begin{array}{l}\text { Constant-current light-sensing system and } \\
\text { improved sensor housing }\end{array}$ \\
\hline 5942814 & Echelon Corporation & Controls & Switching & $\begin{array}{l}\text { Module for controlling current to a load } \\
\text { including sensing of the position of a switch }\end{array}$ \\
\hline 4042903 & Hunt Electronics Company & Controls & Switching & Power control slide switch \\
\hline
\end{tabular}




\begin{tabular}{|c|c|c|c|c|}
\hline $\begin{array}{l}\text { U.S. Patent } \\
\text { Number. }\end{array}$ & Applicant/Company & Category & Subcategory & Patent Title \\
\hline 376130 & Novitas Incorporated & Controls & Switching & Ultrasonic wall switch \\
\hline 380452 & The Watt Stopper & Controls & Switching & Wall-mounted switch \\
\hline 5804991 & The Watt Stopper & Controls & Switching & Zero crossing circuit for a relay \\
\hline 5508878 & Paragon electric Company, Inc. & Controls & Time Clock & Electronic timer using zero power while off \\
\hline 5598000 & Popat, Pradeep P. & Controls & Window treatment & $\begin{array}{l}\text { Dual-mode automatic window covering } \\
\text { system responsive to AC-induced flicker in } \\
\text { ambient illumination }\end{array}$ \\
\hline 5663621 & Popat, Pradeep P. & Controls & Window treatment & $\begin{array}{l}\text { Autonomous low cost automatic window } \\
\text { covering system for daylighting applications }\end{array}$ \\
\hline 6084231 & Popat, Pradeep P. & Controls & Window treatment & $\begin{array}{l}\text { Closed-loop daylight sensing automatic } \\
\text { window covering system insensitive to } \\
\text { radiant spectrum produced by gaseous } \\
\text { discharge lamps }\end{array}$ \\
\hline 416910 & AMX Corporation & Design & Product specific & Remote Control \\
\hline 6166640 & Hubbell Incorporated & Design & Product specific & $\begin{array}{l}\text { Bicolor indicator lamp for room occupancy } \\
\text { sensor }\end{array}$ \\
\hline 5934451 & Leviton Manufacturing Co., Inc & Design & Product specific & $\begin{array}{l}\text { Illuminated rocker switch cover assembly } \\
\text { employing electroluminescent lamp member }\end{array}$ \\
\hline 6011326 & Leviton Manufacturing Co., Inc & Design & Product specific & $\begin{array}{l}\text { Dual switch and/or dimmer system } \\
\text { illuminated with single neon lamp }\end{array}$ \\
\hline 6089893 & Leviton Manufacturing Co., Inc & Design & Product specific & $\begin{array}{l}\text { Illuminated electrical receptacle employing } \\
\text { electroluminescent lamp member }\end{array}$ \\
\hline 6145998 & Leviton Manufacturing Co., Inc & Design & Product specific & Demonstration display for lighting controls \\
\hline 344264 & Lutron Electronics Co., Inc. & Design & Product specific & Control panel for a lighting control unit \\
\hline 381632 & The Watt Stopper & Design & Product specific & Desktop electrical apparatus control unit \\
\hline 6122678 & Leviton Manufacturing Co., Inc. & Systems & Combined sensors & $\begin{array}{l}\text { Local network based multiple sensor device } \\
\text { with electrical load control means and with } \\
\text { temperature sensor that is exposed to } \\
\text { ambient air by diffusion }\end{array}$ \\
\hline 6104963 & $\begin{array}{l}\text { Johnson Controls Technology } \\
\text { Co. }\end{array}$ & Systems & $\begin{array}{l}\text { Communication } \\
\text { methods }\end{array}$ & $\begin{array}{l}\text { Communication system for distributed- } \\
\text { object building automation system }\end{array}$ \\
\hline 6119125 & $\begin{array}{l}\text { Johnson Controls Technology } \\
\text { Co. }\end{array}$ & Systems & $\begin{array}{l}\text { Communication } \\
\text { methods }\end{array}$ & $\begin{array}{l}\text { Software components for a building } \\
\text { automation system based on a standard } \\
\text { object superclass }\end{array}$ \\
\hline 6141595 & $\begin{array}{l}\text { Johnson Controls Technology } \\
\text { Co. }\end{array}$ & Systems & $\begin{array}{l}\text { Communication } \\
\text { methods }\end{array}$ & $\begin{array}{l}\text { Common object architecture supporting } \\
\text { application-centric building automations } \\
\text { systems }\end{array}$ \\
\hline 6154681 & $\begin{array}{l}\text { Johnson Controls Technology } \\
\text { Co. }\end{array}$ & Systems & $\begin{array}{l}\text { Communication } \\
\text { methods }\end{array}$ & $\begin{array}{l}\text { Asynchronous distributed-object building } \\
\text { automation system with support for } \\
\text { synchronous object execution }\end{array}$ \\
\hline 6167316 & $\begin{array}{l}\text { Johnson Controls Technology } \\
\text { Co. }\end{array}$ & Systems & $\begin{array}{l}\text { Communication } \\
\text { methods }\end{array}$ & $\begin{array}{l}\text { Distributed object-oriented building } \\
\text { automation system with reliable } \\
\text { asynchronous communication }\end{array}$ \\
\hline 6028998 & Johnson Service Co. & Systems & $\begin{array}{l}\text { Communication } \\
\text { methods }\end{array}$ & $\begin{array}{l}\text { Application framework for constructing } \\
\text { building automation systems }\end{array}$ \\
\hline 4355303 & Westinghouse Electric Corp. & Systems & $\begin{array}{l}\text { Communication } \\
\text { methods }\end{array}$ & $\begin{array}{l}\text { Receiver for a distribution network power } \\
\text { line carrier communication system }\end{array}$ \\
\hline
\end{tabular}




\begin{tabular}{|c|c|c|c|c|}
\hline $\begin{array}{l}\text { U.S. Patent } \\
\text { Number. }\end{array}$ & Applicant/Company & Category & Subcategory & Patent Title \\
\hline 5408550 & Wireless Control System, Inc. & Systems & $\begin{array}{l}\text { Communication } \\
\text { methods }\end{array}$ & $\begin{array}{l}\text { Remote fiber optic electrical switch using } \\
\text { light pulse signals }\end{array}$ \\
\hline 5844888 & Echelon Corp. & Systems & Control networks & $\begin{array}{l}\text { Network and intelligent cell for providing } \\
\text { sensing, bidirectional communications and } \\
\text { control }\end{array}$ \\
\hline 5703442 & Electronic Lighting Inc. & Systems & Control networks & $\begin{array}{l}\text { Method and apparatus for interfacing a light } \\
\text { dimming control with an automated control } \\
\text { system }\end{array}$ \\
\hline 4209840 & Honeywell Inc. & Systems & Control networks & Data processing protocol system \\
\hline 5971597 & Hubbell Corp. & Systems & Control networks & $\begin{array}{l}\text { Multifunction sensor and network sensor } \\
\text { system }\end{array}$ \\
\hline 6192282 & Intelihome, Inc. & Systems & Control networks & $\begin{array}{l}\text { Method and apparatus for improved building } \\
\text { automation }\end{array}$ \\
\hline 5528215 & Landis \& Gyr Powers, Inc. & Systems & Control networks & $\begin{array}{l}\text { Building automation system having } \\
\text { expansion modules }\end{array}$ \\
\hline 5786993 & $\begin{array}{l}\text { Landis \& Gyr Technology } \\
\text { Innovation Corp. }\end{array}$ & Systems & Control networks & $\begin{array}{l}\text { Apparatus for and method of controlling } \\
\text { and/or regulating process parameters of an } \\
\text { installation }\end{array}$ \\
\hline 6138241 & Leviton Manufacturing Co., Inc. & Systems & Control networks & $\begin{array}{l}\text { Apparatus for and method of inhibiting and } \\
\text { overriding an electrical control device }\end{array}$ \\
\hline 6144993 & Matsushita Electric Works, Ltd. & Systems & Control networks & $\begin{array}{l}\text { Building automation system using common } \\
\text { platform program and common function } \\
\text { package for controlling facility loads and } \\
\text { monitoring terminals }\end{array}$ \\
\hline 5572438 & $\begin{array}{l}\text { TECO Energy Management } \\
\text { Services }\end{array}$ & Systems & Control networks & $\begin{array}{l}\text { Energy management and building } \\
\text { automation system }\end{array}$ \\
\hline 5696695 & TeCom Inc. & Systems & Control networks & $\begin{array}{l}\text { System for rate-related control of electrical } \\
\text { loads }\end{array}$ \\
\hline 5475360 & Thomas Industries, Inc. & Systems & Control networks & Power line carrier controlled lighting system \\
\hline 6216956 & Tocom, Inc. & Systems & Control networks & $\begin{array}{l}\text { Environmental condition control and energy } \\
\text { management system and method }\end{array}$ \\
\hline 6061602 & Creative Lifestyles, Inc. & Systems & Home automation & $\begin{array}{l}\text { Method and apparatus for developing } \\
\text { application software for home automation } \\
\text { system }\end{array}$ \\
\hline 5621662 & IntelliNet, Inc. & Systems & Home automation & Home automation system \\
\hline 6140987 & IntelliNet, Inc. & Systems & Home automation & User Interface for home automation system \\
\hline 6091320 & Microsoft Corp. & Systems & Home automation & $\begin{array}{l}\text { Automated home control using existing } \\
\text { electrical lines as a communications } \\
\text { medium }\end{array}$ \\
\hline 5544036 & Rober J. Brown, Jr. et al & Systems & Home automation & $\begin{array}{l}\text { Energy management and home automation } \\
\text { system }\end{array}$ \\
\hline 5761083 & $\begin{array}{l}\text { Robert J. Brown, Jr., and James } \\
\text { D. Romanowiz }\end{array}$ & Systems & Home automation & $\begin{array}{l}\text { Energy management and home automation } \\
\text { system }\end{array}$ \\
\hline 5579221 & Samsung Electronics Co., Ltd. & Systems & Home automation & $\begin{array}{l}\text { Home automation system having user } \\
\text { controlled definition function }\end{array}$ \\
\hline 5822012 & Samsung Electronics Co., Ltd. & Systems & Home automation & $\begin{array}{l}\text { Home automation apparatus using a digital } \\
\text { television receiver }\end{array}$ \\
\hline
\end{tabular}




\section{APPENDIX B: Controls MANUfACTURERS}

\begin{tabular}{|c|c|c|c|c|c|c|}
\hline $\begin{array}{l}\text { Company } \\
\text { Name }\end{array}$ & $\begin{array}{l}\text { Phone } \\
\text { Number }\end{array}$ & $\begin{array}{c}\text { Fax } \\
\text { Number }\end{array}$ & Web Address & Address & City & State \\
\hline $\begin{array}{l}\text { ABB Control } \\
\text { Inc. }\end{array}$ & $888-385-1221$ & $940-397-7085$ & www.abb-control.com & 1206 Hatton Road & Wichita Falls & $\mathrm{TX}$ \\
\hline $\begin{array}{l}\text { ACE Compact } \\
\text { Electronics }\end{array}$ & $800-375-6355$ & 817-624-8915 & www.ace-ballast.com & 2506 Tillar Street & Fort Worth & $\mathrm{TX}$ \\
\hline $\begin{array}{l}\text { ADB Airfield } \\
\text { Solutions }\end{array}$ & 614-861-1304 & 614-864-2069 & www.sas.siemens.com & 977 Gahanna Parkway & Columbus & $\mathrm{OH}$ \\
\hline $\begin{array}{l}\text { Ademco } \\
\text { Sensor Co } \\
\text { (ASC) }\end{array}$ & $800-467-5875$ & $502-429-0678$ & www.ademco.com & $\begin{array}{l}1230 \text { S. Hurstbourne } \\
\text { Pkwy, Ste } 100\end{array}$ & Louisville & KY \\
\hline \begin{tabular}{|l|} 
Advance \\
Transformer
\end{tabular} & \begin{tabular}{|l|}
$800-372-3331$ \\
$847-390-5000$ \\
\end{tabular} & $847-390-5382$ & $\begin{array}{c}\text { www.advancetransformer. } \\
\text { com }\end{array}$ & $\begin{array}{l}650 \text { W. Grand Ave, Suite } \\
304\end{array}$ & Elmhurst & IL \\
\hline \begin{tabular}{|l} 
Advanced \\
Control \\
Technologies \\
Inc
\end{tabular} & $317-337-0100$ & $317-337-0200$ & www.act-solutions.com & 8076 Woodland Drive & Indianapolis & IN \\
\hline \begin{tabular}{|l|} 
Agilent \\
Technologies \\
\end{tabular} & $800-235-0312$ & $408-654-8575$ & www.agilent.com & PO Box 10395 & Palo Alto & $\mathrm{CA}$ \\
\hline $\begin{array}{l}\text { Alerton } \\
\text { Technologies, } \\
\text { Inc. } \\
\end{array}$ & $425-869-8400$ & $425-869-8445$ & www.alerton.com & 6670-185th Avenue NE & Redmond & WA \\
\hline \begin{tabular}{|l|} 
American-De \\
Rosa Lamparts \\
\end{tabular} & \begin{tabular}{|l|}
$800-777-4440 /$ \\
$323-726-6300$ \\
\end{tabular} & $323-728-0300$ & www.lamparts.com & 1945 Tubeway Ave & Commerce & $\mathrm{CA}$ \\
\hline \begin{tabular}{|l|} 
Amperlite \\
Company Inc
\end{tabular} & \begin{tabular}{|l|}
$800-752-2329 /$ \\
$201-864-9503$ \\
\end{tabular} & 201-864-3955 & www.amperite.com & 600 Palisade Avenue & Union City & $\mathrm{NJ}$ \\
\hline \begin{tabular}{|l|} 
AMX \\
(consolated into \\
PANJA Inc) \\
\end{tabular} & $800-552-6955$ & $469-624-7163$ & www.panja.com & 3000 Research Drive & Richardson & $\mathrm{TX}$ \\
\hline \begin{tabular}{|l|} 
Andover \\
Controls \\
Corporation
\end{tabular} & $978-470-0555$ & $978-470-0946$ & www.andovercontrols.com & 300 Brickstone Square & Andover & MA \\
\hline \begin{tabular}{|l|} 
Antron \\
Compact \\
Electronics Inc
\end{tabular} & \begin{tabular}{|}
$800-375-6355 /$ \\
$800-529-1597$
\end{tabular} & $\mid$\begin{tabular}{|c|}
$817-624-8915 /$ \\
$800-529-1597$
\end{tabular} & www.ace-ballast.com & 2506 Tillar Street & Fort Worth & TX \\
\hline $\begin{array}{l}\text { Architectural } \\
\text { Lighting } \\
\text { Management }\end{array}$ & $\mid$\begin{tabular}{|l|}
$888-446-9137 /$ \\
$847-860-1490$
\end{tabular} & $847-860-0959$ & www.almsys.com & 2480 Delta Lane & Elk Grove Village & IL \\
\hline $\begin{array}{l}\text { Aromat } \\
\text { (Panasonic) }\end{array}$ & $908-464-3550$ & $908-771-5656$ & www.aromat.com & 629 Central Avenue & New Providence & $\mathrm{NJ}$ \\
\hline $\begin{array}{l}\text { Automated } \\
\text { Logic } \\
\text { Corporation }\end{array}$ & $770-429-3000$ & $770-429-3001$ & www.automatedlogic.com & 1150 Roberts Boulevard & Kennesaw & GA \\
\hline $\begin{array}{l}\text { AVAB America } \\
\text { Inc }\end{array}$ & 707-778-8990 & 707-778-8996 & www.avab.com & 434 Payran Street & Petaluma & $\mathrm{CA}$ \\
\hline Blackbird & 812-944-0799 & $812-941-8830$ & None & 219 Grant Line Center & New Albany & IN \\
\hline
\end{tabular}




\begin{tabular}{|c|c|c|c|c|c|c|}
\hline \begin{tabular}{|l} 
Company \\
Name
\end{tabular} & $\begin{array}{l}\text { Phone } \\
\text { Number }\end{array}$ & $\begin{array}{c}\text { Fax } \\
\text { Number }\end{array}$ & Web Address & Address & City & State \\
\hline $\begin{array}{l}\text { Colortran } \\
\text { (Leviton) }\end{array}$ & $503-682-1941$ & $503-404-5600$ & $\begin{array}{c}\text { www.leviton.com (old site } \\
\text { for reference } \\
\text { www.nsicorp.com) }\end{array}$ & 20497 SW Peton & Tualatin & OR \\
\hline Cooper Lighting & $847-956-8400$ & $847-956-1537$ & www.cooperlighting.com & 400 Busse Road & Elk Grove Village & $\mathrm{IL}$ \\
\hline Crestron & \begin{tabular}{|l|}
$800-949-3465 /$ \\
$847-437-6650$
\end{tabular} & $847-437-6052$ & www.crestron.com & $\begin{array}{l}415 \text { West Golf Road, } \\
\text { Suite } 27\end{array}$ & Arlington Heights & IL \\
\hline $\begin{array}{l}\text { Cutler Hammer } \\
\text { (Eaton) }\end{array}$ & \begin{tabular}{|c|}
$630-789-5980$ \\
$1800-272-$ \\
8202
\end{tabular} & 630-789-4999 & $\begin{array}{l}\text { www.cutlerhammer.eaton. } \\
\text { com/apc,www.eaton.com }\end{array}$ & $\begin{array}{l}601 \text { Oakmont Lane, } \\
\text { Suite } 310\end{array}$ & Westmont & IL \\
\hline $\begin{array}{l}\text { Delta Controls } \\
\text { Inc }\end{array}$ & \begin{tabular}{|l|}
$800-335-8221 /$ \\
$604-574-9444$ \\
\end{tabular} & $604-574-7793$ & www.deltacontrols.com & 17850 - 56th Avenue & Surrey & $\mathrm{BC}$ \\
\hline \begin{tabular}{|l} 
Denki \\
Corporation of \\
North America
\end{tabular} & \begin{tabular}{|}
$800-908-8882 /$ \\
$201-393-0722$
\end{tabular} & 201-393-0692 & www.denkicorp.com & $\begin{array}{l}377 \text { Route } 17 \text { South, } \\
\text { Suite } 118\end{array}$ & Hasbrouck Hgts & NJ \\
\hline $\begin{array}{l}\text { Douglas } \\
\text { Lighting } \\
\text { Controls Inc }\end{array}$ & \begin{tabular}{|l|}
$514-342-6581 /$ \\
$604-873-2797$
\end{tabular} & \begin{tabular}{|l|}
$514-342-0133 /$ \\
$604-873-6939$
\end{tabular} & $\begin{array}{c}\text { www.douglaslightingcontro } \\
\text { I.com }\end{array}$ & 345 Isabey Street & St Laurent & QC \\
\hline $\begin{array}{l}\text { EBW } \\
\text { Electronics }\end{array}$ & $800-787-0575$ & $616-786-0513$ & www.ebw-electronics.com & 701 Commerce Court & Holland & Ml \\
\hline \begin{tabular}{|l|} 
Echelon \\
Corporation \\
\end{tabular} & $408-938-5200$ & $408-328-3800$ & www.echelon.com & 4015 Miranda Ave & Palo Alto & CA \\
\hline \begin{tabular}{|l|} 
Electronic \\
Lighting Inc \\
\end{tabular} & \begin{tabular}{|l|}
$800-395-5767 \mid$ \\
$510-795-8555$ \\
\end{tabular} & $510-795-0870$ & www.elinet.com & 37200 Central Court & Newark & $\mathrm{CA}$ \\
\hline \begin{tabular}{|l} 
Electronic \\
Theatre \\
Controls Inc \\
\end{tabular} & $\mid$\begin{tabular}{|l|}
$800-688-4116 /$ \\
$608-831-4116$
\end{tabular} & $608-836-1736$ & www.etcconnect.com & 3030 Laura Lane & Middleton & WI \\
\hline $\begin{array}{l}\text { Elk Products } \\
\text { Inc }\end{array}$ & $800-797-9355$ & $828-397-4415$ & www.elkproducts.com & 3266 US 70 West & Conelly Springs & NC \\
\hline Energy Savings & $847-925-8400$ & $847-925-8490$ & www.esavings.com & & Schaumburg & $\mathrm{IL}$ \\
\hline $\begin{array}{l}\text { Fulham } \\
\text { Company }\end{array}$ & 323-779-2980 & $323-754-9060$ & www.fulham.com & 12705 South Van Ness & Hawthorne & $\mathrm{CA}$ \\
\hline $\begin{array}{l}\text { Future Wave } \\
\text { Technologies }\end{array}$ & $604-990-5325$ & $604-990-0066$ & $\begin{array}{c}\text { www.futurewavetechnologi } \\
\text { es.com/ }\end{array}$ & 133 East Main Street & Marbouough & MA \\
\hline $\begin{array}{l}\text { GE Industrial } \\
\text { Systems }\end{array}$ & \begin{tabular}{|c|}
$800-852-2778 /$ \\
800-TLC- \\
ASST
\end{tabular} & $401-886-6470$ & www.geindustrial.com & 225 Service Avenue & Warwick & RI \\
\hline GE Lighting & \begin{tabular}{|c|}
$800-435-4448$ \\
$($ GE LIGHT $)$
\end{tabular} & $800-327-0663$ & www.gelighting.com & 1975 Noble Road & Cleveland & $\mathrm{OH}$ \\
\hline $\begin{array}{l}\text { Genlyte I } \\
\text { Thomas }\end{array}$ & \begin{tabular}{|c|}
$800-215-1068 /$ \\
$508-679-8131$
\end{tabular} & $508-674-4710$ & www.gennlyte.com & & & \\
\hline GE-Smart & $505-251-6000$ & & www.ge-smart.com & 2100 Summet Court & LasCruces & NM \\
\hline \begin{tabular}{|l} 
Gilbert \\
Manufacturing \\
Company Inc
\end{tabular} & $804-447-8223$ & $804-447-7434$ & none & PO Box 309 & South Hill & VA \\
\hline
\end{tabular}




\begin{tabular}{|c|c|c|c|c|c|c|}
\hline \begin{tabular}{|l|}
$\begin{array}{l}\text { Company } \\
\text { Name }\end{array}$ \\
\end{tabular} & $\begin{array}{l}\text { Phone } \\
\text { Number }\end{array}$ & $\begin{array}{c}\text { Fax } \\
\text { Number }\end{array}$ & Web Address & Address & City & State \\
\hline \begin{tabular}{|l} 
Harrison \\
Toshiba \\
Lighting USA, \\
Inc
\end{tabular} & \begin{tabular}{|}
$877-399-8100 /$ \\
$847-229-8100$
\end{tabular} & $847-229-8124$ & www.toshiba-lighting.com & 1003 Commerce Court & Buffalo Grove & IL \\
\hline \begin{tabular}{|l|} 
High End \\
Systems Inc \\
\end{tabular} & $512-836-2242$ & $512-837-5290$ & www.highend.com & 2217 West Braker Lane & Austin & $T X$ \\
\hline \begin{tabular}{|l} 
Honeywell \\
\end{tabular} & $805-581-5591$ & $805-581-5032$ & www.cas.honeywell.com & 2162 Union Place & Simi Valley & $\mathrm{CA}$ \\
\hline \begin{tabular}{|l} 
Horton Controls \\
/ Watt Stopper
\end{tabular} & $205-271-3400$ & 205-969-0605 & www.hcontrols.com & 4128 Crosshaven Drive & Birmingham & $\mathrm{AL}$ \\
\hline $\begin{array}{l}\text { Howard } \\
\text { Industries Inc }\end{array}$ & \begin{tabular}{|l|}
$800-956-3456 /$ \\
$601-422-0033$ \\
\end{tabular} & $601-422-1652$ & www.howard-ballast.com & PO Box 1590 & Laurel & MS \\
\hline $\begin{array}{l}\text { HUB } \\
\text { Electric/Vara- } \\
\text { Light/Dimatroni } \\
\text { CS }\end{array}$ & $815-455-4400$ & $815-455-1499$ & none & 6207 Commercial Road & Crystal Lake & IL \\
\hline $\begin{array}{l}\text { Hubbell and } \\
\text { Hubbell } \\
\text { Lighting }\end{array}$ & $203-882-4900$ & $800-255-1031$ & $\begin{array}{l}\text { www.hubbell-Itg.com, } \\
\text { www.hubbell.com }\end{array}$ & $\begin{array}{c}185 \text { Plains Road, Milford } \\
\text { PI Corp Ctr }\end{array}$ & Milford & $\mathrm{CT}$ \\
\hline \begin{tabular}{|l|} 
Hunt Controls \\
Systems Inc
\end{tabular} & $970-484-9048$ & $970-493-4125$ & www.huntdimming.com & 200 Rome Court & Fort Collins & $\mathrm{CO}$ \\
\hline \begin{tabular}{|l} 
IBM Mylonas \\
Lighting \\
Company \\
\end{tabular} & & & & 302 A Street & LaPorte & IN \\
\hline \begin{tabular}{|l} 
Irideon Inc \\
(purchased by \\
ETC) \\
\end{tabular} & & & & 301 Regal Row & Dallas & TX \\
\hline \begin{tabular}{|l} 
Johnson \\
Controls
\end{tabular} & $414-274-4000$ & $414-524-4443$ & www.johnsoncontrols.com & 507 East Michigan Street & Milwaukee & WI \\
\hline \begin{tabular}{|l|} 
JRS \\
Technology Inc \\
\end{tabular} & \begin{tabular}{|l|}
$888-922-5527 /$ \\
$607-748-4800$ \\
\end{tabular} & $607-748-7949$ & www.jrstechnology.com & $\begin{array}{c}1061 \text { Perimeter Rd W, } \\
\text { Bldg } 11\end{array}$ & Endicott & NY \\
\hline \begin{tabular}{|l|} 
Keegan \\
Wireless
\end{tabular} & $800-451-2012$ & $909-594-7813$ & & 749 South Lemon Street & City of Industry & CA \\
\hline $\begin{array}{l}\text { Kelar Controls } \\
\text { Inc }\end{array}$ & $800-728-3443$ & $408-365-3289$ & www.kelarcontrols.com & 404-C Umbarger Rd & San Hose & $\mathrm{CA}$ \\
\hline Kingtec Inc & $800-448-5483$ & $201-229-4672$ & $\begin{array}{l}\text { www.kingtec.com (under } \\
\text { const) }\end{array}$ & 85 Challenger $\mathrm{Rd}$ & Ridgefield & NJ \\
\hline \begin{tabular}{|l} 
K-Tronic \\
Ballasts \\
\end{tabular} & 888-458-7664 & $201-488-8480$ & www.k-tronic.com & $\begin{array}{c}290 \text { Vincent Avenue, 3rd } \\
\text { Floor }\end{array}$ & Hackensack & NJ \\
\hline $\begin{array}{l}\text { LEAX Lighting } \\
\text { controls } \\
\end{array}$ & $970-927-4845$ & $970-927-4845$ & \begin{tabular}{|c|} 
www.leaxcontrols.com/CM \\
3 \\
\end{tabular} & 180 Fiou Lane & Basalt & $\mathrm{CO}$ \\
\hline \begin{tabular}{|l|} 
Lehigh Electric \\
Products
\end{tabular} & $610-395-3386$ & $610-395-7735$ & www.lehighdim.com & 6265 Hamilton Blvd & Allentown & PA \\
\hline \begin{tabular}{|l|} 
Leviton (LON \\
products group)
\end{tabular} & $\begin{array}{c}1-800-824- \\
3005\end{array}$ & $\begin{array}{c}1-800-832- \\
9538\end{array}$ & www.leviton.com & $\begin{array}{l}\text { 59-25 Little Neck } \\
\text { Parkway }\end{array}$ & Little Neck & NY \\
\hline \begin{tabular}{|l|} 
Leviton Lighting \\
Control Division
\end{tabular} & \begin{tabular}{|l|}
$800-996-2276 /$ \\
$512-927-7711$ \\
\end{tabular} & $512-927-7799$ & www.leviton.com & $\begin{array}{c}9013 \text { Tuscany Way, Bldg } \\
100\end{array}$ & Austin & $T X$ \\
\hline
\end{tabular}




\begin{tabular}{|c|c|c|c|c|c|c|}
\hline \begin{tabular}{|l} 
Company \\
Name
\end{tabular} & $\begin{array}{l}\text { Phone } \\
\text { Number }\end{array}$ & $\begin{array}{c}\text { Fax } \\
\text { Number }\end{array}$ & Web Address & Address & City & State \\
\hline \begin{tabular}{|l} 
Lithonia \\
Lighting \\
(controls)
\end{tabular} & 770-922-9000 & $770-860-3183$ & www.lithonia.com & One Lithonia Way & Conyers & GA \\
\hline $\begin{array}{l}\text { Lithonia } \\
\text { Lighting } \\
\text { Canada }\end{array}$ & 514-639-3571 & $514-639-3570$ & www.lithonia.com & 1100-50th Avenue & Lachine & $\begin{array}{l}\mathrm{QC} \\
\text { Canada }\end{array}$ \\
\hline $\begin{array}{l}\text { Lumion } \\
\text { Corporation }\end{array}$ & 416-299-5134 & 416-299-7025 & www.lumion.com & 30 North Wind Place & Scarborough & $\begin{array}{l}\text { ON, } \\
\text { Canada }\end{array}$ \\
\hline $\begin{array}{l}\text { Lutron } \\
\text { Electronics Co } \\
\text { Inc }\end{array}$ & \begin{tabular}{|}
$888-588-7661 /$ \\
$610-282-3800$
\end{tabular} & $610-282-8321$ & www.lutron.com & 7200 Suter Road & Coopersburg & PA \\
\hline \begin{tabular}{|l|} 
MagneTek \\
Lighting \\
Products Group
\end{tabular} & \begin{tabular}{|l|} 
615-316-5100/ \\
800-BALLAST
\end{tabular} & 615-316-5162 & $\begin{array}{c}\text { www.magnetek.com/ballas } \\
t\end{array}$ & $\begin{array}{l}26 \text { Century Blvd, Suite } \\
600\end{array}$ & Nashville & TN \\
\hline \begin{tabular}{|l|} 
Marco \\
Electronics \\
(now NSI Corp) \\
\end{tabular} & $503-404-5500$ & $503-404-5600$ & www.nsicorp.com & 20497 Southwest Teton & Tualatin & OR \\
\hline \begin{tabular}{|l|} 
Marinco \\
Industrial \\
Group
\end{tabular} & \begin{tabular}{|l|}
$800-767-8541 /$ \\
$707-226-8600$
\end{tabular} & $707-226-9670$ & www.marinco.com & $\begin{array}{l}2655 \text { Napa Valley } \\
\text { Corporate Drive }\end{array}$ & Napa & $\mathrm{CA}$ \\
\hline $\begin{array}{l}\text { Marlin Controls, } \\
\text { Inc }\end{array}$ & \begin{tabular}{|l|}
$800-788-5750 /$ \\
$214-553-5700$ \\
\end{tabular} & $214-553-1011$ & www.marlincontrols.com & $\begin{array}{l}11011 \text { Regency Crest } \\
\text { Drive, Ste } 200\end{array}$ & Dallas & TX \\
\hline MaxLite & \begin{tabular}{|l|}
$800-555-5629 /$ \\
$973-256-3330$ \\
\end{tabular} & 973-256-9444 & www.maxlite.com & 60E Commerce Way \#E & Totowa & NJ \\
\hline \begin{tabular}{|l|} 
Microlites \\
Scientific \\
\end{tabular} & \begin{tabular}{|l|}
$800-263-8902 /$ \\
$416-299-5301$ \\
\end{tabular} & 416-299-1309 & www.microlites.com & $\begin{array}{l}2370 \text { Midland Avenue } \\
\text { Unit A8 }\end{array}$ & Scarborough & $\begin{array}{c}\text { ON, } \\
\text { Canada }\end{array}$ \\
\hline $\begin{array}{l}\text { Molex } \\
\text { Incorporated }\end{array}$ & 708-969-4550 & $708-512-8620$ & & 2222 Wellington Court & Lisle & IL \\
\hline \begin{tabular}{|l|} 
MYTEC \\
Corporation \\
\end{tabular} & $\begin{array}{c}\text { 800-MYTECH- } \\
\text { C } \\
\end{array}$ & $512-450-1215$ & www.mytech.com & 706 Brentwood & Austin & TX \\
\hline $\begin{array}{l}\text { N.V. ADB-TV } \\
\text { Tech S.A. } \\
\text { (Siemens) }\end{array}$ & $\begin{array}{c}32-2-722-17- \\
11\end{array}$ & $\begin{array}{c}32-2-722-17- \\
64\end{array}$ & & Leuvensesteenweg 585 & $\begin{array}{l}\text { Zaventem, B } \\
\quad 1930\end{array}$ & Belgium \\
\hline $\begin{array}{l}\text { North Point } \\
\text { Engineering } \\
\text { (now Nexlite) }\end{array}$ & 218-828-3700 & 218-824-1568 & www.nexlight.com & $\begin{array}{l}1621 \text { College Road } \\
\text { South }\end{array}$ & Baxter & $\mathrm{MN}$ \\
\hline $\begin{array}{l}\text { Novar Controls } \\
\text { Corporation }\end{array}$ & $330-670-1010$ & $330-670-1029$ & www.novarcontrols.com & 3333 Copley Rd & Copley & $\mathrm{OH}$ \\
\hline Novitas Inc & 310-568-9600 & 310-568-9697 & www.novitas.com & 5875 Green Valley Circle & Culver City & CA \\
\hline \begin{tabular}{|l|} 
Osram \\
Sylvania/ \\
Ballast Division \\
\end{tabular} & \begin{tabular}{|}
$800-654-0089 /$ \\
$847-726-6200$
\end{tabular} & $847-726-6211$ & www.sylvania.com & 800 North Church Street & Lake Zurich & IL \\
\hline Panja Inc & $800-222-0193$ & 469-624-7160 & www.panja.com & 3000 Research Drive & Richardson & TX \\
\hline \begin{tabular}{|l} 
Pass \& \\
Seymour/ \\
Legrand
\end{tabular} & \begin{tabular}{|c|}
$800-223-4162 /$ \\
$315-468-8281$
\end{tabular} & $\mid$\begin{tabular}{|l|}
$800-223-4196 /$ \\
$315-468-8388$
\end{tabular} & $\begin{array}{c}\text { www.passandseymour.co } \\
\mathrm{m}\end{array}$ & PO Box 4822 & Syracuse & NY \\
\hline
\end{tabular}




\begin{tabular}{|c|c|c|c|c|c|c|}
\hline \begin{tabular}{|l|}
$\begin{array}{l}\text { Company } \\
\text { Name }\end{array}$ \\
\end{tabular} & $\begin{array}{l}\text { Phone } \\
\text { Number }\end{array}$ & $\begin{array}{c}\text { Fax } \\
\text { Number }\end{array}$ & Web Address & Address & City & State \\
\hline $\begin{array}{l}\text { PCI Lighting } \\
\text { Control } \\
\text { Systems }\end{array}$ & $\mid \begin{array}{l}800-767-3674 / \\
802-658-6445\end{array}$ & $802-658-6934$ & $\begin{array}{c}\text { www.pcilightingcontrols.co } \\
\mathrm{m}\end{array}$ & 6 Green Tree Drive & South Burlington & VT \\
\hline \begin{tabular}{|l|} 
PLC Multipoint \\
Inc
\end{tabular} & $425-353-7552$ & $425-353-3353$ & www.plcmultipoint.com & $\begin{array}{c}3101 \text { - 111th Street SW, } \\
\text { Suite F }\end{array}$ & Everett & WA \\
\hline $\begin{array}{l}\text { RAB Electric } \\
\text { Mfg Co Inc }\end{array}$ & \begin{tabular}{|l|}
$888-722-1000 /$ \\
$201-784-8600$ \\
\end{tabular} & \begin{tabular}{|l|}
$888-722-1232 /$ \\
$201-784-0077$ \\
\end{tabular} & www.rabweb.com & 170 Ludlow Avenue & Northvale & NJ \\
\hline $\begin{array}{l}\text { Robertson } \\
\text { Worldwide }\end{array}$ & \begin{tabular}{|l|}
$800-323-5633 /$ \\
$708-388-2315$ \\
\end{tabular} & $708-388-2420$ & www.robertsonww.com & 13611 Thornton Road & Blue Island & IL \\
\hline $\begin{array}{l}\text { Semper Fi } \\
\text { Power Supply } \\
\text { Inc }\end{array}$ & $603-656-9729$ & $603-656-9752$ & $\begin{array}{c}\text { www.semperfipowersupply } \\
\text {.com }\end{array}$ & 21 West Auburn Street & Manchester & $\mathrm{NH}$ \\
\hline $\begin{array}{l}\text { SensorSwitch } \\
\text { Inc } \\
\end{array}$ & \begin{tabular}{|l|}
$800-727-7483 /$ \\
$203-265-2842$ \\
\end{tabular} & $203-269-9621$ & www.sensorswitchinc.com & 900 Northrop Road & Wallingford & $\mathrm{CT}$ \\
\hline \begin{tabular}{|l} 
Siebe \\
Enviornmental \\
Controls \\
(Invensys)
\end{tabular} & $815-637-3140$ & $815-637-3000$ & www.invensys.ibs.com & 1354 Clifford Avenue & Loves Park & $\mathrm{IL}$ \\
\hline \begin{tabular}{|l|} 
Siemens \\
Automation \\
\end{tabular} & $770-871-3863$ & $770-448-5662$ & $\begin{array}{l}\text { www.sea.siemens.com/po } \\
\text { wer/product/pdprodlc.html }\end{array}$ & $\begin{array}{c}5405 \text { Metric Place (suite } \\
100)\end{array}$ & Norcross & GA \\
\hline \begin{tabular}{|l|} 
Siemens \\
Building \\
Technologies \\
Ltd \\
\end{tabular} & $847-215-1000$ & $847-215-9907$ & www.sbt.siemens.com & 1000 Deerfield & Buffalo Grove & IL \\
\hline \begin{tabular}{|l|} 
Sine \\
Systems/Pyle \\
Connector Corp \\
\end{tabular} & $630-832-4600$ & $630-782-0700$ & www.sineco.com & $\begin{array}{c}650 \text { W. Grand Ave, Suite } \\
304\end{array}$ & Elmhurst & IL \\
\hline \multicolumn{7}{|l|}{$\begin{array}{l}\text { SLS Industries } \\
\text { UNIDIM }\end{array}$} \\
\hline Smart America & 831-466-9999 & 831-439-5585 & www.smart-america.com & 125 Bethany Drive & Scotts Valley & $\mathrm{CA}$ \\
\hline \begin{tabular}{|l|} 
Square D \\
Company \\
\end{tabular} & \begin{tabular}{|l|}
$800-392-8781 /$ \\
$615-287-3500$ \\
\end{tabular} & $615-287-3409$ & www.squared.com & $\begin{array}{l}295 \text { Tech Park Drive, } \\
\text { Suite } 100\end{array}$ & LaVergne & TN \\
\hline $\begin{array}{l}\text { Sterner } \\
\text { Controls } \\
\end{array}$ & disconnected & & & & & \\
\hline \begin{tabular}{|l|} 
Strand Lighting \\
Canada Inc
\end{tabular} & \begin{tabular}{|c|}
$800-387-3403 /$ \\
$905-677-7130$ \\
\end{tabular} & $905-677-6859$ & & $\begin{array}{c}2430 \text { Lucknow Drive, } \\
\text { Unit } 15\end{array}$ & Mississaugo & $\begin{array}{c}\text { ON, } \\
\text { Canada }\end{array}$ \\
\hline \begin{tabular}{|l|} 
Strand Lighting \\
Inc
\end{tabular} & \begin{tabular}{|l|}
$800-487-0175 /$ \\
$310-637-7500$ \\
\end{tabular} & $310-632-5519$ & www.strandlight.com & $\begin{array}{l}18111 \text { S. Sante Fe } \\
\text { Avenue }\end{array}$ & $\begin{array}{c}\text { Rancho } \\
\text { Dominguez }\end{array}$ & $\mathrm{CA}$ \\
\hline \begin{tabular}{|l} 
Sunpark \\
Electronics \\
Corp \\
\end{tabular} & $\begin{array}{l}888-478-6775 / \\
310-320-7880\end{array}$ & $310-320-7875$ & www.sunpkco.com & $\begin{array}{l}1815 \text { W. 205th Street, } \\
\text { Suite } 104\end{array}$ & Torrance & $\mathrm{CA}$ \\
\hline $\begin{array}{l}\text { Thomas } \\
\text { Lighting }\end{array}$ & $502-894-2400$ & $502-894-2450$ & www.thomaslighting.com & $\begin{array}{c}4360 \text { Brownsboro Road, } \\
\text { Suite } 300\end{array}$ & Louisville & KY \\
\hline Tork & 914-664-3542 & 914-664-5052 & www.tork.com & 1 Grove Street & Mount Vernon & NY \\
\hline $\begin{array}{l}\text { Touch-Plate } \\
\text { Lighting } \\
\text { Controls }\end{array}$ & 219-424-4323 & $219-420-3651$ & www.touchplate.com & $\begin{array}{c}\text { 1830 Wayne Trace, Ste } \\
9\end{array}$ & Fort Wayne & IN \\
\hline
\end{tabular}




\begin{tabular}{|c|c|c|c|c|c|c|}
\hline $\begin{array}{l}\text { Company } \\
\text { Name }\end{array}$ & $\begin{array}{l}\text { Phone } \\
\text { Number }\end{array}$ & $\begin{array}{c}\text { Fax } \\
\text { Number }\end{array}$ & Web Address & Address & City & State \\
\hline Trane & $651-407-3800$ & $651-407-3940$ & www.trane.com & $\begin{array}{l}4831 \text { White Bear } \\
\text { Parkway }\end{array}$ & St Paul & $\mathrm{MN}$ \\
\hline $\begin{array}{l}\text { Trend Group } \\
\text { Inc } \\
\end{array}$ & $908-757-3535$ & $908-756-3636$ & $\begin{array}{l}\text { www.trendgroup.com } \\
\text { (under const) }\end{array}$ & 200-214 West 4th Street & Plainfield & NJ \\
\hline Triatek Inc & \begin{tabular}{|l|}
$888-242-1922 /$ \\
$770-242-1922$ \\
\end{tabular} & $770-242-1944$ & www.triatek.com & 2976 Pacific Drive & Norcross & GA \\
\hline $\begin{array}{l}\text { Tridonic Inc. } \\
\text { (North America) }\end{array}$ & 770-717-0556 & 770-717-7969 & www.tridonic.com & $\begin{array}{c}4405 \text { International Blvd } \\
\text { Suite B103 }\end{array}$ & Norcross & GA \\
\hline $\begin{array}{l}\text { Unenco } \\
\text { (Hubbell) }\end{array}$ & $800-245-9135$ & \begin{tabular}{|l|}
$800-543-0538$ \\
$203-876-3675$ \\
\end{tabular} & www.hubbell-unenco.com & $\begin{array}{c}185 \text { Plains Road, Milford } \\
\text { PI Corp Ctr }\end{array}$ & Milford & CT \\
\hline Vantage & $801-229-2800$ & $801-224-0355$ & www.vantagecontrols.com & 345 East 800 South & Orem & UT \\
\hline $\begin{array}{l}\text { Vantage } \\
\text { Lighting Inc } \\
\end{array}$ & \begin{tabular}{|l|}
$800-445-2677 /$ \\
$415-507-0402$ \\
\end{tabular} & $415-507-0502$ & www.vanltg.com & 175 Paul Drive & San Rafael & CA \\
\hline Veam & $860-274-9681$ & $860-274-4963$ & www.littonveam.com & 100 New Wood Road & Watertown & CT \\
\hline Visonic Inc & $\begin{array}{l}800-223-0020 / \\
860-243-0833\end{array}$ & $860-242-8094$ & www.visonic.com & 10 Northwood Drive & Bloomfield & СT \\
\hline $\mathrm{X}$-10 USA Inc & $201-784-9700$ & $201-784-9464$ & $\begin{array}{c}\text { www.x10.com } \\
\text { (www.x10pro.com) }\end{array}$ & $\begin{array}{c}91 \text { Ruckman Road, Box } \\
420\end{array}$ & Closter & NJ \\
\hline $\begin{array}{l}\text { Xitron } \\
\text { Technologies } \\
\text { Inc }\end{array}$ & $858-530-8099$ & $858-530-8077$ & www.xitron-tech.com & $\begin{array}{c}\text { 9770-A Carroll Centre } \\
\text { Road }\end{array}$ & San Diego & $\mathrm{CA}$ \\
\hline
\end{tabular}




\section{Appendix C: Manufacturers and Their Supporting Protocols}

\begin{tabular}{|c|c|c|c|}
\hline Item & Company Name & Business Category / Focus & Supporting Protocol \\
\hline 1 & ABB Control Inc. & Industrial controls and Instruments & Custom, BACnet, Modicon and LON \\
\hline 2 & Advance Transformer & Ballasts / Transformers & 0-10VDC, 2-wire \\
\hline 3 & $\begin{array}{l}\text { Advanced Control Technologies } \\
\text { Inc }\end{array}$ & Manufacturer of interfaces & LonWorks Independent Developer \\
\hline 4 & Agilent Technologies & HP Subsidiary / Lamps (bulbs) & LAN/WAN Ethernet \\
\hline 5 & Alerton Technologies, Inc. & $\begin{array}{l}\text { DDC Systems for HVAC and } \\
\text { lighting }\end{array}$ & BACnet, Modbus, Ethernet \\
\hline 6 & Andover Controls Corporation & Controls / BAS & BACnet, Ethernet, LON \\
\hline 7 & $\begin{array}{l}\text { Architectural Lighting } \\
\text { Management }\end{array}$ & Controls / dimming & DMX-512 \\
\hline 8 & Automated Logic Corporation & Controls / BAS & $\begin{array}{l}\text { Supports all major communication } \\
\text { protocols including BACnet, } \\
\text { LONworks, MODbus and SNMP. }\end{array}$ \\
\hline 9 & Cooper Lighting & $\begin{array}{l}\text { Fixtures / Inteligent System (ESI } \\
\text { ballast) }\end{array}$ & ESI digital protocol \\
\hline 10 & Crestron & Controls / Software & LAN/WAN/Internet connection \\
\hline 11 & Cutler Hammer (Eaton) & Controls / Home Automation & X-10, CEBus, RS232, INCOM \\
\hline 12 & Delta Controls Inc & Controls & BACnet and ORCAview \\
\hline 13 & Douglas Lighting Controls Inc & Controls /Software & LonWorks \\
\hline 14 & Easylite & Ballast & \\
\hline 15 & Echelon Corporation & Protocol - system developer & LONworks \\
\hline 16 & Electronic Theatre Controls Inc & Controls & $\begin{array}{l}\text { DMX 512, ETCnet, ETClink, MIDI, } \\
\text { BACNet, LONWorks }\end{array}$ \\
\hline 17 & Elk Products Inc & $\begin{array}{l}\text { Controls and components for Home } \\
\text { automation }\end{array}$ & RS232, RS485, SIMPLE \\
\hline 18 & Energy Savings Inc. & Ballast/Controls & Custom \\
\hline 19 & GE Industrial Systems & Lighting Controls & $\begin{array}{l}\text { Ethernet, MODbus, RS485/232, } \\
\text { LONworks }\end{array}$ \\
\hline 20 & GE Lighting & Ballast (Magnetek) & See MagneTek \\
\hline 21 & Genlyte - Lightolier Controls & Controls & RS232, RS485, BACNet, DMX512 \\
\hline 22 & H I Solutions Inc & Controls/Software & LAN Ethernet, RS485 \\
\hline
\end{tabular}




\begin{tabular}{|c|c|c|c|}
\hline 23 & Honeywell & Controls / BAS & $\begin{array}{l}\text { RS232, ARCnet, Ethernet, BACNet, } \\
\text { Modbus, Allen Bradley Data } \\
\text { Highway, Modbus plus, and a wide } \\
\text { variety of other common protocols } \\
\text { are available. }\end{array}$ \\
\hline 24 & Horton Controls / Watt Stopper & Controls & LONworks \\
\hline 25 & Hubbell and Hubbell Lighting & Fixtures / Controls & 0-10VDC, 2-wire \\
\hline 26 & Hunt Controls Systems Inc & Controls & 0-10VDC, 2-wire, ESU super dim \\
\hline 27 & Johnson Controls Inc & Controls / BAS & $\begin{array}{l}\text { BACnet, LONworks, Ethernet and } \\
\text { most other major protocols. }\end{array}$ \\
\hline 28 & JRS Technology Inc & Ballasts/Controls & LONworks, 0-10VDC \\
\hline 29 & LEAX Lighting controls & Lighting Controls & $\begin{array}{l}\text { LONworks, DMX512, RS232, } \\
\text { Analogue Input, Volt free, 2wire, } \\
\text { DALI, 0-10VDC, DSI }\end{array}$ \\
\hline 30 & Lehigh Electric Products & Controls & LONworks, DALI, DSI, 1-10VDC \\
\hline 31 & Leviton (LON products group) & $\begin{array}{l}\text { Building Control System / } \\
\text { Components }\end{array}$ & $\begin{array}{l}\text { 0-10VDC, DMX512, LONworks, } \\
\text { BACnet }\end{array}$ \\
\hline 32 & $\begin{array}{l}\text { Leviton Lighting Control } \\
\text { Division }\end{array}$ & Controls & $\begin{array}{l}\text { LONworks, BACnet, 0-10 VDC, } \\
\text { LAN/WAN Ethernet }\end{array}$ \\
\hline 33 & Lithonia Lighting (controls) & Fixtures / Controls & $\begin{array}{l}\text { BACnet, DMX512, 0-10VDC, 10- } \\
\text { 30VDC }\end{array}$ \\
\hline 34 & Lutron Electronics Co Inc & Controls & $\begin{array}{l}\text { 0-10VDC, 2-wire line coltage } \\
\text { control, 3-wire line coltage control, } \\
\text { DMX 512 }\end{array}$ \\
\hline 35 & $\begin{array}{l}\text { MagneTek Lighting Products } \\
\text { Group }\end{array}$ & Ballasts & 0-10VDC, DMX 512, DALI \\
\hline 36 & MYTEC Corporation & Controls/Software & 2-wire, 24VDC \\
\hline 37 & NexLight & $\begin{array}{l}\text { Building Control System / } \\
\text { Components }\end{array}$ & 2-wire, BACnet \\
\hline 38 & Novitas Inc & Sensors / Controls & 0-10VDC \\
\hline 39 & Osram Sylvania/ Ballast Division & Ballasts & 0-10VDC, DALI \\
\hline 40 & Panja Inc & Controls & DMX512, Ethernet, BACNet \\
\hline 41 & Pass \& Seymour/ Legrand & Controls & 2-wire \\
\hline 42 & $\begin{array}{l}\text { PCI Lighting Control Systems } \\
\text { Inc }\end{array}$ & Controls/Software & RS232, RS 485, DMX 512 \\
\hline 43 & PLC Multipoint Inc & Sensors / Controls & $\begin{array}{l}\text { Direct Serial RS232, FIBER OPTIC } \\
\text { COMMUNICATION, LOCAL } \\
\text { MULTI-DROP COMMUNICATION } \\
\text { BUS }\end{array}$ \\
\hline 44 & SensorSwitch Inc & Sensors /Controls & 1-10VDC \\
\hline
\end{tabular}




\begin{tabular}{|c|l|l|l|}
45 & $\begin{array}{l}\text { Siemens Building Technologies } \\
\text { Ltd }\end{array}$ & Controls & $\begin{array}{l}\text { BACnet, MODbus, LONworks, } \\
\text { DMX 512 }\end{array}$ \\
\hline 46 & Smart America & Controls/Software & Ethernet \\
\hline 47 & Square D Company & Controls & $\begin{array}{l}\text { RS232, RS 485, MODbus, Ethernet } \\
10 \text { Base T Connectivity with TCP/IP } \\
\text { Protocols }\end{array}$ \\
\hline 48 & $\begin{array}{l}\text { The Watt Stopper / Pass \& } \\
\text { Seymour }\end{array}$ & Sensors / Controls & 0-10VDC, LONworks \\
\hline 49 & Tork & Controls & \\
\hline 50 & Touch-Plate Lighting Controls & Controls & BACnet \\
\hline 51 & Triatek Inc & Controls & TRIATEK Link, BACnet, MODbus, \\
Ethernet TCP/IP, RS 485, DALI
\end{tabular}




\section{APPENDIX D: INVESTIGATIONS AND ANALYSIS OF OPERATIONAL PARAMETERS REQUIRED FOR EACH OF THE CONTROL TECHNOLOGIES (OCCUPANCY SENSORS, PHOTOSENSORS, DIMMING ELECTRONIC BALLASTS) IN COMMON COMMERCIAL AND INDUSTRIAL APPLICATIONS}

\section{Exploratory Analysis of Operational Parameters of Controls}

A convenient way of analyzing control devices is to consider a device as a system of inputs and outputs. Inputs to control devices are usually sensors and commissioning set points, and the outputs are control signals that govern the operation of the controlled equipment. Once the inputs and outputs are identified, then the operation of the control device is described by how the inputs affect the outputs. The input/output relationships can either be logical relationships, continuous functional relationships, or a combination of the two. This type of analysis is effective for both small, local control systems such as an occupancy sensor in a room, and for large, distributed control systems where the outputs of devices such as occupancy and photosensors are used as inputs to higher-level building automation control systems. In fact, communication protocols for building automation systems, such as BACnet and LonWorks, are specified in terms of inputs and outputs. This report focuses on occupancy sensors, photosensors, and dimming ballasts, describing them in terms of inputs and outputs.

The information for this report was gathered from the publications cited as well as those listed in the bibliographies, and from manufacturers' web sites, conference seminars, product briefs and interviews with manufacturers.

\section{Occupancy sensors}

- Available sensor inputs:

o Passive infrared (PIR)

o Ultrasonic

o Acoustic

- Other inputs:

o Sensitivity adjustments (for PIR and ultrasonic)

o Time delay

- Outputs:

o On/off power relay

o Bi-level switching on/off power

o Low voltage logic signal (to be used a an input for another controller) 


\section{Input sensing technologies}

Three sensing technologies are used in occupancy sensors for commercial and industrial lighting applications: PIR, ultrasonic, and acoustic. Products are available that use either PIR or ultrasonic, or a combination of ultrasonic or acoustic with PIR sensing. The latter are referred to as "dual technology" products.

\section{Passive Infrared}

PIR sensors respond to movement of infrared sources, such as human bodies in motion. A patterned IR transmitting lens is placed in front of a pyroelectric detector that is sensitive to the infrared blackbody radiation emitted by objects at a temperature near and around human body temperature. The patterned lens, typically of Fresnel design, focuses radiation from adjacent wedge-shaped areas of the room onto the detector. As an IR radiating object moves in and out of each segment of coverage, the signal strength received by the detector changes, signaling the detection of movement. Newer designs use a dual-element pyroelectric detector with the elements connected in series with opposite polarity. Changes in the room temperature then do not affect detection, because for static objects, both elements receive the same signal and cancel one another due to the opposite polarities of their electrical connections. The coverage area and sensitivity of a PIR sensor is greatly affected by the type of patterned lens used. Different lens designs are suited for different applications. An overall wide field of view combined with many narrow field of view segments is used for room occupancy sensors, while fewer, larger segments with an overall narrower field of view are used for corridors to maximize to length of the coverage area.

\section{Advantages}

Passive detection does not emit any potentially harmful or interfering signals. It offers the possibility of consuming very little energy to operate (potential for battery operation).

\section{Limitations}

Historically, PIR sensors have had trouble detecting small hand movements. The size of movement for reliable detection depends on the number and size of each wedge-shaped field of view segment that is focused onto the detector. The smaller the segments, the smaller the differential signal strength as an object moves. This places a limitation on how small the segments can get for a detector of a particular sensitivity. PIR sensors must have an unblocked line of sight to detect motion. Room partitions and furniture can prevent PIR sensors from detecting motion. PIR sensors are most sensitive to movement perpendicular to the direction of the sensor, as this type of movement cuts across the wedge-shaped segments. Sensitivity is lowest for objects moving directly towards and away from the sensor.

\section{Ultrasonic sensors}

Ultrasonic occupancy sensors are active devices that must emit ultrasonic sound energy at a frequency typically in the range from 25 to $40 \mathrm{kHz}$, at sound pressure levels in excess of $90 \mathrm{~dB}$ $(0.63 \mathrm{~Pa})$. Motion is detected by sensing the Doppler shift in the frequency of reflected sound waves when reflected off moving objects. 


\section{Advantages}

Ultrasonic sensors cover the whole volume of the space. A direct line of sight is not required for motion detection, and motion can be detected around partitions such as inside office cubicles and lavatory stalls. Ultrasonic sensors are more sensitive to small movements than PIR sensors.

\section{Limitations}

Emission of relatively high levels of ultrasonic sound energy raises health concerns for long-term exposure, even though most sensor companies claim that their products emit less energy than the safe exposure limits set by organizations such as OSHA and the World Health Organization (WHO). Ultrasonic devices require up to 0.5 watts to generate the ultrasonic signal, so battery operation is not practical. They are also sensitive to air movement as well as body movement; therefore, HVAC system operation can cause false triggering.

\section{Acoustic sensors}

Acoustic sensors are passive devices that respond to sound pressure levels in a space; i.e., a microphone. In commercial and industrial products, these sensors are not used for primary detecting, but rather are used in combination with PIR sensors to reduce the occurrence of false off-triggers. That is, a PIR occupancy detector equipped with an acoustic sensor will only switch off the lights after a period of time during which both the PIR sensor and the acoustic sensor do not indicate occupancy.

\section{Advantages}

Simple passive device.

\section{Limitations}

Since ambient noise levels in buildings vary greatly, and acoustic sounds can travel quite far, acoustic sensors cannot differentiate sounds generated inside a space from sounds generated elsewhere. Also, occupancy does not necessarily generate sound, which would lead to annoying false off-triggers in a quiet space. These facts limit the application of this technology to a secondary role in occupancy detection.

\section{Other inputs of occupancy sensors}

To help reduce the occurrence of false triggers, occupancy sensors usually have sensitivity adjustments to fine-tune operation for specific room conditions. Recent designs are incorporating more sophisticated logic for automatically adjusting sensitivity. The incorporation of microprocessors in the devices makes these new features possible at competitive prices. Sensitivity adjustments can be triggered by at least three different inputs: ambient signal strength, time of day, and on/off state of the output.

\section{Ambient signal strength}

Having sensors automatically compensate for ambient signal strength helps the device distinguish between background "noise" and the more erratic signals generated by occupant movement. Instead of having one sensitivity setting that is a compromise between adequate sensitivity for detection and background "noise” rejection, certain sensors can dynamically adjust the signal level that causes a trigger. For example, the steady signal caused by HVAC air 
movement would cause the device to set a higher trigger threshold to avoid a false trigger. In the absence of a steady signal, when the HVAC system cycles off, the trigger threshold would lower, thereby increasing the likelihood of detecting occupancy.

\section{Time of day}

Sensors with microprocessors are also incorporating real-time clocks that provide a time-of-day and calendar-day input to the device. This information can be used to alter the sensitivity or trigger level of the sensor. Real-time clocks and sophisticated logic are now being combined in a form of artificial intelligence through which the sensor "learns" the daily and weekly routines of motion in the space and uses this information to dynamically adjust sensitivity and/or trigger levels.

\section{On/off state of the output}

A simpler kind of logic added to occupancy sensors permits different trigger levels to be assigned under different conditions. For example, a false trigger that turns off the lights when the room is occupied is a worse mistake than keeping the light on a few minutes longer when the room is unoccupied. Therefore, when the lights are on, a lower trigger level might be used to insure that the smallest of movements will keep the light on. On the other hand, when the lights are off, the trigger level might be set higher so that only a large movement, like someone entering the room, will trigger the lights to turn on.

Variable time delays for turning off the lights are another method used to avoid false off signals. Under one scenario, if the occupancy sensor erroneously switches the lights off while the room is occupied, the occupant will immediately make a large movement to switch the lights back on. The sensor could be programmed so that if a large signal is detected immediately after switching off the lights, the following time delay for switching off the lights for a second time might be extended. Similarly, the sensitivity could be increased, or the trigger level lowered to avoid future false off conditions.

The extent to which these different types of logic are used and how successfully they are implemented undoubtedly varies for different manufacturers. The above information was distilled from the claims manufacturers make in promoting their products and was not further verified. Nevertheless, the claims demonstrate that occupancy sensor technology for lighting products continues to be refined and developed.

\section{Occupancy Sensor Outputs}

The output from an occupancy sensor is a two-state binary signal: occupied or unoccupied. Such a signal can be directly connected to a power relay to switch lights on and off. Occupancy sensors for local control most often incorporate a power-switching relay right into the device, while others provide a logic-level signal that is connected to some other lighting controller. While the signals from occupancy sensors are two-state, the output does not necessarily have to completely switch the light on and off. In many applications where a certain low level of illumination is always required, occupancy sensors are used for bi-level switching of the lighting. For example, occupancy sensors are used in warehouses to switch on the lights over individual aisles to provide high visibility for tasks such as reading labels, while the unoccupied part of the warehouse can remain at lower illumination levels. 


\section{Photosensors}

- Available sensor inputs:
o Illuminance (wide field of view)
o Luminance (narrow field of view)

- Other inputs:
o Sensitivity (gain)
o Light level set point
o Hysteresis (dead band)

- Outputs:
o On/off power relay
o Bi-level switching on/off power
o Continuous dimming level (e.g. 0-10V)

\section{Background}

The input to a photosensor is optical radiation. Loosely speaking, the input is light, but because some photosensors respond to infrared (IR) and ultraviolet (UV) radiation as well, it is necessary to make a distinction between optical radiation that is visible light and other kinds of optical radiation. The response of a photosensor to optical radiation is fully described by the spatial response and the spectral response.

The spatial response describes the sensitivity of the photosensor to incident radiation from different directions - in other words, what the photosensor "sees" at different locations. Spatial response is analogous to a luminaire intensity distribution, but describes sensitivity instead of output.

The spectral response describes the sensitivity of the photosensor to optical radiation of different wavelengths. This is important because only a small part of the optical radiation spectrum is visible. Daylight and fluorescent lighting differ substantially in spectral composition. Daylight has a comparatively uniform distribution of energy over the near-UV, visible, and near-IR regions of the spectrum. Fluorescent lamps, on the other hand, have most of their output concentrated in the region of the spectrum where visual sensitivity is high. This is one reason fluorescent lighting is so efficient. Even though the exact spectrum of daylight changes depending on weather conditions, times of the day and season, as well as being affected by surrounding buildings and foliage, these differences are small compared to the relative differences in UV and IR content between daylight and fluorescent light sources. The greater UV and IR content of daylight, combined with the broader than ideal spectral response of most photosensors, makes most photosensors much more sensitive to daylight than to light from fluorescent lamps. A greater sensitivity means that a photosensor will respond as if more daylight were present than actually exists. This can lead to problems where precise switching or dimming levels need to be realized. 


\section{Illuminance sensors (wide field of view)}

The signal produced from illuminance type sensors is useful for detecting ambient light levels. A wide spatial response corresponds closely to what an illuminance meter would measure.

\section{Advantages}

The advantage of a wide spatial response is that the optical signal sensed by the photosensor is representative of the illumination on the whole workplane, or over the entire room, when the sensor is located on ceiling. The optical signal is also less affected by normal activity in the room than for a narrow response sensor.

\section{Limitations}

The difficulty with a wide spatial response is that the ceiling illuminance does not usually correspond to the workplane illuminance as the balance between daylight and electric light changes. In fact, the ratio of ceiling to workplane illuminance typically changes by a factor of five or more in offices with vertical windows as the proportions of electric light and daylight change. However this non-correspondence in illuminance levels can be largely overcome by the photosensor control algorithm and the above advantages can be realized.

\section{Luminance sensors (narrow field of view)}

Not as common as illuminance type sensors, luminance sensors detect light from a particular direction and over a small field of view. They are used to detect brightness from a distant location; for example, to detect desktop luminance from a mounting position in the ceiling.

\section{Advantages}

The narrower the photosensor's spatial response, the more closely it responds to the luminance (brightness) of the surface at which it is aimed. The luminance of a surface, in turn, is directly proportional to the illuminance falling on the surface provided that the reflectance factor of the surface is constant. Therefore, provided that the reflectance properties of the surface do not change, a narrow spatial response can effectively track illuminance changes; the narrower the response, the better the tracking for a particular location.

\section{Limitations}

The narrower the spatial response, the smaller the sensor's field of view, so what the sensor "sees" may not be representative of the whole surface or workplane. Therefore, a narrow response makes the sensor very sensitive to changes in the reflectance properties of what it is viewing. In practice, the reflectance of the workplane is not constant, but changes depending on the activities going on in the room. Examples include a dark desktop that is sometimes covered with white papers, the colors of peoples' clothing, such as a white shirt versus a dark suit, and even rearrangement of the room's furniture.

Another limitation of a narrow spatial response is increased sensitivity to mirror-like, specular reflections off shiny surfaces. Illuminance on a surface is directly proportional to luminance only for diffusely reflecting surfaces. Most surfaces in a room are diffuse, but some, like a glass table top, can reflect overhead light directly back into the photosensor's field of view causing erratic performance. Specular reflections have proportionally less effect on photosensors with a wider spatial response. 


\section{Other Photosensor Inputs}

The following inputs are set during the commissioning of photosensors. Depending on the type of output (on/off or continuous dimming), as well as the type of control algorithm employed in the particular device, one or more of these inputs will be available to the user. On some photosensor products, the sensitivity and certain set points are combined into one input that controls both together according to some programmed relationship.

Sensitivity (gain)

The spatial sensitivity and the spectral sensitivity of the photosensor characterize the optical gain. Electronic gain amplifies the weak signals from the photocell to practical signal levels. These two gain mechanisms (optical and electronic) determine the sensitivity of the photosensor. Sensitivity adjustments are required for open-loop sensors where the sensitivity adjustment determines the relationship between electric light levels and the sensed signal. For photosensors that do not have a sensitivity adjustment, or those that combine sensitivity with other set-point adjustments, the sensitivity alone can always be adjusted optically by the positioning of the photosensor. While positioning the photosensor differently for different sensitivities is an option, it not very practical and it certainly is not a systematic way of commissioning photosensors.

\section{Set-points}

The signal level that must be attained before an action occurs is known as a set-point. For photosensors, set-points determine the signal level at which lights will be switched, or at what light level dimming will start and/or end. The type and number of set-points that are employed in a photosensor depends on the type of control algorithm used. Simple, open-loop photosensors that switch lights on and off only need to have one set- point that determines the level at which the lights will switch. Often, two set-points are used, however, to give the switch some hysteresis, or a deadband, whereby the light switches on at a higher signal level than that which turns them off. This is to prevent unstable frequent switching when signal levels are near the setpoint.

More complicated closed-loop control algorithms may employ several set-points, which might be measurements that determine sensor and task illuminance ratios used in the algorithm to determine the electric light level.

\section{Photosensor Outputs}

Photosensors fall into two main categories depending on the output. The most familiar and the most prevalent use of photosensor control is on/off control output which is used to turn lights on, or off, based on the light level detected. Far less prevalent is the continuous level output photosensor, which is used with dimming systems to dim the electric lighting level based on some dimming function or control algorithm.

The light-sensing element within a photosensor might be a photodiode, a phototransistor, or a photo-resistive cell. It is important to make a distinction between this, the photocell, and a complete photosensor device that includes additional circuitry to produce the desired output signal(s).

\section{Photosensors for on/off control}

Photosensors for on/off control work most effectively in applications where a large difference in light level exists between the on condition and the off condition. Photosensors for outdoor street lighting is such an example. The set-point at which the output is switched does not have to be 
precise due to the large difference in illumination levels between night and day. Photo-resistive sensing elements are commonly used in this application because of low cost and relatively simple circuit design. Photo-resistive sensors do not have a linear response with light, part-topart consistency is poor, and they have a large temperature dependency. Taken together, these characteristics make precise action at specified set-points difficult. A further disadvantage of photo-resistive devices is that many common types use cadmium, a heavy metal that is considered harmful to the environment.

When switching at precise light levels is needed, silicon photodiode detectors are commonly used. Used in conjunction with an amplifier circuit, these devices offer very predictable and linear output that is stable with time and temperature. This allows switching at precise set-point levels. These types of detectors are useful for indoor applications where the overall range of acceptable light levels is orders of magnitude less than that encountered outdoors.

The output from an on/off sensor is a two-state binary signal. Such a signal can be connected directly to a power relay to switch lights on and off, or used as a low level logic-level signal that is connected to some other lighting controller.

\section{Photosensors for dimming}

Photosensor control for dimming is divided into two main types: open loop and closed loop:

\section{Open loop}

The photosensor does not respond to, or "see" the electric light that it controls. An example of an open-loop system is a photosensor mounted on the outside of a building that controls the electric light level inside the building. In such a case the photosensor is exposed only to daylight. The electric light level is determined from the daylight signal alone. In the case of on/off control, such systems can be designed to simply turn electric lights off when outside daylight reaches a predetermined level. In the case of a dimming system, a signal proportional to the outside daylight instructs the system to dim the electric light by an amount proportional to the amount of available daylight sensed by the photosensor. No feedback control is used for an open loop system.

The drawback of open-loop feedback control is that the system cannot compensate or correct for any changes in the light distribution that affects the constant of proportionality between interior light levels and outside daylight levels. For example, the system will not respond to the use of window blinds, so if the occupant draws the blinds to block direct sunlight, the system will not increase the electric light to compensate for the decreased daylight levels inside the room.

\section{Closed loop}

The photosensor senses and responds to the electric light that it controls. An example of a closed-loop system is a photosensor mounted on the ceiling of the room where the electric lighting is being controlled. In this case the photosensor is exposed to both the daylight and the electric light in the room. The sensing of the electric light forms a feedback loop. Closed-loop systems use negative feedback to respond to changing conditions. Negative feedback is a means of error correcting or compensating whereby an increase in an input signal level causes a decrease in the output signal. Conversely, a decrease in input signal causes an increase in output signal. This is the desired action of photosensor control; an increase in the amount of light in the room causes a decrease in the electric light intensity, and a decrease of daylight causes an increase of electric light. The overall feedback loop of a photosensor system 
must be negative for proper operation. The control algorithm characterizes the negative feedback of a photosensor.

The amount of feedback can vary for different systems and different locations of the system components. In systems where the photosensor is mounted near a window, the feedback is proportionally less than in systems where the photosensor is mounted deep within the room. This is because near the window the proportion of daylight is greater than electric light and the photosensor "sees" proportionally less of the electric light that it is controlling. The opposite is true for a photosensor mounted deep within a room. The amount of feedback is also governed by room geometry and surface reflectances. A room with light-colored finishes will have a greater feedback gain than a room with dark-colored finishes. The gain caused by room geometry and surface reflectances combines with the optical and electrical gain of the photosensor to determine the actual signal level received by the photosensor.

\section{Effect of photosensor output on light level}

For dimming systems, the dimming ballast controls the electric light level based on input from the photosensor. The amount of dimming as a function of input signal is characterized by the dimming response function. For many dimming ballasts, the dimming response function is linear, meaning that it reduces the electric light level in proportion to the input signal. However, the active input dimming range is usually less than the specified range of input control voltage. For example, for a ballast with an input signal specification of zero to $10 \mathrm{~V}$, dimming may actually take place over a more limited range from about $1.5 \mathrm{~V}$ (minimum light output) to $8.5 \mathrm{~V}$ (maximum light output).

\section{Dimming Ballasts}

- Available inputs:
o 1-10V analog signal
$0<0.5$ volt standby signal
o Phase chop angle power line signal
o Digital control interfaces (DALI and SuperDim)

- Outputs:

o Lamp power level

o Ballast status (e.g., lamp failure for DALI equipped ballasts)

\section{Dimming Ballast Inputs}

Control inputs to ballasts are divided into analog and digital categories. Within each of these categories, different signaling protocols and/or conventions are used. Digital control inputs to ballasts have only recently been introduced on the market and currently comprise a very small market share. There is, however, considerable interest in and backing by different ballast manufacturers for the DALI communication protocol for ballasts. (For a detailed listing of the strengths and weaknesses of the different ballast control interfaces see Task 2.9 below) 
Currently, analog control interfaces for ballasts are the most widely available with 0-10V control interfaces being most common. The $0-10 \mathrm{~V}$ interface was the first to be used when dimming electronic ballasts appeared on the market in the early 1990s. The control scheme itself dates back to the early 1970s where it originated in the theatrical lighting controls industry. In fact ANSI has recently approved a 0-10V standard for entertainment technology (Standard E1.32000). However, the implementation of $0-10 \mathrm{~V}$ control in ballasts is different than that used in theatrical controls. ANSI Standard E 1.3-2000 even specifically states that the standard does not apply to fluorescent dimming ballasts. Ballast manufacturers themselves have not adopted a standard for commercial use at this time and it seems likely at this time that none will ever come about. As a result, consistent behavior across different ballasts types or manufacturers is not assured.

The main difference between the implementation of $0-10 \mathrm{~V}$ control for ballasts and that used in the entertainment industry is that the ballast is capable of providing its own signaling voltage while other $0-10 \mathrm{~V}$ devices require the signaling voltage to be provided by a separate controller. The benefit of having a device that provides its own signaling voltage is that it allows the use of very simple control devices that do not require their own power sources. For example, a 0-10V ballast can be dimmed by a simple variable resistor connected across the control wires. Compatibility problems arise when a ballast is connected to a controller that also supplies a signaling voltage. To work properly the controller must be able to conduct current from the higher voltage control wire to the lower voltage wire (sink current). If a controller cannot conduct current in this reverse direction, from the viewpoint of the controller, the ballast will keep the signal high and no dimming will take place.

A deficiency of $0-10 \mathrm{~V}$ control for both ballasts and products covered by the Entertainment Industry standard is that the relationship between dim level and control voltage is not defined. As a consequence, consistent dimming behavior among different ballast types and ballasts made by different manufacturers is not assured. For example, a 5-volt signal for one ballast might result in a 30\% dim level, while the same 5-volt signal might result in a 50\% dim level for another manufacturers ballast. This is problematic for at least two reasons. First, it prevents the mixing of different ballast types within one control area, and second, it complicates the commissioning of dimming systems because each system must be individually calibrated for dimming response. Some manufacturers also provide for analog command regions within the 0 to $10 \mathrm{~V}$ signal range. For example, a control voltage less than 0.3 volts might signal the ballast to shut-down. While these extensions might provide desirable features, they can also lead to compatibility problems with controllers not designed with these features in mind.

Another problem with the $0-10 \mathrm{~V}$ conventions used by ballast manufacturers is that the signal levels are low and thus suspect to interference. Little, if any guidance is given by ballast manufacturers on proper cabling techniques to avoid interference, but anecdotal evidence suggests that control wires must be kept away from power lines and lamp leads, and that the total cable length is a concern.

As an alternative to 0-10V control, two-wire, or ac phase chop dimming is available. This analog approach uses the power lines for signaling. In this control scheme, the rise of the ac signal after each zero-crossing is delayed an amount of time (zero to $8.3 \mathrm{~ms}$ or one-half of the wave form period), which is related to the dim level. Delaying the rise of the ac voltage after each zerocrossing results in a lower rms ac voltage signal whose shape looks as if part of the waveform has been removed, or chopped. This chopping is inexpensively performed by solid-state 
switching devices such as triacs or silicon controlled rectifiers (SCRs). This technique is used for controlling certain electrical loads such as heater coils and incandescent lamps. Operating a nondimming electronic ballast on a phase-chopped voltage could be damaging to the device, but electronic ballasts designed to accept such signals use the phase-chopped signal to set the output power to the lamps.

The major benefit of using power line phase-chop signals for dimming is that no additional wiring is needed to control dimming. For retrofit applications, dimming controllers can replace existing switches and the existing power lines carry both the power and the signal. A secondary benefit is that the signals are less sensitive to interference than low voltage analog signals.

An alternate way of using the existing power lines to carry control signals is by using a power line carrier signal (PLC) at a frequency much higher that than the $60 \mathrm{~Hz}$ power frequency. While somewhat successful for caring digitally encoded signals, such a scheme has not been used for analog control.

For digital control PLC communications have been around for many years, although they have not been very successful in commercial and industrial environments. From the start such systems have been plagued with interference problems and are now considered to be unreliable, except for residential use. Ironically, many of the interference problems are the result of electronic power equipment, such as electronic ballasts, on the same, or nearby circuits. The X-10 protocol for digital control using PLC signals is supported by some lighting equipment manufacturers although no ballast manufacturers are known to have incorporated this directly into their ballast designs.

Another digital communication protocol not supported by ballast manufacturers, but used for lighting equipment is the DMX-512 protocol. DMX-512 is used extensively in theatrical lighting control systems. It is a high-speed, wide bandwidth (250 kbytes/s) method of communication allowing up to 512 points of control per control loop. DMX-512 has not been incorporated directly into ballast designs, most likely because of the relatively high cost of adding such a high-speed communication interface. Also, the existing commands do not lend themselves well to architectural and energy saving applications.

The two digital interface control protocols that are directly incorporated into ballasts are the SuperDim protocol by Energy Savings, Inc. and the Digital Addressable Lighting Interface (DALI), originally conceived by Tridonic, a European lighting equipment manufacturer. Without going into all the details of the protocols, there are some important features that make these protocols useful for commercial lighting control.

Both protocols use two, isolated, low voltage control wires to carry control signals. Twisted pair wiring such as what is used for computer networking is commonly used for cabling. Both use a form of serial communication similar to the common and widely used RS232 method. Data rates are low, 2400 baud for SuperDim and 1200 baud for DALI. The use of relatively low signal rates indicates the need for a robust, low cost network over a higher speed network. Both protocols emphasize the need for both low cost and simple implementation.

The main barrier to overcome when setting up a ballast control network is finding an easy way of commissioning the system. To commission such a system the interconnected ballasts must be logically grouped together to realize different lighting scenes and energy saving strategies. With analog control, the grouping of ballasts is "hard-wired" when the ballasts and control gear are 
installed. This hard-wired approach could also be done with digital controls, but it would not take advantage of one of the main benefits of digital controls, which is the increased flexibility that they offer. Realizing this increased flexibility means that the burden of commissioning has largely been shifted from the installer to a later user of the system. Having a system that can be easily reconfigured seems to be an important selling point of digital control systems over existing analog control systems.

SuperDim and DALI protocols make use of each ballast in a network a having unique address. With the SuperDim protocol a permanent, unique 28-bit address is assigned to every ballast at the time of manufacturer. Part of the commissioning process is then having the addresses of all the connected ballasts input to the controller. Though not mentioned in the communication protocol, ballasts by Energy Savings, Inc., make use of an optical sensing commissioning tool that is used to retrieve the addresses of ballasts by receiving a high frequency modulated light signal from the fluorescent lamps being operated by the particular ballasts. When so instructed, ballasts will output their addresses via high frequency light output modulation. The commissioning tool receives this information when aimed at the ballast of interest and relays the address information to the main controller. In this manner installed ballasts within luminaires can identified and grouped into logical zones for control without dismantling fixtures.

The DALI protocol handles addressing in a different manner. When so instructed, all DALI ballasts on a network will assign each oneself a randomly generated 24-bit address. The controller then determines each ballast's address through an iterative trial-and-error process of trying different addresses until it gets a response. Once the addresses are all known, individual ballast locations can be identified by having the controller send signals to a particular ballast instructing the ballast to flash the lamps on and off, for example. If by chance more than one ballast has the same address, provisions are made for just those ballasts with identical addresses to repeat the randomization process.

\section{Dimming Ballast Outputs}

\section{Lamp power level}

Reducing the power delivered to a fluorescent lamp reduces the light output and effectively dims the lamp. Due to the operational characteristics of fluorescent lamps, power reduction must be done with at least two major provisions to keep the lamp from extinguishing and to preserve long lamp life:

- Maintain a sufficiently high voltage across the lamp to sustain the arc.

- Keep the electrodes properly heated so that they can supply sufficient free electrons to the discharge without being severely damaged.

For these reasons, a specially designed dimming ballast is required to effectively dim fluorescent lamps to levels less than about $70 \%$ of full light output.

The requirements of maintaining lamp voltage and electrode heating prohibit magnetic ballasts from being used successfully as dimming ballasts. While products are on the market that dim magnetic ballasts (e.g., panel-level dimmers), the dimming range is limited to about $50 \%$ of full light output. In addition, long operation times at a low dim level are likely to reduce lamp life. 
High-frequency electronic ballasts have been successfully developed to dim fluorescent lamps to light output levels as low as $1 \%$ of full light output through the use of active electronic components that can dynamically change ballast-operating characteristics as the lamp is dimmed. Lamp voltage can be maintained even at low currents and supplementary electrode heating can be increased to maintain proper electrode heating as the lamp is dimmed.

Even though electronic ballasts are capable of dimming lamps to low power levels while preserving life, the supplemental electrode heating requirements, as well as the power requirements of the additional circuit components, reduce the overall system efficacy of dimming systems compared to non-dimming systems. This is clear from an analysis of the different electronic ballast types currently on the market.

Table 1 lists manufacturer-reported and National Lighting Product Information Program (NLPIP) test data on ballast factor and ballast efficiency factor for instant-start, rapid-start, and dimming ballasts. For each group of similar ballast type, the average ballast factor and average ballast efficiency factor is calculated. In the case of dimming ballasts, the ballast efficiency factor is for the full light output condition. For the same lamp type, and the same number of lamps operated per ballast, ballast efficiency factors can be directly compared to show relative system efficacy. All the ballasts in this analysis are two-lamp ballasts operating T8 lamps. To aid in comparing relative system efficacy, the group averages are shown as a percentage of the highest BEF group, in this case the instant-start ballast group. Relative efficacies are also shown for the dimming ballast group at $40 \%$ and minimum light output levels calculated directly from NLPIP reported light output and system power measurements.

Table 1 shows that, on average, instant-start ballast systems are about $5 \%$ more efficacious than electronic rapid-start systems. This efficacy difference, combined with lower costs and similar lamp life performance, can explain why instant-start electronic ballasts constitute about $80 \%$ of electronic ballast sales based on U.S. census data.

When instant-start systems are compared to dimming ballasts, the discrepancy in efficacy increases to about 9\%. Consequently, from an energy point of view, the average power reduction from dimming would have to be nearly $10 \%$ just to break even on energy consumption if dimming ballasts were used in place of instant-start electronic ballasts. In other words, the user would get $10 \%$ less light from a dimming system than from an instant-start system for the same energy usage.

Most dimming ballasts show a linear relationship between light output and dim level. (See Figure 1 from NLPIP Dimming Ballast Specifier report.) It is important to note that the curve showing this relationship does not intersect the origin, but rather it is offset to the right. This offset is due to a combination of lower lamp efficacies when operated at low power, and energy that is consumed by the ballast, which represents an increasing percentage of the total power consumed by the system as the lamp is dimmed. Therefore, though linear with power, light output is not proportional to power, but shows diminishing returns as the light output is reduced. The data in Table 1 reveal this by showing the relative efficacies for dimming systems at $40 \%$ and at minimum light output levels. The relative efficacies for these conditions are $66 \%$ and $37 \%$, respectively. The reduced efficacy at $40 \%$ light output gives only a $43 \%$ energy savings when the light are dimmed $60 \%$. When compared to non-dimming instant-start systems, the energy savings are only $40 \%$ for a $60 \%$ reduction in light output. 
Because of the diminishing energy savings with dimming, dimming below about $20 \%$ of full light output is ineffective when dimming for energy savings. Below this level, the only way to get further substantial energy savings is to switch off the ballast.

\section{Other dimming ballast outputs}

In addition to lamp power output, digital addressable ballasts using the DALI protocol are capable of a limited form of two-way communication. While at present there doesn't appear to be any clear energy-saving argument for two-way ballast communication, there are instances where feedback from the ballast could improve lighting quality. For example, failed lamps could be automatically reported to facility personnel.

\section{Table 1. Relative Efficacies of 2-lamp, T8 Electronic Ballasts by Type}

Gray indicates NLPIP test results, otherwise manufacturer data

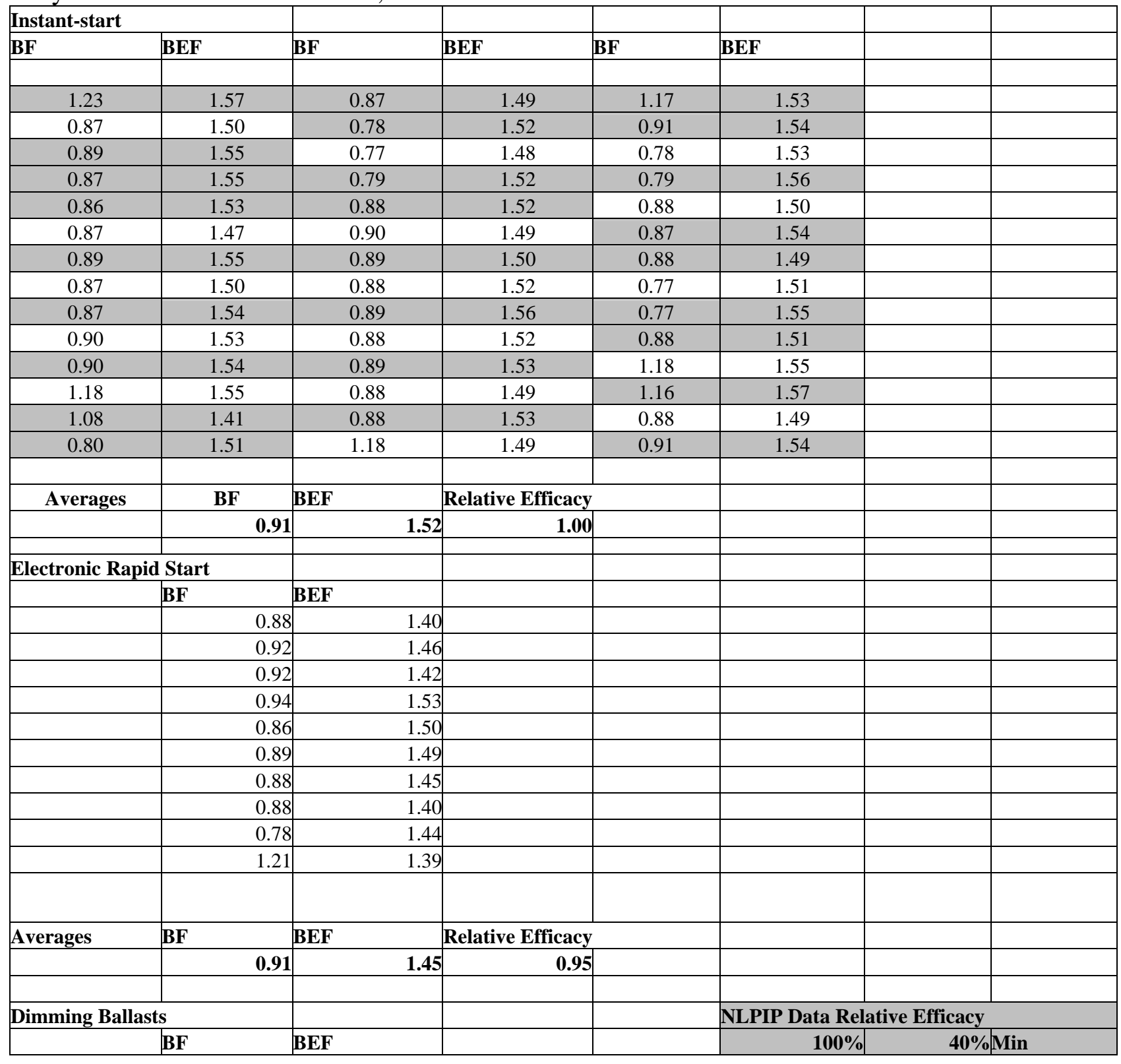




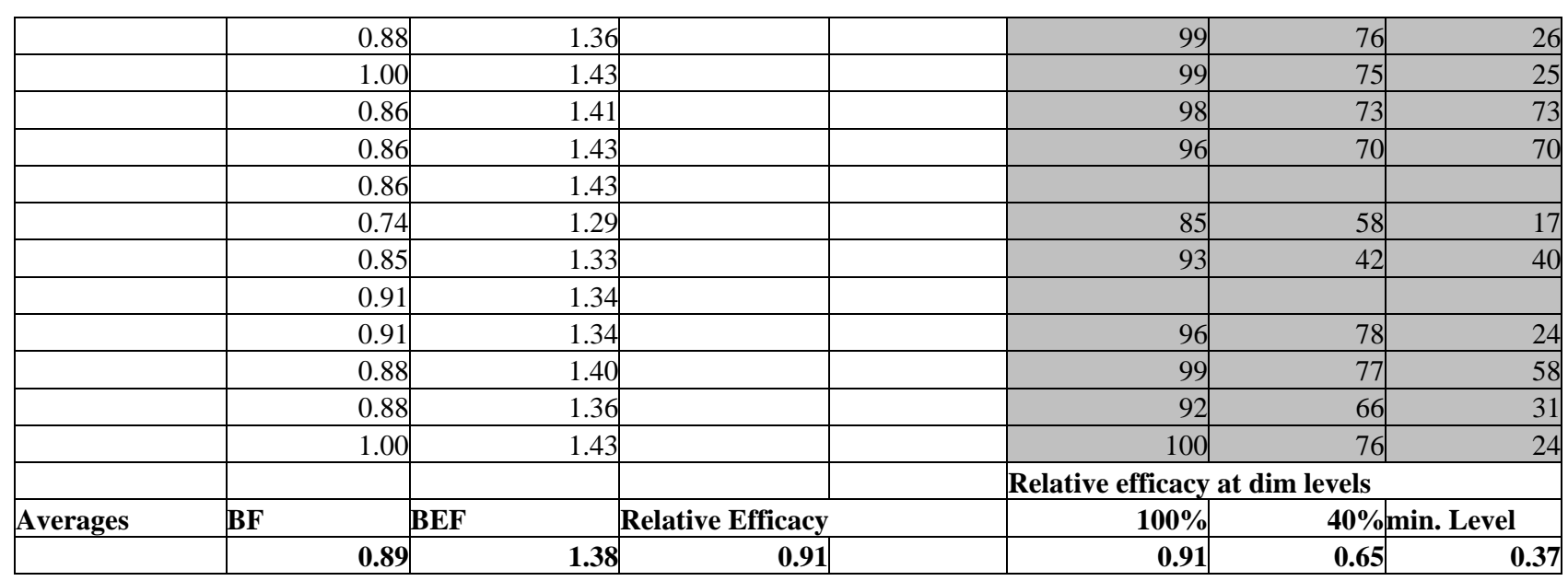

Range of RLO Compared to Active Power Dimming electronic Ballasts for Two F32T8 Lamps

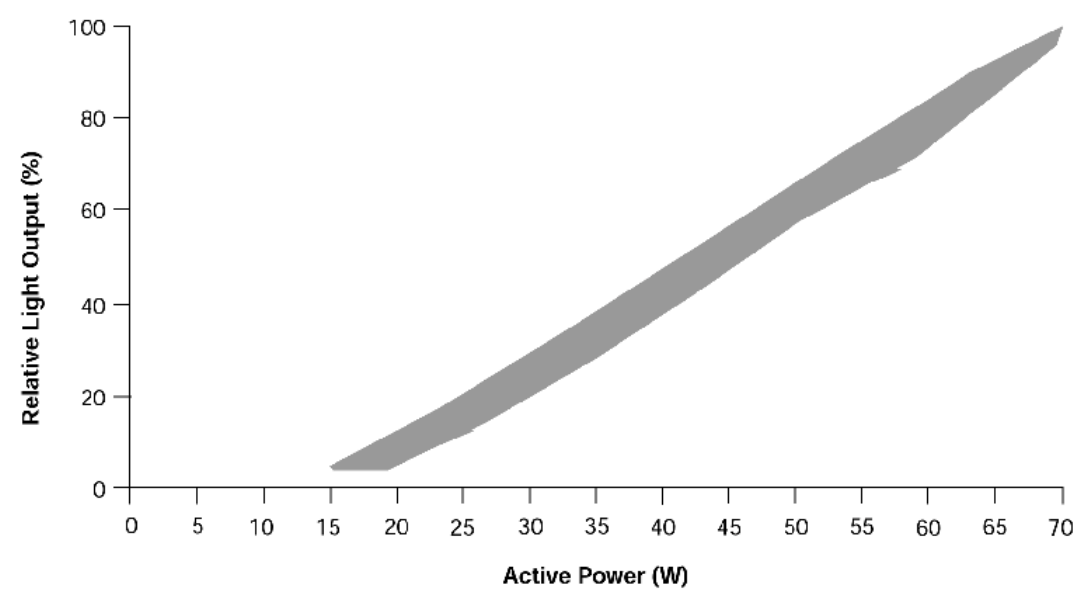

Figure 1. Gray area shows range of relative light output (RLO) plotted against power demand for dimming ballasts tested for NLPIP Specifier Report: Dimming Electronic Ballasts (1999).

\section{References}

National Lighting Product Information Program. 2000. Specifier Reports: Electronic Ballasts Vol (No.). Troy, NY: Lighting Research Center, Rensselaer Polytechnic Institute.

National Lighting Product Information Program. 1999. Specifier Reports: Dimming Electronic Ballasts Vol (No.). Troy, NY: Lighting Research Center, Rensselaer Polytechnic Institute.

National Lighting Product Information Program. 1998. Specifier Reports: Photosensors Vol (No.). Troy, NY: Lighting Research Center, Rensselaer Polytechnic Institute.

National Lighting Product Information Program. 1997. Specifier Reports: Occupancy Sensors Vol (No.). Troy, NY: Lighting Research Center, Rensselaer Polytechnic Institute. 
Lighting Research Center. 2000. Photosensor Tutorial. Troy, NY: Lighting Research Center, Rensselaer Polytechnic Institute.

Rea and Maniccia. Lighting Controls: A Scoping Study, Reference Document 1994. Troy, NY: Lighting Research Center, Rensselaer Polytechnic Institute.

Lighting Research Center. 1998. Report 10073: Photosensor Project Bibliography. Troy, NY: Lighting Research Center, Rensselaer Polytechnic Institute.

A. Bierman and K. Conway. Characterizing daylight photosensor system performance to help overcome market barriers. Illuminating Engineering Society of North America 1999 Annual Conference: Proceedings. IESNA: New York, NY. 413-432.

R.G. Mistrick, C.-H. Chen, A. Bierman and D. Felts. An analysis of photosensor-controlled dimming systems in a small office. Illuminating Engineering Society of North America 1999 Annual Conference: Proceedings. IESNA: New York, NY. 433-448.

D. Maniccia, A. Wadhwa, and R. Longtin. A new method for assessing occupancy sensor performance using robotics. Illuminating Engineering Society of North America 1996 Annual Conference: Proceedings. IESNA: New York, NY. 760-799.

A. Buddenberg and R. Wolsey. Compatibility test of dimming electronic ballasts used in daylighting and environment controls. Illuminating Engineering Society of North America 1995 Annual Conference: Proceedings. IESNA: New York, NY. 1-9. 


\section{Appendix E: Direct MaIl Contractor Survey}

\section{Executive Summary}

This report represents the results of a direct-mail survey conducted from May 15 to June 15, 2001, by the Lighting Research Center. The purpose of the survey was to determine contractors' and controls installers' attitudes about market barriers affecting the acceptance of lighting controls.

This survey confirmed overall what the industry already knows about lighting controls. Two significant results were quite clear:

- The potential market for occupancy sensors, photosensors, and dimming electronic ballasts is much greater than the present market. Installers were willing across the board to say that they could install far more controls than they do, if there were no barriers to market penetration.

- The universally cited barrier to market penetration was lack of customer demand, as expected. Although not directly asked, installers responded that they had difficulty proving that customers would see the payback from lighting controls.

Other market barriers seem relatively unimportant to this group:

- Lighting controls do not seem to cost installers much difficulty, either for time to install, or from returning to the job to adjust the controls. Everyone who completed the surveys commissions as well as installs the controls, so it can be assumed that all the installers are familiar with problems that arise in commissioning.

- Neither difficulty in finding skilled labor, nor a lack of compatible products seem to affect the number of controls this group installs. The majority of installers rate these barriers as "low" in importance across all types of controls, with only a few rating them "medium."

- Information about profitability was unclear; most installers have not broken out the cost of individual controls, as they use fixed contract pricing. However, for all four types of lighting controls, low profitability was cited as a major barrier no more than $20 \%$ of the time.

The results of this survey seem to show that customers must first be convinced that lighting controls will benefit their bottom line. If that happens, little else remains in the way of their widespread use.

Results for each type of lighting control are discussed in detail below.

\section{Survey Demographics}

The survey was mailed to 350 addresses obtained through the web sites of organizations whose members are likely to install lighting controls, including the following:

- National Association of Energy Service Companies

- National Association of Lighting Management Companies

- National Electrical Contractors Association 
- New York Energy Efficiency Council

- Association of Energy Efficiency Engineers

Addresses were evenly distributed across the United States. As an incentive, installers received three Lighting Research Center publications of their choice if they returned the surveys. The response was 20 completed surveys, a return rate of approximately $5.7 \%$. This return rate is in line with average return rates in direct-mail survey work. Note that not all installers who responded work with all types of lighting controls. Thus, the number of responses for each type of control can be less than the total number of surveys returned.

The surveys were more heavily returned from the eastern half of the U.S. Only one set of surveys came in from California, although 23 requests were sent. The rest of the surveys clustered in the Midwest and the upper South. No surveys came from the Northwest or Southwest. The distribution by state is given in Table 1 .

Table 1: Each returned survey's state provenance

$\begin{array}{llll}\text { CA } & \text { KY } & \text { NC } & \text { TN } \\ \text { GA } & \text { MD } & \text { NH } & \text { TX } \\ \text { IA } & \text { MI } & \text { NY } & \text { VA } \\ \text { KS } & \text { MN } & \text { OH } & \text { WI } \\ \text { KS } & \text { MN } & \text { TN } & \text { WI }\end{array}$

Finally, the addresses were evenly distributed in three categories. The first category was independent companies taken from the memberships described above, and the second and third categories were branch offices or affiliated installers from two major controls manufacturers, Siemens Building Technologies, Inc. and Johnson Controls, Inc. The returned surveys included approximately one-third independent companies and one-third each from Siemens and Johnson installers.

\section{Dimming Electronic Ballasts}

(12 responses)

The surveyed group installed relatively few dimming electronic ballasts in the last year, but the potential for increasing this type of control was high. Although 50\% of the group installed fewer than 100 dimming ballasts, more than $40 \%$ thought that they could potentially install 1000 to 50,000 additional ballasts.

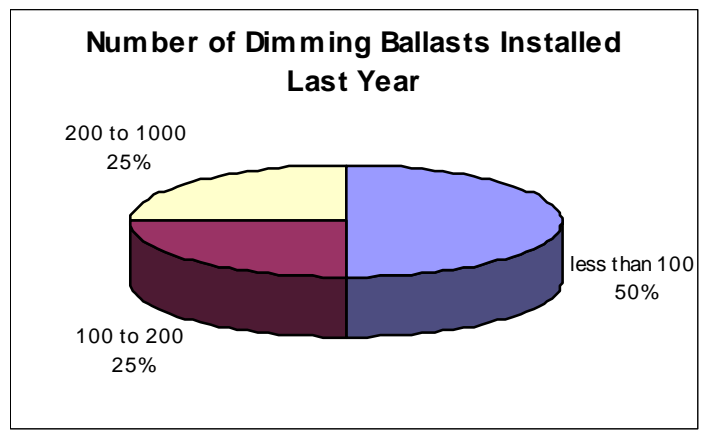

Figure 1: Percentage of installers who cite range of dimming ballasts installed last year. 


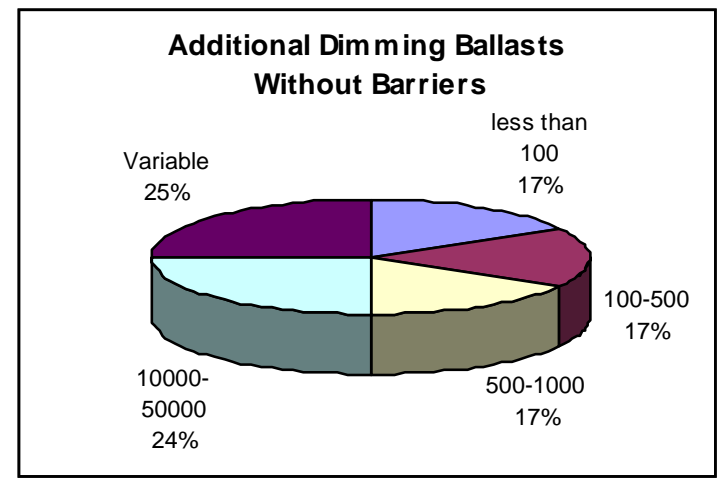

Figure 2: Percentage of installers who cite the range of additional dimming ballasts they could install if no barriers existed.

The major barrier holding back that number, they said, was lack of customer demand-73\% of installers cited this barrier. One installer told us that it is "always difficult to obtain a good payback." He also said, "Dimming controls have always been a compatibility problem."

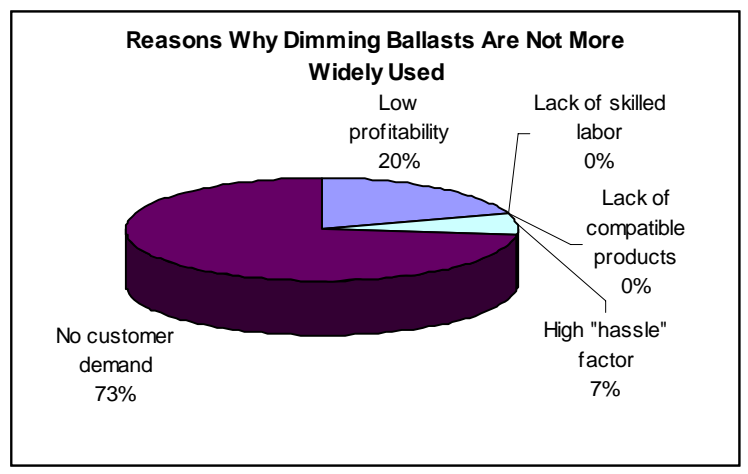

Figure 3: Percentage of installers who cite the importance of a reason for not using dimming ballasts as "high."

Dimming ballasts were time-efficient to install, with 33\% of installers able to complete installation in 30 minutes or less. An additional 25\% could install a ballast in 30 to 60 minutes. The majority of installers charge for dimming ballasts by fixed contract price, with a number using hourly rate as the next most common method. Thus, the average charge they cited varied by contract as well. The most common brand of ballast they use was Advance (4 installers), while Lutron, Howard, and Motorola brands were also mentioned (1 installer each). About half say they sometimes use a different dimming ballast than the one specified by the engineer, architect, or designer.

\section{Occupancy Sensors}

(17 responses)

The surveyed group installed a relatively large number of occupancy sensors in the last year. Three installers (18\%) put in 1000 or more, while four more (23\%) installed between 200 and 1000 occupancy sensors. However, an equal number installed 100 or fewer occupancy sensors. 
Seven installers said that they use Watt-Stopper occupancy sensors, while SensorSwitch and WattMiser were each mentioned once. Again, about half of the installers say they sometimes use a different occupancy sensor than the one specified.

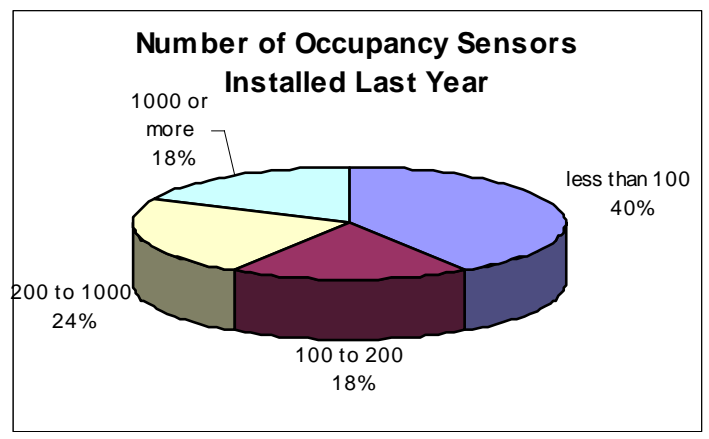

Figure 4: Percentage of installers who cite the range of occupancy sensors installed last year.

If no barriers existed, over 50\% of installers feel they could put in anywhere from 500 to 50,000 additional occupancy sensors.

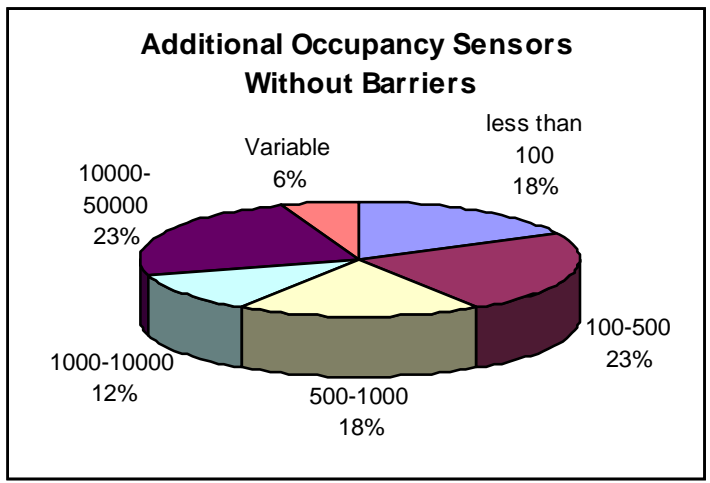

Figure 5: Percentage of installers who cite the range of additional occupancy sensors they could install if no barriers existed.

Lack of customer demand is the greatest barrier, but a high "hassle" factor with the sensors also interferes with growing the market for this type of lighting control. Comments about occupancy sensors from the installers included this: "It is difficult to measure exact savings and also more time-consuming to design, which are two reasons we do not propose them as often." And another said, "[Our] biggest objections are based on poor past customer experience; it's always difficult to predict accurate savings.” 


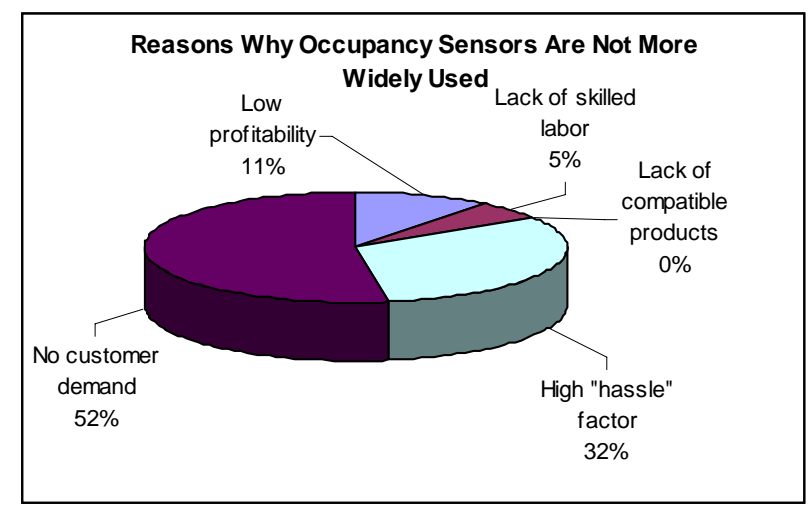

Figure 6: Percentage of installers who cite the importance of a reason for not using occupancy sensors as "high."

It took most installers one to two or more hours to install an occupancy sensor. All respondents said that they themselves commissioned the sensors in addition to installing them. Customers did not often call them back to adjust the sensors once they were installed; $58 \%$ of installers say customers only call them back 1 to $10 \%$ of the time.

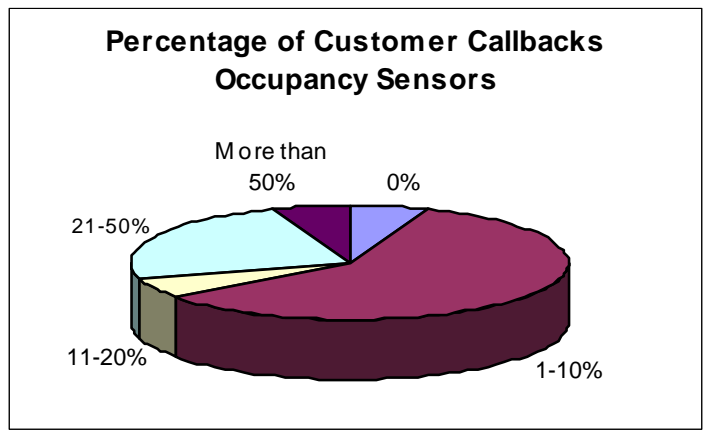

Figure 7: Percentage of installers who report frequency of customer callbacks for occupancy sensor installations.

The installers mostly charged by fixed contract for occupancy sensors, so many could not say what they charged for an individual control. However, one installer did charge a fixed price for a single control (\$20 to \$60) and another charged a unit price per room (\$150 to \$200).

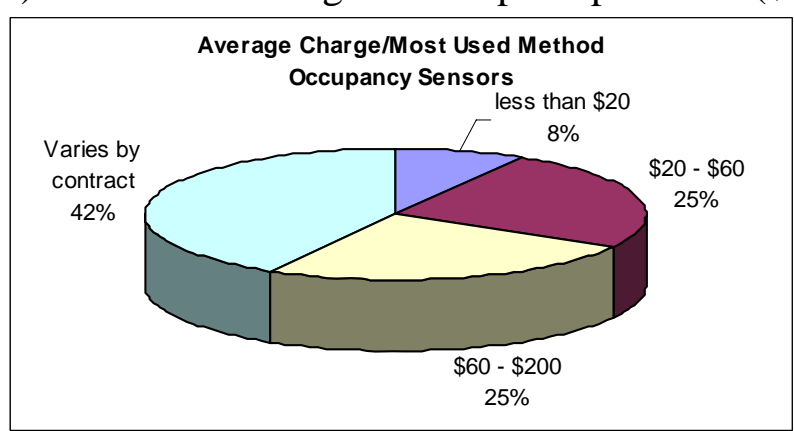

Figure 8: Percentage of installers who report range of average prices for their most frequently used method of charging for occupancy sensors. 


\section{Photosensors}

(9 responses)

Very few installers work with photosensors; only nine returned or filled out this survey. Of those, two said that they did not install any photosensors last year. The most photosensors anyone installed was about 200.

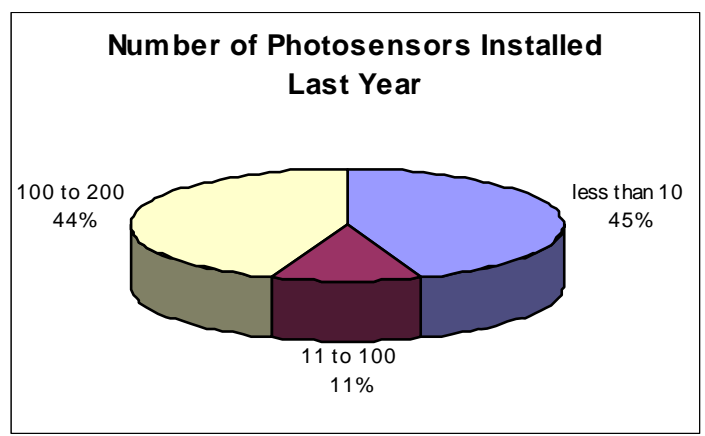

Figure 9: Percentage of installers who cite range of photosensors installed last year.

Unlike occupancy sensors, the installers do not see much additional market potential for photosensors. If no barriers existed, four installers (45\%) felt they could put in fewer than 100 additional photosensors. Three installers, however, did think that they could put in between 100 and 1000 additional sensors.

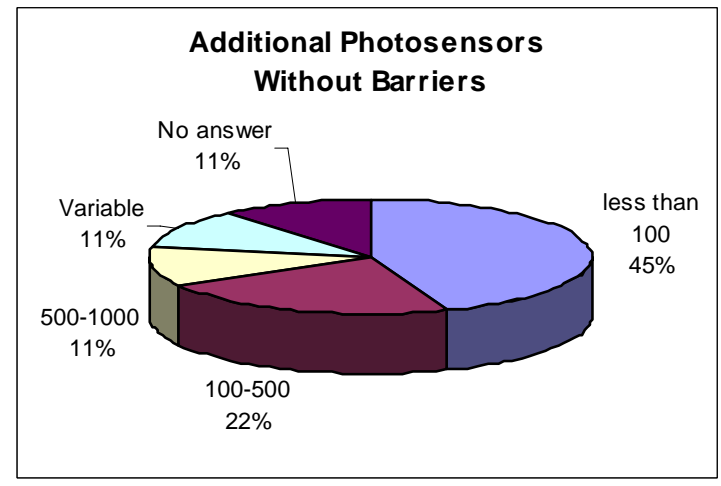

Figure 10: Percentage of installers who cite the range of additional photosensors they could install if no barriers existed.

The two reasons universally cited for resistance to photosensors were lack of customer demand and high hassle factor. However, installers were able install both ceiling- and wall-mounted sensors in less than an hour, and a majority (67\%) were only called back to adjust photosensors 1 to $10 \%$ of the time. One of those who commented on this type of control said, "It is difficult to measure the exact savings, which is one reason we do not propose many of them.” But another commented, "Many simple applications are overlooked where photocontrols are a good fit; it's easy to obtain a good payback. We can install sensors as a standalone project with no other change in technology." Because these facts appear contradictory, it is difficult to ascertain the 
real reasons why photosensors are not more widely used; the sample is too small to draw any conclusions. Perhaps there are simply few installers who have experience with this type of control.

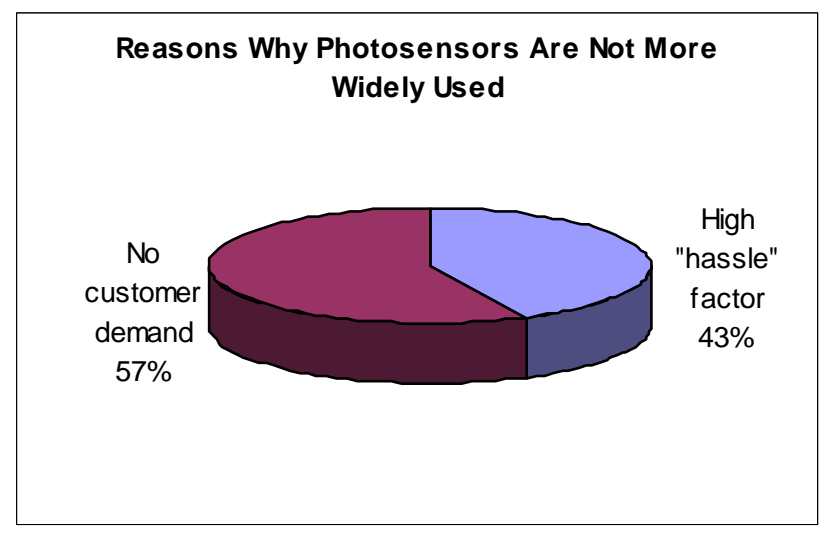

Figure 11: Percentage of installers who cite the importance of a reason for not using photosensors as "high."

\section{Building Automation Systems}

\section{(15 responses)}

Most of the surveyed group install building automation systems. However, they are not using them to control the lighting. Controlling HVAC systems is by far the most common use of automation. More than 73\% of installers say that automation systems control HVAC systems 76 to $100 \%$ of the time. In contrast, $53 \%$ of installers said that lighting is seldom controlled by building automation systems (only $0-25 \%$ of installations). Figure 12 shows how often installers cite each percentage range of installations for each type of building system.

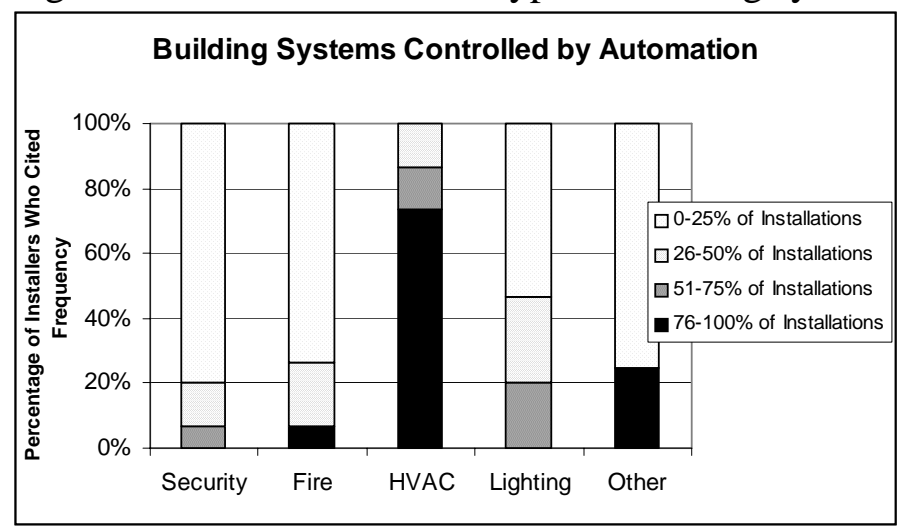

Figure 12: Percentage of installers who cite the frequency with which each building system is controlled by automation systems.

In most cases, the systems they install use twisted pair wire as the method of communication. Sixty percent of installers said they use this method in 76 to $100 \%$ of their systems. Very few installations use existing communication infrastructure as opposed to adding dedicated infrastructure for the automation system. Installers primarily use a proprietary protocol or LAN 
network with Ethernet as the communication protocol in these systems; 53\% of installers use this method 76 to $100 \%$ of the time. BACnet or LonWorks, two other popular protocols were seldom used; in both cases about two-thirds of the installers said they used these protocols in 0 to $25 \%$ of installations.

The installer is most frequently responsible for commissioning the automation system, $46 \%$ of the surveyed group said this was true for 76 to $100 \%$ of their installations. Responsibility for maintaining the system falls to the installer about 51 to $75 \%$ of the time, and to the building's owner 26 to $50 \%$ of the time; about half of the installers gave these responses, as shown in Figures 13 and 14. Two installers said that the manufacturer, which in this case was the same as the installer, was responsible for maintaining the system in all their installations.

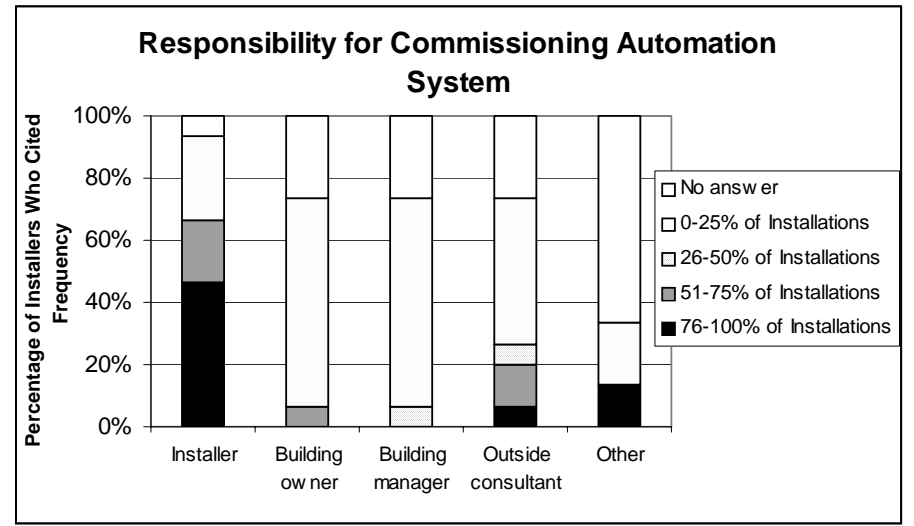

Figure 13: Percentage of installers who cite the frequency with which each category of responsibility commissions building automation systems.

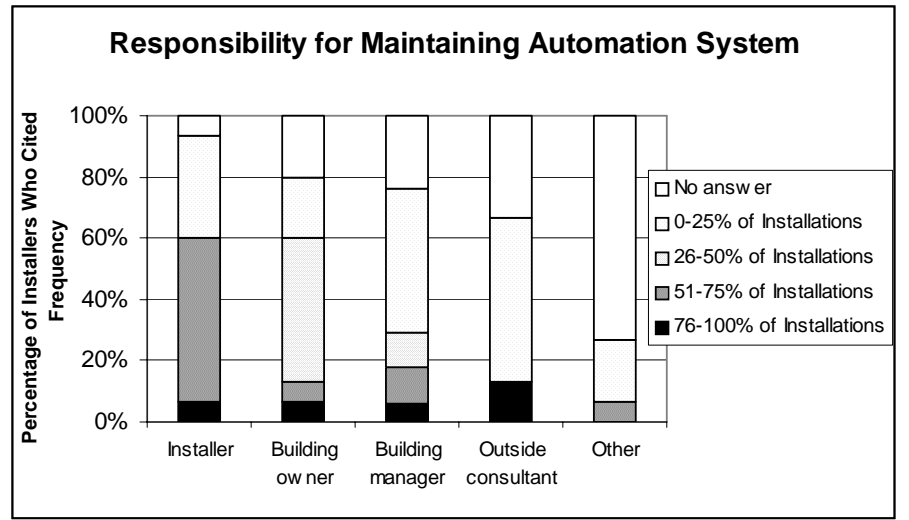

Figure 14: Percentage of installers who cite the frequency with which each category of responsibility maintains building automation systems.

Up to half of their customers ask for and make use of the data that they can obtain from their building automation systems, according to $53 \%$ of the surveyed group. This fact implies that building owners and managers might decide to increase the automation of lighting controls as they get used to seeing the benefits supported by the hard data. 


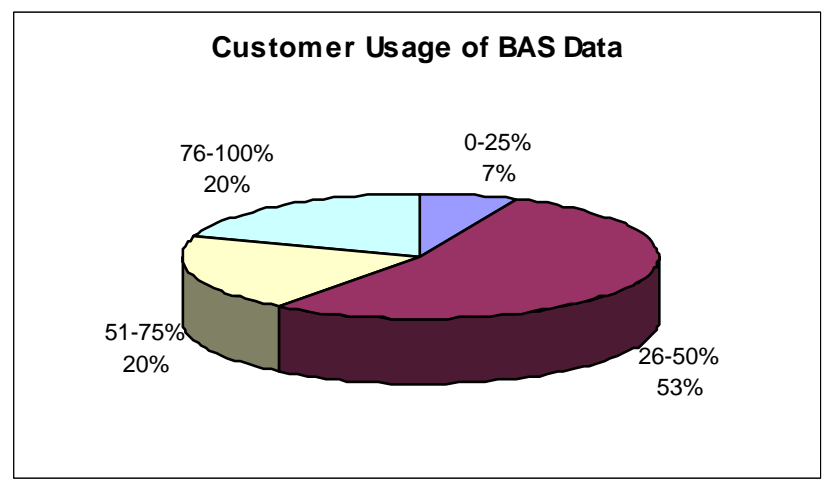

Figure 15: Percentage of installers who report range of customers who ask for and use the data they can obtain from building automation systems.

Again, lack of customer demand is the predominant reason why building automation systems are not more widely used, followed by high initial cost for the customer and low profitability for the installer. These reasons may go far to explain why such systems are seldom used to control lighting; the potential savings from controlling lighting are not enough to justify the investment. One installer commented, "System potential is underutilized."

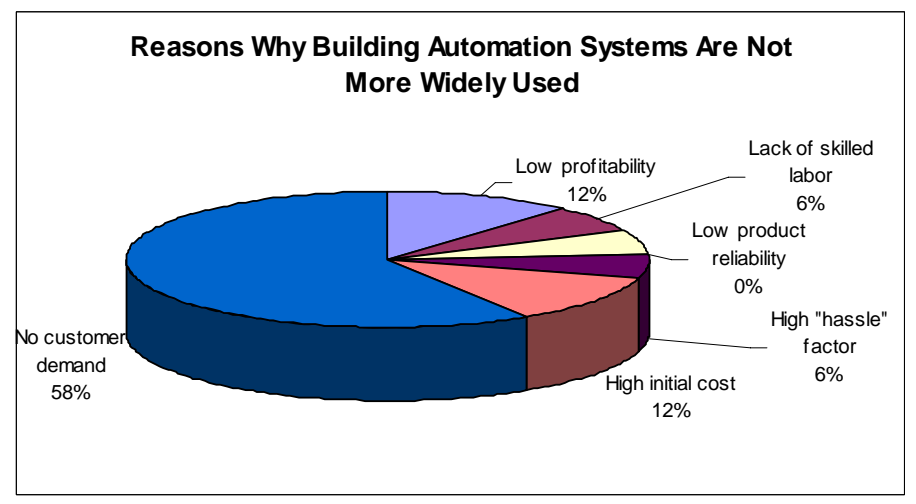

Figure 16: Percentage of installers who cite the importance of a reason for not using building automation systems as "high."

However, 59\% of the surveyed group perceive that customer demand for building automation systems is increasing, whether slowly or rapidly. Another third say that demand is holding steady. The results of this survey indicate that the future is promising for this type of system, but to maximize benefits for lighting loads, customers must be educated in the benefits of controlling their lighting with the systems. 


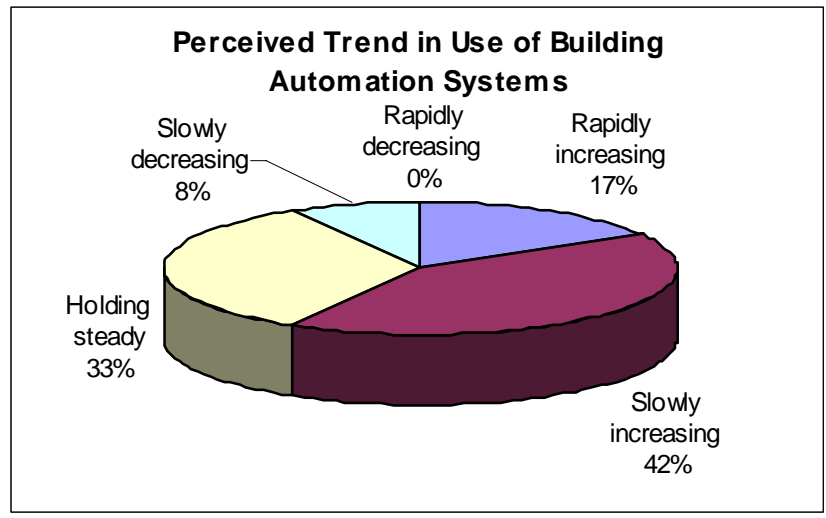

Figure 17: Percentage of installers who perceive trends in the use of building automation systems. 


\section{APPENDIX F: TECHNICAL AND MARKET BARRIERS TO FULL IMPLEMENTATION OF LOAD SHED BALLASTS, OCCUPANCY SENSORS AND PHOTOSENSORS}

The LRC summarized the technologies, barriers and potential opportunities in the following white paper. The LRC also sent a letter to Ron Lewis, Program Manager and at USDOE suggesting names of possible participants in the peer review group. The members of the peer review group are: David Bay (OSRAM SYLVANIA), Jim Benya (Benya Lighting Design), Jim Gallagher (NYS Public Service Commission), Arthur Kressner (ConEdison), Ron Lewis (USDOE), Peter Morante (Northeast Utilities), Dave Peterson (The Wattstopper), Michael Stein (Consultant, Universal Ballast), Edward Yandek (GE Lighting).

\section{Introduction}

The goals of this project are to identify barriers to the penetration of lighting controls into commercial/industrial (C/I) applications that employ fluorescent lamp technologies, and to recommend means for overcoming those barriers. Preliminary recommendations have been developed after discussions with various stakeholders and examinations of published research and patents. Subsequent efforts over the next two years will refine, improve and validate the preliminary recommendations presented here.

There are four categories of barriers that have been identified that include several barriers within each category: lamp-ballast compatibility, component compatibility, installation/commissioning assurance, and marketing. Penetration of lighting controls for fluorescent systems into C/I applications will not be simple or rapid. Indeed, to reach significant penetration into the market a cultural change will have to occur in the lighting industry. This cultural change can be characterized as developing effective communications among all stakeholders in electric energy efficiency and load management, particularly those charged with the responsibility of establishing rational social policies for power generation and transmission.

\section{Lamp - ballast compatibility}

Static, on-off fluorescent lighting systems are the most common technology used in C/I applications. This technology evolved over the middle of the 20th century to become the commodity lighting system because it was more energy efficient and required less maintenance than incandescent technologies. Compatibility among different lamp and ballast manufacturers was another attractive feature of fluorescent systems prior to the 1980s. Virtually any fluorescent lamp could be operated with virtually any magnetic ballast to achieve the same operating performance and life prior to this time.

As a consequence of the energy crisis of the 1970s, significant improvements in the efficacy of fluorescent systems were achieved in the last two decades of the 20th century. The physical properties of fluorescent lamps and ballasts were continually optimized during this era to improve not only efficacy, but also to give them smaller size, better maintenance (lumen depreciation and life), lower mercury content, and higher visual quality (better color rendering and reduced flicker). This evolving sophistication has led to higher and higher expectations of fluorescent system performance in terms of energy efficiency, maintenance, visual satisfaction and, of course, low cost. One down side of this sophistication, however, has been the proliferation of products that provide poor system reliability. Lamps operated on ballasts that do not have optimized starting and operating electrical properties for those lamps will fail 
prematurely, leading to dissatisfaction among specifiers, owners, and facility managers. This was a common situation in the 1980s and early 1990s as the electronic ballast manufacturers learned how to operate lamps produced by the "big three" global manufacturers: GE, Philips and OSRAM. Today, there are still stories of poor performance, but those days are essentially over for static, on-off fluorescent systems, largely because the global manufacturers have taken control of the specifications for ballasts that operate their lamps. This fairly modest but important step reflects the fact that modern fluorescent lamps and ballasts are two parts of the same system. The confidence levels among specifiers, owners, and facility managers for dimming electronic ballasts are not nearly as high as they are for static, on-off fluorescent systems. Anecdotal reports from the field suggest that lamps are failing prematurely when operated on dimming electronic ballasts. Manufacturers have discussed new requirements for lamp seasoning among the, but consensus standards cannot be obtained because the operating characteristics of fluorescent dimming systems are not defined. Presently, a specifier or building facility manager must simply trust the company representative to obtain a reliable fluorescent dimming system. It is important to note that the global manufacturers of lamps and ballasts do not fully understand the basic physics involved in operating fluorescent lamps under dimmed conditions. Coupled with an emphasis on low mercury lamps, there is much more to be understood about fluorescent dimming systems than is currently known. Poor reliability of fluorescent dimming systems, real or imagined, is a significant barrier to the penetration of dimming controls into $\mathrm{C} / \mathrm{I}$ applications, and one that is not being presently addressed by any of the controls manufacturers. This is a problem for control manufacturers because the controls may properly communicate with the ballast, but the ballast may not reliably start and operate the lamp, leading to premature failure or inconsistent operation. An owner or facility manager really won't understand why the system failed, but he/she will swear never do that again!

The global manufacturers are beginning to understand reliable operation of lamps on dimming ballasts, but progress is slow because of low market demand for dimming ballasts. Thus, there is a "Catch 22"; poor demand retards development and retarded development leads to poor demand. Nevertheless, there is some reason for optimism because all three global manufacturers are now taking responsibility for both the lamp and the ballast. And whereas there may not be a complete understanding of the underlying physics in operating lamps under dimmed conditions, it seems likely that the major manufacturers will eventually produce dimming ballasts that work well, if not perfectly, with any commodity fluorescent lamp. If market demand for dimming increased substantially, it seems very likely that reliable fluorescent dimming systems would be produced more quickly. But, again, without that demand, there is little reason to expect commodity fluorescent dimming systems to work reliably in the near future.

\section{Component compatibility}

One barrier that is being effectively addressed by the control manufacturers is the issue of interoperability of control components. On a local level, interoperability means attaining communication to control the ballast. More globally, interoperability concerns communication between individual control devices and, at a higher level, with whole building automation systems (BAS). Lighting controls manufacturers are working successfully to develop products that conform to open communication and command protocols (rather than proprietary systems) for all of the control technologies peripheral to the ballast. Several protocols are being discussed 
and it seems likely that standards will continue to be developed and refined in the next two years that will facilitate utilization of control components in larger systems.

Most lighting control manufacturers offer one or more product types that conform to the popular communications protocols and can be integrated into BASs. LONworks and BACnet have the most support from the lighting controls manufacturers. Devices range from protocol translators to control devices fitted to support the protocol directly. Proprietary protocols from Johnson Controls, Honeywell and Modicon are also supported by the lighting controls manufacturers, but to a lesser extent. At the physical communication level of many of these protocols is the Ethernet protocol. The widespread use and success of Ethernet for computer local area networks (LANs) has undoubtedly aided in establishing Ethernet for building process controls and has helped to lower the cost of hardware components. Other, higher-level communication network protocols from the computer industry are being used and reconfigured for BASs. For example, Microsoft's OPC (Object Linking and Embedding for Process Control) protocol is based on Microsoft's earlier and successful OLE network protocol and it leverages the previously established and widely accepted Windows client/server technology. A review of product literature and advertisements in trade magazines reveals lighting control products offering Internet connectivity, web browser interfaces, and remote monitoring technologies. Therefore, it is evident that controls manufacturers are quick to incorporate the latest crossover technology from the computer industry into their product offerings.

There have also been some recent advances at the more local level of control between individual control devices and fluorescent lamp ballasts, although progress here seems slower than for the higher-level network controls. Direct digital control (DDC) was introduced to the controls industry about 25 years ago and has greatly improved the performance and functionality of building systems. Digital controls operate security, HVAC, fire alarm, and many other specialty systems in modern buildings, yet only in the last year or so has any direct digital control ballast been available on the US market. The lack of a robust and reliable control method with fluorescent lamp ballasts can partly explain why lighting systems are not routinely connected to building energy management systems, except at the electrical panel for simple, on/off control of large areas. Currently, however, there is much activity in the lighting industry regarding digital control interface protocols for fluorescent lamp ballasts. The ESI Superdim DDC protocol and ballast is presently the only system available in the US market that offers one-way communication with ballasts for both on/off and dimming control. The market share of ESI ballasts, however, is very small. DALI is a current protocol used in Europe that has recently been accepted as an ANSI standard. Although no products that incorporate DALI yet exist on the US market, the major fluorescent lamp and ballast manufacturers have shown support for incorporating the standard protocol in future products, and the leading ballast manufacturer in Europe, Tridonic, has plans to begin marketing a DALI-equipped ballast in the US.

DDCs for ballasts will offer many advantages over the existing hardware available for lighting control by providing reliable, consistent control and simplified installation. More importantly, a uniform implementation of a protocol based on a widely accepted standard can increase market penetration of control technologies by raising people's confidence in using products that bear a seal of conformity. A widely accepted dimming control protocol such as DALI could serve these purposes.

One significant problem, however, is that these controls manufacturers are not effectively discussing the issues of controls outside their own community. There is no discussion with the ballast manufacturers on reliable operation of fluorescent lamps and ballasts. There is little if any 
discussion with installers, and, as will be emphasized later, there is no discussion with utilities and government agencies charged with the responsibility of providing reliable electric power to the public. Without these discussions, there will be little demand for control systems no matter how robust the controls protocols. Effective interaction with all stakeholders, particularly with the utility industry and related government agencies, is essential to creating market demand and thus overcoming the major barrier to the penetration of fluorescent lighting controls into $\mathrm{C} / \mathrm{I}$ applications.

\section{Installation/commissioning assurance}

The ability to install and commission controls ranges from very good to nonexistent. The reliable installation of on-off controls into C/I applications is simple and can be accomplished easily by trained technicians. Time controls, occupancy sensors, and manual switching are reliable and easy to install. There is not, however, significant market demand for on-off lighting controls beyond simple switches in C/I applications. This barrier will be discussed in the next section. Dimming systems, however, are both expensive to acquire and difficult to install. Commissioning photosensors is prohibitively time-consuming and, consequently, photosensors are almost never installed. Recent development by the Lighting Research Center of a simplifiedcommissioning photosensor to take advantage of daylight may, however, overcome this barrier. Even if this photosensor technology becomes commercially available, there are still nagging small barriers to installing and commissioning lighting controls, particularly dimming systems. These barriers include wiring uncertainty, identification and zoning issues for groups of ballasts, the need for manual overrides, and the training of installation personnel.

As mentioned previously, DDC ballasts are essentially non-existent in the United States.

Therefore, the only options today for dimming control are either a $0-10 \mathrm{~V}$ analog control interface or a line voltage phase-chop signal interface, neither of which conform to any open standards. 0$10 \mathrm{~V}$ control is by far the most common for dimming ballasts. At first glance, the $0-10 \mathrm{~V}$ control appears to follow the newly introduced standard for 0-10V control in the entertainment industry (ANSI E1.3-2000). Closer inspection reveals, however, that the 0-10V control used with fluorescent lamp ballasts in C/I applications differs significantly from the entertainment industry standard. In fact, the ANSI E1.3 standard draws attention to this point.

Even if dimming fluorescent lamp ballasts did conform to this particular standard, problems would remain. One problem is that the standard does not detail the functional relationship between the control signal level and the light output level of the ballast. Therefore, a consistent dimming level across different ballast types is not assured. For example, a $5 \mathrm{~V}$ signal from one ballast manufacturer may result in a $30 \%$ dim level, while that same $5 \mathrm{~V}$ signal may result in a $50 \%$ dim level for another manufacturer's ballast. Controls must be field-calibrated to work consistently in many cases, and mixing ballasts from different manufacturers under the same controller will likely result in different light levels.

Perhaps a bigger problem with the $0-10 \mathrm{~V}$-control scheme as implemented in ballasts today is the lack of application guidelines that result in poor field performance. These problems arise from the fact that $0-10 \mathrm{~V}$ signals are susceptible to electrical interference, yet little or no information is given to installers concerning proper cabling practices. Examples of wiring installation practices that lead to performance problems include lamp flickering, lamp brightness striations, and lamp extinguishing at low dim levels. These problems are the result of running the control wires in close proximity to power lines and/or lamp leads, excessive control wire lengths, and reversing polarity of one or more control wires when operated on parallel circuits. The wide skepticism 
among lighting specifiers about fluorescent dimming systems could be reduced by educating installers about the special wiring requirements for dimming ballasts, providing clear and readily available literature on system installation, and improving product design to eliminate these field problems.

In addition to hardware and wiring problems, there are logistical problems when installing and commissioning large installations with dimming ballasts. For conventional analog input ballasts, the task of zoning must be done when the ballasts are installed. In other words, the zoning is "hard-wired" for the life of the lighting system. This makes the zoning difficult to change should the space be renovated or used for a different purpose. For the newer DDC ballasts appearing on the market, zoning is done after installation as part of the commissioning process. This makes rezoning much easier as the space functions change, but zoning is still a laborious task because each ballast location must be manually identified to the controller. While different communication protocols offer different methods of assigning addresses, all methods involve time-consuming human interactions that increase cost as well as the potential for problems. Finally, attention must be paid to insure that all systems are equipped with manual overrides for unpredictable events. Occupants want to have control over their lighting during unusual work schedules or events. However, engineers and facility managers seem hesitant to provide manual overrides, because it is assumed that people will use them too often and energy savings will be lost. In general, and as discussed below, automatic controls should be used to turn lights off when no one occupies the space. Manual controls should be used to turn lights on when the space is occupied. These principles consistently meet the demands for occupant satisfaction and energy savings. Automatic dimming during occupancy is still an area of uncertainty, because no principles have been established for occupant acceptance of the amount of dimming and the rate of dimming. This uncertainty further undermines the confidence of engineers and facility managers in dimming systems, but could be addressed with a program of human factors research.

\section{Marketing}

First cost remains a barrier for lighting controls. Often the person or department that pays for the installation of lighting equipment does not receive the benefit from the reduced electrical bills. Also, in existing buildings, managers do not want the "hassle factor" of implementing a change. First cost and hassle factor have been overcome for other energy-efficient lighting technologies with demand-side management (DSM) incentive programs, market transformation program procurement, shared-savings marketing by energy service companies (ESCOs), and new code requirements. Combinations of these approaches increase market demand for energy-efficient lighting products, leading to lower product cost and market transformation. Products such as T8 lamps, electronic ballasts, and LED exit signs are examples of successful lighting market transformations. Lighting controls, however, have yet to benefit substantially from these market forces because of the difficulty in predicting the energy savings resulting from control use. DSM program developers and evaluators, code developers, and ESCOs do not have adequate information to predict expected savings by control measures. They need to know, for example, when and for how long the control switches off or dims the lights. There are case study examples, but robust, statistically significant control use factors need to be developed that will afford the program developers confidence that a specific control installation will deliver a specific energy savings. With this confidence, controls will more likely find their way into lighting incentive programs, code provisions, and ESCO strategies. 
The lighting industry is a fragmented collection of component manufacturers. No sector of the lighting community, private, public, or non-profit, is the recognized champion for lighting. Each agency works very hard to communicate the value of its own product or service. However, that communication is most often to other lighting component agents. Consequently, the lighting industry members do not and cannot communicate the value of lighting to specific markets such as auto manufacturers, health care professionals, sports and recreation, community development, etc. Remarkably, the industry remains largely uneducated about the perceived value of lighting to these sectors, focusing on market share in traditional markets rather than growing new markets. This is a significant cultural barrier to the penetration of fluorescent lighting controls into the $\mathrm{C} / \mathrm{I}$ market.

In particular, lighting controls manufacturers fail to communicate the value of lighting of their products. The lighting controls manufacturers are insulated not only from the public, but also from other sectors of the lighting industry (e.g., lamp and ballast manufacturers) and from specifiers. Lutron is one notable exception. They have established themselves not as a component or even system manufacturer, but, rather, they have successfully reached the specification community by providing assurances that their lighting controls will work and will deliver value to their customers. The majority of the lighting controls manufacturers remain focused on components, which drives down price. With little profit, there is little innovation or marketing. Consequently, there has been little penetration of lighting controls into the $\mathrm{C} / \mathrm{I}$ market.

The overwhelming sentiment among the installers of controls technologies, other than those produced by Lutron, is that they can make controls work, but there is no market demand. They believe that some agency needs to repeatedly and effectively communicate the value of lighting controls to building owners and facility managers. This sentiment is essentially correct, but misses perhaps the largest opportunity for fluorescent lighting controls, both static and dimming. To understand this potential, one must first ask the basic question: "What is the value of lighting controls?” Lutron has identified a need for lighting controls in multi-use spaces, particularly conference rooms, and fulfilled it in the marketplace. There is indeed a real need for controls in these spaces. Often the conference room is a space designed to create a positive impression with customers and clients and perhaps, dynamic lighting during a meeting, meal or formal presentation. Most often AV needs in a conference or meeting room require multiple lighting levels. Lutron has focused on this market and has established a reputation for reliable solutions, albeit at a cost well in excess of the components involved.

There seems to be little or no need, however, for multiple lighting scenarios in the largest C/I spaces, private and open-plan work areas. Tasks performed by office workers do not demand sophisticated lighting controls, with one clear exception: control of sunlight and, to a lesser extent, daylight. A recent study of private offices showed that workers rarely used dimmers on a regular basis, although the majority of workers did prefer to set electric light levels in daylight spaces below those found in interior offices (approx. $500 \mathrm{~lx}$ ). Thus, even though building occupants may perceive individual lighting control to be of some value, that perception is not a major driver for widespread implementation of control technologies.

The main driver for lighting controls is most often assumed to be energy savings. However, there is always the concern that controls may compromise the productivity of the workers in controlled spaces. Common wisdom for saving energy, therefore, requires turning lights off when the space is unoccupied. Certainly, on-off strategies such as time clocks, occupancy sensors, and even manual switching are effective and commonly employed strategies for saving energy. Indeed, 
there is no technical barrier to implementing these tried-and-true techniques except establishing awareness on the part of owners considering a lighting retrofit or renovation. Again, the installers of lighting controls identified this failure to communicate the value of (static) lighting controls to the important sectors of the building industry as the major barrier to their penetration into the C/I market.

To ensure against disappointment in this communication of the value of lighting controls, however, there are some important principles to be considered for simple on-off strategies that have emerged from research into lighting controls. First, the goal of static controls is to turn lights off when no one is in the space. This strategy cannot annoy the occupant or compromise the worker's productivity in the space because the space is unoccupied. Because no technology is completely foolproof, however, it is essential that there be a local, manual override to any automatic strategy to turn the light "off.” For example, if a worker is working late one evening and the automatic time clock initiates an "off" command, the worker must be able to manually override the automatic command in the work space and in areas needed for exiting the work space. Again, these simple principles and the technologies for achieving energy savings are well established; there are no technical or human barriers to turning the lights off in unoccupied spaces to achieve energy savings. There is simply "a failure to communicate" that static, on-off lighting control technologies that turn lights off in unoccupied spaces are reliable and cost effective.

It is much more difficult; however, to garner energy savings in occupied spaces. People do not like lights to go on or off unpredictably. Although some pilot data are available, little is known about the acceptance of automatic dimming in occupied spaces. There are limits to the amount of dimming and the rate of dimming that occupants will accept, but again, no guiding principles have been established. A program of human factors research could provide valuable information. Daylight dimming is the most obvious strategy for dimming in occupied spaces. Ideally, the natural light offsets electric light to save energy and the dimming goes largely unnoticed by the occupant. However, daylight dimming represents several significant barriers to energy savings. Most notably, it is difficult or impossible to commission commercially available photocells. Recent research and development at the Lighting Research Center offers some promise for a simplified-commissioning technology. Nevertheless, the energy saved by photocells is limited largely to windowed spaces, and these spaces represent only about $20 \%$ of the commercial building stock. The amount of energy saved by dimming electric lighting in daylit spaces is also small. One study suggests that the incremental energy savings from dimming over simple switching in daylit spaces may be only $10 \%$. Further, the level of dimming is not usually proportional to the amount of power reduction in the lighting system. Rapid-start fluorescent lighting systems are usually employed with dimming systems because filament heat must be provided to the lamp during operation. Thus, the ballast continues to use some fixed amount of power at every level of dimming. Finally, the price of electricity is a barrier to energy savings. Electric energy is inexpensive relative to the incremental cost of dimming ballasts. Presently, dimming ballasts cost $100 \%$ more than static ballasts. All of these economic factors lead to a very long return on investment (ROI) for daylight dimming, usually more than a decade. Other strategies such as lumen depreciation and manual dimming for energy savings are also costprohibitive, again, not just in terms of first costs but also in terms of commissioning costs. Finally, the effects of dimming on lamp life are not, as yet, well understood by the lamp-ballast manufacturers. Anecdotal reports of premature lamp failures and other problems such as flicker 
and brightness striations permeate the industry and create a perception of high risk for fluorescent dimming systems.

These issues paint a very bleak picture for fluorescent dimming systems. There is little perceived value of dimming by the occupants, and there is no cost incentive for widespread investment. The simple conclusion is that the barriers are too high to be overcome to have a significant effect on energy efficiency. However, there is an important angle that has not been addressed by the lighting industry, reflecting again their insular behavior. Some states and regions in the United States are struggling to meet the growing demand for electric power at traditionally low prices. Utilities must build power capacity for peak demand, not for average demand. In California for example, peak demand coupled with inadequate supply and price caps for exceedingly high energy costs have led to sporadic blackouts and high wholesale energy costs. Dimming fluorescent systems used in conjunction with utility communication systems offer a potentially valuable means of keeping businesses operating during periods of peak demand while maintaining relatively low energy prices and, most significantly, while avoiding construction of new power plants and transmission corridors.

Central to understanding the potential value of dimming systems is an examination of the real costs of electric energy. The cost of electric energy usage for lighting can be measured in several ways, and each measurement differentially affects the value, or potential value, of lighting controls. To understand the value of lighting controls, it is necessary to delineate the costs and the benefits for all of the stakeholders when maximizing the use of electric energy with lighting controls.

The most obvious measure is the cost of the electricity used to operate the lighting system. The cost of electricity is measured in $\$ / \mathrm{kWh}$. Depending upon the retail power supplier and the type of $\mathrm{C} / \mathrm{I}$ customer, this rate will typically vary between $\$ 0.06 / \mathrm{kWh}$ and $\$ 0.12 / \mathrm{kWh}$. Both the amount of power needed to operate the lighting and the time the lights are operated will affect the annual cost of operating the lighting. Static, on-off lighting controls can reduce both the time of operation and the number of systems operating. Dimming (step or continuous) controls can reduce the power required to operate the lighting system.

Another common measure is based upon the building peak energy use during a billing period. These peak demand charges are presently used to support the cost of installing and maintaining power infrastructure to the C/I customer. Before deregulation of the utility industry, peak demand charges also included the cost of generating and supplying power during peak periods of operation, but this is no longer the case. Static, on-off lighting controls can reduce peak demand by turning off unnecessary lighting. Dimming controls can reduce power demand without turning the lights completely off.

Both of these measurements are in place in most $\mathrm{C} / \mathrm{I}$ applications and are reasonably well understood by all stakeholders when performing economic analyses. Depending on the cost of energy and peak demand, reflected in the utility billing structure, the C/I customer will or will not select lighting controls for installation. It is fair to say, however, that these measures have not been major drivers to the penetration of lighting controls into C/I applications. Less than $2 \%$ of $\mathrm{C} / \mathrm{I}$ applications have more than simple manual switching for lighting control.

Although the cost of electric energy and peak demand are important, these are not the only measures that can affect the value of lighting controls. The cost of purchasing power can vary throughout the day and throughout the year. The cost of power is strictly a matter of supply and demand. As demand increases and base load is exhausted, the cost of adding additional electric power increases, sometimes exponentially. It has been estimated by the New York State Public 
Service Commission that during the hottest day of the year 2000, the last 100MW of power demanded in NYC cost ratepayers $\$ 100 \mathrm{M}$. This is $\$ 1 / \mathrm{W}$ ! If that peak demand for power could be reduced or if the real cost of using power during the peak was passed on to the customers that use it, the yearly average energy-use rate paid to electric utilities by C/I customers could be significantly reduced. Unfortunately, this is not the case, because there is no strong incentive to customers to eliminate wasteful energy use during peak periods of system demand. Moreover, as wasted lighting energy increases, the need for additional power generation increases to meet the ever-growing peak demand. To pay for this added capacity, the average electricity rates increase, creating increased operating costs for all C/I customers and a net economic downturn. Lighting controls offer a significant potential benefit to ratepayers, and to the economy, if two things occur. First, the peak system demand must be reduced reliably and predictably. Second, customers demanding power during peak periods should actually pay for the added costs of supplying that power, either in the bidding process or for construction of new power-plants. It is not necessarily obvious, however, how guaranteed peak load reductions could be obtained in $\mathrm{C} / \mathrm{I}$ applications. Certainly no employer would want to turn off computers, copiers, or even lights in most spaces. Few would want to increase ambient temperatures in air-conditioned spaces during hot summer days when peak demand is greatest. However, it is possible to dim the lighting in these spaces without significantly compromising the productivity of employees. In fact, no other technology that depends upon electricity is so readily amenable to load reductions. Not only will people accept some dimming, the technology for dimming is largely in place. Moreover, utility real-time pricing communication systems have been developed and are already being used in some applications. Neither the dimming nor the communication systems have been optimized and integrated.

Table 1 provides several dimming fluorescent lighting control scenarios using current pricing structures for electric energy and power demand, together with the value of avoiding expensive power and costs of adding new generating capacity. For example, consider a New York City office with luminaires containing two 32-watt lamps that are dimmed by $30 \%$ during peak demand. The price of energy is $\$ 0.10 / \mathrm{kWh}$ and demand charges are $\$ 18.00 / \mathrm{kW} / \mathrm{month}$. Demand charge savings would be $\$ 4.08$ per year and energy savings are $\$ 1.62$ per year for that dimmed luminaire, plus the value of the system demand reduction is $\$ 1.81$. If the lamps are operated 50 hours a week and are dimmed 33\% of this time, the ten-year present value of a load-shedding ballast would be $\$ 55.34$. The cost of a dimming electronic ballast is currently between $\$ 50.00$ and $\$ 60.00$, while a static electronic ballast is about $\$ 20.00$. Thus, the incremental cost of a dimming electronic ballast is between $\$ 30.00$ and $\$ 40.00$. In this example the payback for dimming electronic ballasts is at least five years. It is important to note, however, that the estimated incremental cost to manufacturers for producing a load-shedding electronic ballast that would dim to just $30 \%$ is currently between $\$ 0$ and $\$ 10$. This would significantly shorten payback periods to between six months and two years.

Table 1 shows that there is a value to ratepayers for not using electric energy for lighting. Presently, however, there are several barriers to capturing that value. First, the cost of dimming is prohibitive for several reasons. The first cost for all load-shedding control technologies is high, especially for fluorescent dimming systems. Architectural dimming ballasts cost $100 \%$ more (or higher) than static, on-off ballasts. Installation and commissioning costs are also quite high, as is the perceived increased risk for poor performance due to lamp-ballast compatibility. Second, presently there is no infrastructure (hardware and software) that guarantees load shedding from lighting controls when it is needed. In the late 1980s, guaranteed energy savings were ensured 
through utility rebate programs that removed less efficient (T12/magnetic ballast) lighting systems for more efficient (T8/electronic ballast) lighting systems. Without guaranteed savings from lighting controls, there is little chance for significant market penetration of lighting controls systems. New communication technologies need to be developed and coordinated with dimming fluorescent lighting systems. Third, the people who would pay for technologies that garner energy savings and load reduction are often not the ones who would benefit from those investments. High demand during peak hours is averaged over all ratepayers, rather than assigned to those who actually create the high demand. New methods of billing, such as realtime pricing, need to be implemented before there is a significant cost incentive for load reduction and energy savings. Fourth, utility rate structures only pay for load offset from current generation and transmission, not for the potential for new power plant and transmission corridor planning and construction. The US Department of Energy's Annual Energy Outlook forecasts the need for at least 1300 new power plants nationwide by 2020, about half of which could be avoided by increased energy efficiency. Yet there is no way to capture the value of lighting controls when offsetting construction of new power generation and transmission systems. Clearly then, a combination of technical and policy barriers must be overcome before the value of lighting controls can be captured. 
Table1: Dimming fluorescent lighting control scenarios for electric energy and power demand

\begin{tabular}{|c|c|c|c|c|c|c|c|c|c|c|c|c|c|c|}
\hline & & & & & & & & & & & $\begin{array}{l}10 \text { year } \\
\text { present } \\
\text { value }\end{array}$ & $\begin{array}{l}10 \text { year } \\
\text { present } \\
\text { value }\end{array}$ & $\begin{array}{l}10 \text { year } \\
\text { present } \\
\text { value }\end{array}$ & $\begin{array}{l}10 \text { year } \\
\text { present } \\
\text { value }\end{array}$ \\
\hline measure & power & occupancy & $\begin{array}{l}\text { dim on } \\
\text { demand }\end{array}$ & $\begin{array}{l}\text { measure } \\
\text { penetration }\end{array}$ & $\begin{array}{c}\text { measure } \\
\text { savings }\end{array}$ & $\begin{array}{l}\text { energy } \\
\text { rate }\end{array}$ & $\begin{array}{l}\text { demand } \\
\text { charge }\end{array}$ & $\begin{array}{l}\text { demand } \\
\text { savings }\end{array}$ & $\begin{array}{l}\text { energy } \\
\text { savings }\end{array}$ & $\begin{array}{l}\text { turn off } \\
\text { when } \\
\text { needed }\end{array}$ & $\begin{array}{l}\text { enery } \\
\text { savings PV }\end{array}$ & $\begin{array}{l}\text { demand } \\
\text { savings PV }\end{array}$ & $\begin{array}{l}\text { turn off } \\
\text { when } \\
\text { needed }\end{array}$ & $\begin{array}{l}\text { total } 10 \\
\text { year } \\
\text { PV/6\%int }\end{array}$ \\
\hline 2 lamp/ 20\% dimming & 63 & 50 & 20 & 33 & 12.6 & 0.10 & 18.00 & 2.72 & 1.08 & 1.21 & 7.96 & 20.03 & 8.90 & 36.89 \\
\hline 2 lamp/ 20\% dimming & 63 & 50 & 20 & 33 & 12.6 & 0.10 & 13.00 & 1.97 & 1.08 & 1.21 & 7.96 & 14.47 & 8.90 & 31.33 \\
\hline 3 lamp/ 20\% dimming & 93 & 50 & 20 & 33 & 18.6 & 0.10 & 18.00 & 4.02 & 1.60 & 1.79 & 11.75 & 29.57 & 13.14 & 54.46 \\
\hline 3 lamp/ 20\% dimming & 93 & 50 & 20 & 33 & 18.6 & 0.10 & 13.00 & 2.90 & 1.60 & 1.79 & 11.75 & 21.36 & 13.14 & 46.24 \\
\hline 2 lamp/ 30\% dimming & 63 & 50 & 30 & 33 & 18.9 & 0.10 & 18.00 & 4.08 & 1.62 & 1.81 & 11.94 & 30.05 & 13.35 & 55.34 \\
\hline 3 lamp/ 50\% dimming & 93 & 50 & 50 & 33 & 46.5 & 0.10 & 18.00 & 10.04 & 3.99 & 4.46 & 29.36 & 73.92 & 32.86 & 136.14 \\
\hline 2 lamp/ 20\% dimming & 63 & 50 & 20 & 33 & 12.6 & 0.08 & 18.00 & 2.72 & 0.86 & 1.21 & 6.37 & 20.03 & 8.90 & 35.30 \\
\hline 2 lamp/ 20\% dimming & 63 & 50 & 20 & 33 & 12.6 & 0.08 & 13.00 & 1.97 & 0.86 & 1.21 & 6.37 & 14.47 & 8.90 & 29.73 \\
\hline 3 lamp/ 20\% dimming & 93 & 50 & 20 & 33 & 18.6 & 0.08 & 18.00 & 4.02 & 1.28 & 1.79 & 9.40 & 29.57 & 13.14 & 52.11 \\
\hline 3 lamp/ 20\% dimming & 93 & 50 & 20 & 33 & 18.6 & 0.08 & 13.00 & 2.90 & 1.28 & 1.79 & 9.40 & 21.36 & 13.14 & 43.89 \\
\hline 2 lamp/ 30\% dimming & 63 & 50 & 30 & 33 & 18.9 & 0.08 & 18.00 & 4.08 & 1.30 & 1.81 & 9.55 & 30.05 & 13.35 & 52.95 \\
\hline 3 lamp/ 50\% dimming & 93 & 50 & 50 & 33 & 46.5 & 0.08 & 18.00 & 10.04 & 3.19 & 4.46 & 23.49 & 73.92 & 32.86 & 130.27 \\
\hline 2 lamp/ 20\% dimming & 63 & 50 & 20 & 33 & 12.6 & 0.12 & 18.00 & 2.72 & 1.30 & 1.21 & 9.55 & 20.03 & 8.90 & 38.48 \\
\hline
\end{tabular}




\begin{tabular}{|c|c|c|c|c|c|c|c|c|c|c|c|c|c|c|}
\hline 2 lamp/ 20\% dimming & 63 & 50 & 20 & 33 & 12.6 & 0.12 & 13.00 & 1.97 & 1.30 & 1.21 & 9.55 & 14.47 & 8.90 & 32.92 \\
\hline 3 lamp/ 20\% dimming & 93 & 50 & 20 & 33 & 18.6 & 0.12 & 18.00 & 4.02 & 1.92 & 1.79 & 14.09 & 29.57 & 13.14 & 56.81 \\
\hline 3 lamp/ 20\% dimming & 93 & 50 & 20 & 33 & 18.6 & 0.12 & 13.00 & 2.90 & 1.92 & 1.79 & 14.09 & 21.36 & 13.14 & 48.59 \\
\hline 2 lamp/ 30\% dimming & 63 & 50 & 30 & 33 & 18.9 & 0.12 & 18.00 & 4.08 & 1.95 & 1.81 & 14.32 & 30.05 & 13.35 & 57.72 \\
\hline 3 lamp/ 50\% dimming & 93 & 50 & 50 & 33 & 46.5 & 0.12 & 18.00 & 10.04 & 4.79 & 4.46 & 35.24 & 73.92 & 32.86 & 142.02 \\
\hline
\end{tabular}

Assumptions: controls reduce demand at times of peak demand of both the building and the system.

Assumption: $\$ 8 / \mathrm{kW} / \mathrm{mo}$ given to shed load when needed to offset new plant construction and current power at expensive rates. 


\section{Preliminary Recommendations}

The following recommendations are ordered in terms of the relative effort needed to overcome identified barriers to the penetration of lighting controls into C/I markets. The first two recommendations are focused on existing, on-off lighting controls that have been demonstrated to be cost effective and reliable. These two recommendations can be implemented immediately. The remaining recommendations represent significant investment of resources to support widespread penetration of fluorescent dimming systems. As noted in this document, there are many significant barriers to the use of dimming fluorescent systems in C/I applications. These barriers will not be overcome using traditional economic models, and should not be attempted without a much stronger financial incentive. A new economic model that incorporates the cost to society for building new power generation infrastructure does offer a realistic financial foundation for overcoming the barriers to fluorescent dimming systems.

1. Support data collection that provides fixed numbers for expected energy savings and load reductions for various static on-off lighting control technologies in various applications.

2. Support programs that overcome the inertia to first costs to static on-off lighting controls, working with building owners, professional societies, code developers, ESCOs, and conservation program developers.

3. Support functional and substantive means of communication among lamp-ballast manufacturers, controls manufacturers, controls installers, building automation system manufacturers, utilities, and government agencies charged with maintaining public benefit. The economics of load management needs to be widely understood by all stakeholders in lighting controls. The first task would be to identify key decision-makers in these different sectors, and then to provide incentives for those disparate groups to cooperatively work together. These incentives could be in the form of demonstration projects specifically designed to cooperatively link the key sectors. Incentives could also include support for cooperative technology development. In particular, two development projects are immediately envisioned:

3a. Develop an inexpensive, highly efficacious dimming ballast that responds to loadshed signals from the utility.

3b. Support development of open communications systems that link utility need for shedding load to building fluorescent dimming systems. This implies a standard ballastutility interface for load shedding.

4. Support a human factors research program that establishes the principles for dimming levels and dimming rates in occupied spaces.

5. Develop a long-term labeling and testing program that provides assurances to all stakeholders that (a) the dimming ballast reliably operates the lamp under all conditions, (b) all control system components work reliably together, (c) installation and commissioning of dimming systems are reliable, (d) articulates the value of lighting controls for energy and cost savings that reflect their social value by ensuring an inexpensive and reliable supply of electrical power. 


\title{
APPendix G: PeER ReVIEW group MeEting Minutes
}

\section{Minutes of the Peer Review Group Meeting Revised February 2002}

\author{
Meeting date: December 12, 2001 \\ Location: $\quad$ Lighting Research Center, Troy, NY \\ Time: Noon to $4: 00 \mathrm{pm}$ \\ Participants: David Bay (OSRAM SYLVANIA) \\ Jim Benya (Benya Lighting Design) \\ Jennifer Brons (LRC) \\ Andrew Bierman (LRC) \\ Mariana Figueiro (LRC) \\ Claudia Hunter (LRC) \\ Russ Leslie (LRC) \\ Ron Lewis (USDOE) - unavoidably late \\ Peter Morante (Northeast Utilities) \\ Dave Peterson (WattStopper) \\ Steve Purdy (independent consultant) - unavoidably late \\ Mark Rea (LRC) \\ Mike Stein (consultant - Universal Ballast)
}

The meeting started at 12:30 pm. Mark Rea presented the agenda (see attached) for the afternoon and set the goals for the meeting, which included achieving consensus on a set of priorities for the project, setting a date for the round table to be held in February, and developing a list of invitees for that meeting.

All the participants introduced themselves. Mark Rea summarized the barriers of lighting control usage and the recommendations described in the white paper. A copy of the presentation is attached.

Russ Leslie opened the discussion for comments and suggestions. He clarified that the goal of the meeting was to define what to do - not how to do it.

Four members were asked to give their perspectives on issues that would complement the information included in the white paper.

Jim Benya (Understanding the triad of building operations: owner, manager, and tenant):

- $\quad$ Energy-efficient retrofit: There is a triangle among the building owner, manager and tenant. Each has a different financial budget, and the largest barrier is convincing each that it is worth spending additional resources on implementing an energy management strategy. The owner is concerned about the investment of capital, the manager is concerned about the effective operation of the building, and the tenant is concerned about the cost of the lease and the electric bill. One building in LA, for example, has been losing about \$2 million per 
year because the owner, manager and user each have different interests. It is important to find mechanisms that address everyone in that triangle.

Mike Stein (Updates on status of standards):

- Update on standards pertaining to dimming:

- As fluorescent lamps dim, their behavior changes. Europeans have standardized the characteristics of lamp filaments for linear T5 and most of the single ended lamps. A dimming standard proposal for these lamps based on the "Sum of Squares" method developed at IEC workshops will be issued shortly. ANSI C78 committee will be adopting these IEC standards as ANSI standards.

- $\quad$ The US manufacturers would like to standardize filaments for T8 lamps, but the design and manufacturing equipment for T8 lamps in this country is very different, thus a standard may not be viable. When dimming lowers the light level below the $50 \%$ threshold, filament characteristics affect performance. Resolution to this problem appears distant.

- $\quad$ IEC and ANSI have analog $(0-10 \mathrm{v})$ dimming ballast standards. The market is heading toward digital control (DALI). Europeans have been working on this standard for 10 years. The IEC standard is about to be approved, and ANSI will probably adopt this IEC standard.

- Controls: Nothing has been written about controlling the DALI ballast. Theoretically, the ballast, not controls, should be interchangeable. Ballast and control manufacturers are discussing the possibility of matching the ballast and controls. Bacnet is considering working to become compatible with DALI.

Jim Benya made two criticisms of DALI - it has limited zone capability and the protocol characteristics are based on European building design. Dave Peterson added that manufacturers need the same ballast suppliers and that DALI has reduced capability (up to 64 addresses) due to commissioning. Benya cautioned that we might be racing to a digital solution to solve an analog problem. He added, however, that the important matter at hand is to define the standard for protocol, and to determine later whether an analog or digital approach is appropriate.

Dave Peterson (Updates on penetration of controls on C/I markets):

- Dave Peterson showed the results of a market research survey on lighting controls, which indicated that lighting contributes substantially to energy use. In educational facilities, lighting energy use ranges from $\$ 0.38 / \mathrm{ft}^{2} /$ year to $\$ 0.92 / \mathrm{ft}^{2} /$ year.

- Of the \$131 million market for lighting controls, daylight controls represent a small piece (\$1 million), panels represent \$71 million, and occupancy sensors represent \$59 million.

- $\quad$ New construction (added or renovated space) accounts for 65 billion square-feet. Three to four percent of the lighting control market is used for new construction.

- Commercial office space, which includes school and retail office space, has a penetration of about 2 to 5\%, perhaps $10 \%$. Specifiers say that in education, $65 \%$ of floorspace in educational facilities and $60 \%$ of floorspace in commercial spaces (new construction) has some type of lighting control. 
- Factors influencing the use of lighting controls are the owners' requests, increasing energy savings, and complying codes. Currently, there is a low level of interest for low shedding of power supplied by a utility.

- The vast majority (94\%) of control users are using occupancy sensors. Sixty percent are using scheduling systems.

- Satisfaction: The least acceptable control strategy is daylighting controls usage, and the most acceptable is time clocks.

- Scheduling systems usage is the least problematic control strategy, and daylighting is the most problematic.

- Occupancy sensor: The number one barrier is failure to maintain detection, followed by non-familiarity.

- Photosensors: The number one barrier is that users desire for more lighting with photosensors.

- Impact on industry: The communications protocol systems did not work together. Another barrier is the cost of dimming ballasts. Plug-and-play solutions (integrated solutions) are starting to be known.

- How would one do it? The majority would use step, rather than continuous, switching due to cost, though continuous is preferable because it is less perceivable.

- In summary, 1) Controls are being used in new construction. 2) There is low interest in load shedding. 3) A strong attraction to dimming exists, but high cost is a barrier.

Mark Rea: Although load shed interest is low, if customers were aware of what they were being charged, they may become interested. The key is to change the way pricing costs are delivered to the customer.

Peter Morante (The customer's perspective on load management):

- A copy of the presentation is enclosed.

- There is a customer pull for lighting controls. The customer wants to keep the building operating. In terms of the pricing signal, there is currently no deregulation. In the future, states will be deregulated.

- Currently, under regulation, the customer pays the highest month's the demand charge for each of the12 months. Customers are unaware of this charge. They still do not understand how to achieve savings. The key issue is to define who pays the bill, who pays for the investment, and who has the incentive.

- The problem with electricity is that its price is determined by the capacity of the system. Electricity cannot be stored, thus, it must be paid for on a daily basis. Price doubles on normal days when the capacity reaches its maximum. At 100\% capacity, the price can reach \$1000 MWh.

- In Peter Morante’s opinion, customers will start requesting load management when they understand how much they are paying during peak demand. 
Jim Benya: The California energy crisis caused increase concern about energy savings. The market is not all that free yet. Deregulation has ended in the northwest. Electricity has a very high price. The demand for the technology will rapidly increase when customers understand how much they are paying for energy use. Cost is the number one barrier, followed by lack of standards and familiarity. Incentive finances will help overcome the cost barrier. Benya thinks we need to help eradicate the cost and familiarity barriers. External forces, such as deregulation, will help.

There are no barriers for the use of static controls (automatic shut-off). California will eliminate the below 5000 square-foot exemption for required automatic controls from Title 24 in 2005. The technology is cost-effective. The lighting industry does not have enough data to support how much energy can be saved with controls. The technology exists, and is cost effective.

Mark Rea: Static controls (automatic shut-off) are robust, should be implemented, and there are no major barriers. Consensus of the group regarding that recommendation: New construction and retrofit should be considered separately. Hard numbers can have an impact on retrofit. An educational aspect is necessary to help increase the market. Support programs should overcome barriers of installation and awareness. The term "Automatic shut-off controls" should be used rather than "static controls."

\section{Recommendation 3 (Load shed management):}

Dave Peterson: A strong economic model of the multitude of benefits from load-shed management is needed.

Mike Stein: It is important to differentiate between dimming and step dimming. A step dimming solution may be achieved more rapidly.

Jim Benya: Step dimming already exists in California. Lack of standard protocol is the reason why dimming ballasts will not be required in the 2005 Title 24 code revisions.

David Bay: Minimal lamp and ballast R\&D efforts are required to achieve lamp/ballast compatibility when dimming from $100 \%$ down to $75 \%$. Some R\&D effort will be required to achieve lamp/ballast compatibility dimming down to $50 \%$. A significant amount of lamp and ballast R\&D will be required to achieve lamp/ballast compatibility for dimming to levels below $50 \%$.

Peter Morante: The customer's opinion regarding load management is important. The design community has accepted automatic shut-off. We need supporting programs that help overcome lack of awareness.

Jim Benya - The dimming ballast is the back end. We need to focus on the front end as well, which is how the signal is generated. No controls industry exists to deal with the problem. An industry needs to grow in order to provide the front end (controls). Integration is a problem. He handles the wiring technique by being open-minded and envisioning technologies that are easy to install and commission. Other obstacles exist besides the ballast.

Ron Lewis questioned the interactions of lighting and heating and cooling. David Bay: The focus on end user is missing. Build in Jim Benya's triangle (explained above). Mark Rea: Is it worth worrying about societal benefit? It is clear that we need a near-term strategy that will try to articulate more clearly and succinctly the automatic shut-off controls. Is it worth worrying about dimming today? What are we doing about dimming?

Jim Benya: Dimming is a good option for energy and management. We should work on a lowcost ballast and prepare the market for these technologies. It is important to speed up standards to use dimming. 


\section{Consensus}

Automatic shut-off controls are ready for implementation. There is a need to conduct demonstrations projects that will help increase the impact of this technology in the retrofit market. An educational program to increase awareness of the benefits of these products is also necessary.

Load management strategies: Research and development activities are still necessary to help develop a technology that is low-cost, easy to install and commission, and reliable. In parallel, it is necessary that the customer understands how much he is being charged for energy, which will increase interest and demand for load-shedding.

\section{Next Steps}

The next roundtable was set for February 5th in Washington, DC (NEMA's headquarter). Mark will contact each member to sketch out an agenda and finalize the list of invitees individually. The LRC will sort out some of the economic models, address customer perspectives, and revise the white paper accordingly.

\section{Postscript to the minutes}

The LRC staff followed up with everyone at the meeting, including those who could not attend. Impressions of the meeting were obtained and additional suggestions were solicited. Everyone was complementary but felt there was too little time to discuss all the issues. Based upon this feedback it was decided, in full collaboration with the peer review group, to concentrate on the automatic-off controls on February 5, 2002 and on load-shed ballast specifications on January 23, 2002. In late February or early March of 2002, a meeting will be held to discuss future strategies for peak load reduction amongst regulators, electric power suppliers, and controls manufacturers. In August 2002 a general meeting will be held to integrate all three of these "break-out” sessions.

The LRC staff participated in a meeting with James Gallagher (Chief, Retail Competition Section, Office of Electricity \& Environment - Public Service Commission, New York State), a member of the peer review group. They had an in-depth discussion on the future for peak load reduction and how load-shed, lighting controls could play an important role in reducing energy use and peak demand in commercial and industrial applications. They also outlined an agenda for a meeting in the first quarter of 2002. Invitees will share their perspective on load management and will develop an action-plan for integrating lighting controls into load management strategies.

In addition, the LRC staff has set up a meeting of key people in the manufacturing side (David Bay, OSRAM SYLVANIA; Al Russeau, Philips Lighting; Bob Erhardt and Sree Venkit, Advance Lighting; Mike Stein, Universal ballast; Ed Yandek, GE Lighting) on January 23 in Troy, NY to develop performance specifications for load-shed ballasts. This new technology would require a very small incremental cost but would be able to reduce electric power on demand. 


\section{Load-shed Management}

The peer review group recommended that the project be bifurcated and that automatic shut-off be treated separately from dimming. As a result, the LRC decided to conduct two roundtables: one on barriers to widespread use of automatic shut-offs; the other on load-shedding technologies.

The LRC held a meeting on January $23^{\text {rd }} 2002$ with the three major lamp and ballast manufacturers to discuss the development of a performance specifications for load-shed fluorescent systems. This new technology would require a very small incremental cost but would be able to reduce electric power on demand.

In the meeting held on January $23^{\text {rd }} 2002$, manufacturers helped develop a performance specification for load-shed fluorescent systems. The representatives of the manufacturers agreed to go back to their organizations and get feedback on the issues discussed in the meeting. LRC agreed to gather information from utility personnel involved with communications (power line carriers - PLC) on current performance of utility-to-customer signaling strategies. In addition, the LRC will also contact parties specifically interested in load management to talk about future pricing of energy to the customers.

The LRC plans to bring these three groups of people together late in 2002 to discuss the ways to overcome the barriers to the widespread use of load-shed dimming controls. 


\section{Meeting Minutes}

\section{Load Shed Ballast Performance Specification Drafting}

January 23, 2002

Project: Reducing Barriers to Efficient Lighting Systems

Sponsor: US Department of Energy

\section{Meeting attendees}

Ed Yandek (GE Lighting)

Mike Stein (Universal Ballast)

David Bay (OSI)

Roger Hunt (OSI)

Al Rousseau (Philips)

Stuart Berjansky (Advance Transformer)

Mark Rea (LRC)

Andrew Bierman (LRC)

Francisco Garza (LRC)

Mariana Figueiro (LRC)

Conan O’Rourke (LRC)

Russ Leslie (LRC, arrived late)

The meeting started at approximately 9:00 am

Mark summarized the project, its goals and objective, its tasks, and its outcomes.

At this point in the project, there are two distinct activities: Automatic shut-off controls and dimming/load shedding. For automatic shut off there are no barriers for new construction, but some barriers on retrofit. Meeting on Feb $5^{\text {th }}$ to try to reduce these barriers.

This meeting concentrated on load shedding. We are trying to be ahead of the curve, because we believe that there will be a greatly increasing market for load-shedding in the near future. Mark will be meeting with parties specifically interested in load management to talk about future pricing of energy to the customers. The unregulated side of the utility is going to be the primary focus of the discussion at this time. We see a potential penetration of controls for load management if we are ready with a technology that the market wants. Mark asked Ed to summarize discussions from the previous evening.

Ed: We have a choice. Either do typically what we have been doing, that is, moving slowly (glacial movement) and working apart, or we can get a group of people together and get things to happen more quickly and show how things are going to happen. A consortium can make an impact (e.g., Bluetooth) if we decide that's what we want to do, and be committed to do it. To make things happen quickly we need a complete system, that is, specify how the whole system works from A (utility) to B (building) to C (ballast)

David: Bold is to look into the future and at technology at the same time. Ed: Utilities must be committed to implement this type of load shedding. David: Who is going to benefit from it [this new load shed technology]? 
Mark: Reduce the power consumption during peak demand - utility customers and society as a whole will benefit

Roger: It takes a large volume to make this type of product financially possible. It's not only about the product, but all the pieces involved with the product.

Mike: We need to, as an industry, know what we are doing and propose a solution that all of us agree on and go to them with a solution. We have to come up with a solution that benefits the manufacturers too.

Roger: There are a limited number of ballasts and sockets... what are you going to displace by using load shed ballast?

Mark: It depends on whether you think load management is important to people in the future. If we don't believe that, we are wasting our time. We think that load management is going to rise in importance and popularity and be a major economic driver in the near future.

Stewart: Utilities already have a rebate program for load shed on air conditioners and heaters.

$\mathrm{Al}$ : An upfront approach may be more beneficial than waiting until things happen.

Ed: Using IT [information technology] model, we [the lighting industry] are going to design a protocol and put it in the market. We don't work that way historically. The forces are lining up and we are getting ready to provide the market with a solution.

Mark: We would be in a much stronger negotiation position if we come together as an industry and present a solution.

David: This could be similar to what happened with the FCC regulations for high frequency lamp operation. In that case we got together and presented a plan to the FCC, which they adopted, to all our benefit.

Two drivers: regulated and unregulated side. Reducing peak demand for power is already a market. The regulated side is still unclear, but we are anticipating that they are going to real time pricing.

Andrew: Let's work on the definition of load shed. What is it? Is it a load management device controlled by whoever is responsible for the building? Is it a system that is fairly secure, or do you need to give this access to the facility manager so that he can, for example, react to real-time pricing?

Ed: Guidelines - control the range of operating scenarios. Outside this range is not included in the guarantee [on the performance of the system, e.g. lamp life].

Mark diagrammed the signaling path for load shed from A (utility) to B (building) to C (ballast). Mike: There might be a D and E, not just a C. In other words, inside the building there could be many parts.

Mark: Let's work on the curve [cost versus amount of dimming]. How much dimming can we get at a reasonable cost? Then we can work on the specification, then on the signal to the ballast (what are we putting in the ballast that makes it a load-shed ballast).

How much can lamps be dimmed? 50\%?

[General question and discussion] 50\% of what ballast factor? What is the baseline ballast? Most commercial ballasts have a ballast factor of 0.88 . T-8 lamps are designed for operation on a reference ballast at $260 \mathrm{~mA}$. Typical full output of commercial high frequency ballasts range from 140 to $180 \mathrm{~mA}$. Lamps are optimized for conditions that are rarely used.

Ed: If this [load-shed] becomes a big thing, I can imagine optimizing the lamp for load shed. This provides an incentive to re-optimize T-8s to run, at say, $180 \mathrm{~mA}$.

Andrew: We can look at two scenarios; load shed often and load shed rarely. 
Ed suggested that we base the amount of load shed on Peter Morante's data of actual building load curves.

Assumption - we can go down lower if load shed occurs less frequent. Based on Peter Morante's data for an office building, if $30 \%$ of load is shed, this will happen $12 \%$ of the time. Mike: We must keep in mind that we don't want to rob occupants of proper light levels.

Ed: Let's test this on a less expensive solution (only the $20 \%$ reduction with an instant-start system) and test the solution with the utilities. If it works, we can expand the work. Program start ballasts are being pushed to the people that are using occupancy sensors. Programmed start can be used for greater dimming because it heats the electrodes.

Andy presented data from Specifier Reports. In going to a rapid start system there is a loss in efficacy. However, we don't know about life. We can get the demand savings, but the cost of running may be higher.

[General consensus] We can do $20 \%$ now without affecting lamp life so much. In the future we can try to go lower, if this is successful.

Mike: The 0.88 ballast factor is the platform we should agree on. Concerned that we are talking about $50 \%$ dim. $25 \%$ of ballasts have a ballast factor of 0.78 .

David wrote the following list on the blackboard.

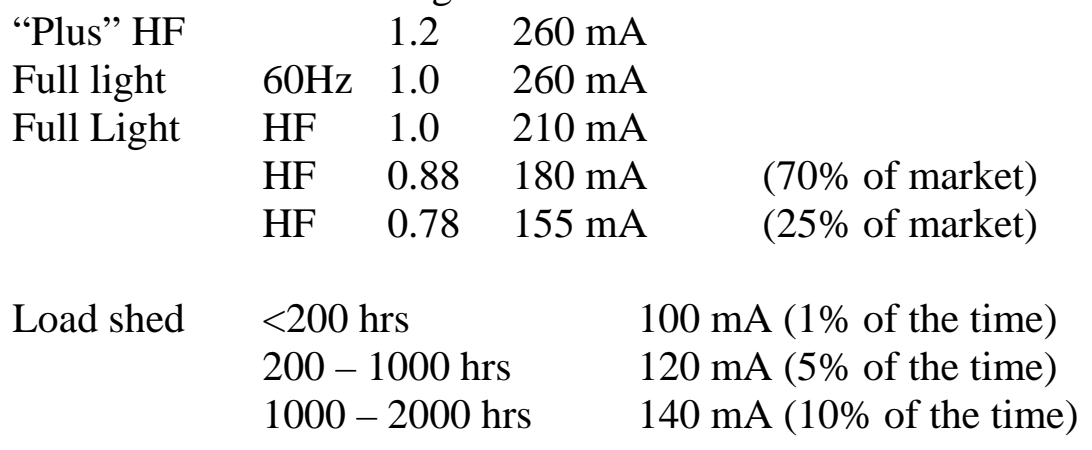

Above table is for rated life of 20,000 hours. Rated life is for 3 hrs on, 20 minutes off. Nobody actually operates lamps on this short a cycle, so life is extended.

As long as lamp current stays above $140 \mathrm{~mA}$, the $10 \%$ of the time operation could be $90 \%$ without decreasing life.

A safety margin is important for line voltage fluctuations, component tolerances and temperature variations.

Ed drew a graph showing how life slowly decreases as current is reduced up until some threshold, or knee in the plot $(130 \mathrm{~mA})$ where life decreases rapidly with further reductions in current. $130 \mathrm{~mA}$ is the lower limit of his comfort zone.

Mike: The system to consider must be for a retrofit scenario - ballast factor of $0.88,180 \mathrm{~mA}$ as baseline.

Assume that we want to do it at $5 \%$ of the time. There will be zero risk, minimal impact on lamp life if it goes down to $120 \mathrm{~mA}$ at nominal line voltage $(120 \mathrm{~V})$ for on average $5 \%$ of the time (off a 0.88 ballast factor instant-start ballast).

Magnetic market is about $80 \%$, but it is difficult to know for sure. $50 \%$ of sales are still magnetic ballasts. Maybe we can motivate the market to retrofit the magnetic ballast. $66 \%$ installed or more is T12.

Russ Leslie and others did some calculations of demand savings using data presented at the DOE project roundtable meeting in December, 2001 (Table 1 of handout). As an example, a 3-lamp 
ballast capable of reducing power by $30 \%$ corresponds to a $\$ 6 /$ ballast/year building demand savings and a \$3/ballast/year system demand reduction savings. The future value of such savings would be about $\$ 65$ over a 10 year ballast life. The above numbers assume a $\$ 8 / \mathrm{kW} / \mathrm{month}$ utility incentive to shed load when needed and an $\$ 18 / \mathrm{kW} /$ month building peak demand cost. The $5 \%$ of time limitation placed on dimmed lamp operation easily absorbs all the time of high system demand when load shedding would occur under a real-time pricing scenario.

We need to go to the utilities' people and ask them if the technology excites them and if they are willing to support that. Everyone here needs to go back and check the proposed loadshed specification.

We need to talk to the utility industry about their rebate and load control programs.

The discussion turned to the topic of signaling and communications

Power line carriers (PLC) may be a solution. How low cost can it be? Once I am in the building, everybody will do the same thing. The advantage is that we are talking about little information. We want to minimize interference. Look at the utility programs and determine what are the signals and equipment that they are using. This may be used for the lighting signal. Search on the products that are out there before making any assumptions on the types of signals, etc.

Ballast receives a signal every 10 minutes.

Must have means so cycling the power on/off does not defeat that load shed. How well can PLC signals be isolated within buildings and for a particular tenant? Problems may arise when signals extend beyond the building/tenant.

Zoning becomes too costly though.

The problem can be solved with contracting. The utility will know whether the demand was actually reduced.

Signaling, metering, PLC technology - people from utilities that understand about these issues can help us solve the problem. Push a lot of the information/intelligence to the control; instead of on the ballast is a good idea. Keep the ballast as low cost as possible. Basically the ballast only contains a switch and the necessary circuitry to decode the PLC signal.

Two inputs to the controller may be needed to initiate load shed: one based on building power demand and one for a utility generated signal. This could be implemented as a simple or-gate. Next, go to utility people to discuss with them interfacing with large customers. They will validate our economical perspective as well. They can share with us what works and what does not work. Utilities will likely want a pilot program.

David: Incremental cost is around $\$ 1$ a ballast; incremental price is $\$ 2$ a ballast for PLC reception.

\section{Summary}

- Assignment - manufacturers to go back and make sure those are likely numbers.

- Signaling- ballast is only able to decode the signal it is receiving. Manufacturers need to go back and find out the cost of the options. Minutes to be distributed by end of business day on Friday. Two weeks for the manufacturers to get back with information. 
- LRC will gather information from utility personnel involved with communications (PLC) on current performance of utility-to-customer signaling strategies.

- Next meeting may be in a utility site in a month to discuss this specification.

- A Phase 2 could potentially include T8 and T5 lamps (T5 is optimized for lower current). 


\section{Automatic Shut-off}

The LRC has been working with market transformations groups, federal and state agencies, and manufacturers to promote the development of programs that will increase the widespread use of automatic shut-off controls. A short white paper was developed summarizing the findings during the first year on automatic shut-off and a few recommendations were made to increase the market penetration of automatic shut-off controls in retrofit applications. The findings and recommendations detailed in the white paper were distributed to all the round table participants and discussed during the meeting. A summary of the recommendations that the group agreed upon is provided below.

The white paper summarizing the findings and recommendations for automatic shut-off controls is below.

\section{Overcoming Barriers to Widespread Use of Lighting Controls in Commercial/Industrial Applications}

\section{Part 1: Automatic Shut-off Controls Background}

Under funding from the US Department of Energy, the Lighting Research Center (LRC) is identifying barriers to the widespread penetration of lighting controls in commercial and industrial (C/I) applications and making recommendations to overcome these barriers. Since fluorescent lamps are the predominant technology in $\mathrm{C} / \mathrm{I}$ applications, the focus of this project is the control of fluorescent lighting systems.

The LRC reviewed existing research, technology, patents, and market data related to lighting controls, interviewed control manufacturers, surveyed control installers, and conducted a peer group review to improve and validate a set of preliminary recommendations. Two distinct categories of controls were identified, each with their own set of barriers: dimming controls (architectural dimming, load shed dimming, and photosensor-activated daylight dimming) and automatic shut-off controls (occupancy sensors and timers). Dimming controls still have substantial technical barriers and are dependent upon changes in electricity pricing systems to be cost-effective in many applications. Automatic shut-off controls, however, are technologically robust, less expensive, and ready for widespread installation.

Because these differences between dimming controls and automatic shut-off controls need to be addressed by different stakeholders, the peer group review recommended a bifurcation of the project. This paper and roundtable only addresses the barriers to the widespread penetration of automatic shut-off controls. A parallel effort is being made to reduce the barriers to widespread use of dimming controls.

Penetration of automatic shut-off controls for fluorescent lighting systems into C/I applications can be increased, particularly if market transformations groups, specifiers, government agencies, and manufacturers collaborate to help reduce the remaining barriers to the use of this robust control technology. 


\section{Automatic shut-off controls}

Automatic shut-off controls turn off lamps when a signal is received from an occupancy sensor ("unpredictable scheduling") or from a time-of-day scheduling device ("predictable scheduling”). There are several different ways this can be accomplished, including the use of building automation systems, energy management systems, individual time clocks, wallbox mounted devices, and various combinations of relays, sensors and control panels. Since lamps are not dimmed the increased cost of the dimming ballast and controller is avoided. There are several issues relevant to widespread deployment of automatic shut-off controls:

\section{Market Penetration: Retrofit vs. New Construction}

Automatic shut-off controls are becoming commonplace in new construction; some estimates indicate that $60 \%$ of commercial projects now utilize them. Current and planned energy-code provisions, the ease of installation in new construction, and the potential energy savings will continue to increase the penetration of automatic shut-off controls in new construction. To capture additional penetration of automatic shut-off controls in new construction, the most effective approach to widespread penetration includes specifier education and more pervasive code requirements and/or enforcement.

However, according to the Energy Information Agency, annual new construction represents only $1-2 \%$ of the total floor space lit in the US. Penetration of automatic shut-off controls in existing buildings is poor.

Retrofitting existing buildings with automatic shut-off controls presents several barriers not present in new construction:

- Wiring is usually more difficult and expensive in a completed building compared to wiring while a building is under construction;

- In new construction, automatic controls can be purchased instead of manual controls; in retrofit construction, the manual controls they are replacing have already been purchased;

- Lighting and electrical plans are drawn for new construction, providing an opportunity where automatic controls can be easily considered and integrated into the plans. Electrical or lighting plans for renovations in existing buildings are seldom redrawn, especially for minor alterations.

- Energy code provisions often do not apply to existing buildings unless they are undergoing major renovations;

- "Hassle factor” is perhaps the greatest barrier. Building owners and tenants are focused on their businesses and seldom have the time or interest to consider redoing their lighting or control systems.

The overwhelming sentiment among the installers of automatic shut-off controls is that they can make controls work, but there is a small market demand in existing buildings. They believe that some agency needs to repeatedly and effectively communicate the value of lighting controls to building owners and facility managers. Partnerships between manufacturers and public benefit market transformation parties need to be formed to develop programs to retrofit buildings with automatic shut-off controls. These partnerships are necessary to reap the large untapped, energy savings potential in existing buildings. 


\section{Lamp/Ballast System Reliability}

As a consequence of the energy crisis of the 1970s, significant improvements in the efficacy of fluorescent systems were achieved in the last two decades of the 20th century. The physical, electrical, and photometric properties of fluorescent lamps and ballasts were continually optimized during this era to improve not only efficacy, but also to give them smaller size, better maintenance (lumen depreciation and life), lower mercury content, and higher visual quality (better color rendering and reduced flicker). This evolving sophistication has led to higher and higher expectations for fluorescent system performance in terms of energy efficiency, maintenance, visual satisfaction and, of course, low initial cost. One down side of this sophistication, however, has been the proliferation of products that provide poor system reliability. Lamps operated on ballasts that do not have optimized starting and operating electrical properties for those lamps will fail prematurely, leading to dissatisfaction among specifiers, owners, and facility managers. This was a common situation in the 1980s and early 1990s as the electronic ballast manufacturers learned how to operate lamps produced by the "big three” global manufacturers: GE, Philips and OSRAM, but those days are essentially over for C/I on-off fluorescent lighting systems, largely because the global manufacturers have taken control of the specifications for ballasts that operate their lamps. Today, the stories of poor reliability mostly refer to fluorescent systems operated with dimmers and/or residential-grade products. The remaining lamp/ballast compatibility issue for automatic shut-off controls is the effect of frequent switching on lamp life. BAS, panel controls, and timers are usually not configured so that the lamps are frequently switched. On the other hand, occupancy sensors set for short time delays and located in frequently used locations such as stairways, large restrooms, and hallways could cause frequent switching and therefore, shortened lamp life. Premature lamp failures in these instances could create a generalized barrier to the use of automatic shut-off controls.

\section{The Human Element: Manual On and Overrides}

Some important principles for automatic shut-off controls have emerged from research into lighting controls. The goal of automatic shut-off controls is to turn lights off when no one is in the space. This strategy will not annoy the occupant or compromise the worker's productivity in the space because the space is unoccupied. In general, manual controls should be used to turn lights on when needed; automatic controls should be used to them off when no one occupies the space. This manual on/automatic off approach reduces false triggering of occupancy sensors and prevents lights from being turned on needlessly when daylight is sufficient for the occupants or when the lights are not otherwise needed in the space.

Because no technology is completely foolproof, however, it is essential that there be a local, manual override to any automatic strategy. For example, if a worker is working late one evening and the automatic time clock initiates an "off” command, the worker must be able to manually override the automatic command in the work space and in areas needed for exiting the work space. However, engineers and facility managers seem hesitant to provide manual override, because it is assumed that people will use them too often and energy savings will be lost. To address their concern, manual overrides capabilities should be designed to automatically reset to automatic shut-off mode after each use.

\section{Programs to Overcome Barriers}

First cost remains a barrier for lighting controls. Often the person or department that pays for the installation of lighting equipment does not receive the benefit from the reduced electrical bills. 
Also, in existing buildings, managers do not want the "hassle factor" of implementing a change. First cost and hassle factor have been overcome for other energy-efficient lighting technologies with demand-side management (DSM) incentive programs, market transformation procurement programs, shared-savings marketing by energy service companies (ESCOs), and new code requirements. Combinations of these approaches increase market demand for energy-efficient lighting products, leading to lower product cost and market transformation. Products such as T8 lamps, electronic ballasts, and LED exit signs are examples of successful lighting market transformations. Lighting controls, however, have yet to benefit substantially from these market forces, at least in part, because of the difficulty in predicting the energy savings resulting from control use.

DSM program developers and evaluators, code developers, and ESCOs do not have adequate information to predict expected savings by control measures. They need to know, for example, when and for how long the control switches off the lights. There are case study examples, but robust control use factors need to be developed that will afford the program developers confidence that a specific control installation will deliver a specific energy savings. With this confidence, controls will more likely find their way into lighting incentive programs, code provisions, and ESCO strategies.

Again, there are no technical or human barriers to turning the lights off in unoccupied spaces to achieve energy savings. There is simply "a failure to communicate" that automatic shut-off lighting controls that turn lights off in unoccupied spaces are reliable and cost effective. These principles consistently meet the demands for occupant satisfaction and energy savings.

\section{Preliminary Recommendations}

Aggressively encourage the widespread deployment of automatic shut-off lighting controls in existing and new $\mathrm{C} / \mathrm{I}$ buildings.

The following actions, listed in descending order of priority, overcome the identified barriers to the penetration of lighting controls into C/I markets. They are focused on existing, automatic shut-off lighting controls that have been demonstrated to be cost effective and reliable. These recommendations can be implemented immediately.

1. Bring together various stakeholders interested in increased penetration of automatic shutoff lighting controls (manufacturers, specifiers, government, public benefit energy efficiency groups, building owners) to prioritize approaches and form implementation partnerships that overcome the hassle factor and/or first cost barriers to automatic shutoff lighting controls in existing buildings, such as:

a. campaigns for specifiers and customers

b. education/training of qualified technicians

c. rebate programs

d. procurement programs for large real estate

e. labeling programs that will assure quality of the products

f. model specifications and standards.

2. Support data collection that provides fixed numbers for expected energy savings and load reductions for various automatic shut-off lighting control technologies in various 
applications. Develop simple guidelines for setting time delays on occupancy sensors used in spaces that frequently change from occupied to unoccupied.

a. research and development

b. demonstrations and evaluations.

3. Encourage use of automatic shut-off controls that:

a. have manual on/automatic off capabilities

b. reset to automatic off mode after each manual override

c. for retrofit occupancy sensors, can be installed without power wiring in a switch run and operate fluorescent lighting systems.

4. Encourage more stringent code provisions requiring automatic shut-off lighting controls for new construction and substantial renovations.

\section{Roundtable: Recommendations from Attendees February 5, 2002}

Despite the diverse backgrounds of the attendees, there was much agreement about the most important aspects of automatic shut-off lighting controls. In general, the group agreed that industry should aggressively encourage the widespread deployment of automatic shut-off lighting controls in existing and new C/I buildings. Specific recommendations were focused on five main aspects, 1) the need for outreach/education, 2) the need to provide more information for decision makers, 3) the importance of code requirements, 4) the need for technology performance specifications, and 5) the need for a coordinated effort.

\section{Outreach / Education}

- Develop "best practice” documents: Specifiers and installers need best practice documents based on consensus. This document would explain which technologies are most appropriate for each class of application, how/where they should be installed, and appropriate features.

2. Decision making

- Collect data: Building owners / decision makers need defensible estimates about how much they can expect to save in their building. Data collection is necessary, to provide fixed numbers for expected energy savings and load reductions for various automatic shut-off lighting control technologies in various applications.

- Owner focus group: A focus group with large customers needs to be convened.

- Aggregate data and articulate to decision makers: Present the value of automatic shut-off lighting controls to decision makers, in their terms.

3. Code compliance

- Encourage nationwide code requirements: More stringent code provisions and enforcement need to be established, requiring automatic shut-off lighting controls for new construction and substantial renovations. 


\section{Technologies}

- Develop, peer review, and disseminate performance specifications for automatic shut-off lighting controls.

5. Coordinated effort

- Establish coordination: Under direction from DOE, identify a national market transformation group to bring together all the stakeholders interested in increased penetration of automatic shut-off lighting controls. This group would prioritize approaches and form implementation partnerships between manufacturers, specifiers, government, public benefit energy efficiency groups, and building owners. 


\section{APPENDIX H: COMMUNICATION PROTOCOLS TO MEET THE NEEDS OF A FULLY INTEGRATED LIGHTING AND WHOLE BUILDING CONTROL PROTOCOL}

\section{Introduction}

The lighting market in the United States has not experienced wide penetration of the use of whole building integrated lighting controls. While such lighting controls could undoubtedly reduce energy consumption for lighting, it must be realized that other, less expensive, lighting controls are available, and those markets have not seen pervasive utilization either. To determine whether the slow adoption of whole building lighting controls is due to technological barriers or other economic and market forces, it is necessary to take a critical look at the necessity of these controls and what economic benefits they offer. Then, an examination of what is currently available and what is being developed will determine if these needs are being met.

The success of lighting control products on the market must allow systems to achieve three main goals:

1. Achieve sufficient functionality to meet the key requirements of their main market.

2. Allow a significant cost reduction compared to current market standard systems. Cost should take into account: hardware capital cost including wiring, design time required by the specifier and the control system manufacturer, installation time required from the electrician, and commissioning time and remedial time required from the electrician and end user.

3. Minimize ongoing perceived overhead costs and inconvenience to the end user, or in other words, systems should be simple to understand and use.

\section{What benefits do whole building systems provide?}

Available control strategies differ widely in terms of functionality and cost; some are much simpler than others. Some operate locally on a small number of fixtures, and some collectively over a large number of fixtures. Some require continuous monitoring and immediate actions, while others are not so time sensitive. For analysis purposes, it is helpful to divide lighting control strategies into two groups based on similar communication needs. The groups are local control and collective control.

Local controls should respond to local conditions. They require continuous monitoring and often immediate (very short time delay) responses. Control strategies in this group include:

Occupancy sensors

Photosensor control

Manual switching and dimming 
Collective controls respond to global, or external, signals. Exact timing of actions is not important because no immediate action is required. Many fixtures are often grouped together all responding to the same signal. Control strategies in this group include:

Scheduling (sweep off functions)

Load shed

Local control functions do not need to be integrated into a whole building system in order to work effectively. For monitoring purposes they might be connected, but their value for energy savings is not clear. Problems encountered when trying to integrate local control into large networks include:

1. Too many control nodes: the large number of individual ballasts and lighting fixtures adds excessive cost and complexity to systems. Such individualism is usually not needed anyway because fixtures are mostly operated/controlled in groups as part of an overall lighting design.

2. The need for immediate response/action places excessive demand on bandwidth and processing ability of a networked system. For example, a signal from an occupancy sensor must turn on a light within half a second or less. This is easily accomplished in a local system with a dedicated signal path, but in a networked system, a lot of processing and communication has to be done to determine which sensor is signaling, what action should be taken, addressing each component, receiving confirmations, carrying out network protocols, and gaining access to the communication medium. As more devices are added to the network, the reliability of getting a signal through within a given period of time diminishes.

3. Attention to reliability is of much greater importance for an integrated system than for individual controls, which adds considerable cost. Problems with an integrated system threaten the whole operation of a building, while local control isolates problems to small areas.

Collective controls could benefit by being integrated with other building communication systems. With collective control schemes, the number of control nodes is greatly reduced by collecting individual lighting ballasts/fixtures into groups. This does not strain the limitations of existing networks. Also, large groupings of fixtures usually do not require immediate action/response. The cost-benefit of integrating collective control is that existing hardware and network infrastructure can be shared across all building control systems.

Trying to apply a single solution that is capable of handling all the requirements of the different control strategies is not cost effective. Such a strategy will result in an overbuilt system where most of the resources are hardly, if ever used, and so consequently the system is too expensive and/or complicated to use. Therefore, it should be recognized that whole building lighting control systems might not be appropriate for all buildings now, until costs come down. The ultimate level of control being pursued by the lighting industry is the individually addressable ballast, ideally with full-range dimming, as well as switching capabilities. Listed below (Table 1) are the key features and applications of a ballast/control system that meets this ideal. Meeting these features goes well beyond ballast design and hardware issues. They are also critically dependent upon the communication protocol used for implementing wholebuilding control, the commissioning of the system, and other building aspects such as daylight availability and auxiliary control devices. 
It is not expected that all buildings need, or even desire this level of control, but the more features that can be packaged into a system presumably increases its market appeal, provided that other factors, namely cost and reliability, are not affected. The other advantage of an all-inone approach is that makes life simpler for the specifier and improves interoperability because fewer product types need to be offered. Manufacturers also benefit from having to support fewer product lines. Most likely, though, cost and reliability are affected by making a system more universal. In this case the market will eventually sort out what features are most beneficial and cost effective. Assuming that the trends for increasing space use intensification, energy costs, and state and federal building code legislation continue, many of these features that are now considered nonessential may become perceived as mandatory, as they already are to some extent in other countries for intensively used, urban office space.

\begin{tabular}{|l|l|}
\hline \multicolumn{2}{|c|}{ Table 1 - Key features and applications for individually addressable luminaires ${ }^{A}$} \\
\hline Testing and monitoring of emergency & Photosensing \\
luminaires & Control of window shading devices \\
Load shedding & Interchangeability \\
Lamp failure reporting & Occupant control from desktop \\
Reduced / zero wiring & \\
$*$ See notes for further explanation & \\
\hline
\end{tabular}

Notes:

A. Individually addressable luminaires allow end-users to organize workplace lighting into functional groups, so that switching / scene setting corresponds to the organization of the workplace. Intensively-used (usually urban) office space is frequently repartitioned or reorganized to adapt to the changing requirements of businesses and addressable systems allow the lighting to be reconfigured easily as this happens.

The end users should be able to either carry out simple lighting reconfiguration themselves (adding new luminaires, changing functional groups, changing scene settings), or have reconfiguration carried out by their regular qualified electrician, rather than having to contact the control system manufacturer. This is desirable both in order to reduce wasted time for the end-user, and to reduce the overhead costs of lighting controls manufacturers, which are recovered by adding (often very significantly) to the capital cost of lighting controls hardware, thus raising a barrier to more widespread use.

Because large buildings often house high salary workers at high occupancy densities, disruption is costly and, as far as possible, readdressing of luminaires should be carried out without access to the ceiling being required.

B. Lamp failure reporting facilitates quick replacement of failed lamps, which minimizes occupant dissatisfaction, maintains the architectural appearance of the building, and fulfils the end-user's health and safety obligations.

C. Assuming that he cost of electrical and electronic hardware will continue to fall in comparison to the cost of labor, the necessity of installing additional signal wiring will become an increasingly significant barrier to the use of control systems. This problem 
may soon be solved by wireless communications, but in the short term it is desirable for lighting control protocols either to allow the amount of wiring to be minimized, and/or for their signals to be sufficiently robust to allow signal wires to be run with lighting power in order to minimize the cost or cable or conduit.

Under the National Electric Code (NEC) 2002, conductors of different circuits rated at $600 \mathrm{~V}$ or less (both ac and dc) are permitted to run in the same cable (300.3(C1)), so barring regional variations - wiring of this type, though not common, is technically feasible.

D. Interchangeable systems are those in which components from a variety of different suppliers can be used as part of an integrated system. Conversely, proprietary systems are those with which only a single supplier's components are compatible. Due to the technical difficulty of achieving interoperability, and the absence of a perceived need for interoperability among lighting specifiers, proprietary systems remain the worldwide norm for lighting (though not for HVAC systems, which don't require instant responses and usually require far fewer addresses). Nevertheless, interchangeability remains a desirable goal for clients nervous about having only a single source for future replacement or upgraded parts. However, it remains to be seen whether interchangeability will founder on the contractual rocks of having to allocate responsibility or blame when components turn out not to be interchangeable in real life. The related issues of "interoperable” systems are a partial solution not addressed in this report.

\section{Do established and newly-developed protocols meet these functionality requirements at a reasonable cost?}

A major cost not included in this discussion is the cost of system commissioning. Currently major systems can be commissioned only by the lighting controls manufacturers (the anticipated cost of this is built into the price quotation), or in rare cases by a professional systems integrator. It is likely that part of the motivation for the development of the DALI system was to transfer commissioning cost from the manufacturers to the contractors, but from the point of view of the client the cost will not change significantly.

Another cost category that is often overlooked in the discussion of whole building controls that must be considered is the foundation costs of controls, including the cost of peripheral control technologies (e.g., photoelectric sensors or manual dimmers) as well as the more expensive dimming ballasts that is needed to provide heat to the electrodes while dimmed. Together, these foundation costs can easily be as much as an additional \$150.00 per luminaire.

\section{Analogue 1-10V protocol}

\section{Functionality}

The $1-10 \mathrm{~V}$ protocol allows continuous dimming of one or more luminaries, to the same light level. The protocol is technically ill-defined and there is no agreed statement of the exact parameters for impedance or current, and consequently this protocol has developed a reputation for inconsistent and unpredictable light levels from different luminaires, depending on the length (hence signal voltage drop) of each signal wire, and upon the particular permutation of control system and ballast from different manufacturers. 
Hardware cost

The 1-10V protocol carries no addressing information, so if luminaries are to be individually addressable, each pair of signal wires must be terminated into a local control system outlet, which in turn is connected to the rest of the system by a different (usually proprietary) addressable bus. To avoid excessively complex wiring, no more than a handful of luminaries can be connected into the same local outlet box, so the 1-10V protocol carries a high overhead of ceiling-mounted lighting hardware, which adds mainly to capital cost but also to design time and installation time. In the absence of any other barriers, this cost barrier is sufficient to prevent the widespread uptake of lighting control systems.

Wiring cost

The signal carried on $1-10 \mathrm{~V}$ wires is highly susceptible to $60 \mathrm{~Hz}$ interference, and so should not be run in the same conduit or cable as lighting power. The necessity for different cable routing adds to the wiring cost and the wiring complexity of the system. The maximum length of signal wire permitted before voltage drop begins to severely affect the system is debatable, and depends how much discrepancy in luminaire output the client is prepared to tolerate, as well as the number of ballasts connected. Where this wire length is exceeded, signal repeater units must be used to boost the signal.

\section{Design cost}

Usually, not all the luminaries in a given area need to be individually addressable. To save cost in 1-10V systems, luminaries in common areas of the workplace can be grouped together and set to the same dimming level. This is also often the case with a row of luminaries parallel to the windows in a daylight-linked system. In these cases the grouping and wiring of the luminaries must be exactly specified during the design stage, and must satisfy the requirements of both the architect and the electrical engineer. This process involves the issuing of extra drawings and the necessity for additional project group meetings. There is also a need to consult with the control system manufacturer about maximum cable lengths, the necessity for signal repeaters, and potentially other wiring issues.

\section{Reconfiguration cost}

If a fully-addressable $1-10 \mathrm{~V}$ system is installed, it should be as easy to readdress the luminaries, as it would be with any other system. However, 1-10V systems very seldom have full addressability, in which case reconfiguration involves access to the ceiling void and re-wiring of parts of the control system. Usually this is a complex process and would require the original ceiling plan to be referenced.

\section{Non-addressable digital dimming protocols (SuperDim, DSI etc)}

These protocols are topologically the same as $1-10 \mathrm{~V}$, but provide specific technical advantages, including accurately specified and predictable light output, no problems with voltage drop, zero susceptibility to radiated interference, and the ability to report lamp or ballast failures (either from an individual luminaire, or from somewhere within a group). However, they still do not allow individual dimming and so incur the same high overheads as $1-10 \mathrm{~V}$ systems. Due to the resistance of the US market to factory-prewired components including modular wiring, these 
cost overheads are much more significant in the US than in other countries, and are not likely to be overcome.

\section{DALI}

Functionality

DALI is a protocol developed by a partnership of major ballast manufacturers. It allows addressing, dimming, grouping, lamp error feedback, and will allow a variety of other functionalities in the future. DALI-compliant ballasts and input devices from different suppliers can be used interchangeably in the same installation.

\section{Hardware cost}

The DALI protocol is based on the DSI digital protocol, but with additional bi-directional communication that allows 64 separately addressable luminaires to be connected on a single free-topology bus. This feature allows addressability to be achieved with far fewer ceilingmounted boxes. The overall cost of DALI components is low because they are standardized between manufacturers.

The facility for emergency testing and monitoring is incorporated into the DALI protocol, and load shedding can be carried out very simply. However, due to the low speed of the bus, DALI's ability to convey continual level signals from photosensors is very limited. This limitation can be overcome by using fewer photosensors (or even just one single photosensor for the whole building), which also minimizes installation and maintenance effort.

The DALI protocol is very rigid in order to ensure interchangeability between ballasts and input devices from any DALI-compliant supplier. Additional functionality can be built into individual systems, but because the protocol has been specifically developed for the lighting industry, writing specialist applications is a specialist business.

\section{Wiring cost}

The protocol operates at a low speed of 1200 baud and is therefore highly resistant to interference, so it can be carried in the same conduit or cable as the lighting power. Furthermore, it does not require regular twists in the wires (as is the case with some protocols), so standard (cheap) RJ11 or RJ45 cabling can be used to reduce on-site wiring (though cable insulation must of course be rated for plenum ceilings where relevant). There question of whether low-voltage signal wires can be run in the same cable as lighting power appears to be a gray area - if this were allowed it would further reduce the incremental cost of lighting controls.

\section{Design cost}

Because the wiring of the luminaries is not determined by their functional groupings, the time required to design cable routing is minimal, and the routing can be adapted to suit the space requirements of other ceiling services. The absence of voltage drop problems allows more

19 A low-cost communication interface does not necessarily translate into a low system cost. Dimming ballasts are expensive in terms of lamp operation circuit design, and decreased efficiency in operating the lamp over non-dimming ballast designs. Peripherals that add functionality to the system, such as sensors and user interfaces, also contribute greatly to the overall cost. This can make a DALI system, or any other control system, expensive independent of the isolated communication protocol cost. 
flexibility in the siting of boxes remotely from the luminaries they control. Only the electrical engineer need be involved in designing the wiring, so there is little need for consultation between the engineer, the architect and the control system manufacturer.

Reconfiguration cost

Because all major ballast manufacturers support the DALI protocol, electrical contractors are likely to become sufficiently familiar with the system to undertake minor reconfigurations without having to consult the manufacturer of the particular components used. This means that the end-user will likely be able to use their regular electrician, who is already familiar with the building, to carry out the work. Many end-users will be able to carry out the work in-house. In the longer term this will result in a significant saving in post-installation site visits by control system manufacturers, reducing their overhead costs.

\section{LBNL IBECS}

Functionality

IBECS is a protocol under development at Lawrence Berkeley National Laboratories. It offers addressability over a twisted pair bus, and potentially the same range of functionality as DALI, with the advantage that the higher baud rate allows more devices to be connected, and allows the attachment of devices such as photosensors which send out continuous level signals.

Hardware cost

IBECS interface chips can be built in to ballasts in the same way that 1-10V and DALI systems are at present, but for much lower cost. (See note in section 4.3.2.)

Due to the fast baud rate of the bus, IBECS is susceptible to the high degree of harmonic and radiated interference produced by high frequency and dimming ballasts. For this reason it is necessary to incorporate an opto-isolator into each module. The opto-isolator adds only marginally to the cost of the system, and apparently solves the interference problem completely.

The interface between the IBECS microLAN and the high level TCP-IP network is made via a readily-available and cheap standard RS232 gateway.

Wiring cost

Up to 100 devices can be connected to each microlan, making the wiring cost comparable with DALI. IBECS can be made to run over a wireless network, probably more cheaply than will be possible with DALI, because the microlan is more akin to standard IT industry networks.

Design cost

As with DALI, because the wiring of the luminaries is not determined by their functional groupings, the time required to design cable routing is minimal, and the routing can be adapted to suit the space requirements of other ceiling services. The absence of voltage drop problems allows more flexibility in the siting of boxes remotely from the luminaries they control. Only the electrical engineer need be involved in designing the wiring, so there is little need for consultation between the engineer, the architect and the control system manufacturer.

Reconfiguration cost 
IBECS runs on an IT industry-standard high speed bi-directional bus, known as MicroLAN, which allows a virtually unlimited number of separately addressable luminaries, and a similarly unlimited variety of other potential functions such as emergency testing and monitoring, load shedding, and communication with other components such as shading device actuators. The protocol is potentially much less rigid than DALI, and since it is based on a very common technology, specialist applications are likely to be easier to design. However, for the same reason, some question mark exists over the interchangeability of components.

\section{Summary}

Realizing that whole building controls are not the only solution to an assumed latent market demand for energy-saving, and/or comfort-enhancing lighting controls, the added functionality of using such controls for lighting was presented. In terms of this added functionality of the whole-building approach, the capabilities of existing control protocols were analyzed. This analysis reveals that existing lighting controls protocols are functionally capable of meeting the needs of current and future market demand for lighting control systems, though the ease and expense of specifying, designing, purchasing and installing a lighting control system is significantly affected by the protocol choice. The current move in the market is towards protocols, which require fewer pieces of associated lighting control hardware - the ultimate goal being the "addressable ballast".

In particular, the DALI protocol offers high functionality and low hardware cost, and is well along the road to widespread use outside the US, although the requirements of the National Electrical Code, and inertia associated with electrical engineering practice raise questions over whether it can cheaply be implemented in this country. The IBECS protocol from Lawrence Berkeley National Labs may offer similar functionality at an ever lower cost, though no large IBECS systems have yet been made or tested in the laboratory. Such testing would have to be conducted before IBECS could be viewed as a viable technology for lighting control.

The current market standard 1-10V dimming protocol is widely considered to be unreliable and excessively expensive, and progress in energy-efficient lighting is unlikely to be made until improved technology becomes widely available and widely understood by specifiers and electricians.

The industry drive for individually addressable ballast systems is making progress in the US market despite there being no clear demand at this present time for the functionality it offers. The added cost, complexity, and market wariness associated with dimming ballasts along with the overall high first-costs associated with putting together a complete functional system most likely limits broader acceptance. Continued development and investment to lower these costs is occurring even though the economic drivers behind this investment are not obvious to everyone. Codes and standards must also be considered because, if adopted, they could radically change the current economic picture, making greater penetration of controls required in new construction and major renovation. 
APPENDIX I: ENERGY SAVINGS FOR LOAD-SHEDDING BALLAST FOR FLUORESCENT LIGHTING SYSTEMS

\section{Draft Report: $\quad$ ENERGY SAVINGS FOR LOAD-SHEDDING BALLAST FOR FLUORESCENT LIGHTING SYSTEMS}

Sponsored by: Connecticut Light and Power

Prepared by: $\quad$ Yukio Akashi, Ph.D.

Jason Neches

Andy Bierman, MS 


\section{Introduction}

Load shedding ballasts could be used as an effective means of load management by reducing the peak demand for electricity needed for lighting. This is especially true for the brief and infrequent times when demand for electricity approaches the capacity of the power supply system and prices soar as more expensive means of generation an/or transmission are called upon to meet the demand. Lighting offers the opportunity to reduce demand without impacting productivity and normal business activities by dimming to lower power levels. Thereby, lighting is still provided to preserve function, but at a reduced level. However, before such a load-shed technique is applied to the real world, it is important to understand occupants' light level requirements with respect to dimming.

A recent study at the Lighting Research Center (LRC) investigated a detectable range of illuminance change and suggested that occupants could not detect up to $20 \%$ illuminance reduction regardless of initial illuminance or dimming speed within the experimental conditions (Kryszczuk, 2001). A similar study, in which the subjects were more sensitive to illuminance change than in Kryszczuk's study, also suggested that illuminance could be changed by up to $20 \%$ from the initial value without being detected by occupants when they were devoted to tasks (Shikakura, 2001).

However, it is unknown what cues occupants use to detect illuminance change and whether the reducible illuminance range defined in the above studies, up to $20 \%$ change from the initial illuminance, could be extended if slower dimming speeds or smoother dimming functions are employed. To detect such illuminance changes, two cues-memory of the initial illuminance and the transient change in illuminance-are likely decision factors. Sensitivity to the transient illuminance change occurring over time periods shorter than around 3 seconds has already been well investigated by a series of studies on flicker (e.g. Kelly, 1961, 1971). Longer, more drawn out illuminance changes occurring over many seconds, as could easily be done for load shed, has not been investigated as completely. The first experiment of this study seeks to determine if memory of initial illuminance levels is the main clue to detecting dimmed light levels. If so, then less effort could be placed on investigating different dimming rates and functions and more effort focused on determining the appropriate ultimate dim level. With regard to the above question of whether using slower dimming speed and or smoother dimming functions can extend the reducible illuminance range, this study focused on the effects of dimming functions rather than that of very slow dimming speed. This is because fast dimming speed, or short dimming periods that are presumably less than 15 seconds, allow the use of less expensive dimming techniques than long dimming periods, and very long dimming periods, such as hours in length, would not be responsive enough for load shed.

Knowing what people can detect in terms of illuminance reduction is the first step in understanding what occupants' dimming requirements are. Beyond detection, and perhaps more relevant to load shed dimming, is determining what level of dimming is acceptable to occupants. Acceptable dim levels must be at least as low as what is a detectable change in illuminance, and quite possibly they are much lower depending on the context, thereby enabling a greater load shed potential. Since acceptability may vary according to motivation to energy savings, this study also investigated the effects of subjects' bias on acceptable dimming range.

\section{Objectives}

To aid in specifying the dimming parameters of load shedding ballasts, the following objectives were defined:

- To investigate the mechanism of how occupants detect illuminance changes: memory study

- To investigate the effect of different dimming functions on detectable and acceptable dimming ranges: dimming curve study 
- To investigate the effect of motivation on the acceptable dimming range: bias study.

\section{Literature survey}

Recently, the LRC had conducted an experiment on the detection of dimming (Kryszczuk, 2001). The experiment measured when subjects perceived a reduction in luminance on a target and its surroundings under different conditions of initial illuminance, dimming speed, and task context. While conducting a task, each subject signaled the detection of illuminance reduction, as soon as the subject noticed it, by pressing a manual switch. The time necessary for the subject to detect the illuminance change was recorded from which the amount of illuminance reduction was calculated. The results suggested that regardless of the initial illuminance, dimming speed (ranging from 3.7 to 340 lux per second), and task context, the illuminance could be reduced by up to $22 \%$ without being noticed by subjects.

Another recent study conducted similar experiments (Shikakura et al., 2001). The study tackled the same question of to what degree illuminance could be reduced without detection by occupants under different initial illuminance levels, target illuminance levels, and dimming speeds. The results of the experiment suggested:

- When subjects conducted no tasks, $50 \%$ of the subjects could not notice an illuminance change of up to $7 \%$, regardless of the initial illuminance and dimming speed. The subjects hired in Shikakura's experiment seemed more sensitive to illuminance change than subjects in the LRC experiment, or the particular conditions in the experiment heightened their sensitivity.

- When conducting a visual search task or VDT task, or when being interviewed, $50 \%$ of the subjects could not notice the illuminance change by up to $20 \%$ from the initial level.

These studies imply that lighting levels may be reduced by about $20 \%$ without compromising occupant satisfaction with the lighting because such a change would barely be detectible. However, these studies do not deal with occupants' acceptance of possibly greater illuminance change, or the effects of dimming curve functions and occupants' motivation to energy conservation on acceptability. To investigate these factors, the following three experiments were carried out.

\section{Experiments}

In this study, three experiments-memory study, dimming curve study, and bias study-were conducted. The memory study addressed the question of whether one can memorize the initial illuminance level and how long this memory is sustained. The dimming curve study investigated the effects of dimming function on detectability and acceptability of illuminance reduction. The bias study addressed the question of how occupants' motivation influences the acceptability of illuminance reduction. All three experiments used the following experimental setup:

\subsection{Experimental setup}

The experiments used a windowless private office and an adjacent room. Figure 1 shows the private office viewed from the adjacent room. Although, in this picture the door is open, the door remained closed during the experiment. Figure 2 illustrates the plan of the private office and the experimental system in the adjacent room. The interior wall and ceiling of the private office was painted white (reflectance: 85\%). The reflectance of the floor carpet was about $30 \%$. The office was furnished with a bookshelf, a desk and a chair. The office was equipped with three-direct/indirect pendant luminaires suspended from the ceiling. Figure 3 shows the ceiling with the luminaires and the luminous intensity distribution of the luminaires. The experimental apparatus in Figure 2 was composed of the above described luminaires with dimming ballasts and T8 fluorescent lamps; an operating system—a desktop computer, a picoammeter, a photo-sensor, and a DC power supply; a communication system-two 
telephone sets and a "hands-free" phone tool; and a monitoring system—a TV monitor, a digital video camera with a transmitter, and a receiver. Table 1 summarizes the details of the experimental system.

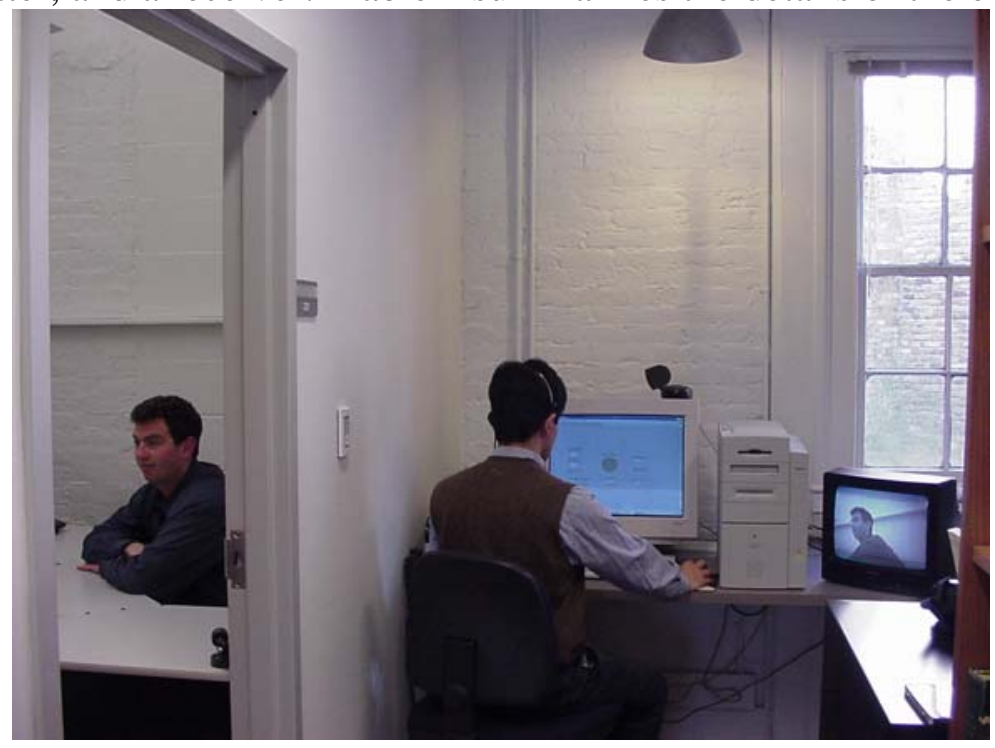

Figure 1. Windowless private office and adjacent room used in all three experiments

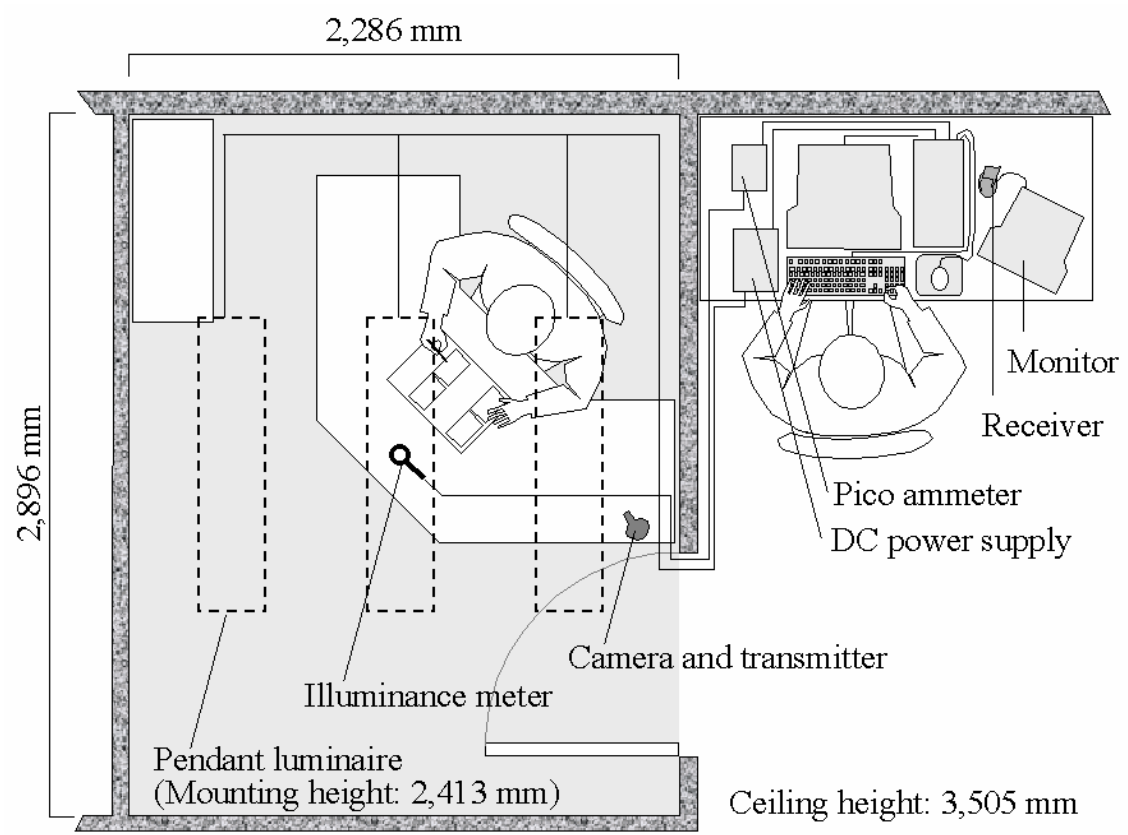

Figure 2. Room plan and experimental setup 

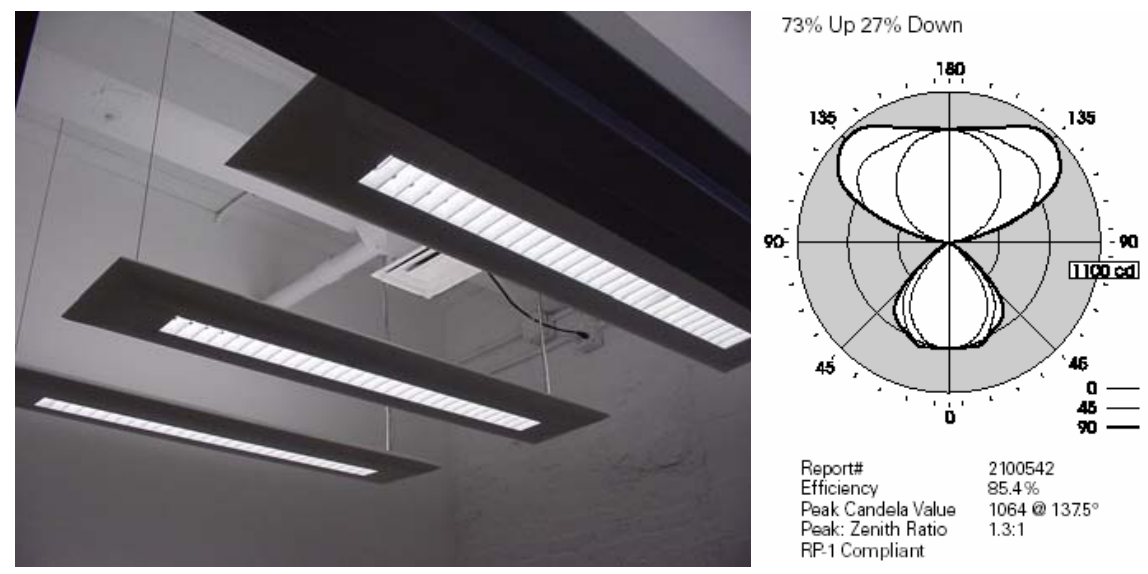

Figure 3. Direct/indirect pendant luminaires (Crescent, Ledalite)

Table 1. Experimental system.

\begin{tabular}{l|l|l}
\hline Components & Product name & Manufacturer \\
\hline 3 Luminaires & Crescent (8316T02PN) & Ledalite \\
3 Ballasts & M2-PD-T8-5C-B-120 Gold Edition & Motorola \\
& Programmed Start, dimming 5 110\% & \\
6 Fluorescent lamps & FO32/835/XP & Osram Sylvania Inc. \\
1 Computer & P5-166 & Gateway \\
1 Picoammeter & 485 Auto ranging Picoammeter & Keithley \\
Software & Lab View 6.0 & National Instruments \\
1 DC power supply & E3632A & Hewlett Packard \\
1 Photo sensor & 268P photopic, cosine response & Graseby Optronics \\
1 Color TV monitor (13”) & PC1342 & CRAIG \\
1 Camera and transmitter & XC10A & X10, Inc. \\
1 Receiver & VR30A & X10, Inc. \\
2 Telephones & DX2NA-12CTXH TEL (BK) & Nitsuko America Co. \\
1 Hands-free phone tool & Vista, M12 & Plantronics \\
\hline
\end{tabular}

\subsection{Memory study}

The memory study investigated whether subjects could memorize the initial illuminance level and how long the memory of the subjects was sustained.

\subsubsection{Experimental conditions}

Table 2 summarizes the experimental conditions employed in the memory study. As independent variables, the target illuminance and eye closure time varied. The initial illuminance was constant at 500 lx. The dependent variable of the experiment was subjective evaluation of whether the illuminance at a given moment is different from the initial illuminance. The three target illuminance levels that are higher than the initial illuminance (500 lx) were used as balancing conditions.

Table 2. Experimental conditions.

\begin{tabular}{l|l}
\hline Variables & Range \\
\hline Target illuminance (lx) & $976,781,625,500,400,320,256$ \\
Eye closure period (seconds) & 3,100 \\
\hline
\end{tabular}




\subsubsection{Experimental procedure}

Twelve subjects, ranging from 22 to 41 years of age, participated in the experiment. An experimenter escorted a subject to the private office and seated the subject in the chair. The subject was exposed to the initial illuminance of $500 \mathrm{~lx}$, for about five minutes so that he/she would adapt to this lighting condition. During this adaptation time, the subject read and signed an informed consent form. The experimenter gave instructions about the experimental procedure to the subject. Then, the experiment began. First, the subject closed his/her eyes for a given period, 3 or 100 seconds. When closing their eyes, the subject used an eye mask to prevent light from coming through their eyelids. While the subject sat with his/her eyes closed, the experimenter changed the initial illuminance level to one of the target illuminance levels. Second, the experimenter asked the subject to open his/her eyes and answer whether the illuminance level was changed from the initial illuminance level. The subject was allowed to choose one from three choices- “down”, "same” or "up”. The orders of the target illuminance and the eye closure time were randomized across subjects. All $14(7 \times 2)$ conditions were repeated three times for each subject.

\subsubsection{Experimental results}

For each of the 14 experimental conditions, all 36 responses (three answers from each of the twelve subjects) were analyzed. Figure 4 shows the percentage of subjects who responded "down” for the 14 conditions. Figure 4 suggests:

- Subjects are able to memorize the brightness under the initial illuminance and can reliably detect reductions greater than $20 \%$.

- As eye closure time increases, memory may fade and therefore the sensitivity to illuminance change may decrease. This is based on the sensitivity to reduction after the 3-second eye closure being higher than that after the 100-second eye closure. For instance, the comparison between the 100 - and 3-second eye closure periods at 50\% probability, when $50 \%$ of the subjects detected illuminance changes, suggests that illuminance reduction by up to $20 \%$ and $30 \%$ of the initial illuminance was undetectable respectively.

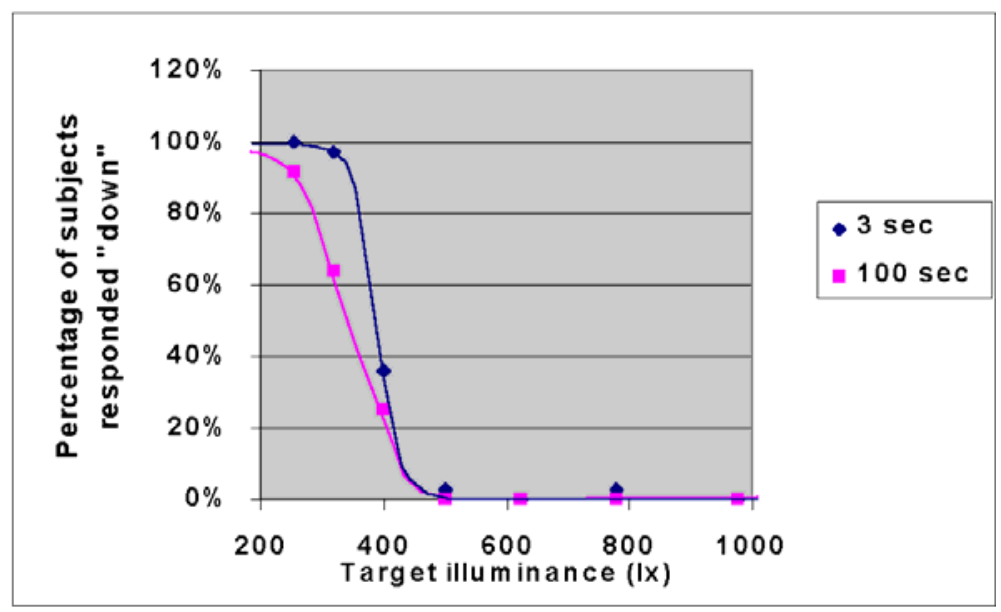

Figure 4. Percentage of subjects who responded “down”.

A two-factor analysis of variance (ANOVA) with replication was conducted using all the subject data. Table 3 shows the results of the ANOVA. Table 3 shows that the ANOVA found significant differences 
between different target illuminance levels and between eye closure period conditions, and in their interaction. These statistical results supported all the above suggestions derived from Figure 4.

Table 3. Two-factor analysis of variance with replication.

\begin{tabular}{lcccccc}
\hline Source of Variation & SS & df & MS & F & P-value & $\mathbf{F}_{\text {crit }}$ \\
\hline Target illuminance & 24.78571 & 6 & 4.130952 & 184.1984 & $1.29 E-67$ & 2.157911 \\
Eye closure period & 0.291667 & 1 & 0.291667 & 13.00536 & 0.000419 & 3.902557 \\
Interaction & 0.5 & 6 & 0.083333 & 3.715818 & 0.001769 & 2.157911 \\
Within & 3.453704 & 154 & 0.022427 & & & \\
Total & 29.03108 & 167 & & & & \\
\hline
\end{tabular}

\subsubsection{Discussion:}

The results of the memory experiment support the hypothesis that people can detect changes of illuminance greater than about $20 \%$ based solely on memory of the initial light level. Therefore, over the range of times tested, the actual dimming speed, or function, presumably has little effect on detecting illuminance reductions.

The data also suggest that subjects could remember the initial illuminance more precisely after the 3second eye closure period than after the 100-second eye closure. This implies that as eye closure time increases, the memory of the initial illuminance fades. However, an experimental confound, dark adaptation, might have influenced the sensitivity of subjects to illuminance changes. Some of the subjects reported that all the illuminance presentations looked brighter after the 100-second eye closure than those after the 3-second eye closure. This might be because the 100-second period allowed the subjects time to adapt to the dark condition of covering their eyes, while less adaptation occurred for the 3-second period. This confound, which proved very difficult to eliminate from this type of experiment, works to make decreases in illuminance less detectable over longer periods of time. Therefore, we cannot conclude at this point whether the further decreases in illuminance for the same level of detection result from the adaptation confound or from some other effect over time. Nevertheless, for the time periods tested, 3 and 100 seconds, we can put a limit on the additional amount of dimming possibly gained by the time factor, which in this case is an additional $10 \%$, and conclude that the dominant effect is the memory of the initial level.

Figure 5 compares the results of this study for 3-second eye closure period with those of the Kryszczuk study. This figure illustrates percentages of subjects who detected illuminance changes as a function of change in illuminance. In the Kryszczuk study, the initial illuminance was $475 \mathrm{~lx}$ and the dimming period ranged from 3.3 seconds to 120 seconds. The line shows averaged data for all his experimental conditions. Both the lines in Figure 5 show similar trends. This supports the earlier conclusion that the memorized initial illuminance is the most dominant factor. However, Figure 5 indicates that the subjects in this study were less sensitive to the illuminance changes than those in the Kryszczuk study. The difference in sensitivity between the two experiments hints that subjects might use another clue, presumably transient change in illuminance. 


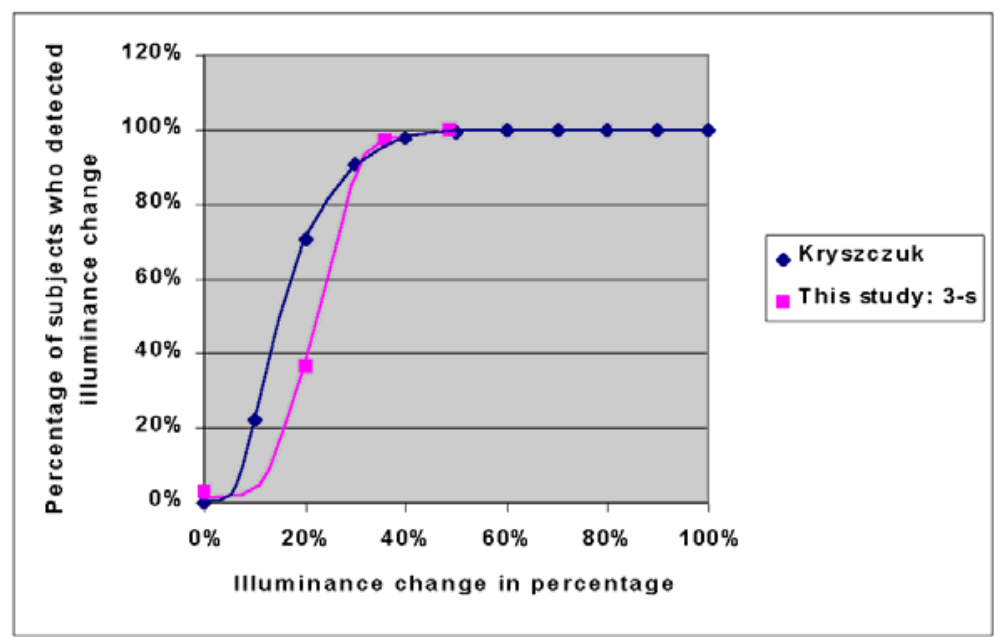

Figure 5. Percentage of subjects who detected illuminance

changes vs. the degree of illuminance changes in percentage

\subsection{Dimming curve study}

The dimming curve study investigated the effects of shape, or curvature of dimming curves on detectability and acceptability of illuminance reduction. Another objective of the dimming curve study was to investigate the effect of task conditions on detectability and acceptability.

\subsubsection{Experimental conditions}

Table 4 shows the experimental conditions employed in the dimming curve study. The independent variables of the experiment were dimming curve function, target illuminance level, and task condition. The initial illuminance and dimming period were constant at $500 \mathrm{~lx}$ and 10 seconds respectively. This experiment used Equation 1 to vary the curvature of dimming curve. Constants $a$ and $b$ were determined according to each target illuminance. As a constant $c$ that determines the curvature, $0.1,0.2$, and 0.4 were used. As the constant decreases, the function becomes more curvilinear. The constant 0.4 made the curve straighter than the constant 0.2 or 0.1 . In this experiment, dimming period: $p$ was constant at 10 (seconds). This is because a pilot study, done using two subjects to determine the dimming period, could not find any difference in detectable range of illuminance reduction while the dimming period ranged between 3 seconds and 120 seconds. Additionally, since a long dimming period, presumably longer than 15 seconds, for load shedding ballasts requires more sophisticated and therefore expensive control systems, the ten-second dimming period was selected as a constant experimental condition.

$$
E=a \times e^{-\frac{t}{c \times p}}+b
$$

where $E$ : illuminance (lx)

$a, b$ : constants

$c$ : a constant to change curvature

$p$ : dimming period (second)

$t$ : time (second)

This experiment used two task conditions: no-task and paper-task conditions. Under the no-task condition, subjects were allowed to freely gaze anywhere in the room. Under the paper task condition, subjects conducted word puzzles. Figure 6 shows a sample of the word puzzle. Four sets of word 
puzzles were printed in each page. Each puzzle had a 12×12 array of 12-point capital letters and a list of words. The goal of subjects was to find and mark the listed words among the $12 \times 12$ array of letters. This experiment used eleven target illuminance levels. Five of the eleven, which were higher than the initial illuminance (500 lx), were used as dummy conditions to make the probabilities of increase and decrease conditions identical.

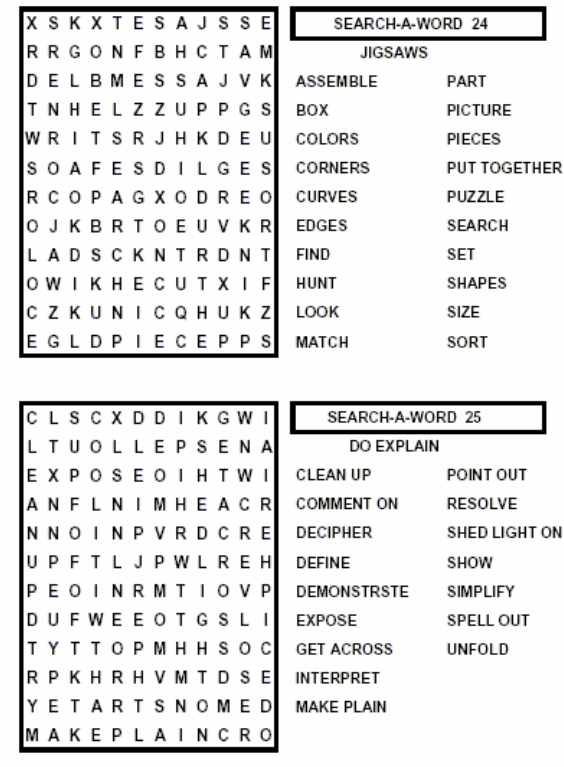

\begin{tabular}{|c|c|c|}
\hline SEARCH-A-V & ORD 26 & $N L Y R I C T M G Y V E$ \\
\hline POETRY & & $Y O I R E X E Q E N D F$ \\
\hline ALLEGORY & METER & $F D \perp N E T E N N O S J$ \\
\hline ALLITERATION & MOTIF & $O N O T E G S \mid R R X V$ \\
\hline BALLAD & ODE & $R B M R A T A V E \mid Y Y$ \\
\hline CANTO & PARALLELISM & $M A N \mid A R E M E E W R$ \\
\hline ELEGY & PARODY & EL JNFPEU IR VO \\
\hline FORM & POEM & $O L Z E H F \perp T O M S G$ \\
\hline GENRE & REFRAIN & PARALLEL ISME \\
\hline HAIKU & SONNET & C D I N KENOTL L L \\
\hline IMAGERY & STANZA & C K SO T Y GMGRL L \\
\hline IRONY & & $U O T N A C N Y F B O A$ \\
\hline $\begin{array}{l}\text { LINE } \\
\text { LYRIC }\end{array}$ & $\begin{array}{l}\text { TONE } \\
\text { VERSE }\end{array}$ & \\
\hline SEARCH-A-WO & ORD 27 & $B E Y G E K J K Q C A T$ \\
\hline CHECKINC & & $C O C Z Z T N U O C C A$ \\
\hline ACCOUNT & DATE & $A R \cup I B S Y A D O J X$ \\
\hline AMOUNT & DEPOSIT & $S D E N \vee E K A B B B C$ \\
\hline BALANCE & ENDORSE & HEE DCRTKACKH \\
\hline BANK & FUNDS & $C Z N V I E E L T H Y E$ \\
\hline воок & INTEREST & A MOUNTAS IASC \\
\hline BOUNCE & MONEY & ERUTANG ISRF K \\
\hline CARD & NUMBER & T D R A C I VDOGHS \\
\hline CASH & SERVICE & I S PENDNDPEPO \\
\hline CHARGE & SIGNATURE & RREBMUNYENOM \\
\hline & SPEND & $W E G C F E D M D Q N$ \\
\hline CREDIT & WRI & \\
\hline
\end{tabular}

Figure 6. Word puzzle on a page.

The dependent variable was a subjective evaluation of whether illuminance level at a given moment was different from the initial illuminance level and whether the illuminance change was acceptable. The subjects also evaluated the acceptability using an eleven-step scale, from zero (very unacceptable) to five (neutral) and ten (very acceptable).

Table 4. Experimental conditions

\begin{tabular}{l|l}
\hline Variables & Range \\
\hline Dimming curve function & Linear, 0.4, 0.2, 0.1 \\
Target illuminance (lx) & 833, 752, 679, 613, 554, 500, 451, 408, 368, 332, 300 \\
Task condition & Paper task (word puzzle), no task (free gaze) \\
\hline
\end{tabular}

\subsubsection{Experimental procedure}

Twenty subjects, ranging from 22 to 41 in age, participated in the experiment. In the experiment, an experimenter escorted a subject to the private office and seated the subject in the chair. The subject adapted himself/herself to the brightness of the initial illuminance, $500 \mathrm{~lx}$, for about five minutes. During the adaptation, the subject read and signed an informed consent form. The experimenter gave instructions about the experimental procedure to the subject. The instructions emphasized that the subject should evaluate not the final illuminance but the whole illuminance presentation in which the illuminance might or might not had changed. After the instruction, the experiment began. The experiment was divided into two sessions-paper-task and no-task sessions. The order of the sessions was counterbalanced across subjects. The subject started performing either the paper-task or no-task under the initial illuminance level of 500 lx. After 5-10 seconds the illuminance was or was not gradually dimmed according to one of the four dimming functions for ten seconds. After the ten second presentation, the experimenter asked the subject the following three questions-(1) whether the illuminance changed ("up", "same”, or “down”), (2) whether the illuminance change (if the subject 
detected) was acceptable (“yes" or "no"), and (3) how acceptable was the illuminance change (if the subject detected). To respond to the third question the subject used an eleven-step scale from 0 to 10 0: "very unacceptable”, 5: "neutral”, and 10: "very acceptable”.

\subsubsection{Experimental results}

Figure 7 shows the results of the detectability of illuminance reduction. Figure 7 suggests that the dimming curvature and task conditions have little influence on the detection of illuminance reductions. Regardless of the dimming curvatures or tasks, $50 \%$ of the subjects could detect the change in illuminance after about a $15 \%$ reduction from the initial illuminance. Figures 8 and 9 show the results of the two acceptability evaluations-yes-or-no and rating evaluations. Figure 8 suggests that the dimming curvatures and tasks little influenced the acceptability of illuminance reductions. $50 \%$ and 80 $\%$ of the subjects accepted illuminance reductions up to about $40 \%$ and $20 \%$ respectively. Figure 9 shows a similar trend to Figure 8.

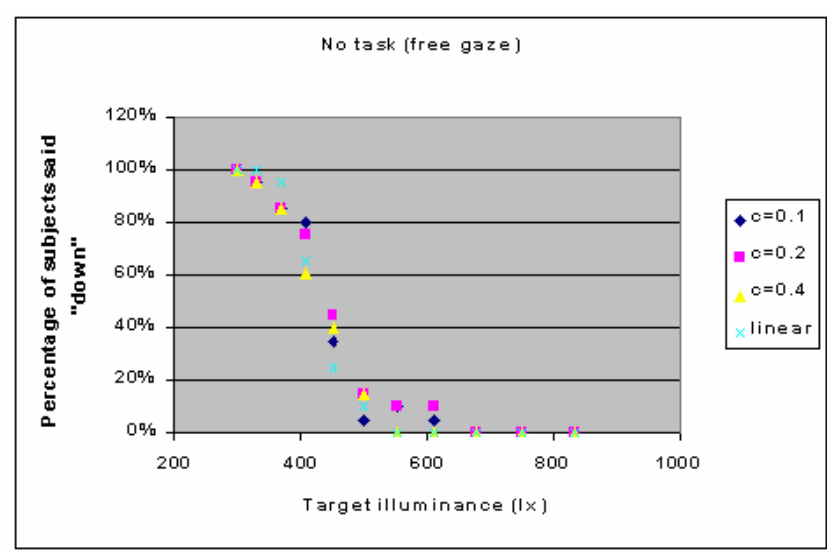

(a) No task (free gaze)

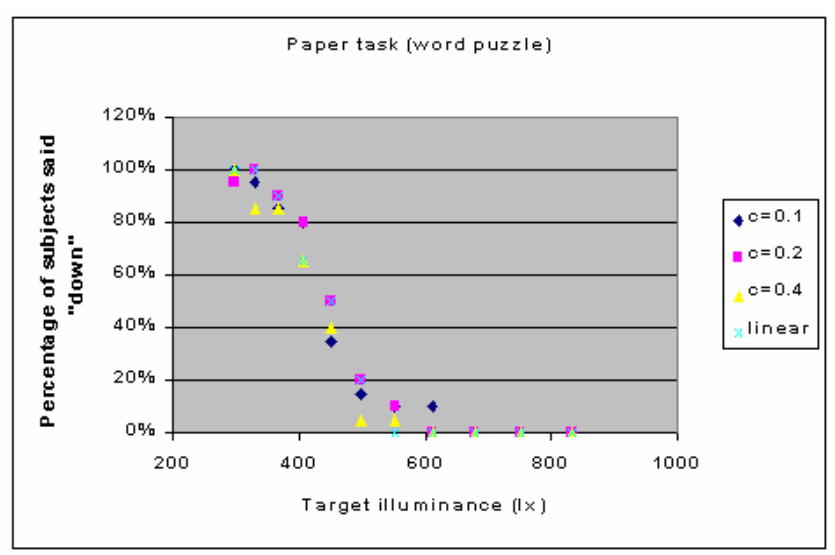

(b) Paper task (word puzzle)

Figure 7. Detectability of illuminance reduction.

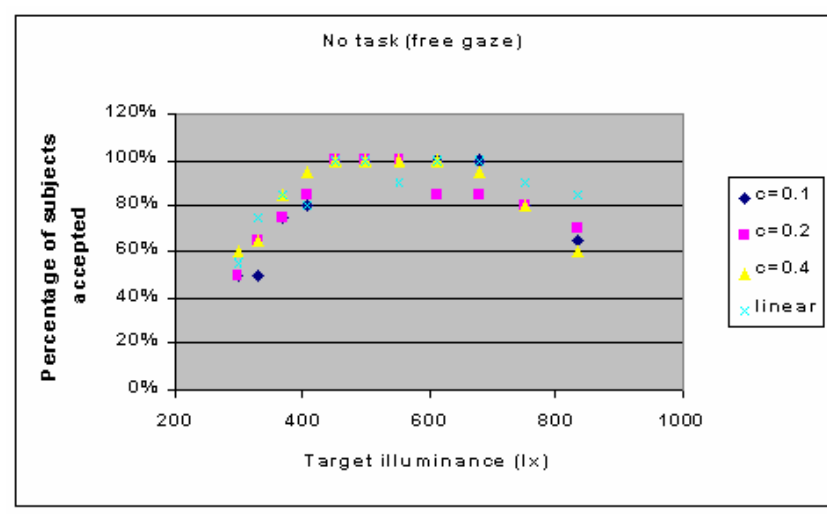

(a) No task (free gaze)

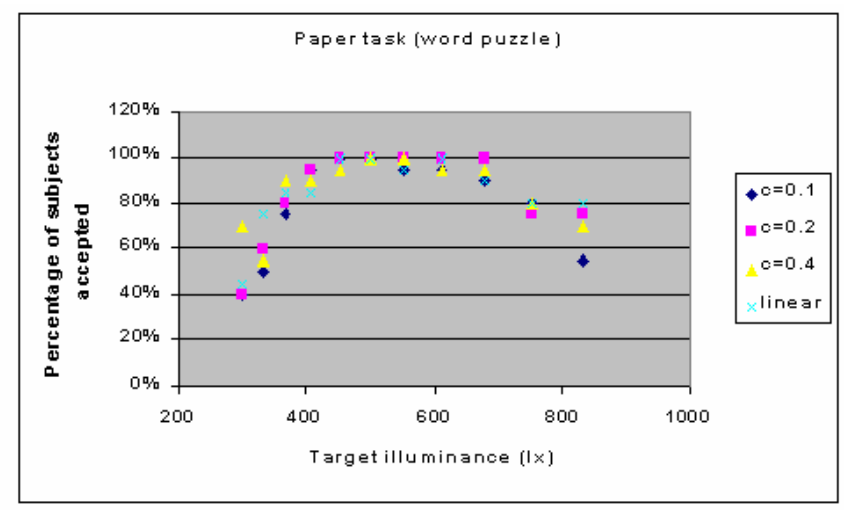

(b) Paper task (word puzzle)

Figure 8. Acceptability of illuminance reduction. 


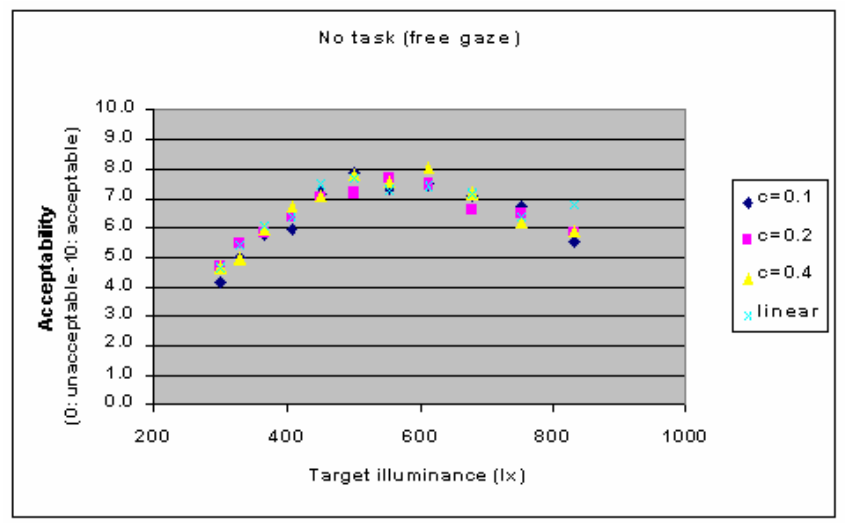

(a) No task (free gaze)

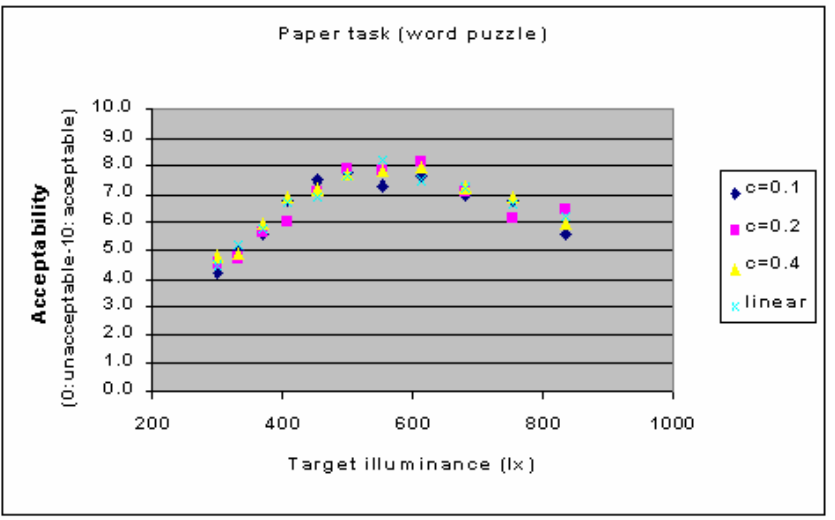

(b) Paper task (word puzzle)

Figure 9. Acceptability of illuminance reduction (0 to 10 scale).

\subsubsection{Discussion}

Figure 10 diagrams the relationship between the percentage of subjects who accepted the change in illuminance and the acceptability ratings. Figure 10 illustrates a linear relationship with high $\mathrm{R}^{2}$ value: 0.83 between the two evaluations and therefore implies the consistency of evaluation of all the subjects.

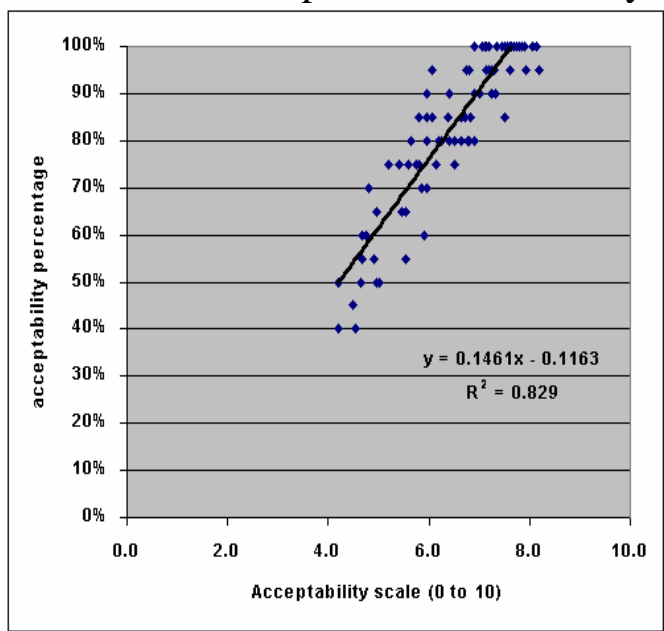

Figure 10. Relationship between acceptability rating and percentage.

\subsection{Bias study}

The bias study investigated how bias given to subjects influences the acceptability to illuminance change. Another objective of the bias study was to investigate how tasks performed by subjects influence the acceptability of illuminance change.

\subsubsection{Experimental conditions:}

Table 5 summarizes the experimental conditions. As independent variables, the target illuminance, task condition, font size, and bias condition varied. A wider range (20 lx to 1,000 lx) than the dimming curve study was employed because it was expected that biased subjects might accept lower target illuminance levels. For both the paper and VDT tasks, the same word puzzles as the dimming curve study were used. For the VDT task, a desktop personal computer (DELL, OptiPlex) with a 16” CRT screen was used. The dimming period and initial illuminance were constant at 10 seconds and 500 lx respectively. The dependent variable was subjective evaluation of whether the illuminance level at a given moment was different from the initial illuminance level and whether the illuminance change was acceptable. The 
subjects also evaluated the acceptability using the same eleven-step scale as the dimming curve study, from zero (very unacceptable) to five (neutral) and ten (very acceptable).

Table 5. Experimental conditions

\begin{tabular}{l|l}
\hline Variables & Range \\
\hline Target illuminance (lx) & $1000,900,820,740,660,580,500,420,340,260,180,100,20$ \\
Task condition & Paper task, VDT task \\
Font size (point) & 6,12 \\
Bias & No-bias, bias \\
\hline
\end{tabular}

\subsubsection{Experimental procedure}

Four subjects, ranging 25 to 28 in age, participated in the experiment. In the experiment, an experimenter escorted a subject to the private office and seated the subject in the chair. The subject adapted himself/herself to the brightness of the initial illuminance, $500 \mathrm{~lx}$, for about five minutes. During the adaptation, the subject read and signed an informed consent form. The experimenter gave instructions about the experimental procedure to the subject. The experiment was divided into two sessions, one for a no-bias session and the other for a bias session. All the subjects started with the nobias session. In each session, the subject conducted a paper task and a VDT task. Both tasks used the same word puzzles as the dimming curve study. Two font sizes, 6 and 12 points were used for the puzzles in both the tasks. The order of the tasks and font sizes were counterbalanced across subjects. For each of the four combinations ( 2 tasks $\times 2$ font sizes), thirteen target illuminance levels were presented to the subject. In each presentation, the illuminance was gradually reduced from the initial illuminance (500 lx) to each of the thirteen target illuminance levels for ten seconds following linear dimming functions. The order of the target illuminance levels was randomized. The subject started performing either the paper task or the VDT task under the initial illuminance level, $500 \mathrm{~lx}$. After the ten-second dimming presentation, the experimenter asked the subject whether the illuminance change was detectable, and if so, acceptable ("yes” or "no"). After completing the no-bias session, the experimenter gave an instruction sheet to the subject. The instructions given to subjects are listed below. All three following paragraphs were given to the subject as one general bias:

- Economic effect: Assume you are working for a company in the capital district of NY that is on the verge of blackouts. To avoid building more power plants, we have to find a way to cut peak electricity consumption. Some feasibility studies have shown that dimming the lighting is an effective way to cut the peak load without compromising productivity. Such load shedding could reduce your company's electricity bill by about $\$ 1,500 / 10 \mathrm{~kW} /$ year.

- Global effect: Simulations show that every new power plant will lead to an increase of $\mathrm{CO}_{2}$ gas, which will cause a global warming effect. We have already seen such greenhouse effects such as an increase of the sea level, climatic change, and more frequent floods. Demand side management through load shedding ballasts can reduce the number of new power generators and therefore reduce additional contribution to the greenhouse effect.

- Local effect: New power plants and transmission lines need to go somewhere. For a variety of reasons, health, aesthetic and economic, people generally do not want such structures built near them. The reality is that people have little control over their community, and a new plant or transmission line may be built near you. Knowing that load shedding ballasts will reduce the number of new plants and transmission lines needed, how acceptable are the following dimming levels?

After reading the instructions, the subject followed the same procedure as the no-bias session. Each session took a subject about 30 to 40 minutes. The subject conducted the two sessions on different days. 


\subsubsection{Experimental results}

Figure 11 shows the results of the acceptability evaluations to the illuminance changes under the four task conditions-(1) paper task with 6-point font size, (2) paper task with 12-point font size, (3) VDT task with 6-point font size, and (4) VDT task with 12-point font size. Figure 11 suggests that the instruction given to the subjects between the two sessions somewhat affected the subject responses. It is apparent that the acceptable dimming range by biased subjects is wider than that by non-biased subjects, although the probability curves are not smooth because of the small sample size. Figure 12 compares acceptable dimming ranges between biased subjects and non-biased subjects. Each of the two lines shows the average of the percentages for all four task conditions. The comparison of illuminance reductions, which all four subjects accepted, between the non-biased subjects and the biased subjects shows that the acceptable target illuminance of the biased subjects was lower by one to two steps, $80 \mathrm{~lx}$ to $160 \mathrm{~lx}$ respectively, than that of the non-biased subjects. The comparison of the acceptable target illuminance levels between the four task conditions in each of the graphs (a) and (b) in Figure 11 suggests that the acceptable target illuminance for the VDT task tends to be lower than that for the paper task. It also suggests that the lager font size might lower the acceptable target illuminance.

The results of this study imply that if the motivation of occupants towards energy savings is raised, occupants may accept larger reductions in illuminance. However, further studies need to be done with more subjects to obtain more reliable data.

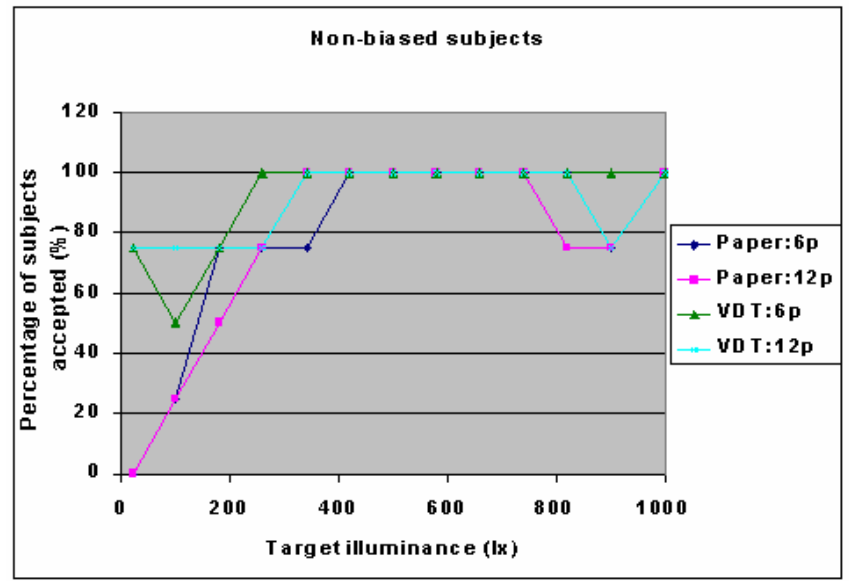

(a) Non-biased subjects

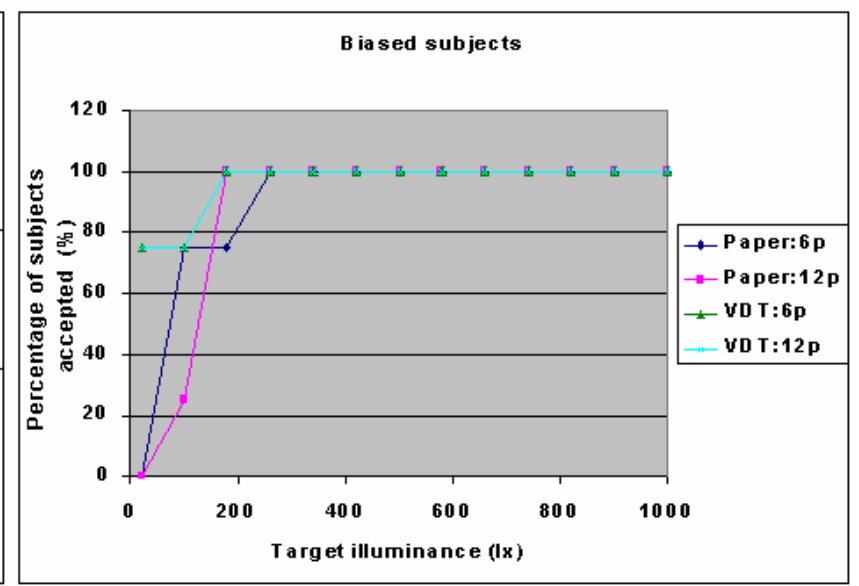

(b) Biased subjects

Figure 11. Acceptability of illuminance reduction in the bias study

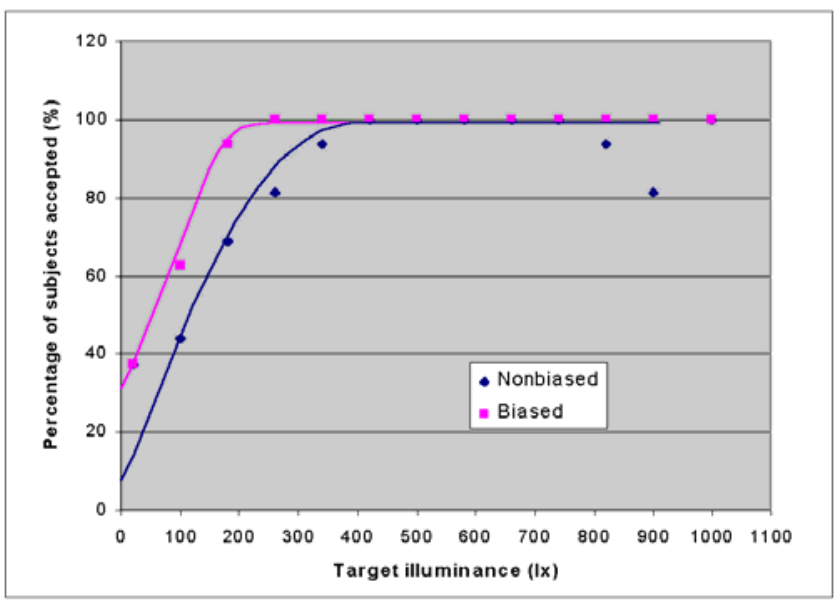

Figure 12. Acceptability of illuminance reduction

biased subjects and non-biased subjects 


\section{Conclusions}

The memory study indicates that occupants are able to memorize the initial brightness of their surroundings and respond to illuminance changes based on this memory. Lengthening the time in the dark from exposure to the initial lighting conditions appears to decrease sensitivity to illuminance reduction, but this result is potentially confounded with light adaptation, thereby possibly diminishing the magnitude of this effect. The dimming curve study suggests that the dimming curvature and task conditions have little influence on the detection of illuminance reductions. It was also found that regardless of the dimming curvatures or tasks, $50 \%$ of the subjects could detect a reduction of initial illuminance of about a $15 \%$ or greater. With regard to acceptability, the experimental results suggest that the dimming curvatures and tasks had little influence on the acceptability of illuminance reductions. The bias study suggested that the acceptable dimming range of biased subjects is wider than that of non-biased subjects, offering an additional 20 to 30\% acceptable illuminance reduction. More precise results with smoother probability curves, especially for the specific task analyses require a larger number of subjects. That data is now being collected as this work continues.

\section{Implications for load shedding}

If the amount of power reduction for effective load management requires dimming by more than $15 \%$ then there is a high probability that people will notice the change in illuminance, no matter how that change is accomplished. Fortunately, changes of this magnitude and up to about $25 \%$ are acceptable to most people. Furthermore, by educating people on the reasons for and benefits of load shedding, which is called biasing when under experimental conditions, the acceptable dimming range can be extended to $50 \%$ or more. This provides great insight into how to manage a successful load-shed program. To maximize demand savings, education of the public, or affected persons, should be a high priority.

\section{Further study}

It is important to add more subjects to the bias study to specify acceptable dimming ranges for biased and non-biased occupants. It is also useful to investigate how dimming periods longer than two minutes affect the acceptability of illuminance reduction. Eventually, based on our findings, we will have to conduct field studies in real commercial offices to verify whether occupants are comfortable with the specified requirements of the dimming range, curve, and speed and evaluate how much electric energy can be saved through the load shedding system.

\section{Acknowledgements}

The authors would acknowledge Connecticut Light and Power for funding this research. The authors would like to acknowledge Peter Boyce and Mark Rea for their comments on the experiments. The authors would like to thank Lei Deng, Jean Paul Freyssinier, Chao Ling, and Francisco Garza for their technical support.

\section{References}

Kelly, D.H. (1961) Visual response to time-dependent stimuli, Journal of the Optical Society of America, No. 51

Kelly, D.H. (1971) Theory of flicker and transient responses, Journal of Optical Society of America, No. 61

Kryszczuk, K. (2001) Detection of slow light level reduction, 2001 IESNA Conference Proceedings, pp.315-322 
Shikakura, T., Morikawa, H., Nakamura, Y. (2001), Research on the perception of lighting fluctuation in a luminous offices environment, Journal of the Illuminating Engineering Institute of Japan, 85, 5, pp. 346-351 
APPENDIX J: THE EFFECT OF BIAS TOWARDS ENERGY SAVINGS ON OCCUPANTS' DIMMING REQUIREMENTS

\section{Draft Report: $\quad$ The effect of bias towards energy savings on occupant's dimming requirements}

Sponsored by US Department of Energy

Prepared by: $\quad$ Yukio Akashi, PhD

Jason Neches 


\section{Introduction}

Load shedding ballasts could be used as an effective method for load management by reducing the peak electricity demand needed for lighting. However, before they are applied to the real world, it is important to understand occupants' light level requirements with respect to dimming.

A recent study at the Lighting Research Center (LRC) investigated a detectable range of illuminance change and suggested that occupants could not detect up to $20 \%$ illuminance reduction regardless of initial illuminance or dimming speed within the experimental conditions (Kryszczuk, 2001). A similar study, in which the subjects were more sensitive to illuminance change than in Kryszczuk's study, also suggested that illuminance could be changed by up to $20 \%$ from the initial value without being detected by occupants when they were devoted to tasks (Shikakura, 2001). More recently, two studies at the LRC investigated what cues occupants used to detect illuminance change and whether the reducible illuminance range defined in the above studies, up to $20 \%$ change from the initial illuminance, could be extended if slower dimming speeds or smoother dimming functions are employed (Akashi et al., 2002). The first Akashi et al. study sought to determine whether memory of the initial illuminance level is the main cue to detecting dimmed light levels. The results of this study suggested that occupants were able to memorize a room's brightness under the initial illuminance and could reliably detect reductions greater than $20 \%$ when the dimming period was relatively short. As the period of dimming increased, memory may have faded and therefore the sensitivity to illuminance change decreased. These results suggested that memory is the primitive decision factor. The results also led to a hypothesis that longer dimming periods, and therefore a slower rate of illuminance change, might lead to decreased sensitivity in detecting illuminance reduction.

With regard to the above question of whether slower dimming speeds and/or smoother dimming functions can extend the undetectable illuminance range, the second Akashi et al. study focused on the effect of dimming functions. The study also investigated acceptable range of illuminance reductions because, beyond detection, and perhaps more relevant to load shed dimming, is determining what level of dimming is acceptable to occupants. The results indicated that dimming function had little effect on the detectability and acceptability of illuminance change. The subjects accepted larger illuminance reductions than the above mentioned undetectable illuminance reduction-e.g., $50 \%$ of the subjects accepted illuminance reductions up to about $40 \%$ of the initial illuminance level while they could detect illuminance reductions greater than $15 \%$ of the initial illuminance.

Since acceptability may vary according to one's motivation for energy savings, it is important to investigate the effect of subjects' bias on the acceptable dimming range. Therefore, in this study, the LRC attempted to investigate whether a pre-assigned bias towards energy savings could increase the acceptability of dimming through load shedding ballasts. Another objective of this study was to investigate how tasks performed by subjects influence the acceptability of illuminance change.

\section{Experiment}

\section{Experimental setup:}

The experiment used a windowless private office and an adjacent room. Figure 1 shows the private office viewed from the adjacent room. Although, in this picture the door is open, the door remained closed during the experiment. Figure 2 illustrates the plan of the private office and the experimental system in the adjacent room. The interior wall and ceiling of the private office was painted white (reflectance: 85\%). The reflectance of the floor carpet was about $30 \%$. The office was furnished with a bookshelf, a desk and a chair. The office was equipped with three-direct/indirect pendant luminaires suspended from the ceiling. Figure 3 shows the ceiling with the luminaires and the luminous intensity distribution of the luminaires. The experimental apparatus in Figure 2 was composed of the above 
described luminaires with dimming ballasts and T8 fluorescent lamps; an operating system-a desktop computer, a picoammeter, a photo-sensor, and a DC power supply; a communication system- two telephone sets and a "hands-free" phone tool; and a monitoring system — a TV monitor, a digital video camera with a transmitter, and a receiver. Table 1 summarizes the details of the experimental system.

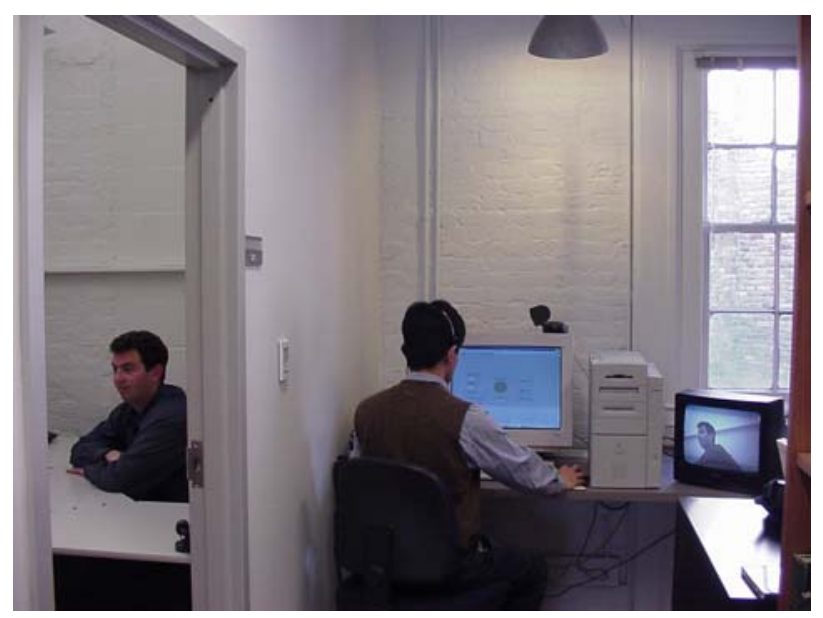

Figure 1. Windowless private office and adjacent room used in all three experiments

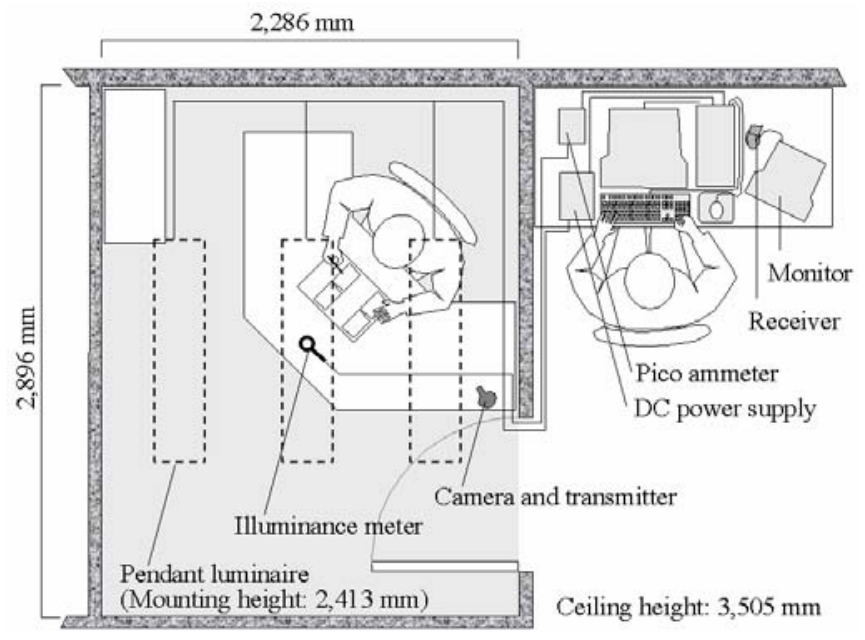

Figure 2. Room plan and experimental setup
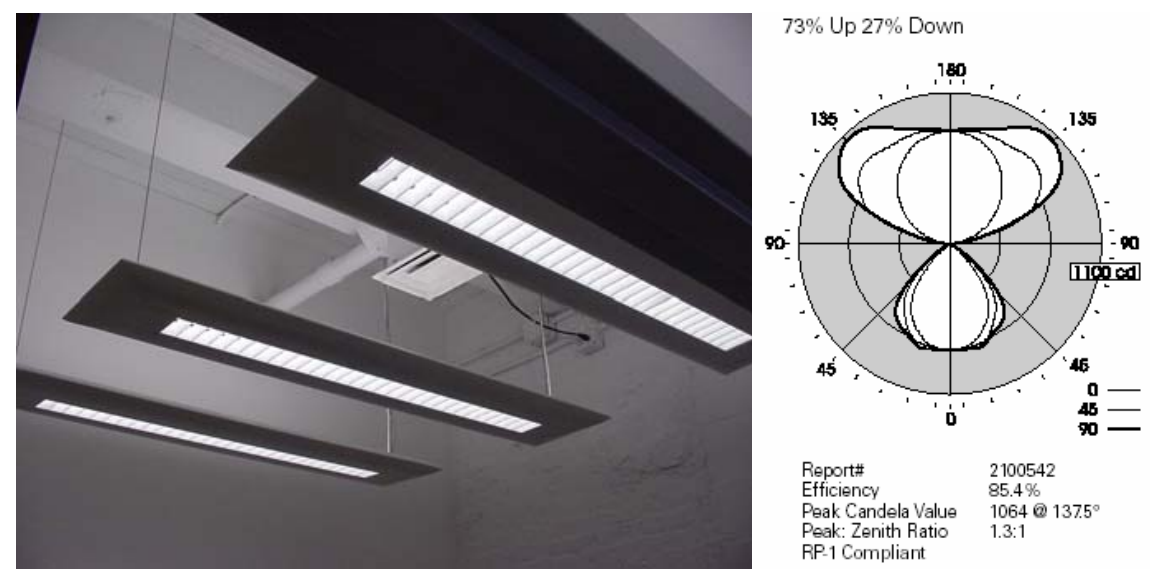

Figure 3. Direct/indirect pendant luminaires (Crescent, Ledalite) 
Table 1. Experimental system.

\begin{tabular}{l|l|l}
\hline Components & Product name & Manufacturer \\
\hline 3 Luminaires & Crescent (8316T02PN) & Ledalite \\
3 Ballasts & M2-PD-T8-5C-B-120 Gold Edition & Motorola \\
& Programmed Start, dimming 5 110\% & \\
6 Fluorescent lamps & FO32/835/XP & Osram Sylvania Inc. \\
1 Computer & P5-166 & Gateway \\
1 Picoammeter & 485 Auto ranging Picoammeter & Keithley \\
Software & Lab View 6.0 & National Instruments \\
1 DC power supply & E3632A & Hewlett Packard \\
1 Photo sensor & 268P photopic, cosine response & Graseby Optronics \\
1 Color TV monitor (13”) & PC1342 & CRAIG \\
1 Camera and transmitter & XC10A & X10, Inc. \\
1 Receiver & VR30A & X10, Inc. \\
2 Telephones & DX2NA-12CTXH TEL (BK) & Nitsuko America Co. \\
1 Hands-free phone tool & Vista, M12 & Plantronics \\
\hline
\end{tabular}

\section{Experimental Conditions:}

Table 2 summarizes the experimental conditions. As independent variables, the target illuminance, task condition, font size, and bias condition varied. Both the paper and VDT tasks used a word puzzle as shown in Figure 4. The VDT task used a desktop personal computer (DELL, OptiPlex) with a 16” CRT screen. The dimming period and initial illuminance were constant at 10 seconds and 500 lx respectively. The dependent variables were an evaluation of whether the illuminance level at a given moment was higher, lower, or identical to the initial illuminance level and whether the illuminance change was acceptable. The subjects also evaluated the acceptability using an eleven-step scale, from zero (very unacceptable) to five (neutral) and ten (very acceptable). 
Table 2. Experimental conditions

\begin{tabular}{l|l}
\hline Variables & Range \\
\hline Target illuminance (lx) & $1000,900,820,740,660,580,500,420,340,260,180,100,20$ \\
Task condition & Paper task, VDT task \\
Font size (point) & 6,12 \\
Bias & Non-biased, biased \\
\hline
\end{tabular}

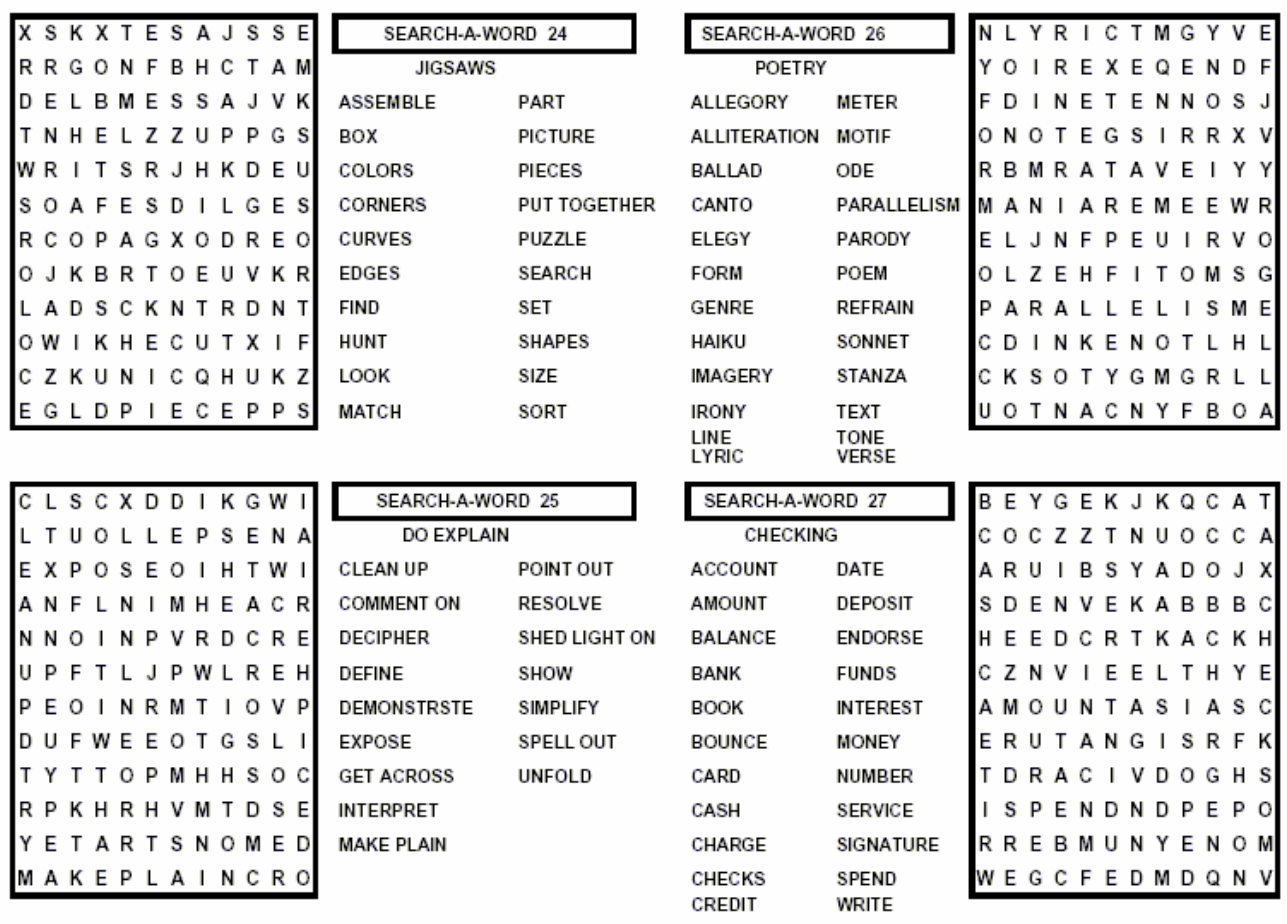

Figure 4. Word puzzle on a page.

\section{Experimental procedure:}

Twenty subjects, ranging from 20 to 40 in age, participated in the experiment. In the experiment, an experimenter escorted a subject to the private office and seated the subject in the chair. The subject adapted himself/herself to the brightness of the initial illuminance, $500 \mathrm{~lx}$, for about five minutes. During the adaptation, the subject read and signed an informed consent form. The experimenter gave instructions about the experimental procedure to the subject. The experiment was divided into two sessions, one for a non-biased session and the other for a biased session. Half the subjects started with the non-biased session, the other half started with the biased section. In each session, the subject conducted a paper task and a VDT task. Both tasks used word puzzles. Two font sizes, 6 and 12 points were used for the puzzles in both the tasks. The order of the tasks and font sizes were counterbalanced across subjects. For each of the four combinations ( 2 tasks $\times 2$ font sizes), thirteen target illuminance levels were presented to the subject. In each presentation, the illuminance was gradually increased or reduced from the initial illuminance $(500 \mathrm{~lx}$ ) to each of the thirteen target illuminance levels for ten seconds following linear dimming functions. The order of the target illuminance levels was randomized. The subject started performing either the paper task or the VDT task under the initial illuminance level, $500 \mathrm{~lx}$. After the ten-second dimming presentation, the experimenter asked the subject whether the illuminance change was detectable, and if so, acceptable ("yes" or "no"). Before undertaking the biased session, the experimenter gave an instruction sheet to the subject. The instructions given to subjects are listed below. All three following paragraphs were given to the subject as one general bias: 
Economic effect: Assume you are working for a firm in the capital district of New York. This area is on the verge of a power shortage. Power shortages cause an increase in the price of electricity due to the laws of supply and demand. Some feasibility studies have shown that dimming the lighting is an effective way to cut the peak electricity load without compromising worker productivity. Such load shedding could reduce your company’s electricity bill by about \$1,500/10kW/year.

Global effect: Simulations show that every new power plant will lead to an increase in CO2 gas, which will cause a global warming effect. We have already seen such greenhouse effects such as an increase in the sea level, climatic change, and more frequent floods. Electricity demand side management through load shedding ballasts can reduce the number of new power generators and therefore reduce your contribution to the greenhouse effect.

Local effect: New power plants and transmission lines need to go somewhere. For a variety of reasons, health, aesthetic and economic, people generally do not want such structures built near them. The reality is that people have little control over their community, and a new plant or transmission line may be built near you. Knowing that managing electricity demand through lighting will reduce the number of new plants and transmission lines needed, how acceptable are the following dimming levels?

After reading the instructions, the subject followed the same procedure as the non-biased session. Each session took a subject about 30 to 40 minutes. The subject conducted the two sessions (biased and nonbiased) on different days.

\section{Experimental results:}

Figure 5 shows the results of the detectability of illuminance change. Figure 5 confirmed the results of the former studies that occupants could not detect up to $20 \%$ illuminance reduction and obviously shows that detectability of illuminance reduction is unaffected by bias. This should be the case, as detectability is a lower order response unrelated to bias feelings.

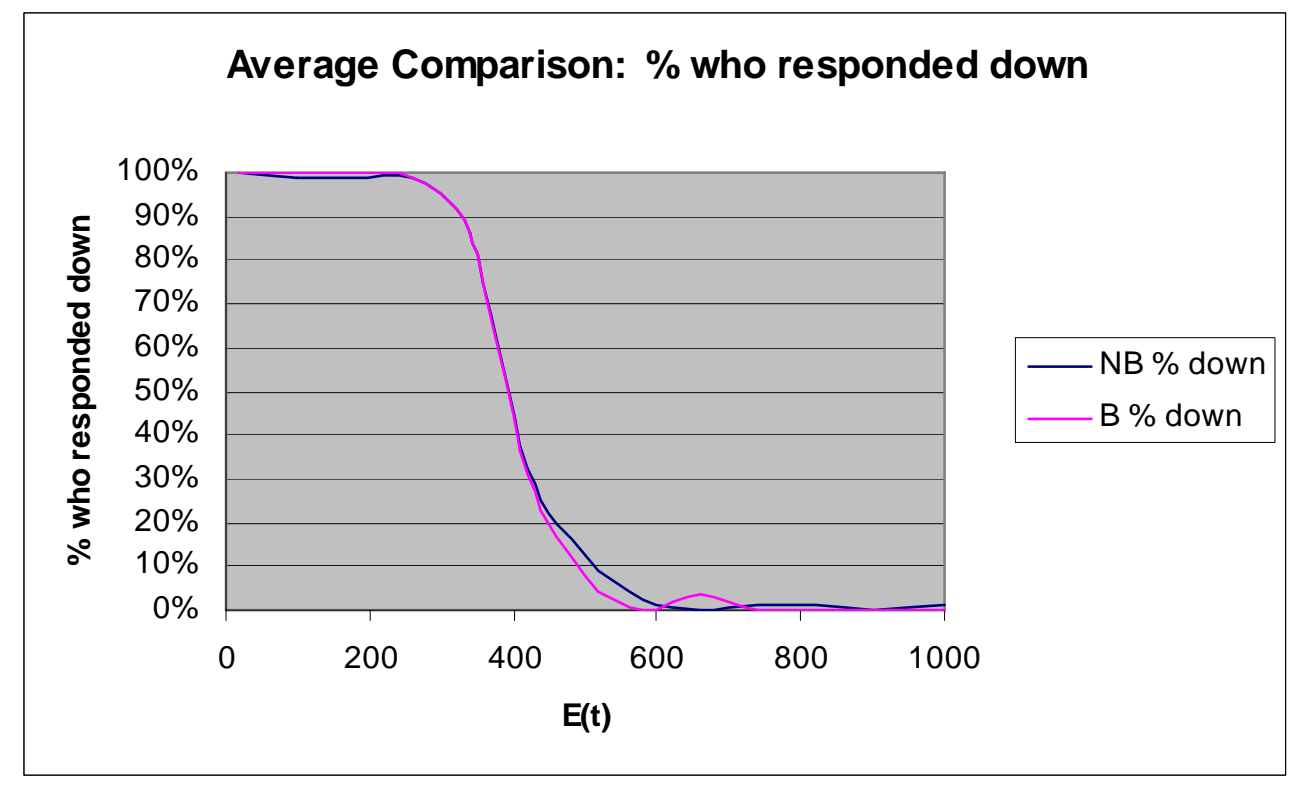

Figure 5. Detectability of illuminance change in the bias study for biased (B) and non-biased (NB) subjects 
Figures 6 and 7 show the results of acceptability evaluations to illuminance changes under the four task conditions - (1) paper task with 6-point font size, (2) paper task with 12-point font size, (3) VDT task with 6-point font size, and (4) VDT task with 12-point font size. Figures 6 and 7 suggest that the instruction given to subjects between the two sessions somewhat affected their responses. It is apparent that the acceptable dimming range for biased subjects is wider than that for non-biased subjects. Figure 8 compares acceptable dimming ranges between biased subjects and non-biased subjects, averaged for all four task conditions. Figure 8 shows that the acceptable target illuminance of biased subjects was lower (by approximately 80 lux) than that of non-biased subjects. For instance, $50 \%$ of the biased subjects accepted illuminance reduction by $380 \mathrm{~lx}$ (76 \% of the initial illuminance from $500 \mathrm{~lx}$ ) while 50 $\%$ of the non-biased subjects accepted illuminance reduction by $300 \mathrm{~lx}$ (60\% of the initial illuminance from $500 \mathrm{~lx}$ ). The $80^{\text {th }}$ percentile acceptable illuminance reductions are $270 \mathrm{~lx}$ for biased subjects and $190 \mathrm{~lx}$ for non-biased subjects. Comparison of Figures 6 and 7 suggests that the acceptable target illuminance for the VDT task tends to be lower than that for the paper task. We can assume that this difference was due to the self-luminous VDT screen. Interestingly, very little effect was shown with reducing the font size within a given task. Most likely, for both the point sizes of 6 and 12, the visual performance was already plateaued for such short-term tasks. Figures 9 through 11 show subjective ratings (scale $0-10$ ) which where asked of subjects as a method to verify acceptability evaluations. These figures indicate similar tendencies to the above results of the yes/no appraisals.

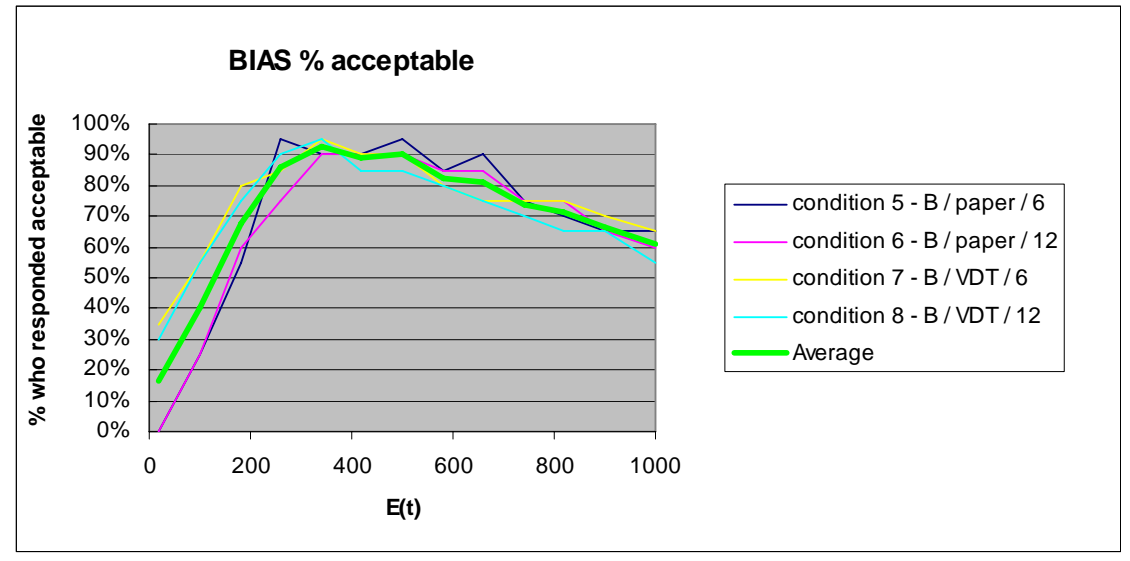

Figure 6. Acceptability of illuminance change for biased (B) subjects

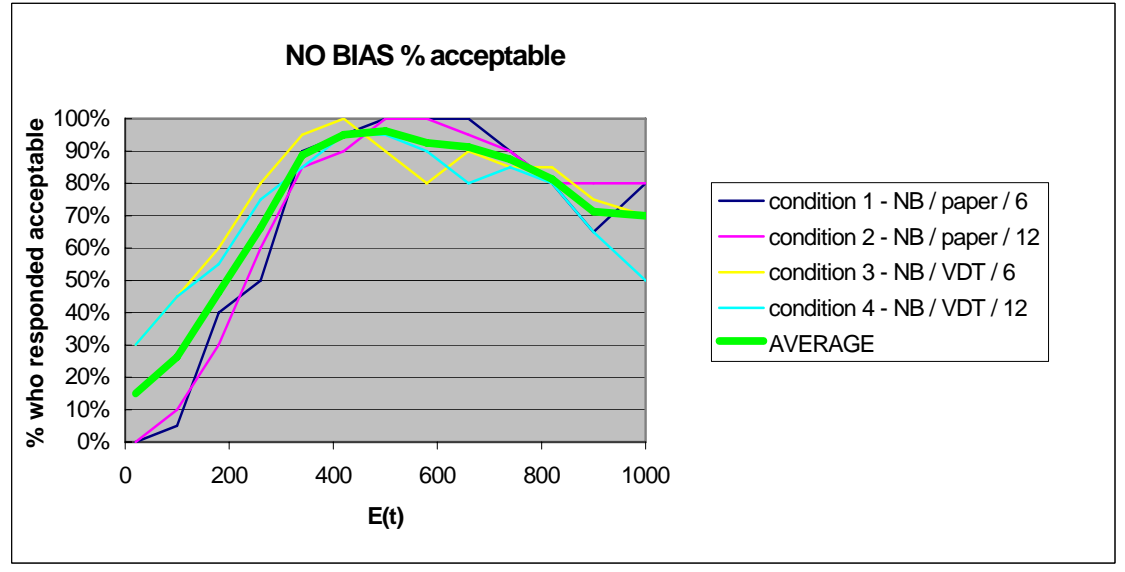

Figure 7. Acceptability of illuminance change for non-biased (NB) subjects 


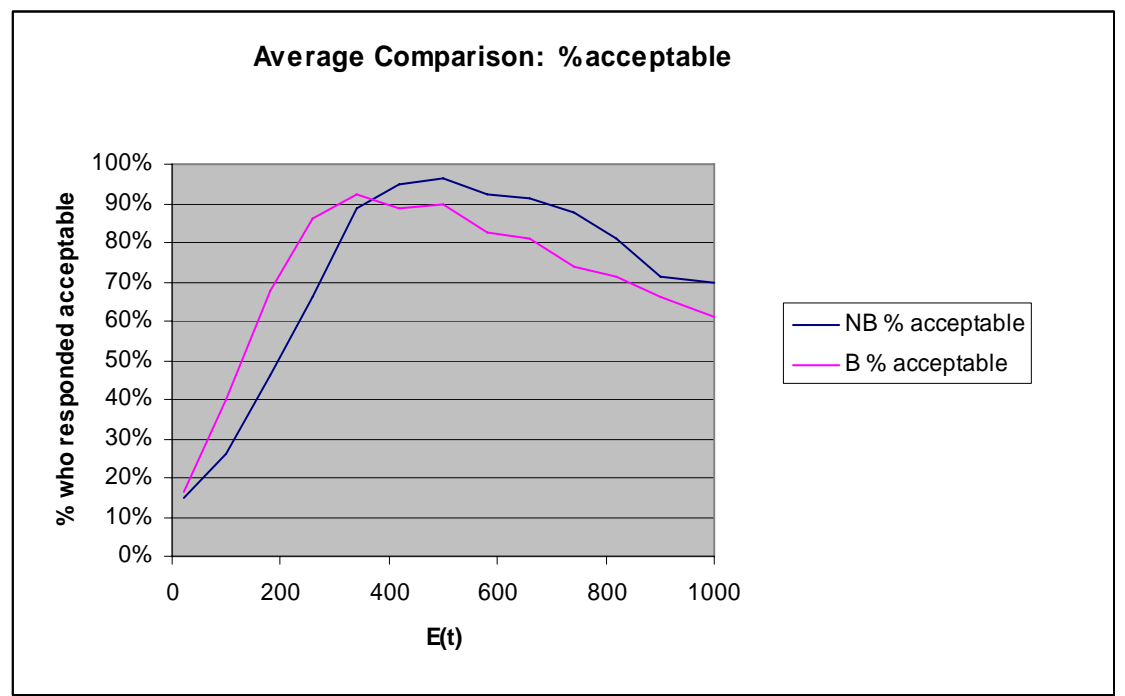

Figure 8. Acceptability of illuminance change for biased (B) and nonbiased (NB) subjects, averaged for all four tasks

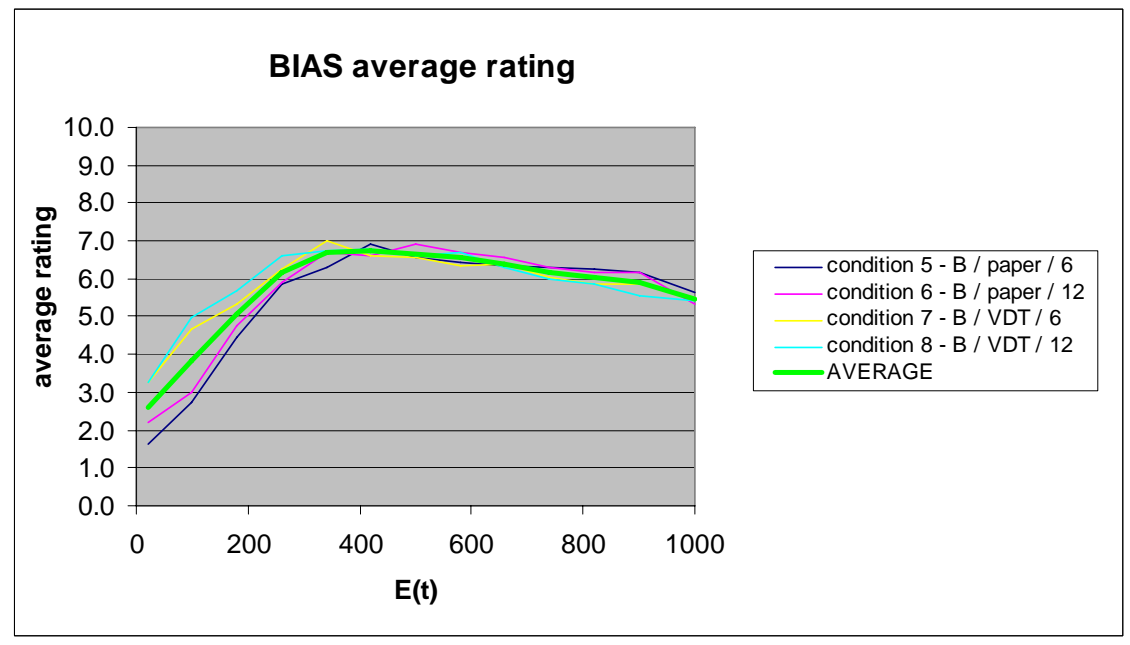

Figure 9. Subjective rating of illuminance change for biased (B) subjects

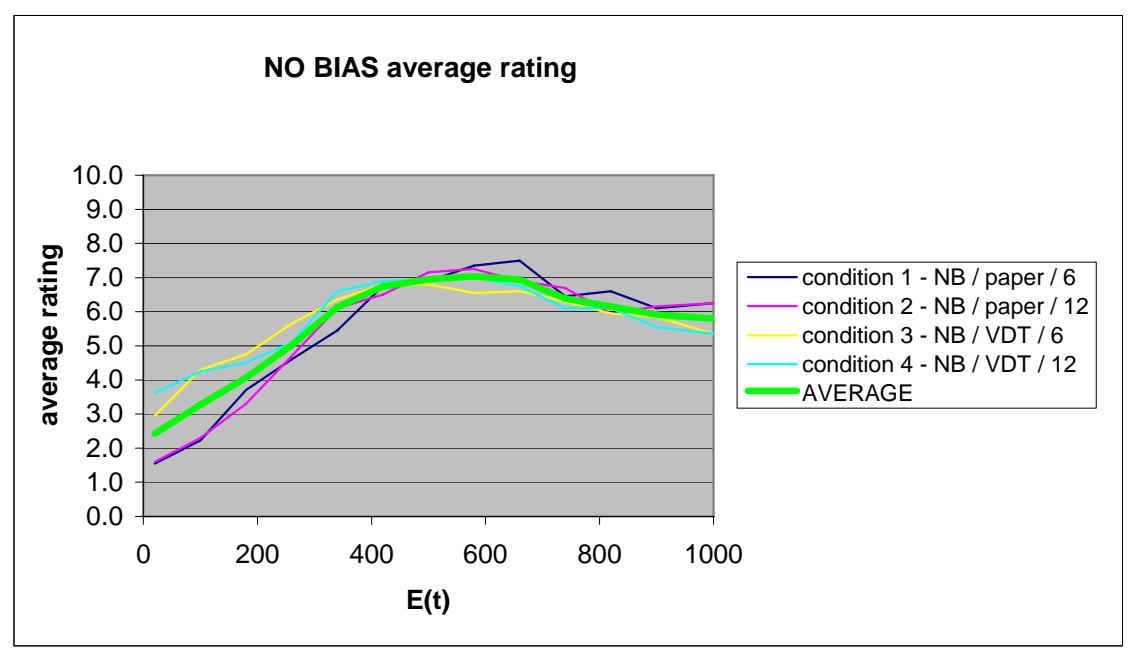

Figure 10. Subjective rating of illuminance change for non-biased (NB) subjects 


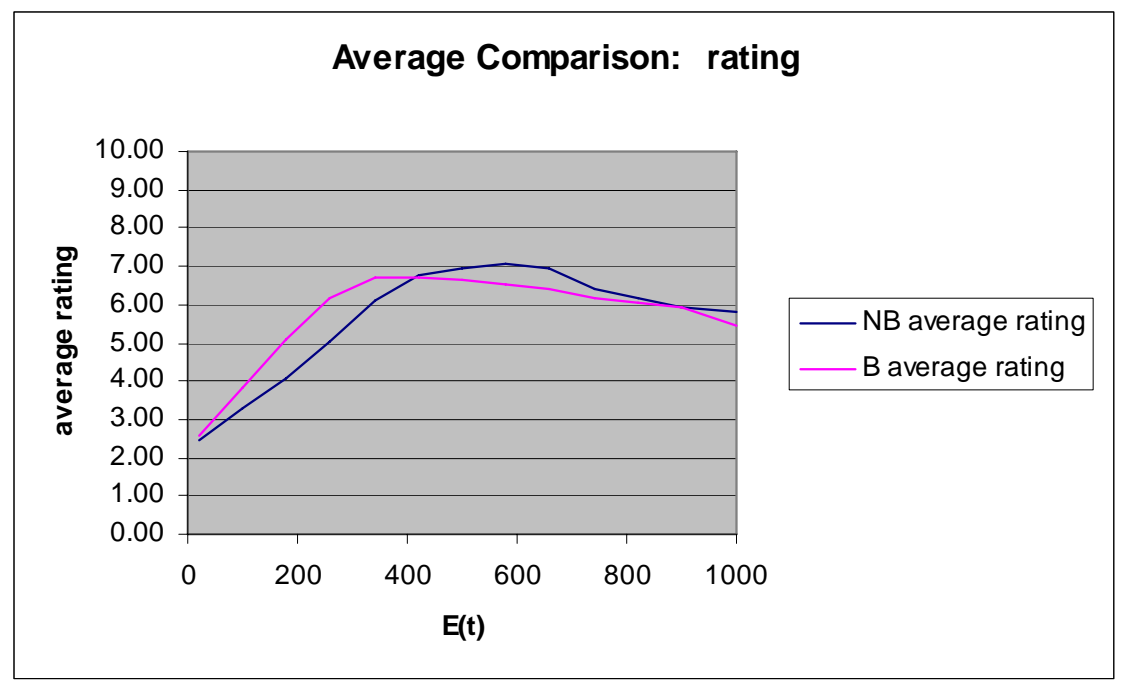

Figure 11. Average rating of illuminance change for biased $(B)$ and nonbiased (NB) subjects, averaged for all four tasks

\section{Conclusions}

The results of this study on detectability were similar to the results of the previous studies-occupants detected illuminance reductions greater than $15 \%$ of the initial illuminance. This detectable range of illuminance change was not influenced by the bias given to subjects. This study also found that the acceptable dimming range of biased subjects was wider than that of non-biased subjects. For instance, the $50^{\text {th }}$ percentile acceptable illuminance reduction was $380 \mathrm{~lx}$ (76\% of the initial illuminance) for the biased subjects while $300 \mathrm{~lx}$ (60\% of the initial illuminance) for the non-biased subjects. The $80^{\text {th }}$ percentile acceptable illuminance reductions are $270 \mathrm{~lx}$ for the biased subjects and $190 \mathrm{~lx}$ for the nonbiased subjects. These results imply that if the motivation of occupants towards energy savings is raised, occupants may accept larger reductions in illuminance.

\section{References}

Kryszczuk, K. (2001) Detection of slow light level reduction, 2001 IESNA Conference Proceedings, pp.315-322

Shikakura, T., Morikawa, H., Nakamura, Y. (2001), Research on the perception of lighting fluctuation in a luminous offices environment, Journal of the Illuminating Engineering Institute of Japan, 85, 5, pp. 346-351

Akashi, Y., Neches J., Bierman, A. Energy saving load-shedding ballast for fluorescent lighting systems: occupant's dimming requirements. Draft report submitted to Connecticut Light and Power on September $16^{\text {th }}, 2002$. 


\section{APPENDIX K: INVESTIGATION OF THE EFFECTS OF DIMMING ON FLUORESCENT LAMP}

\section{LIFE}

\section{Introduction}

The overall objective of this project is to identify and seek to reduce the barriers to wide acceptance and use of energy-saving daylighting, electric lighting, and control technologies, including occupancy sensors, photosensors, dimming electronic ballasts, and whole building integrated control systems. The following are detailed descriptions of the scope of work from tasks 4.3, 4.4, and 4.5:

- Task 4.3 - Investigate critical performance factors, such as optimal electrode voltage vs. percent rated current required maintaining electrode hot spot temperature. The focus will be on popular 32 Watt, T8 lamps, and on the performance of rapid start dimming ballasts.

- Task 4.4 - Investigate impact on lamp life of starting lamps when dimmed, i.e., at lower than rated current. This is related to lamps starting under dimmed conditions.

- Task 4.5 - Investigate the allowable dimming range that can be achieved without additional electrode heating voltage. This will enable lower cost, instant start, dimming ballasts for load shedding, lumen maintenance, etc. This task requires investigation of instant start, dimming ballasts for load-shedding applications.

Overall, these three tasks require the LRC to investigate the interaction between dimming ballasts and fluorescent lamps, and to identify the impacts of dimming on fluorescent lamp life. This knowledge will be used to help reduce the barriers to widespread use of electronic dimming ballasts.

The first section reviews research on the topic of fluorescent lamp failure mechanism, electrode characteristics, ballast operation and starting on lamp life. This section provides necessary background information on fluorescent lamps, ballasts, and their interactions. The sections that follow relate directly to specific tasks as follows:

The section, Optimum electrode heating as a function of the discharge current, provides a direct answer to Task 4.3, detailing how dimming affects fluorescent lamp life and how to reserve dimmed lamp life by supplying optimum electrode heating.

The section, Impact on lamp life of starting lamps when dimmed, focuses on task 4.4, providing theoretical analysis and laboratory testing on how starting lamps when dimmed may reduce lamp life.

The section, Allowable dimming range without additional electrode heating, investigates the allowable dimming range that can be achieved without additional electrode heating voltage. These results are a direct answer to task 4.5.

Appendix-4.3 - A presents a market analysis for load-shedding ballasts, which is needed in task 4.5 in order to decide the allowable dimming range in such applications.

\section{Fluorescent Lamp Life}

\section{Lamp failure mechanism}

The failure of fluorescent lamps is caused mainly by the loss of the electron emissive coating of the lamp electrodes (den Hoek, 2002, Verderber, 1985, Waymouth, 1971). Under certain circumstances, such as high frequency operation and frequent starting on instant start ballasts (“cold ignition”), fracture of the tungsten coil is also observed, which causes the lamp to fail (Haverlag, 2002). Electrode temperature directly affects the evaporation and erosion of the emitting material and thus the lamp life. 
A very high electrode temperature (greater than $1000^{\circ} \mathrm{C}$ ) will reduce lamp life due to evaporation of the emitting material, and a low electrode temperature (less than $700{ }^{\circ} \mathrm{C}$ ) will reduce lamp life due to erosion of the emitting material by sputtering (Ji, 1998).

With the introduction of low-mercury content lamps it is possible that lamp life, when over 20,000 hours, might also be influenced by a reduction of available mercury within the lamp. Since there is a strong movement in the industry to limit the mercury content of fluorescent lamps, some new lamp designs are manufactured with near the minimum amount of mercury needed for an efficient mercury discharge. Over time, free mercury in the lamp bonds to other materials within the lamp envelope, especially the sputtered and/or evaporated electrode material that gets deposited over the inside lamp walls (personal conversation with lamp manufacturer representative). For lamps with excess mercury added, mercury absorption is not an operational problem because the lamp electrodes limit life before the excess available mercury is removed. However, lamps with marginal amounts of mercury dosing, combined with severely sputtered and/or evaporated electrodes may show different end-of-life behavior.

\section{Electrodes for fluorescent lamps}

\section{Electrode characteristics}

Fluorescent lamps operated on alternating current have two identical electrodes that serve alternately as the anode and the cathode. The electrode at the negative end of the tube (the cathode) and its associated discharge region (the negative glow) serve the function of injecting the necessary electron current into the discharge column. The positive electrode (the anode), on other hand, must extract electrons from the discharge column at the other lamp end. The sketch in Figure 1 (Waymouth, 1971) identifies the main discharge regions and electric potentials for a fluorescent lamp.

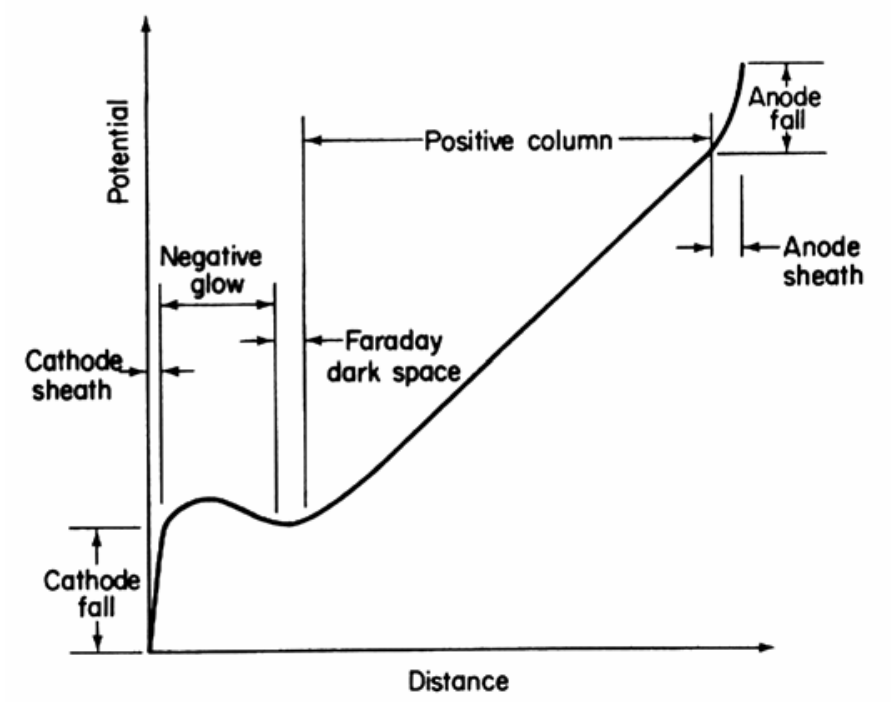

Figure 1. Sketch identifying the main discharge regions and potentials across them (Waymouth, 1971)

Fluorescent lamps used for general lighting applications are known as hot cathode lamps. These are different than cold cathode lamps used for "neon" signs and other special applications. Hot cathode lamps produce light much more efficiently than cold cathode lamps. Hot cathode operation is what is generally referred to as fluorescent lighting. Therefore, this discussion will consider only hot cathode lamps.

Electrode temperature is an important factor that affects lamp operation and life. The majority of the electrons emitted by the cathode result from the process of thermionic emission, whereby thermally excited electrons have enough energy to free themselves from the material. The work function is a 
property of a material that determines the energy needed for an electron to escape the material. Fluorescent lamp electrode filaments are coated with an emission mix, made from calcium (Ca), barium (Ba), and strontium (Sr) oxides, that has a very low work function, ranging from 0.9 to $1.1 \mathrm{eV}$, compared to that of the bare tungsten filament whose work function would be about $4.5 \mathrm{eV}$. For coated filaments, temperatures of about $900{ }^{\circ} \mathrm{C}$ are high enough to create thermionic emission of electrons sufficient for the discharge current. Without the emissive coating, thermionic emission is insufficient for the discharge current, which, if maintained, would lead to the destruction of the electrode and lamp failure.

The emissive coating on the electrodes is removed by two processes: evaporation and sputtering. Evaporation is the continual, temperature dependent removal of material into the low pressure atmosphere inside the lamp envelope. The removed material is deposited on nearby cooler surfaces, such as the lamp wall, that results in lamp end-darkening. Sputtering is the removal of material by the impact of positively charged ions accelerated toward the cathode. Sputtered material also gets redeposited on nearby surfaces, including the electrode.

Operating life, therefore, is limited by evaporation and sputtering of electrode coating. If the electrode temperature is too high, lamp life is reduced by evaporation of the emissive coating. While a low electrode temperature will reduce the evaporation of the emissive coating, it may increase lamp electrode sputtering. Sputtering increases at low electrode temperatures because alternate processes take the place of thermionic emission for generating the supply of electrons for the discharge. These processes require a drop in electric potential adjacent to the electrode, which is responsible for accelerating ions, which impact the electrode. This drop in electric potential is called cathode fall voltage. Near end of life, when the emission mix is depleted from the electrode filament, the work function of the electrode material increases up to about $4.5 \mathrm{eV}$, that of bare tungsten. To sustain the discharge, the sharp drop in electric potential at the cathode increases dramatically to aid in extracting electrons. Large increases in cathode fall voltage result in either catastrophic sputtering of the electrode, or the ballast failing to sustain or initiate the discharge due to the higher overall lamp voltage.

\section{Cathode fall voltage}

Cathode fall voltage is a fundamental property of the discharge and always indicates the electrode sputtering level (Hammer, 1995). Cathode fall voltage is the drop in electric potential from the cathode to the end of the cathode sheath region; a distance of about $0.1 \mathrm{~mm}$. The cathode fall voltage accelerates the electrons emitted from the electrode toward the lamp arc stream and enables ion generation when these accelerated electrons collide with the mercury and argon atoms in the gas atmosphere of the lamp. Once produced, these ions are accelerated by the cathode fall voltage and strike the cathode. The ion bombardment heats the cathode surface, raising the electrode temperature and increasing the emission of electrons. High cathode fall voltage will cause ions to strike the cathode so forcefully that a great deal of emissive coating sputtering occurs. Alternately, low cathode fall voltage indicates an abundance of free electrons from which the rate of thermionic emission is high. This implies that the electrode is operating at an excessively high temperature with a high electrode-evaporation rate that eventually will shorten lamp life.

Theoretically, the thermionic emitting properties of the electrode can be used to indicate cathode fall voltage and the resulting degree of sputtering during lamp operation. The cathode at its operating temperature with no external electric field has a certain thermionic emission of electrons, called zerofield thermionic emission. A higher zero-field thermionic emission, implies little or no sputtering. Several researchers developed different methods to measure the zero-field thermionic emission and electrode temperature, while other researchers developed models to predict these values (Waymouth, 1971, Soules et al 1989, Watanabe 1995). However, these researchers never related their measurement to real lamp-life data. Thus, it is still not clear how these properties relate to fluorescent lamp life. 
Research on cathode fall voltage has progressed beyond measurement and models to relate the cathode fall voltage data to lamp life (Hammer 1989 and 1995, Misono 1992, Watanabe 1993).

Figure 2 shows the postulated lamp-life relationship with cathode fall voltage. Figure 3 shows

Misono's data on the relation between cathode fall voltage and lamp life for experimental lamps. At 60 $\mathrm{Hz}$ operation, the acceptable range of peak cathode fall voltage is approximately 11.0 to $14.5 \mathrm{~V}$. However under high frequency operation ( $>20 \mathrm{kHz}$ ), this range will be as low as 7 to 10V (Hammer 1989).

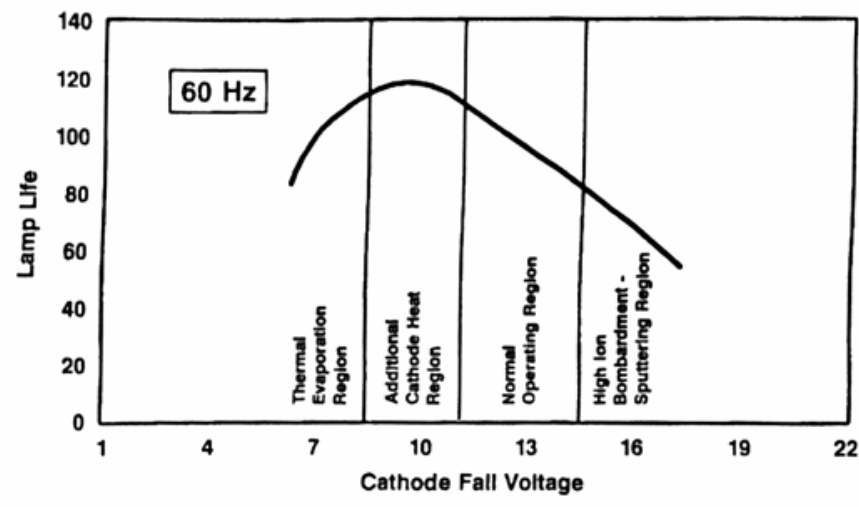

Figure 2. Postulated lamp life (\%) vs. cathode fall voltage (V) at $60 \mathrm{~Hz}$ (Hammer, 1995)

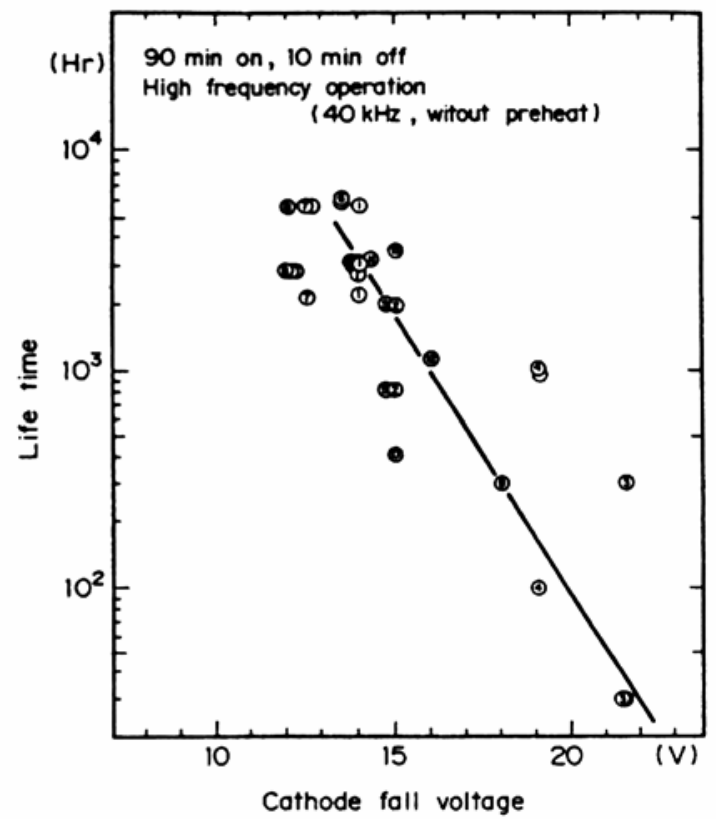

Figure 3. Relationship between lamp life and cathode fall voltage (Misono, 1992) 


\section{Electrode thermal model}

In alternating current (ac) operation, when no separate heating of the cathode is provided, most of the cathode heating arises from electron bombardment during the half-cycle when the electrode is acting as anode (Lowry, 1951). Some additional heating results from ion bombardment during the half-cycle when the electrode is acting as cathode and from the resistive losses (loss $=I^{2} R$ ) in the tungsten wire core caused by the discharge current flowing in through the leads and out through the cathode surface. In these cases, the cathode heat developed is related to the arc current being drawn.

For both low frequency lamp operation $(60 \mathrm{~Hz})$ and high frequency operation $(>20 \mathrm{kHz})$ most of the cathode heating comes from the anode cycle because the energy dissipated (lost) at the anode is much larger than that lost at the cathode. According to Watanabe (1993), for $50 \mathrm{~Hz}$ operation, anode cycle loss is about 6 times the cathode cycle loss. For $43 \mathrm{kHz}$ operation, anode cycle losses decrease due to the lack of the anode fall voltage, however, the anode loss from the work function of the electrode material is still about one and a half times larger than the cathode fall loss.

The main electrode heat loss mechanisms are conduction to adjacent elements, electron emission cooling, radiation cooling, and convection cooling of the electrode surface. The reduced demand on the electrode to supply electrons resulting from dimming the lamp can reduce the heat loss from electron emission cooling. However, this reduction of heat loss cannot completely compensate for the reduction of electrode heating due to dimming.

The inherent bombardment type of cathode heating that occurs on the lamp electrodes ordinarily results in what is known as "hot spot" operation. Since points on the cathode will vary in potential depending on their distance from the lead wire supplying power, and since it is a practical impossibility to maintain all areas of the cathode surface at exactly the same electron emitting efficiency, the discharge tends to localize at the most efficient emitting areas and also at areas nearest the lowest electric potential. The result is that this type of cathode does not operate at a uniform temperature throughout its length. A "hot spot” develops, which comes to a temperature and area equilibrium depending on the arc current being passed. During lamp life, emission mix material leaves the electrode by evaporation. Due to the relatively high temperature of the "hot spot," the emission mix evaporates more in that location. This results in the hot spot moving like a candle flame from one end of the electrode to the other. When all of the emission mix material is consumed, the lamp will quickly extinguish. Soules (1989) has presented an excellent simulation of this process. Investigations at the LRC support this "candle flame" movement of the hot spot occurring over the life of lamps operated on instant-start ballasts (See Figure 4).

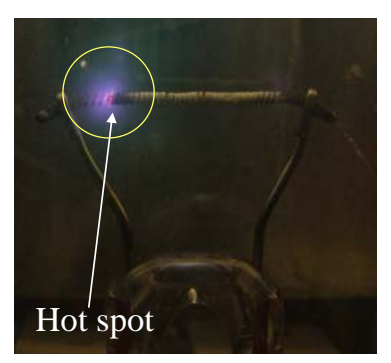

$64 \mathrm{~mA}$

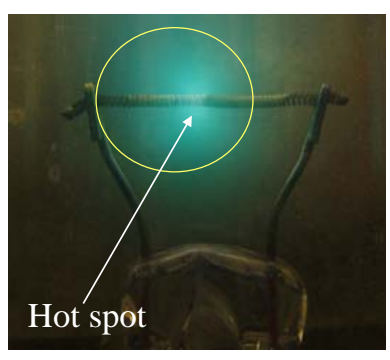

$121 \mathrm{~mA}$

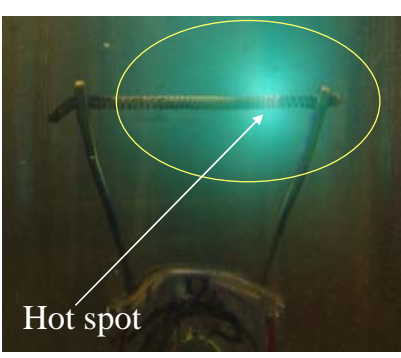

$180 \mathrm{~mA}$ 


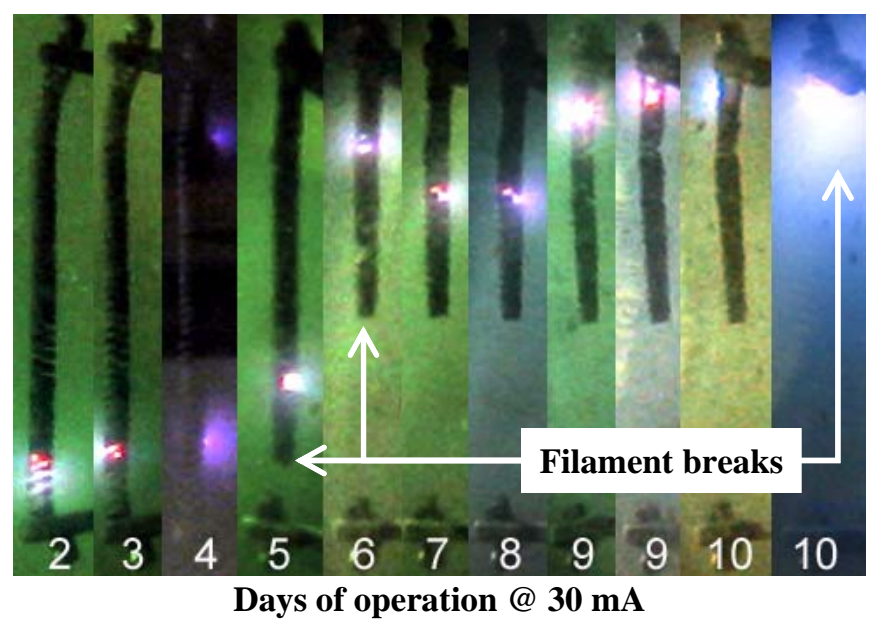

Figure 4. Movement of Hot Spot for Instant Start Fluorescent Lamp under Dimmed Condition

\section{Electrode heating under $60 \mathrm{~Hz}$ and high frequency operations}

When a fluorescent lamp operates on alternating current, the electrode alternately operates as both cathode and anode. During the anode cycle, since the anode does not emit ions, a negative space charge builds up in front of the electrode and an anode fall voltage is formed, analogous to cathode fall voltage. Under $60 \mathrm{~Hz}$ operation, the anode fall voltage averages approximately $5 \mathrm{~V}$ and is marked by oscillations in voltage about this level (Waymouth, 1971). Electrons accelerated by this anode fall are collected by the anode and contribute a significant fraction of the energy to heat the emitter coil. Since no ions exist in front of the electrode during the anode cycle at $60 \mathrm{~Hz}$ operation, the anode current flows uniformly into the electrode. Thus, the electrode is heated rather uniformly during the anode cycle.

When a fluorescent lamp is operated at high frequency ( $>20 \mathrm{kHz}$ ), the electrons and ions formed in front of the electrode during its cathode cycle are hardly removed during the following anode cycle because of the short length of time of the half-cycle period. The anode can draw electrons from the free electrons remaining from the period in which the electrode was a cathode. As a consequence, the anode fall voltage vanishes and the anode cycle loss decreases due to the lack of the anode fall voltage. The average electrode temperature therefore is lower under high-frequency operation than under lowfrequency operation due to the drop in the anode cycle loss. However, the electrode hot-spot temperature under high-frequency operation may be still higher than the electrode temperature under low-frequency operation. This is supported by the fact that the cathode fall voltage under high frequency operation is lower than that under $60 \mathrm{~Hz}$ operations (Hammer, 1995). Watanabe (1993) explained this peculiar sounding phenomenon as follows:

Ion space charges are formed in front of the cathode spot position during the cathode cycle. When the polarity is changed, the ion space charges are quickly neutralized by the electrons from the positive column, and the electric field vanishes. Then, mercury ions in front of the cathode-spot position diminish mainly through the diffusion process during the anode cycle... Therefore mercury ions randomly travel a distance of $240 \mu \mathrm{m}$ during the anode cycle. Since this distance is comparable to the size of the cathode spot, it is reasonably concluded that the ion space charges in front of the cathode-spot position are virtually not removed during the anode cycle.

Due to the ion space charge in front of the cathode spot, the anode cycle loss is concentrated at one particular spot on the electrode in the case of high frequency operation. Thus, the cathode-spot is efficiently heated during the anode cycle under high frequency operation. In contrast, uniform heating by the anode cycle loss under low frequency operation is less effective for increasing the cathode-spot 
temperature. In this case, high frequency operation will not only increase the lamp efficacy due to the elimination of anode fall voltage, but also may enhance lamp life due to the effective heating of the cathode spot (Watanabe, 1993).

\section{Ballast operating effects on fluorescent lamp life}

There are three operating parameters that may affect fluorescent lamp life: lamp current crest factor (CCF), supplemental electrode heating voltage, and lamp operating current. The ballast that operates the lamp mainly determines these three parameters.

Lamp current crest factor is the ratio of peak lamp current to the root mean square lamp current. A higher CCF indicates a distorted wave shape with the potential for high peak current, which can damage the lamp electrode and reduce lamp life. Fortunately, most electronic ballasts have satisfactory CCF of less than 1.7, which is regarded as an acceptable limit by ANSI ballast performance standards. However, this limit is based on $60 \mathrm{~Hz}$ operation, therefore applying it to high frequency operation is questionable. Perhaps higher CCF values are acceptable for high frequency operation due to the more efficient heating of the electrodes under high frequency operation.

Supplemental lamp electrode voltage is the voltage across the electrode filament at each lamp end that is supplied by certain ballast types to heat the electrodes during lamp operation. For Instant start and modified rapid start ballasts, this voltage is $0 \mathrm{~V}$ (non-existent), but some electronic rapid start ballasts, and most magnetic rapid start ballasts continue to provide about $3.5 \mathrm{~V}$ across the electrodes during lamp operation. Although this increases the active power of the system, it can diminish or possibly avoid the sputtering of the electrode emissive material that occurs if the electrode temperature drops below 700 ${ }^{\circ} \mathrm{C}$. The electrode temperature will likely drop to below $700^{\circ} \mathrm{C}$ when the lamp is dimmed.

Lamp operating current is the current flowing through the lamp during operation. The Ballast factor (BF) is the ratio of the luminous flux emitted by a lamp operated on a given ballast to the flux emitted by the same lamp when operated on a reference ballast. These two parameters are directly related in that reducing the lamp operating current reduces the light output of the lamp and therefore reduces the BF. ANSI sets maximum limits on lamp operating current to minimize the evaporation of the electrode emissive coating and minimum limits to avoid electrode sputtering. ANSI does not address the issue of dimming the light output of lamps. Lamp voltage increases slightly as lamp current is decreased, making the direct relationship between lamp current and light output deviate slightly from being directly proportional.

\section{Starting effects on fluorescent lamp life}

There are four main methods to start fluorescent lamps: preheat start (or switch-start), rapid start (includes modified rapid start), programmed start, and instant start.

In a preheat start system, a starter switch diverts lamp current across the filaments at the lamp ends to preheat the lamp electrode for several seconds. Then, when the starter's switch opens, the ballast provides approximately 200 to $300 \mathrm{~V}$ across the lamp to strike the arc. This method is obsolete in the U.S. and is rarely used in commercial and industrial lighting systems.

Rapid-start ballasts include an electrode heating circuit that provides a low filament heating voltage (about $3.5 \mathrm{~V}$ ) to obtain an electrode temperature ranging from about 700 to $1000{ }^{\circ} \mathrm{C}$ within a one- or two-second starting-period while at the same time applying a starting voltage of 200 to $300 \mathrm{~V}$ to the lamp. Most rapid-start ballasts continue to supply the electrode heating voltage even after the lamp has started, which results in power efficiency losses of about 1.5 to 3 watts per lamp. There is also a ballast type that uses a rapid start starting method, called cathode disconnect (or modified rapid start), that removes the supplemental heating after the lamp has started.

Programmed start, being recently introduced to the market, is defined in ANSI 2002 standards as follows: 
Those systems in which the sequence for starting hot-cathode electric discharge lamps is as follow: (1) the lamp cathodes are initially preheated to a temperature sufficient for adequate electron emission and without establishing local ionization across the cathodes; (2) this cathode heating is accomplished by supplying the required energy from a voltage or current source in the ballast itself, while during the preheated period the voltage across the lamp is kept below a level to initiate a glow discharge; (3) after the preheating period the voltage across the lamp is increased to a sufficient level to initiate the arc breakdown discharge; and (4) cathode heating may be reduced or removed after the lamp is in full conduction.

Supplying the correct amount of supplemental heating is critical for the above starting methods. If the electrode heating voltage is too high, the overall temperature of the electrode may exceed $1000{ }^{\circ} \mathrm{C}$, thus reducing lamp life due to a high electrode coating evaporation rate, especially if the supplemental electrode heating is not removed after the lamp starts. If the electrode heating voltage is too low, the temperature of the electrode may be too low for sufficient thermionic emission, thus reducing lamp life due to excessive coating loss through sputtering. The electrode $\mathbf{R h} / \mathbf{R} \mathbf{c}$ ratio is often used to measure whether or not the electrode is heated to an appropriate temperature during starting. Rc is the cold lamp electrode resistance at room temperature $\left(25^{\circ} \mathrm{C}\right) . \mathbf{R h}$ is the hot lamp electrode resistance at the end of the preheat period but just before the glow to arc transition. Ji (Ji, 1998) illustrated that $\mathbf{R h} / \mathbf{R c}$ ratio correlates well with rapid start fluorescent lamp life (See Figure 5). A higher $\mathbf{R h} / \mathbf{R c}$ ratio enables more starts.

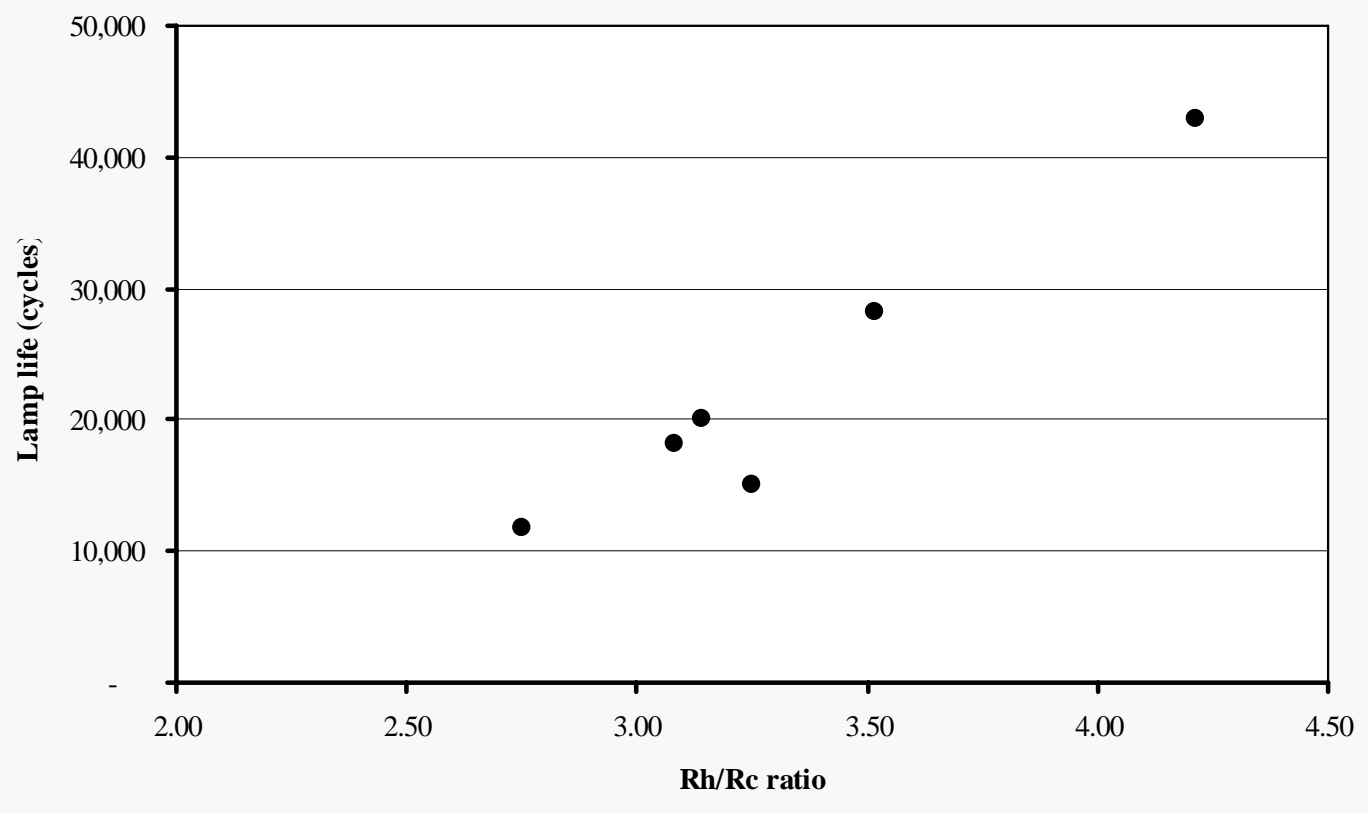

Figure 5. $\mathbf{R h} / \mathbf{R c}$ ratio vs. lamp life (Ji, 1998)

Instant start ballasts supply a high initial voltage (over $400 \mathrm{~V}$ for 4-foot lamps) to strike the arc and start the lamp. The high voltage is required to initiate the discharge between the unheated electrodes. Since no supplemental heating is provided to the electrodes either before or during lamp operation, instant start ballast systems are more efficient than rapid start systems. According to the U.S. Census Bureau (2001), $85 \%$ of the electronic ballasts sales for fluorescent lighting systems are instant start. This is despite the fact that rated life claims for lamps operated on instant start ballasts are less than that for rapid start operation. However, rated life may not take into account operation at high frequency which provides better self-heating of the electrodes and the longer cycle times found in many applications (on for 8 to 
10 hours at a time compared to the three-hours-per-start cycle for rating purposes). Recent life test results show that high frequency electronic instant start systems have nearly the same lamp life as rapid start systems (Ji, 1998). The widespread market acceptance of instant start electronic ballasts can be attributed to the benefits of the instant start method, including higher efficiency (5\% higher than rapid start), faster starting and lower initial costs (3 - 9\%).

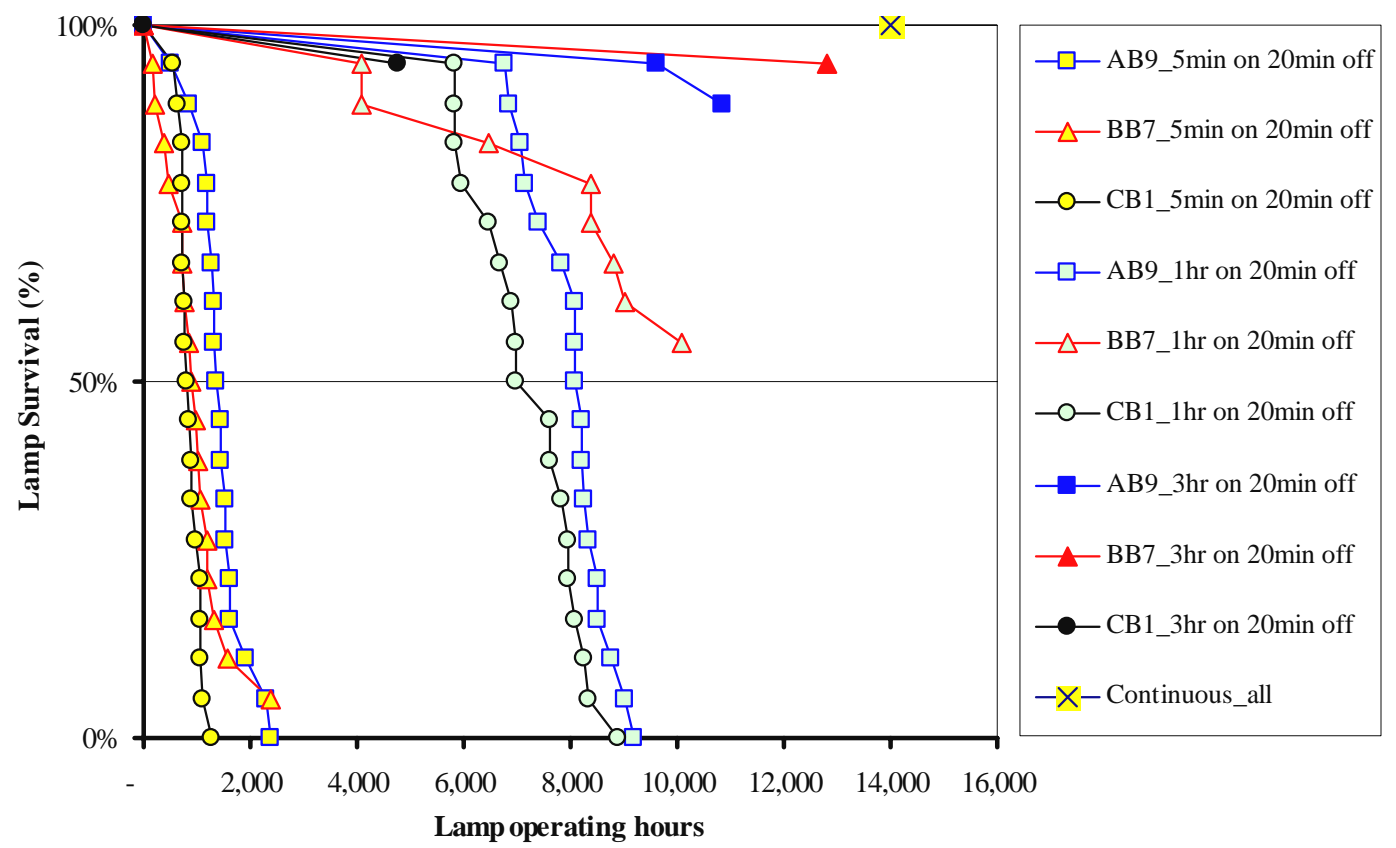

Figure 6. Instant start fluorescent lamp life at different operating cycles

As previously stated, the operating life of fluorescent lamps is determined in most cases by the rate of the electrode emitter depletion. There are two different contributions to this process: depletion during steady-state operation and depletion due to starting. Damage to the cathode from the starting process is generally more serious than steady-state operation. Waymouth (1971) found that starting could reduce the life of the fluorescent cathode by a factor of about 2 to 3. Figure 6 shows the Lighting Research Center (LRC) life test results for T8 lamps operated on high frequency instant start electronic ballasts at different operating cycles. The lamps used in the study were manufactured by GE, Philips and OSI. The difference in life is more than a factor of four comparing the 5-minute-on per-start to the 1-hour-on per-start. It is important to note that frequent switching of lamps operated on instant start ballasts shortens life significantly, yet the market still chooses instant-start ballasts over other ballast types by a large margin. This demonstrates that lamp-life is just one of many factors considered when choosing a particular technology. Consumers apparently accept the damaging effects of starting using instant-start ballasts because they gain energy savings and lower initial costs.

\section{Optimum electrode heating as a function of the discharge current} (task 4.3)

Fluorescent lamp electrodes are designed to operate at a temperature high enough to support thermionic emission of electrons when they are operated near the rated discharge current. Dimming a fluorescent lamp by reducing the lamp current will reduce the cathode hot spot temperature. The consequence will be an increase in cathode fall voltage that is sufficient to increase the electrode temperature again by increasing the energy of the ions reaching the electrode. This may result in a higher electrode sputtering rate and lead to an early lamp failure. At the same time, however, the reduced discharge current requires less electron emission from the cathode. This reduced requirement somewhat offsets the lack of cathode heating, but because cathode electron emission is highly non-linear with electrode temperature, cathode 
fall voltage still rises upon dimming. At lamp discharge currents in the range of a few percent of the rated current value, lamp life may drop down to values of only a few days.

In order to avoid these negative effects on lamp life when dimming, there is the possibility of applying supplemental electrode heating current, which goes through the electrode filament and heats it by resistance heating to the temperature that is necessary for thermionic emission. Obviously, the lower the dimming level at which the lamp is operated, the higher the supplemental electrode heating current required. However, the electrode heating current should not be too high. The guiding principles are:

a. Too little heat reduces lamp life due to sputtering.

b. Too much heat reduces lamp life due to a high rate of the emission-mix evaporation.

Following these arguments, an optimum supplemental electrode heating current (or electrode voltage) exists for lamp dimming as a function of the discharge current and must be maintained in order to keep lamp life within a reasonable range. Various approaches for the determination of this optimization are possible through the following measurements:

a. Lamp life at various levels of the discharge current and different values for the electrode heating current

b. Electrode temperature (overall hot spot location) at different discharge and supplemental heating currents

c. Evaporation of barium from the electrode at different discharge and supplemental heating currents

d. Cathode-fall voltage measurements at different settings of discharge and heating currents made by the following techniques:

1. Langmuir probe

2. Capacitive coupling

3. Measuring the lamp operating voltage

The first method yields the most reliable results as it is a direct measure of life and can match conditions as closely as possible to the situation the lamp user would experience. Unfortunately, results from such measurements will only be available after two to three years of testing with much effort and at great expense.

Hilscher (2002), from OSRAM GmbH, found a way of acquiring cathode-fall voltage of a fluorescent lamp operated at different discharge and electrode heating current values by measuring the lamp operating voltage. The lamps used in the experiment were $32 \mathrm{~W}$, triple tube compact fluorescent (OSRAM DULUX T/E 32W), operated on $25 \mathrm{kHz}$ electronic ballasts. Figure 7 shows the maximum observed cathode fall voltage, Ucf (i.e. the cathode fall voltage that corresponds to a minimum or no supplemental heating current) as a function of the discharge current. This clearly indicates that the cathode fall voltage increases exponentially with the reduction of lamp discharge current.

The measured values of the cathode fall voltage were used to determine minimum, target, and maximum values for the cathode-heating current. In practice it is difficult to isolate supplemental cathode heating current from lamp current, since the same wires that attach to the lamp carry both currents at the same time. An approximate value that represents the effect of the heating current is calculated as the sum of the squares (SOS) of the current in each lamp-end lead wire. These so called SOS values, given in terms of lamp current, are used as design guides for proper supplemental electrode heating. For the triple tube, 32W compact fluorescent lamp, Hilscher (2002) obtained:

$$
\begin{aligned}
& \text { SOS }_{\text {min }}=0.518-0.544^{*} \text { Id (A) } \\
& \text { SOS }_{\text {target }}=0.163-0.255^{*} \text { Id (A) } \\
& \text { SOS }_{\text {max }}=0.181+0.267 * \text { Id (A) }
\end{aligned}
$$




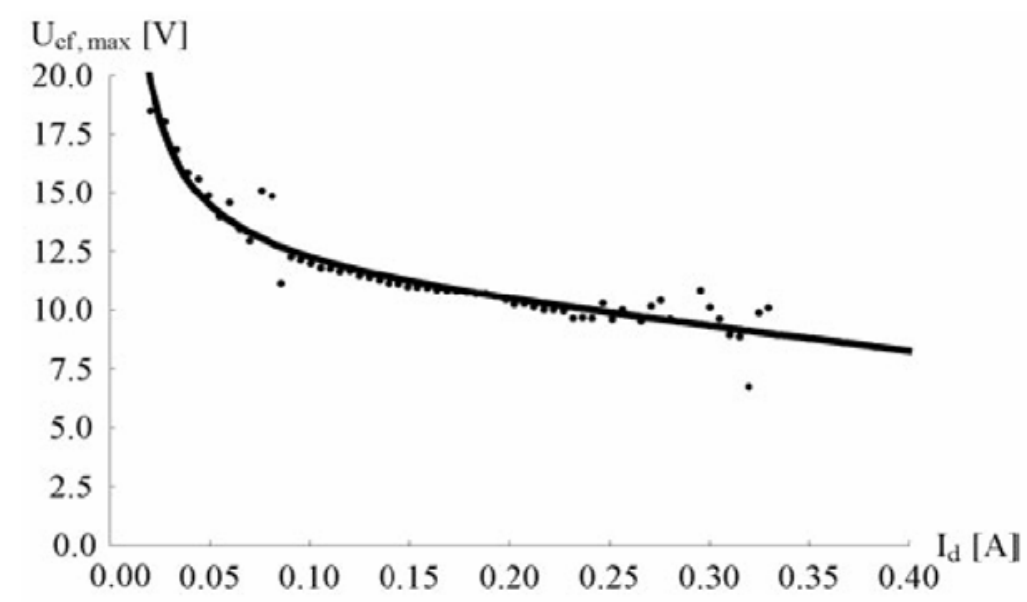

Figure 7. Maximum cathode fall voltage (Ucf) as a function of lamp discharge current (Id) (Hilscher, 2002)

Tetri (2000) conducted a life test on dimming T8 fluorescent lamps operated on dimming ballasts that provided cathode heating. Tetri found that when the lamps were operated at static dim levels of $1 \%, 5 \%$ or 15\% luminous flux, or dimmed dynamically up and down according to daylight illuminance levels, the lamp would reach the nominal lamp life and lumen maintenance factor. Thus, Tetri concluded that dimming with proper cathode heating would not impact fluorescent lamp life. Examination of the life data from this experiment reveals a trend of decreasing lamp life for higher output levels that was not discussed by the authors. This hints at the possibility that the shortest life might be at an intermediate dim level. A probable reason for this is that at the lowest dim levels of $1 \%$ and $5 \%$, very little current is demanded from the electrodes, so precise heating is not critical. As the current increases for higher levels of light output, heating becomes more critical, but the lamp current is still insufficient for selfheating of the electrodes.

\section{Impact on lamp life of starting lamps when dimmed} (task 4.4)

During the instant start and rapid start starting processes, the lamp undergoes a transition from cold cathode glow discharge to hot cathode discharge. The cold cathode glow discharge is formed when the cathode is capable of emitting electrons only by ion-impact-induced secondary electron emission. Even though the discharge might be self-sustaining, the current near the cathode is carried mainly by positive ions. As a result, each electron that leaves the cathode must cause the production of many ions at a point near the cathode. For this to occur, a very high cathode-fall-voltage must exist-often several hundred volts.

In the case of hot cathode discharge, the current at the cathode is carried mainly by thermionicallyemitted electrons; positive ions are needed only to neutralize electron space charge and provide a modest electron-accelerating field at the cathode surface. The necessary ion production is much less than one ion per electron. The cathode fall voltage is therefore very small, typically on the order of the ionization potential of the atoms of the gas ( 5 to $10 \mathrm{~V}$ ). Figure 8 illustrates the potential versus distance for cold cathode and hot cathode discharges operated at the same current in similar tubes at the same gas pressure $\left(60 \mathrm{~Hz}\right.$ ) (Waymouth, 1971). The positive column potential drop, $\mathbf{V}_{\mathbf{p}}$, and anode fall, $\mathbf{V}_{\mathbf{A}}$, are the same, but cathode fall voltage, and therefore total potential, are substantially greater for the cold cathode discharge. 


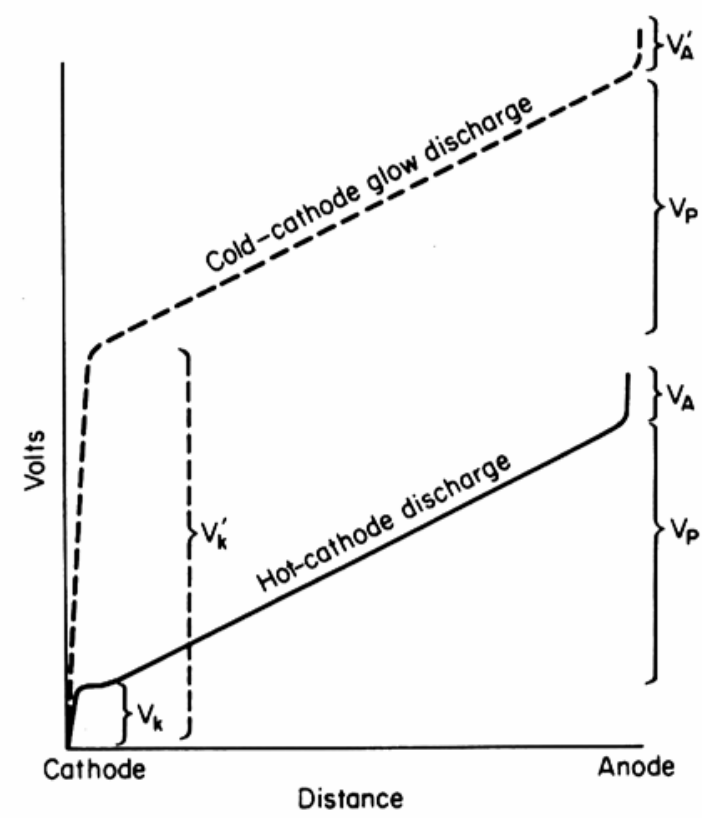

Figure 8. Potential versus distance for cold cathode and hot cathode discharges (60 Hz operation)

In the usual range of glow discharge conditions encountered in the process of lamp starting, the cathode fall voltage increases with rising current (Brown, 1959). This helps the transition from cold to hot cathode discharge by increasing the energy input to the cathode from the discharge. The positive ions reaching the cold cathode strike it with kinetic energies equal to the charge times the accelerating potential, which can be as high as the full cathode fall voltage. Most of this energy goes into heating the cathode, which increases the cathode temperature. The higher the cathode fall voltage and the ion current, the faster the cathode is heated to thermionic emitting temperature and the sooner the discharge is converted to a hot-cathode discharge.

When starting the lamp with an instant-start ballast at its rated lamp current, a sufficiently high potential is applied to the lamp to ionize the gas and to reach full operating current without a temporary pause in the glow state. The discharge current and lamp light output reach their normal operating values typically well before the $100 \mathrm{~ms}$ time limit measured after the application of the open-circuit voltage as defined by ANSI. The instantaneous energy input to the cathode during the transient glow-dischargelike condition that persists while current is increasing to normal operating current is very high. The rate of change of cathode surface temperature has been described as "almost of explosive violence" (Waymouth, 1971). As a result, during lamp starting, simple blasting off of chunks of cathode material by the drastic temperature rise will reduce the amount of coating remaining and would be expected to reduce lamp life. It is not known whether this potentially explosive heating reduces lamp life in practice, however. Most fluorescent lamp electrodes are designed with a coiled-coil or triple coil structure to help restrain the emission material so the lamp can last its rated life (typically 20,000 hours at 3 hours per start for T8 lamps) with no problem.

In the case of starting lamps when dimmed, i.e. at lower than rated current, the transition from coldcathode glow discharge to hot-cathode discharge will most likely be extended due to the decrease of lamp current and the corresponding decrease in heating power available to the electrode. Although this may alleviate somewhat the violent cathode damage due to the explosive temperature rise, overall it is much more damaging on the cathodes because of the potential for a relatively extended period of glow discharge ion bombardment (sputtering). In the extreme case, when the lamp is dimmed to levels below about $20 \%$, the current in the steady-state glow discharge is too low to heat the cathode to a sufficient temperature to convert it to the hot cathode mode. The discharge will "hang up" in the glow state. The high-energy ion bombardment in the glow discharge knocks away surface atoms and erodes the cathode 
to the point of destruction in a short time. Waymouth (1971) explained this sputtering damage during glow discharge at starting as follows:

Under glow-discharge conditions, with cathode fall voltages of $200 \mathrm{~V}$ or so, sputtering rates of 0.1 atom removed per incident ion would be quite typical. Besides, sputtering also damages the thermionic emission of the surface (even without significant material removal) in two ways. First, since the cathode is cold, atoms are sputtered away, bounced back on the cathode surface by the gas, and re-deposited helter-skelter instead of in an orderly way. Destruction of the ordered surface of the crystal must be expected to increase the work function and thus reduce thermionic emission. Second, tungsten atoms sputtered from the surface of exposed tungsten are deposited on the surface of the oxide almost as readily as on the tungsten, "plating" the oxide with a metallic covering and increasing its work function. The resulting reduction of accelerating field emission of the cathode means that the cathode fall voltage in the discharge must be higher to increase the ion current; the increased power input to the cathode increases its temperature to bring the electron emission up to the required level. The increased cathode fall voltage, ion current, and temperature persist for ten minutes to an hour after the damage, until the effects of the damage have been 'annealed out'.

Figure 9 illustrates the relationship between lamp current and starting time for one Sylvania OCTRON FO32/735 T8 lamp operated by a high-frequency electronic ballast. It clearly indicates that start time increases exponentially with the reduction of lamp current.

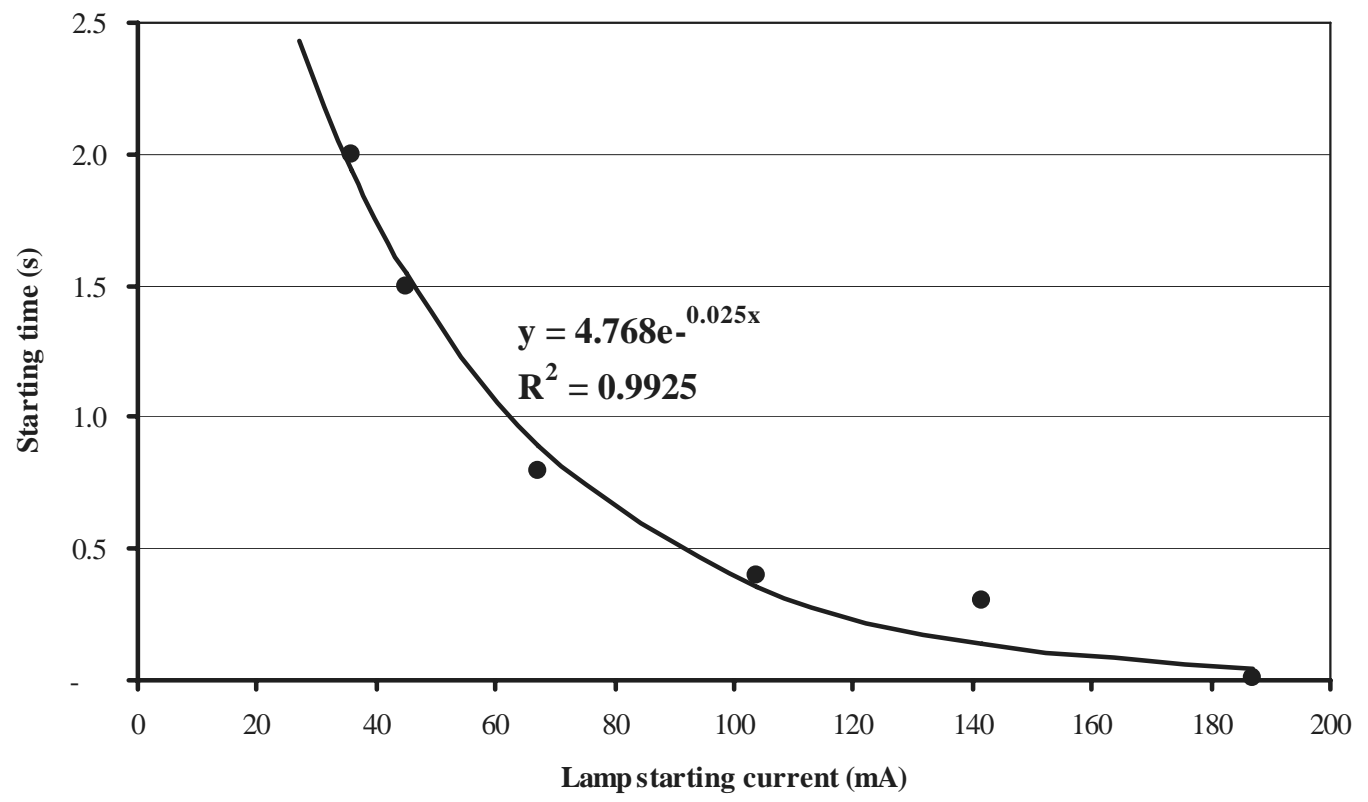

Figure 9. Relationship between lamp starting current (mA) and starting time (s)

Also, it is observed after each start that the cathode operates for a significant time at a higher temperature than it does under continuous-burning conditions. This period of increased temperature greatly increases the evaporation rate of cathode coating material, and consequently the overall loss rate of coating material, leading to shortened lamp life.

Technically, it is possible to apply supplemental electrode heating current to heat the electrode before starting lamps when dimmed. This is similar to the situation of rapid start or programmed start fluorescent lamp. However, this is at the expense of efficiency, and/or initial cost. The possibility also exists that the intended supplemental electrode heating current is not effectively applied in all cases. LRC researchers found that in actual applications, connections and excessively long, tightly bundled 
wire leads reduce the amount of heating that actually takes place. On at least one LRC project this condition seriously reduced lamp life when traditional dimming ballasts that employ supplemental electrode heating were used (LRC, to be published).

Therefore, starting lamps when dimmed, i.e., at lower than rated current, is not recommended. To ensure long life, especially when supplemental electrode heating is not applied (e.g., instant start), a procedure of starting the lamp at its nominal full output level and then dimming it down to the desired light levels is necessary. Starting at full power will minimize the damaging effects of starting. This is advisable whether supplemental electrode heating is applied as part of the starting sequence, but most critical without supplemental electrode heating.

\section{Allowable dimming range without additional electrode heating} (task 4.5)

In ac operation, when no separate heating of the cathode is provided, most of the cathode heating arises from electron bombardment during the half-cycle when the electrode is acting as anode. Some additional heating results from ion bombardment and from the resistance losses (loss $=\mathrm{I}^{2} \mathrm{R}$ ) in the tungsten wire core caused by the discharge current flowing in through the leads and out through the cathode surface. In these cases, the cathode heat developed is positively related to the arc current being drawn. The main heat loss comes from the conduction to adjacent elements, the electron emission cooling, radiation cooling, and convection cooling of the electrode surface. Although dimming can reduce the electrode heat loss from the reduction of electron emission cooling, this reduction of heat loss does not compensate completely for the reduction of electrode heating.

Obviously, a certain amount of variation in lamp current is entirely permissible and results in no unfavorable performance. This is demonstrated by the fact that ballasts with ballast factors ranging from 1.3 to 0.77 are widely used and appear to maintain lamp life acceptably. Nevertheless, any cathode operates best at some definite value of lamp current. A cathode that is properly designed for best operation at $225 \mathrm{~mA}$, for example, will run too hot at $400 \mathrm{~mA}$ and entirely too cold at $75 \mathrm{~mA}$. If such a cathode is operated at too high a current and temperature, results may include excessive vaporization of barium, discoloration in the form of anode spots, and reduced lamp life. If the cathode is operated at too low of a current, too high of a percentage of the total emission must be obtained by ion bombardment, field emission, or some mechanism other than thermionic emission. This method of operation is conducive to an excessive cathode-fall voltage, greatly increased cathode sputtering by ion bombardment, and in extreme cases, very short life.

It has also been found that sputtering under continuous operating conditions is small under fairly wide variations of lamp current. Wehner (Stuart and Wehner, 1962) found that at a cathode fall voltage less than $20 \mathrm{~V}$, the electrode sputter field is quite small, about $10^{-6}$ atoms removed per incident ion. Also there is little cathode temperature dependence in the sputtering yield (Stuart and Wehner, 1962). A great deal of the electrode heating energy comes from the collection of electrons in the anode half-cycle, and due to thermal inertia, the electrode does not cool down between its half-cycle of cathode duty. During operation, evaporation normally occurs at about a factor of 20 times greater than the rate of removal by sputtering. Therefore, sputtering can be ignored entirely in comparison with evaporation as a cause of coating loss under continuous burning conditions at nominal lamp currents. A lamp should be able to preserve a reasonable life within fairly wide variations of lamp current if it is started at its full rated current, and then dimmed down to the desired light level after it has reached its full light output. Now the question is: how much dimming is allowable for such reasonable lamp life?

In order to answer this question regarding the effect of lamp current on high frequency instant start fluorescent lamp life, a life test was designed at the Lighting Research Center. The life test included a total of 80 lamps at eight different lamp current conditions: ten lamps at each lamp current. All lamps were operated on $120 \mathrm{~V}$ ballasts for one lamp. The lamp current conditions selected were $180 \mathrm{~mA}, 121$ $\mathrm{mA}, 64 \mathrm{~mA}, 49 \mathrm{~mA}, 41 \mathrm{~mA}, 30 \mathrm{~mA}, 24 \mathrm{~mA}$, and $21 \mathrm{~mA}$. 
All ballasts were operated at $120 \mathrm{~V}$, were from the same manufacturer, and had the same model number. The ballasts were rated to operate one lamp at $180 \mathrm{~mA}$, which corresponds to a ballast factor of about 0.9, taking into account high frequency lamp operation. In order to achieve the different, reduced, lamp currents required by the life test design, a high voltage ceramic capacitor was placed in series with the lamp. The value of the capacitor, along with the specific ballast characteristics (e.g., operating frequency, output circuit details) determined the lamp current for each capacitor-ballast system. All of the capacitor-ballast systems were tested with a T8 fluorescent lamp to ensure that they operated the lamp closely to the selected lamp current.

In order to avoid the adverse effect of starting lamps under dim mode, the capacitors were connected in series with the lamp through a shorting device to bypass the capacitor during starting. After the lamps started, the shorting devices were manually removed, thereby dimming the lamps.

Calibrated capacitor-ballast systems were installed on two metallic racks that served as the support structure for holding the lamps. Each rack had eight levels, one level for each lamp current condition. One rack held six ballast-capacitor-lamp systems (48 lamps total). The other held four systems (32 lamps total). Figure 10 illustrates one such life test rack. Both racks were powered by a regulated 120 V power supply.

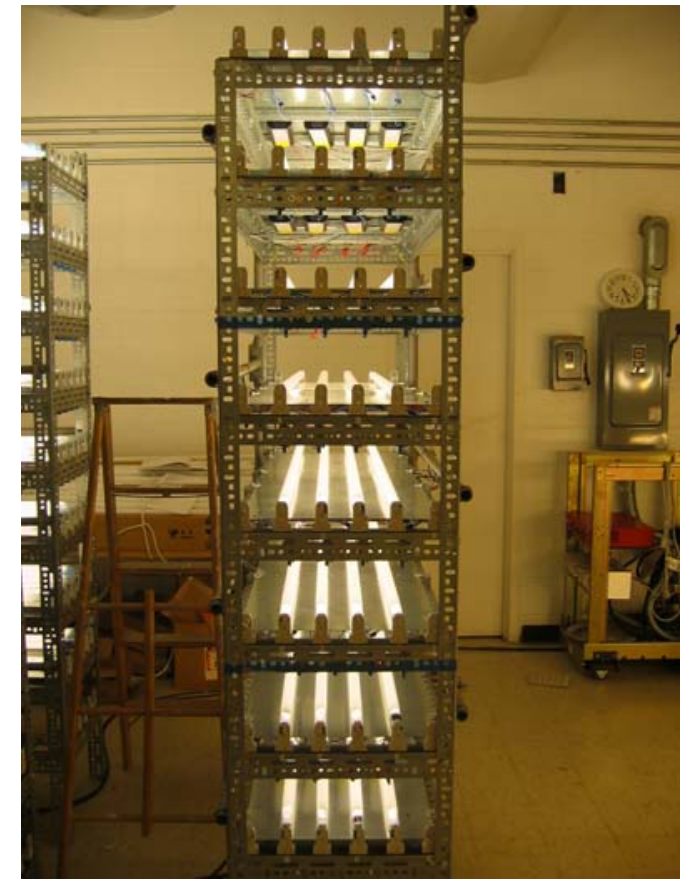

Figure 10. Instant start fluorescent lamp life test rack

The life test was started with the capacitors shorted, thus all systems were started at their nominal lamp current. After 30 minutes of operation, the capacitor-shorting devices were removed, dimming the test lamps to their intended lamp currents. At this point, measurements of the lamp operating time began. Hours of operation for each lamp were accumulated until the lamp failed. A lamp was considered failed when it had no visible light output to the experimenter's bare eye. The average lamp life was defined as the number of hours at which $50 \%$ of the test lamps at a given operating current failed.

As of November $6^{\text {th }}$, 2002, the lamps had accumulated 93 days of operation (2139 hours). By this date, all lamps operated at $49 \mathrm{~mA}$ and below had reached their median life (50\% had failed). At the other extreme, all lamps operated at or above $121 \mathrm{~mA}$ were still operating. For the group at $64 \mathrm{~mA}$ operating current, only one lamp had failed by this date. Figure 11 summarizes the results for the dimming testing of instant start fluorescent systems. The results show that dimming to about one third of rated current (121 mA), a T8 fluorescent lamp operated with an instant start circuit still keeps a reasonable long life (more than 2139 hours with no failures). 
Using current electricity pricing practices and load shed programs, LRC researchers found that load shed-related dimming of 100 hours per year would capture most of the economic benefit (see Appendix 4.3 - A). If the damage to the electrodes during dimmed operation is simply cumulative, meaning that no effects that accelerate the damage caused by dimming are occurring, then 2000 hours of dimmed operation is sufficient for load shedding applications. For example, a 20,000-hour lamp life corresponds to about 5.5 years of operation at 10 hours per day. If load shed dimming amounts to 100 hours per year, then 550 hours of dimmed operation is required. This dimmed operation would be expected to reduce the life of the lamp by about 25\% (550/2100), equivalent to a 15,000 hour life. A more exact calculation, taking into account the reduced dimmed operation time due to the reduced life yields a life reduction of only $19 \%$, corresponding to a life of 16,200 hours.

One interesting point worth mentioning is that impact of dimming on instant start fluorescent lamp life is quite analogous to frequent switching effects on life. Figure 12 is a re-plot of Figure 4, which shows the Lighting Research Center (LRC) life test results for T8 lamps operated on high frequency instant start electronic ballasts at different operating cycles. Frequent switching of lamps operated on instant start ballasts significantly shortens life, yet the market still chooses instant-start ballasts (85\% shipment in 2001) over other ballast types by a large margin. This demonstrates that lamp-life is just one of the many factors considered when choosing a particular technology. Consumers apparently accept the damaging effects of starting using instant-start ballasts because they gain the energy savings and lower initial costs. Based on the above calculation, dimming 100 hours per year to $67 \%$ rated current levels is expected to reduce overall life by less than 20\% (20,000 hour life reduced to 16,000 hours).

Considering the energy savings, it should be reasonable for consumers to accept low cost instant start load shedding ballasts (about $\$ 9$ cost increment on the standard instant start ballast) that enable the user

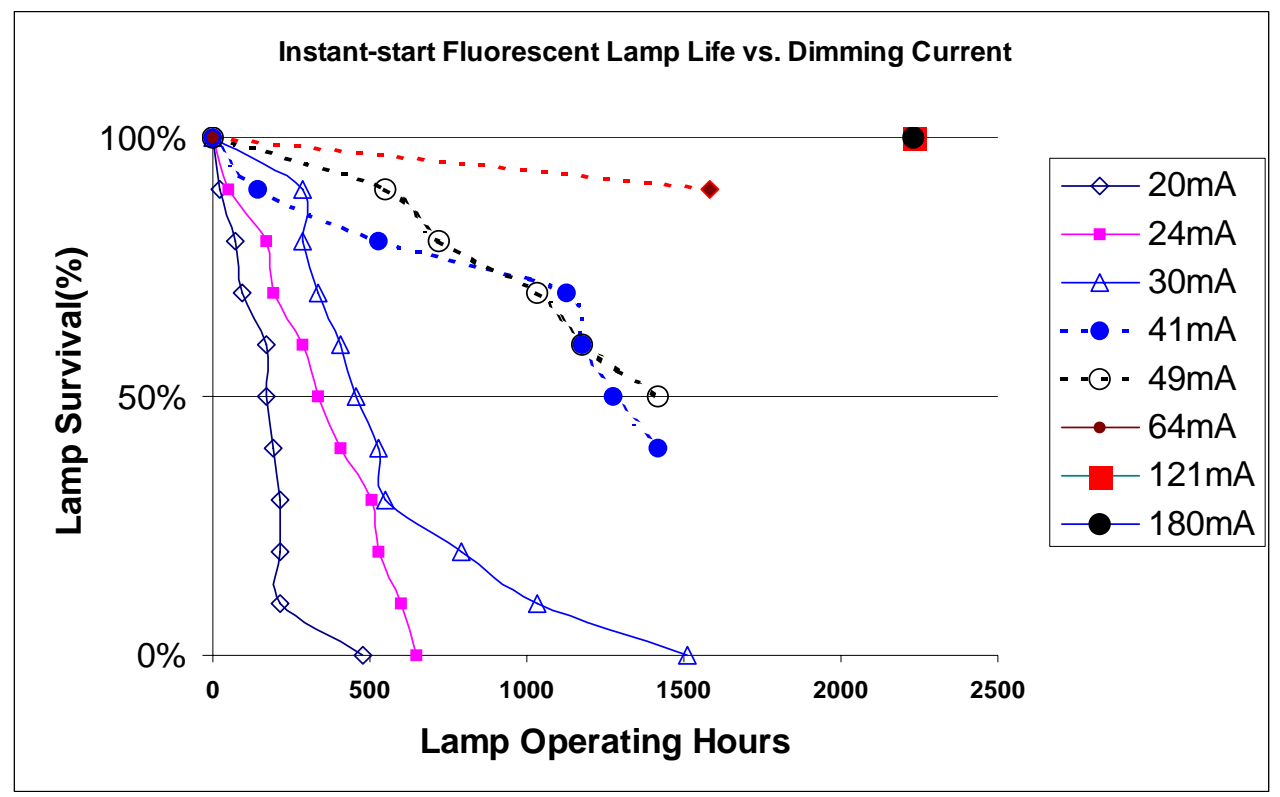

to dim the lamp to $67 \%$ rated current level.

Figure 11. Instant start fluorescent lamp life vs. dimming current 


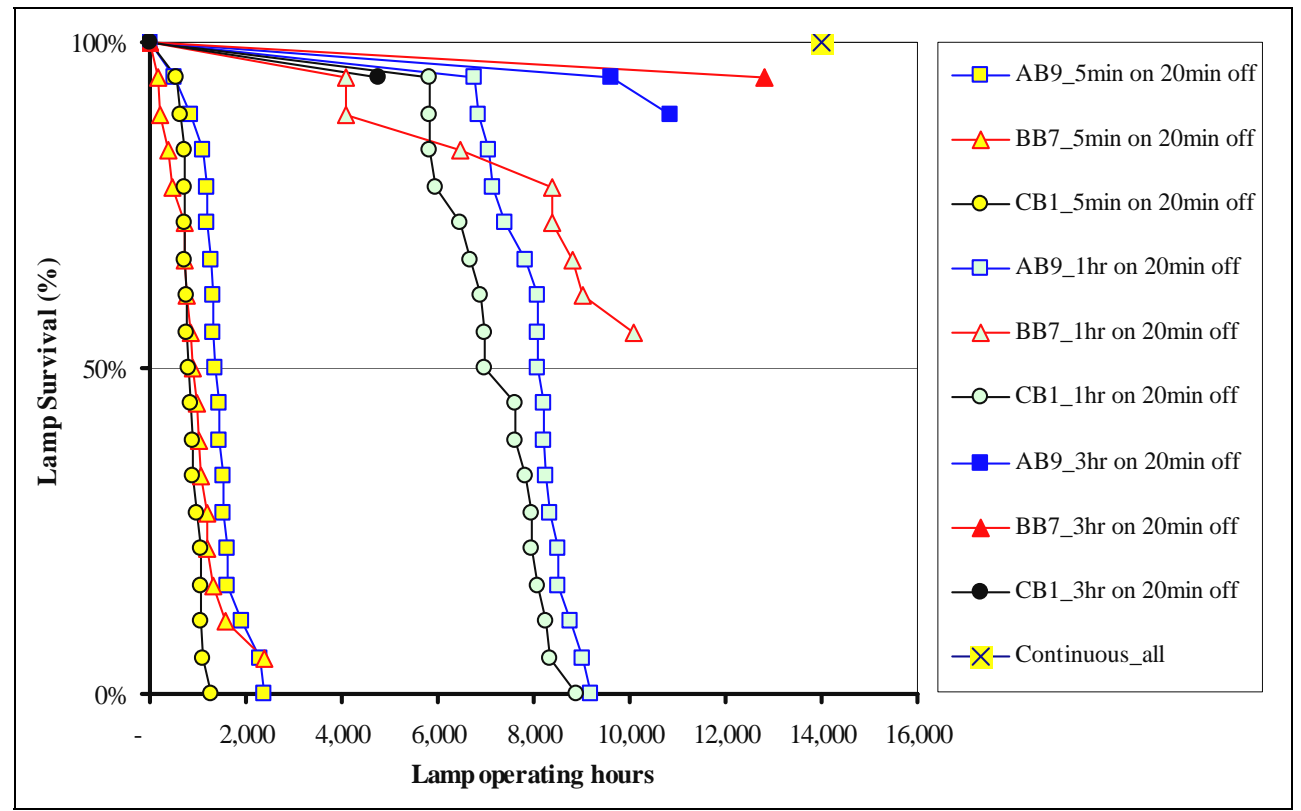

Figure 12. Re-plot of Instant start fluorescent lamp life at different operating cycles

\section{References}

ANSI C82.11 Consolidated-2002, ANSI standard for lamp ballast-High frequency fluorescent lamp ballasts -supplements, American National Standard Lighting Group, NEMA

Brown, S.C. Basic data of plasma physics (Cambridge, MA: M.I.T. Press, 1959)

Hammer, E.E. "Comparative starting operating characteristics of typical F40 systems." Journal of the Illuminating Engineering Society 18, no. 1 (1989): 63-69.

Hammer, E.E. “Cathode fall voltage relationship with fluorescent lamps.” Journal of the Illuminating Engineering Society 24, no. 1 (1995): 116-122.

Hilscher, A. "Determination of the cathode fall voltage in fluorescent lamps by measurement of the operating voltage.” Journal of Physics D: Applied Physics 35, (2002): 1707-1715.

Lowry, E.F. “Thermionic Cathodes for Fluorescent Lamps and Their Behavior.” Illuminating Engineering 46 (1951): 288-294.

Misono, K. "Cathode fall voltage of low-current fluorescent lamps.” Journal of the Illuminating Engineering Society 20, no. 2 (1991): 108-115.

Lighting Research Center "Integrated skylight luminaire.” Field Test Delta 1, no. 1 (to be published).

Davis, RG., Ji, Y. “Fluorescent lamp-ballast systems.” ESEERCO Final Report 94-40, part 1, 1998. Troy NY, Lighting Research Center.

Soules, T.F., Ingold, J.H., Bhattacharya, A.K., Springer, R.H. "Thermal model of the fluorescent lamp electrode.” Journal of the Illuminating Engineering Society 18, no. 2 (1989): 81-92.

Stuart, R.V., Wehner, G.K. “Sputtering at Very Low Bombarding Energies.” Journal of Applied Physics 33, no. 7 (1962): 2345-2352.

Tetri, E., "Daylight linked dimming: effect on fluorescent lamp performance." Lighting Research and Technology 34, no.1 (2000): 3-10.

US Census Bureau, “Fluorescent lamp ballasts: 2001.” Current Industrial Reports MQ335C (01)-5, (July 2001).

Watanabe, Y., Murayama, S. "Cathode fall characteristics of fluorescent lamps under high-frequency operation.” Japanese Journal of Applied Physics 32, Pt. 1, no. 8 (1993)

Watanabe, Y. "Dependency of cathode temperature on operation frequencies in fluorescent lamps." Journal of the Illuminating Engineering Society 24, no. 1 (1995): 48-57.

Waymouth, J.F. Electric discharge lamps (Cambridge, MA and London, England: M.I.T. Press, 1971). 


\section{Appendix 4.3 A: Market Analysis for Load-Shedding Ballasts}

\section{Introduction}

Market penetration for a load-shedding ballast and its associated communication link is a function of the incremental cost of the ballast/communication link and the potential customer savings on their electric bills from participation in load management programs or reducing monthly demand. Some portions of the country (mainly the northeast and west coast) also offer the customer a payment (conservation rebate) for installing load-shedding devices. Table 1 presents an analysis of possible customer savings from seven utilities for instituting load shedding.

This analysis examines these conservation rebates but does not use them in determining the maximum incremental cost of a load-shed ballast in the marketplace. The offering and amount of conservation rebates changes frequently and should not be relied upon in determining market penetration. Load management programs are in their infancy. Their use and value to customers are expected to grow over the next five years as the deregulated electricity market matures and stabilizes.

Bottom line - If a load shed ballast and its associated communication link can be sold to the customer for an incremental cost of $\$ 9$ or less over an instant-start ballast (the most common ballast used today), the market for this ballast would be approximately $10 \%$ of all ballasts sold into the new construction/remodeling market.

\section{Customer Savings/Payments}

There are three possible revenue streams from which the customer can receive a savings or payments for reducing their peak electric demand. These payments come from the utility or state agency that offers conservation rebates or from the utility or transmission system operator for participation in load management programs. Customer savings are from reducing demand and thereby reducing the monthly electric bill.

\section{Conservation Rebate Programs}

There are some locations within the U.S. where customers are offered rebates if they install loadshedding equipment. Most of these rebates can be found in the northeast or west coast. The rebates vary widely and are subject to change or program cancellation/suspension annually. Current rebates range from approximately $\$ 5$ to $\$ 40$ per ballast in those locations where they are offered.

Because of the transitory nature of conservation programs and rebates, it is recommended that these incentives to customers not be included in determining market penetration of load-shed ballasts. Where the rebates are available, they will assist in the early years of adoption of the technology and, therefore, increase sales projections.

\section{Load Management Programs}

Load management programs exist either through a utility's load curtailment rates or through the transmission system operator payment to the customer for shedding load. However, not all utilities or transmission operators have these kinds of rates or programs in place today. Assuming a $20 \%$ to $30 \%$ reduction in wattage for each light fixture equipped with a load-shed ballast, the customer can expect to receive between \$0.30 and \$1.50 per year per ballast in payments for reducing their lighting load. It is expected the value for load shedding will rise in future years by as much as $50 \%$ as the deregulated electric marketplace begins to place stronger values on load management. 


\section{Demand Reduction Savings}

A customer who can control his/her monthly peak demand will garner savings through reduced electric bills. Assuming a $20 \%$ to $30 \%$ reduction in wattage for each light fixture equipped with a load-shed ballast, the customer can expect to save, with today's electric rates, between $\$ 1.03$ and $\$ 8.03$ per year per ballast installed. The amount depends on the electric utility's demand charge and how many months the customer is willing to control his or her demand. For example, Consolidated Edison of New York's demand charges are much higher than those found at Public Service Electric and Gas in New Jersey. A customer participating in a load management program cannot double count the demand reduction for that program with savings on their electric bill associated with controlling monthly peak demand. Therefore, the customer's benefits for reducing demand is either the amount available from a load management program OR from reduced electric bills. In most cases, the greater savings today comes from controlling the monthly peak demand and obtaining a savings on the monthly electric bill. This savings averages about $\$ 3.20$ per year per ballast.

\section{Ballast and Communication Link Costs}

It is assumed a customer would be willing to accept a three year payback on the investment of a loadshed ballast. The customer savings associated with a demand reduction of $20 \%$ to $30 \%$ of the light fixture wattage is approximately $\$ 9$ based on the net present value of an annual saving stream of $\$ 3.20$ and a $6 \%$ discount rate. Therefore, the ballast and its communication link must cost the customer (not manufacturing cost) no more than $\$ 9$ over what he/she would have paid for a ballast without the load shedding capability. For the new construction/remodeling market, the $\$ 9$ is incremental to the price of a standard ballast. For the retrofit market, the customer would only be willing to pay a total ballast cost of approximately $\$ 12$, $\$ 9$ for the load shedding and a $\$ 3$ premium to obtain a new ballast.

\section{Market Analysis}

Based on the above information, the new construction/remodeling market across the country would be the primary market for the load-shed ballast. Currently, such a ballast would have to be sold based on reducing monthly demand and thereby reducing monthly electric bills. Future changes in the deregulated electric industry may produce greater customer savings associated with load management programs. A conservative market penetration of $10 \%$ of ballasts sold into the new construction/remodeling market is anticipated within five years of ballast introduction. In Connecticut, this $10 \%$ market penetration would translate into 10,000 ballasts per year. The market size is not known for the remainder of the country.

The retrofit market of existing/operational ballasts would be included only where conservation rebate programs exist. These programs are within Connecticut, Massachusetts, New York, New Jersey, California, Oregon, Washington, Wisconsin, and a few other states. Other than Connecticut, where there are about 6 million ballasts within commercial floor space, the size of this market is unknown. Penetration into the retrofit market is expected to be smaller. An approximation of about $1 \%$ of all existing ballasts by year five is considered reasonable for those states in which conservation rebates are available. 


\section{Table 1. Analysis of possible utility customer savings for instituting load shedding.}

\begin{tabular}{|c|c|c|c|c|c|}
\hline \multicolumn{6}{|c|}{$\begin{array}{l}\text { LOAD SHEDDING DIVIDEND } \\
\text { (or What the Customer is willing to Pay for Load Shedding Controls) }\end{array}$} \\
\hline UTILITY & $\begin{array}{l}\text { Rebate Incentive } \\
\text { for Ltg or Controls }\end{array}$ & $\begin{array}{l}\text { Load Reduction } \\
\text { Program Payments }\end{array}$ & $\begin{array}{c}\text { Normal Billing } \\
\text { Demand Reductions }\end{array}$ & $\begin{array}{l}3 \text { Year Load } \\
\text { Shedding Dividend }\end{array}$ & $\begin{array}{l}\text { \# Hours Required by } \\
\text { Load Reduction Pgm }\end{array}$ \\
\hline ConEdison & $\begin{array}{l}\$ 12.12 \text { per ballast } \\
\text { from NYSERDA program }\end{array}$ & $\begin{array}{l}\$ 0.75 / \mathrm{yr} . / \mathrm{ballast} \\
\text { from NY-ISO }\end{array}$ & $\begin{array}{c}\$ 4.01 \text { to } \$ 8.03 / \text { yr./ ballast } \\
\text { Rate } 9,6 \text { mo to } 12 \text { mo. } \\
\text { reductions }\end{array}$ & $\begin{array}{l}\$ 22.84 \text { to } \$ 33.56 / \\
\text { ballast. Dependant on } \\
\text { \# mo. curtailing }\end{array}$ & $\begin{array}{l}4 \mathrm{hr} . / \text { day. Must be } \\
\text { available every day }\end{array}$ \\
\hline Ohio Edison & none & none & $\begin{array}{c}\$ 2.64 \text { to } \$ 5.28 / \text { yr./ ballast } \\
\text { Rate GS-Large, } 6 \text { mo to } 12 \\
\text { mo reductions }\end{array}$ & $\begin{array}{l}\$ 7.06 \text { to } \$ 14.12 \text { / } \\
\text { ballast. Dependant on } \\
\text { \# mo. curtailing }\end{array}$ & $\begin{array}{c}\text { Assumed } 8 \mathrm{hrs} / \mathrm{mo} \text { or } \\
96 \mathrm{hrs} \text { per yr. }\end{array}$ \\
\hline $\begin{array}{l}\text { ComEd } \\
\text { (Chicago) }\end{array}$ & none & $\begin{array}{l}\text { Curtailable Coop } \\
\$ 1.05 / \text { yr/ballast }\end{array}$ & $\begin{array}{l}\$ 1.47 \text { to } \$ 4.95 / \mathrm{yr} / \mathrm{ballast} \\
\text { Rate } 6 \mathrm{~L}, 3 \mathrm{mo} \text { to } 12 \mathrm{mo} \\
\text { reductions }\end{array}$ & $\begin{array}{l}\$ 3.93 \text { to } \$ 13.20 \text { / } \\
\text { ballast. Dependant on } \\
\text { \# mo. curtailing }\end{array}$ & $\begin{array}{l}\text { Max. required } 120 \mathrm{hrs} \\
\text { during } 3 \text { summer mo }\end{array}$ \\
\hline Georgia Power & none & $\begin{array}{c}\$ 0.45 / \text { yr } / \text { ballast } \\
\text { Real Time Pricing } \\
\text { Rate }\end{array}$ & $\begin{array}{c}\$ 1.80 \text { to } \$ 3.60 / \text { yr./ ballast } \\
\text { Rate PLL-3, } 6 \text { mo to } 12 \\
\text { mo reductions }\end{array}$ & $\begin{array}{l}\$ 4.81 \text { to } \$ 9.62 \text { I } \\
\text { ballast. Dependant on } \\
\text { \# mo. curtailing }\end{array}$ & $\begin{array}{l}\text { Assumed } 8 \mathrm{hrs} / \mathrm{mo} \text { or } \\
96 \mathrm{hrs} \text { per } \mathrm{yr} \text {. }\end{array}$ \\
\hline CT Light \& Power & $\begin{array}{l}50 \% \text { of cost of ballast } \\
\text { differential }\end{array}$ & $\begin{array}{l}\$ 0.30 / y r / b a l l a s t \\
\text { from ISO-NE }\end{array}$ & $\begin{array}{l}\$ 1.49 \text { to } \$ 2.98 / \text { yr./ ballast } \\
\text { Rate } 55,1 \text { mo to } 12 \text { mo } \\
\text { reductions }\end{array}$ & $\begin{array}{c}50 \% \text { cost of ballast } \\
\text { plus } \$ 3.98 \text { to } \$ 7.97 \text { I } \\
\text { ballast. Dependant on } \\
\# \text { mo curtailing }\end{array}$ & $\begin{array}{l}\text { ISO unspeciified \# hrs } \\
\text { Assumed } 8 \mathrm{hrs} / \mathrm{mo} \text { or } \\
96 \mathrm{hrs} \text { per yr. }\end{array}$ \\
\hline $\begin{array}{c}\text { PSE\&G } \\
\text { (New Jersey) }\end{array}$ & $\$ 40 /$ ballast & $\begin{array}{c}\text { \$0.75/yr./ballast } \\
\text { from PJM or } \\
\$ 1.17 / y r . / \text { ballast } \\
\text { from PSE\&G Curtailable } \\
\text { Load Rider }\end{array}$ & $\begin{array}{l}\$ 1.03 \text { to } \$ 2.81 / \text { yr./ ballast } \\
\text { Rate LPL, } 4 \text { mo to } 12 \text { mo } \\
\text { reductions }\end{array}$ & $\begin{array}{l}\$ 42.75 \text { to } \$ 47.51 / \\
\text { ballast. Dependant on } \\
\text { \# mo. curtailing }\end{array}$ & $\begin{array}{c}\text { PJM unspeciified \# hrs } \\
\text { Curtailable Rider, about } \\
\text { 25hr./yr } \\
\text { Assumed } 8 \mathrm{hrs} / \mathrm{mo} \text { or } \\
96 \mathrm{hrs} \text { per yr. }\end{array}$ \\
\hline PG\&E & $\$ 22 /$ ballast & $\begin{array}{l}\$ 1.50 / y r / b a l l a s t \\
\text { from CA-ISO }\end{array}$ & 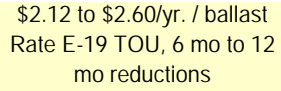 & $\begin{array}{l}\$ 27.67 \text { to } \$ 28.95 / \\
\text { ballast. Dependant on } \\
\text { \# mo curtailing }\end{array}$ & $\begin{array}{c}\text { Max. } 24 \text { hrs } / \mathrm{mo} \text {, all mo. } \\
\text { Assumed } 8 \text { hrs/mo or } \\
96 \text { hrs per yr. }\end{array}$ \\
\hline
\end{tabular}

Notes:

Information is from current published electric rates or conservation programs of each utility, ISO or state energy agency.

Each load shed ballast can reduce demand by 30 watts based on T-8, electronic 3 lamp fixture

In these examples, the customer is assumed to want to recover their initial investment in 3 years.

Payments will come from either the ISO or utility load reduction program or from reducing demand on the normal rates but not from both

The 3 year load shedding dividend is the sum of any rebate and the NPV (3 yrs./6\%) of the higher of load reduction program of reducing demand under normal rates.

Therefore, the amount the customer is willing to pay for a load shedding ballast is shown in the 3 year load shedding dividend column. 


\section{Appendix 4.4 A: Load-shed Ballast Specification \\ Revised Draft Performance Specification - Loadshed Ballasts}

\section{Operational definition of loadshed}

What: $\quad$ A ballast specification for reducing power demand by dimming fluorescent lighting (T-8 lamps) from a centralized point of control.

Why:

To manage load on the electrical grid. Used by electricity suppliers/grid operators as a substitute for power generating resources in times of critical peak system demand, and for managing demand at any time for more efficient system operation and risk avoidance.

How: $\quad$ Dimming and/or switching T-8 fluorescent lamps to reduce luminaire power input by $33 \%$ or $50 \%$, and aggregating those loads via a building-wide signaling system. There are two scenarios for control of dimming:

Scenario 1. Dimming is controlled by the electricity supplier/grid operator via remote signaling and distributed automatically to the luminaires (i.e., ballasts). Dimming times and payments are determined through negotiated contracts between the utility/grid operator and electricity customers. Payments to customers are based on minimizing the customer's electric demand while reducing operating risk for utility/grid operator.

Scenario 2. Dimming is controlled by the building owner/manager and used as a load management resource to limit peak electrical demand, resulting in lower electrical demand charges. An automated system that tracks electrical demand and initiates load shedding is envisioned.

When: $\quad$ As needed up to a maximum of 100 hours per year of lamp operating time.

Where: $\quad$ The technology is applicable to all C/I applications except where light-dependent critical tasks or processes are conducted (e.g., hospital operating rooms)

\section{Loadshed Ballast Specifications}

\section{Non-loadshed condition}

The loadshed ballast, operating under non-loadshed conditions, shall perform equally well, in terms of energy efficiency and reliability, as the popular instant-start ballasts that now dominate the fluorescent lighting market. A loadshed ballast must meet or exceed most existing ballast performance specifications so that it can be installed everywhere where traditional ballasts are installed.
Ballast type
Any (e.g., instant start, programmed start)
Ballast factor
0.88 (nominally $180 \mathrm{~mA}$ high frequency operation)
Power factor:
$>0.9$ 
THD:

Ballast efficiency factor:

Loadshed condition

Input power

Ballast efficiency factor

THD:
$<20 \%$ (ANSI definition)

$\left.\begin{array}{l}\text { 2-Lamp }>1.48 \\ \text { 3-Lamp }>1.00\end{array}\right\} \begin{aligned} & \text { Matching that of standard } \\ & \text { instant start ballasts. }\end{aligned}$

Reduce operating power by $33 \%$ or $50 \%$

2-Lamp $>1.35\}$ Maintain efficiency when dimmed

3-Lamp $>0.92\}$ comparable to standard dimming ballasts.

$<32 \%$ relative to the fundamental $(60 \mathrm{~Hz})$ input current.

\section{Loadshed Signal}

The signal to loadshed originates at the electricity supplier/grid operator, or within the building depending on the control scenario. Signals originating outside the building are distributed to building by various established means (e.g., internet, telephone modem), which are not covered in this specification. Once inside the building, the signal is distributed to a network of Ballast Control Modules, that in turn, distribute the signal to individual ballasts. The ballast contains circuitry to decode the signal from the Ballast Control Module and to dim or switch the lamps upon command.

\section{Ballast Control Module Inputs}

Outputs

Refresh rate of signal to ballasts

Default state signal at least 1 (receiving load shed signal from primary control center within building)

PLC signal covering at least 10,000 sq. ft. of building space

at least once every 10 minutes (perhaps continuous signal during load shedding)

non-loadshed (full light output)

The method of distribution of the loadshed signal inside a building to the Ballast Control Modules is yet to be determined, but it seems probable that an existing building automation protocol using twisted pair wiring could be used.

\section{Ballast characteristics} Starting of lamps

Lamps always start at full power levels

Response time (Dim/switch and restore)

$<1$ minute after receiving signal

The physical nature of the PLC signal and encoding scheme are yet to be determined. A PLC signaling scheme from the Ballast Control Module to the individual ballasts seems most promising based on current knowledge. An example of the signaling scheme might be frequency shift keying (FSK) at 42 and $52 \mathrm{kHz}$, encoding an alternating sequence of ones and zeros for either analog or digital decoding circuitry. 


\section{Task 4.6 Develop recommendations for improved components and systems as evaluated in Task 2, based on defined performance characteristics, and defined acquisition, installation and commissioning costs}

\section{Summary of recommendations:}

In the course of Task 2 of this project, three technologies were identified as having the potential to achieve greater market share, if certain barriers could be overcome. These technologies were the prototyped load-shed ballast, occupancy sensors, and an innovative easily-commissioned photosensor. To maximize their market potential, these technologies must all demonstrate their cost-effectiveness, their practicality in real installations, and their suitability to the market. Based on subsequent development of these technologies and analysis of the market, we recommend the following courses of action:

\section{Load-shed ballasts:}

- Perform large scale field trials of load-shed ballasts. These trials should aim to verify the acceptability of illuminance reductions to occupants, both when the reasons for load shedding have been explained to occupants, and when they have not. The process of informing occupants of the environmental and cost benefits of load shedding is referred to as "biasing".

- $\quad$ Test the electromagnetic compatibility (EMC) of load-shed ballasts in their two normal operating states (i.e., full on and dimmed), and when switching between states.

- Work with one or more ballast manufacturers to produce a commercially viable load-shed ballast based on electronic instant-start technology. To be financially attractive to customers, the price of this ballast should be no more than $\$ 9$ more than a regular instant-start ballast.

- $\quad$ Test the prototype ballast as part of the LRC's National Lighting Product Information Program (NLPIP), and make the result of the testing available on the NLPIP website.

- Work with one or more ballast manufacturers to develop a suitable method to carry signals from a central controller within the building, to the individual ballasts. ("stage 2" signaling). This signal should not cause disruption (e.g., flicker) to the other lighting in the building.

- Ensure that the signals sent out by electricity suppliers are suitable to be received by lighting load-shed controllers ("stage 1" signaling). Both the format and the medium for these signals should be considered in detail.

- Develop a performance specification for load-shed ballasts that can be easily followed by commercial ballast manufacturers, yet ensures minimum performance standards.

- Leverage current and future market forces to create demand for load-shed ballasts. This can be achieved by promoting the electricity cost savings achieved using load-shed ballasts, and by promoting load-shed ballasts as an environmentally friendly and therefore desirable feature of a building, especially new-builds. This may take place through published articles, seminars, demonstration projects and voluntary accreditation schemes.

\section{Occupancy sensors:}

- Publicize the aggregate figures for energy savings compiled as part of Task 4. Everyone in the building industry should be aware of typical figures for energy saving achievable by the use of occupancy sensors. 
- Work with control system manufacturers to produce a commissioning standard for occupancy sensors. If all sensors can be commissioned using the same procedure, the likelihood of installers making mistakes on site will be much reduced, and these mistakes account for a lot of the failings of systems in situ.

\section{Easily-commissioned photosensor:}

- Continue to use existing market channels, such as the LRC's NLPIP program and outreach education, to support the use of photosensing in those applications for which it is commercially viable. This is usually only in owner-occupied buildings with large window area and high occupation density. 


\title{
APPENDIX L: REDUCING BARRIERS TO THE USE OF LIGHTING CONTROLS; PROBLEMS ENCOUNTERED DURING INSTALLATION AND COMMISSIONING OF SENSORS
}

\author{
AUTHOR: Andrew Bierman \\ AUDIENCE: DOE program administrators \\ REPORT SECTION: Best Practices for Installing Occupancy Sensors
}

\section{Background}

Andrew Bierman, a researcher at the Lighting Research Center in Troy, NY, traveled to Titusville, New Jersey to witness occupancy sensor installations at the a company's headquarters. The work was conducted the night of June 27, 2002 from 5:00pm until 2:30am. The following are his observations, problems encountered, and recommendations for improving efforts to reduce barriers to the use of lighting controls.

\section{Customer familiar with sensors}

The contractor had installed ultrasonic occupancy sensors throughout most of the building's private offices and some of the open plan offices approximately four years earlier. Now, the customer wanted more occupancy sensors installed in the remaining open plan offices and hallways, and photosensors installed in the throughways between buildings and daylit reception areas. The contractor explained that this particular job of installing sensors would be more time consuming than typical installations because the easy application areas for occupancy sensors had already been exploited, and what remained were the more challenging locations.

\section{Training customer's maintenance staff before installation}

On the day of the installation, the installer met with the customer and some of the maintenance staff to present a basic training session about operating the new sensors. The installer appeared to have a good relationship with the customer, but a lack of communication appeared to exist between the customers' management and maintenance staff. During a question and answer session, the customer discovered that maintenance personnel had removed a number of the existing occupancy sensors because they had encountered problems. Management, not informed about the removals until the training session, had assumed that all of the sensors were being utilized. Also, during the training session, the customer's staff displayed a distrust of and annoyance with the sensor products. The information most sought by the maintenance staff was how to override, or disable the device when it fails or causes complaints.

\section{Installation time difficult to estimate}

The time required to install occupancy and photosensors is difficult to estimate and prone to take longer than expected because of the complexity and uniqueness of each installation. Finding and tracing circuits takes time, and the installers report that "as-built" drawings are rarely accurate. For most products installing the sensor part of the control is only half the job. Connecting to the lighting circuit that the sensor will control is the other half and it is not trivial. As an example, a photosensor was installed the previous night with the low voltage signal wiring run back to the electrical panel box. The installer had about two additional hours of work consisting of drilling into the main panel box and add an auxiliary box for the necessary power packs that supply electricity to the sensors and contain the switching relays, adding new wires that must be connected to the appropriate circuit breakers and the new power packs, connecting the low voltage wires to the power pack relays and securing all wires and components. This work is time consuming partly because each panel box is somewhat unique in its size, shape, wiring and location making custom fittings necessary. 


\section{Problems encountered}

In this installation, the contractors had to break circuits and put in special wiring and relay controls on panel boxes to control atrium area lights. One technician installed only two sensors during the evening...but neither worked when finished. He did not know for sure what the problem was and decided to return during the day to commissioned the sensors. In another example, three technicians worked four hours to install two occupancy sensors in a hallway, plus get an open-plan office area wired with relay power packs so that occupancy sensors could be installed the following night.

When wiring the hallway, the installers tried to use sensors manufactured by one manufacturer and power packs (with relays) manufactured by another manufacturer. Neither manufacturer supplied circuit schematics or documentation explaining the inputs and outputs to the sensors and power packs, and each used different wire colors. This resulted in trial-and-error installation and troubleshooting, which was very time consuming. Even more time would have been used if the installers had to turn off the electricity each time they tried a different wiring combination as the instructions suggest. After several failed attempts, the installers replaced the power packs with Novitas equipment and the sensors worked. Although I was not present when these particular sensors were commissioned, I did witness the technicians commissioning other sensors and this too is a trial-and-error technique, which obviously was expedited by the installers' experience using this particular product. Had an inexperienced technician installed the sensors, commissioning would have been much more difficult.

Lighting controls are often installed in two stages; first the power packs and relays are installed, then the sensors are added. Installers rely on overriding the system after the power packs and relays are installed so the lights will work while awaiting installation of sensors. However, the installers often don't know how to override because they don't have sufficient documentation by manufacturer. The installers guessed at which connections would turn the lights on with no sensor attached, unaware of any damage that may be incurred by shorting different wires together in a trial-and-error approach.

When walking around the installation site (11:00pm), some private office lights were on with no one present. The technicians checked and found many of the previously installed sensors' sensitivity adjustments were turned all the way up, perhaps by facilities technicians who were trying to avoid complaints. Oversensitive sensors tend to keep the lights on all the time. The technicians also pointed out that some of the installations of wall mounted occupancy sensors in the private offices were not the ideal location for such a sensor because of obstructions such as file cabinets and book cases. In these cases a ceiling mounted sensors would probably have worked better, but would have been about twice as expensive to install.

The installers offered an important tip: Never disable manual controls when installing automatic shut-off controls. If a hallway, for example, already has a light switch, add the automatic control in series with the existing switch. In this way, the automatic control supplements the light switch rather than replacing it. The light switch does not override the sensor. Rather, the light switch and the sensor work in combination, either one able to switch the lights.

\section{Commissioning}

The commissioning procedure used by the installers for the occupancy sensors is as follows:

1. Turn time delay to minimum and put sensitivity dial in middle position (the dial has no other indication).

2. Walk through the area monitored by the sensors. If the light does not turn on, increase the sensitivity.

3. Wait for light to turn off (after minimum time delay) and test again.

4. If the lights turn on, but then don’t turn off, turn down the sensitivity. 
5. After the sensitivity is set correctly, set the desired time delay remembering that lower sensitivity can be somewhat compensated by increased time delay.

These repetitive tasks take time, as the installers must repeatedly wait for the lights to turn off before they can retest the sensitivity. The objective is to adjust the sensitivity so the lights turn on only when needed, but not when not needed. The installers commented that even for similar applications, similar settings do not guarantee similar performance because dial positions do not correspond to similar sensitivity settings from sensor to sensor.

\section{Lessons learned}

The following lessons learned were gleaned from observations of, and discussions with the installers, product information, manufacturers' web sites and Training Videos provided by Sensor Switch, Inc.

1. Professional installers are needed to properly install and commission occupancy sensors.

2. Installer: "People should realize that sensors can not be placed just anywhere. Some areas are just not appropriate for sensors."

3. Current commissioning procedures take too much time! The time delay features alone require waiting for the lights to turn off before the installers can retest the sensor for movement detection.

4. Blueprints cannot be trusted. Installers often find circuits are different than the drawings indicate so wire paths must always be verified.

5. Open plan areas with cubicles need sufficient numbers of sensors placed appropriately. Too few sensors are commonly used in an attempt reduce costs, resulting in lack of coverage and poor performance.

6. A lot of judgment goes into placement of sensors. Installers must assess each situation individually. The experience of a professional installer is very beneficial.

7. Installers should place occupancy sensors near areas where small movements are made...near desks, over bathroom stalls, etc., and not worry about sensors picking up large motions such as opening doors, people entering rooms, which sensors easily detect.

8. A trick used by installers in hallways is to put two sensors, one at each end, pointing toward the center of the controlled area. This provides good coverage in the hallway and minimizes the risk of the lights turning on when someone walks near, but not in, the hallway.

9. The type of wiring affects how easily circuits can be broken. Conduit requires more circuit tracing because of multiple wires within the conduit. BX cable, which is armored, is easier to use.

10. Modular wiring can be problematic. One problem is by cutting cable doesn't provide enough slack, so they have to piece in new wiring. This requires junction boxes, extra cost, extra time, etc.

11. Since installation is often done at night, so as not to interfere with company's business activities, installers don't have manufacturer support available during installation.

12. Ceiling mounted sensors are nearly twice the cost of wall switch replacement sensors, because you have to install the sensor and the power pack, plus the extra wiring involved and more difficult access.

13. During commissioning or re-commissioning, when turning down sensor sensitivity, installers should increase the time delay to somewhat compensate for the reduced sensitivity to motion. 
Although a longer time delay is not a substitute for sensitivity, longer time delays allow the lights to stay on longer without demanding movement.

14. Device failures: Alan Rhode estimated product failure rates at less than two percent. Most failures are immediate and are dealt with during installation. Actual callbacks for failed devices are estimated by Alan Rhode to be less than $1 / 4$ of one percent.

\section{Recommendations}

Based on Andrew Bierman's observations and on knowledge gained at the Lighting Research Center, we recommend the following:

1. Manufacturers should include labeled circuit schematics on the devices or in the packaging identifying the signals, and/or function of all the electrical connections. Presently, manufacturers seem to keep information from contractors, perhaps to encourage them to purchase all components from same manufacturer, or from fear of confusing installers. The more information installers have, the better they can cope with all the different situations that arise in the field.

2. Industry should develop a diagnostic interface that installers/facility technicians can plug into each power pack or sensor to speed up commissioning and diagnosing problems. The currently practice of trial-and-error is much too time consuming and prone to non-optimal solutions.

3. All manufacturers should include a "zero delay" setting on the sensor to allow faster commissioning. This would allow the lights to turn off immediately so installers would not wait several seconds or minutes between each test.

\section{Bottom line}

The lighting industry does not seem to have the financial resources, or the willingness to develop low cost, sophisticated sensor systems. If as much money were spent on lighting control systems as is spent on cell phone development, for example, barriers to controls use could be quickly and greatly reduced. 
APPENDix M: OCCUPANCY SENSOR SELECTION GUIDE
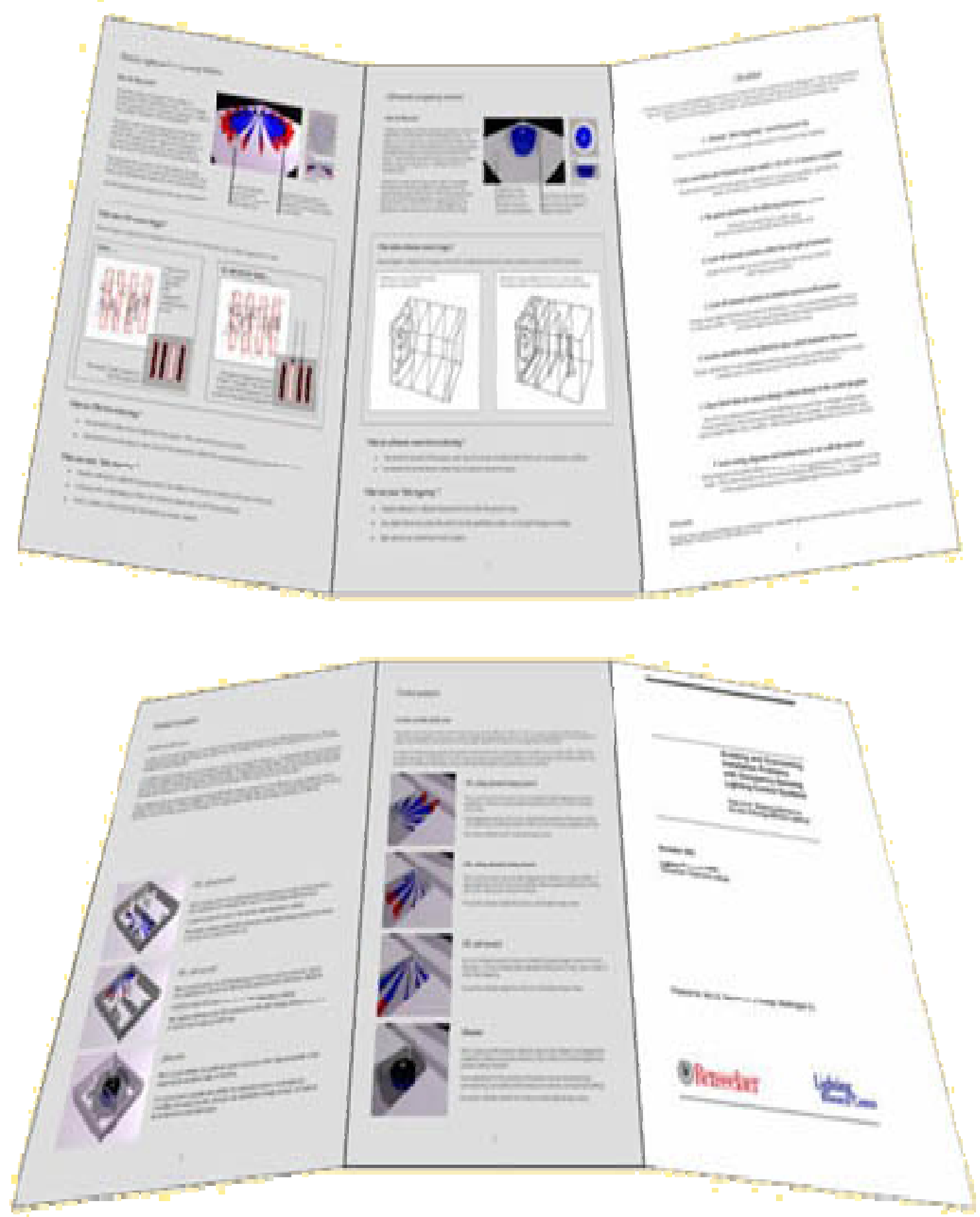


\section{APPENDIX N: TECHNOLOGY TRANSFER PLAN}

\section{OBJECTIVE}

Develop a viable technology transfer plan to bring to market improved, integrated lighting and control systems that will be embraced by end users and will therefore significantly reduce energy used for lighting in commercial buildings.

\section{FACTUAL INFORMATION}

The proposed Energy Policy Act, 2003, places renewed emphasis on load management and real time pricing. Lighting loads in commercial buildings are some of the easiest and best electric loads to control during periods of high electrical usage. Lighting control systems such as the load-shedding ballast, which are responsive to customer needs and cost-effective, will greatly enhance the ability of lighting to be used successfully for load management purposes.

Real time pricing will expose business customers to the actual high costs of electricity during times of peak electrical usage. This pricing will require customers to seek cost effective alternatives to reduce electric loads during these peak times. Lighting and its appropriate controls offer customers, regardless of size, the ability to control electric loads and, thereby, control costs.

While most of the United States currently has sufficient electric generating and transmission capacity, there are pockets across the country where neither generation nor transmission are robust enough to provide electricity efficiently during periods of high demand. These areas include southwest Connecticut, New York City, Long Island, NY, major metropolitan areas of Texas and the Bonneville Power Administration (WA, ID, OR, MT) area. The BPA situation is somewhat unique in that it involves electric loads which peak in the winter. Areas in the northern US such as Idaho and Minnesota experience summer electric peaks of such short duration that generation and transmission alternatives are prohibitively expensive.

To ensure the security and availability of electricity in these pockets of electric capacity need and to overcome the growing problem of the "not in my back yard" syndrome of sighting generation or transmission assets, load management alternatives must be developed. Lighting and lighting controls offer a cost-effective, viable alternative that can be employed in the near future. Lighting controls such as load-shedding ballasts and the self-commissioning photosensor have the potential to reduce lighting loads by $30 \%$ in all types and sizes of businesses when the demand for electricity is greatest.

Organizations and companies within these pockets of electric capacity need are charged with improving the efficiency and load management of electric using equipment. To leverage DOE's funding to reduce the barriers to using high efficiency lighting systems, collaboration with these regional organizations to further develop technologies, conducting demonstration projects and communicating the viability and value of these technologies to building designers and owners makes logical sense.

Market research conducted for the LRC for a daylighting project revealed the following:

- Building designers and owners/developers have a positive attitude toward building design which makes widespread use of natural light.

- The final decision about using daylighting and its associated lighting controls is a financial decision made by building owners/developers.

- Building designers and owners/developers indicated the major benefits of daylighting are the occupants of the building feeling better/more comfortable, reduced energy consumption, increased employee productivity, improved building appearance/aesthetics and increased marketability of the building to tenants or buyers. 
The greatest barrier to the expanded use of daylight is the capital costs involved; the second largest hurdle was problems with technologies including lighting controls.

Building designers and owners/developers said they most often get their information about daylighting from industry publications. Other sources included seminars, web sites, sales people/vendors, case studies and trade shows.

Market research conducted by the LRC for a load-shedding ballast project revealed the following:

- To attract business customers to load management programs, the cost to participate must be in line with the customer benefits.

- Lighting is an excellent load to control because there is so much of it and there is little impact on building occupants or operations if controlled properly. Lighting also provides a predictable amount of load control.

- An ideal lighting control scheme must allow the lights to be dimmed from a single location and must be easy to use.

- Load-shedding ballasts must be cost effective and be easy to install.

- Other important, required characteristics of load-shedding ballasts are: minimal impact on lamp life with no impact on the manufacturer's warranty; a "good housekeeping” seal of approval beyond the normal UL listing (such as the ENERGY STAR ${ }^{\circledR}$ symbol); the signal to dim cannot create interference with other customer equipment; and technical support of the ballast and signaling equipment after installation.

- Customers would allow utilities to control load-shedding ballasts directly if customers had override capabilities.

- Customers want demonstrated proof that the load-shedding technology works.

- Customers would first look to their utility for information regarding load-shedding ballasts and a related load management program and then to their trade associations.

- Electronic communications regarding information on load-shedding ballasts or load management programs is not desired by customers.

\section{MEASURING SUCCESS}

Leverage DOE efforts and funding to reduce barriers to the use of high efficiency lighting systems by developing and securing proposals to further reduce barriers through demonstration and evaluation of lighting controls such as load-shedding ballasts and self-commissioning photosensors.

Strategic regional organizations/corporations, where immediate needs such as electrical generation or transmission constraints can be alleviated by the use of lighting controls, participate with the LRC in technical transfer efforts thereby further leveraging DOE funds. These areas include southwest Connecticut, New York City, Long Island, extreme northern U.S. such as Idaho and Minnesota and the BPA in Northwest US.

National organizations concerned with energy efficiency are convinced of the value lighting controls can play to the continued security and availability of electric energy and have agreed to include lighting controls in their energy efficiency agendas. These organizations include the National Electrical Manufacturers Association (NEMA), National Electrical Contractors Association (NECA), National Association of Energy Service Companies (NAESCO), Association of Energy Service Professionals (AESP), Building Operators and Managers Association (BOMA), Natural Resources Defense Council (NRDC) and American Council for an Energy Efficient Economy (ACEEE) These organizations represent the spenders, users, doers, producers, and enablers depicted in the following technology transfer model. 
DOE states at the end of the project that the LRC has met all of its obligations and the project has succeeded in promoting DOE's agenda for greater use of lighting controls. 


\section{TECHNOLOGY TRANSFER MODEL}

Successful technology transfer must recognize the technical foundation and market enabling mechanisms that are in play with each new technology and the organizations, companies and representatives (or players) that interact with these mechanisms. By knowing where a technology is in relationship to these mechanisms and who the drivers/players are to move the technology through the remaining steps of the mechanisms, a collaborative plan can be developed. The plan will reflect the strengths of each organization/player in their area of expertise. The plan coordinator must tailor communication efforts that are of interest to each organization so they willingly participate in the plan. The figure below depicts the key players and activities they pursue under today's current practices of technology transfer. The ultimate goal of technology transfer is to have the technology so widely used that the impact of the technology is written into laws, regulations, codes and/or standards.

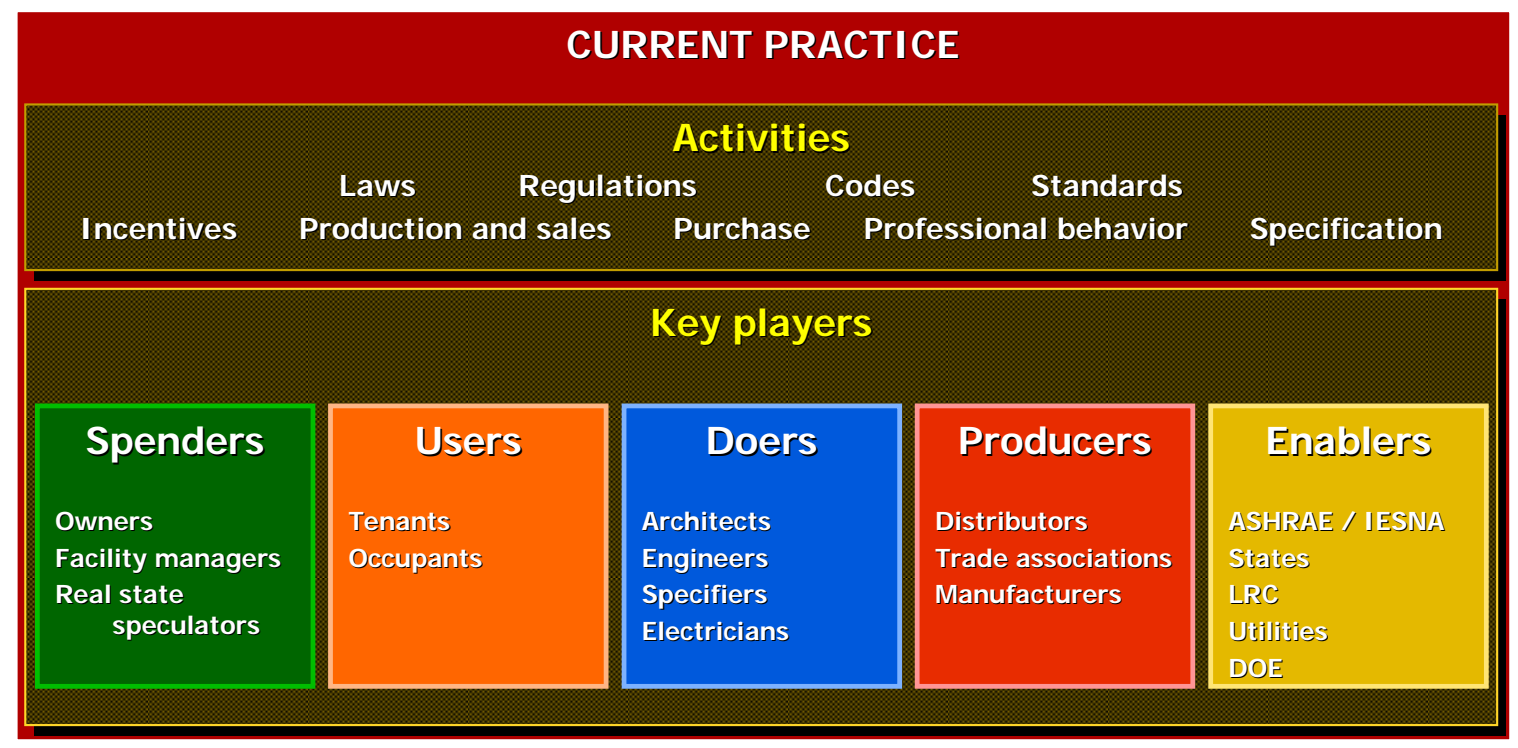

Key players include:

Spenders - These are building owners, facility managers, real estate speculators and developers. They make the ultimate buying decision but are influenced by the other types of key players and what they read and see in different types of communication outlets.

Users - These are the ultimate users of the technologies such as building tenants and occupants. They must be satisfied with the technology's performance and the ability to deliver its claims.

Doers - These are the architects, consulting engineers, specifiers and installers of the technology. They have great influence over the spenders. When doers recommend or install a technology, they risk their reputation on whether product will perform as advertised.

Producers - These are the people who make and distribute the technology and their respective associations/organizations. They must have a reliable and marketable technology that will earn profits for the company. Besides the key role of manufacturing the technology, they act as marketer.

Enablers - These are the organizations that write laws, regulations, codes and/or standards such as state and federal governments, American Society of Heating, Refrigeration and Air Conditioning Engineers (ASHRAE), National Electrical Manufacturers Association (NEMA) or Illuminating Engineering Society of North America (IESNA). Also included are organizations who influence law, regulation, code and/or are standard writers such as Natural Resources Defense Council (NRDC) or American Council 
for an Energy Efficiency Environment (ACEEE). Enablers are also represented by organizations such as the Lighting Research Center who has relationships and partnerships with all of the key players within an industry and is able to bring these dissimilar types of players together in collaborative and mutually beneficial efforts to successfully perform technology transfer. Also included with this grouping are utilities and state/regional organizations such as Xcel Energy and the Northwest Energy Efficiency Alliance, which are charged with the responsibility for implementation of energy efficiency programs.

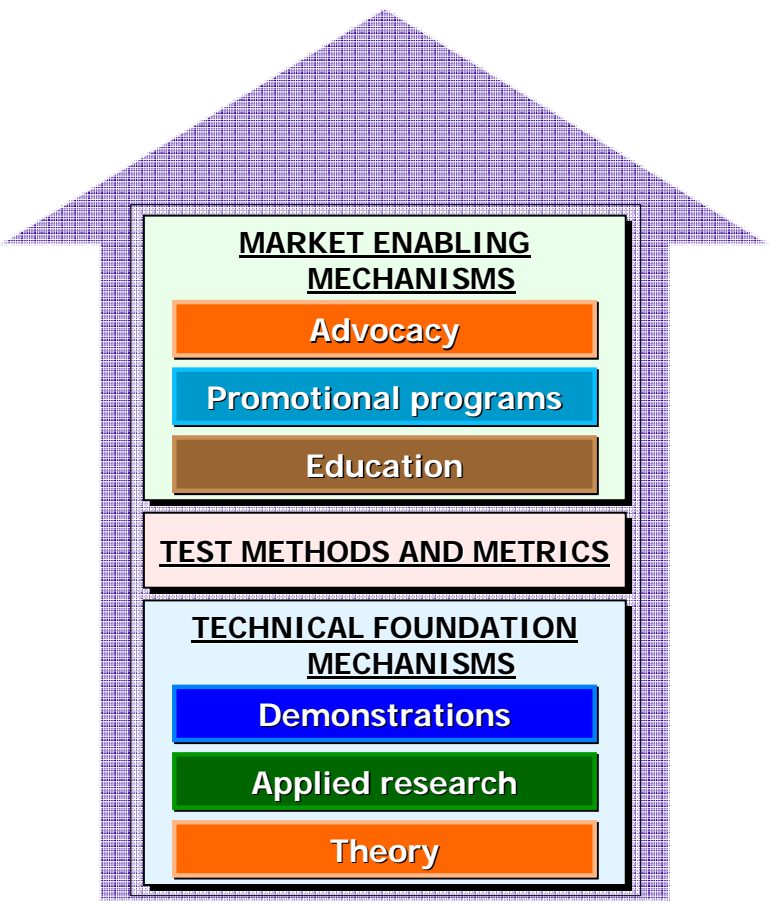

The transformation mechanisms shown are methodical, step-by-step processes for developing, demonstrating, evaluating, commercializing and, finally, customer acceptance of a technology. The successful achievement of each step's goals is a prerequisite to moving onto the next step. Failure to meet these goals or an attempt to skip a step reduces the chances of the technology being accepted by the user.

The mechanisms include:

Theory - This mechanism identifies a possible technology to satisfy an unfulfilled customer or user need. The technology gets defined and researchers determine if the technology is achievable at some reasonable cost to the user. While research organizations like the LRC and other producers are the primary players for this mechanism, input from and funding to the other key players is mandatory. Applied Research - This mechanism must prove the technical and economic concepts of the technology through technical and market research. Prototypes are developed as part of meeting this step's goals. Again, close collaboration with all key players is necessary as the research or manufacturing organizations endeavor to meet the goals of this mechanism. As one of its goals, the applied research mechanism identifies manufacturers willing to commercialize the technology and begin performing the necessary engineering for manufacturing.

Demonstrations - Full scale demonstrations are necessary to "show the world" the technology works and delivers the promised benefits. The demonstrations must answer any questions or concerns raised by users or spenders during the market research phase. The proof must be of the technology and its economics as applied to an actual, rather than laboratory, situation. Successful demonstrations with multiple locations or situations increase the acceptance of the technology. Funding and defining the measured metrics for this phase must come from other key players. 
Test Methods and Metrics - The method and metrics on which the evaluation is conducted of a technology, especially during the demonstration step, must be acceptable and agreed to by the research community as well as all the key players. Test results must withstand the rigors of review. While this mechanism may seem intuitive, it may be the most important step of all transformation mechanisms. It sets the stage as to how believable the claims made by the technology will be to the spenders, users, doers, producers and enablers and, ultimately, how successful the technology will be in the marketplace. Also, it provides a uniform means to measure results utilizing the same technology in different labs and facilities.

Education - The education mechanism is meant to be broad in scope to include the necessity to educate all the key players about the benefits, abilities and limitations of the technology and why it should be adopted in the marketplace, i.e. what it will do to help society. This is the first step in bringing the technology to the market. Yet, many times organizations responsible for technology development do not view the educational function as part of their responsibilities. Funding for education should be included as part of any technology development or else the technology may never advance to the market.

Promotional Programs - If the rigors of the test methods and metrics are met and if a successful education program is funded and executed, then, and only then, will the producers, enablers, utilities and state/regional agencies charged with the conduct of energy efficiency programs promote the use of the technology to the spenders, doers and other users. Promotional programs can take many forms such as utility incentive programs or endorsements.

Advocacy - This is the final step in the technology transfer model but does not occur until after the technology has reached some level of acceptance within the marketplace. It only occurs when there is public consensus of the use and benefits of the technology. Advocates then request that the benefits of the technology are included in regulations, codes and/or standards.

The technology transfer model works best when combining current practices with transformation mechanisms. One must identify the key players for each of the mechanisms for each technology to be transferred to the marketplace. As a result, this will allow for the development of a transfer plan with targeted audiences and messages.

The model works by identifying where the technology is along the mechanism steps and who the key players are to move it to the next step.

If the technology is in relatively wide use, approach the enablers for the advocacy of the benefits of the technology to promote regulations, codes and standards.

If the technology is ready for widespread use, use promotional programs to convince the doers and spenders of the benefits of the technology.

If the technology has been developed and demonstrated to be of value but is not in use, convince utilities and others to promote the technology.

If the technology has been developed but is not proven on a wide scale, seek funding for demonstration and evaluation projects and to educate spenders, users, doers and enablers of the unbiased results. If the technology is in the applied research stage, seek funding to prove/disprove the technology and its economics. Also seek someone from the producer segment to manufacture the product.

In all cases, ensure communications across all key player types of the technologies possibilities, evaluations, etc. 


\section{WHERE LIGHTING CONTROLS TECHNOLOGIES STAND TODAY}

\section{Occupancy Sensors}

Current status - This technology is commercially available, viable and proven to save energy. Discussions concerning the amount of energy saved persist as well as how to select and install the correct occupancy sensor for a given application.

Future technology transfer efforts - This technology is ready for inclusion into codes and standards. Communication efforts must include providing decision makers (doers and spenders) with predictable energy savings as well as selection and installation practices.

\section{Self-commissioning Photosensors}

Current status - This technology has been developed, is easily installed compared to other photosensors and as been shown to save energy under a small demonstration scenario. Economic viability under daylight conditions is marginal because of the cost of a fully dimming ballast.

Future technology transfer efforts - The technology needs to undergo the rigors of a full demonstration and evaluation to quantify energy savings, economically justify the technology and communicate the results. Funding for these activities should be sought from regional organizations (users) where electric capacity issues exist. Also, follow-up with manufacturers (producers) is necessary to fully commercialize this product.

\section{Load-shedding Ballasts}

Current status - This technology is under development with a cost-effective ballast operation proven in the laboratory. The communication link from a central building controller to each ballast is also needed.

Future technology transfer efforts - Seek funding from regional organizations (users) to continue the development of this technology. A manufacturer (producer) willing to commercialize this product must be found if the product is technically and economically viable.

\section{DETAILED PLAN}

The plan must meet the technology transfer objective and achieve the measures of success identified in this plan by meeting the needs of each key player for the technology in question (as partially identified in paragraph II, Factual Information). Using the model presented above for each of the three technologies (occupancy sensor, self-commissioning photosensor and load-shedding ballast) will facilitate identification of all key players needed to move the technology through the remaining steps of technology transfer. The plan presents a model for each technology and all of its key players and then identifies specific actions to be taken with specific key players, targeting completion during Year 3 of this project.

Since it is impossible to interact with each individual spender, user, doer, producer or enabler, the LRC must interact with organizations whose members represent individual companies, professionals, owners, etc.

\section{General Actions}

The LRC will:

Seek review of this plan and concurrence with proposed actions from state and regional energy efficiency organizations, lighting specifiers and other lighting decision makers. 
Develop and maintain a dedicated web site directed toward state and regional lighting decision makers to assist them in using lighting control technologies, keeping them informed as new/improved technologies become available on the market for the duration of this project.

\section{Occupancy Sensor}

\begin{tabular}{|c|c|c|c|c|c|c|}
\hline & \multirow{2}{*}{ Status } & \multicolumn{5}{|c|}{ Key Players } \\
\hline & & Spenders & Users & Doers & Producers & Enablers \\
\hline Advocacy & Ongoing & & & $\begin{array}{l}\text { NAESCO, } \\
\text { AESP }\end{array}$ & NEMA & $\begin{array}{l}\text { ACEEE } \\
\text { NRDC }\end{array}$ \\
\hline $\begin{array}{l}\text { Promotional } \\
\text { Programs }\end{array}$ & Ongoing & & & & manufacturers & $\begin{array}{c}\text { Utilities, } \\
\text { NYSERDA, } \\
\text { CEC, NEEA }\end{array}$ \\
\hline Education & Ongoing & BOMA & BOMA & $\begin{array}{l}\text { ASHRAE, } \\
\text { NECA, } \\
\text { IESNA, AIA } \\
\text { NAESCO }\end{array}$ & NEMA & $\begin{array}{c}\text { DOE, LRC } \\
\text { Utilities, } \\
\text { NYSERDA, } \\
\text { CEC, NEEA }\end{array}$ \\
\hline $\begin{array}{l}\text { Test Methods \& } \\
\text { Metrics }\end{array}$ & Complete & & & & & \\
\hline Demonstrations & Complete & & & & & \\
\hline $\begin{array}{l}\text { Applied } \\
\text { Research }\end{array}$ & Complete & & & & & \\
\hline Theory & Complete & & & & & \\
\hline
\end{tabular}

\section{Actions}

Prepare and publish an article for BOMA or a similar building owner/manager publication regarding the use and energy savings of occupancy sensors in office buildings.

Prepare and publish the application and installation guide to occupancy sensors developed in Task 4 of this project and disseminate through NECA or similar organization of electrical contractors.

Prepare and present a paper on the application and energy saving estimates of occupancy sensors developed as part of Task 4 of this project to ASHRAE, IESNA, AESP or similar professional organizations or to regional organizations charged with improving energy efficiency.

Prepare and present the findings of the "Reducing Barriers to Use of High Efficiency Lighting Systems" project to NEMA, ASHRAE, IESNA or similar industry standard organization.

Prepare and present the findings regarding the use of occupancy sensors to ACEEE, NRDC or similar energy efficiency advocacy groups so they will advocate the inclusion of occupancy sensors in future codes or standards. 


\section{Self-commissioning Photosensor}

\begin{tabular}{|c|c|c|c|c|c|c|}
\hline & \multirow{2}{*}{ Status } & \multicolumn{5}{|c|}{ Key Players } \\
\hline & & Spenders & Users & Doers & Producers & Enablers \\
\hline Advocacy & Not started & & & & NEMA & $\begin{array}{l}\text { ACEEE, } \\
\text { NRDC }\end{array}$ \\
\hline $\begin{array}{l}\text { Promotional } \\
\text { Programs }\end{array}$ & Not started & & & & Manufacturers & $\begin{array}{c}\text { Utilities, } \\
\text { NYSERDA, } \\
\text { CEC, NEEA }\end{array}$ \\
\hline Education & Not started & BOMA & BOMA & $\begin{array}{c}\text { ASHRAE, } \\
\text { NECA, } \\
\text { IESNA, AIA, } \\
\text { NAESCO }\end{array}$ & NEMA & $\begin{array}{c}\text { DOE, LRC, } \\
\text { Utilities, } \\
\text { NYSERDA, } \\
\text { CEC, NEEA }\end{array}$ \\
\hline $\begin{array}{l}\text { Test Methods \& } \\
\text { Metrics }\end{array}$ & Ongoing & & & & NEMA & LRC \\
\hline Demonstrations & Ongoing & BOMA & BOMA & & Manufacturers & $\begin{array}{c}\text { LRC, DOE, } \\
\text { Utilities, } \\
\text { NYSERDA, } \\
\text { CEC, NEEA }\end{array}$ \\
\hline Applied Research & Complete & & & & & \\
\hline Theory & Complete & & & & & \\
\hline
\end{tabular}

\section{Actions}

Develop and submit a proposal for a full-scale demonstration project using the self-commissioning photo sensor to utilities, NYSERDA, CEC, NEEA or similar regional organizations charged with improving energy efficiency to measure energy savings and the ease of installation. This will leverage DOE expenditures in reducing barriers to the use of high efficiency lighting systems.

Develop and submit a proposal for project funding on lamp/ballast compatibility testing under dimming conditions.

Prepare and present the uses and benefits of a self-commissioning photosensor to utilities or regional organizations charged with improving energy efficiency.

Develop and present the benefits and uses of the self-commissioning photosensor to lighting professionals and codes/standards writers. 
Load-shedding Ballast

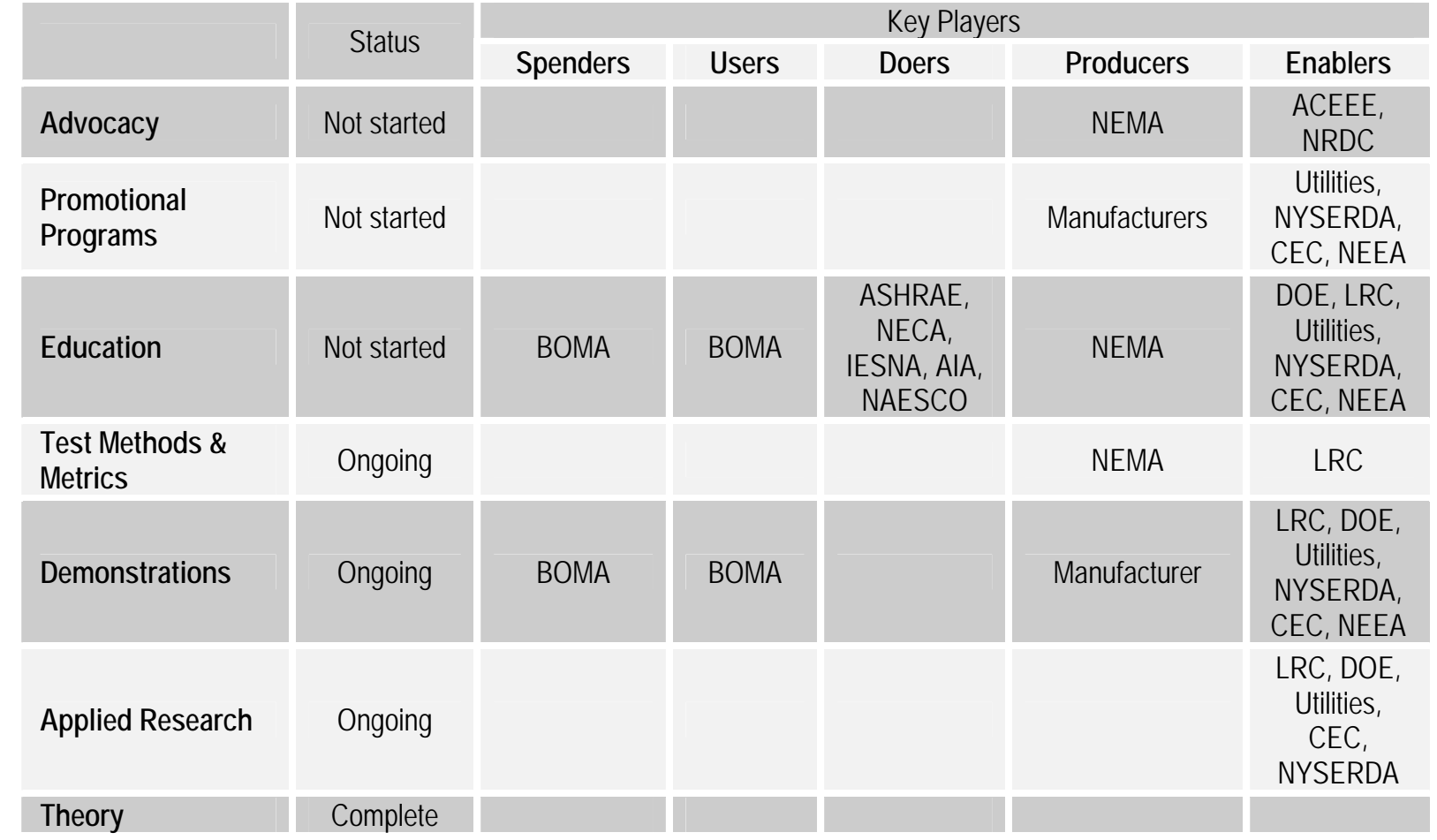

\section{Actions}

Leverage DOE funding for the "Reducing Barriers” project by developing and submitting a proposal to develop the communication links for load-shedding ballasts to utilities or regional organizations charged with improving energy efficiency in states or regions where electric capacity is constrained.

Develop and present to NRDC, ACEEE, or similar advocacy organization information on the value of using lighting as a load management tool and the lighting control schemes utilizing load-shedding ballasts that will encourage businesses to shed lighting loads so they may advocate their use in codes and standards.

Prepare and publish an article for a BOMA or similar building owner/manager publication or NAESCO or similar doer publication on the values of using lighting systems for electric load shedding. 


\section{RECOMMENDATIONS FOR CONTINUATION OF INFORMATION DISSEMINATION}

The plan above clearly indicates a need for continuation of information flow in the form of published articles, presentations, education and a dedicated web site to move occupancy sensors, selfcommissioning photosensors, load-shedding ballasts and other lighting controls through the technology transfer model. As technologies are developed, demonstrated and evaluated, information on their values and uses must be presented to the spenders, users, doers, producers and enablers so they are eager to commercialize the technology and feel comfortable with the potential accomplishments of the technology. Communication with these groups creates a "pull” for the technology from the spenders and users and a "push" from doers, producers and enablers.

As a minimum, the dedicated web site should be maintained and updated quarterly with existing and emerging lighting control technology information. The information should include factual data on the technical and economic merits of the technologies. The web site can be turned over to DOE at the end of this project for continued maintenance or DOE can issue a request for proposal for the maintenance of the site.

It is further recommended that any future DOE grants for the development of lighting control technologies include funding for technology transfer activities such as the publication of articles in professional journals, presentations of the technology to regional and national organizations and seminars or other education opportunities to teach spenders, users and doers to properly specify, install and use the technology. Development of a technology, by itself, will not ensure successful introduction into the marketplace. Dissemination of accurate, defendable information is necessary to garner acceptance. 
Appendix O: Association of Energy Service Professionals Presentation (begins on following page) 


\section{REDUCI NG BARRI ERS TO USE OF HI GH EFFI CI ENCY LI GHTI NG SYSTEMS}

Information Regarding Occupancy and Photo Sensors

Project Sponsor: U.S. Department of Energy

Project Executed by: Lighting Research Center 


\section{MARKET RESEARCH Understanding What Customers Want}

- Cost effectiveness

- Easy to install, operate and maintain

- Fully developed and tested products with a "Good Housekeeping" seal of approval

- Substantiated proof of any product claims

- Successful employment of the technology has occurred elsewhere

- Educate/Communicate with building owners/developers and designers (tech transfer) 


\section{ANSWERI NG THE QUESTI ONS}

- How well do the sensors work?

(technology issues)

- What do they cost/save? (economic issues)

- What barriers still exist to their use?

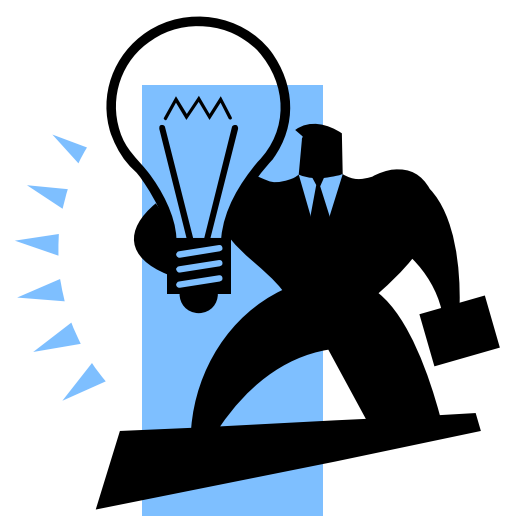
(technology transfer issues) 


\section{OCCUPANCY SENSOR TECHNOLOGY ISSUES}

- Two technology types - infra red \& ultrasonic

- Both are mature technologies

- Both work well with proper selection and positioning

- CONCLUSION (from a technology view point): Occupancy sensors are ready for codes/ standards inclusion 


\section{OCCUPANCY SENSOR ECONOMI C ISSUES}

- How much energy do occupancy sensors save? from LRC literature review:

Owned

Space

Sporadic Use:

Scheduled Use:
Shared

Space

$32 \%$

$41 \%$

$32 \%$

However, savings for a specific space may vary dramatically from these mean numbers. 


\section{BARRIERS TO OCCUPANCY SENSOR USE (Tech Transfer Issues)}

- Misunderstanding (mistrust?) of cost effectiveness

- Choosing the correct sensor

- Positioning the sensor within the space properly

- No standardization on wiring or commissioning/recommissioning

- Non user-friendly systems

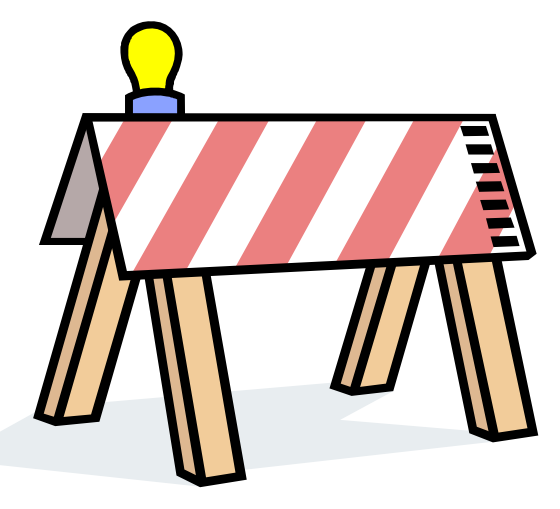




\section{OVERCOMI NG THE BARRI ERS (conducting tech. transfer tasks)}

- Publish energy saving information

- Develop easy to understand selection and positioning guide

- Work with manufacturers to standardize wiring and commissioning as much as possible

- Discuss results of market research regarding customer needs (conduct additional research)

- Make sure end-user knows how to re-commission the sensor 


\section{PHOTOSENSORS FOR NON-ARCHITECTURAL DI MMI NG}

- For use in daylighting applications or lumen maintenance.

- Architectural dimming should not be included in energy codes. The application objectives are for aesthetics. 


\section{PHOTOSENSOR TECHNOLOGY ISSUES}

- Analog design of control algorithm makes it difficult to accurately adjust the photosensor during commissioning

- Lack of manual controls to adjust photosensor to individual preferences

- Minimal integration with other lighting controls, i.e. occupancy sensors 


\section{PHOTOSENSOR ECONOMI C ISSUES}

- Daylighting Application thru Windows

10 sight survey of office use, standard 10 hours per day, 5 days per week operation, northeastern U.S.

$30 \%$ savings or approximately $80 \mathrm{kWh} / \mathrm{yr} /$ fixture

- Daylighting Application thru Skylights

1 sight survey of warehouse, 20 hours per day, 5 days per week operation, northeastern U.S.

$\mathbf{3 0} \%$ savings or approximately $\mathbf{8 0 0}$

kWh/ yr/ skylight 


\section{PHOTOSENSOR ECONOMI C I SSUES}

- Cost effectiveness has two parts - the savings and the cost to obtain the savings

- Incremental cost of full dimming ballast and its fixture share of the photosensor control is approximately $\$ 40$.

- Energy savings of approximately $80 \mathrm{kWh} / \mathrm{yr}$ produces different dollar savings across the country

NYC $\$ 16.32 / \mathrm{yr}$ or $2.45 \mathrm{yr}$ payback

Ohio $\$ 7.05 / \mathrm{yr} \quad$ or $\quad 5.67 \mathrm{yr}$ payback

SF $\quad \$ 8.17 / y r \quad$ or $\quad 4.90 \mathrm{yr}$ payback

Bottom line - daylighting not economical from energy savings only 


\section{BARRI ERS TO PHOTOSENSOR USE (Tech Transfer I ssues)}

- Technology must satisfy customer needs

- Cost effectiveness must improve

- Proof of technology continual operation beyond commissioning

- Proof of benefits

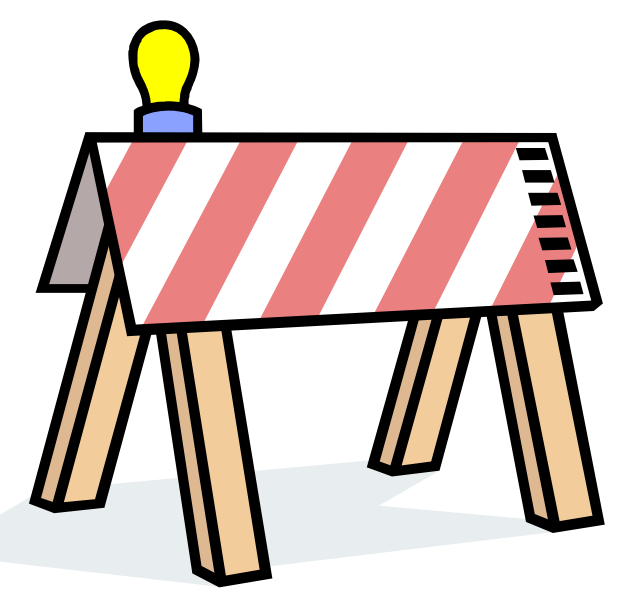




\section{OVERCOMI NG THE BARRI ERS Technology}

Features of Self-commissioning Photosensor

- Sliding set point algorithm

- Wireless, remote sensor

- Self-commissioning (minimal user interaction)

- Occupant preference adjustment independent of commissioning

- Switching in addition to dimming control

- Compact size (replaces std. switch)

- Efficient operation ( 1.2 watts @ 120 Vac)

- Microprocessor based

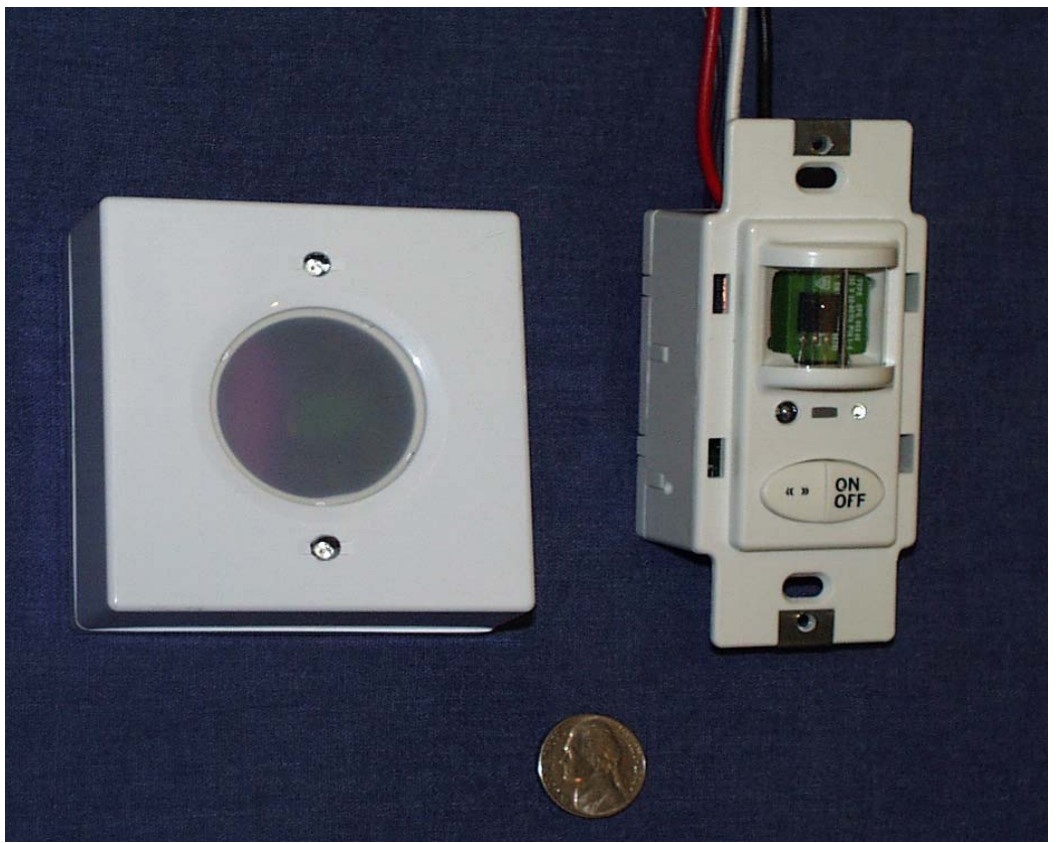

Commissioning time $<3 \mathrm{~min}$. on most cases 


\section{OVERCOMI NG THE BARRI ERS Technology}

- Survey/interview results (8 subjects)

- 5 noticed dimming, none found it distracting

- 3 noticed on/off switching, one found it slightly distracting

- 3 felt they had more control, 5 reported no change

- 6 had no suggestions for improvements, one suggested better buttons, one for better appearance

- 6 would like the photosensor in new office, 2 said it didn't matter

- Overall, no negative comments 


\section{OVERCOMI NG THE BARRI ERS Economics}

Decrease cost or I ncrease benefits or both

Major program underway sponsored by:

- US Dept. of Energy

- New York State Energy Research \& Development Auth.

- I owa Energy Center

- California Energy Commission

- Connecticut Light \& Power Co.

- Northwest Energy Efficiency Alliance

- North Carolina Solar Center

Daylight Dividends 


\section{ON THE TECHNOLOGY FRONT}

- Dayswitch

- Blind Controls

- Lamp / Dimming Ballast Compatibility 


\section{ON THE ECONOMI C FRONT Cost reduction or benefit increase}

- Groundbreaking study about to be released

- be out in about 6 weeks

- Prepared by Dr. Peter Boyce \& reviewed by 4 leading daylighting, productivity, health experts

- Most research to date shows increase in productivity due to increase in light, not necessarily daylight

- Can increase in productivity be measured through the circadian system (maybe the only way to measure)?

- Do buildings that incorporate daylight have a greater value than non-daylit buildings?

- Is there an inherent need for humans to be connected to the outside world? 


\section{ON THE ECONOMI C FRONT (cont.) Cost reduction or benefit increase}

- What is the added cost, if any, to construct a daylit facility?

- Does the use of daylight increase HVAC energy needs?

- What are the barriers in the use of skylights? 


\section{NTEGRATED SKYLI GHT LUMI NAI RE Lighting Research Center

\section{ISL Components}

Simple bubble skylight, double-dome, 4'x4' Sunlight diffusing box Photosensor Controls hub Fluorescent luminaires
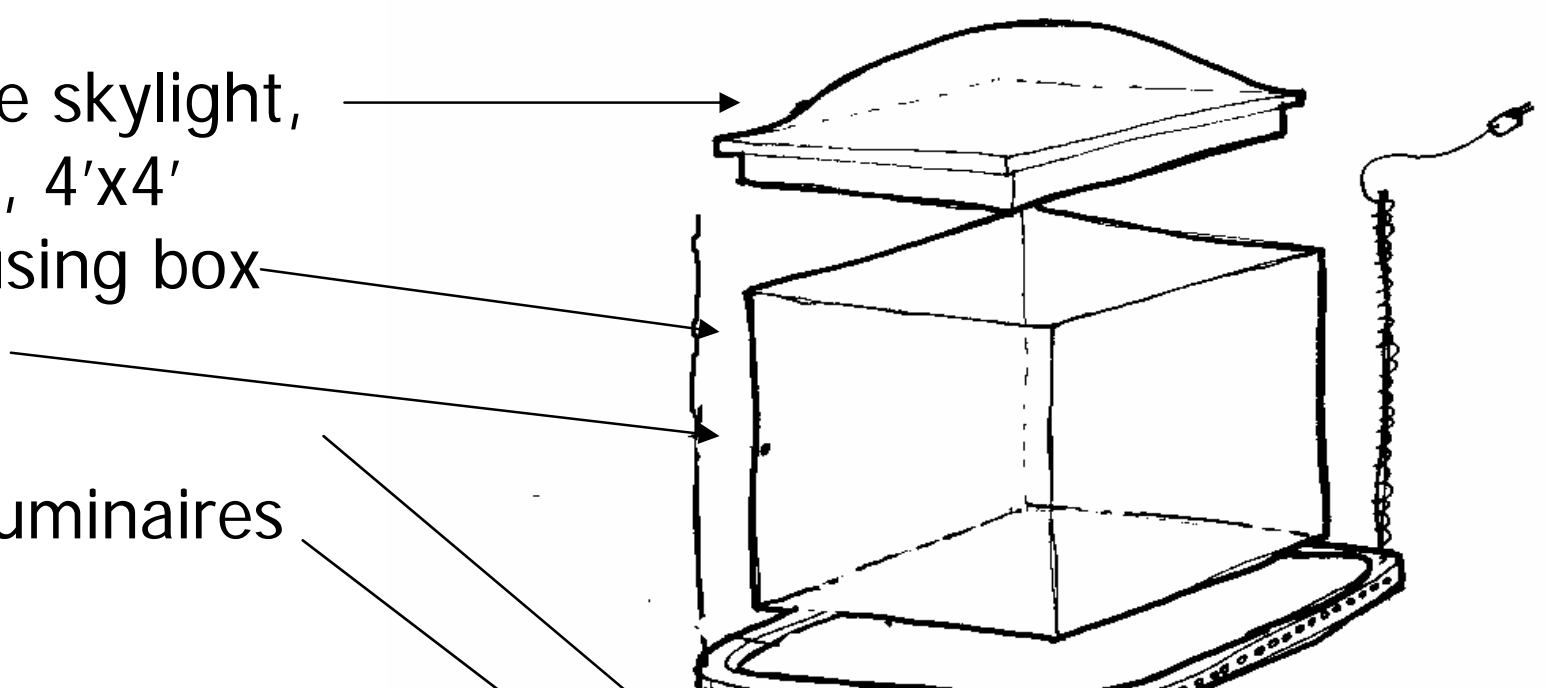


\section{HOW DO PEOPLE LI KE IT?}

"(The ISL) fulfills lighting requirements better than a single light because it is a larger area of illumination"

- Lightolier employee

" I think they're great. When I come in in the morning, they're on and I don't bump into things"

- Lightolier employee the lighting is good

Overall, it is easy to see what I need to see

Overall, the lighting is comfortable

The light fixture is attractive to

look at

The light fixture is uncomfortable to look at directly

The lighting is sometimes too bright

The lighting is sometimes too

The lighting flickers The lighting creates strong shadows in some areas

The switching of the fluorescent lighting is disturbing

\begin{tabular}{|c|c|c|}
\hline $\begin{array}{c}\text { Tube } \\
\text { storage } \\
\text { by day } \\
(n=17)\end{array}$ & $\begin{array}{c}\text { Tube } \\
\text { storage } \\
\text { by night } \\
(n=11)\end{array}$ & $\begin{array}{c}\text { Staging } \\
\text { area at } \\
\text { any } \\
\text { time } \\
(n=15)\end{array}$ \\
\hline $100 \%$ & $83 \%$ & $85 \%$ \\
\hline $100 \%$ & $90 \%$ & $100 \%$ \\
\hline $100 \%$ & $82 \%$ & $85 \%$ \\
\hline $69 \%$ & $50 \%$ & $54 \%$ \\
\hline $24 \%$ & $45 \%$ & $73 \%$ \\
\hline $18 \%$ & $18 \%$ & $47 \%$ \\
\hline $35 \%$ & $45 \%$ & $27 \%$ \\
\hline $13 \%$ & $18 \%$ & $20 \%$ \\
\hline $0 \%$ & $20 \%$ & $29 \%$ \\
\hline $18 \%$ & $\begin{array}{c}\text { not } \\
\text { applicable }\end{array}$ & $\begin{array}{c}\text { applicable } \\
\end{array}$ \\
\hline
\end{tabular}




\title{
LIGHTING CONTROLS:
}

\section{WHERE ARE WE AND WHERE ARE WE GOING}

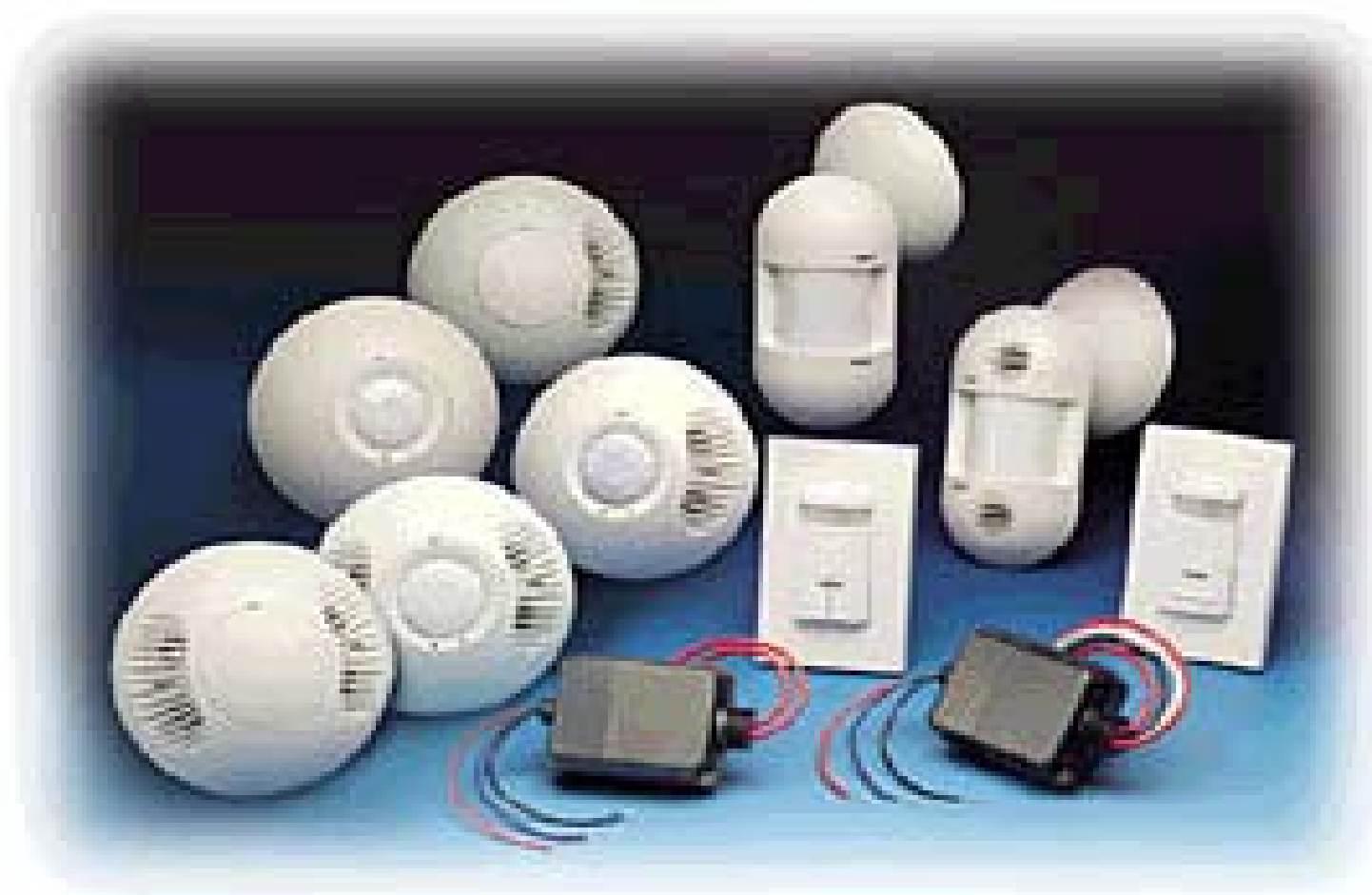

\section{Presentation to:}

Association of Energy Service Professionals International $14^{\text {th }}$ National Energy Services Conference and Exposition

December 9, 2003

\author{
Peter M. Morante \\ Andrew Bierman \\ Director of Energy Programs \\ Specialist \\ Lighting System \\ Lighting Research Center \\ Lighting Research \\ Center
}




\section{LIGHTING CONTROLS: WHERE ARE WE AND WHERE ARE WE GOING}

\section{INTRODUCTION}

The Lighting Research Center (LRC) is part of Rensselaer Polytechnic Institute and is the world leader in university based lighting research. With funding from the U.S.

Department of Energy, the LRC has conducted extensive research on the use and barriers of lighting controls. This paper limits its discussions to lighting controls, current and future, used in non-residential applications and for fluorescent lighting systems.

Successful market transfer and technology acceptance occur through a process that recognizes the roles of key market players and the market enabling mechanisms that are in play with each new technology. The LRC has developed a technology transfer model for lighting controls to help it understand where a lighting technology is currently in the process of market acceptance and who the key players are to move the technology to full customer use. For example, motion sensors have achieved technological acceptance and their energy savings provide users with positive economic outcomes if properly employed. However, the specification and installation of occupancy sensors still needs to be explained to achieve full market acceptance. The solution is education for specifiers and installers, or what the technology transfer model calls doers.

This paper discusses two commercialized lighting controls, motion sensors and photo sensors for dimming fluorescent lights in the presence of daylight. An emerging lighting control technology will also be discussed. Digital Addressable Lighting Interface (DALI) is beginning to be commercialized. Lighting ballast and control manufacturers believe it is the next big breakthrough since the introduction of electronic ballasts. Whether customers will be willing to pay a premium price for this technology is not yet clear. The last technology discussed is in the developmental stages. Load management, also referred to as distributed resources, will play a major role meeting the needs of peak electric demands in both regulated and deregulated electric markets. Therefore, a cost effective load shedding ballast is being developed. 


\section{MARKET RESEARCH}

Understanding what customers need and want is an important first step in developing new lighting control technologies. The Lighting Research Center, as part of its research contracts, has conducted a series of focus groups and surveys to understand the market and, thereby, direct technology development. Too many organizations rush to the technology development stage without due consideration of what the customer wants. This leads to product failures in the marketplace because the technology is too complicated, does not provide an economic return or its features are not wanted. The lighting industry sometimes suffers from the lack of giving the customer what he/she wants.

\section{Primary Market Research Findings}

There is consistency among all surveys conducted by the LRC on the basic needs of customers.

Above all else, customers want a reasonable return on any investment they make regarding lighting or any other energy efficiency improvement.

The technology must be easy to install, operate and maintain. Customers do not want to have to employ specially trained personnel to make the technology work properly. Customers want products to be fully developed and tested prior to entering the marketplace. They do not want the manufacturer to find it necessary to further modify the technology after it has been installed.

Customers want substantiated proof of any technology or economic claim. Customers want to know/see that the technology has been successfully employed by others (the "first to be second" syndrome).

While these findings are pretty much intuitive and common sense, they seem to get lost when a technology is being developed. Yet, these findings can explain lighting technology acceptance or rejection within the marketplace.

For example, the electronic ballast became widely accepted because it satisfied all of the customer needs. It is very cost effective. The technology can be installed by most electrical contractors without any special skills. It operates without additional human intervention. While electronic ballasts had some technological problems after their market introduction, they were easily corrected. Today electronic ballasts make up the majority of ballast sales.

Conversely, photo sensors for dimming fluorescent lighting have not made inroads in the marketplace because of the high cost of the dimming ballast, the complexity in correctly commissioning the photo sensor, the horror stories that persist in their continued use and lack of substantiated proof of their benefits.

\section{Daylight Dividends Market Research}

Market research conducted for the LRC and its sponsoring organizations for a program in daylighting called "Daylight Dividends" revealed the following": For the full report of the market research findings and much more information on daylighting, please review the Daylight Dividends website at www.daylightdividends.org. 
Building designers and owners/developers have a positive attitude toward building design that makes widespread use of natural light.

The final decision about using daylighting and its associated lighting controls is a financial decision made by building owners/developers.

Building designers and owners/developers indicated the major benefits of daylighting are the occupants of the building feeling better/more comfortable, reduced energy consumption, increased employee productivity, improved building appearance/aesthetics and increased marketability of the building to tenants or buyers.

The greatest barrier to the expanded use of daylight is the capital costs involved; the second largest hurdle was problems with technologies including lighting controls.

Building designers and owners/developers said they most often get their information about daylighting from industry publications. Other sources included seminars, web sites, sales people/vendors, case studies and trade shows.

\section{Load Management Market Research}

The LRC conducted a market research effort to understand customer needs regarding using lighting controls to reduce peak electric demand. This project, funded by the California Energy Commission, was conducted as part of the development of a cost effective load shedding ballast for fluorescent lighting. Results revealed ${ }^{2}$ :

To attract business customers to load management programs, the cost to participate must be in line with the customer benefits.

Lighting provides a predictable amount of load control and, if controlled properly, has little impact on building occupants or operations.

An ideal lighting control scheme must allow the lights to be dimmed from a single location and must be easy to use.

Load-shedding ballasts must be cost effective and be easy to install.

Other important, required characteristics of load-shedding ballasts include:

minimal impact on lamp life with no impact on the manufacturer's warranty;

a "good housekeeping" seal of approval beyond the normal UL listing (such as the ENERGY STAR ${ }^{\circledR}$ symbol);

the signal to dim cannot create interference with other customer equipment;

manufacturers must provide technical support of the ballast and signaling equipment after installation.

Customers would allow utilities to control load-shedding ballasts directly if customers had override capabilities.

Customers want demonstrated proof that the load-shedding technology works.

Customers would first look to their utility for information regarding load-shedding ballasts and a related load management program and then to their trade associations. Electronic communications regarding information on load-shedding ballasts or load management programs is not desired by customers.

\section{TECHNOLOGY TRANSFER MODEL ${ }^{3}$}

The technology transfer model was developed to help the LRC visualize where existing lighting technologies stood in their technical and market developments, what is needed to move the technology toward market acceptance and who could assist in moving the technology forward. The model reflects the LRC's understanding of information 
discovered during the market research phase such as customers want to see the technology working. This is included in the model by ensuring there is a step to demonstrate the properties and economics of the technology.

Successful technology transfer must recognize the technical foundation and market enabling mechanisms that are in play with each new technology and the organizations, companies and representatives (or players) that interact with these mechanisms. By knowing where a technology is in relationship to these mechanisms and who the drivers/players are to move the technology through the remaining steps of the mechanisms, a collaborative plan can be developed. The plan will reflect the strengths of each organization/player in their area of expertise. The plan coordinator must tailor communication efforts that are of interest to each organization so they willingly participate in the plan.

Figure 1 below depicts the key players and activities they pursue under today's current practices of technology transfer. The ultimate goal of technology transfer is to have the technology so widely used that the impact of the technology is written into laws, regulations, codes and/or standards.

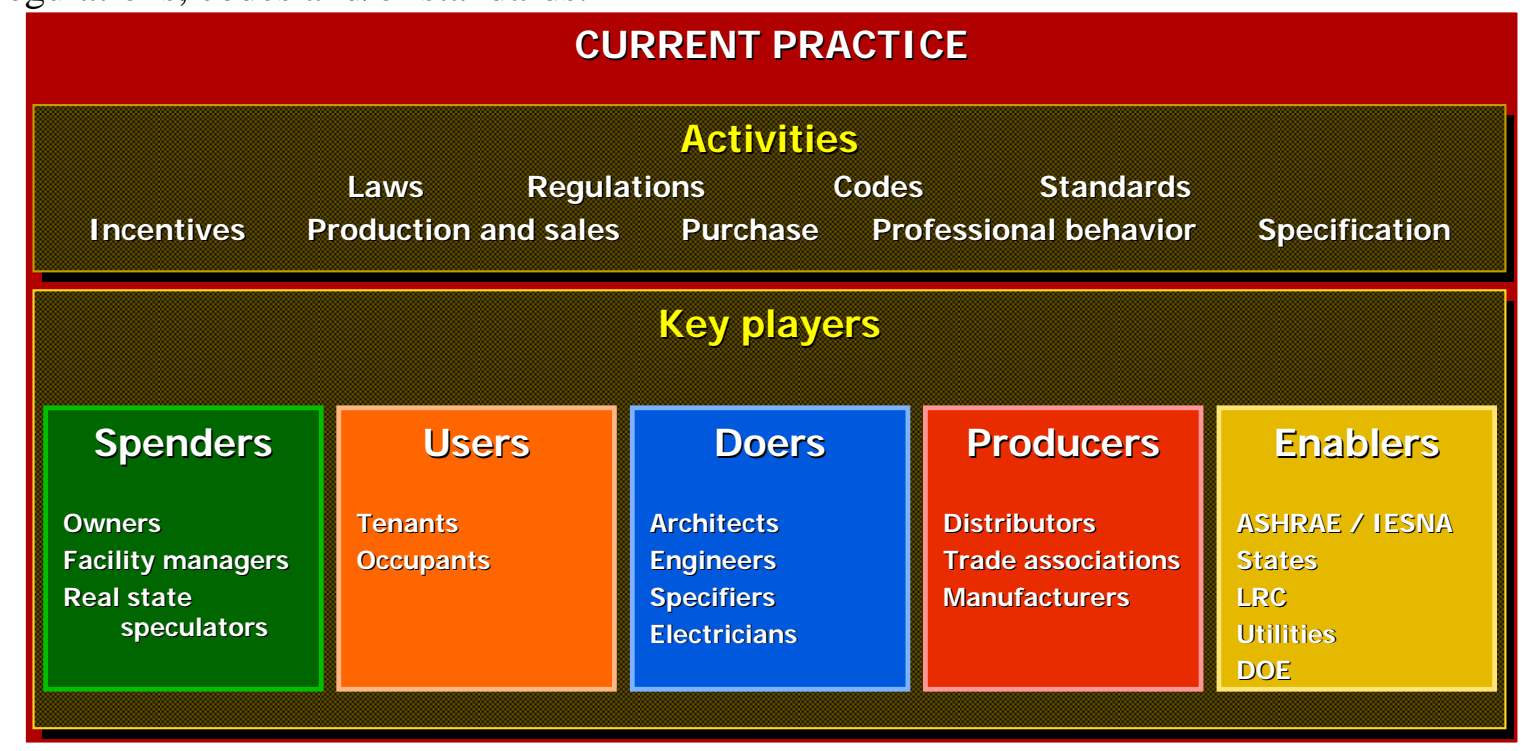

Figure 1

\section{Key players include:}

Spenders - These are building owners, facility managers, real estate speculators and developers. They make the ultimate buying decision but are influenced by the other types of key players and what they read and see in different types of communication outlets. Users - These are the ultimate users of the technologies such as building tenants and occupants. They must be satisfied with the technology's performance and its ability to deliver its claims.

Doers - These are the architects, consulting engineers, specifiers and installers of the technology. They have great influence over the spenders. When doers recommend or install a technology, they risk their reputation on whether the product will perform as advertised. 
Producers - These are the people who make and distribute the technology and their respective associations/organizations. They must have a reliable and marketable technology that will earn profits for the company. Besides the key role of manufacturing the technology, they act as marketers.

Enablers - These are the organizations that write laws, regulations, codes and/or standards such as state and federal governments, American Society of Heating, Refrigeration and Air Conditioning Engineers (ASHRAE), National Electrical Manufacturers Association (NEMA) or Illuminating Engineering Society of North America (IESNA). Also included are organizations that influence laws, regulations, or codes, and/or write standards such as the Natural Resources Defense Council (NRDC) or American Council for an Energy Efficiency Environment (ACEEE). Other Enablers include organizations that have relationships and partnerships with industry key players and bring these dissimilar types of players together. These collaborative efforts to perform technology transfer are mutually beneficial. Finally, utilities and state/regional organizations that are charged with the responsibility for implementation of energy efficiency programs, are Enablers. These include Xcel Energy and the Northwest Energy Efficiency Alliance.

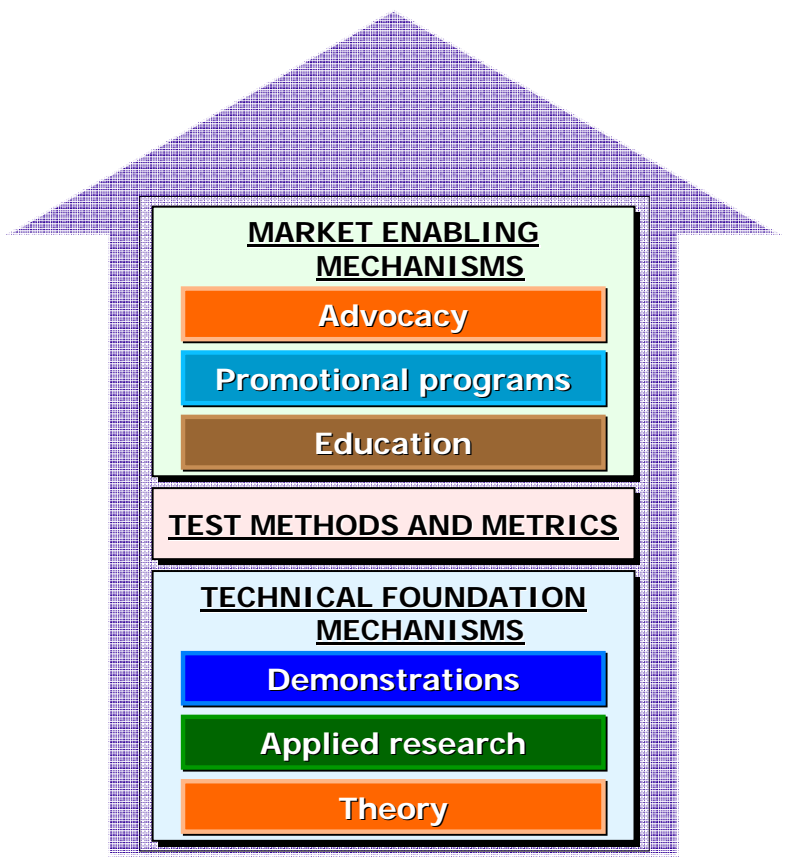

Figure 2

The transformation mechanisms shown in Figure 2 are methodical, step-by-step processes for developing, demonstrating, evaluating, commercializing and, finally, customer acceptance of a technology. The successful achievement of each step's goals is a prerequisite to moving onto the next step. Failure to meet these goals, or an attempt to skip a step, reduces the chances of the technology being accepted by the spender, user or doer.

The mechanisms include: 
Theory - This mechanism identifies a possible technology to satisfy an unfulfilled customer or user need. The technology gets defined and researchers determine if the technology is achievable at some reasonable cost to the user. While research organizations like the LRC and other producers are the primary players for this mechanism, input and funding from the other key players is mandatory.

Applied Research - This mechanism must prove the technical and economic concepts of the technology through technical and market research. Prototypes are developed as part of meeting this step's goals. Again, close collaboration with all key players is necessary as the research or manufacturing organizations endeavor to meet the goals of this mechanism. As one of its goals, the applied research mechanism identifies manufacturers willing to commercialize the technology and begin performing the necessary engineering for manufacturing.

Demonstrations - Full scale demonstrations are necessary to "show the world" that the technology works and delivers the promised benefits. The demonstrations must answer any questions or concerns raised by users or spenders during the market research phase. The proof must be of the technology and its economics as applied to an actual, rather than laboratory, situation. Successful demonstrations with multiple locations or situations increase the acceptance of the technology. Funding and defining the measured metrics for this phase must come from other key players.

Test Methods and Metrics - The method and metrics on which the evaluation is conducted of a technology, especially during the demonstration step, must be acceptable and agreed to by the research community as well as all the key players. Test results must withstand the rigors of review. While this mechanism may seem intuitive, it may be the most important step of all transformation mechanisms. It sets the stage as to how believable the claims made by the technology will be to the spenders, users, doers, producers and enablers and, ultimately, how successful the technology will be in the marketplace. Also, it provides a uniform means to measure results utilizing the same technology in different labs and facilities.

Education - The education mechanism is meant to be broad in scope to include the necessity to educate all the key players about the benefits, abilities and limitations of the technology and why it should be adopted in the marketplace, i.e. what it will do to help society. This is the first step in bringing the technology to the market. Yet, many times organizations responsible for technology development do not view the educational function as part of their responsibilities. Funding for education should be included as part of any technology development or else the technology may never advance to the market. Promotional Programs - If the rigors of the test methods and metrics are met and if a successful education program is funded and executed, then, and only then, will the producers, enablers, utilities and state/regional agencies charged with the conduct of energy efficiency programs promote the use of the technology to the spenders, doers and other users. Promotional programs can take many forms such as utility incentive programs or endorsements. Manufacturers also play a role in promoting their products through advertisements and sales personnel.

Advocacy - This is the final step in the technology transfer model but does not occur until after the technology has reached some level of acceptance within the marketplace. It only occurs when there is public consensus of the use and benefits of the technology. 
Advocates then request that the benefits of the technology are included in regulations, codes and/or standards.

Combining the market enabling mechanisms and the key players allows for the identity of players for each step of the process.

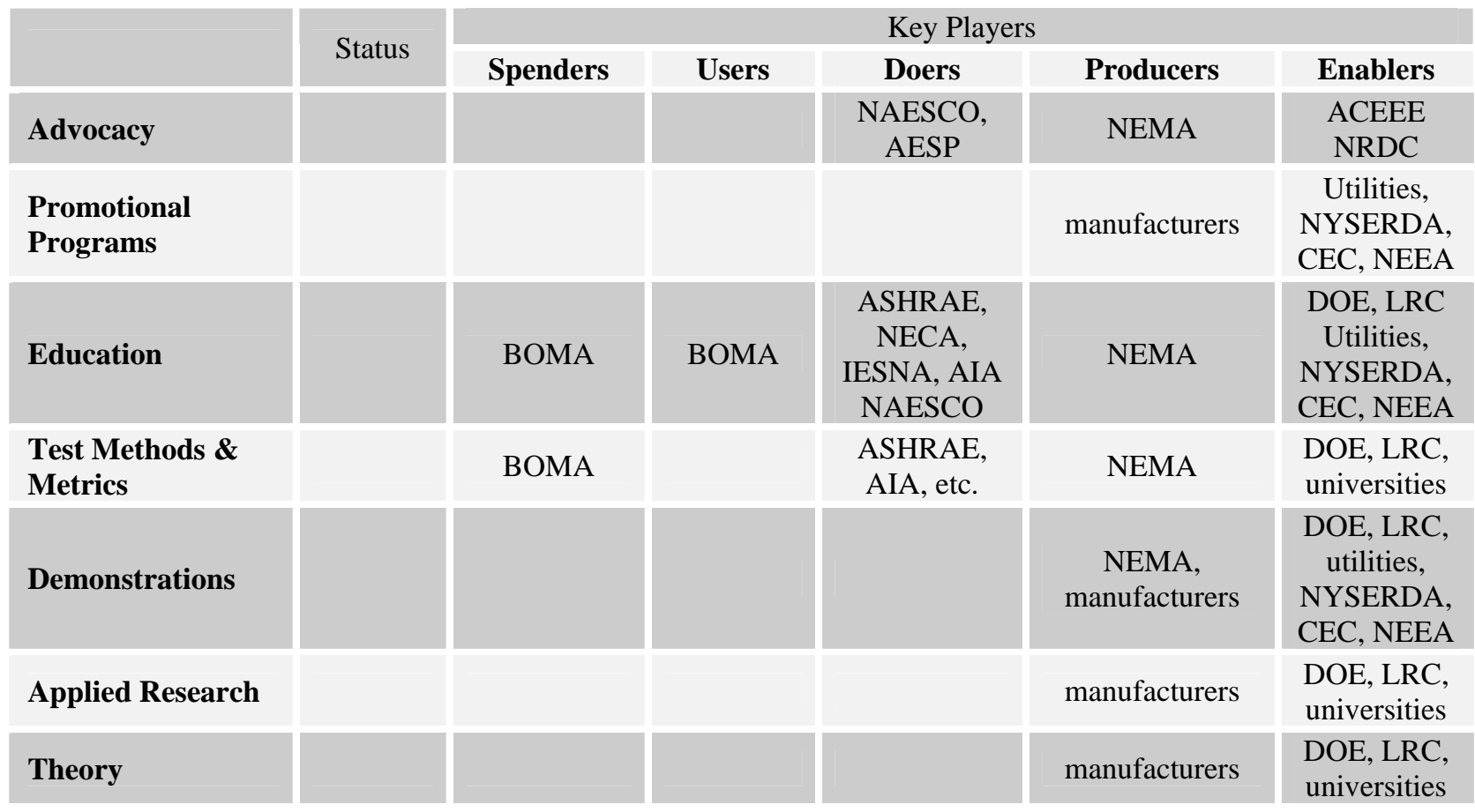

Figure 3

The technology transfer model works best when combining current practices with transformation mechanisms. One must identify the key players for each of the mechanisms for each technology to be transferred to the marketplace. As a result, this will allow for the development of a transfer plan with targeted audiences and messages. The model works by identifying where the technology is along the mechanism steps and who the key players are to move it to the next step.

If the technology is in relatively wide use, approach the enablers for the advocacy of the benefits of the technology to promote regulations, codes and standards.

If the technology is ready for widespread use, use promotional programs to convince the doers and spenders of the benefits of the technology.

If the technology has been developed and demonstrated to be of value but is not in use, convince utilities and others to promote the technology.

If the technology has been developed but is not proven on a wide scale, seek funding for demonstration and evaluation projects and to educate spenders, users, doers and enablers of the unbiased results.

If the technology is in the applied research stage, seek funding to prove/disprove the technology and its economics. Also seek someone from the producer segment to manufacture the product.

In all cases, ensure communications across all key player types of the technologies possibilities, evaluations, etc. 


\section{MOTION SENSORS}

Motion sensors turn lighting fixtures either on or off based on existence of someone being present in a space controlled by the occupancy sensor. The sensor detects motion either through changes in the infra-red radiation pattern or actively through detection of reflected ultrasonic microwave signals. The lack of detection causes the sensor to signal a controller to turn off the lights. Occupancy sensors are commonplace today in new construction (estimates of up to $60 \%$ of commercial construction projects now utilize them) but much of the existing floor space has not been retrofitted with these devices. 


\section{The Technology Transfer Model for Motion Sensors ${ }^{3}$}

Figure 4

\begin{tabular}{|c|c|c|c|c|c|c|}
\hline & \multirow{2}{*}{ Status } & \multicolumn{5}{|c|}{ Key Players } \\
\hline & & Spenders & Users & Doers & Producers & Enablers \\
\hline Advocacy & Ongoing & & & $\begin{array}{c}\text { NAESCO, } \\
\text { AESP }\end{array}$ & NEMA & $\begin{array}{l}\text { ACEEE } \\
\text { NRDC }\end{array}$ \\
\hline $\begin{array}{l}\text { Promotional } \\
\text { Programs }\end{array}$ & Ongoing & & & & manufacturers & $\begin{array}{c}\text { Utilities, } \\
\text { NYSERDA, } \\
\text { CEC, } \\
\text { NEEA }\end{array}$ \\
\hline Education & Ongoing & BOMA & BOMA & $\begin{array}{c}\text { ASHRAE, } \\
\text { NECA, } \\
\text { IESNA, } \\
\text { AIA } \\
\text { NAESCO }\end{array}$ & NEMA & $\begin{array}{l}\text { DOE, LRC } \\
\text { Utilities, } \\
\text { NYSERDA, } \\
\text { CEC, } \\
\text { NEEA }\end{array}$ \\
\hline $\begin{array}{l}\text { Test Methods } \\
\text { \& Metrics }\end{array}$ & Complete & & & & & \\
\hline Demonstrations & Complete & & & & & \\
\hline $\begin{array}{l}\text { Applied } \\
\text { Research }\end{array}$ & Complete & & & & & \\
\hline Theory & Complete & & & & & \\
\hline
\end{tabular}

The model tells us motion sensors are a proven technology that require additional education of building owners/occupants on the benefits of this technology and specifiers and electrical contractors (doers) on selection, location and installation of the sensors. This education is best provided by the manufacturers (producers) and utilities and others charged with operating energy efficiency programs (enablers).

\section{Energy Savings}

Debate over the potential energy savings achievable through the use of occupancy sensors has created doubt in the minds of building owners/occupants (spenders/users) as to the benefits of the technology. Therefore, based on market research (customers want proof), most customers are not retrofitting existing buildings.

The LRC consulted its extensive resource collection to compile a group of 26 case studies and claims by manufacturers regarding the effectiveness of occupancy sensor technologies. We organized the data in different ways in the various studies, often by specific room type (restrooms, hallways, coffee break rooms, conference rooms). We reviewed the literature and organized studies into broader occupancy categories based upon private (“owned”) vs. shared and scheduled vs. sporadic use:

Private ("Owned”) Spaces (such as single-person offices or spaces in which the user takes “ownership”)

Shared Spaces, Scheduled use (such as classrooms)

Shared Spaces, Sporadically used (such as public spaces, open-plan offices, corridors, bathrooms, storage rooms)

Private (“Owned”), scheduled occupancy was not a legitimate category. 
For private offices, all data were averaged leading to an average savings of $31.7 \%$. This average represents a wide range of hours of use, so we also examined the percentage of energy savings in those studies that were based upon documented "core hours" between 7.5 to 10 hours per day. This analysis yielded a lower average energy savings of $26 \%$, as expected because the former analysis was dominated by studies using longer hours of use as a base line. This latter percentage (26\%) is perhaps a better estimate of potential energy savings from occupancy sensors because, for buildings showing wasted energy use outside the core hours, other technologies such as time clocks are usually more cost effective than occupancy sensors. The LRC recommends $25 \%$ as an easy to remember best estimate of energy savings for private offices with sporadic use.

For shared spaces that are used sporadically, the average savings was 40.8\%. Again, there was a wide range of hours of use, but it seems inappropriate to base energy savings on "core hours" (7.5 to 10 hours per day) because hallways, stairs, etc. can be used at any time day or night. Therefore, we recommend $40 \%$ energy savings as the best estimate for shared, sporadically occupied spaces. Note, however, that some spaces should be illuminated without occupancy if lighting is used to signal potential occupants that a business is open or if the lights being on provide occupants with a sense of security or safety.

For shared, scheduled spaces, in particular classrooms, it is often difficult to ascertain the hours of use. Classrooms are used not only for teaching during the day, but also for community activities during the night. Often more than one teacher uses the space but does not "own" the classroom. Since these spaces are sporadically used and do not have a clear "owner," occupancy sensors are a good choice for reducing wasted lighting energy. The average energy savings was $31.7 \%$. We recommend $30 \%$ energy savings as an easy to remember best of estimate for shared, scheduled, spaces. Note, however, that one study of teacher "owned" classrooms reviewed for this task showed that more energy waste due to the time delays on the occupancy sensors. Consequently, the specifier must have a clear understanding of classroom use before applying occupancy sensors to reduce wasted energy.

While these saving estimates are averages based on extensive research, the potential savings for a specific application will vary based on the occupancy and behavioral patterns of a particular space.

\section{Percent Savings from Occupancy Sensors (Mean Values)}

\begin{tabular}{ll|c|} 
& \multicolumn{1}{c}{ Private ("Owned") } & Shared \\
\cline { 2 - 3 } Sporadically Use: & 25 & 40 \\
\cline { 2 - 3 } Scheduled Use: & & 30 \\
\hline
\end{tabular}

Table 1 - Recommended values for the three types of spaces. ${ }^{4}$ 


\section{Tips for Manufacturers and Installers}

Here are a few tips for manufactures and installers that could help reduce the installation and commissioning time and hassle:

Manufacturers should:

Supply circuit schematics or documentation explaining inputs and outputs to the sensors and power packs.

Use the same wire color scheme across manufacturers. This will facilitate the installation of sensors and power packs or any other components that are not from the same manufacturer.

Supply documentation on how to override the system after the power packs are installed. In general, power packs are installed before the sensor and installers do not have enough information about how to override the system after the sensor is installed.

Develop a diagnostic interface that can be plugged in each power pack and sensor to speed up commissioning and diagnosing problems.

Include zero-delay setting to the sensor to allow faster commissioning.

Set up a 24-hour hotline customer service to allow nightshift installers to have customer support at night.

Installers should:

Never disable manual controls when installing motion sensors; existent light switches should be supplemented, not replaced, by the motion sensor.

Provide a sufficient number of sensors, placed appropriately, in open plan offices.

Saving money by reducing the number of motion sensors used in open-plan areas may result in poor performance and thus, dissatisfaction and rejection.

Place occupancy sensors close to areas where small movements are made (e.g., near desks, over bathroom stalls) to improve sensitivity to these motions.

Put two sensors in hallways, one at each end, pointing toward the center of the controlled area to provide good coverage and avoid false triggering.

For more information on occupancy sensors, please go to the LRC website: www.lrc.rpi.edu/programs/NLPIP/publications.asp and look under Specifier Reports. 


\section{PHOTO SENSORS}

Photo sensors discussed here are for dimming fluorescent lighting in non-residential applications when sufficient daylight is present. This is not about architectural dimming for aesthetic purposes or multi-medai spaces. The purpose of dimming is to improve the energy efficiency of the building.

\section{The Technology Transfer Model for Photo Sensors ${ }^{3}$}

\begin{tabular}{|c|c|c|c|c|c|c|}
\hline & Ctotu & & & Key Playe & & \\
\hline & status & Spenders & Users & Doers & Producers & Enablers \\
\hline Advocacy & Not started & & & & NEMA & $\begin{array}{l}\text { ACEEE, } \\
\text { NRDC }\end{array}$ \\
\hline $\begin{array}{l}\text { Promotional } \\
\text { Programs }\end{array}$ & Not started & & & & Manufacturers & $\begin{array}{c}\text { Utilities, } \\
\text { NYSERDA, } \\
\text { CEC, NEEA }\end{array}$ \\
\hline Education & Ongoing & BOMA & BOMA & $\begin{array}{l}\text { ASHRAE, } \\
\text { NECA, } \\
\text { IESNA, AIA, } \\
\text { NAESCO }\end{array}$ & NEMA & $\begin{array}{c}\text { DOE, LRC, } \\
\text { Utilities, } \\
\text { NYSERDA, } \\
\text { CEC, NEEA }\end{array}$ \\
\hline $\begin{array}{l}\text { Test Methods \& } \\
\text { Metrics }\end{array}$ & Ongoing & & & & NEMA & LRC \\
\hline Demonstrations & Ongoing & BOMA & BOMA & & Manufacturers & $\begin{array}{c}\text { LRC, DOE, } \\
\text { Utilities, } \\
\text { NYSERDA, } \\
\text { CEC, NEEA }\end{array}$ \\
\hline Applied Research & Complete & & & & & \\
\hline Theory & Complete & & & & & \\
\hline
\end{tabular}

Figure 5

Current commercialized photo sensor technology does not meet customer needs. They are not easy to install or maintain and customer payback, based on energy savings, exceeds customer acceptable levels. Therefore, photo sensors have not been widely accepted. Even where they have been installed problems persist with building occupant satisfaction because of the difficulty of maintaining sufficient lighting levels. The technology transfer model tells us insufficient demonstrations and testing occurred.

\section{Current Photo Sensor Technology}

All commercialized photo sensors use analog technology to establish the dimming algorithm necessary to maintain a constant lighting level on the work surface. Analog technologies require manual intervention to commission the photo sensor. This is a tedious process that, at best, is trial and error. Complicating matters is that the same signal level from the photo sensor to ballasts produces different levels of dimming for different manufacturers' products. i.e. This technology does not meet identified customer needs, not easy to install, use or maintain.

Dimming ballasts are approximately three time as expensive as an instant start ballast which is the current market standard. The instant start ballast has captured over $85 \%$ of the electronic ballast market and cost approximately $\$ 10$ while the full dimming ballast is 
about \$45. Can energy savings pay for the added cost of the ballast over a reasonable time? The answer is it depends on what the customer is paying for electricity. Table 2 below expresses the energy and cost savings for the use of a dimming system that responds to the presence of daylight. ${ }^{5}$ Explanations and assumptions include:

The analysis is conducted on a per light fixture basis.

The light fixture used is a three lamp, T-8, electronic ballast, parabolic fixture. This type of fixture is currently the most commonly used in new construction or remodeling.

Lights are on 10 hours per day and 260 days per year.

Based on initial findings in the photo sensor project, the annual energy savings is $30 \%$ of the light fixture energy use with no daylighting controls. This amounts to $78 \mathrm{kWh}$ per year.

Credit is included in the energy cost savings for a reduction in peak electric demand due to the light fixture being dimmed. Since most buildings' peak demand occurs on sunny days due to solar heat gain, assuming the fixture would be in a dimmed mode is reasonable.

Actual electric rates from utilities located in the sampled regions are used to calculate savings.

The differential cost of a full dimming ballast versus a instant start electronic ballast is $\$ 35$. An additional \$5 per fixture in incremental investment is included to compensate for the control portion, i.e. photo sensor. This makes the total incremental investment $\$ 40$ which must be offset by the energy savings.

\section{Table 2}

Location

New York City

Ohio

Chicago

Georgia

Connecticut

New Jersey

San Francisco

Washington State

\begin{tabular}{|c|c|}
\hline Annual Savings per Fixture & Simple Payback \\
\hline$\$ 16.32$ & 2.45 years \\
\hline$\$ 7.05$ & 5.67 years \\
\hline$\$ 10.36$ & 3.86 years \\
\hline$\$ 7.20$ & 5.56 years \\
\hline$\$ 7.58$ & 5.28 years \\
\hline$\$ 9.68$ & 4.13 years \\
\hline$\$ 8.17$ & 4.90 years \\
\hline$\$ 5.36$ & 7.20 years \\
\hline
\end{tabular}

Most commercial type end use customers have an investment criteria which will allow for capital improvements that produce a simple payback of three years or less. This criteria changes based on the general business climate. During economic downturns, businesses will shorten paybacks wanting to recover their investments in shorter periods of time. Conversely, during economic upturns, the payback period may be extended. Therefore, the use of daylighting (and its accompanying photo sensor) for most of the United States must be justified on more than the economics associated with energy savings. Environmental, health and productivity benefits must be proven, quantified and added to those of energy. 
For more information on dimming ballast and controls please go to the Lighting Research Center website at www.lrc.rpi.edu/programs/NLPIP/technologies.asp.

\section{New Photo Sensor Technologies}

Photo sensors must enter the digital age. The LRC has developed a photo sensor that is as cost effective as current technologies but is self commissioning to meet customer needs for ease of installation and operation through funding provided by Connecticut Light and Power Company..

The LRC photosensor consists of two separate devices: a wall-switch controller and a remote, wireless sensor. Figure 6 shows a photograph of the two devices. The wall-switch controller, installed in an electrical gang-box, replaces the traditional light switch located near the entrance of a room. This controller could be place elsewhere in larger open offices. The wall-switch controller receives power and is connected to the dimming ballasts in the room via the power line wires and dimming control wires brought to the electrical gang-box. The wall-switch controller can then control the dimming of the fluorescent lighting as well as switch the power supplied to the dimming ballasts for on/off control. The wall-switch controller also incorporates a manual on/off switch, a user preference/override input button, and three LED status indicators.

The remote sensor is a wireless, self-powered device that senses and transmits light level information to the wall-switch controller via an infrared optical transmitter. The wallswitch controller receives the transmitted signals, processes the information according to the programmed control algorithm, and dims and/or switches the fluorescent lighting. The remote sensor also transmits a simple commissioning instruction when a button on the device is pressed, initiating the commissioning process.

Commissioning is accomplished by first placing the remote sensor on the work surface and pressing the commissioning button. The sensor takes light readings with the artificial lights off (daylight only) and then with the lights on fully. This allows for the calculation of the differences between the two sources. The remote sensor is then placed at its permanent location and the commissioning button is depressed again. The sensor again cycles through the same readings. All this information is transmitted to the wall unit which contains a memory chip and processor. The readings allow the processor to develop an algorithm that will maintain light levels on the work surface. Commissioning also requires the processor to learn the dimming values of the ballast. This is necessary because different manufacturers' dimming ballasts have different dimming response curves. The wall-switch controller must know what control voltage corresponds to a certain amount of light output from the lamps. The entire commissioning process takes less than three minutes and produces results in dimming the fluorescent lighting to maintain either constant work surface illumination or increasing work surface illumination as daylight enters depending on occupant preference. ${ }^{6}$ 


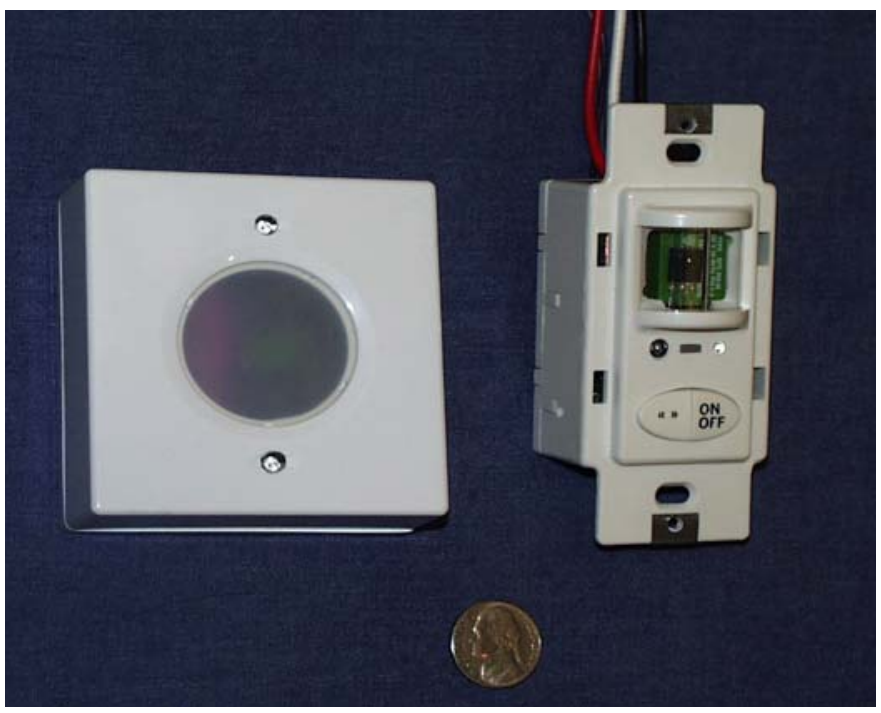

Figure 6

Even this technology must be linked to a costly dimming ballast. While the new technology overcomes customer concerns with installation and operation, it does not meet the customers' economic criteria. Currently, the only means of overcoming the economic issues is to not use a full dimming ballast. If an approach is taken to save a little less energy by only turning off the lights in the presence of sufficient daylight, then one can employ an instant start ballast with a photo sensor that turns off the light fixture. Initial research by the LRC indicates the difference in energy savings is approximately 10 percentage points. The reduction in costs to achieve savings is substantial. Initial economic analyses indicate this approach can meet customer economic needs. The LRC is developing a "dayswitch" (Figure 7) that utilizes the technology developed for the digital, self commissioning photo sensor so the "dayswitch" will be easy to install and operate as well as meeting customer economic needs.

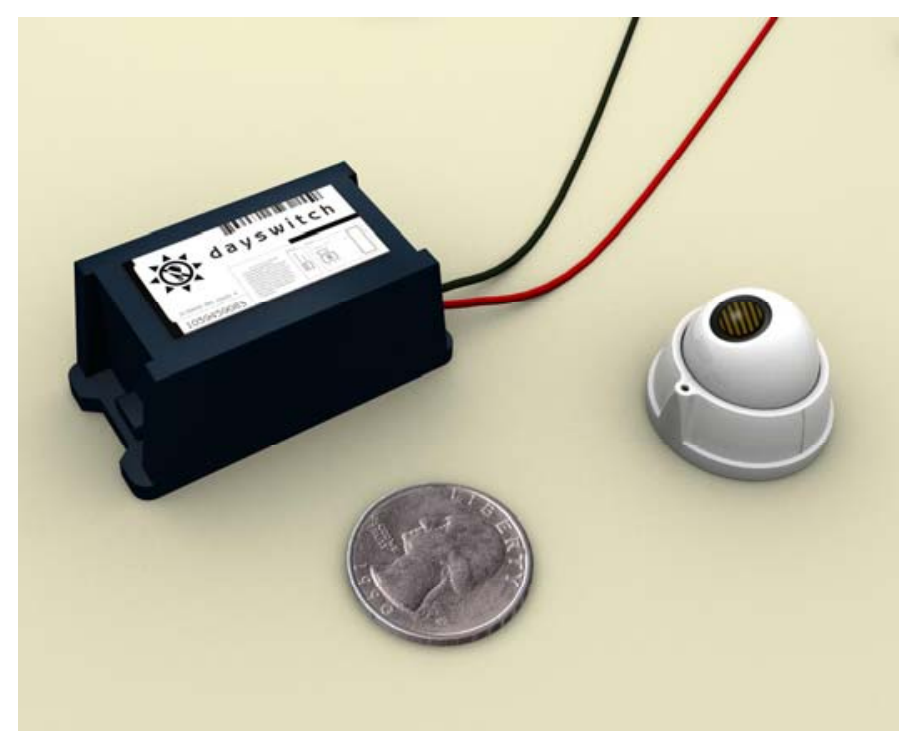

Figure 7 


\section{DIGITAL ADDRESSABLE LIGHTING INTERFACE (DALI)}

DALI, which is a standard developed in Europe, is coming to America. "The DALI standard includes addressable control of individual and groups of ballasts, as well as easier installation and maintenance due to the ability to mix and match DALI components seamlessly from multiple manufacturers into complete systems”, states Odile Ronat of International Rectifier which manufactures components for DALI ballasts. ${ }^{7}$ Manufacturers agree to build these ballasts and lighting controls so a single communication protocol will work with all components.

DALI components use a twisted pair of communication wiring between components and a controller. The controller has the ability to control up to 64 lighting ballasts or control points. The controllers can be integrated into larger groups through the building's automation system. Each ballast or control is given a digital address. A software program can then group different addresses into different lighting groupings without rewiring devices when a building change takes place. Since the ballasts and controls use digital technology, there are fewer components required. The communications that occurs is two-way which allows the ballast to indicate its status back to a central location. Advantages of the DALI system over current lighting control systems are:

Reduced installation costs due to simpler wiring requirements.

Capability of monitoring lamp and ballast status from a central location. This may reduce maintenance costs.

Increased flexibility in programming different lighting fixtures into groupings without having to rewire the fixtures.

The ability to use any DALI manufacturers' components with another manufacturer's DALI equipment.

DALI has advantages where lighting system controls beyond simple on/off control are desired. However, the jury is still out as to customer acceptance of this new technology. Does DALI meet the needs and wants of business customers? Is it cost effective? Discussions above regarding photo sensors and dimming ballasts indicate less than desirable returns on customer investments based on simply energy savings. Is it easy to install and maintain? DALI requires extensive software programming to gain the greatest advantages from the technology. This is normally not a skill set found in the average building facility personnel. Is DALI a proven technology? It certainly has worked well in Europe.

\section{LIGHTING AND LOAD MANAGEMENT}

Electrical load management creates winning situations for many constituencies including the end use customer, energy supplier, the environment and the distribution utility. The end use customer benefits are monetary in nature to reflect his/her willingness to reduce electrical loads when requested by a utility, energy supplier or regional transmission organization. The energy supplier mitigates their risks for the purchase of high priced electricity during periods of shortages by having the customer reduce the amount of electricity used. The environment benefits because reductions of peak electrical loads means less efficient, higher polluting generators will not run. The distribution utility may be able to postpone the need for expanded transmission or distribution capacity which is 
getting exceedingly harder to acquire as consumers say “not in my back yard”. The Federal Energy Regulatory Commission (FERC)

has recognized the value load management must play in providing for future electrical needs by including it as an integral part of its proposed Standard Market Design for the wholesale electric market. Hopefully, regulatory agencies responsible for retail electrical markets will also adopt the FERC's suggestions regarding load management.

Can lighting meet the needs of the customer as well as the other players in the load management equation? Lighting and lighting controls certainly possess many positive attributes required for it to be successful in the load management marketplace. The majority of businesses have lighting loads so the number of customers eligible to participate is substantial. Reductions in lighting loads, unlike other manageable loads, are very predictable and repeatable, two highly desirable qualities for load management programs. With the development of additional advanced lighting controls, lighting loads become easy to control from either a remote location or at the point of use. Dimming, rather than turning off lights, has the added advantage of avoiding or minimizing any productivity losses.

What is missing in either the market or lighting technologies to allow for the successful use of lighting and its controls in the load management marketplace? A simple, low cost fluorescent dimming ballast and the communication links capable of communicating with a central controller are not commercially available. Whatever technology is developed must be easy to install and maintain and simple to operate. It also must be cost effective. The simple system should be easily integrated into accepting expanded features such as remote system monitoring and two way communications if the customer so desires. The lighting industry is somewhat hesitant to invest in this technology development because it is uncertain of the economics associated with electric load management. Any technology development research efforts must also include demonstrations of the economic impact on the end use customer when the technology is employed. Both the customer and lighting manufacturers want proof through demonstration projects that the technology works, that it is acceptable to the customer (meets the customer needs listed above) so a market pull can be created and that the technology is economically justifiable to the customer and the manufacturer. i.e. the customer receives a reasonable return on his/her investment and the manufacturer can sell sufficient numbers of the technology to earn a profit. The final step, which should be considered in any research funding, is the successful market transfer of the technology and the underlying customer economic analysis. This will require funding for education of the lighting industry, end use customers, building design community, energy suppliers and energy service companies to create both market pull and push. This last step is extremely important and requires adequate funding if lighting is to be accepted as a load management technology. 


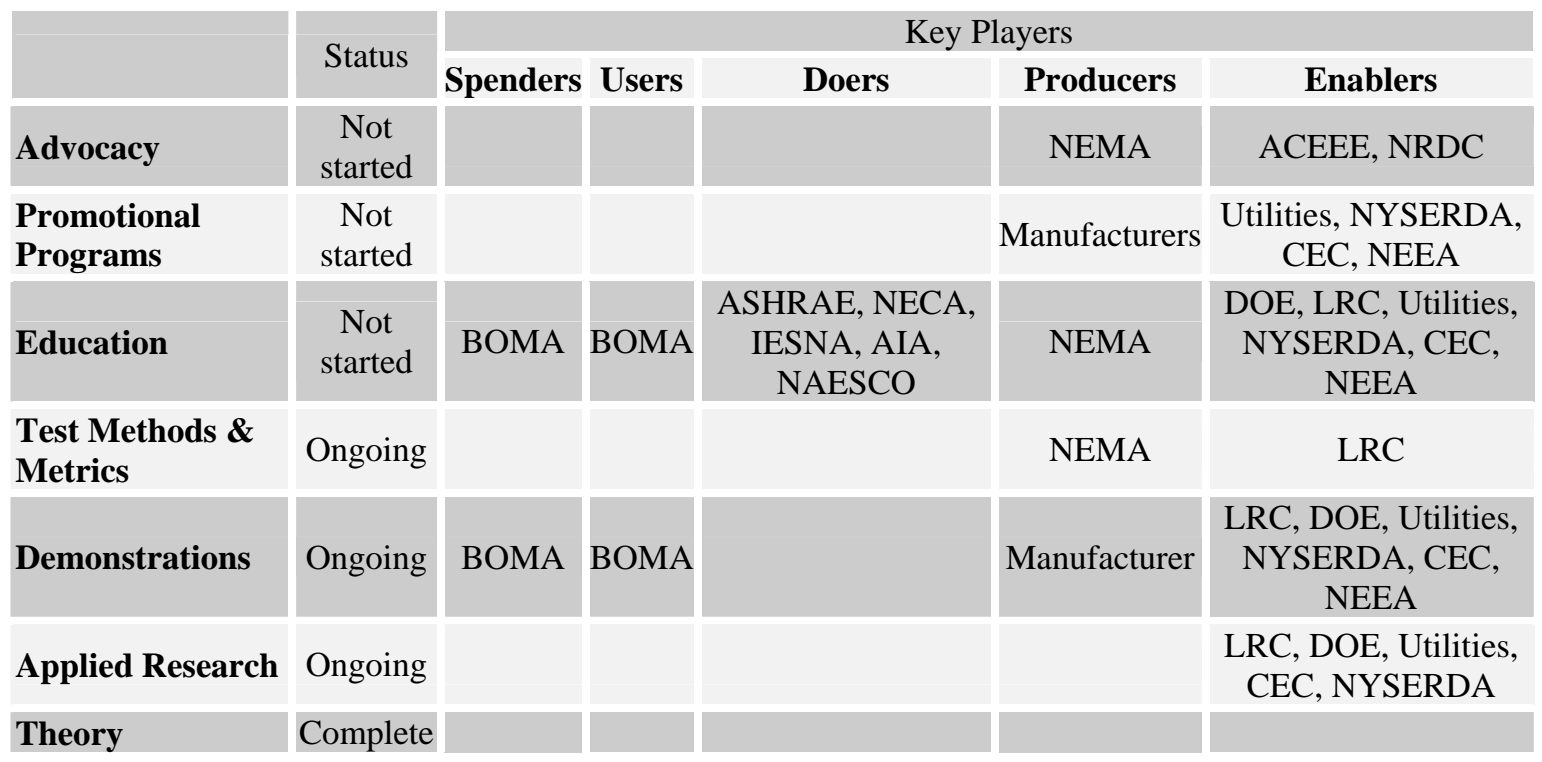

Figure $8^{3}$

Current LRC efforts, which have been funded by U.S. Department of Energy, Connecticut Light and Power Company, California Energy Commission and New York State Energy Research and Development Authority, have lead to the development of a cost effective load shed ballast and communication system and is seeking manufacturers to commercialize these technologies. A full scale demonstration of the technologies will occur in 2004 with assistance from Consolidated Edison of New York and will prove the technical and economic merits.

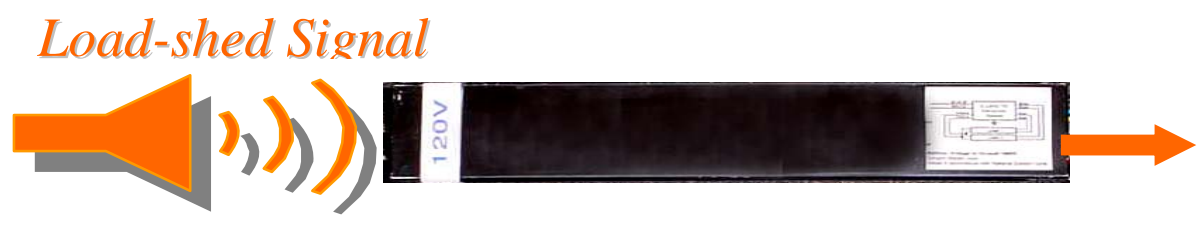

Figure 9

Since the load shed ballast is built on the low cost instant start ballast platform, its incremental cost is approximately $\$ 8.00$ per ballast compared to a full dimming ballast incremental cost of approximately $\$ 35$. Added ballast circuitry allows the ballast to receive a signal from a central controller and reduce current to the lamps by approximately 33\%. Dimming occurs over approximately 3 second period so building occupants do not see an abrupt change. LRC research indicates that people will accept up to a $30 \%$ decrease in illumination levels for short periods of time and up to $50 \%$ decrease if informed of the social/environmental benefits of load shedding. 


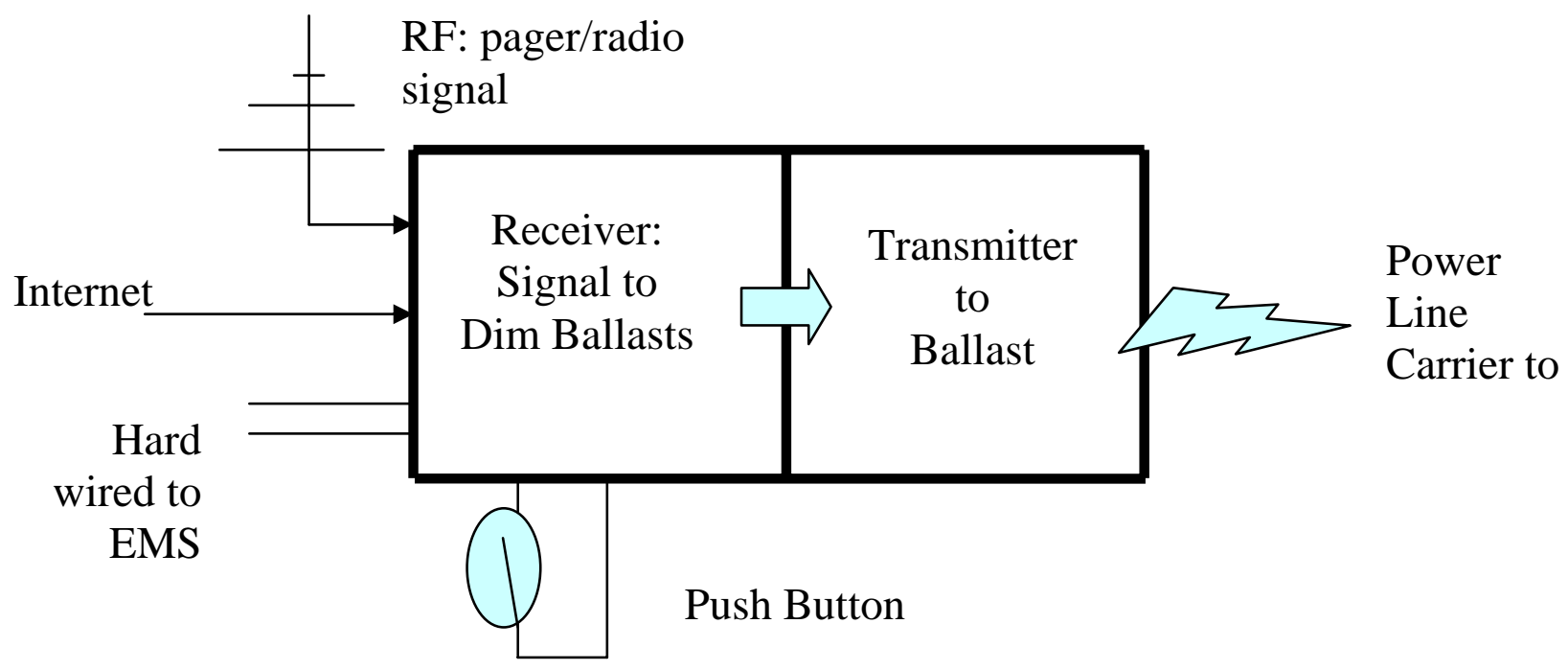

Figure 10

The load shed controller communicates to a series of ballasts via power line carrier. Since the ballast only requires a simple on/off signal, minimal bandwidth will suffice. This is an ideal situation for power line carrier communications. This solution has the added advantage of not requiring additional wiring. The controller, while physically located within the facility, can be controlled locally by the customer or remotely via a communication link by a utility or transmission system operator. This load management system provides a reasonable payback to many electric customers today. The following table provides examples of simple paybacks at different locations across the country.

Table 2: Economic Benefits of the Load Shed Ballast ${ }^{8}$

New York City

Benefit $\quad \underline{\text { Simple Payback }}$

Ohio $\$ 4 /$ yr. 1.5 yrs.

Chicago $\$ 3.50 / y r$. 2.25 yrs.

Georgia $\$ 2.80 / y r$. 2.6 yrs.

Connecticut $\$ 3 /$ yr.

3.2 yrs.

New Jersey

$\$ 2.80 / \mathrm{yr}$.

3.0 yrs.

California

$\$ 3.50 / y r$.

3.2 yrs.

2.6 yrs.

\section{CONCLUSIONS/RECOMMENDATIONS}

Motion sensors meet customer needs, are technically feasible and cost effective. They are ready for prime time implementation as long as education on selection and installation is done. 
Current photo sensor technologies are not conducive to meeting customer needs for ease of use and cost effectiveness. New digital technologies need to be employed.

The cost of full dimming ballasts must be lower to meet customer payback criteria or the benefits received from the use of daylighting must increase. More exploration is needed into the productivity, health and school children education improvements that may occur with daylighting.

The successful introduction of the DALI system is dependant on it defining and meeting customer needs at a reasonable price. Market research is needed to better position this product.

Utilization of lighting as a load management tool must be explored in a manner to satisfy customer cost effectiveness and ease of installation and operation needs. Demonstration of the full load shed ballast system in a commercial building setting must be accomplished to prove the technology and economics to customers.

\section{REFERENCES}

${ }^{1}$ Daylight Dividends Focus Group Research Project Final Report, Prepared by Spectrum Associates, May 12, 2003

${ }^{2}$ Roundtable Load Shedding Proceedings Report, PIER Lighting Research Program, Peter Morante, Lighting Research Center, April 16, 2003

${ }^{3}$ Reducing Barriers to Use of High Efficiency Lighting Systems: Technology Transfer Plan, Peter Morante, Lighting Research Center, May 27, 2003

${ }^{4}$ Overcoming Barriers to Widespread Use of Automatic Shut-off Controls in Commercial/Industrial Applications, Mariana Figueiro, Lighting Research Center, August 29, 2003

${ }^{5}$ Daylighting and Photosensor Economic Analysis, Peter Morante, Lighting Research Center, January 9, 2003

${ }^{6}$ Development and Demonstration of an Improved, Energy-Efficient, Photosensor Lighting Control, Final Project Report, Andrew Bierman, March 31, 2003

${ }^{7}$ The Digital Addressable Lighting Interface (DALI): An Emerging Energy-Conserving Lighting Solution, Technical Paper, Odile Ronat, International Rectifier, April 9, 2003

${ }^{8}$ Economic Analysis of a Load Shed Ballast, Peter Morante, 2002 
APPENDIX Q: ASHRAE 90.1 PRESENTATION

(begins on following page) 


\section{ENERGY EFFI CI ENT \& LOAD}

RESPONSI VE LI GHTI NG
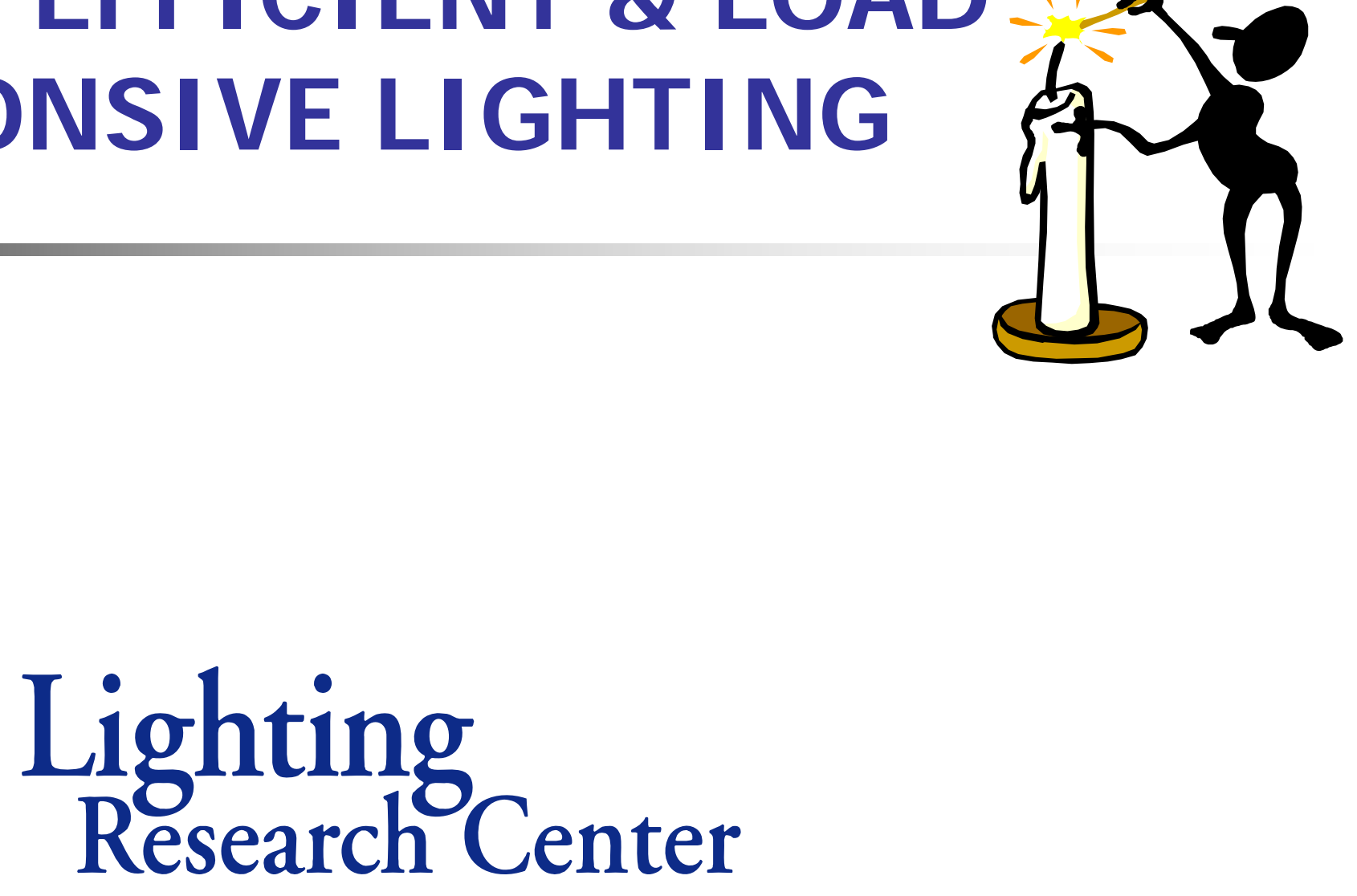


\section{DISCUSSI ON TOPI CS}

- What do customers want

- Motion sensors

- Photo sensors and dimming

- Digital Addressable Lighting I nterface (DALI)

- Panel level power reducers

- Lighting \& load management

- Conclusions 


\section{What Customers Want}

- Reasonable return on their investment

- Easy to install, operate and maintain

- Fully developed $\&$ tested products

- Substantiated proof of any product claims

- Successful employment of the technology has occurred elsewhere

- Educate/communicate with building owners/developers \& designers 


\section{ANSWERI NG THE QUESTIONS}

- How well does the technology work? (technology issues)

- What do they cost/save? (economic issues)

- What barriers still exist to their use? (technology transfer issues)

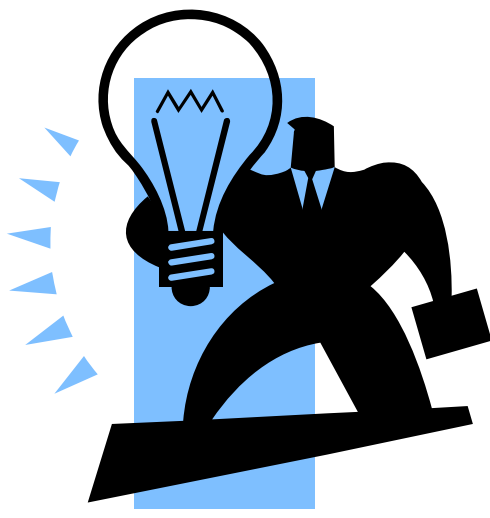




\section{MOTI ON/ OCCUPANCY SENSOR TECHNOLOGY ISSUES}

- Two technology types - infra red \& ultrasonic

- Both are mature technologies

- Both work well with proper selection and positioning

- CONCLUSION (from a technology view point): Occupancy sensors are ready for codes/ standards inclusion

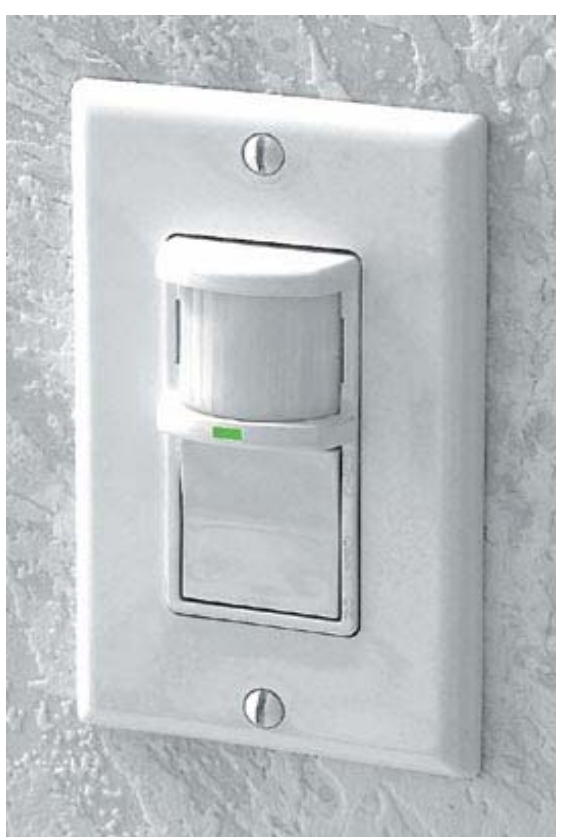




\section{MOTI ON SENSOR ECONOMI C I SSUES}

- How much energy do occupancy sensors save?

from LRC literature review:

Owned

Space

- Sporadic Use:

- Scheduled Use:
Shared

Space

$25 \%$

$40 \%$

$30 \%$

- However, savings for a specific space may vary dramatically from these mean numbers. 


\section{BARRI ERS TO MOTION SENSOR USE (Tech Transfer Issues)}

- Misunderstanding (mistrust?) of cost effectiveness

- Choosing the correct sensor

- Positioning the sensor within the space properly

- No standardization on wiring or commissioning/recommissioning

- Non user-friendly systems 


\section{Motion Sensor brochure}

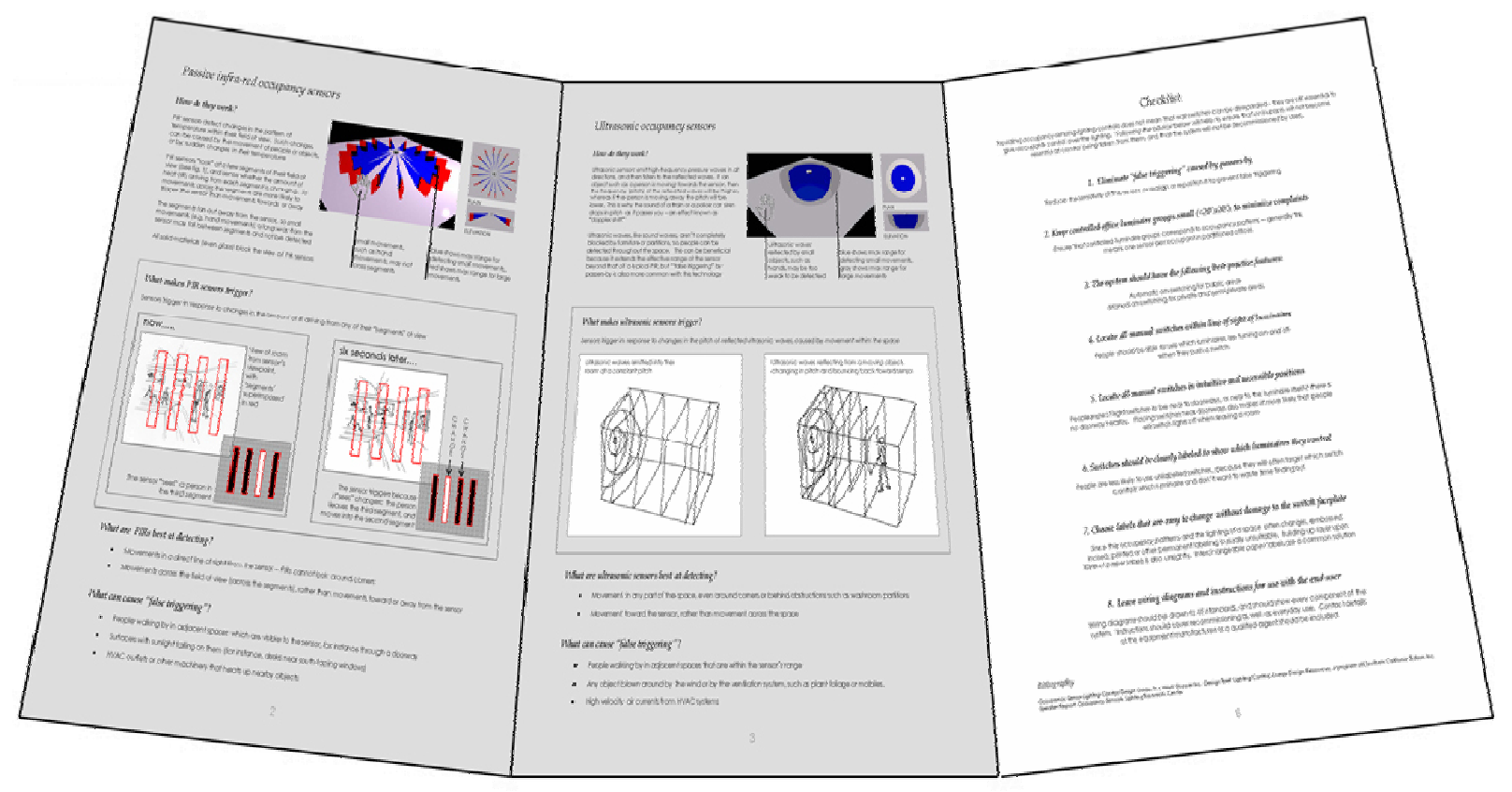

Lighting 


\section{PHOTOSENSOR TECHNOLOGY ISSUES}

- Analog design of control algorithm makes it difficult to accurately adjust the photosensor during commissioning

- Lack of manual controls to adjust photosensor to individual preferences

- Minimal integration with other lightinn controls, i.e. occupancy sensors 


\section{PHOTOSENSOR ECONOMI C I SSUES}

- Cost effectiveness has two parts - the savings and the cost to obtain the savings

- Incremental cost of full dimming ballast and its fixture share of the photosensor control is approximately $\$ 40$.

- Energy savings of approximately $80 \mathrm{kWh} / \mathrm{yr}$ produces different dollar savings across the country

$\begin{array}{lcccc}\text { - } & \text { NYC } & \$ 16.32 / \mathrm{yr} & \text { or } & 2.45 \mathrm{yr} \text { payback } \\ \text { - } & \text { Ohio } & \$ 7.05 / \mathrm{yr} & \text { or } & 5.67 \mathrm{yr} \text { payback } \\ \text { - } & \text { SF } & \$ 8.17 / \mathrm{yr} & \text { or } & 4.90 \mathrm{yr} \text { payback } \\ \text { - } & \text { Bottom line - daylighting not economical from energy } \\ & \text { savings only }\end{array}$




\section{BARRIERS TO PHOTOSENSOR USE (Tech Transfer I ssues)}

- Technology must satisfy customer needs

- Cost effectiveness must improve

- Proof of technology continual operation beyond commissioning

- Proof of benefits

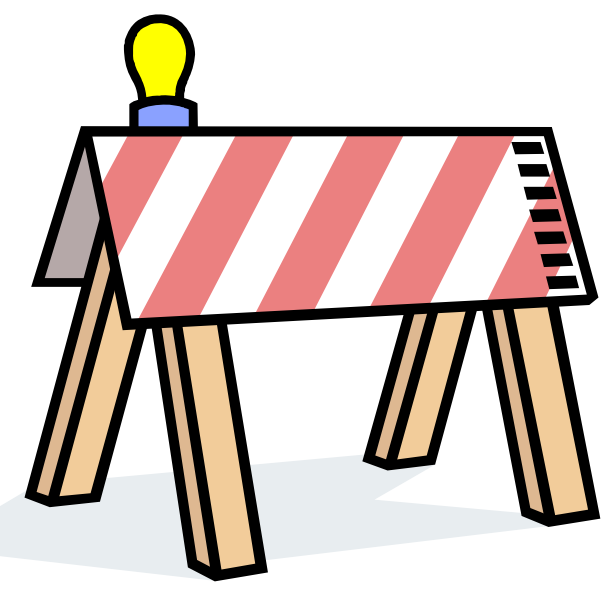




\section{OVERCOMI NG THE BARRI ERS Technology}

- Features of self-commissioning photosensor

- Sliding set point algorithm

- Wireless, remote sensor

- Self-commissioning (minimal user interaction)

- Occupant preference adjustment independent of commissioning

- Switching in addition to dimming control

- Compact size (replaces std. switch)

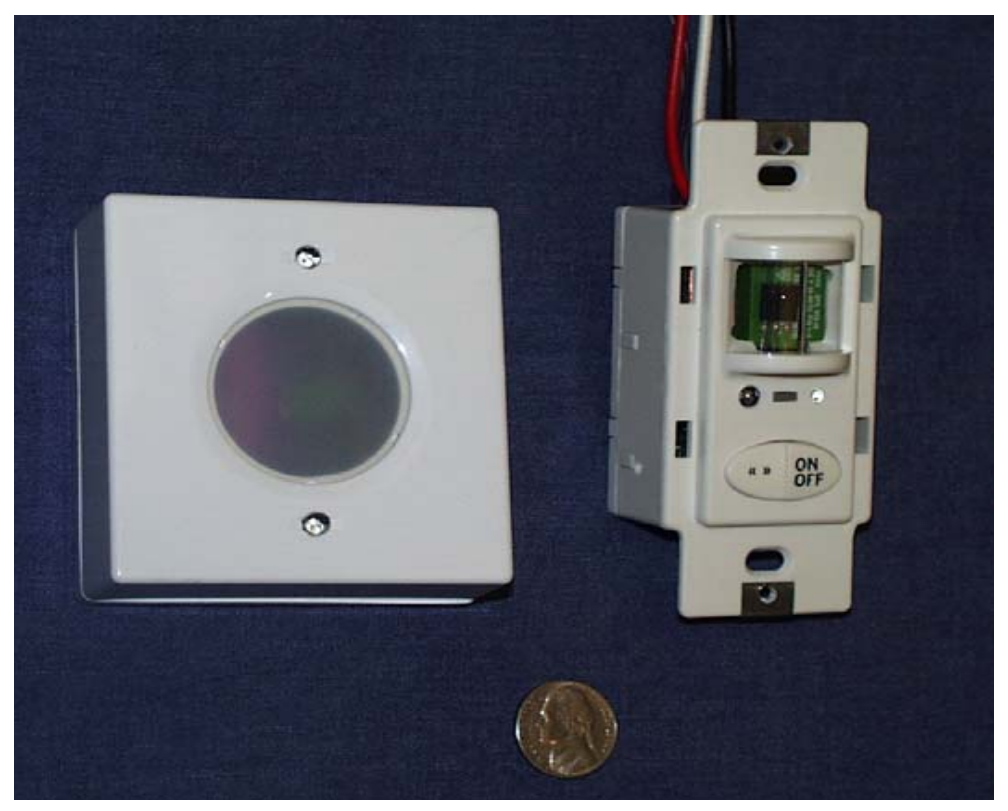

- Efficient operation ( 1.2 watts @ 120 Vac)

- Microprocessor based 


\section{OVERCOMI NG THE BARRI ERS Economics}

Decrease cost or increase benefits or both

- Dayswitch

- Blind controls

- Is productivity affected by daylighting?

- Do buildings that incorporate daylight have a greater value than non-day lit buildings?

- Is there an inherent need for humans to be connected to the outside world?

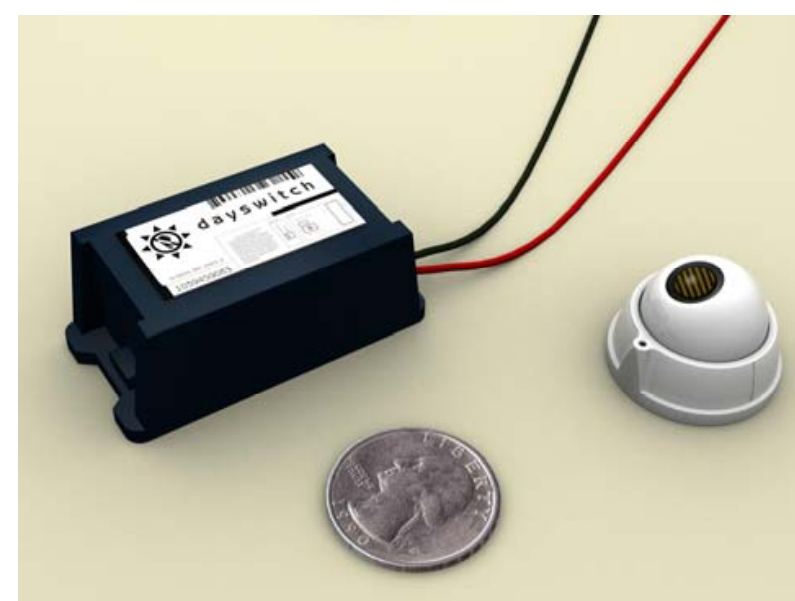




\section{GITAL ADDRESSABLE LI GHTI NG I NTERFACE (DALI) WHAT IS IT?}

- Lighting System - ballast, controls, communications and software for fluorescent lighting

- Two-way communication - digital commands and responses

- Open architecture - all manufacturers' equipment are interchangeable

- Addressable control of individual and groups of ballasts

- Programmable to allow for changes without rewiring

- Can dim or turn off lights based on presence of daylight or for load control

- Does require communication wiring

- Costs are about \$25 greater than today's dimming ballasts 


\section{DALI \\ Will it make it in the marketplace?}

- Customers want return on investment - if the standard dimming ballast is not cost effective, can DALI be?

- Customers want easy to use systems - DALI requires programming each ballast separately

- Customers want substantiated proof of claims - too soon to tell. Documented case studies required.

- Customers want fully developed products - DALI is still very new and manufacturers are still learning how to employ the technology. 


\section{GHTI NG AND LOAD MANAGEMENT}
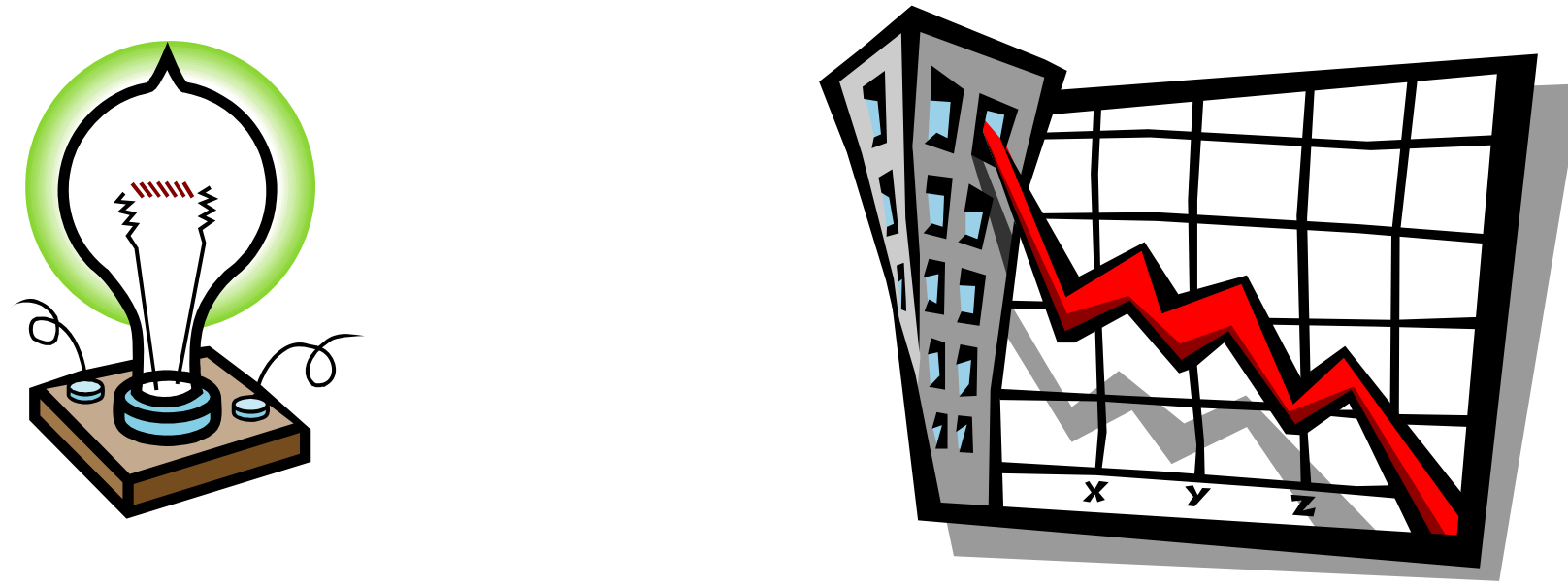


\section{POSI TION: Lighting is an ideal electric end use for load management}

- Available in virtually every building

- Easy to control

- Easy to measure the results

- The load reduced is repeatable

- Can reduce the amount of lighting rather than turn it off, thereby preserving productivity 


\section{I ssues to the Use of Lighting for Load Management}

- Today, control is mainly through manually turning off the lights

- It is hard to be productive in the dark

- Dimming ballasts are expensive (simple payback exceeds 10 years) 


\section{SOLUTION: Develop a cost effective ballast \& controller that will:}

- Dim lights / reduce demand for power

- Retain worker productivity

- Not effect lamp life

- Control from central location, internal or external to building

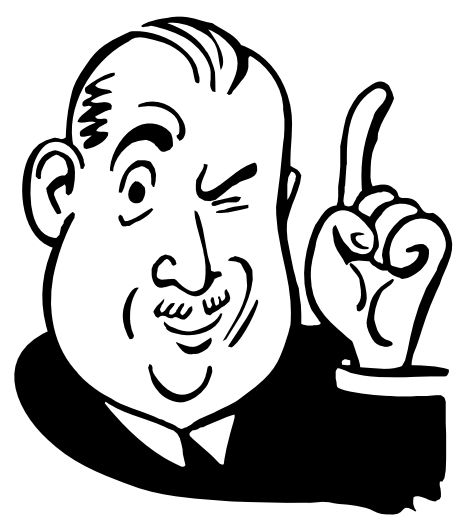

- Pay for itself within 3 years 


\section{With funding from: \\ U. S. Dept. of Energy; California Energy Commission; Connecticut Light \& Power; \\ New York State Energy Research \& Development Authority}

\section{The Lighting Research Center has developed a load shed ballast}

- Instant start ballast platform add circuitry to reduce current to lamps by fixed amount

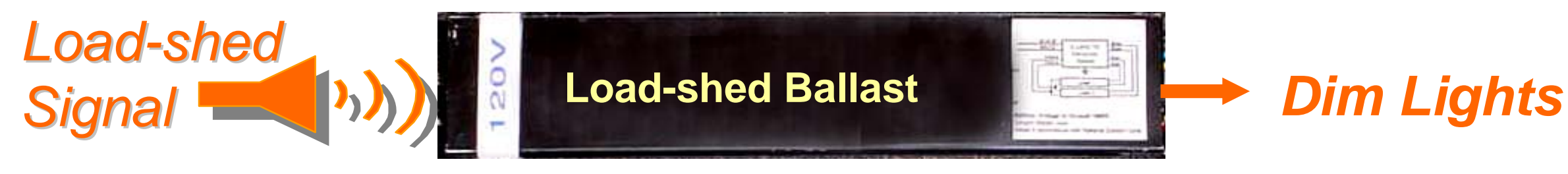

- Receiver for dimming signal

-Dimming occurs over 10 seconds

- Incremental cost of no more than \$8 


\section{CONTROLLER}

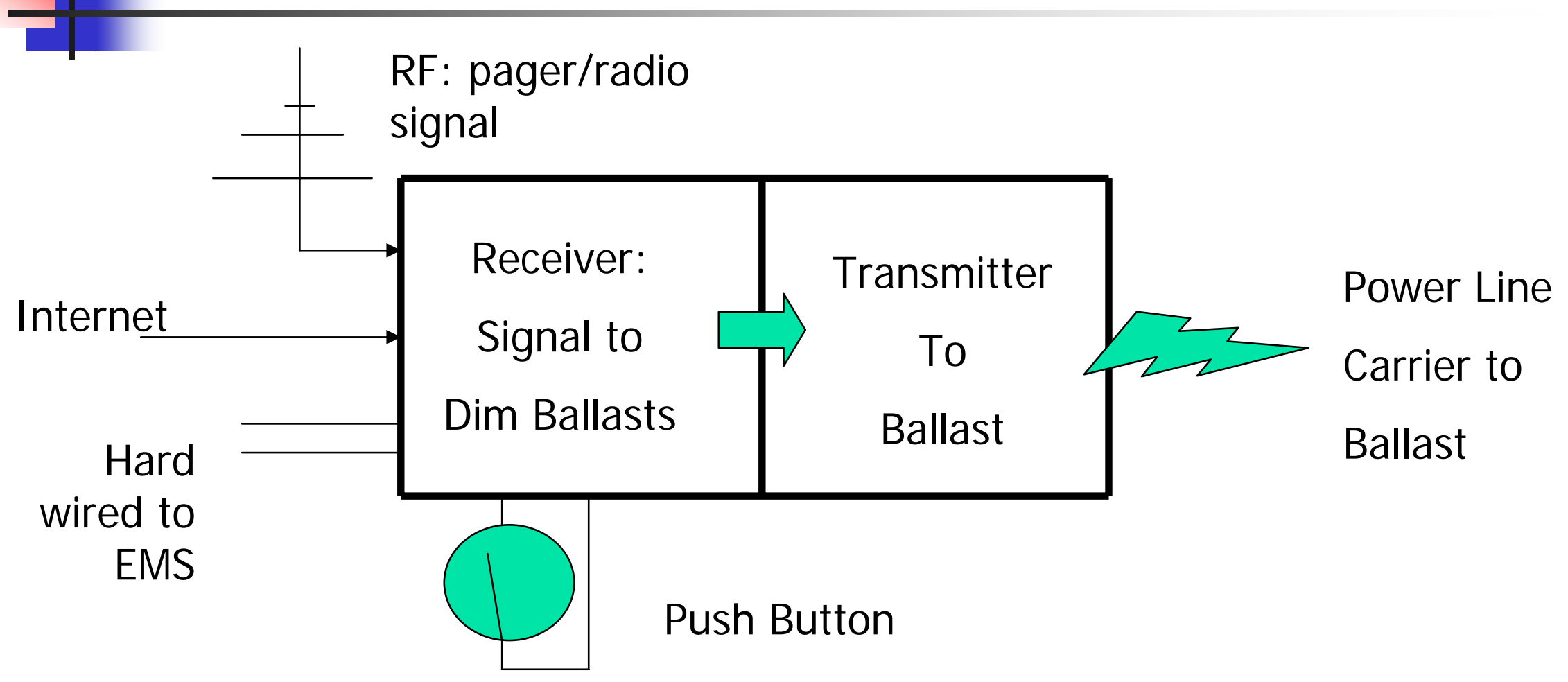

- Interchangeable circuits for how signal is received

- Minimum data transferred to ballast (on/off)

-PLC allows for no additional wiring

- Estimated cost $\$ 1$ per ballast 


\section{WHERE IS IT?}

-Ballast is developed; a large ballast mfr. Has agreed to build prototype load shed ballast

- Controller being tested; manufacturer interested

- Full scale demonstration in NY in early 2005, utility to ballast

- Seeking funding for an additional demonstration site

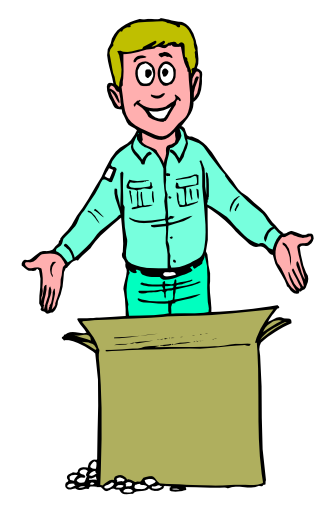

- Commercialization late 2005 


\section{CONCLUSI ONS}

- When considering lighting controls - MUST remember what customers want

- Motion sensors are ready for prime time

- If photo sensors and dimming ballasts are to catch on, improvements in technology and economic benefits are required.

- The jury is still out on the market acceptance of DALI

- Lighting can play a key role in electric load management.

$$
\text { www.Irc.rpi.edu }
$$




\section{Thank You . . .}

Lighting

Research Center 
APPENDIX R: IESNA LIGHTING CONTROL PROTOCOL SUBCOMMITTEE PRESENTATION

(begins on following page) 


\section{Lighting Controls:}

\section{Reducing Market Barriers to the Widespread Use of Energy Efficient Lighting Systems}

Sponsor:

U.S. Department of Energy 


\section{Goals of the Project}

- Identify barriers to the widespread use of lighting controls for commercial and industrial (C\&l) applications that employ fluorescent lamp technologies, and to recommend means for overcoming these barriers.

- This project is not about:

- HID and incandescent

- Architectural dimming

- Residential 


\section{Barriers}

- Lamp/ballast dimming performance

- Cost, reduced lamp life, performance issues

- Components and compatibility

- Cost, hassle, uncertainty

- Installation and commissioning

- Cost, hassle, uncertainty

- Marketing

- Confused message, occupant acceptance, cost 


\section{Philosophy}

\section{Cost/benefit curve}

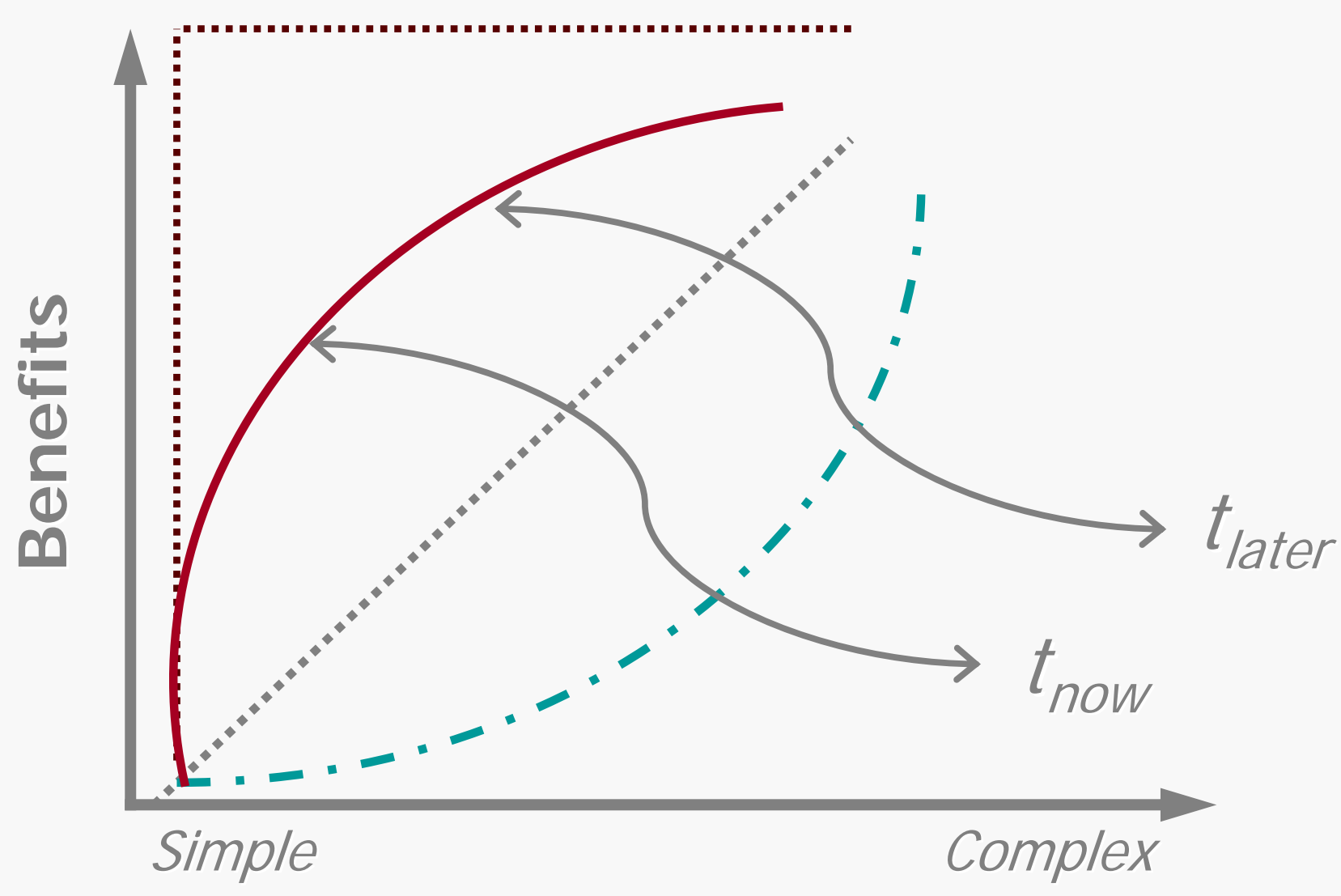

\section{Cost}




\section{Building Automation}

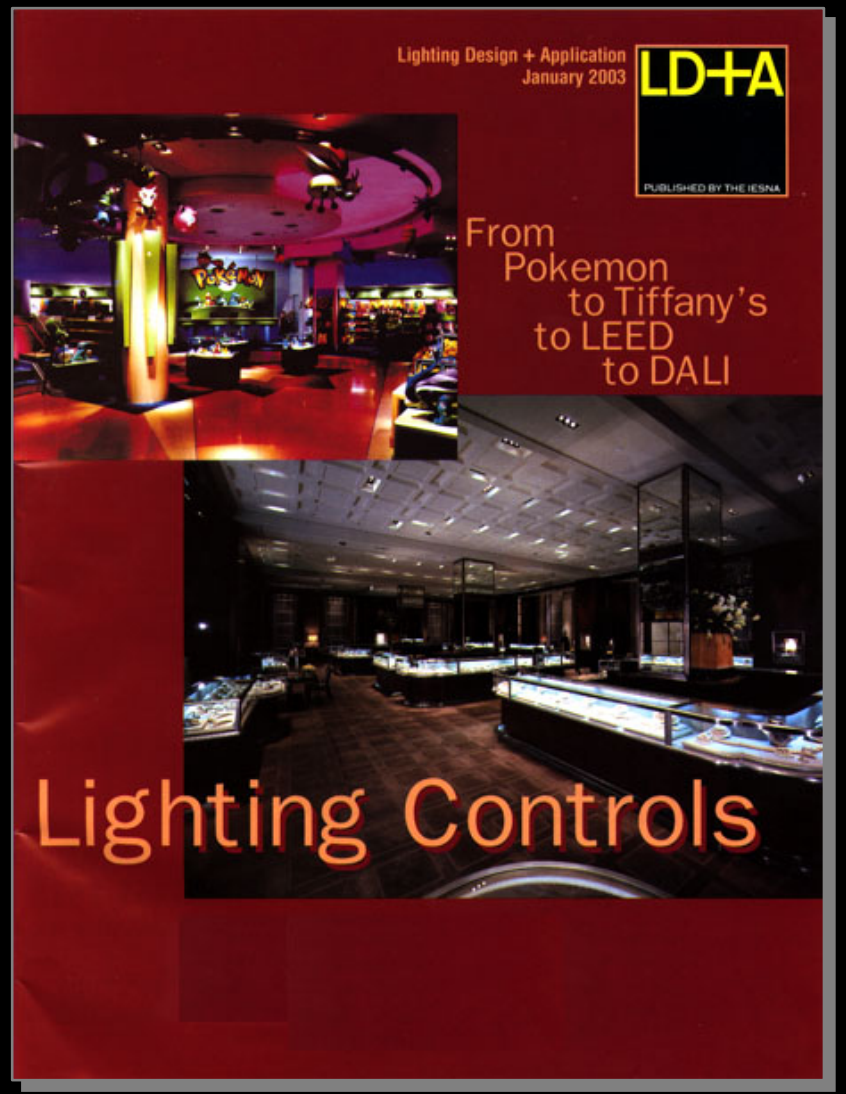

LD+A

J anuary 2003
- Happening without help due to vested manufacturer interest - little we can or should do.

- But...

- Expensive platform

- Costly commissioning

- And

- No obvious benefits to mainstream C\&l (productivity, satisfaction, etc.) 


\section{Automatic shut-off}

- Time clocks

- Occupancy sensors

- Panel relays

- Centralized controls

- These work,

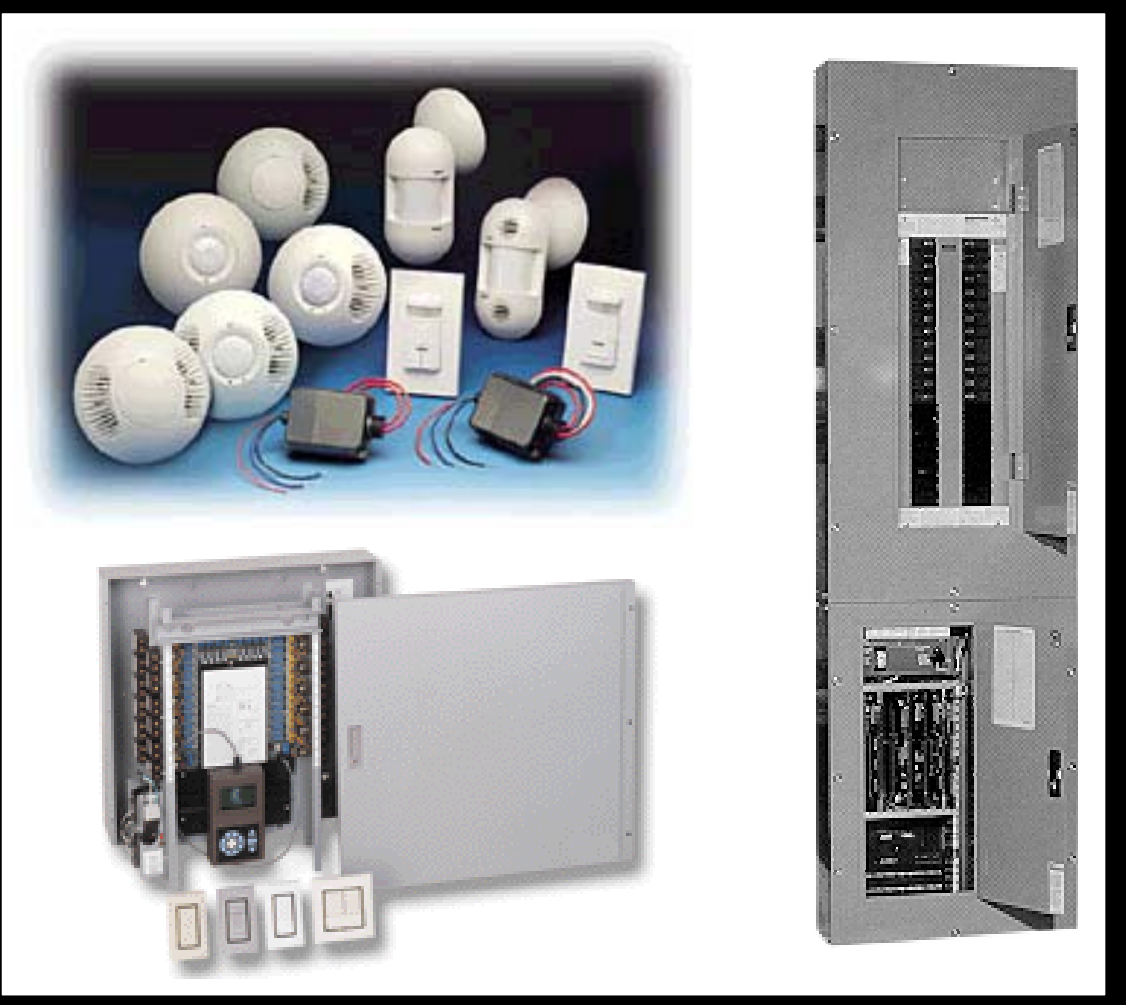
but implementation depends on wiring, installation, commissioning, etc. 


\section{Automatic Shut-off}

- Aggressively encourage the widespread deployment of automatic shut-off lighting controls in existing and new C\&l buildings

- Best practices document

- Approved values for savings calculations

Survey of 26 case studies and manufacturers' claims

\section{Percent savings from occupancy sensors (mean values)}

Private

Shared

("owned")

Scheduled use:

30 


\section{Load Management}

\section{Energy efficiency is not just about kWhr anymore!}

Energy efficiency is becoming more about when power is needed than how long power is needed; the real cost of power for certain days and certain times of the day can approach $\$ 1 / \mathrm{W}$ !

Plus... Society doesn't want to build new power stations or transmission lines. (Especially "not in my backyard!")

Electric market clearing prices

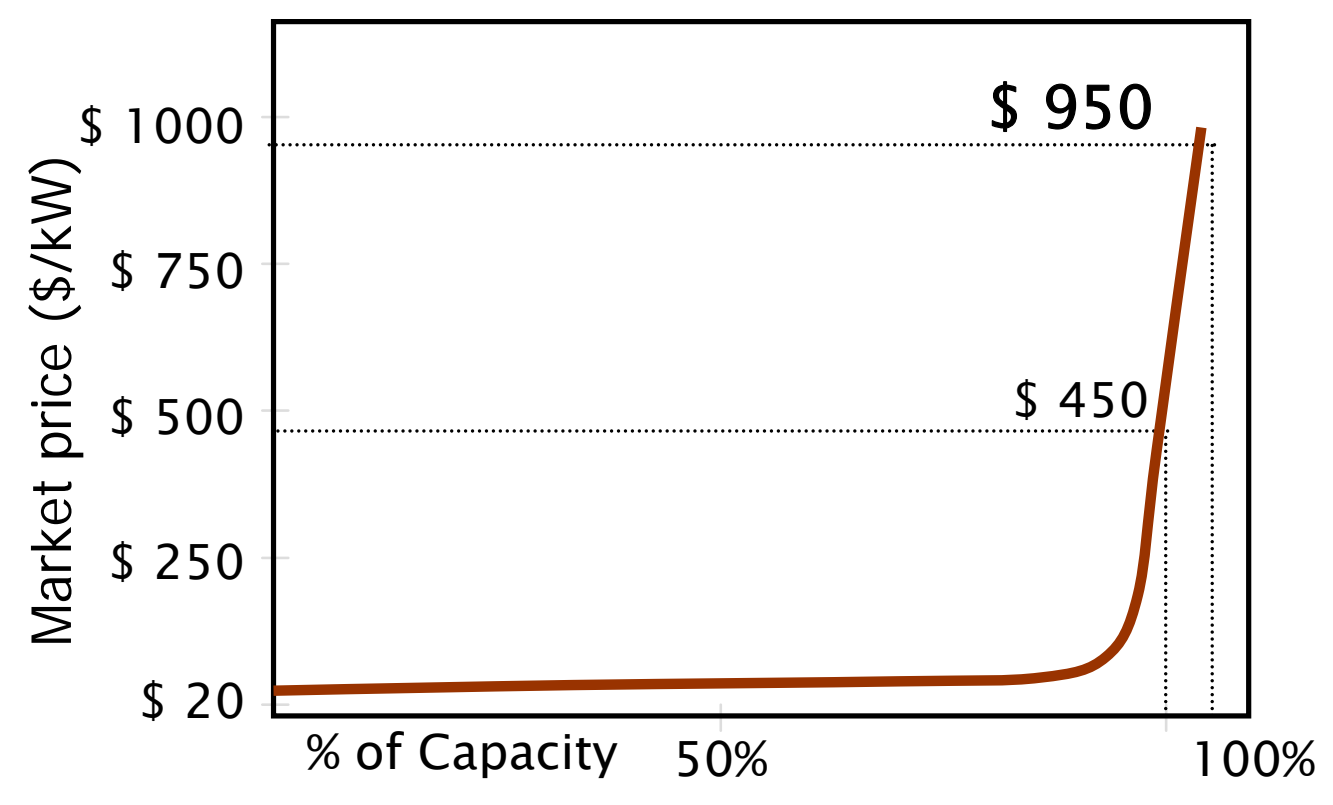




\section{Load Management}

Why does energy grab fewer headlines that it did during energy crises of the 1970s? Because it costs less as a share of disposable household income these days. And the burden should continue to lighten.

One big contributor: an enormous increase in energy efficiency since the first oil shock in 1973.

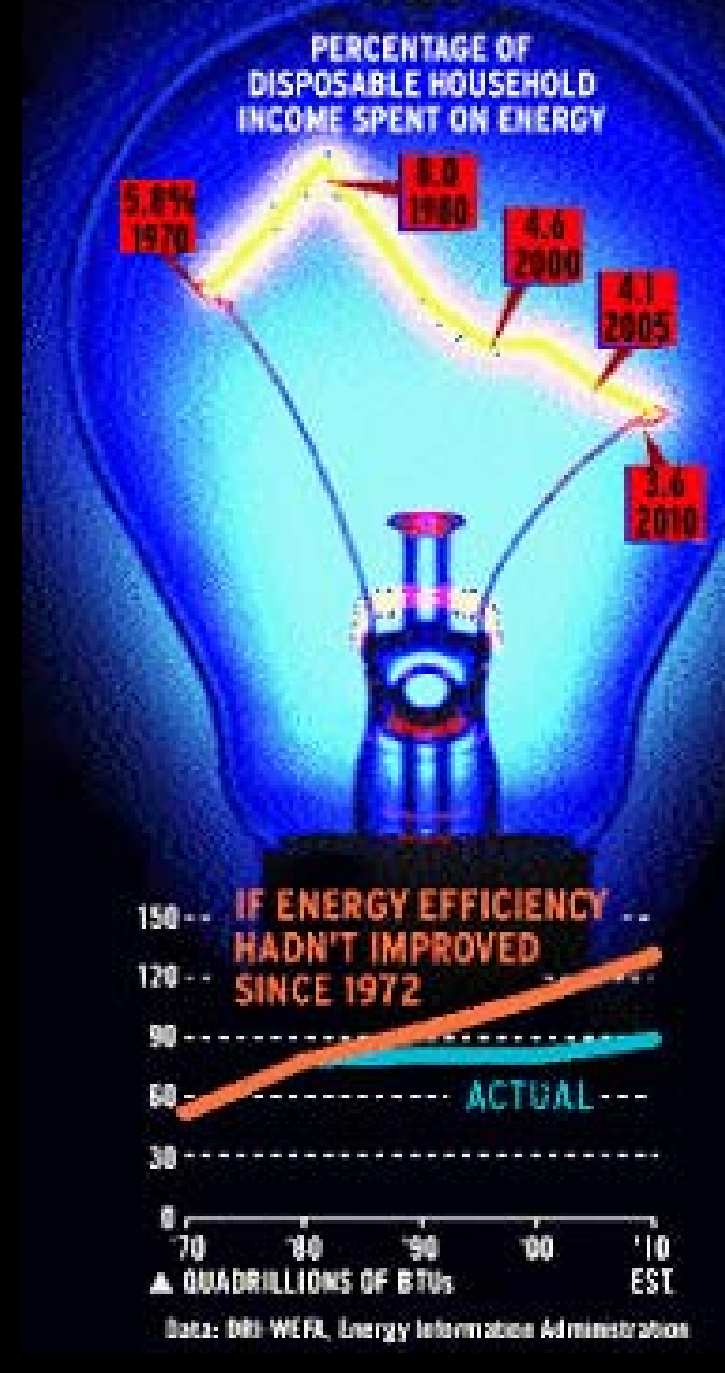




\section{Lighting is ideal for load-shedding}

- Lighting is the ultimate distributed resource for load management

- Non-disruptive (especially with daylight)

- Non-polluting

- Constitutes a large load ( $40 \%$ of commercial load) with large periods of use

- Distributed load, high reliability

- Immediate response with no "rebound"

- Can be aggregated into larger loads

- But requires a third party organization to do this

Joel S. Gilbert, P.E., CEO APOGEE Interactive, Inc. LRC Partner's Day 2002 


\section{Barriers to Lighting Load-shedding}

- Problems with existing dimming ballasts

- High cost

- Reliability concerns

- Increased energy consumption (average 9\% increase over instant-start ballasts)

- Aggregating load

- Individually small, widely distributed load

- Lack of controls within price range for load shedding applications 


\section{LRC Approach to Lighting Load-shedding}

- Keep it simple and inexpensive

- Instant-start ballast platform for dimming or on/off

- Reliable

- Energy efficient

- Simple to install

- Low first costs (>75\% of new electronic ballast sales are instant-start)

- PLC communication

- No new infrastructure

- Meets speed requirements

- Low cost

- Available communication channel (few FCC regulations)

- Can be made reliable 


\section{Initial data suggests that instant-start systems can be dimmed}

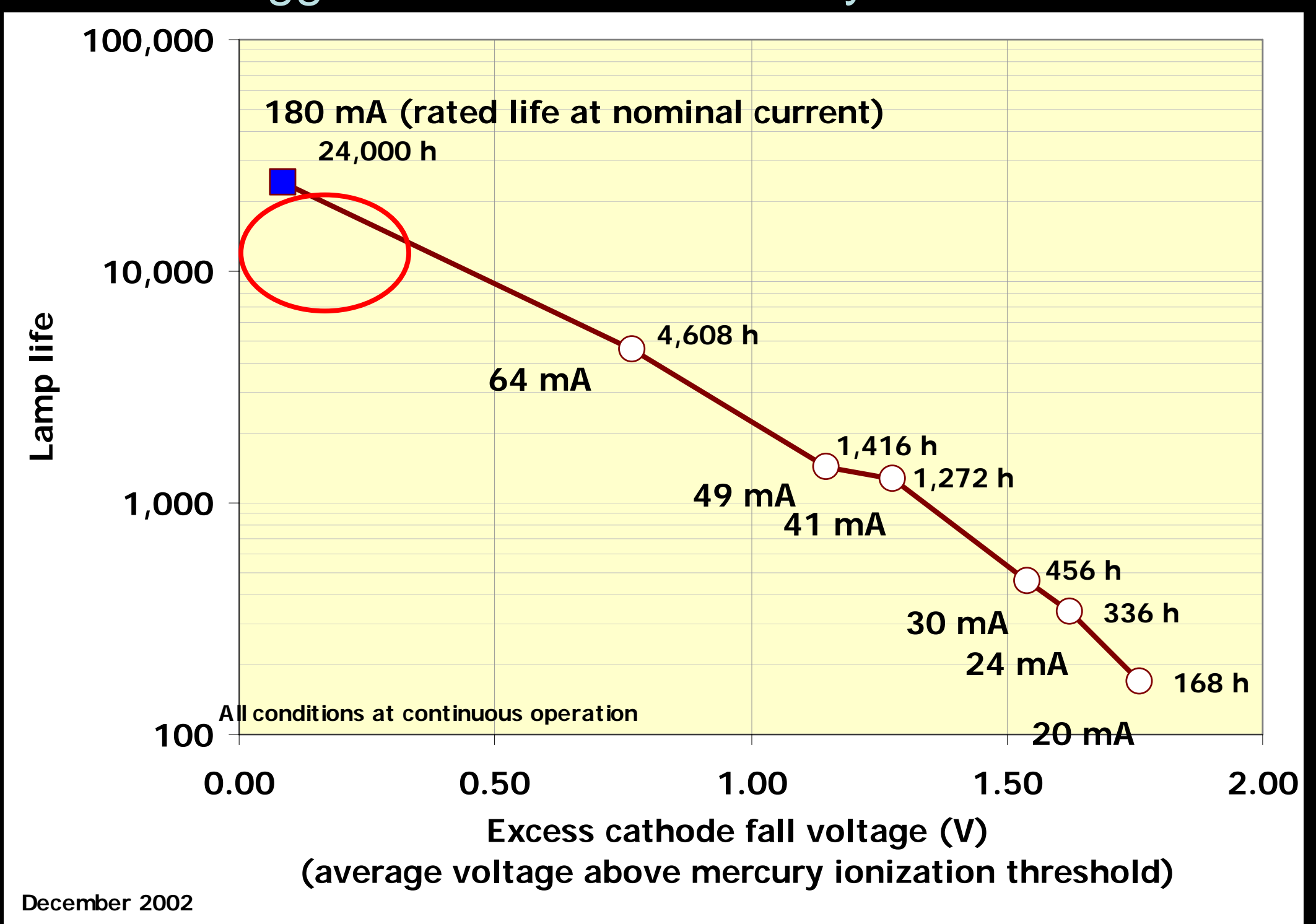




\section{Signaling a Load-shed Ballast and Aggregating Load}
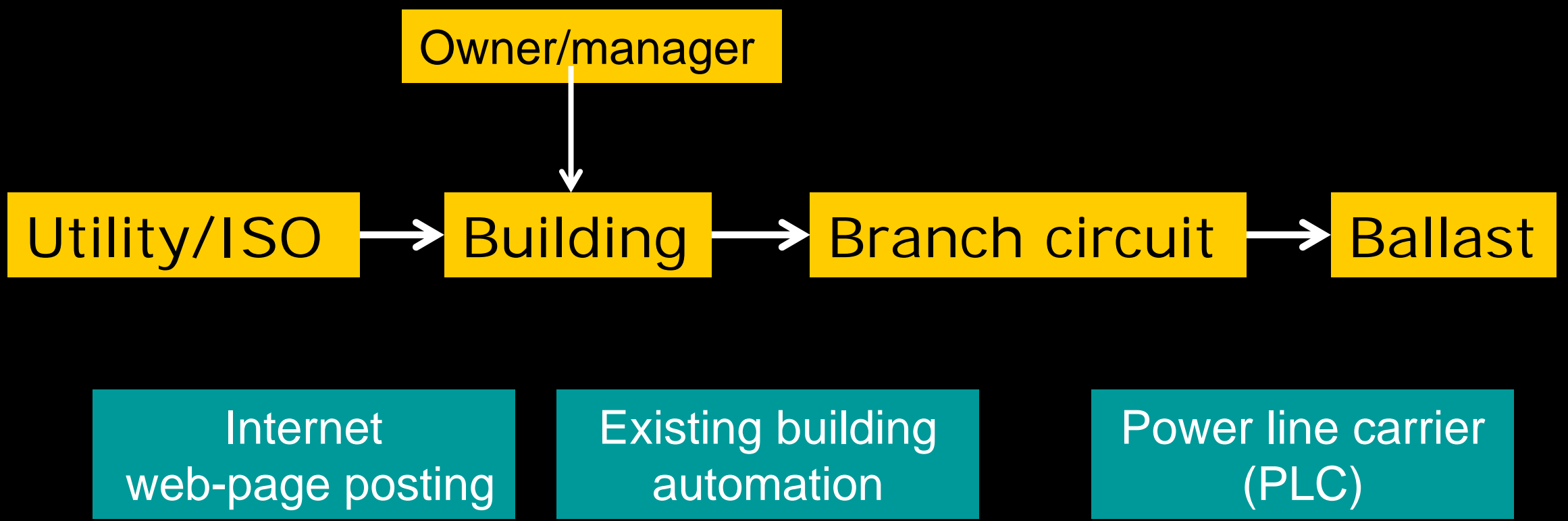
Existing building automation

\section{Power line carrier}

(PLC)

Technology 


\section{Signaling Requirements}

- One-way communication

- on/off binary signal

- Extremely low speed (1 bit per minute)

- Reliability commensurate with existing controls

- Does not conflict/interfere with existing systems 


\section{Load-shed ballast and retrofit device}

\section{Load-shed Ballast}

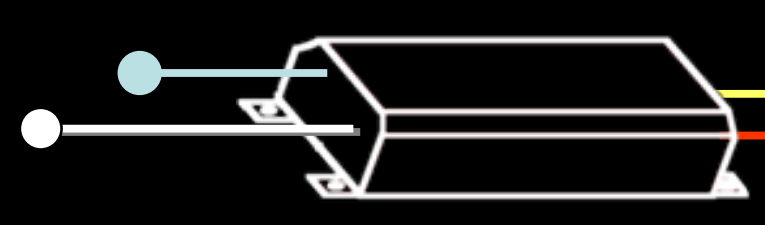

Fluorescent lamp

Power line

$+$

PLC signal
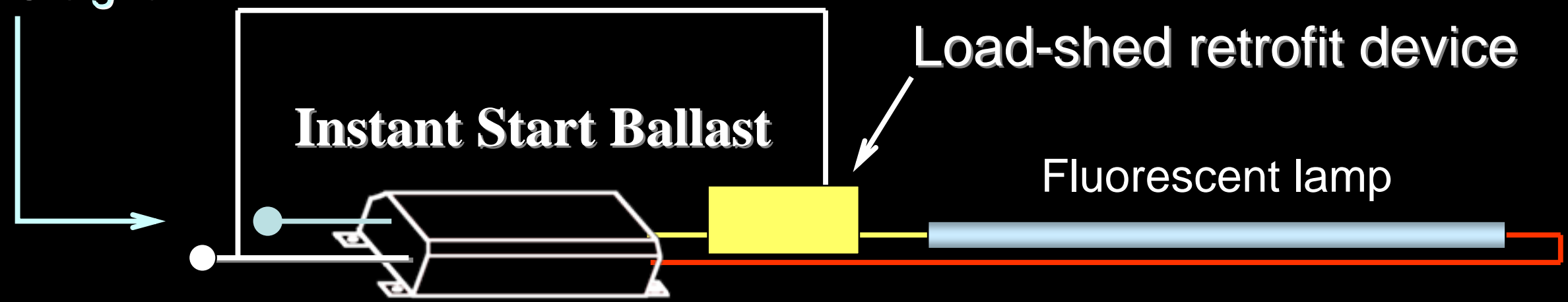


\section{Lighting Load-shedding Specification}

- LRC is working with lamp and ballast manufactures to develop a performance specification for lighting loadshedding

- Defines

- What is lighting load-shedding

- Limits on hours of use

- Dimming levels (67 and 50\% light output levels

- Method of signaling

- System architecture

- Type of PLC signal (frequency, modulation method)

- Ballast performance

- Efficiency requirements

- Power quality 
ApPendix S: PeAK loAd MANAgEMENT ALlianCE PRESENTAtion

(begins on following page) 


\section{LIGHTING AND LOAD MANAGEMENT}
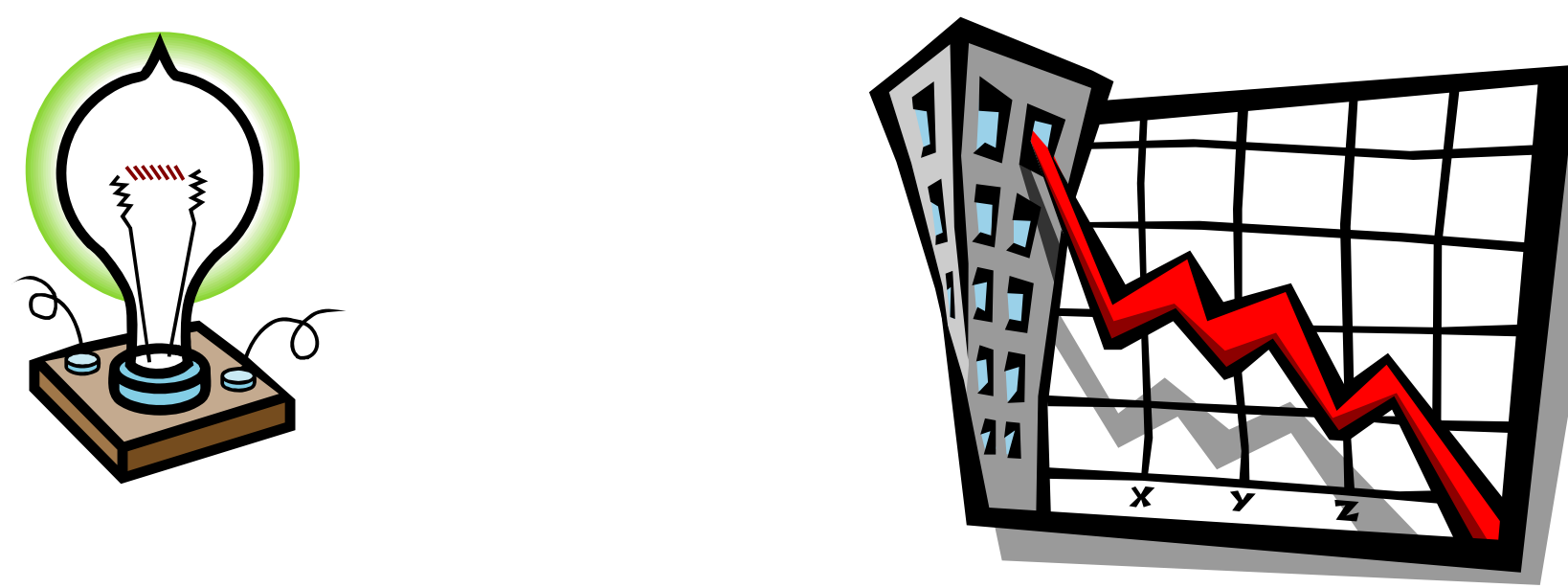

Peter Morante, Director of Energy Programs, Lighting Research Center, Troy, NY 


\section{POSITION: Lighting is an ideal electric end use for load management}

- Available in virtually every building

- Easy to control

- Easy to measure the results

- The load reduced is repeatable

- Can reduce the amount of lighting rather than turn it off, thereby preserving productivity 


\section{HOW IS LIGHTING CONTROLED TODAY}

- Manual control - turn off the light switch

- Through the building's energy management system - turn off the lights

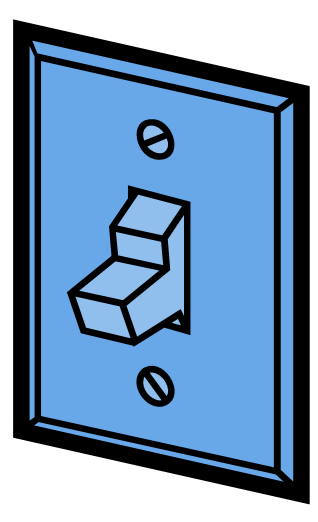

- Issues

- People aren't as productive in the dark

- Cost of maintenance personnel to manually turn on \& off lights 


\section{CAN CUSTOMERS BENEFIT FROM LOAD MANAGEMENT?}

Benefits received from utilities or system operators are less than adequate to recover costs of current lighting technologies

- Full dimming ballasts are costly

- Loss of productivity, when turning off the lights is high

- Simple paybacks exceed 10 years 


\section{SOLUTION: Develop a cost effective ballast \& controller that will:}

- Dim lights / reduce demand for power

- Retain worker productivity

- Not effect lamp life

- Control from central location, internal or external to building

- Pay for itself within 3 years 


\section{With funding from: \\ U. S. Dept. of Energy; California Energy Commission; Connecticut Light \& Power; \\ New York State Energy Research \& Development Authority \\ Lighting Research Center has developed a load shed ballast}

- Instant start ballast platform Add circuitry to reduce current to lamps by fixed amount

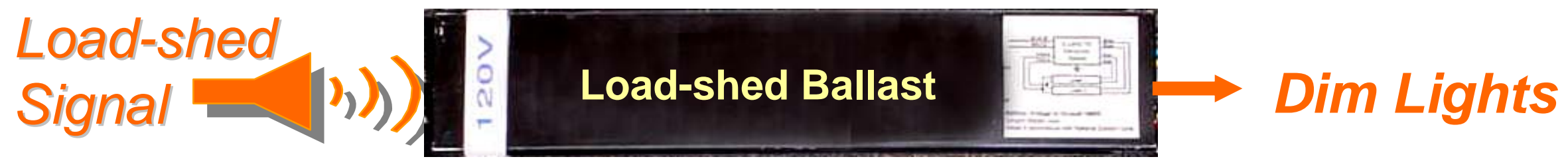

- Receiver for dimming signal

-Dimming occurs over 10 seconds

- Incremental cost of no more than \$8 


\section{CONTROLLER}

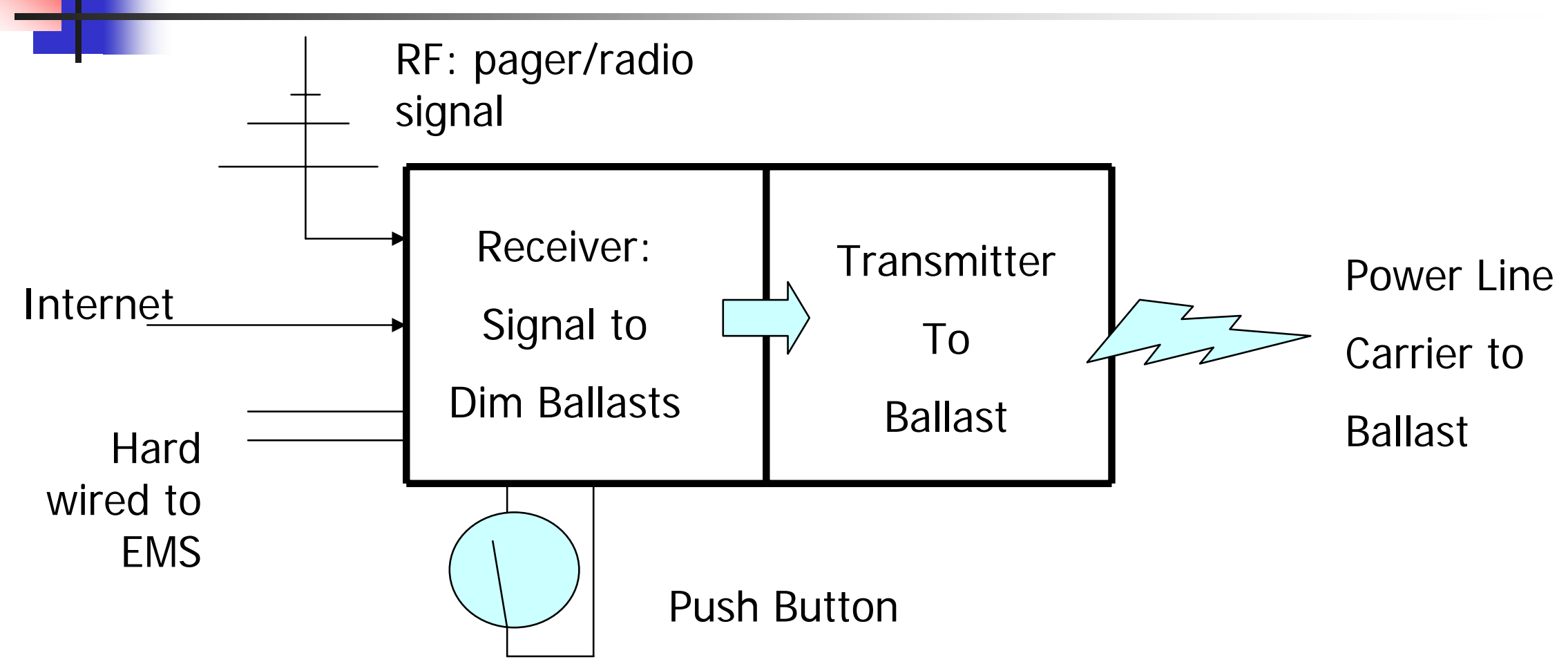

- Interchangeable circuits for how signal is received

- Minimum data transferred to ballast (on/off)

-PLC allows for no additional wiring

-Estimated cost $\$ 1$ per ballast 


\section{PAYBACK TO CUSTOMER}

- Dependent on location, utility demand charges or system operator incentives

- Cost of $\$ 9$ per ballast

Benefit

New York City

Ohio

Chicago

Georgia

Connecticut

New Jersey

California
$\$ 6 / y r$.

$\$ 4 / y r$.

$\$ 3.50 / y r$.

$\$ 2.80 / y r$.

$\$ 3 / y r$.

$\$ 2.80 / y r$.

$\$ 3.50 / y r$.
Simple Payback

$1.5 \mathrm{yrs}$.

2.25 yrs.

$2.6 \mathrm{yrs}$.

3.2 yrs.

3.0 yrs.

3.2 yrs.

$2.6 \mathrm{yrs}$. 


\section{WHERE IS IT?}

-Ballast is developed; discussions with large mfr. underway

- Controller being tested; manufacturer interested

-Full scale demonstration in NYC in 2004, Utility to ballast

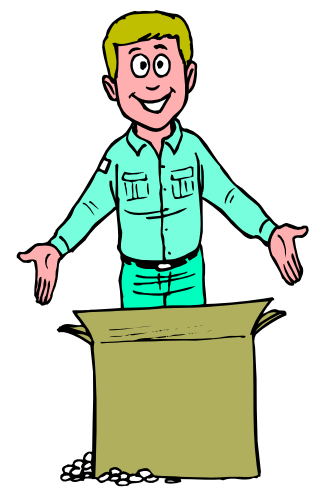

-Seeking funding for 3 additional demonstration sites

-Commercialization late 2004 
APPENDIX T: HUMAN FACTORS RESEARCH PRESENTATION, IESNA CONFERENCE

(begins on following page) 


\section{Detectability and Acceptability of Illuminance Reduction for Load Shedding}

Yukio Akashi, Ph.D.

Jason Neches, M.S.

Lighting Research Center

Rensselaer Polytechnic Institute

August 5th 2003

IESNA 2003 Conference, Chicago 


\section{AAcknowledgements}

Connecticut Light and Power sponsored this study.

Technical support

- Andrew Bierman

- Peter Boyce

- Lei Deng

- Jean-Paul Freyssinier

- Francisco Garza

- Claudia Hunter

- Russell Leslie

- Peter Morante

- Mark Rea 


\section{ZWhat is Load Shedding?}

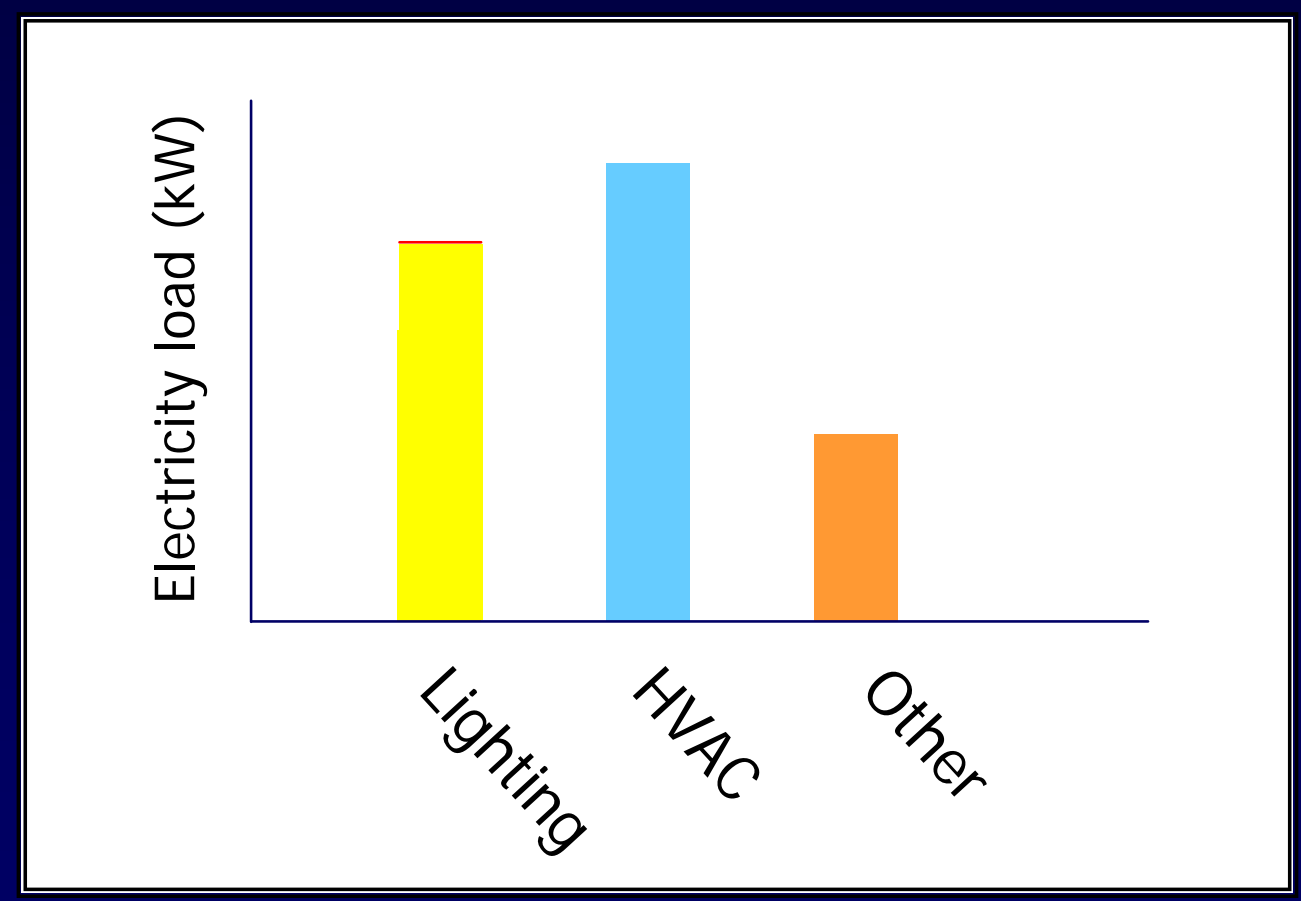




\section{Why is Load Shedding Important?}

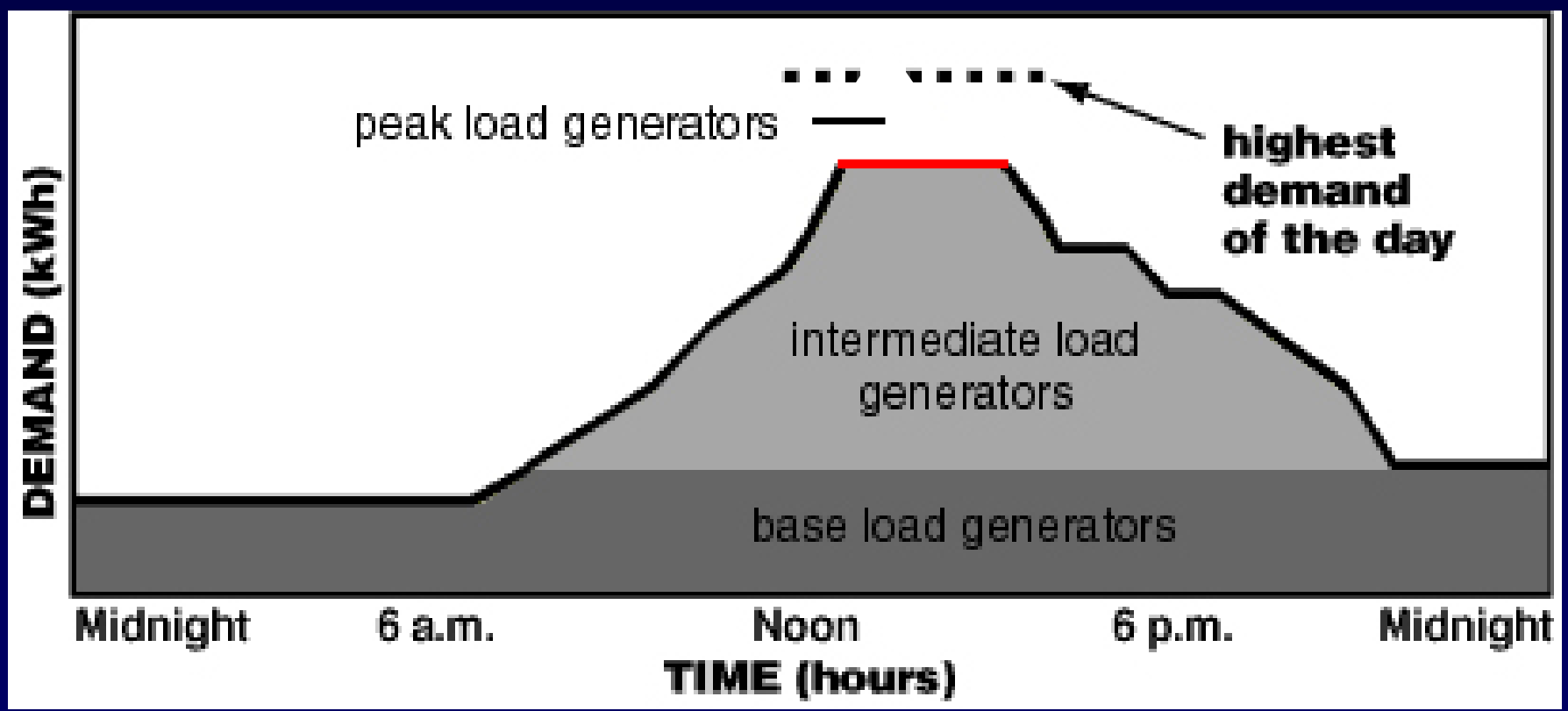




\section{Why is load shedding important?}

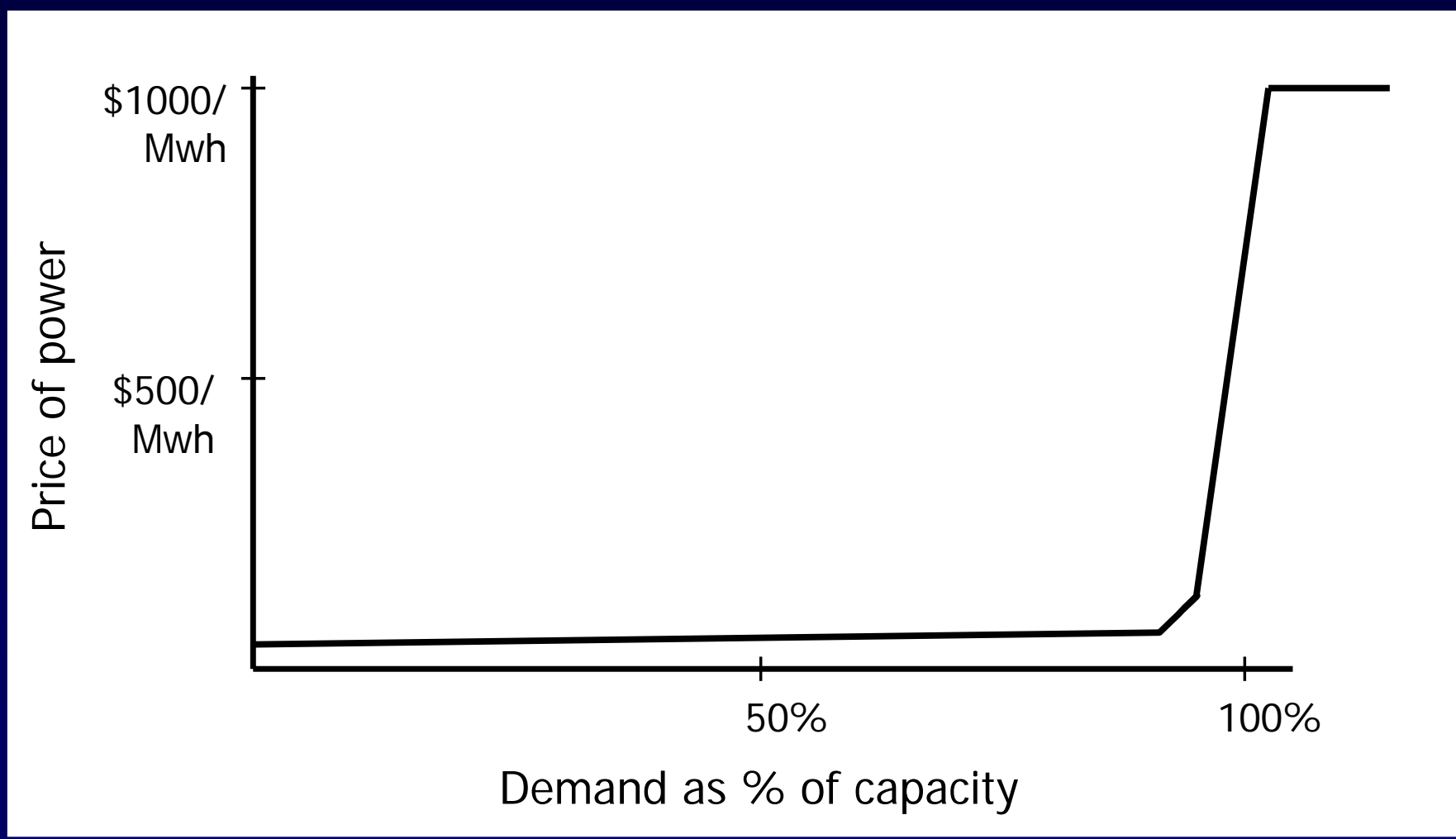

(Source: Peter Morante, Lighting Research Center, 2003) 


\section{AWhy load-shed lighting?}

- Other loads cannot be "dimmed."

- Dimmed lighting can give immediate energy savings that is predictable and repeatable.

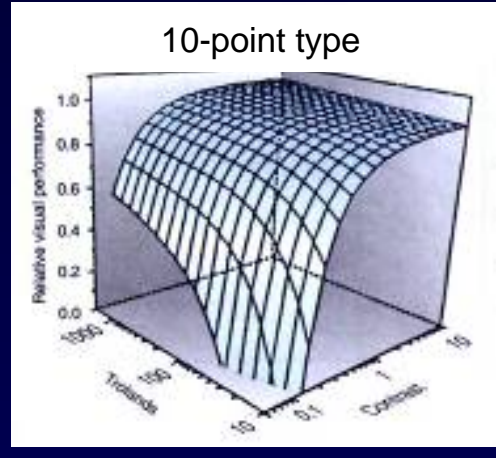

(Source: IES Handbook, 2000)

- VP studies showed that a small decrease in illuminance did not greatly affect visual performance for a task of medium to high contrast.

- How much illuminance can be reduced without being noticed? 


\section{AHow do we detect illuminance changes?}

- Detection of change in illuminance may be governed by neural detectors that sense:

- Dynamic stimuli-rate of change triggers detection (transient mechanism)

- Sustained stimuli-absolute level of change triggers detection (memory mechanism)

- Detectors may engage in "detector race"

- Depending on rate of change, either the transient or memory detector may "fire," resulting in the perception of the illuminance variation. 
Lterature survey

\section{Kally, 1961}

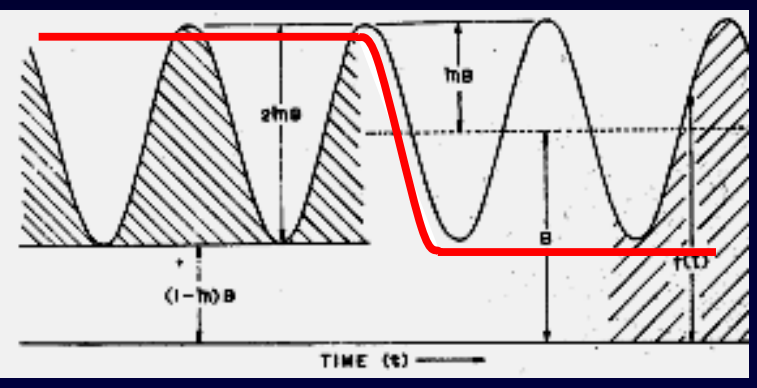

- Investigated sensitivity to sine wave "flicker" for a uniform visual field.

- Measured human sensitivity to temporal changes in light levels, but at a fairly high frequency of modulation.

- Transient mechanism

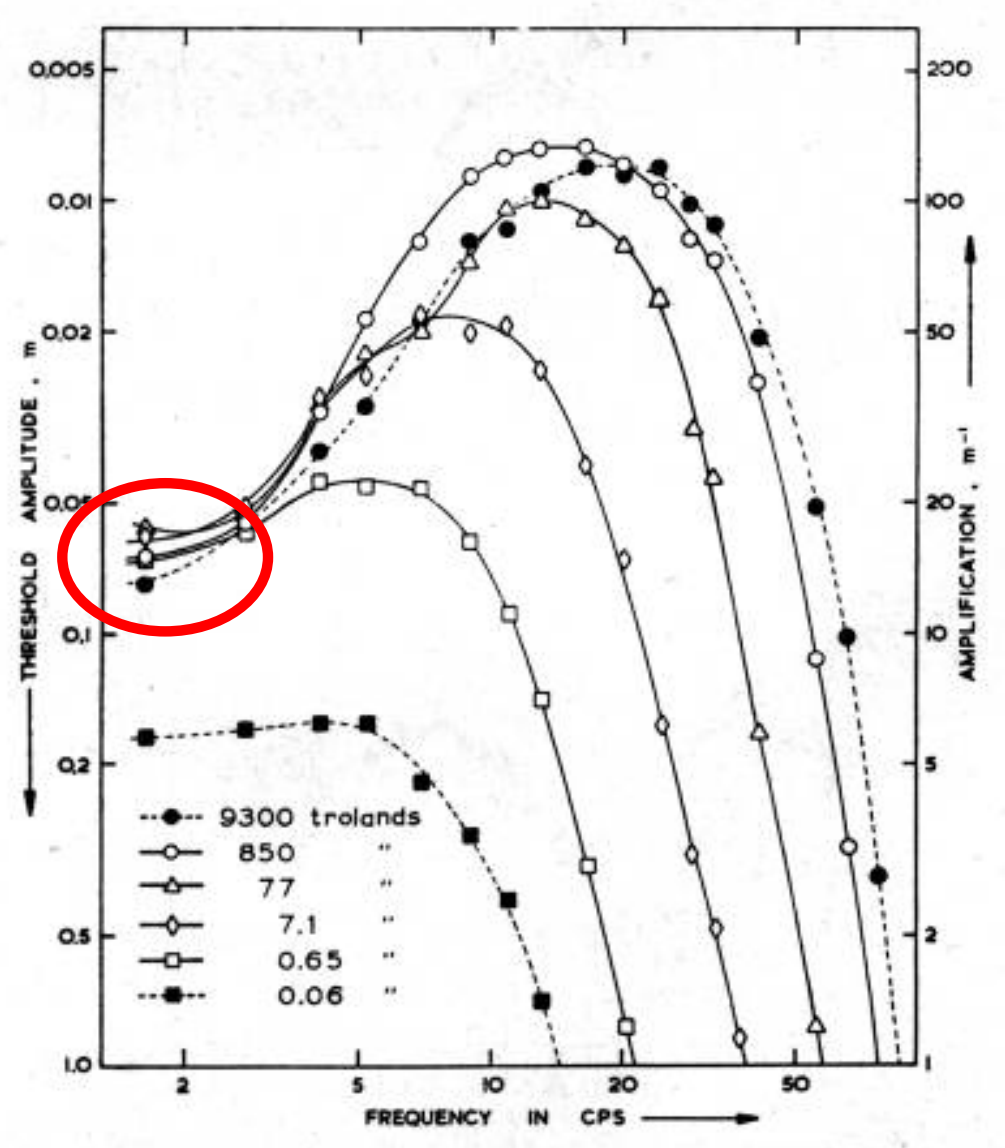




\section{Literature survey}

\section{A Kryszczuk \& Boyce, 2001 \& 2002}

- Lower frequency

- Investigated how fast illuminance could be reduced before change is noticed.

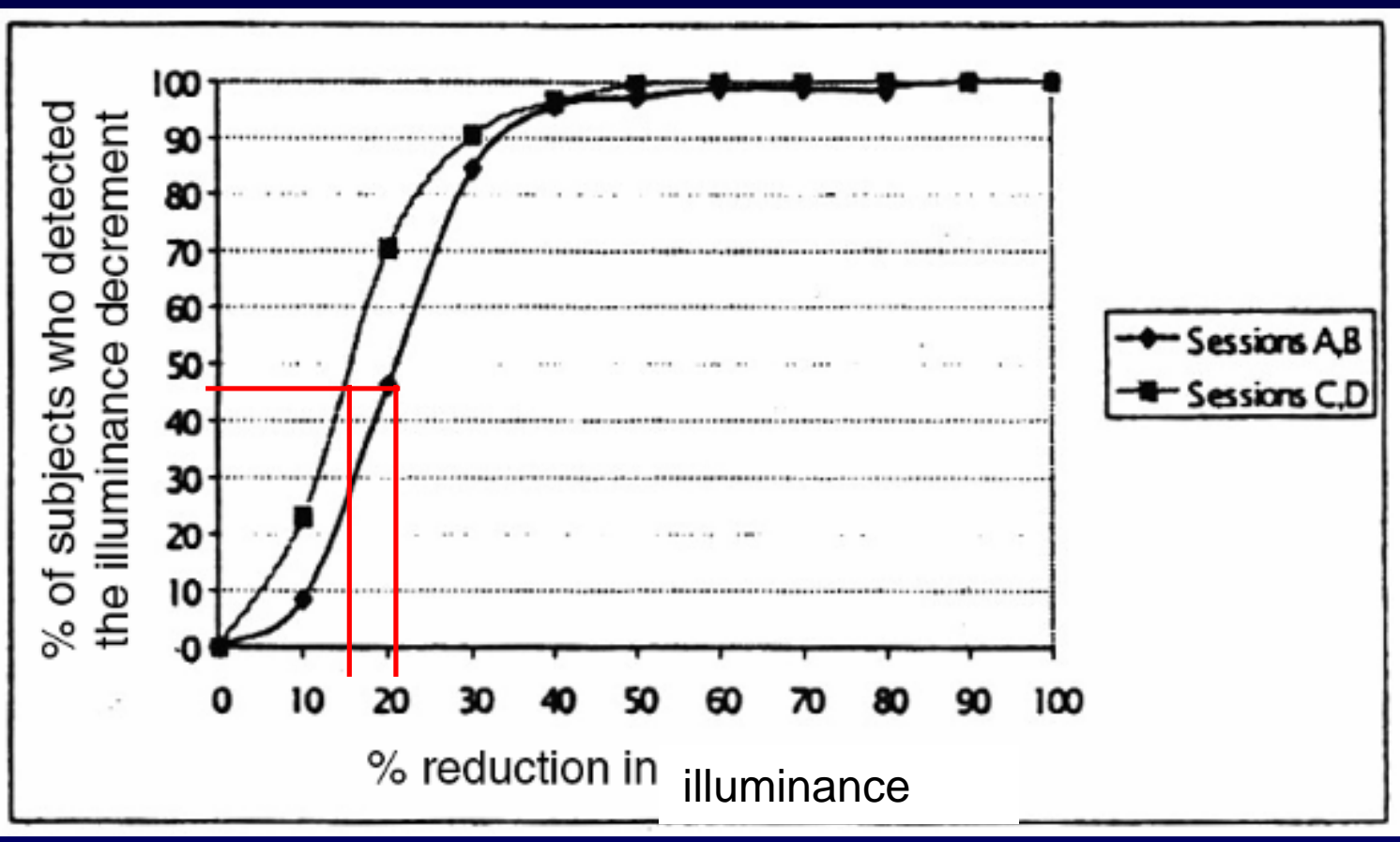

1000 Ix initial

500 Ix initial 
Lterature survey

\section{A Kryszczuk \& Boyce, 2001 \& 2002}

- Detection threshold for slow light level reduction is independent of the rate of change.

- Once the frequency of change exceeds the threshold of transient detector's response, the memory mechanism dominates.

- Found about $15-20 \%$ reduction in illuminance is undetectable regardless of the rate of change. 
Lterature survey

\section{AOther recent studies}

Shikakura et al., 2001:

- Detectability study similar to Kryszczuk's.

- Eight-sec dimming period condition confirmed Kryszczuk's study-about $20 \%$ reduction in illuminance is undetectable.

Newsham et al., 2002:

- Slower reduction $(0.5 \% / \mathrm{min})$

- Let subjects increase illuminance when the illuminance becomes too low.

- Subjects allowed illuminance to fall by $40-50 \%$ before choosing to increase the light level. 
Lterature survey

\section{APrevious studies}

Kryszczuk \& Boyce (2001); Shikakura (2001)

- Focused only on detectability and didn't include acceptability.

- Used only linear dimming functions.

Newsham (2002)

- The effect of inertia on illuminance choices was unknown.

- Used only a linear dimming function. 


\section{ZGoals of this study}

- Maximize the energy savings through load shedding,

- Identify mechanisms of illuminance change detection

- Minimize detectability of dimming process,

- Maximize acceptability of dimming process,

- Create dimming recommendations for a load shedding ballast. 


\section{AObjectives}

Memory study:

- Investigate mechanisms of detecting illuminance change-whether ones can memorize the initial illuminance and how long the memory is sustained.

Dimming curvature study:

- Identify detectable and acceptable ranges of illuminance change

- Investigate how dimming curvatures and task types affect detectability and acceptability. 
Memory study 
Memory study

\section{AExperimental conditions}

Independent Variables:

- Target illuminance (Ix): $976,781,625$,

$500,400,320,256$

- Eye closure period (s): $\quad 3,100$

Dependent Variables

- Subjective judgment: down / same / up 
Memory study

\section{AExperimental Setup}
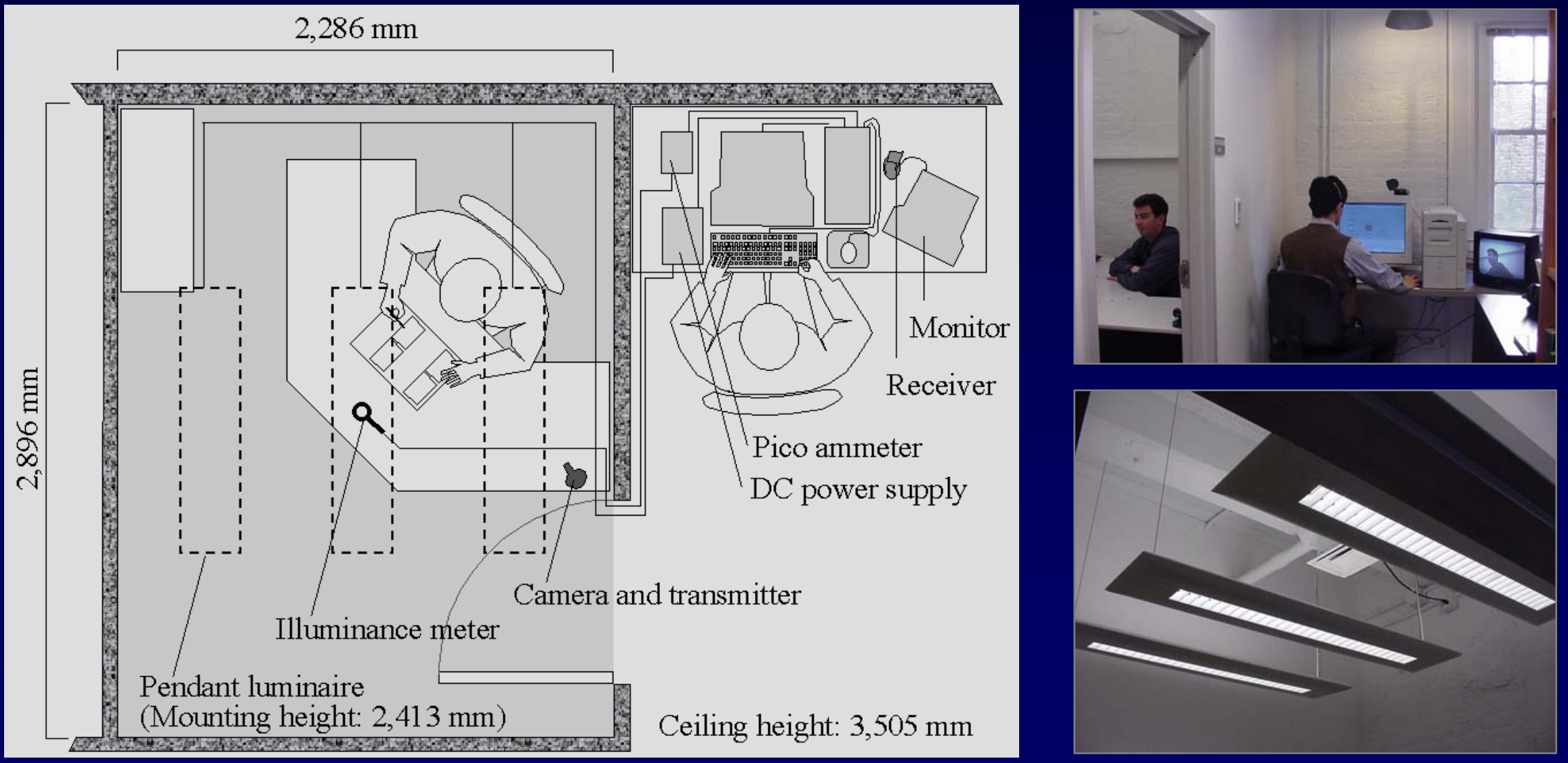
Memory study

\section{AProcedure}

- Twelve subjects, ranging from 23 to 41 in age.

- Subjects adapted to the initial illuminance of $500 \mathrm{~lx}$ for 5 minutes.

- Subjects closed their eyes and wore an eye mask for either 3 or 100 seconds.

- Subjects opened their eyes and answered whether the lighting had become brighter, darker, or remained unchanged.

- Illuminance was reset to $500 \mathrm{~lx}$, the initial level.

- Approximately 20-30 seconds separated conditions. 
Memory study

\section{AResults}

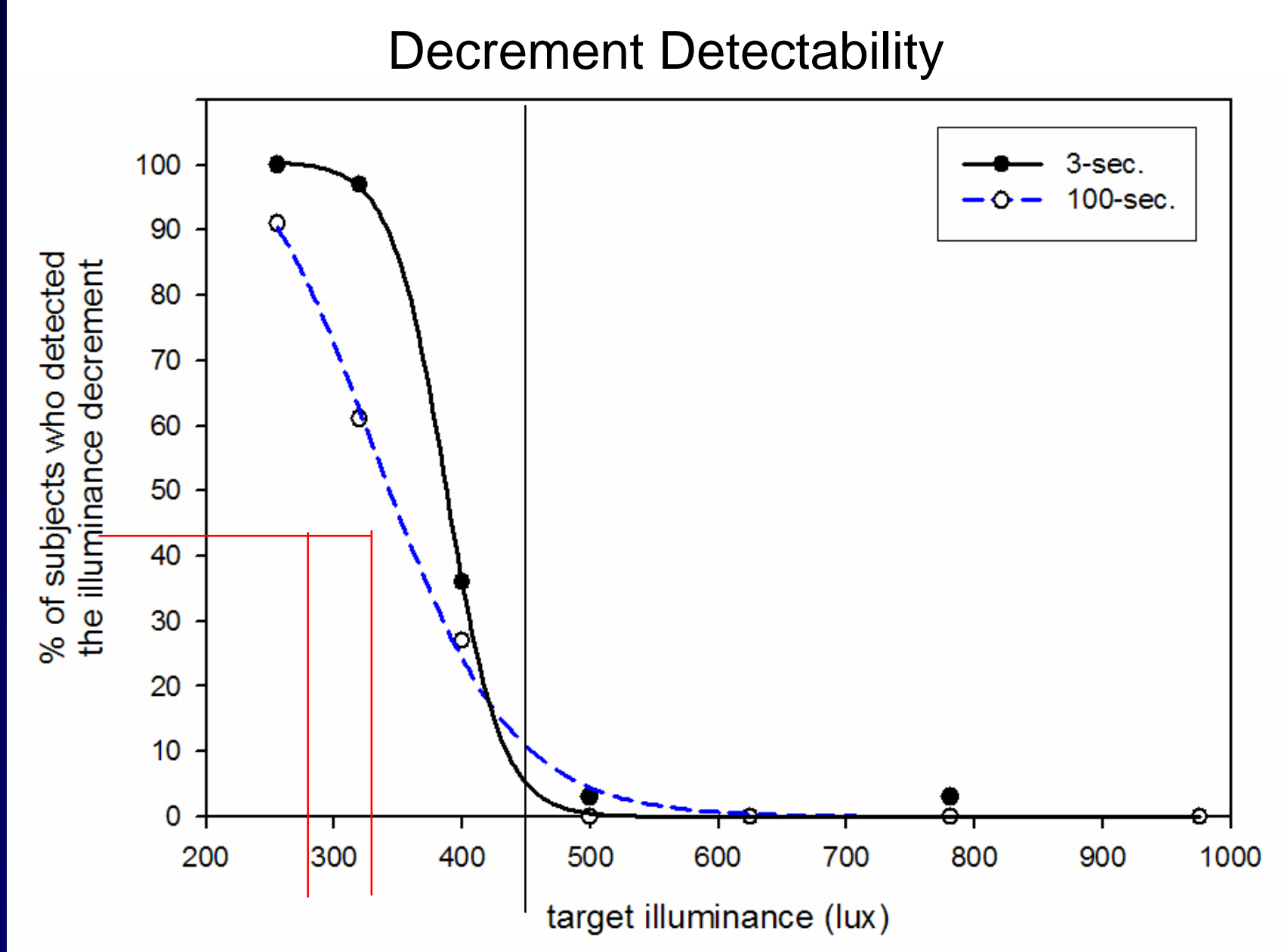




\section{ATwo-way ANOVA}

\begin{tabular}{l|c|c|c|c|c}
\hline \multicolumn{1}{c|}{$\begin{array}{c}\text { Source of } \\
\text { variation }\end{array}$} & SS & df & MS & F & P-value \\
\hline Target E & 24.79 & 6 & 4.13 & 184.20 & $0.000^{*}$ \\
\hline Period & 0.29 & 1 & 0.29 & 13.01 & $0.000^{*}$ \\
\hline Interaction & 0.5 & 6 & 0.08 & 3.71 & $0.001^{*}$ \\
\hline Within & 3.45 & 154 & 0.02 & & \\
\hline Total & 29.0 & 167 & & & \\
\hline
\end{tabular}


Memory study

AConclusions

- Proved previous study results-20\% of illuminance reduction is undetectableeven if subjects close their eyes.

- Memory of initial illuminance may be affected by longer dimming periods.

- Confound was introduced through subjects dark-adapting when closing their eyes for 100 seconds, but not after 3 seconds.

- Dark adaptation might have influenced the sensitivity of subjects to illuminance changes. 


\section{Curvature study}




\section{Curvature study}

\section{AExperimental conditions}

Independent variables

- Dimming curvature: Linear, $0.4,0.2,0.1$

- Target illuminance (Ix): $833,752,679,613,554,500$,

$451,408,368,332,300$

- Task condition: Paper task (word puzzle),

No task (free gaze)

Dependent Variables

- Detectability:

down / same / up

- Acceptability: 
Memory study

\section{AExperimental Setup}
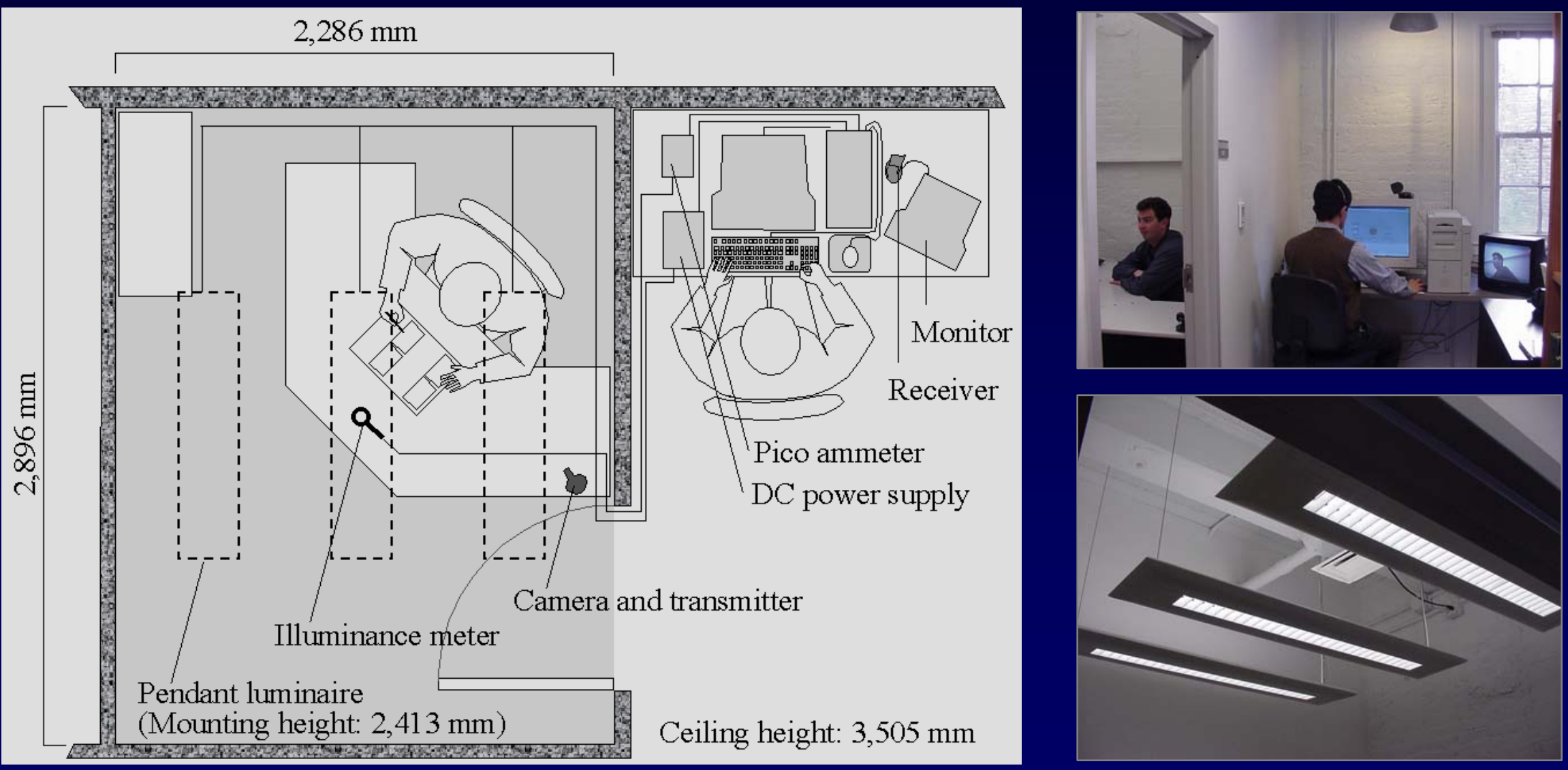
Curvature study

\section{A Dimming Curvatures (10 sec):}

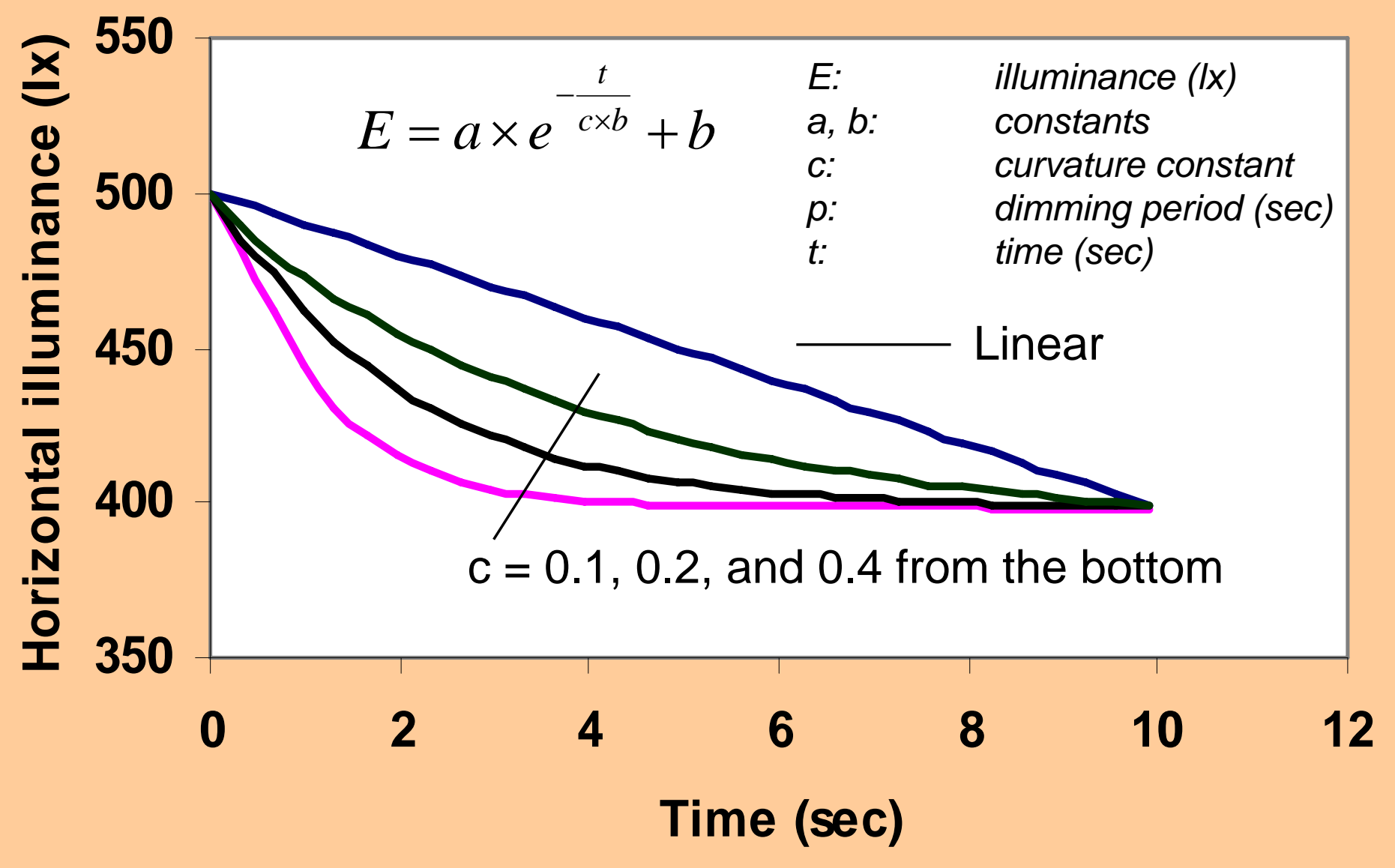




\section{Curvature study}

\section{APaper task (word puzzle)}
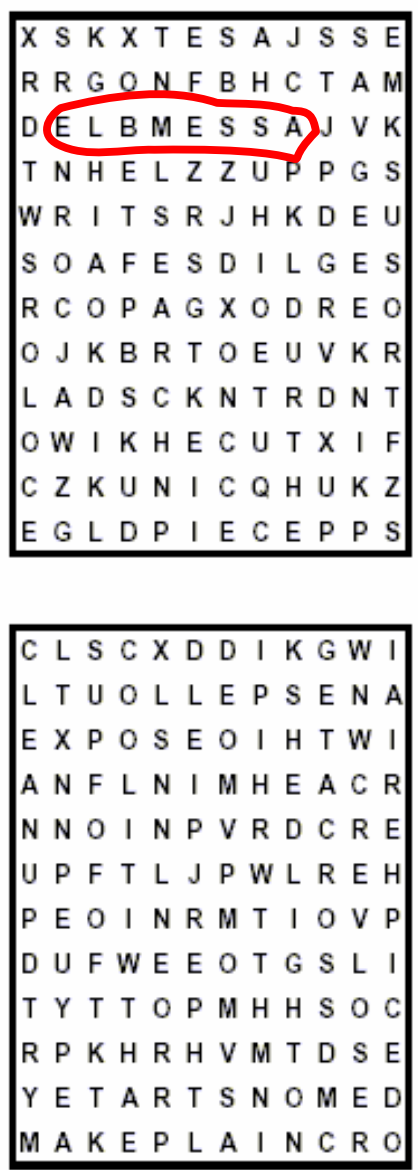

\begin{tabular}{l}
\hline SEARC \\
\hline \multicolumn{2}{|r}{ JIG } \\
ASSEMABLE \\
BOX \\
COLORS \\
CORNERS \\
CURVES \\
EDGES \\
FIND \\
HUNT \\
LOOK \\
MATCH
\end{tabular}

ARCH-A-WORD 24 JIGSAWS

COLORS

DGES

HUNT

MATCH

\section{SEARCH-A-WORD 25}

DO EXPLAIN

CLEAN UP

COMMENT ON

DECIPHER

DEFINE

DEMONSTRSTE

EXPOSE

GET ACROSS

INTERPRET

MAKE PLAIN
PART

PICTURE

PIECES

PUT TOGETHER

PUZZLE

SEARCH

SET

SHAPES

SIZE

SORT

POINT OUT

RESOLVE

SHED LIGHT ON

SHOW

SIMPLIFY

SPELL OUT

UNFOLD

\begin{tabular}{ll}
\hline \multicolumn{2}{|c|}{ SEARCH-A-WORD 26} \\
POETRY \\
ALLEGORY & METER \\
ALLITERATION & MOTIF \\
BALLAD & ODE \\
CANTO & PARALLELISI \\
ELEGY & PARODY \\
FORM & POEM \\
GENRE & REFRAIN \\
HAIKU & SONNET \\
IMAGERY & STANZA \\
IRONY & TEXT \\
LINE & TONE \\
LYRIC & VERSE \\
\hline
\end{tabular}

SEARCH-A-WORD 27 CHECKING ACCOUNT AMOUNT

BALANCE

BANK

BOOK

BOUNCE

CARD

CASH

CHARGE

CHECKS CREDIT

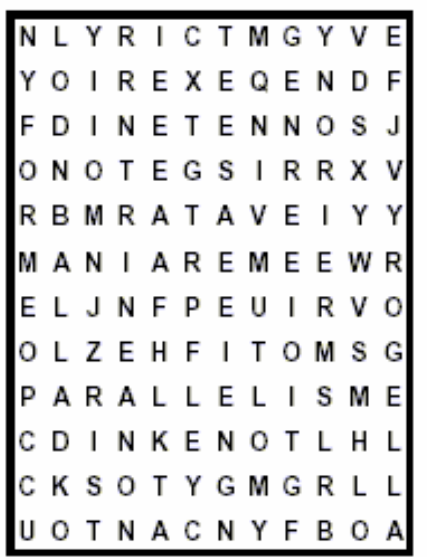

U O T N A C N Y F B O A

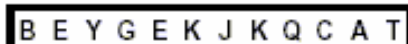

C O C Z Z T N U O C C A $A R U$ I B S Y A D O J X $S D E N V E K A B B$ B C H E E D C R T K A C K H C Z N V I E E L T H Y E A MOUNT A S I A S C ER U T A N G I S R F K T D R A I V D O G H S I S P EN D N D P P O RRE B M UN Y E N O M WE G C F E D M Q N V 


\section{Curvature study}

\section{A Procedure}

- Twenty subjects, ranging from 22 to 41 in age.

- Subjects seated themselves in the office and adapted to the initial illuminance of 500 lx for five minutes.

- Subject started performing either the paper task or free gaze under the initial illuminance.

- After a short time (5 to 10 seconds) the illuminance was or was not gradually dimmed according to one of the four dimming functions for 10 seconds.

- Illuminance was reset at 500 lx, the initial level.

- Approximately 20-30 seconds separated conditions. 


\section{Curvature study}

\section{A Procedure}

After each 10-second presentation, the experimenter asked the subject the following three questions:

1. Whether the illuminance was changed-up, down, or no change.

2. Whether the illuminance change, if the subject detected, was acceptable-yes or no.

3. How acceptable was the illuminance change, if detected- $0=$ very unacceptable, $5=$ neutral, $10=$ very acceptable 


\section{Cunvature study}

\section{AResults: detectability}
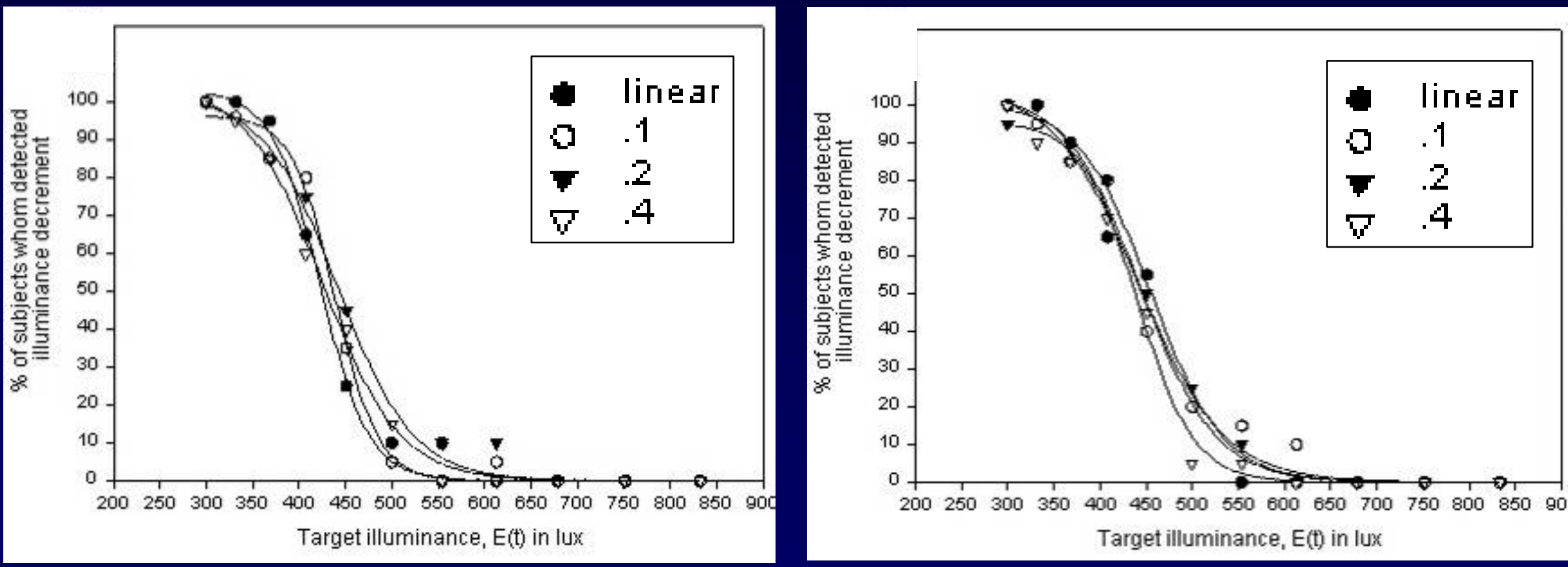

Free gaze

Paper puzzle 


\section{Cunvature study}

\section{ZAcceptability: yes or no}
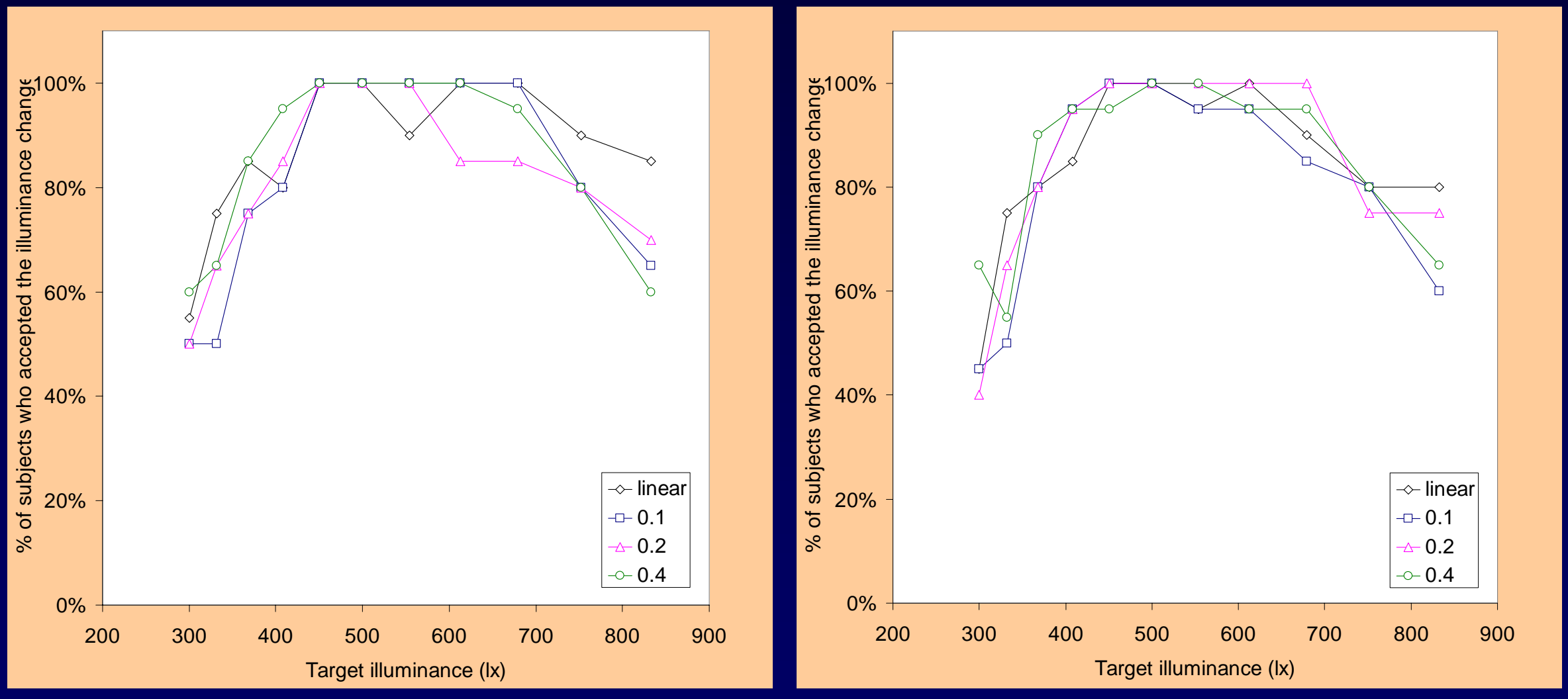

Free gaze

Paper puzzle 


\section{Cunvature study}

\section{AAcceptability: 0-10 rating}

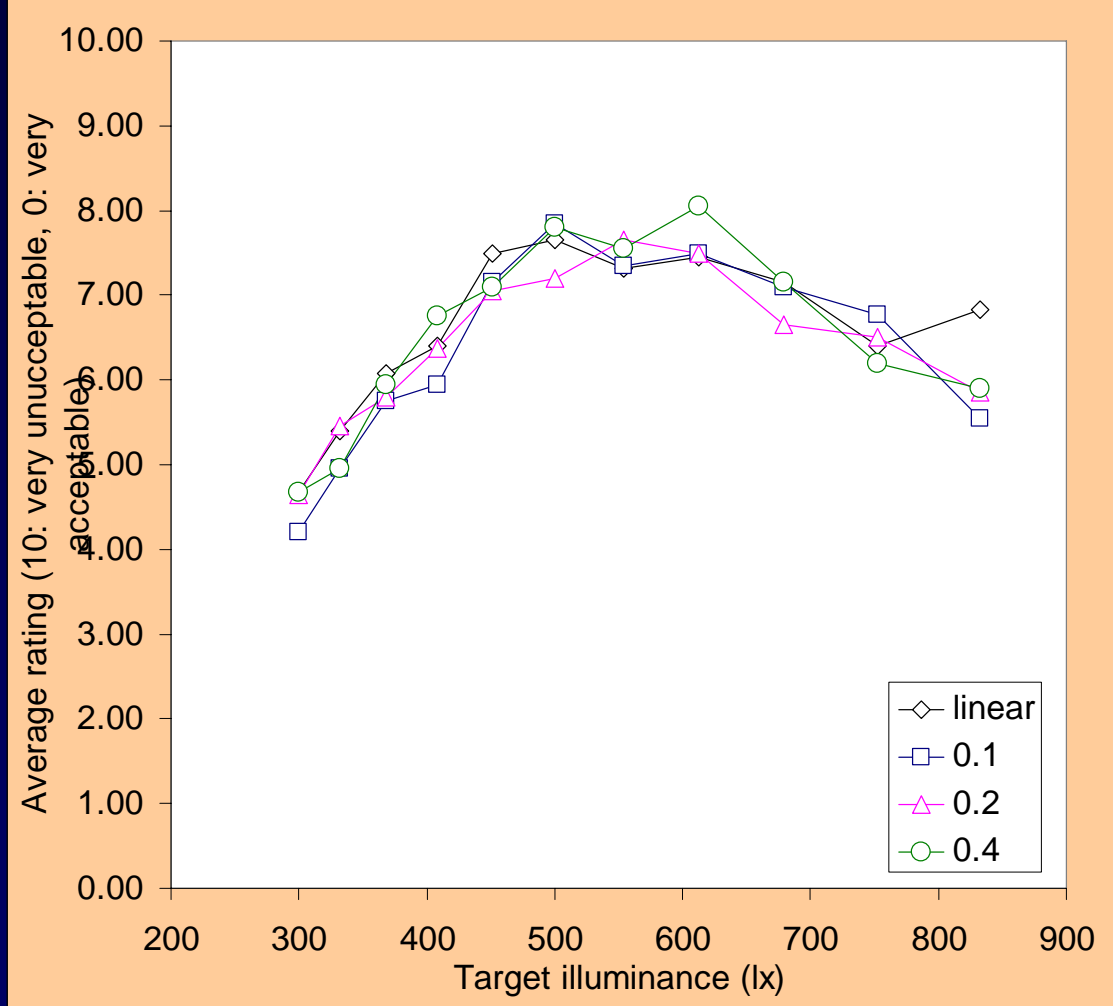

Free gaze

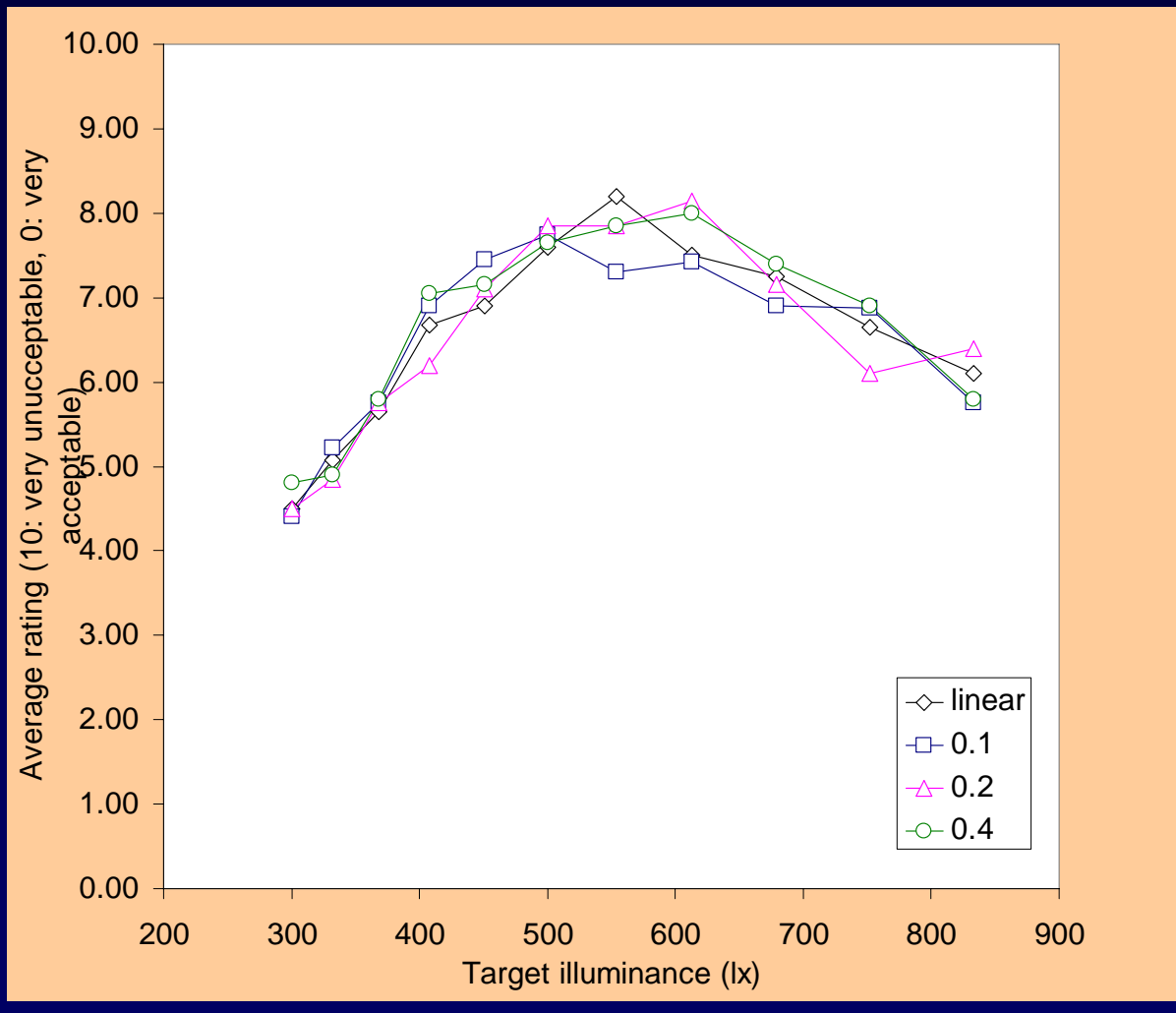

Paper puzzle 


\section{Curvature study}

\section{AAcceptability Correlation}

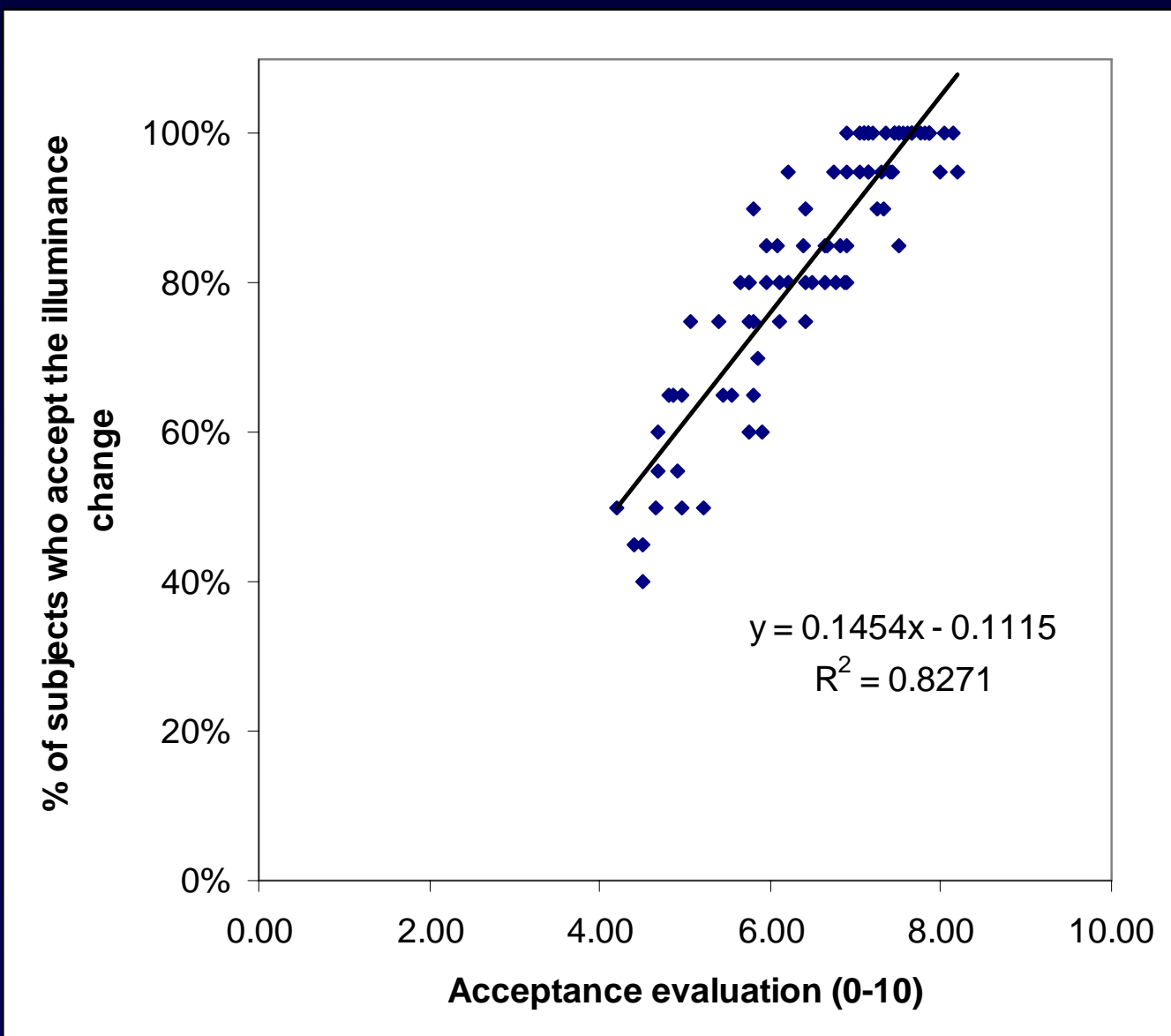


Curvature study

AConclusions

- Dimming curvature little affected the detectability and acceptability of illuminance change.

- Regardless of task, $50 \%$ of subjects could detect illuminance change after about 15\% reduction from the initial illuminance.

- Regardless of task, $50 \%$ and $80 \%$ of subjects accepted illuminance reduction up to about $40 \%$ and $20 \%$ respectively. 
Overa ll Disc ussion

\section{Zupdate to Kelly's 1961 model}

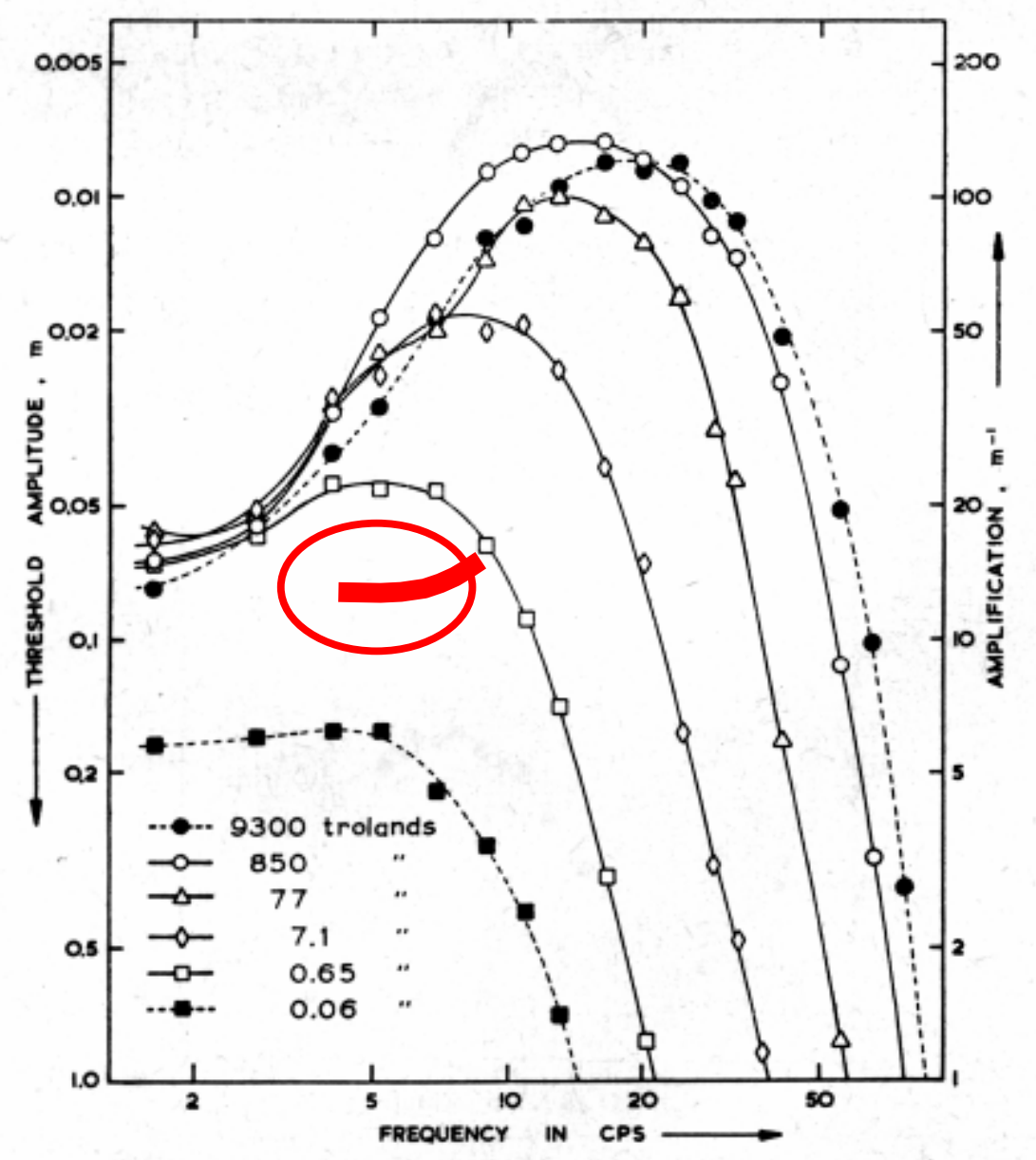




\section{Overa II Disc ussion}

\section{Z Detectability: Illuminance increase}

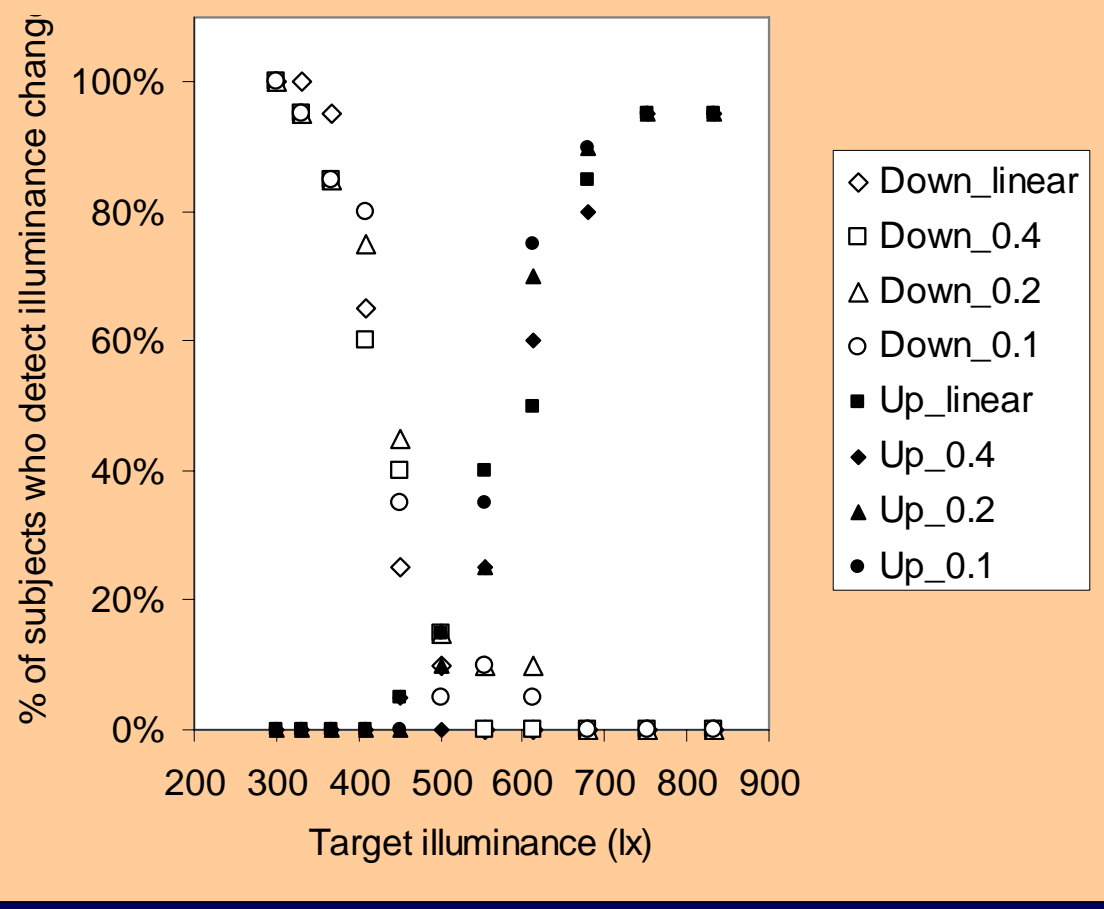

Free gaze

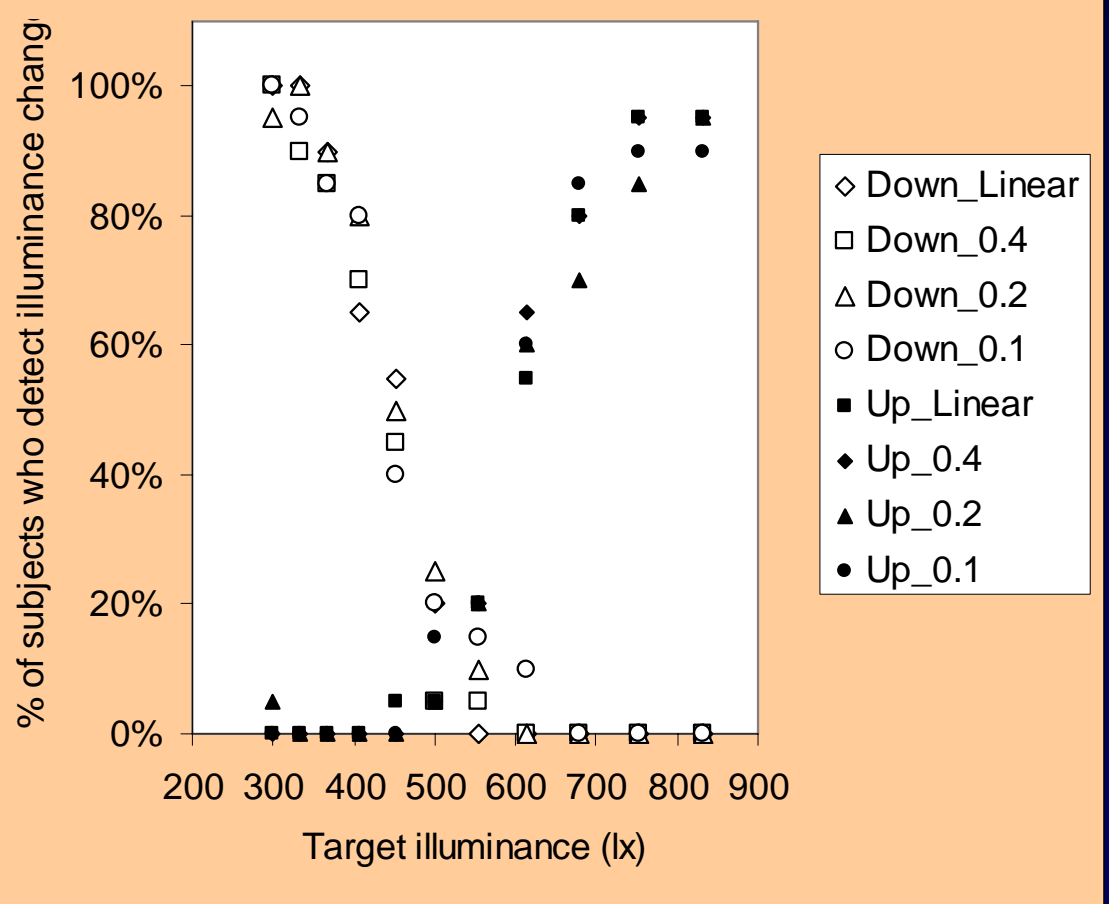

Paper puzzle 